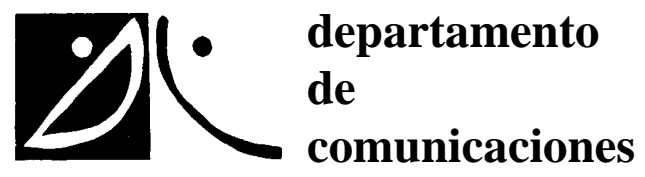

\title{
ESTUDIO DE EFECTOS NO LINEALES EN DISPOSITIVOS FOTÓNICOS Y SU APLICACIÓN EN SISTEMAS RADIO SOBRE FIBRA ÓPTICA
}

Autor: Francisco Ramos Pascual

Director: Dr. J avier Martí Sendra

DEPARTAMENTO DE COMUNICACIONES

UNIVERSIDAD POLITÉCNICA DE VALENCIA

Valencia, junio de 2000 



\section{Agradecimientos}

En primer lugar, deseo agradecerle a mi director de tesis J avier el haberme sabido transmitir desde bien temprano su afán por la investigación, sus buenos consejos y ayudas en momentos puntuales que salvan grandes obstáculos y todo lo que he aprendido trabajando a su lado. Asimismo quiero agradecer a los miembros del Grupo Radio-Fibra J osé Miguel Fuster, J uan Luis Corral, Paco Martínez y Luis Sempere todo el apoyo prestado y el buen compañerismo existente. En especial a mis colegas de trabajo y también miembros del grupo Valentín, Roberto y Amadeo por los buenos momentos pasados. Y sobre todo a mis padres por todo lo que me han apoyado y animado a lo largo de la sol itaria labor que supone la redacción de una tesis doctoral. Gracias a todos. 

A mis padres y a mi hermano 



\section{Contenido de la Tesis}

Introducción a la Tesis

1 Introducción a los sistemas de comunicaciones radio sobre fibra óptica

1.1 Introducción 13

1.2 Evolución de la red de acceso 17

1.2.1 XDSL: digitalización del bucle de abonado 20

1.2.2 Bucle local inalámbrico 24

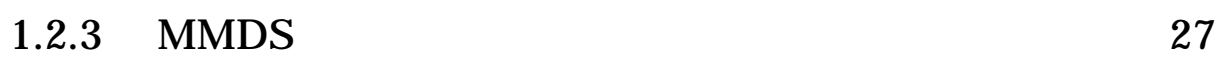

1.2.4 LMDS/MVDS 30

1.2.5 Introducción de la fibra óptica en la red de acceso 37

1.2.6 Redes HFC 40

1.3 Modelado de los sistemas ópticos analógicos 46

1.3.1 Sistemas WDM-SCM 46

1.3.2 Modulación directa frente a modulación externa $\quad 49$

1.3.3 Sistemas radio sobre fibra a frecuencias milimétricas 52

1.3.4 Medida de calidad: ruido y distorsión 56

2 Distorsión no lineal introducida por la fibra óptica 63

2.1 Introducción 63

2.2 Modelado de la fibra óptica 65 
2.2.1 Modelo lineal de la fibra 65

2.2.2 Modelo no lineal de la fibra 67

2.2.3 Split-step Fourier method 68

$\begin{array}{ll}2.3 \text { Distorsión no lineal } & 72\end{array}$

2.3.1 Distorsión no lineal producida por dispersión 73

2.3.2 Distorsión no lineal producida por efecto Kerr 76

2.3.3 Resultados de distorsión no lineal 78

2.4 Penalización de potencia inducida por la fibra óptica 84

2.4.1 Análisis teórico 84

2.4.2 Resultados experimentales 87

2.5 Margen dinámico 92

2.5.1 Cálculo de la expresión del SFDR 92

2.6 Estudio de distorsión en sistemas radio-fibra 96

2.6.1 Sistema CATV 96

2.6.2 Sistema MMDS 99

2.6.3 Sistema LMDS 102

2.6.4 Sistema MVDS 107

2.6.5 Comparación entre sistemas 110

2.7 Técnicas de compensación de dispersión 114

2.7.1 Estudio de distorsión no lineal en la modulación SSB 114

2.7.2 Breve introducción a las redes de difracción sobre fibra óptica 117

2.7.3 Comparación de las técnicas SSB y CFG 121

2.8 Conclusiones y contribuciones 128

3 Aplicación del SPM inducido por la fibra óptica para reducir los efectos de la dispersión cromática

3.1 Introducción 131

3.2 Función de transferencia de fibras ópticas monomodo 134

3.2.1 Expresión teórica de la función de transferencia de fibras ópticas dispersivas y no lineales $\quad 134$

3.2.2 Resultados teóricos de la función de transferencia 137

3.2.3 Resultados experimentales de la función de transferencia $\quad 140$

3.3 Aplicación del SPM inducido por la fibra 144

3.3.1 Modelo de chirp del SPM 144

3.3.2 Reducción del efecto de supresión de la portadora 147 
3.3.3 Limitaciones de la técnica 151

3.4 Conclusiones y contribuciones 154

4 Compensación de distorsión no lineal mediante la técnica de inversión espectral

4.1 Introducción 157

4.2 Análisis de la técnica OPC 160

4.2.1 Compensación de dispersión cromática y SPM $\quad 160$

4.2.2 Configuraciones y arquitecturas empleadas $\quad 163$

4.2.3 Generación de la señal conjugada 167

4.3 Resultados de compensación de distorsión no lineal $\quad 170$

4.3.1 Ecualización de los efectos de GVD y SPM 170

4.3.2 Compensación de distorsión no lineal en sistemas radio-fibra

4.4 Consideraciones adicionales sobre la técnica OPC $\quad 186$

4.4.1 Sensibilidad de la técnica OPC 186

4.4.2 Compensación exacta de dispersión y SPM 196

4.4.3 Influencia de la dispersión de segundo orden 205

4.5 Conclusiones y contribuciones 208

5 Construcción de conjugadores ópticos 211

5.1 Introducción 211

5.2 FWM en fibras ópticas monomodo 214

5.2.1 Origen del FWM en fibras ópticas 214

5.2.2 Ecuaciones del FWM en fibras ópticas monomodo 217

5.2.3 Influencia de la condición de fase 223

5.2.4 Influencia de otros efectos no lineales 226

5.3 FWM en amplificadores de semiconductor 237

5.3.1 Origen del FWM en SOAs 237

5.3.2 Ecuaciones del FWM en SOAs 239

5.3.3 Influencia de diferentes variables y parámetros 242

5.4 Construcción de conjugadores ópticos 247

5.4.1 Consideraciones de un conjugador óptico basado en FWM 247

5.4.2 Estudio comparativo de conjugadores basados en DSF/SOA en sistemas ópticos analógicos 
5.4.3 Compensación del efecto de supresión de la portadora empleando un conjugador óptico basado en DSF

5.5 Conclusiones y contribuciones

6 Conclusiones y líneas abiertas 269

6.1 Introducción 269

6.2 Conclusiones y contribuciones originales 270

6.3 Líneas abiertas 274

Apéndices:

I Respuesta en frecuencia de los sistemas de comunicaciones ópticas analógicos 277

I.1 Introducción 277

I.2 Función de transferencia de la fibra óptica 279

I.2.1 Análisis teórico de la propagación a través de la fibra 279

1.2.2 Funciones de conversión y cálculo de la función de transferencia de la fibra 281

I.3 Influencia del chirp del transmisor óptico 287

I.3.1 Modelado del chirp 287

I.3.2 Respuesta de la fibra óptica en presencia de chirp 293

I.4 Modulación óptica en banda lateral única 298

I.4.1 Generación de la modulación SSB empleando un MZM dual-drive

I.4.2 Modelado y respuesta de la fibra con modulación SSB

I.5 Inversión espectral 305

I.5.1 Modelado del conjugador de fase 305

1.5.2 Respuesta en frecuencia de sistemas con inversión espectral 306

I.5.3 Influencia del chirp en sistemas con inversión espectral

I.6 Fibras de dispersión desplazada 312

I.6.1 Análisis teórico de la propagación a través de DSFs 
II Funciones de Bessel de orden imaginario puro

II.1 Introducción

II.2 Funciones de Bessel modificadas de orden imaginario

II.2.1 Ecuación diferencial de Bessel modificada

II.2.2 Soluciones de la ecuación de Bessel modificada de orden imaginario puro

II.2.3 Propiedades de las funciones de Bessel modificadas

III Publicaciones internacionales del autor

III.1 Publicaciones relacionadas con la Tesis

III.2 Otras publicaciones del autor 326

III.2.1 Publicaciones en revista internacional 326

III.2.2 Actas de congresos internacionales

327

Bibliografía de la Tesis 


\section{Introducción a la Tesis}

\section{MARCO DE LA TESIS}

Esta Tesis se ha desarrollado íntegramente en el seno del Grupo de Sistemas Radio-Fibra de la Universidad Politécnica de Valencia, dentro de una línea de investigación dedicada al estudio de efectos no lineales en dispositivos fotónicos. El trabajo presentado a continuación resume una labor de investigación de más de tres años que comenzó gracias a una beca de Formación de Personal Investigador de la Conselleria de Cultura, Educació i Ciència de la Generalitat Valenciana.

Durante los dos primeros años, el autor trabajó conjuntamente en varios proyectos de investigación directamente relacionados con los sistemas radio sobre fibra óptica. Uno de ellosi, subvencionado por la Comisión Interministerial de Ciencia y Tecnología (CICYT), consistía en el desarrollo de una plataforma para la transmisión de señales de alta velocidad empleando las técnicas de multiplexación en longitud de onda y de

i proyecto TIC96-0611 
subportadoras eléctricas multiplexadas, lo que se conoce habitualmente como WDM-SCMii. Por otra parte, en otro proyecto de investigación se construyó un demostrador de sistema de distribución punto a multipunto a $28 \mathrm{GHz}^{i i i}$ para la transmisión de señales de vídeo y datos. Asimismo, se propusieron y demostraron técnicas de alimentación óptica de redes LMDS remotas.

En marzo de 1999, el autor se incorporó al Departamento de Comunicaciones de la Universidad Politécnica de Valencia, donde actualmente imparte la asignatura de Radiocomunicaciones. Desde esa fecha, ha continuado desempeñando su labor investigadora en la búsqueda de nuevas aplicaciones de las no linealidades de los dispositivos fotónicos en sistemas ópticos analógicos.

\section{MOTIVACIÓN DE LA TESIS}

La introducción de los nuevos servicios multimedia en el entorno residencial o empresarial requiere de una gran cantidad de ancho de banda que debe ser soportado por la red de acceso. EI principal candidato para permitir estos servicios es, sin lugar a dudas, la fibra óptica. Sin embargo, los operadores se encuentran con un alto factor de riesgo: deben realizar un gran desembolso económico para montar toda una infraestructura de red que llegue a cada uno de los abonados. Además, dado el actual nivel de competencia que existe en la red de acceso del usuario, lo que cualquier operador desea es comenzar a dar servicio lo más rápidamente posible. En este caso, la única solución es optar por los sistemas de acceso vía radio.

Ahora bien, la creciente demanda de servicios de banda estrecha en los actuales sistemas radio ha reducido las posibilidades de alojar en el espectro radioeléctrico la gran capacidad que demandan estos sistemas inalámbricos de banda ancha. Para contrarrestar esta limitación, se está considerando la

\footnotetext{
ii wavelength division multiplexing-subcarrier multiplexing

iii sistemas denominados LMDS
} 
utilización de enlaces radio a frecuencias milimétricas $(26-100 \mathrm{GHz})$ para aplicaciones en sistemas micro/picocelulares de banda ancha, sistemas fijos de distribución punto a multipunto o redes de área local inalámbricas [Zys96, Hon97, Bit99]. La complejidad de estos enlaces radio puede simplificarse compartiendo los equipos transmisores y receptores de milimétricas entre los distintos canales radio empleando la técnica de subportadoras multiplexadas (SCM) [Ols89]. Si además las señales radio se transportan sobre la fibra a frecuencias milimétricas, todavía se simplifican más las estaciones base, dado que el equipamiento de éstas se reduce a componentes optoelectrónicos y amplificadores eléctricos, sin necesidad de equipos conversores de frecuencia. Una posible desventaja sería, sin embargo, el coste asociado al fotodiodo de banda ancha.

De este modo, entregando las señales directamente desde la estación de control y a través de la fibra, se evita la necesidad de generar la portadora de alta frecuencia en la estación base, el cual suele ser además un emplazamiento de difícil acceso. Luego la principal ventaja de estos sistemas es su capacidad para concentrar la mayoría del equipamiento de alta frecuencia más costoso en un lugar centralizado (estación de control), permitiendo que el equipamiento de la estación base sea simple, de pequeño tamaño y con un bajo consumo. Además, el operador no necesita desplegar una enorme infraestructura de fibra óptica, sino que esta se limita a unos cuantos enlaces troncales entre el centro de control y las diferentes estaciones base.

Surgen así los sistemas de comunicaciones radio sobre fibra óptica, caracterizados por combinar dispositivos de radiofrecuencia con enlaces de fibra óptica [Coo90, Wak93]. Básicamente, los sistemas radio sobre fibra se aprovechan de la fibra óptica para transportar señales directamente hasta un punto de radiación remoto (estación base). Algunas de las aplicaciones de estos sistemas en las actuales redes de acceso son:

- Aplicaciones satélite: redes VSAT (Very Small ApertureTerminals)

- Sistemas inalámbricos de banda ancha punto a multipunto: MMDS (Multichannel Multipoint Distribution System), LMDS (Local 
Multipoint Distribution System) o MVDS (Multipoint Video Distribution System)

- Sistemas celulares: GSM/DECT, MBS (Mobile Broadband System)

- Aplicaciones telemáticas: redes de área local inalámbricas

- Aplicaciones comerciales o militares

Ahora bien, la gran mayoría de dispositivos fotónicos empleados en estos sistemas de comunicaciones ópticas se comportan de forma no lineal ante niveles de potencia óptica elevados [Agr87, Agr89, Chr90]. Por ejemplo, potencias ópticas del orden de $+10 \mathrm{dBm}$ a la entrada de fibras ópticas o de amplificadores ópticos de semiconductor son suficientes para inducir efectos no lineales apreciables.

Básicamente, los procesos no lineales que ocurren en los dispositivos fotónicos se clasifican atendiendo a su naturaleza y características. En particular, los principales efectos no lineales que han sido estudiados hasta el momento son:

Automodulación de fase (self-phase modulation, SPM): se manifiesta como una modulación de fase de la envolvente de la señal óptica inducida por la modulación de intensidad de la propia señal. Está presente en fibras ópticas $y$ en amplificadores de semiconductor y es especialmente perjudicial en sistemas MI-DDiv cuando se combina con la dispersión cromática de la fibra [Agr89, Ste90].

Modulación de fase cruzada (cross-phase modulation, XPM): idéntico al anterior, salvo que en este caso la modulación de fase se induce como consecuencia de la modulación de intensidad de una portadora óptica vecina. Es el doble de eficiente que el SPM, aunque sólo aparece en sistemas ópticos multicanal como WDM [Agr89, Car98b, Phi99].

Mezclado de cuatro ondas (four-wave mixing, FWM): este proceso no lineal de tercer orden da lugar a la aparición de nuevas frecuencias ópticas del tipo

iv modulación de intensidad/detección directa 
$f_{1}+f_{2}-f_{3}$ a la salida del dispositivo. La conversión se produce de forma eficiente en regiones de dispersión nula, como por ejemplo en amplificadores ópticos de semiconductor o en fibras de dispersión desplazada operando en tercera ventana, encontrando gran número de aplicaciones como la construcción de conjugadores ópticos o convertidores de longitud de onda en redes WDM [Shi87, Agr88, Agr89, Ino92].

Scattering estimulado de Brillouin (SBS): Ia potencia óptica se refleja en el interior de las fibras como consecuencia del proceso no lineal de SBS, el cual genera una onda de Stokes desplazada respecto a la frecuencia de la onda incidente en una cantidad que depende del medio no lineal. Este proceso se ve influenciado por el ancho de línea de la portadora óptica, y limita la potencia que puede aplicarse a la entrada de la fibra óptica [I pp72, Aok88, Agr89].

Scattering estimulado de Raman (SRS): similar al anterior, pero en este caso el scattering es en ambas direcciones y necesita mayores niveles de potencia para ser eficiente. Limita las prestaciones de los sistemas ópticos multicanal debido a que la energía de un canal se transfiere a los canales vecinos [Agr89, Phi99].

Modulación de ganancia cruzada (cross-gain modulation, XGM): este proceso no lineal surge en amplificadores ópticos de semiconductor, y se manifiesta en una modulación dinámica de la ganancia del amplificador inducida por la modulación de intensidad de una señal que satura el dispositivo. Su principal aplicación se ha destinado a la elaboración de convertidores de longitud de onda, al igual que el FWM [Agr89, Agr97].

Todos estos procesos no lineales han sido estudiados en detalle en el contexto de los sistemas ópticos digitales [Chr90, Ian96], donde la señal de información digital modula directamente un láser, o externamente un modulador Mach-Zehnder o de electroabsorción. No obstante, menor atención se ha prestado a la mayoría de ellos en sistemas de comunicaciones ópticas analógicos, salvo en el caso del SPM [Phi91, Des94, Wil96, Gop96] o SBS [Mao92, Wil94, Zha97]. Adicionalmente, todos los estudios se han 
enfocado a analizar principalmente las degradaciones que producen estos efectos no lineales [Yos93, Wan95, Phi99], sin entrar a valorar sus posibles aplicaciones. Hasta el momento, no se conocen aplicaciones de las no linealidades en sistemas radio sobre fibra salvo contadas excepciones [Shi96, Fre97, Yao98, Yao98b, Kho99]. Así pues, existe un campo importante de investigación que puede aportar resultados de interés en el área de los sistemas de comunicaciones ópticas analógicos.

\section{OBJETIVOS DE LA TESIS}

El principal objetivo de esta Tesis, como ya se ha esbozado anteriormente, es realizar un completo estudio de algunos efectos no lineales que ocurren en determinados dispositivos fotónicos en el contexto de los sistemas radio sobre fibra óptica. Y todo ello en busca de posibles aplicaciones que pueden tener estos efectos como compensación de dispersión cromática, generación de subportadoras de milimétricas, o incluso compensación de la degradación introducida por efectos no lineales a partir de las no linealidades de los dispositivos. Así, en base a este objetivo se han desarrollado las líneas de trabajo que se enumeran a continuación:

\section{1) Elaboración de un completo modelo, tanto teórico como de simulación, de la propagación de señales de RF a través de fibras ópticas dispersivas y no lineales.}

La propagación de señales a través de fibras ópticas monomodo dispersivas y no lineales se rige por la ecuación de Schrödinger no lineal [Agr89]. Esta ecuación no tiene una solución cerrada salvo para situaciones muy concretas, como es en el contexto de los solitones [Dod82]. Sin embargo, estas soluciones sólo tienen aplicación en el caso de transmisión digital y no sirven para modelar la propagación de señales de radiofrecuencia (RF) a través de la fibra.

Por este motivo, se ha particularizado el estudio para el caso de modulación en pequeña señal [Wan92, Car98], y se ha llegado así a expresiones cerradas 
que puedan modelar la respuesta de fibras ópticas en sistemas con modulación analógica, incluyendo otros efectos como pueden ser el chirp de frecuencia introducido por los transmisores ópticos [Koy88, Sri95]. En especial, el estudio se ha extendido desde frecuencias de microondas hasta frecuencias milimétricas $\left(\mathrm{f}_{\mathrm{m}}>18 \mathrm{GHz}\right)$.

En el caso de simulación, se ha empleado el algoritmo conocido como splitstep Fourier method [Har73, Agr89]. Este al goritmo se ha aplicado con éxito para modelar la propagación de pulsos a través de fibras ópticas y, al mismo tiempo, ya se ha demostrado su validez en los sistemas SCM [Des94, Mar97, Ram98a].

En esta línea de investigación se ha realizado una publicación [Ram00] donde se presenta una expresión teórica para caracterizar la función de transferencia de fibras ópticas monomodo dispersivas y no lineales. Esta expresión, basada en funciones de Bessel de orden imaginario puro, es válida tanto para modulación óptica en doble banda lateral como en banda lateral única, e incluye además los efectos del chirp del transmisor óptico y las pérdidas de la fibra.

\section{2) Estudio de la distorsión no lineal introducida por la fibra óptica en sistemas radio-fibra a frecuencias milimétricas.}

El efecto conjunto de la dispersión cromática y del SPM da lugar a la aparición de distorsión no lineal a la salida del fotodetector en sistemas multicanal radio sobre fibra óptica [Yon91, Ber91, Cro94, Des94]. Esta distorsión no lineal genera productos de intermodulación que degradan la calidad del sistema. Los parámetros que se utilizan para medir esta degradación son el CSO (composite second-order) y el CTB (composite triple beat), definidos como la relación entre las potencias de los términos de distorsión de segundo y tercer orden, respectivamente, y la potencia de señal útil.

La influencia del SPM en la distorsión no lineal se ha modelado con anterioridad [Des94] en el caso de sistemas CATV. Además, existen algunos 
trabajos que presentan resultados experimentales [Wil96, Gop96]. No obstante, este análisis no se ha extendido a otro tipo de sistemas como pueden ser los sistemas MMDS, LMDS o MVDS. Estos sistemas tienen gran aplicación para la difusión de vídeo y datos, dada la congestión que existe actualmente en el espectro radioeléctrico. Como aportación, se pretende estudiar la influencia que tiene la fibra óptica como medio de transporte en arquitecturas donde la señal multicanal a frecuencias milimétricas se distribuye hasta un nodo remoto, y después se convierte al dominio eléctrico para poder ser radiada por medio de una antena (alimentación de antenas remotas).

En esta línea de investigación se ha realizado una publicación [Ram99a], donde se comparan la técnica de modulación óptica en banda lateral única (singlesideband, SSB) y el uso de redes de difracción sobre fibra óptica (chirped fiber gratings, CFGs) en relación con la compensación de distorsión no lineal introducida por la dispersión de la fibra en sistemas ópticos multicanal LMDS.

\section{3) Compensación de la distorsión no lineal introducida por la fibra óptica empleando la técnica de inversión espectral.}

Para compensar la distorsión no lineal introducida por la fibra óptica en sistemas multicanal radio sobre fibra se propone la técnica de inversión espectral en el dominio óptico. Esta técnica se ha aplicado con éxito en sistemas ópticos digitales [Wat93, Wat94, Zha95], pero no ha recibido demasiada atención en el caso de los sistemas analógicos, salvo algunos trabajos realizados recientemente por Kitayama y Sotobayashi [Kit99, Sot99, Sot99a]. Debemos indicar que esta Tesis ha dado lugar, por primera vez, a publicaciones donde se propone la técnica OPC ${ }^{v}$ para compensar la distorsión no lineal introducida por la fibra en sistemas SCM [Mar97, Ram98a].

\footnotetext{
${ }^{\vee}$ optical-phase conjugation, en lengua inglesa
} 
El elemento clave utilizado en la técnica de inversión espectral es el conjugador óptico, también conocido como OPC (optical-phase conjugator), y que se sitúa generalmente en mitad del enlace de fibra. Su función principal es la de invertir la fase de la señal óptica, lo cual se realiza por medio de procesos paramétricos no lineales. Bajo ciertas condiciones, la distorsión introducida en el primer trayecto de fibra se compensa mediante la propagación por el segundo tramo de fibra colocado tras el bloque OPC [Wat96].

A diferencia de otras técnicas de compensación de dispersión, como pueden ser las fibras compensadoras de dispersión [Ven93] o las redes de difracción sobre fibra [Mar97a], esta técnica también compensa la dispersión en presencia de SPM. Además, no requiere de complejos circuitos de ajuste de la fase o de estabilización de la polarización del modulador como otras técnicas, ya que es independiente de la modulación utilizada. Otra de sus ventajas es su reducida sensibilidad frente a variaciones de la longitud del enlace de fibra, lo cual permite situar el bloque OPC en un lugar común y compartirlo con multitud de usuarios. De este modo, se consigue eliminar la duplicación de recursos, puesto que el OPC es un dispositivo relativamente costoso.

Los resultados que se han obtenido son relativos a la compensación de CSO, CTB y penalización de potencia en sistemas ópticos analógicos: CATV, MMDS, LMDS y MVDS. Además, también se ha analizado de forma teórica la influencia que tienen distintos parámetros de la técnica como longitudes de fibra o potencias ópticas en los resultados de ecualización.

\section{4) Estudio del proceso no lineal de mezclado de cuatro ondas para la elaboración de conjugadores ópticos.}

Dado que el conjugador óptico es el el emento clave en la técnica de inversión espectral, interesa que esté bien caracterizado. Este elemento se construye normalmente a partir de FWM en una configuración de fibra de dispersión desplazada (dispersion-shifted fiber, DSF) [Wu94, Zha96] o de amplificador óptico de semiconductor (semiconductor optical amplifier, SOA) [Duc96, 
Shi96]. En especial, en esta Tesis se pretende analizar en profundidad el fenómeno de FWM en ambas configuraciones dentro del contexto de las señales analógicas. En particular, se evalua cuál de las dos posibles configuraciones, SOA/DSF, es más ventajosa en el caso de los sistemas radio sobre fibra desde diversos puntos de vista: pérdida de inserción, ancho de banda de conversión, distorsión no lineal, ruido, etc.

En esta línea de investigación se han publicado resultados experimentales de compensación del efecto de supresión de la portadora empleando un conjugador basado en DSF y construido en el laboratorio [Ram99].

\section{5) Aplicación del SPM para reducir los efectos de la dispersión cromática de la fibra.}

Los sistemas radio sobre fibra óptica es interesante que operen en tercera ventana (1550 nm) debido a la reducida atenuación de la fibra óptica a esta longitud de onda y a la existencia de amplificadores ópticos de fibra dopada con erbio. No obstante, la dispersión cromática de la fibra limita el producto ancho de banda-distancia como consecuencia del fenómeno de supresión de la portadora [Sch95]. En particular, se propone el SPM como una solución para reducir este efecto perjudicial. Mediante el SPM, se induce un chirp de frecuencia de signo negativo que permite alejar los nulos de transmisión de la respuesta de la fibra hacia frecuencias mayores, de forma similar a como se realiza en [Smi97] empleando el chirp de un modulador Mach-Zehnder. Este efecto ha sido demostrado experimentalmente y ha dado lugar a la publicación [Ram98]. Adicionalmente, se demuestra la transmisión de señales de RF tolerantes a la dispersión de la fibra empleando la técnica SPM. 


\section{ESTRUCTURA DE LA TESIS}

Para alcanzar los objetivos planteados en la sección anterior, la Tesis se estructura en 5 capítulos que se describen a continuación brevemente.

El capítulo 1 supone una introducción a los sistemas radio sobre fibra óptica, donde se analiza la evolución que han sufrido las redes de acceso para acomodar los nuevos servicios de banda ancha, se estudiarán diferentes arquitecturas y técnicas utilizadas en los sistemas ópticos analógicos y, finalmente, se introducen diferentes parámetros utilizados para evaluar la calidad de estos sistemas. En especial, se realiza un completo estudio de los sistemas fotónicos que operan a frecuencias milimétricas.

La problemática de la distorsión no lineal introducida por la fibra óptica se estudia en el capítulo 2. En particular, se presentan resultados relativos a distorsión no lineal, penalización de potencia y margen dinámico para diversos sistemas ópticos multicanal: CATV, MMDS, LMDS y MVDS. Finalmente, se realiza un análisis comparativo entre dos técnicas tolerantes a la dispersión cromática, la modulación óptica SSB y las CFGs, donde se evalúa la capacidad de cada una de ellas para compensar la distorsión producida por la dispersión de la fibra.

El capítulo 3 trata la primera de las aplicaciones de los efectos no lineales en sistemas radio sobre fibra. En el mismo se demuestra la aplicación del SPM inducido por la fibra óptica para reducir el efecto de supresión de la portadora y, de este modo, extender el alcance del sistema. Se presentan medidas experimentales de la función de transferencia de fibras ópticas monomodo dispersivas y no lineales para distintos val ores de potencia óptica y se calcula una fórmula teórica cerrada para caracterizarla. Por último, se demuestra experimentalmente la transmisión tolerante a la dispersión empleando la técnica SPM.

La técnica de inversión espectral, propuesta para compensar la distorsión no lineal y la penalización de potencia introducidas por la fibra en sistemas 
ópticos multicanal, se estudia en detalle en el capítulo 4. En este capítulo se obtienen resultados de ecualización en sistemas multicanal radio-fibra y se realiza un completo modelado de la técnica OPC en sistemas ópticos analógicos. En especial, se estudia la sensibilidad de la técnica frente a diversos parámetros del sistema. El dispositivo conjugador se supone ideal, dejando para el capítulo siguiente su análisis detallado.

El capítulo 5 aborda el estudio del proceso no lineal de mezclado de cuatro ondas en fibras de dispersión desplazada y amplificadores de semiconductor. Y todo ello como aplicación en la elaboración de conjugadores ópticos utilizados por la técnica OPC en sistemas analógicos. De este modo, se realiza un análisis comparativo entre las configuraciones DSF/SOA utilizando parámetros de calidad típicos de estos sistemas: linealidad, CNR, etc. Por último, se demuestra experimentalmente la compensación del efecto de supresión de la portadora empleando un conjugador óptico basado en FWM en una DSF.

Adicionalmente, se incluyen dos apéndices para complementar los contenidos presentados en los diferentes capítulos. En el primero de ellos se modela en detalle la respuesta en frecuencia de sistemas ópticos analógicos, mientras que en el segundo se introducen algunas definiciones y propiedades de las funciones de Bessel de orden imaginario puro. 


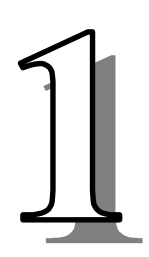

\section{Introducción a los sistemas de comunicaciones radio sobre fibra óptica}

\subsection{INTRODUCCIÓN}

Los sistemas de comunicaciones radio sobre fibra óptica, caracterizados por combinar dispositivos de radiofrecuencia (RF) con enlaces de fibra óptica, encuentran cada vez más aplicaciones en las actuales redes de telecomunicación [Coo90, Ahm97, Noe97, Bra98]. El futuro parece dirigirse hacia comunicaciones inalámbricas con una mayor movilidad, al tiempo que los operadores intentan, cada vez más, acercar su infraestructura de fibra óptica hacia los usuarios. En este sentido, los sistemas radio sobre fibra proporcionan la sinergia adecuada entre la óptica y la radio, permitiendo la fusión entre estos dos tipos de tecnología tan dispares [Wak93, Wil95 (cap. 8)]. 
Básicamente, los sistemas radio sobre fibra se aprovechan de la fibra óptica para transportar señal es directamente hasta un punto de radiación remoto: estación base. Dependiendo de la aplicación considerada, las señales radio pueden ser VHF, UHF, microondas o incluso ondas milimétricas. En cuanto a la fibra óptica, éste es el medio de transmisión ideal por varias razones. En primer lugar, sus bajas pérdidas (inferiores a 0,25 dB/km para una longitud de onda de $1550 \mathrm{~nm}$ ) unido a la existencia de amplificadores ópticos (por ejemplo, de fibra dopada con erbio') permite alcanzar grandes distancias. En segundo lugar, su inmunidad frente a las interferencias electromagnéticas. $Y$ en tercer lugar, su gran ancho de banda de transmisión únicamente limitado por la dispersión cromática.

La creciente demanda de servicios de banda estrecha en los actuales sistemas radio ha reducido las posibilidades de alojar en el espectro radioeléctrico la gran capacidad que demandan los sistemas inalámbricos de banda ancha. Para contrarrestar esta limitación, se está considerando la utilización de enlaces radio a frecuencias milimétricas $(26-100 \mathrm{GHz})$ para aplicaciones en sistemas micro/picocelulares de banda ancha, sistemas fijos de acceso inalámbrico o redes de área local inalámbricas [Zys96, Hon97]. La complejidad de estos enlaces radio puede simplificarse compartiendo los equipos transmisores y receptores de milimétricas entre los distintos canales radio empleando la técnica SCM (subcarrier multiplexing) [OIs89].

En estas redes inalámbricas, la elevada atenuación atmosférica a frecuencias milimétricas permite desarrollar arquitecturas celulares con mejoras en el reúso de frecuencias y, por lo tanto, una gestión espectral más sencilla. No obstante, dado que el área de cobertura es inferior, el requisito de un mayor número de estaciones base obliga a que éstas sean funcionalmente simples en su diseño. Si las estaciones base se conectan a una estación de control, las funciones de enrutamiento y procesado estarán centralizadas, simplificando de este modo el equipamiento de las estaciones base. EI medio ideal para realizar estas conexiones es la fibra óptica por las tres razones anteriormente enumeradas. Si además las señales radio se

\footnotetext{
' EDFA (erbium doped fiber amplifier)
} 
transportan sobre la fibra a frecuencias milimétricas, todavía se simplifican más las estaciones base, dado que el equipamiento de éstas se reduce a componentes optoelectrónicos y amplificadores eléctricos, sin necesidad de equipos conversores de frecuencia. De este modo, entregando las señales directamente desde la estación de control y a través de la fibra, se evita la necesidad de generar la portadora de alta frecuencia en la estación base, que puede tener además un emplazamiento de difícil acceso.

En cualquier sistema de comunicaciones, cuestiones prácticas como pueden ser el tamaño, peso, fiabilidad, coste o consumo de potencia del equipamiento remoto son de vital importancia. La principal ventaja de los sistemas radio sobre fibra es su capacidad para concentrar la mayoría del equipamiento de alta frecuencia más costoso en un lugar centralizado (estación de control), permitiendo que el equipamiento de la estación base sea simple, de pequeño tamaño y con un bajo consumo. Así pues, estos y otros motivos hacen que estos sistemas encuentren gran número de aplicaciones en las actuales redes de acceso. En la figura 1.1 se representa en un diagrama de bloques simplificado cuál sería la estructura del sistema radio sobre fibra.

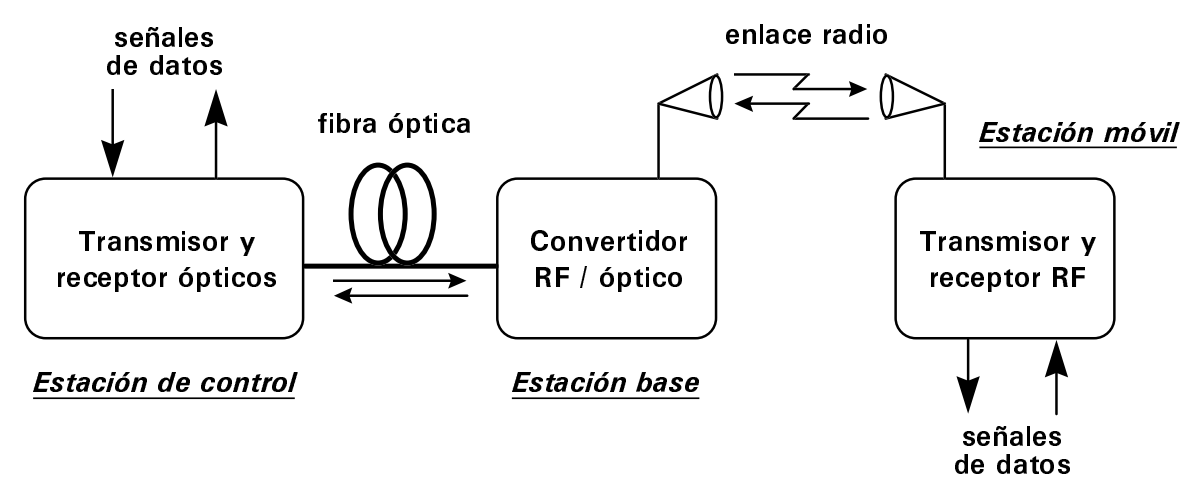

Figura 1.1 Configuración de un sistema de comunicaciones radio sobre fibra óptica.

Adicionalmente, la técnica de multiplexación por longitud de ondaii permite simplificar la arquitectura de red y mejorar la implantación de estaciones

\footnotetext{
ii WDM (wavel ength-division multiplexing)
} 
base, ya que es posible establecer comunicaciones dúplex con varias estaciones base empleando una única fibra [Smi98]. Además, el enrutamiento puede realizarse en el dominio óptico a partir de la longitud de onda de las señales, lo cual proporciona gran flexibilidad.

El objetivo principal de este capítulo es proporcionar una visión general de los sistemas radio sobre fibra óptica, ya que la tesis se desarrolla en el marco de este tipo de sistemas. La sección 1.2 comienza analizando la evolución que han sufrido las redes de acceso para acomodar los servicios de banda ancha que demandan los usuarios. Aquí se estudian diferentes tipos de sistemas, como por ejemplo XDSL, bucle local inalámbrico, MMDS, LMDS o MVDS. En especial, se estudia la forma de implantar la fibra óptica en estas redes de acceso, llegándose a arquitecturas con distintos niveles de penetración de la fibra: FTTC, FTTB o FTTH. Un caso particular lo constituyen las redes HFC de distribución de señales CATV [Way99], caracterizadas por combinar enlaces de fibra óptica con trayectos de cable coaxial. Por otro lado, en la sección 1.3 se aborda el modelado de los sistemas de comunicaciones ópticas analógicos, los cuales engloban a los sistemas radio sobre fibra. En esta sección se estudian los sistemas híbridos WDM-SCM, junto con las dos posibilidades que existen para inyectar la señal de RF en la fibra óptica: modulación directa/externa. También se trata en detalle el caso particular de sistemas radio sobre fibra que operan a frecuencias milimétricas, donde se analizarán diferentes técnicas para generar la subportadora de milimétricas. En particular, una de ellas se basa en la conversión PM-IM que se produce en dispositivos dispersivos como son las redes de difracción sobre fibra óptica [Mar99]. Por último, se definirán expresiones relativas al cálculo de ruido y distorsión, dos parámetros fundamentales para determinar la calidad de los sistemas ópticos analógicos [Cox97]. 


\subsection{EVOLUCIÓN DE LA RED DE ACCESO}

La red de acceso constituye la parte de la red de telecomunicaciones en contacto directo con los usuarios. Por ello, se convierte en elemento clave para la implantación de servicios de banda ancha. Recientemente, se ha experimentado una creciente demanda de servicios tales como acceso a Internet, TV por cable, TV interactiva, vídeo bajo demanda,

TABLA I Tasas de transmisión de varios servicios de banda ancha [H on97].

\begin{tabular}{|l|c|c|}
\hline $\begin{array}{l}\text { Servicio digital de } \\
\text { banda ancha }\end{array}$ & $\begin{array}{c}\text { ancho de banda } \\
\text { descendente }\end{array}$ & $\begin{array}{c}\text { ancho de banda } \\
\text { ascendente }\end{array}$ \\
\hline $\begin{array}{l}\text { Difusión de vídeo } \\
\text { Difusión de TV } \\
\text { Pago por visión } \\
\text { mejorado }\end{array}$ & 1,5 a $6 \mathrm{M} \mathrm{bit/s} \mathrm{por} \mathrm{canal}$ & Nulo o tel efónico \\
\hline $\begin{array}{l}\text { Vídeo interactivo } \\
\text { Vídeo bajo demanda } \\
\text { TV interactiva } \\
\text { J uegos interactivos } \\
\text { Servicios de información }\end{array}$ & $64 \mathrm{kbit} / \mathrm{s}$ a $6 \mathrm{M} \mathrm{bit/s}$ & 9,6 a $64 \mathrm{kbit} / \mathrm{s}$ \\
\hline $\begin{array}{l}\text { Acceso a internet } \\
\text { (www, FTP, Telnet) } \\
\text { Voz } \\
\text { Difusión }\end{array}$ & $14,4 \mathrm{kbit} / \mathrm{s} \mathrm{a} 10 \mathrm{Mbit} / \mathrm{s}$ & 14,4 a $128 \mathrm{kbit} / \mathrm{s}$ \\
\hline $\begin{array}{l}\text { Datos simétricos } \\
\text { Escritorio multimedia } \\
\text { Teletrabajo } \\
\text { Videoconferencia } \\
\text { Videotelefonía }\end{array}$ & $9,6 \mathrm{kbit} / \mathrm{s} \mathrm{a} 2 \mathrm{Mbit} / \mathrm{s}$ & $9,6 \mathrm{kbit} / \mathrm{s}$ a $2 \mathrm{M} \mathrm{bit/s}$ \\
Fax & & \\
\hline $\begin{array}{l}\text { Servidor de información } \\
\text { de Internet }\end{array}$ & 9,6 a $384 \mathrm{kbit} / \mathrm{s}$ & $64 \mathrm{kbit} / \mathrm{s}$ a $1,5 \mathrm{Mbit} / \mathrm{s}$ \\
\hline & & \\
\hline
\end{tabular}


videoconferencia, etc, algunos de los cuales tienen un importante consumo de ancho de banda como se refleja en la tabla I. Conforme cambian las necesidades de los usuarios, así como sus requisitos de ancho de banda, la red de acceso debe adaptarse añadiendo o modificando su infraestructura. Dada la competencia por ofrecer más y mejores servicios, los operadores se están planteando la introducción de la fibra óptica tan cerca del usuario como sea posible. Como se ha comentado en la introducción, la fibra óptica presenta numerosas ventajas con respecto a otro tipo de medios de transmisión. Sin embargo, el principal problema para la implantación de la tecnología óptica a gran escala es el coste. Las características clave de cualquier red de acceso son un bajo coste inicial, flexibilidad en la provisión de servicios, bajos costes de operación o mantenimiento, y alta capacidad/ancho de banda. Por lo tanto, resulta muy difícil predecir la evolución y penetración de las comunicaciones ópticas en las redes de acceso.

Si dejamos de un lado por el momento las soluciones basadas en fibra óptica, existen tres importantes tipos de redes de acceso en la actualidad:

- Par trenzado. Constituye el llamado bucle de abonado de la red tel efónica conmutada. Consiste en un acceso de usuario analógico de 3,4 $\mathrm{kHz}$ de ancho de banda sobre par trenzado de cobre. Se caracteriza por el hecho de que una gran inversión se ha realizado durante las últimas décadas en todo el mundo para crear esta infraestructura de comunicaciones. La digitalización de esta red se conoce como RDSI, mediante la cual es posible conseguir velocidades de $128 \mathrm{kbit} / \mathrm{s}$ para un acceso básico o de $2 \mathrm{Mbit} / \mathrm{s}$ para un acceso primario. Recientemente, se están aplicando técnicas englobadas bajo la denominación xDSL para conseguir aumentar el ancho de banda.

- Coaxial. Originariamente instalado para proporcionar servicios de televisión por cable (CATV), la red se caracteriza por una estructura arborescente con ramificaciones que permiten gran ancho de banda en el enlace descendente pero muy reducido en el enlace ascendente. Los porcentajes de penetración de estas redes de acceso varían de unos países a otros: un 30 \% en Reino Unido y un 99 \% en Bélgica. Sin embargo, en 
Estados Unidos es donde existe una mayor infraestructura instalada, la cual ha ido evolucionando progresivamente hacia lo que se conoce como red HFC.

- Radio. Las tecnologías inalámbricas, tanto terrestres como satélite, constituyen otra forma de competir en las redes de acceso. Además, frente al rápido crecimiento del mercado de comunicaciones móviles, esta tecnología de acceso es lógicamente la única solución. En Europa, los estándares GSM y DECT para telefonía móvil se encuentran bastante establecidos. En el caso de los PCs, las redes de área local inalámbricas serían su equivalente. Aunque los porcentajes de penetración varían enormemente, la TV por satélite es un claro competidor de la tecnología CATV en aplicaciones de distribución. Las constelaciones de satélites también se han anunciado como una posible alternativa para el desarrollo de redes interactivas de banda ancha en aplicaciones multimedia de cobertura mundial, como por ejemplo Internet. Las posibles arquitecturas para estas redes se basan en el uso de satélites de órbita baja a una altitud de aproximadamente $1500 \mathrm{~km}$ y con distintos haces que cubren zonas de unos $300 \mathrm{~km}$ de radio. Finalmente, para la distribución inicialmente de vídeo y en la actualidad también de datos, destacan los sistemas inalámbricos punto a multipunto MMDS, LMDS y MVDS, los cuales se diferencian principalmente en las frecuencias de trabajo y constituyen el Ilamado bucle local inalámbrico.

Considerando que, en el caso del par trenzado y coaxial, el $80 \%$ del coste de la red se encuentra en la parte de acceso, es de crucial importancia la óptima reutilización de las infraestructuras ya instaladas para escenarios futuros. Por otro lado, las tecnologías inalámbricas permiten realizar un despliegue de red mucho más rápido que con arquitecturas basadas en cable. Así pues, todos estos son factores que deberá tener en cuenta cualquier operador a la hora de proporcionar sus servicios. 


\subsubsection{XDSL: digitalización del bucle de abonado}

XDSL son un conjunto de tecnologías que permiten la transmisión de servicios de banda ancha a usuarios individuales u organizaciones sobre el par de cobre trenzado telefónico. Dentro de la familia xDSL se encuentran: ADSL (Asymmetric Digital Subscriber Line), SDSL (Symmetric DSL), RADSL (Rate Adaptative DSL), VDSL (Very-High-Bit-Rate DSL) y HDSL (High-Bit-Rate DSL). Varios son los motivos por los cuales los operadores públicos de tel efonía están contemplando el uso masivo de estas tecnologías:

- reutilización del gran tendido de cobre que existe en la actualidad,

- acceso a redes de banda ancha a través de la línea telefónica manteniendo intacto el canal vocal,

- atender a la demanda del mercado residencial y de pequeñas y medianas empresas con tarifas atractivas,

- en particular, utilización del bucle de abonado como un acceso barato a servicios basados en ATM, frente a un acceso basado en fibra óptica.

En el año 1989 se desarrolló la tecnología ADSL, equivalente a una línea de abonado digital asimétrica. La denominación de asimétrica se debe a la existencia de un canal de alta capacidad (6-8 M bit/s) en sentido descendente (de la central local hacia el abonado), y de uno de capacidad media/baja (640 kbit/s-1 M bit/s) en sentido ascendente (del abonado hacia la central local). No obstante, para que una línea tel efónica convencional pueda soportar una transmisión utilizando ADSL, es necesario que se cumplan unos ciertos requisitos. Las distintas velocidades que ofrece ADSL tienen una clara dependencia con la longitud del cable tel efónico y con el estado del mismo. Según las características de esta tecnología, para alcanzar velocidades del orden de 1,5-2 Mbit/s son necesarias longitudes de bucle de abonado inferiores a los 5,5 km. En el caso de centros urbanos o periferias de grandes ciudades no sería una limitación, pero puede darse el caso de pequeños pueblos o zonas rurales distantes más de $10 \mathrm{~km}$ de la central tel efónica. A medida que la distancia entre los módems ADSL aumenta, la velocidad de transferencia al canzable disminuye. 
La modalidad VDSL es la más rápida de las tecnologías de la familia XDSL, alcanzando velocidades de entre 13 y $52 \mathrm{Mbit} / \mathrm{s}$ desde la central hasta el abonado y de 1,5 a 2,3 Mbit/s en sentido contrario, por lo que se trata también de un tipo de conexión asimétrica. Es la tecnología idónea para suministrar, en un futuro, señales de TV de alta definición, aunque la distancia entre dos módems VDSL no debe superar los 1,371 km.

La tecnol ogía HDSL es simétrica, por lo que la velocidad desde la central al usuario y viceversa es la misma. Esta es la tecnología más avanzada de todas, debido a que se encuentra instalada en fábricas donde existen grandes redes de datos que necesitan transportar información de un punto a otro a muy alta velocidad. Es posible alcanzar velocidades de 1,544 M bit/s utilizando dos pares de cobre y de 2,048 $\mathrm{Mbit} / \mathrm{s}$ sobre tres pares, aunque la distancia máxima de $4,5 \mathrm{~km}$ es algo menor que en ADSL.

Finalmente, la variante SDSL es muy similar a la tecnología HDSL, puesto que soporta transmisiones simétricas pero con dos particularidades: utiliza un único par de cobre y tiene un alcance máximo de 3,048 km. Dentro de esta distancia es posible mantener una velocidad similar a HDSL, aunque esta norma se encuentra todavía en desarrollo.

Además, existe otra variante de ADSL, conocida como R-ADSL, que funciona en los mismos márgenes de velocidad pero que tiene la ventaja de adaptarse de forma dinámica a las condiciones de la línea y a su longitud. De este modo, la velocidad final de conexión utilizando esta variante de ADSL puede seleccionarse cuando la línea se sincroniza, bien durante la conexión o como resultado de una señal procedente de la central tel efónica.

Al igual que ocurre con los módems tradicionales, los módems ADSL se encargan de realizar una transformación de la señal para que pueda ser transmitida sobre las líneas telefónicas convencionales. Para ADSL existen dos sistemas de modulación que son rivales entre sí, hasta tal punto de haber creado grupos de partidarios a favor de una u otra alternativa. Estos sistemas de modulación son CAP (Carrierless Amplitude/ Phase) y DMT (Discrete MultiT one). Los sistemas DMT toman el ancho de banda disponible 


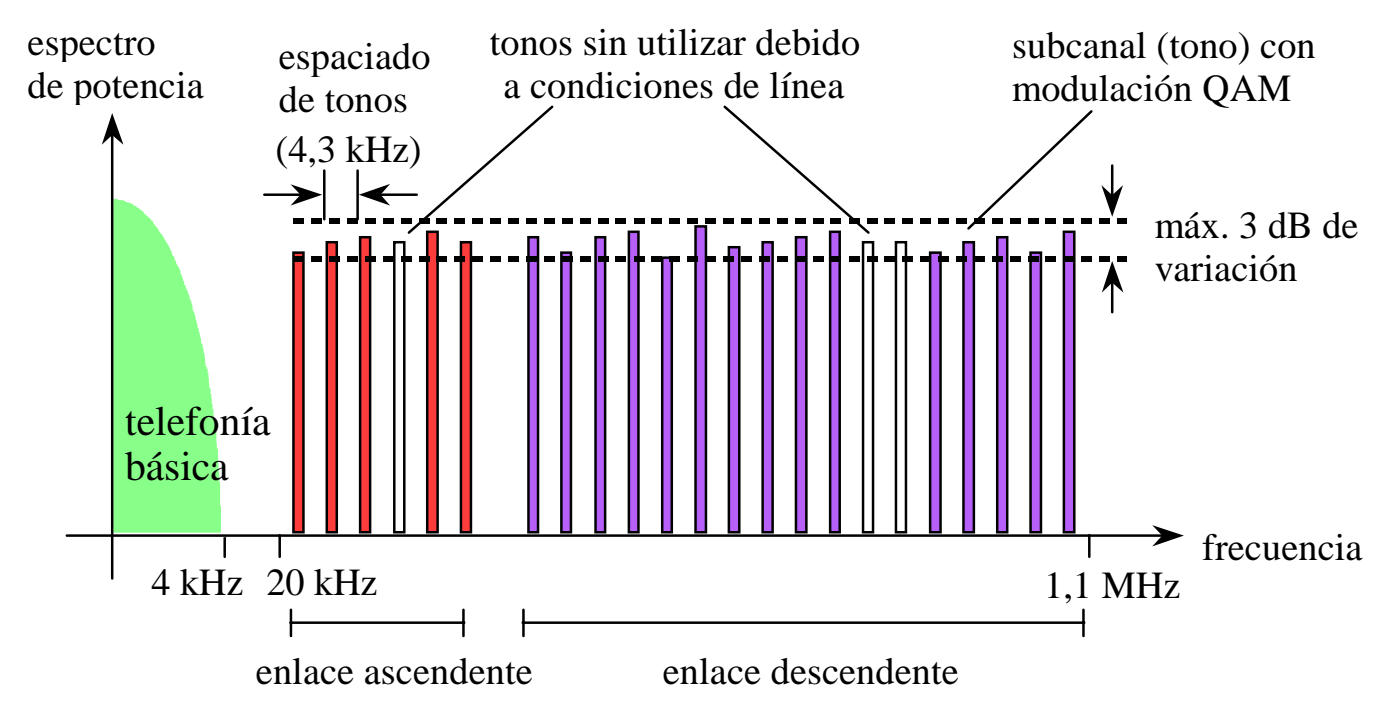

Figura 1.2 Espectro de la modulación DMT utilizada en el sistema ADSL.

de la línea y lo dividen en múltiples subcanales de $4 \mathrm{kHz}$. Después, tras analizar las características del medio de transmisión, distribuyen los datos sobre estos subcanales de la forma apropiada para optimizar las prestaciones. En la figura 1.2 se puede ver cuál sería el espectro de la señal de un sistema ADSL basado en la técnica de modulación DMT. En teoría, para una línea en particular y una velocidad de transmisión dada, un sistema CAP o QAM monoportadora puede diseñarse para alcanzar las mismas prestaciones que un sistema DMT multiportadora. Pero en realidad, debido a la gran varianza que presentan las líneas y a que las velocidades oscilan entre los $64 \mathrm{kbit} / \mathrm{s}$ y los $8 \mathrm{Mbit} / \mathrm{s}$, un sistema monoportadora no alcanza las prestaciones óptimas para el rango de líneas y velocidades que maneja ADSL. Además, otra ventaja del sistema DMT es una mejor adaptación frente a condiciones de línea cambiantes, de tal forma que el sistema realiza una monitorización continua y cambia los datos de un subcanal a otro cuando detecta un aumento en el nivel de ruido.

La estructura de un sistema que emplea la tecnología ADSL se muestra en la figura 1.3. EI módem ADSL situado en el lado del usuario se conecta, a través de un cable tel efónico convencional, a un módem ADSL (o a una pila de tales módems) situado en las centrales del operador. Éste, a su vez, se 
enlaza con un conmutador Ethernet, un router o un conmutador ATM que crea una conexión a Internet o a otra red de datos. Varios fabricantes trabajan en el diseño de multiplexores que integrarán esa funcionalidad.

A pesar de las variantes de XDSL que existen, algunas de ellas ya probadas y comenzándose a implantar y otras aún en proceso de desarrollo, ninguna de ellas ha sido oficialmente reconocida por ningún organismo para adoptarla como estándar. El grupo de trabajo T1E1.4 del ANSI (American National Standards I nstitute) ha aprobado un estándar ADSL a velocidades de hasta 6,1 Mbit/s (estándar ANSI T1.413), al que contribuyó también el ETSI (European Telecommunications Standards Institute) con un anexo en el que se exponen las necesidades y variantes europeas. Éste admite un tipo de terminal con interfaz única en el lugar de destino. En la actualidad, el grupo de trabajo está estudiando incluir en el estándar una interfaz multiplexada en el lugar de destino, protocolos de gestión y configuración de red, etc. La UIT (Unión Internacional de Telecomunicaciones) alcanzó un principio de acuerdo en noviembre de 1998, denominado ADSL G.Lite, que contaba con el respaldo de grandes empresas como Microsoft, Intel o Compaq. ADSL G.Lite es una versión de la tecnología ADSL también conocida como splitterless (sin filtro voz/datos) o DSL universal. En octubre

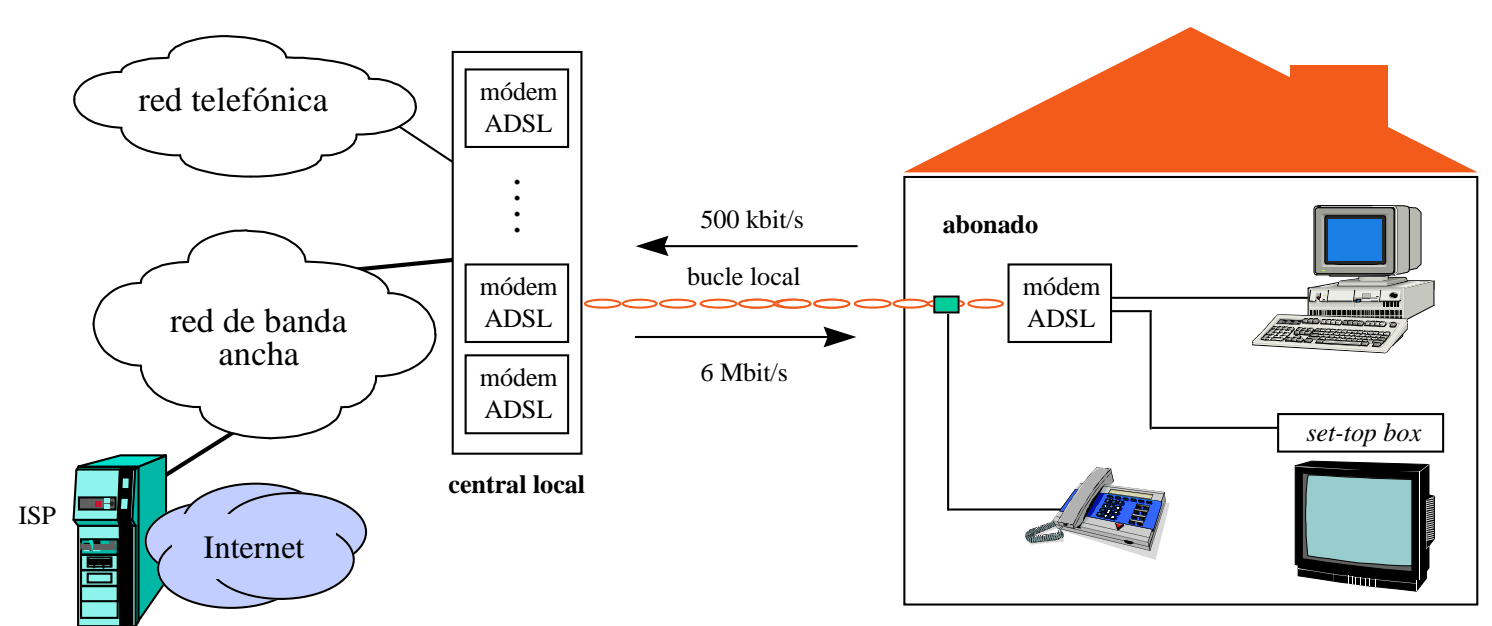

Figura 1.3 Diagrama esquemático de la arquitectura de un sistema ADSL para la provisión de servicios de vídeo y datos a través del par trenzado telefónico. 
de 1998, G.922.2 fue adoptado por la UIT como el estándar que recogía a la tecnología G.Lite. La ratificación definitiva del G.922.2 tuvo lugar en junio de 1999.

\subsubsection{Bucle local inalámbrico}

Las soluciones basadas en tecnol ogía radio juegan un papel importante en la nueva red de acceso debido a que permiten el despliegue de la red de forma más rápida y flexible que con soluciones cableadas, proporcionando un tipo y calidad de servicios en consonancia con dichas soluciones cableadas. Normalmente, los operadores de cable optan por esta tecnología en una fase inicial para poder capturar rápidamente cuota de mercado y, posteriormente, se dedican a ir desarrollando progresivamente la infraestructura de fibra óptica de su red. Las soluciones fijas de acceso radio son conocidas bajo distintos acrónimos, siendo los más populares los de FWA (acceso inalámbrico fijo, Fixed Wireless Access) y WLL (bucle local inalámbrico, Wireless Local Loop).

La figura 1.4(a) muestra la evolución prevista del mercado mundial de acceso radio (en millones de nuevas líneas). Es necesario remarcar que a partir del año 2000 empieza a ser significativo el mercado de acceso radio en países desarrollados. Este mercado, en el período 1999-2003, representa aproximadamente el $15-20 \%$ del mercado total. Basado en la oferta de servicios de banda ancha, el principal motor del mercado de acceso radio es el precio por línea y, fundamentalmente, la rapidez de despliegue de la red que permite ofrecer el servicio a un usuario final en el mínimo tiempo posible. Esto además se ve favorecido instalando las estaciones base en lugares elevados para lograr la máxima cobertura de usuarios.

Por otra parte, la figura 1.4(b) muestra la fragmentación del mercado en función de la banda de frecuencias utilizada y del tipo de servicio ofrecido. Así, se tienen los mercados conocidos como BE (banda estrecha) y BA (banda ancha), que incluyen servicios al usuario final de acceso basico 2B+D RDSI y líneas alquiladas de $n \times 64 \mathrm{~Kb} / \mathrm{s}(\mathrm{n}<15)$, respectivamente. 


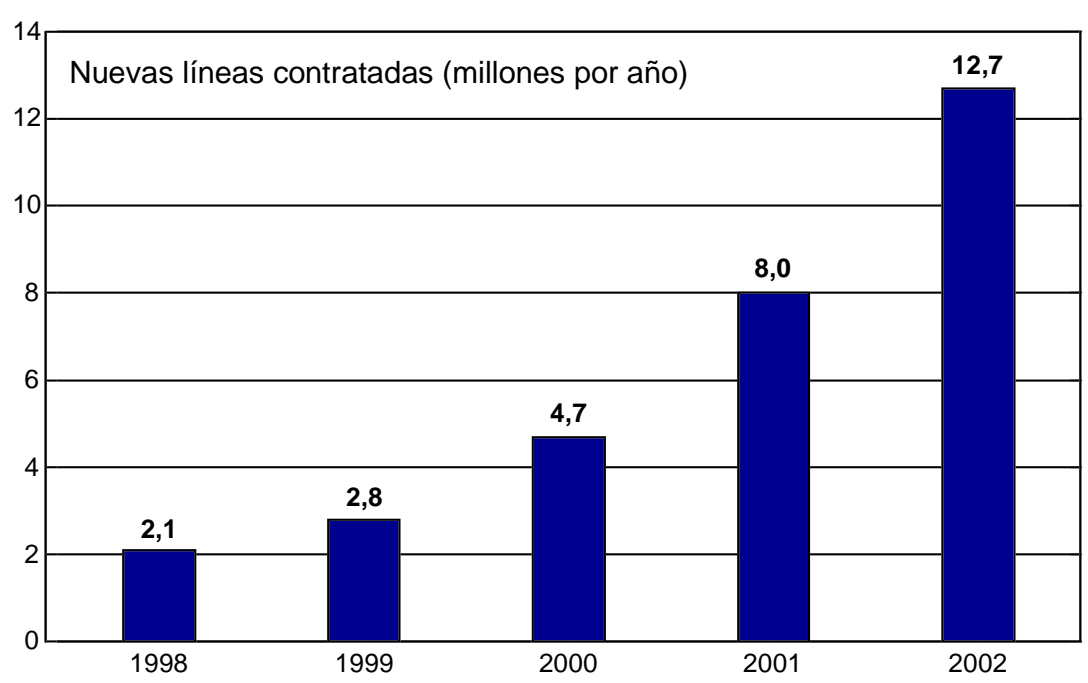

(a)

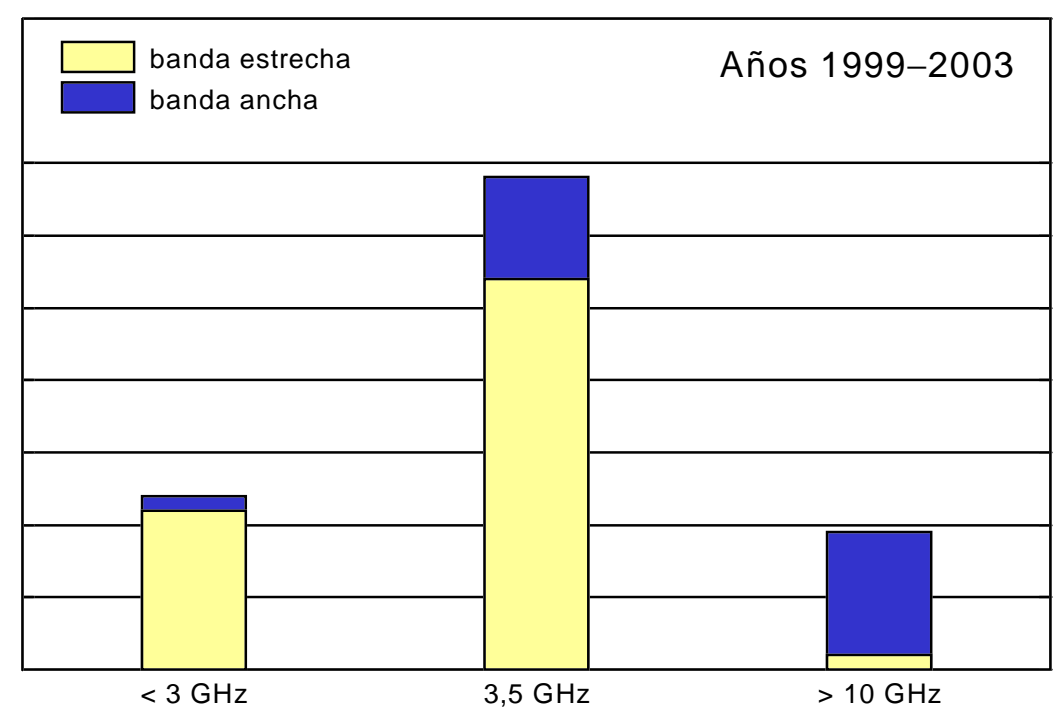

(b)

Figura 1.4 Evolución del mercado de bucle local inalámbrico [Bit99]. (a) Nuevas líneas contratadas y (b) fragmentación del mercado.

El empleo de distintas bandas de frecuencia para sistemas fijos de acceso radioeléctrico se deriva de factores técnicos y regulatorios. Las bandas de frecuencia más altas $(10,26,28$ y $40 \mathrm{GHz})$ son más problemáticas desde el punto de vista de propagación, aunque como ventaja disponen de un gran ancho de banda que las hace adecuadas para la transmisión de servicios de banda ancha. En un punto intermedio se encuentra la banda de $3,5 \mathrm{GHz}$. EI uso de esta banda proporciona una serie de ventajas inherentes con respecto 
a otras bandas como 26 y $28 \mathrm{GHz}$. Por una parte, la circuitería de radio está más desarrollada, y lo que es más importante, este desarrollo está adquiriendo un ritmo cada vez más acelerado como consecuencia de las resoluciones adoptadas por los reguladores de frecuencia que sitúan a la banda de 3,5 GHz como la banda natural para las aplicaciones WLL de banda estrecha, en especial en los países desarrollados y en entornos urbanos. Esta mayor madurez tecnológica se traduce en ventajas importantes de coste para los componentes de RF. Además, otro aspecto importante se refiere a las características de propagación radioeléctrica, con unas pérdidas menos severas que a 26 ó $28 \mathrm{GHz}$. Adicionalmente, las condiciones de planificación radioeléctrica no son tan estrictas, ya que la Iluvia no afecta prácticamente a $3,5 \mathrm{GHz}$ y las directividades de las antenas no necesitan ser tan elevadas (anchos de haz de unos 20 grados).

No obstante, dado el ancho de banda disponible a 3,5 $\mathrm{GHz}^{\mathrm{iii}}$, las arquitecturas radio de banda ancha requieren el uso de esquemas de modulación muy eficientes para poder acomodar las capacidades típicas de $32 \mathrm{Mbit} / \mathrm{s}$ por sector en configuraciones de 4 sectores por estación base. Esto Ileva a pensar en modulaciones 16QAM o incluso mejores, al menos en el enlace descendente que es el que normalmente soporta la mayor velocidad de transmisión. El enlace ascendente podría transportar velocidades inferiores, del orden de los $8 \mathrm{Mbit} / \mathrm{s}$, lo que permite encontrarse con esquemas de modulación mixtos: 16QAM descendente/QPSK ascendente.

Por último, dado que el despliegue típico de estos sistemas requiere de topologías multicelda y multisector para mitigar en lo posible los problemas derivados de las interferencias adyacente y cocanal, resulta conveniente recurrir a mecanismos flexibles de asignación dinámica de recursos. De este modo, se elimina la necesidad de planificación de frecuencias y se simplifica enormemente la incorporación de nuevos abonados al sistema.

\footnotetext{
iii normalmente bloques de $25 \mathrm{MHz}$ ( 2 × $25 \mathrm{MHz}$ para transmisión bidireccional)
} 


\subsubsection{MMDS}

Los sistemas MMDS (Multipoint Multichannel Distribution System) surgieron en la década de los 80 como una evolución de los sistemas MDS (Microwave Distribution System), que constituyeron la primera explotación comercial de la banda de $2 \mathrm{GHz}$ para la distribución directa al abonado de un canal de TV de pago. Posteriormente, se concedieron licencias para el servicio multicanal ocupando el espectro entre 2,150 y $2,682 \mathrm{GHz}$ con un ancho de banda de $500 \mathrm{MHz}$, aunque ciertas subbandas dentro de estas frecuencias son compartidas con instituciones educativas dependiendo de factores tales como la zona o la demanda. Estos sistemas son típicos de Estados Unidos, y la mayor parte de las licencias están dedicadas a la transmisión de señales de TV analógica, por lo que a este servicio se le denomina también "cable inalámbrico" (wireless cable).

Los sistemas MMDS han experimentado un crecimiento relativamente rápido en la década de los 90, pasando de aproximadamente 200.000 abonados en 1992 al millón en 1999. Sin embargo, este número palidece frente a los más de 65 millones de suscriptores a la TV por cable y a los más de 5 millones de la TV multicanal por satélite. Muchos observadores atribuyen la escasa penetración relativa de los sistemas MMDS al hecho de que el ancho de banda disponible permite transmitir un máximo de 33 canales de vídeo analógico (6 MHz/canal en el sistema NTSC americano), frente a los 80 canales analógicos disponibles en el cable y a los 150 canales digitales disponibles en los sistemas DTH (Direct To Home) por satélite, por lo que no puede haber competición directa respecto al tipo de servicio ofrecido. Esto ha hecho, precisamente, que los sistemas MMDS hayan tenido mas éxito comercial en zonas rurales o de baja densidad de abonados, donde el coste de la distribución por cable no justifica la inversión requerida.

Sin embargo, alrededor de 1995 surgieron dos tecnologías que han modificado la situación de los sistemas MMDS de forma considerable: la disponibilidad de equipos de compresión digital a coste relativamente bajo, y la disponibilidad de sistemas de acceso con ancho de banda compartido para la transmisión bidireccional de datos. La tecnología de compresión digital de 
canales de TV permitió multiplicar la capacidad de los sistemas MMDS de 33 a cerca de 200 canales (compresión 5:1). En estos sistemas se utiliza modulación 64QAM, la cual permite unos $30 \mathrm{M}$ bit/s por cada canal analógico de $6 \mathrm{MHz}$ previamente ocupado. Por otro lado, las tecnologías de transmisión de datos con acceso compartido empezaron a utilizarse en 1997, en un principio mediante equipos de transmisión inalámbrica con multiplexación TDM en el enlace descendente: modulación 64QAM, tres portadoras de $10 \mathrm{Mbit} / \mathrm{s}$ por cada canal de $6 \mathrm{MHz}$, y con retorno por la línea tel efónica. Pero dada la popularidad de este tipo de servicio, Ia FCC autorizó el uso del espectro para la transmisión inalámbrica bidireccional incluyendo el camino de retorno desde el abonado a la estación base. En la actualidad, el retorno se realiza normalmente en la banda de 2,150-2,162 GHz empleando modulaciones QPSK o DQPSK. EI radio típico de celda en los sistemas MMDS varía en torno a los 50-75 km, dependiendo de la orografía del terreno y de la situación de las antenas. Se deduce, pues, que es perfectamente viable cubrir una ciudad completa utilizando una única estación base.

Por idénticas razones a las ya comentadas en el caso de la banda de 3,5 $\mathrm{GHz}$, la tecnología utilizada en los sistemas MMDS, tanto para aplicaciones de TV analógica, digital $\mathrm{o}$ de datos bidireccionales, se encuentra relativamente avanzada con respecto a la de otros sistemas inalámbricos de banda ancha. El diagrama de bloques de un sistema MMDS típico se muestra en la figura 1.5. La cabecera del sistema normalmente incluye equipos de generación y modulación de vídeo similares a los de una cabecera de cable para canales analógicos, o a los de una cabecera de TV digital tipo DTH para canales digitales. También incluye los módems de cabecera, el sistema de gestión de red y la conexión a la red de datos o Internet. La cabecera se encuentra en la mayoría de los casos conectada por medio de fibra óptica o un enlace radio a la estación base, donde se encuentra un transmisor multicanal y un receptor de conversión en bloque, generalmente conectados por medio de un duplexor a antenas omnidireccionales o sectoriales, dependiendo de la configuración de la celda. 


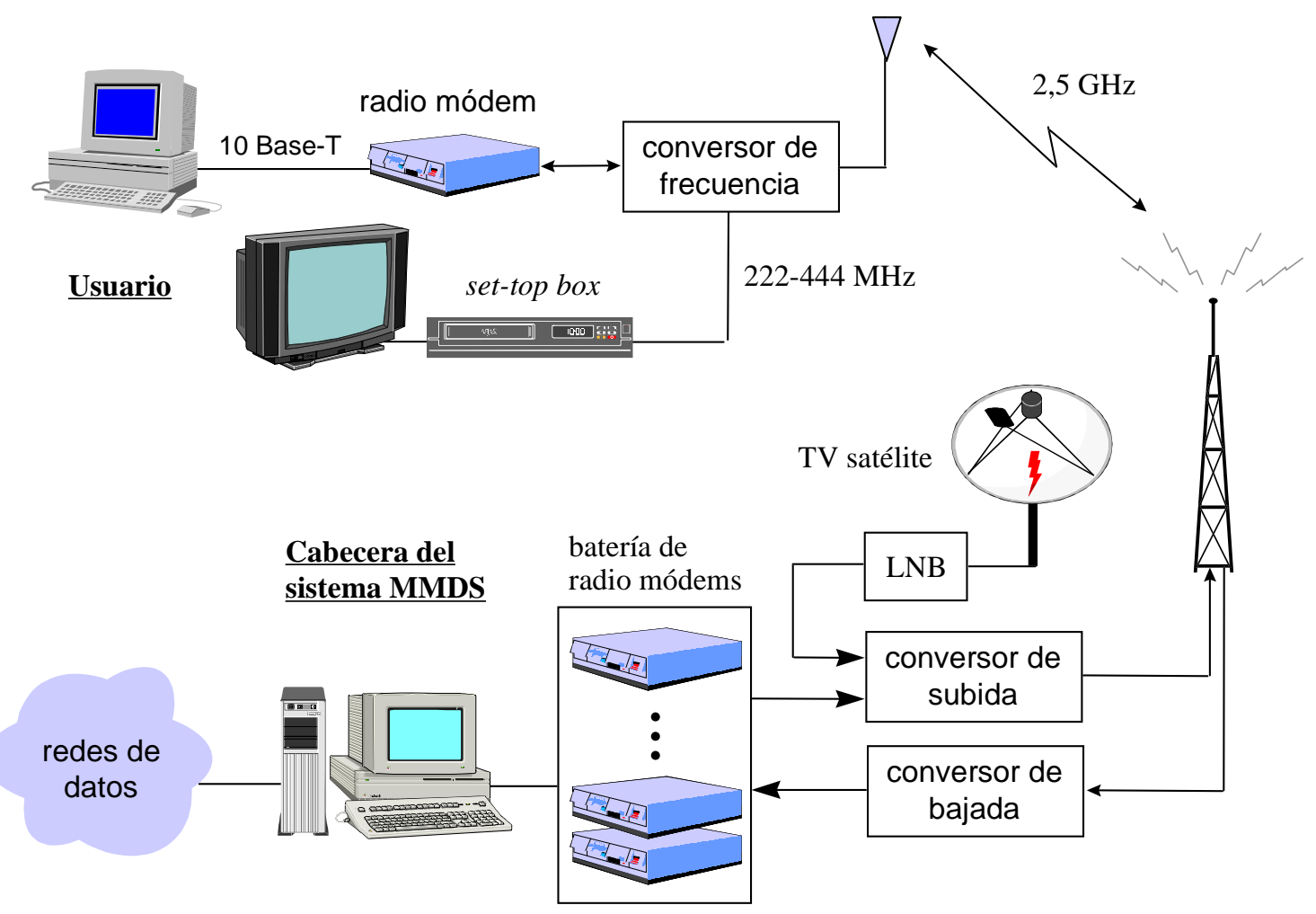

Figura 1.5 Diagrama de bloques de un sistema MMDS de distribución de TV satélite y conexión a Internet.

Los transmisores multicanal modernos son unidades de banda ancha que permiten multiplexar simultáneamente todas las portadoras (vídeo analógico, vídeo digital y datos) en una única señal en la banda de 222-422 $\mathrm{MHz}$, evitando de esta manera la necesidad de multiplexar varios transmisores monocanal a frecuencias de microondas. Por otra parte, los equipos de abonado consisten en una unidad exterior de RF integrada con una antena parabólica tipo parrilla. Esta unidad de RF está formada por un transmisor QPSK de 100 mW de potencia, integrado con el duplexor, y un convertidor de bajada similar al LNB ${ }^{\text {iv }}$ utilizado en la TV por satélite. La frecuencia de salida del convertidor se encuentra en la banda de 222 a 422 $\mathrm{MHz}$, y la de entrada en la banda de 10 a $50 \mathrm{MHz}$. Finalmente, la unidad interior de abonado puede configurarse como un set-top box analógico (si los canales analógicos vienen codificados), un set-top box digital, y/o un módem de datos con conexión directa Ethernet 10-BaseT al PC o a una LAN.

\footnotetext{
iv low-noise block
} 


\subsubsection{LMDS/MVDS}

Originariamente, el acrónimo MVDS (Multipoint Video Distribution System) se utilizó para designar los primeros sistemas celulares que aparecieron de difusión de canales de TV analógica. En Europa, la CEPT publicó en 1992 la recomendación TR/52-01 en donde se identificaba la banda de 40,5 a 42,5 $\mathrm{GHz}$ para tales servicios. Sin embargo, la nueva generación de sistemas digitales tiene ahora un objetivo más amplio, dado que ahora se trata de transmitir señales de TV, voz y datos, con el consiguiente canal de retorno. En Estados Unidos, estos sistemas se encuentran bastante establecidos en la actualidad, aunque allí reciben la denominación LMDS (Local Multipoint Distribution System) y operan en la banda de $28 \mathrm{GHz}$. En general, las administraciones no tienen demasiado claro qué banda utilizar, por lo que muchas empresas europeas están optando por fabricar equipos para ambas frecuencias: 28 y $40 \mathrm{GHz}$. La problemática de los $28 \mathrm{GHz}$ es que parte se encuentra reservada para radioenlaces digitales y existe el riesgo de interferencias con las futuras generaciones de satélites de órbita baja que operen en la banda Ka. Es por ello que el ETSI ha desarrollado un estándar para la recomendación de la CEPT, el EN 300748 v.1.1.2, en el que se cita expresamente la banda recomendada por la CEPT pero indicando que es aplicable a cualquier frecuencia por encima de los $10 \mathrm{GHz}$. Desde este momento, nos referiremos con el acrónimo LMDS al sistema que opera a 28 $\mathrm{GHz}$ y con MVDS al sistema que trabaja a $40 \mathrm{GHz}$.

La introducción de nuevos servicios multimedia en el entorno residencial o empresarial, tal es como vídeo bajo demanda, videoconferencia, etc., requiere de una gran cantidad de ancho de banda que debe ser soportado por las redes de acceso. El principal candidato para permitir estos servicios es sin lugar a dudas la fibra óptica. No obstante, la operadora se encuentra con un alto factor de riesgo: debe realizar un gran desembolso económico para montar su infraestructura de red. Además, dado el actual nivel de competencia que existe en la red de acceso del usuario, lo que cualquier operadora desea es comenzar a dar servicio lo más rápidamente posible. De esta forma, LMDS aparece como una prometedora tecnología de gran valor estratégico en el marco de las comunicaciones inalámbricas de banda ancha. 
En comparación con las tecnol ogías basadas en cable, los sistemas LMDS se pueden instalar muy rápidamente, al tiempo que la naturaleza intrínsecamente modular de su arquitectura permite una ampliación progresiva en función de las necesidades y del aumento de la cuota de mercado. Además, la tecnología digital empleada y los anchos de banda disponibles permiten comunicaciones de alta velocidad, comparables a las al canzables por medio de la fibra óptica.

Como ya se ha comentado, la principal diferencia de los sistemas LMDS con respecto a otras tecnol ogías de acceso vía radio es su margen de frecuencias

TABLA II Frecuencias del sistema LMDS/LMCS/MVDS en al gunos países.

\begin{tabular}{|l|c|c|}
\hline \multirow{3}{*}{ País } & rango de frecuencias (GHz) & ancho de banda (MHz) \\
\cline { 2 - 3 } & $27,50-28,35$ & 850 \\
\cline { 2 - 3 } & $29,10-29,25$ & 150 \\
\hline \multirow{3}{*}{ Venezuela } & $31,00-31,30$ & 300 \\
\cline { 2 - 3 } & $27,50-29,50$ & 2000 \\
\hline \multirow{4}{*}{ Argentina } & $31,00-31,30$ & 300 \\
\cline { 2 - 3 } & $25,35-28,35$ & 3000 \\
\cline { 2 - 3 } & $29,10-29,25$ & 150 \\
\hline Canadá & $31,00-31,30$ & 300 \\
\hline Rumanía & $37,00-40,00$ & 3000 \\
\hline Corea & $25,85-29,85$ & 4000 \\
\cline { 2 - 3 } & $27,50-28,50$ & 1000 \\
\hline Inglaterra & $24,25-27,50$ & 3250 \\
\hline Francia & $40,50-42,50$ & 2000 \\
\hline España & $40,50-42,50$ & 2000 \\
\hline Rusia & $27,50-29,50$ & 2000 \\
\hline Filipinas & $27,50-29,50$ & 2000 \\
\hline
\end{tabular}


de trabajo. En este caso, LMDS opera a frecuencias milimétricas en torno a los $28 \mathrm{GHz}$ (banda Ka). Dada la congestión espectral que existe a frecuencias bajas, ésta es la única forma de conseguir anchos de banda elevados. En particular, anchos de banda de unos $2 \mathrm{GHz}$ son típicos en estos sistemas. En la tabla II se resumen las bandas de trabajo de los sistemas LMDS/MVDS dependiendo del país. La utilización de las bandas de frecuencia más el evadas del espectro ha tenido lugar tradicionalmente en el ámbito de sectores especializados como defensa, o en el sector espacial, debido sobretodo a la complejidad y coste de los equipos. Sin embargo, los rápidos avances en tecnología de semiconductores, y concretamente en Arseniuro de Galio (AsGa), han permitido la obtención de circuitos integrados monolíticos de microondas, así como procesadores avanzados de señal, que han propiciado que los costes disminuyan considerablemente para fabricación en grandes volúmenes.

Ahora bien, las elevadas frecuencias utilizadas en estos sistemas limitan enormemente la cobertura como consecuencia de la atenuación introducida en el trayecto de propagación radio. Esto les confiere una estructura celular, de tal forma que existen estaciones base distribuidas por toda la zona que se desea cubrir. Los radios de las celdas oscilan entre los 2 y los $7 \mathrm{~km}$, dependiendo de la potencia de los transmisores, y en el interior de éstas se agrupan un determinado número de usuarios. Por ejemplo, para una disponibilidad de servicio del $99,9 \%$ se pueden alcanzar distancias de hasta $14 \mathrm{~km}$. Los usuarios situados a $14 \mathrm{~km}$ de la estación base no recibirán servicio en una media de 8 horas por año, mientras que los usuarios más cercanos tendrán una disponibilidad mejor. Si la disponibilidad aumenta al 99,99\%, el alcance se reduce a $5 \mathrm{~km}$ (valor típico), mientras que para el 99,999 \% se tendría una distancia máxima de tan solo 2,5 km. A diferencia del resto de sistemas de comunicaciones celulares como DECT o GSM, en este caso se trata de equipos fijos, donde las antenas se sitúan normalmente en la azotea de los edificios.

Adicionalmente al problema de la atenuación, existe el problema de las reflexiones. A estas frecuencias, las ondas de radio se reflejan en obstáculos tales como edificios, árboles, etc., por lo que no existe difracción y se necesita 
visión directav entre las antenas transmisora y receptora. Este hecho provoca la aparición de zonas de sombra que no pueden ser cubiertas. Dado que son sistemas fijos, la situación de los equipos de usuario es perfectamente conocida y este problema es menor que en el caso de los sistemas de tel efonía móvil. No obstante, hay que intentar reducir las zonas de sombra al máximo dado que perdemos potenciales usuarios. De este modo, lo que se suele realizar es un cierto solapamiento entre celdas, o bien utilizar transmisores secundarios (repetidores), reflectores o amplificadores dependiendo de la situación.

Por último, las moléculas de agua introducen una atenuación adicional a estas frecuencias, por lo que en condi ciones de lluvia es necesario aumentar considerablemente la potencia de transmisión para cubrir una determinada zona. Normal mente, se utilizan sistemas de potencia variable que, asociados a equipos de detección de lluvia, aumentan la potencia de transmisión de forma automática cuando se produce este fenómeno. Otros agentes meteorológicos como el hielo o la nieve no afectan significativamente a las señales. Para un completo modelo del canal de propagación radio en sistemas LMDS se puede consultar la referencia [Pap97].

Así pues, se observa que los sistemas LMDS tienen que pagar un precio elevado por el hecho de operar a frecuencias tan altas. Sin embargo, todos estos problemas se resuelven creando una estructura celular con celdas de radio variable en función de la potencia de la estación base o densidad de usuarios, y convenientemente solapadas para evitar al máximo las zonas de sombra. Las antenas, situadas en lugares elevados, consiguen una mayor cobertura. Como se representa en la figura 1.6, un sistema LMDS consiste en un conjunto de estaciones base interconectadas entre sí y que cubren cel das de diferente tamaño y con distintas densidades de usuarios. El centro de control es el lugar donde se procesan las distintas señales a transmitir. En especial, se reciben señales de TV digital satélite y existen enlaces de alta capacidad con otras redes externas. Para la alimentación de las señales hacia las estaciones base existen multitud de posibilidades. Por una parte,

v line of sight (LOS) 


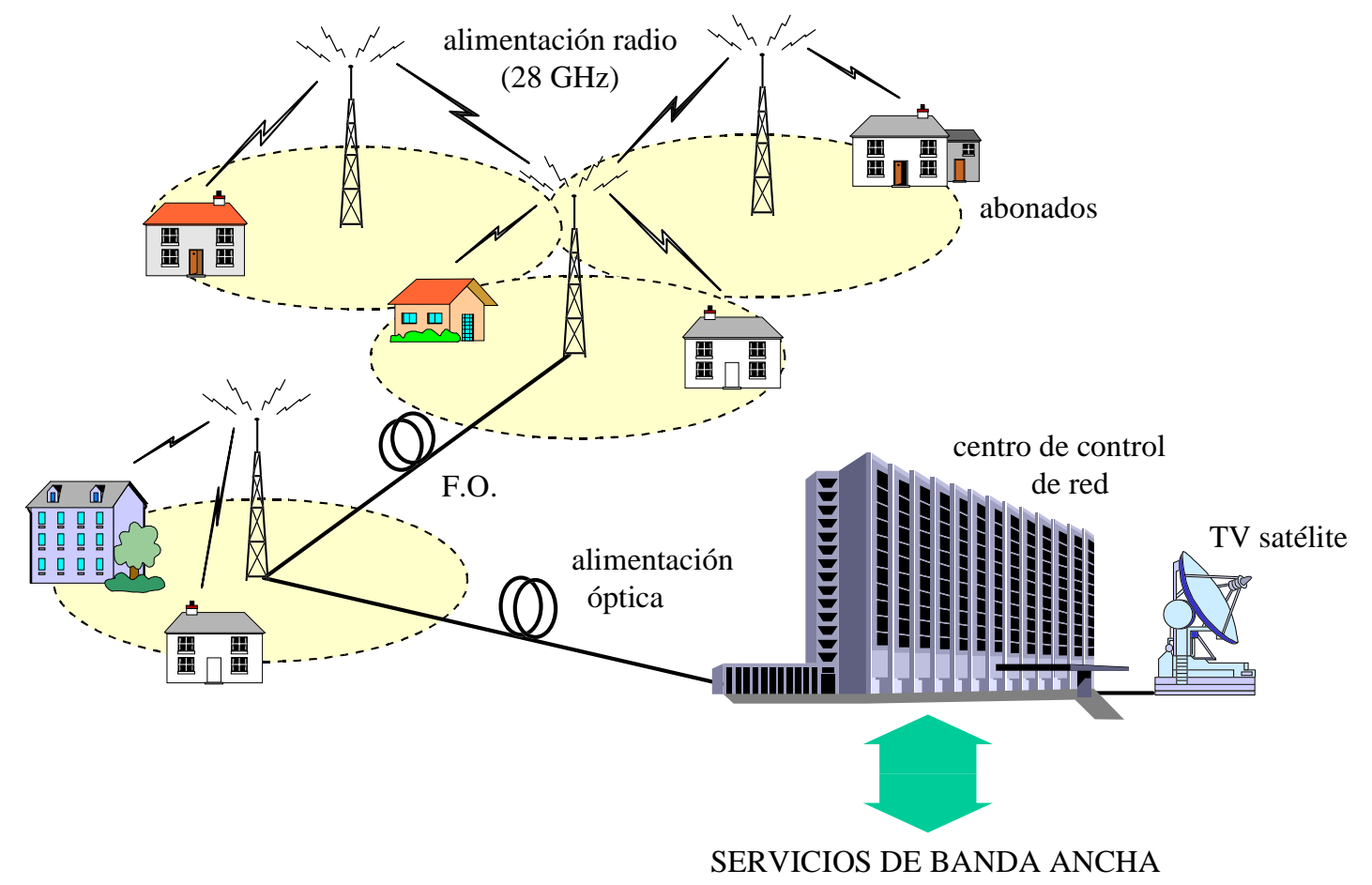

Figura 1.6 Arquitectura del sistema celular fijo LMDS para la provisión de servicios bidireccionales de banda ancha a $28 \mathrm{GHz}$.

la estación base puede encontrarse en el propio centro de control. Por otra parte, la señal se puede distribuir hasta la estación base empleando un enlace punto a punto por medio de cable (fibra óptica/coaxial) o radio. Al mismo tiempo, la estación base puede actuar como repetidor de otra estación base para cubrir una determinada zona de sombra.

Las comunicaciones en los sistemas LMDS se realizan en forma de radiodifusión desde la estación base hacia los usuarios, en lo que se conoce como punto a multipunto. Al mismo tiempo, dado que la comunicación es bidireccional, los usuarios también pueden establecer enlaces punto a punto con dicha estación base. Para el enlace descendente suele emplearse TDMA como técnica de acceso. Por otra lado, para el enlace ascendente se utilizan combinaciones de acceso múltiple TDMA y FDMA. La transmisión de los datos se realiza con modulaciones QPSK o QAM, aunque en el canal de retorno se utiliza siempre modulación QPSK. EI ancho de banda ocupado por una conexión a $2 \mathrm{Mbit} / \mathrm{s}$ empleando esta técnica de modulación es de tan sólo 1,4 MHz. Algunas velocidades típicas del enlace descendente utilizando 
canales de $6 \mathrm{MHz}$ de ancho de banda serían: $10 \mathrm{M} \mathrm{bit/s}$ con QPSK, $20 \mathrm{Mbit} / \mathrm{s}$ con 16QAM ó $30 \mathrm{M}$ bit/s con 64QAM.

Las antenas utilizadas en los sistemas LMDS difieren bastante dependiendo de que nos encontremos en la estación base o en el emplazamiento de usuario. En el caso de este último, se emplean antenas directivas para conseguir la máxima ganancia. Una bocina cónica con un diámetro de apertura de unos 15 ó $20 \mathrm{~cm}$ suele ser una antena de usuario típica. Para reducir la longitud de la antena sin introducir un excesivo error de fase se utiliza una lente colocada en la apertura de la bocina. En el caso de la estación base, debido a la transmisión punto a multipunto, se emplean o bien antenas omnidireccionales que cubren completamente la celda, o bien un conjunto de antenas sectoriales con anchos de haz que pueden variar entre los 30 y los 180 grados. Las antenas sectoriales, además de poseer una mayor ganancia, permiten reutilizar las frecuencias del sistema. De este modo, se obtiene una multiplicación de la capacidad del sistema en términos del número de abonados a los que se les puede dar servicio. Además, empleando polarizaciones distintas entre sectores adyacentes, se consigue optimizar el aislamiento entre los mismos. Las polarizaciones utilizadas en estos sistemas son lineales: horizontal $(H)$ y vertical $(V)$. También es posible diseñar celdas con cobertura cuadrada modificando convenientemente el diagrama de radiación de las antenas. De hecho, esta configuración es la típica en LMDS. Por último, los anchos de banda de las antenas oscilan entre 1 y $2 \mathrm{GHz}$.

Pueden considerarse al menos dos tipos de arquitecturas distintas para implementar los equipos transmisores, las cuales han sido utilizadas en los equipos comerciales existentes. Por una parte, una estructura monocanal de subida en frecuencia, amplificación y transmisión, con canales de ancho de banda estrecho, reducida potencia y que se combinan antes de ser radiados. O alternativamente, también se puede utilizar una estructura de banda ancha donde se suban en frecuencia y se amplifiquen simultáneamente todos los canales. Como ventajas de la estructura monocanal se tienen una mayor linealidad (los amplificadores se emplean más eficientemente) y una mayor eficiencia espectral dado que se puede combinar con el uso de antenas 
sectorizadas. Sin embargo, el transmisor es excesivamente modular y existe redundancia de equipos. Por otro lado, en el caso multicanal se puede entregar un mayor ancho de banda a la antena, aunque a costa de una limitación de potencia como consecuencia de la intermodulación introducida por el amplificador de potencia.

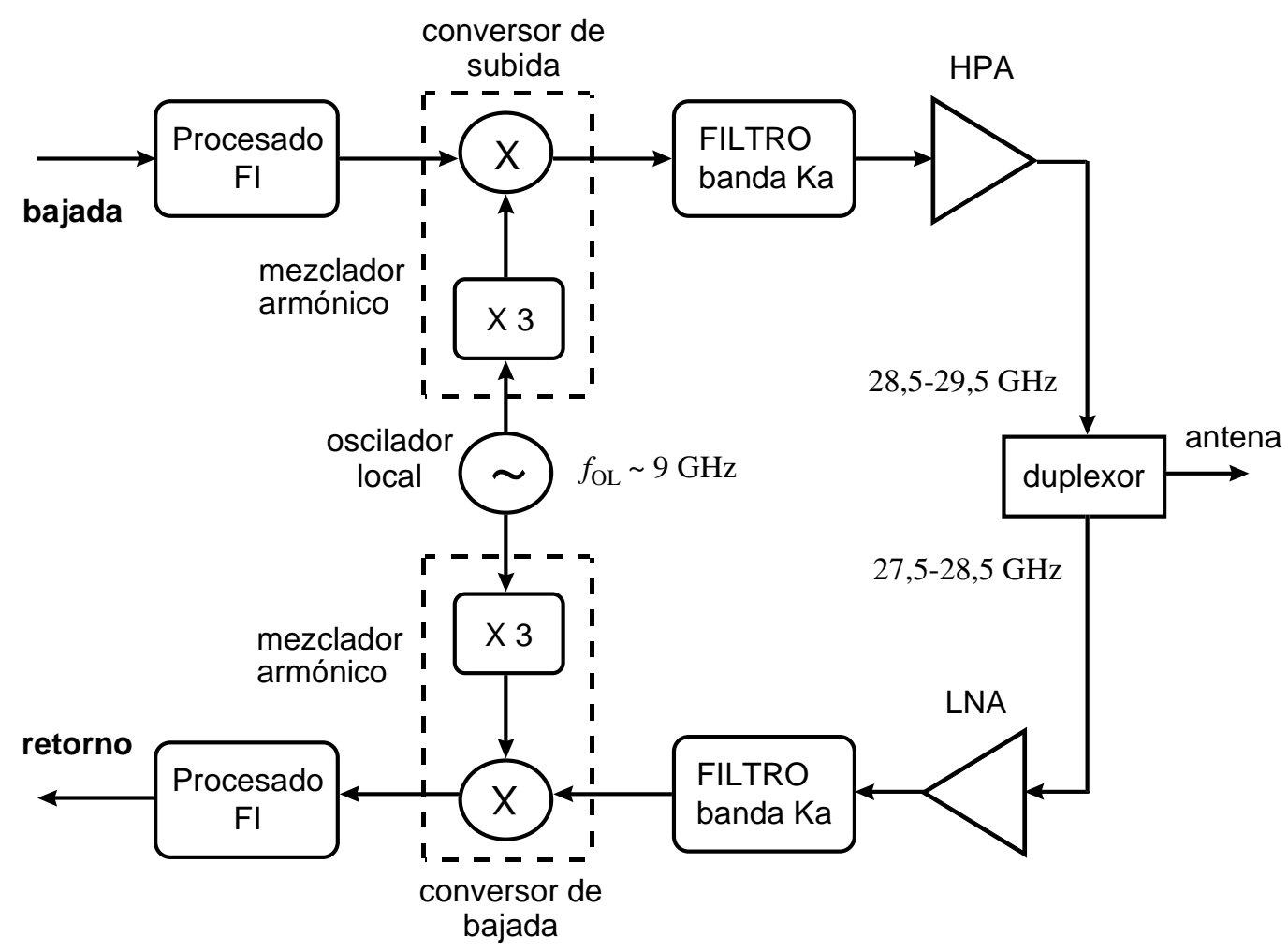

Figura 1.7 Transceptor LMDS típico de un usuario. Obsérvense como elementos principales el oscilador local y el mezclador armónico de milimétricas.

En lo que respecta al equipamiento receptor, éste se encarga de recoger las señales del enlace de subida de cada uno de los usuarios. Por lo tanto, para optimizar la potencia transmitida desde el emplazamiento de usuario, interesa minimizar la figura de ruido del equipo receptor. Con la tecnología MMIC (Monolythic Microwave Integrated Circuit) actual, es posible obtener amplificadores de bajo ruido con figuras de ruido de unos $5 \mathrm{~dB}$, que se colocan inmediatamente a la salida de la antena. 
El equipamiento del emplazamiento de usuario se resume en el diagrama de bloques del transceptor LMDS de la figura 1.7. Los enlaces ascendente y descendente deben separarse por medio de un duplexor colocado a la salida de la antena. En este tipo de esquema es imprescindible evitar la interferencia del enlace ascendente sobre el descendente, ya que la diferencia de potencias es considerable y el aislamiento del duplexor no es perfecto. Por ello, los $2 \mathrm{GHz}$ de ancho de banda se dividen y se toman bandas diferentes para cada uno de los sentidos. Los bloques de procesado de FI se encargan de trabajar con las señales de frecuencia intermedia que serán posteriormente subidas en frecuencia a la banda Ka (upconversion) o que previamente fueron bajadas de dicha banda (downconversion). En muchos casos, la señal multicanal recibida suele bajarse a banda $L$, por lo que el conversor de bajada tendría la misma funcionalidad que un LNB de TV satélite, pudiéndose conectar el set-top box directamente a su salida. EI elemento clave encargado de realizar las conversiones de frecuencia es el mezclador de milimétricas. Las estructuras que suelen emplearse son las basadas en el mezclado armónico. De este modo, la frecuencia de oscilador local que alimenta al mezclador es un submúltiplo de la frecuencia de portadora, obteniéndose un ahorro económico importante. Así, suponiendo un mezclador de tercer armónico y una frecuencia de portadora de $27 \mathrm{GHz}$, sería necesario un oscilador a tan sól o $9 \mathrm{GHz}$. Para obtener esta frecuencia, que debe ser muy precisa, se utilizan osciladores sintetizados enganchados a un reloj de Rubidio de referencia. Otras alternativas incluyen el uso de tonos piloto de RF transmitidos desde la estación base. Finalmente, otros elementos serían filtros en banda Ka y amplificadores de milimétricas. En el caso de estos últimos, se utiliza un amplificador de potencia para alimentar la señal a la antena y un amplificador de bajo ruido para amplificar la señal proveniente de la antena, ambos realizados normalmente en tecnología MMIC.

\subsubsection{Introducción de la fibra óptica en la red de acceso}

Una red de acceso se compone esencialmente de una red de alimentación y de una red de distribución. Los sistemas basados en fibra se han utilizado con éxito en esta red de alimentación [Way99], la cual cumple una función 
de transporte de los servicios originados en la estación de control o central de conmutación. Al mismo tiempo, la red de distribución que une la parte de alimentación con los usuarios se ve bastante limitada en el coste, ya que éste se comparte entre un grupo reducido de abonados. Por ello, resulta crucial aprovechar las infraestructuras de distribución existentes para obtener una solución económicamente viable.

Dependiendo del factor de penetración de la fibra óptica en la red de acceso, existen diferentes tipos de escenarios que reciben la denominación FTTx. En el caso de que la fibra óptica ll egue hasta el hogar del usuario se tiene FTTH (Fiber To The Home). Si llega hasta el edificio y después existe distribución por cable, entonces se denomina FTTB (Building). Finalmente, si la fibra llega hasta una manzana de edificios o hasta una central de conmutación tenemos diferentes denominaciones: FTTC (Curb), FTTCab (Cabinet) o FTTExch (Exchange). En la tabla III se resumen algunas de las características de los diferentes esquemas de introducción de fibra óptica en la red de acceso. El último trayecto hasta el hogar del usuario suele realizarse mediante tecnología xDSL. En la figura 1.8 se representan esquemáticamente las diferentes alternativas. En el caso de FTTH, el nodo

TABLA III Características de las diferentes alternativas de acceso basadas en fibra óptica.

\begin{tabular}{|l|c|c|c|c|}
\cline { 2 - 5 } \multicolumn{1}{c|}{} & $\begin{array}{c}\text { unidad } \\
\text { óptica }\end{array}$ & $\begin{array}{c}\text { alcance } \\
\text { cobre }\end{array}$ & velocidad (Mbit/s) & $\begin{array}{c}\text { máx. núm. } \\
\text { accesos }\end{array}$ \\
\hline FTTH & ext./int. & - & $<25 \downarrow$ & 1 \\
\hline FTTB & interior & $<500 \mathrm{~m}$ & - & $8-16$ \\
\hline FTTC & exterior & $<500 \mathrm{~m}$ & $\begin{array}{c}<25-52 \downarrow / 2-6 \uparrow \\
\text { residencial } \\
12 \downarrow / 12 \uparrow \text { empresas }\end{array}$ & $32-64$ \\
\hline FTTCab & exterior & $<1,5 \mathrm{~km}$ & $\begin{array}{c}<25 \downarrow / 2 \uparrow \text { residencial } \\
<2 \downarrow / 2 \uparrow \text { empresas }\end{array}$ & $64-128$ \\
\hline FTTExch & interior & $<5 \mathrm{~km}$ & $\begin{array}{c}<1,5-6 \downarrow / 64-640 \mathrm{kbit} / \mathrm{s} \uparrow \\
\text { residencial } \\
<0,5 \downarrow / 0,5 \uparrow \text { empresas }\end{array}$ & $64-128$ \\
\hline
\end{tabular}


óptico se encuentra en el propio hogar del abonado, por lo que se reduce el número de usuarios afectados en caso de fallo y aumenta la capacidad de renovación de la red. No obstante, el coste adicional de la infraestructura debe reducirse empleando equipos de bajo coste.

En el caso de los sistemas inalámbricos, la penetración de la fibra óptica en la red de acceso da lugar a lo que se conoce como sistemas híbridos fibraradio (HFR). Aunque pudiera parecer que bajo la denominación de sistemas radio sobre fibra óptica se incluyen únicamente los sistemas HFR, en general suelen considerarse también en el mismo bloque otras variantes como FTTC/XDSL o HFC. Así, en este estudio consideraremos sistemas radio sobre fibra óptica a todos aquellos que se aprovechen de la fibra óptica para transmitir señales de RF empleando modulación analógica en el transmisor óptico.

La figura 1.9 muestra una arquitectura típica de sistema radio sobre fibra para la provisión de servicios de banda ancha. Por la red de alimentación con topología en anillo viajan multiplexadas en longitud de onda las señales

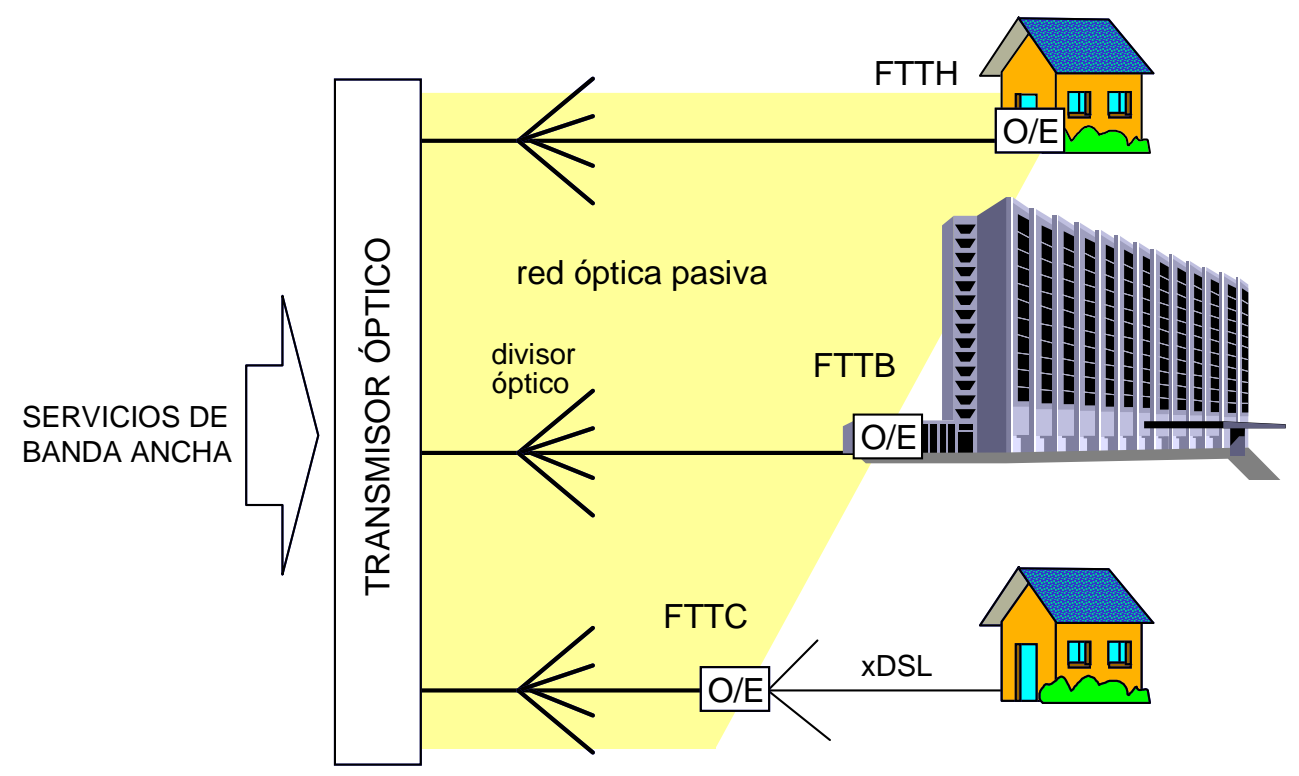

Figura 1.8 Diferentes niveles de penetración de la fibra óptica en la red de acceso: FTTC, FTTB o FTTH. 


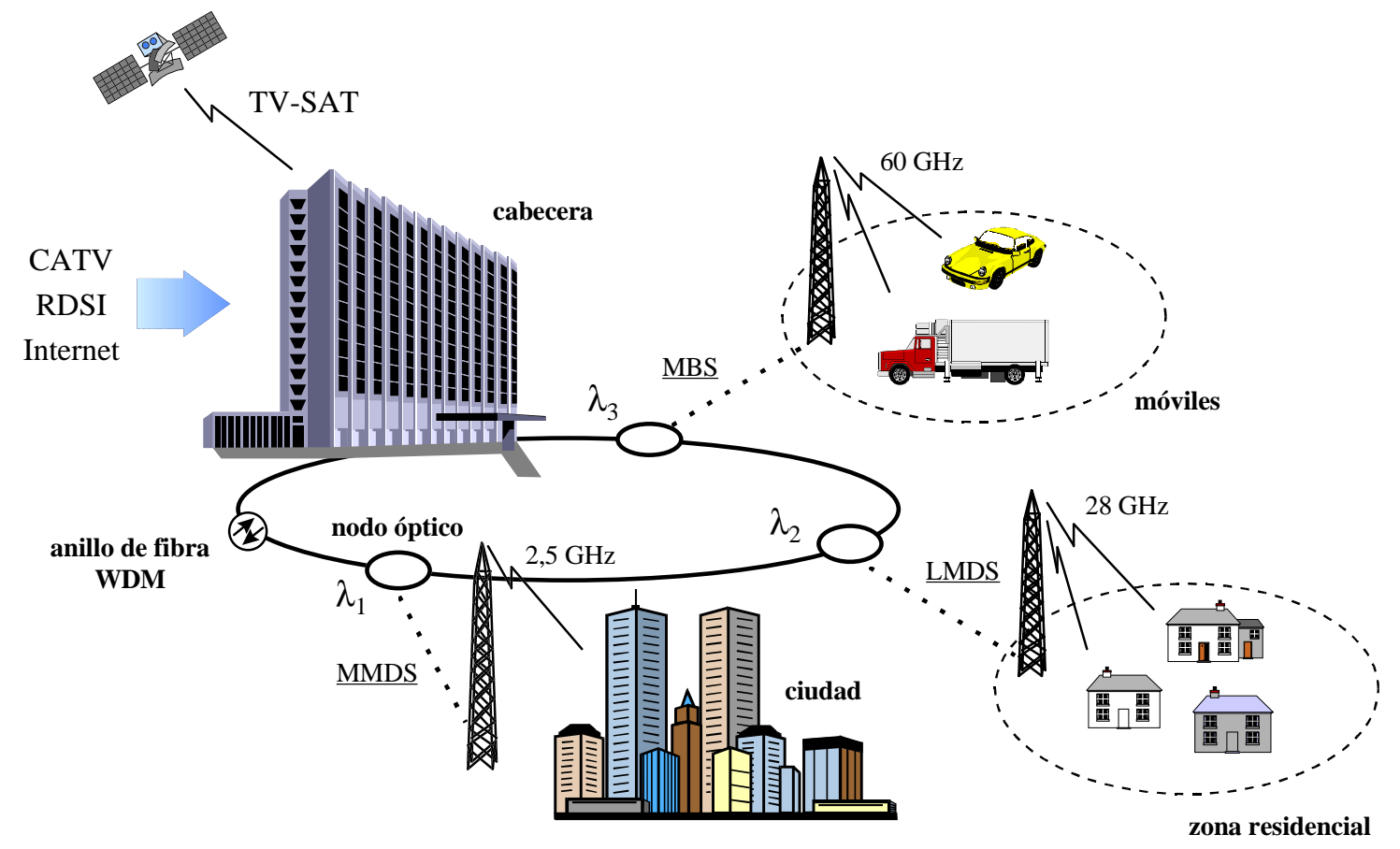

Figura 1.9 Sistema radio sobre fibra óptica WDM-SCM para proporcionar servicios bidireccionales de banda ancha MMDS, LMDS y MBS.

de los diferentes sistemas: MMDS, LMDS/MVDS y MBS (Mobile Broadband System). La principal ventaja de este esquema conjunto WDM-SCM es su flexibilidad, ya que el enrutamiento de las señales de los diferentes sistemas puede realizarse en el dominio óptico.

\subsubsection{Redes HFC}

Históricamente, las redes CATV se diseñaron como sistemas de difusión unidireccionales utilizando la topología de red más adecuada en este caso: la topología en árbol mostrada en la figura 1.10(a). El sistema consistía en una cabecera de red que se encargaba de captar las señales a transmitir, usualmente TV satélite, y posteriormente distribuirlas sobre cable coaxial hasta los usuarios finales.

La cabecera de la red CATV es el centro desde el que se gobierna todo el sistema, y su complejidad depende de los servicios que deba prestar la red. Por ejemplo, para el servicio básico de distribución de canales de TV, tanto analógicos como digitales, dispone de una serie de equipos de recepción de 
TV terrena, satélite y microondas, así como de enlaces con otras cabeceras o estudios de producción. Las señales analógicas se acondicionan para su transmisión por el cable y se multiplexan en frecuencia en la banda comprendida entre los 86 y los $606 \mathrm{MHz}$. Las señales digitales de vídeo, audio y datos que forman los canales de TV digital se multiplexan para formar el flujo de transporte MPEG (Motion Picture Experts Group). Una vez añadida la codificación para corrección de errores, se utiliza un modulador QAM para transmitir la información hasta el equipo terminal de abonado. Los canales digitales de TV, así como otros servicios de datos, se ubican en la banda comprendida entre los 606 y $862 \mathrm{MHz}$. Finalmente, la cabecera es también la encargada de monitorizar la red y supervisar su correcto funcionamiento.

Dado que la distancia entre la cabecera de red y un usuario final es de varias decenas de kilómetros, para compensar la atenuación y mantener una buena calidad de señal se hace necesaria la presencia de múltiples amplificadores (incluso hasta 30) a lo largo del trayecto. Sin embargo, estas numerosas cascadas de amplificadores se convierten en un importante problema de fiabilidad, a la vez que introducen distorsión y limitan el ancho de banda disponible como consecuencia de su frecuencia de corte. Por ello, para aumentar la capacidad del sistema, la fiabilidadad, la calidad de la señal y la resistencia a interferencias, disminuyendo a su vez los costes de mantenimiento, los operadores de CATV están reemplazando en sus redes de forma progresiva largas secciones de cable coaxial por fibra óptica. De hecho, los nuevos operadores que se incorporan despliegan directamente la infraestructura de fibra óptica con niveles de penetración que pueden llegar prácticamente hasta el hogar del abonado. La red resultante de esta modificación se conoce con el nombre de red híbrida de fibra óptica y coaxial (HFC, Hybrid Fiber Coaxial). De este modo, instalando enlaces de fibra óptica de bajas pérdidas se eliminan los amplificadores del tramo de red troncal, dejando únicamente del orden de dos o tres amplificadores en la red de distribución final de coaxial que tiene una longitud reducida. La red troncal suele presentar una estructura en forma de anillos redundantes de fibra óptica que unen un conjunto de nodos primarios. Esta estructura puede emplear tecnología PDH o SDH (J erarquía Digital Plesiócrona o 
Síncrona, respectivamente), lo que permitiría construir redes de cable basadas en ATM.

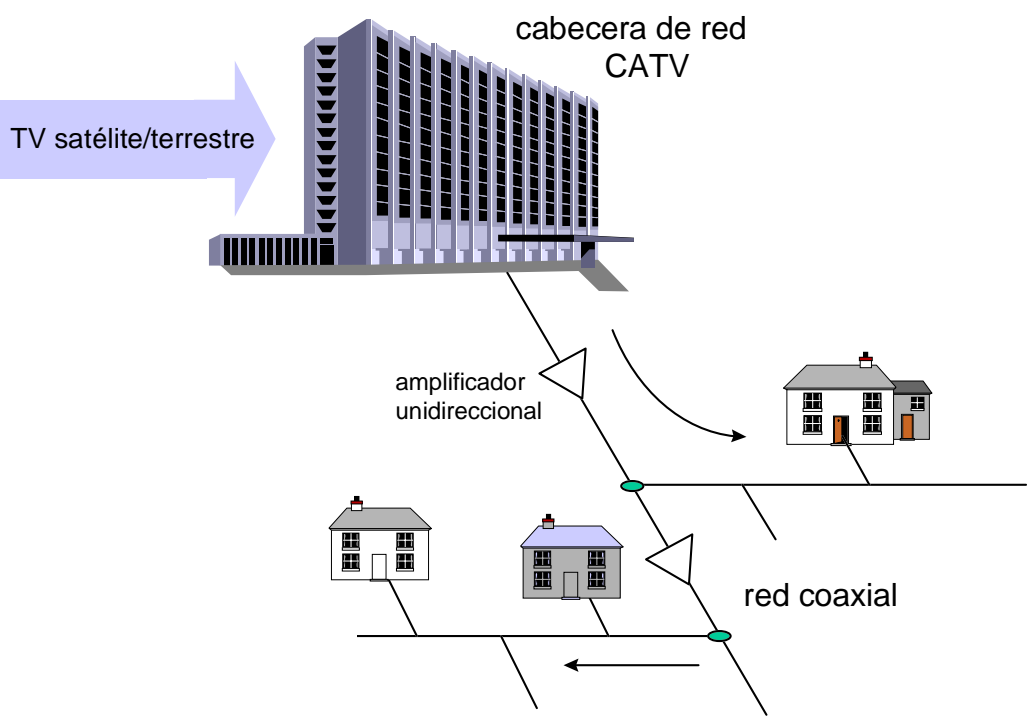

(a)

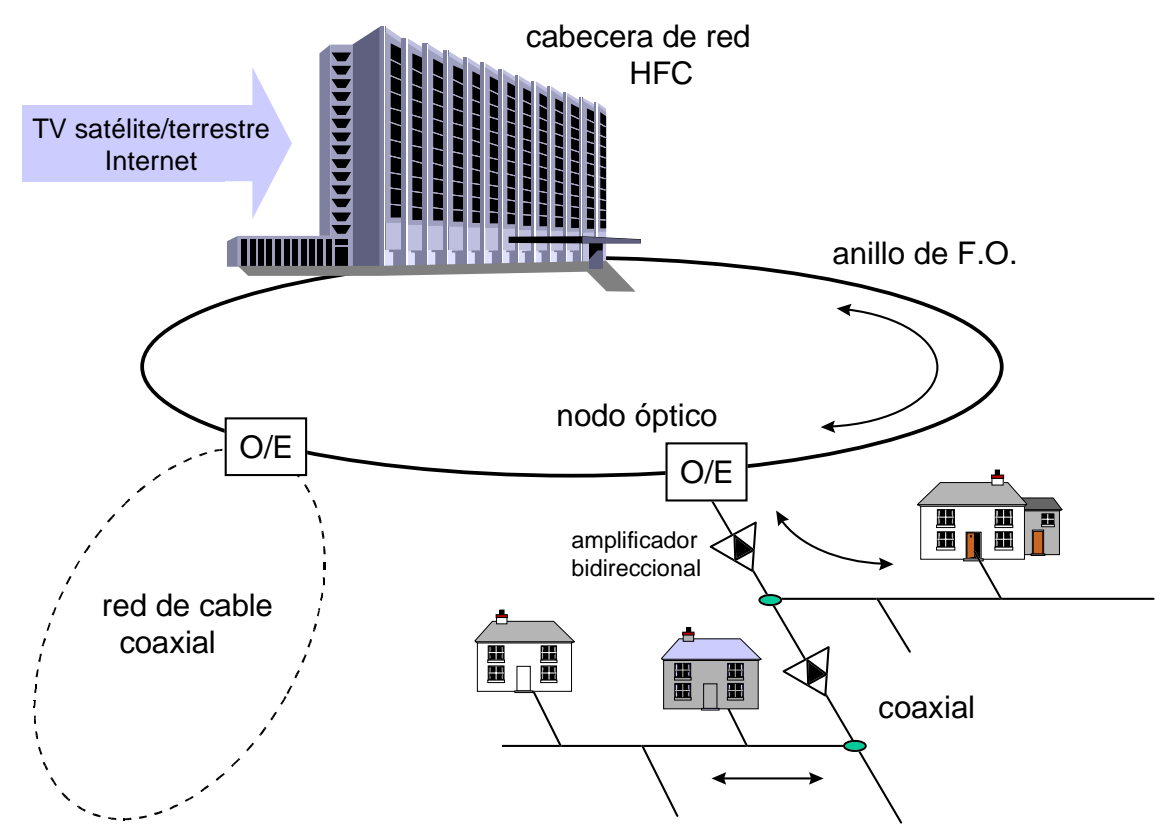

(b)

Figura 1.10 Evolución de (a) la red CATV original a (b) una arquitectura de red HFC bidireccional. 
Las modernas redes de telecomunicaciones HFC deben estar preparadas para poder ofrecer un amplio abanico de aplicaciones y servicios a sus abonados. La mayoría de estos servicios requieren de la red la capacidad para establecer comunicaciones bidireccionales entre la cabecera y los equipos terminales de abonado, y por lo tanto exigen la existencia de un canal de comunicaciones ascendente o de retorno. Así, los operadores de CATV deben reemplazar sus amplificadores unidireccionales por amplificadores bidireccionales que permitan el enlace ascendente desde el usuario a la cabecera de red. Durante la instalación de la fibra óptica, esto puede realizarse directamente para los amplificadores ópticos que se sitúen en el trayecto, aunque puede llevar más tiempo en el caso de los amplificadores eléctricos situados en la red de coaxial cercana a los usuarios. En la figura 1.10(b) se muestra la arquitectura de una red HFC bidireccional. Los nodos ópticos se encargan de realizar la conversión entre la señal óptica y eléctrica para el enlace descendente y viceversa para el enlace de retorno, por lo que necesitan de un transmisor óptico. El canal de retorno en las redes HFC ocupa el espectro comprendido entre 5 y $42 \mathrm{MHz}$. Este ancho de banda lo comparten todos los hogares servidos por un mismo nodo óptico. Los retornos de distintos nodos llegan a la cabecera por distintas vías o multiplexados a distintas longitudes de onda. De este modo, una señal generada por el equipo terminal de un abonado recorre la red de distribución de coaxial en sentido ascendente, atravesando amplificadores bidireccionales hasta llegar al nodo óptico. Aquí convergen las señales de retorno de todos los abonados, las cuales se convierten en señales ópticas por medio del láser de retorno y se transmiten hacia la cabecera.

Con objeto de poder utilizar los mismos receptores que se emplean con la TV terrena, las redes de cable utilizan para el enlace descendente la misma banda de frecuencias, aunque los sistemas HFC expanden esta banda desde aproximadamente los $550 \mathrm{MHz}$ hasta los $860 \mathrm{MHz}$ o incluso $1 \mathrm{GHz}$, dejando la parte baja del espectro (5-42 en Europa y 5-65 en Estados U nidos) como canal de retorno para órdenes, debido a que esta región se encuentra más sujeta a interferencias. En la banda útil se pueden incluir un gran número de canales de TV analógica si se tiene en cuenta que, con el estándar americano NTSC un canal de TV ocupa un ancho de banda de $6 \mathrm{MHz}$, 
mientras que con el europeo PAL o SECAM éste se incrementa tan sólo hasta los $8 \mathrm{MHz}$. Con un canal RF de $6 \mathrm{MHz}$, se puede proporcionar un flujo de datos de $27 \mathrm{Mbit} / \mathrm{s}$ desde la cabecera de red hasta el usuario final haciendo uso de técnicas de transmisión que utilicen 64QAM, o incluso mayor (hasta $36 \mathrm{Mbit} / \mathrm{s}$ ) si se emplease 256QAM. En sentido inverso, del usuario a la cabecera de red se utilizan modulaciones más simples como QPSK, que proporciona una velocidad suficiente entre $256 \mathrm{kbit} / \mathrm{s}$ y un máximo de $10 \mathrm{Mbit} / \mathrm{s}$, y no requiere sofisticados equipos en el hogar del usuario. Como alternativa, se puede utilizar también DPSK, que resulta algo más lenta pero ofrece mayor inmunidad frente a interferencias.

El acceso a Internet a velocidades cada vez mayores es uno de los grandes negocios de las nuevas redes de acceso de banda ancha. Las redes HFC, mediante el uso de módems especialmente diseñados para las comunicaciones digitales en redes de cable, tienen capacidad para ofrecer servicios de acceso a redes de datos como Internet a velocidades cientos de veces superiores a las que el usuario medio está acostumbrado: hasta 64 kbit/s a través de la red tel efónica. En realidad, los módems de cable están convirtiendo las redes CATV/HFC en verdaderos proveedores de servicios de telecomunicación de vídeo, voz y datos.

Un módem de cable típico se caracteriza por ser asimétrico: recibe datos a velocidades de hasta $30 \mathrm{Mbit} / \mathrm{s}$ y puede transmitir hasta $10 \mathrm{Mbit} / \mathrm{s}$. Se conecta a la red HFC mediante un conector de cable coaxial tipo F, y al PC del abonado por medio de una tarjeta Ethernet 10Base-T. La recepción de datos se realiza por un canal de entre 6 y $8 \mathrm{MHz}$ de la banda $606-862 \mathrm{MHz}$, normalmente con modulación digital 64QAM. EI módem de cable demodula la señal recibida y encapsula el flujo de bits en paquetes $E$ thernet. De esta forma, el abonado ve la red HFC como si fuera una enorme LAN. En sentido ascendente, el módem de cable descompone los paquetes Ethernet que recibe del PC y los convierte en celdas ATM o en tramas con formato propietario. Posteriormente, utilizando un canal de unos $2 \mathrm{MHz}$ del espectro de retorno, los datos son transmitidos hacia cabecera empleando modulación QPSK. 
Los estándares aplicables a este tipo de módems han ido evolucionando con el tiempo, hasta el punto de existir tres generaciones. Los estándares de la segunda generación de módems de cable se recogen en la especificación DOCSIS (Data Over Cable Service Interface Specification), que constituye el estándar de facto en Estados Unidos. En Europa, DAVIC/DVB (Digital Audio-VIsual Council/ Digital Video Broadcasting) es el estándar predominante para los set-top box, y ahora también, para los módems de cable. La terecera generación, promovida por el IEEE y contenida en su norma IEEE 802.14, pretende ser un estándar internacional ambicioso y a prueba de futuro. Se basa en el uso de ATM como la mejor manera de proporcionar la calidad de servicio (QoS) garantizada que requieren las comunicaciones integradas de voz, datos y vídeo sobre redes de cable. 


\subsection{MODELADO DE LOS SISTEMAS ÓPTICOS ANALÓGICOS}

Los sistemas radio sobre fibra óptica se incluyen dentro de los llamados sistemas ópticos analógicos, los cuales se caracterizan por una modulación analógica en el transmisor óptico, independientemente de que la señal eléctrica de modulación pueda transportar distintas subportadoras con modulación digital [Bra98a]. En particular, en esta sección y en el resto de la tesis nos centraremos en los sistemas MI-DD, caracterizados por modulación de intensidad (MI) en el transmisor óptico y detección directa (DD) en el receptor óptico. Otras posibilidades incluirían la modulación de frecuencia o la detección coherente.

\subsubsection{Sistemas WDM-SCM}

Los sistemas de subportadoras multiplexadas (SCM) se utilizan comúnmente para la transmisión de señales de RF sobre enlaces de fibra óptica [Dar86, Ols87, Ols89, Dar90, Wak93, Agr97 (cap. 7)]. SCM es una técnica en la que una señal de banda ancha compuesta por diversos canales multiplexados en frecuencia se aplica directamente a un láser modulado en intensidadvi. Estos sistemas se caracterizan por acomodar en el espectro tanto canales analógicos como digitales, transportando señales de voz, datos, vídeo, audio digital, TV de alta definición, y cualquier combinación de servicios que se desee. Cada usuario puede servirse con una única subportadora, o bien distribuir una señal multicanal entre todos los usuarios. Su enorme flexibilidad los hace muy atractivos en el caso de aplicaciones de banda ancha, especialmente si los servicios se originan desde distintos proveedores utilizando esquemas de modulación y anchos de banda diferentes.

La configuración básica de un sistema SCM se muestra en la figura 1.11. Un gran número de subportadoras de microondas, $\mathrm{f}_{\mathrm{i}}$, moduladas con las señales a transmitir, se combinan para generar una señal multiplexada en frecuencia. Esta señal compuesta se aplica ahora junto con la corriente de

\footnotetext{
vi existe también la posibilidad de inyectar la señal de RF por medio de un modulador externo, aunque originariamente se empleaba modulación directa
} 
polarización a un láser de semiconductor o modulador externo, el cual actuará como transmisor óptico del sistema. La señal óptica modulada en intensidad se transmite sobre un enlace de fibra óptica monomodo y finalmente se fotodetecta utilizando un fotodiodo InGaAs p-i-n de banda ancha.

En el caso de aplicaciones que requieran mayor sensibilidad en el receptor, tales como redes de distribución con múltiples etapas de repartidores pasivos o troncales de CATV, se puede utilizar un fotodiodo InGaAs APD de banda ancha que presenta una mayor ganancia. La señal de microondas recibida puede amplificarse por medio de un amplificador de banda ancha y bajo ruido o un receptor $\mathrm{p}$-i-n-FET. Comercialmente, existen amplificadores

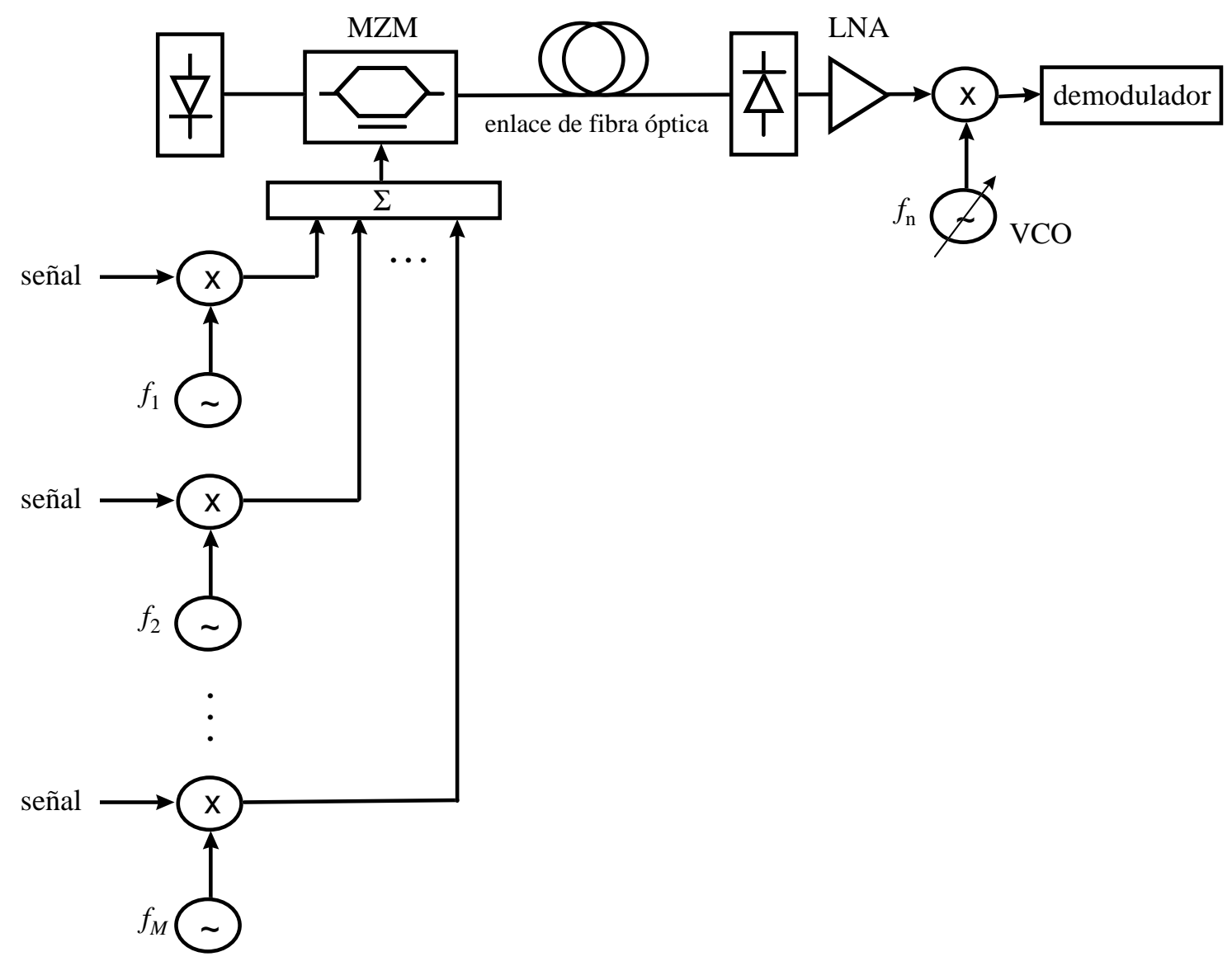

Figura 1.11 Diagrama de bloques de un sistema SCM típico formado por M subportadoras eléctricas. En el receptor se selecciona el canal n-ésimo para la demodulación. 
de bajo ruido con anchos de banda de $2-8 \mathrm{GHz}$ y figuras de ruido menores que $2 \mathrm{~dB}$. En el caso de un receptor p-i-n-FET con ancho de banda de DC a 8 $\mathrm{GHz}$, es posible un valor rms de corriente de ruido en torno a los $12 \mathrm{pA} / \mathrm{VHz}$, equivalente a una figura de ruido de un amplificador de 2,5 dB. Esta solución sería válida en un sistema SCM híbrido que combinara una señal digital banda base junto con canales de alta frecuencia.



Figura 1.12 Espectro de un sistema de comunicaciones ópticas híbrido WDM-SCM.

En un sistema de distribución a abonados, como por ejemplo CATV, sólo necesitamos seleccionar un canal para la demodulación. Por tanto, se puede utilizar simultáneamente un oscilador local sintonizable, un mezclador y un filtro de banda estrecha para seleccionar el canal SCM deseado y bajarlo a una frecuencia intermedia más conveniente para realizar finalmente la demodulación. En el caso de los receptores de TV satélite, todos estos componentes electrónicos se integran dentro del elemento conocido como LNB vii.

\footnotetext{
vii low noise block: consiste en un amplificador con $1 \mathrm{~dB}$ de figura de ruido, un mezclador y un oscilador local de banda $\mathrm{X}$
} 
Sin embargo, todavía es posible aumentar un escalón más el nivel de multiplexación si consideramos las señales SCM generadas por diversos transmisores ópticos. En este caso, señales SCM a distintas longitudes de onda podrían multiplexarse en el dominio óptico empleando un combinador e inyectarse posteriormente en una misma fibra. Surgen así los sistemas híbridos WDM-SCM [Sha91]. En la figura 1.12 se representa el espectro óptico típico de uno de estos sistemas. En especial, alguna de las portadoras ópticas podría estar modulada directamente por una señal digital en banda base. La ventaja de este esquema de multiplexación conjunto es que permite enrutar cada portadora hacia un determinado nodo óptico en función de la longitud de onda, y posteriormente seleccionar la subportadora de cada usuario en el dominio eléctrico, por lo que aumenta enormemente la flexibilidad. En la figura 1.9 anterior ya se pudo ver la arquitectura de uno de estos sistemas. En [Des90, Smi98], por ejemplo, se proponen también al gunas otras aplicaciones utilizando redes ópticas pasivas.

\subsubsection{Modulación directa frente a modulación externa}

Para inyectar la señal de RF a transmitir por el sistema de comunicaciones ópticas existen dos alternativas claramente diferenciadas, las cuales se representan de forma esquemática en la figura 1.13. Por una parte, la señal de RF puede aplicarse junto con la polarización en la entrada de modulación de un láser, lo cual se conoce con el nombre de modulación directa. Básicamente, todos los sistemas MI-DD con modulación directa utilizan diodos láser con dos diseños de cavidad diferentes: Fabry-Perot (FP) o DFB, aunque algunos enlaces de bajo coste y reducidas prestaciones pueden usar LEDs. La pendiente de la eficiencia de acoplamiento de los láseres disponibles comercialmente varía entre 0,1 y 0,32 W/A [Cox97]. Para modulación directa, la ganancia del enlace se define como

$$
G_{R F}=\frac{Z_{\text {rec }}}{Z_{\text {las }}}\left(\frac{d P}{d l}\right)^{2} t_{\text {opt }}^{2} R^{2}
$$

donde $\mathrm{dP} / \mathrm{dl}$ es la pendiente de la curva potencia óptica-corriente eléctrica del láser (W/A), $t_{\text {opt }}$ representa la atenuación del enlace de fibra, $R$ es la responsividad del fotodetector $(A / W), Z_{\text {las }}$ la impedancia de entrada del láser 


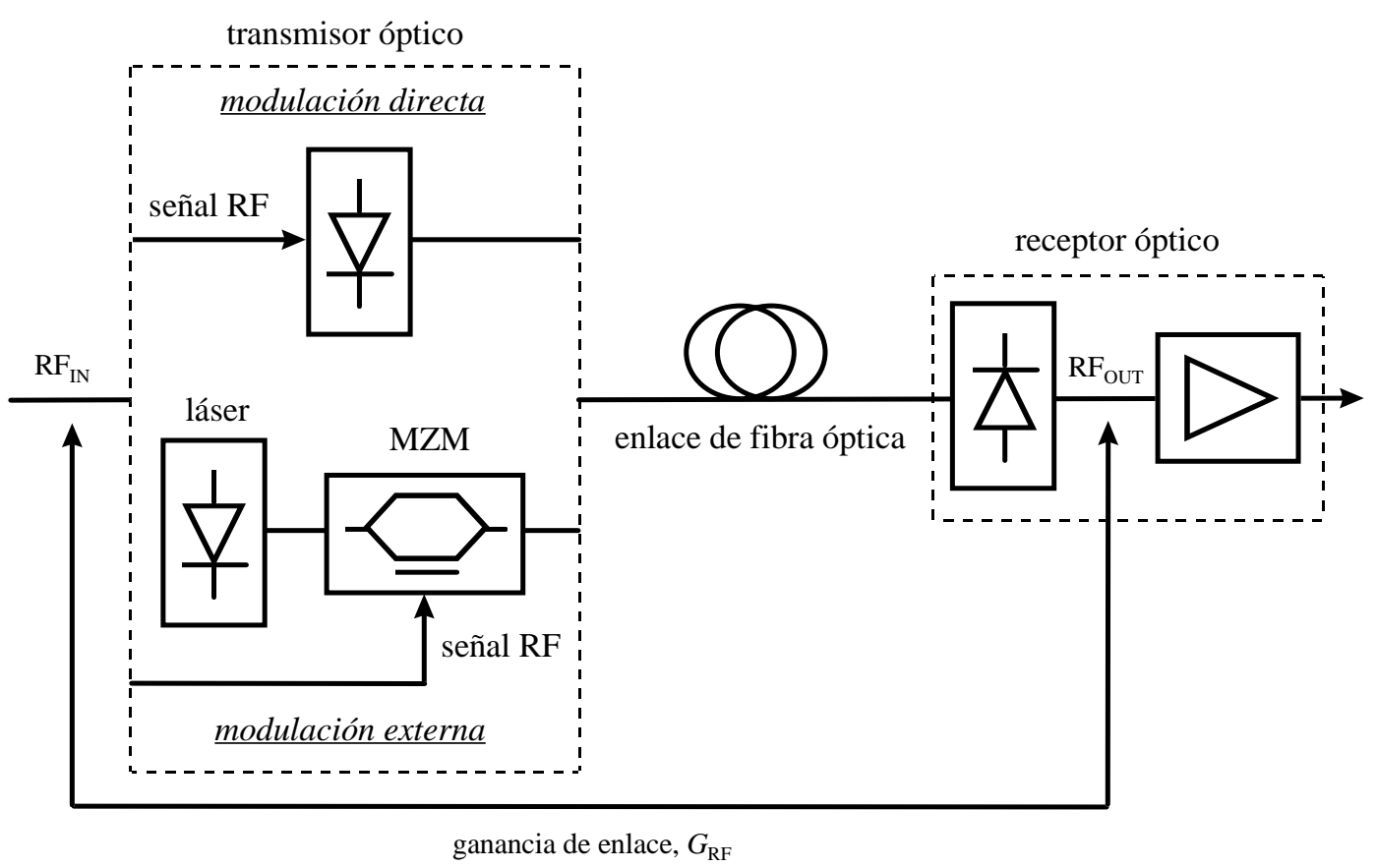

Figura 1.13 Diagrama de bloques comparativo entre sistemas de comunicaciones ópticas analógicos con modulación directa y modulación externa.

y $Z_{\text {rec }}$ la impedancia de entrada del receptor situado tras el fotodiodo. En [Cox97] se presentan medidas de la ganancia de enlace obtenida en diferentes experimentos.

No obstante, uno de los principales problemas de la modulación directa viene determinado por el ancho de banda de modulación limitado que poseen los diodos láser. Los valores típicos se encuentran por debajo de los $10 \mathrm{GHz}$, aunque se han conseguido frecuencias máximas de modulación de hasta $20-30 \mathrm{GHz}$ pero en prototipos de laboratorio. Adicionalmente, su respuesta intrínsecamente no lineal da lugar a niveles de distorsión apreciables, especialmente si se trabaja cerca de la frecuencia de las oscilaciones de relajación [Kuo92a]. Finalmente, en los diodos láser la modulación de intensidad viene acompañada siempre de una modulación de frecuencia que se conoce con el nombre de chirp. Este efecto es directamente proporcional a la amplitud de la señal moduladora y, como se verá en el capítulo 2, da lugar a niveles de distorsión considerables a la salida del 
fotodetector cuando se combina con la dispersión cromática de la fibra [Phi91].

Como alternativa, la modulación externa presenta una serie de ventajas con respecto a la modulación directa de los láseres: es posible conseguir una mayor linealidad y ancho de banda, a la vez que el chirp puede llegar a eliminarse completamente. En este caso, la señal moduladora se inyecta por el puerto de entrada de RF del modulador electroóptico, el cual se polariza por medio de una señal de DC. Ahora, para caracterizar el chirp del modulador externo suele utilizarse el parámetro $\alpha$ [Koy88]. De entre las distintas alternativas para realizar la modulación externa, destacan claramente los moduladores Mach-Zehnder (MZMs) y los moduladores de electroabsorción (EAMs). Los primeros se basan en una estructura interferométrica construida en Niobato de Lítio $\left(\mathrm{LiNbO}_{3}\right)$, mientras que los segundos se basan en la dependencia con el campo eléctrico de la frontera de la banda de absorción del semiconductor, siendo generalmente más no lineales que los MZMs. No obstante, en [Wel95] se demostró que balanceando la influencia del efecto Franz-Keldysh con el efecto Stark de cuantos confinados, es posible fabricar un EAM incluso más lineal que un MZM estándar. Como desventaja, los EAMs admiten valores de potencia óptica a su entrada inferiores. Así, se ha conseguido inyectar en un MZM una potencia óptica de hasta $400 \mathrm{~mW}$, frente a $34 \mathrm{~mW}$ en un EAM [Sun95]. En el caso de modulación externa, la ganancia del enlace viene dada por

$$
\mathrm{G}_{\mathrm{RF}}=\mathrm{Z}_{\mathrm{rec}} \mathrm{Z}_{\mathrm{mod}}\left(\frac{\mathrm{P}_{0} \pi}{2 \mathrm{~V}_{\pi}}\right)^{2} \mathrm{t}_{\mathrm{opt}}^{2} \mathrm{R}^{2}
$$

siendo $Z_{\text {mod }}$ la impedancia de entrada del modulador, $V_{\pi}$ el voltaje de media onda y $\mathrm{P}_{0}$ la potencia óptica media a la salida. Las ganancias de enlace obtenidas en algunos experimentos con modulación externa también se resumen en [Cox97].

Tanto en el caso de modulación directa como externa, el campo eléctrico a la salida del transmisor óptico puede finalmente expresarse como 


$$
E(t)=\sqrt{P_{0}} \sqrt{1+m_{i} \cos \left(2 \pi f_{m} t\right)} \exp \left\{j\left[\omega_{0} t+m_{f} \cos \left(2 \pi f_{m} t+\varphi\right)\right]\right\},
$$

donde $\omega_{0}$ es la pulsación de la portadora óptica, $f_{m}$ es la frecuencia de modulación, $m_{i}$ y $m_{f}$ son respectivamente los índices de modulación de intensidad y de fase, y $\varphi$ representa un desfase entre ambas modulaciones. Estos dos últimos parámetros caracterizan el chirp del dispositivoviii.

\subsubsection{Sistemas radio sobre fibra a frecuencias milimétricas}

Los sistemas radio sobre fibra en la banda de frecuencias milimétricas no se encuentran excesivamente desarrollados, en gran medida debido a la dificultad de obtener dispositivos optoelectrónicos adecuados. Sin embargo, ya se ha comentado que tales sistemas encuentran importantes áreas de aplicación como consecuencia de una serie de ventajas: tamaños de antena más reducidos, gran ancho de banda disponible, menor nivel de regulación, o mayor absorción atmosférica que permite reutilizar la frecuencia en el caso de sistemas celulares. Por lo tanto, para desplegar estos sistemas a gran escala, es necesario encontrar métodos de bajo coste para la generación y detección de la señal óptica a frecuencias milimétricas.

En la actualidad, la máxima frecuencia de modulación de los diodos láser se encuentra en torno a los $30 \mathrm{GHz}$ [Ral94], por lo que no resulta factible la modulación directa en el caso de frecuencias milimétricas. Por otro lado, aunque existen disponibles comercialmente moduladores electroópticos trabajando a frecuencias de hasta $50 \mathrm{GHz}$, o incluso optimizados en una banda en particular (por ejemplo, $60 \mathrm{GHz}$ [Min98]), estos dispositivos son caros y requieren altos voltajes. No obstante, se está investigando activamente la modulación óptica a frecuencias milimétricas utilizando componentes optoelectrónicos de baja frecuencia más baratos. De hecho, la investigación se enfoca al descubrimiento de nuevas técnicas en lugar de a intentar mejorar las prestaciones o funcionalidad de los dispositivos existentes. En especial, se han estudiado diferentes métodos: modulación de las resonancias de la respuesta de un láser [Geo94], generación armónica [Rei92, Moo95] o mezclado óptico coherente [Oga91]. 
La primera de las técnicas se ha demostrado utilizando láseres de cavidad externa, donde se realzan aquellas frecuencias que ocurren a múltiplos del tiempo de recorrido de la cavidad (round-trip time). Por otro lado, las técnicas de generación armónica permiten utilizar componentes optoelectrónicos a frecuencias relativamente bajas como parte integrante del sistema de milimétricas. En todos estos componentes se aprovecha su respuesta óptica no lineal ante la señal eléctrica de entrada. Así, tanto láseres como moduladores electroópticos pueden utilizarse para este fin, obteniéndose una generación de frecuencias milimétricas eficiente empleando armónicos de órdenes elevados. Finalmente, el mezclado óptico coherente también puede utilizarse para generar señales milimétricas. Si dos portadoras ópticas coherentes inciden en un mismo fotodiodo, entonces se generará una componente de señal a la salida del mismo a la frecuencia diferencia entre ambas portadoras. Por ejemplo, una diferencia de 0,5 nm a una longitud de onda central de $1550 \mathrm{~nm}$ producirá un batido de señal a una frecuencia ligeramente por encima de los $60 \mathrm{GHz}$. No obstante, esta técnica sufre en general de problemas tales como estabilidad o pureza espectral de las señales generadas. La generación armónica, por el contrario, produce señales con una pureza espectral derivada del oscilador de referencia utilizado, por lo que se puede llegar a sintetizar señales con anchos de línea inferiores a $1 \mathrm{~Hz}$.

En lo referente a la detección, los fotodiodos de alta velocidad disponibles comercialmente son caros y poseen baja eficiencia. La búsqueda de fotodiodos para frecuencias milimétricas de alta eficiencia y potencialmente bajo coste de fabricación es también objeto de investigación. Algunos ejemplos incluyen los fotodiodos integrados monolíticamente con preamplificadores ópticos y, los fototransistores bipolares de heterounión (photoHBTs).

Un diagrama de bloques de la configuración de transceptor de milimétricas típico se muestra en la figura 1.14. La señal óptica entrante se fotodetecta y se filtra una de sus bandas laterales para posteriormente amplificarla y

viii consúltese el apéndice I 


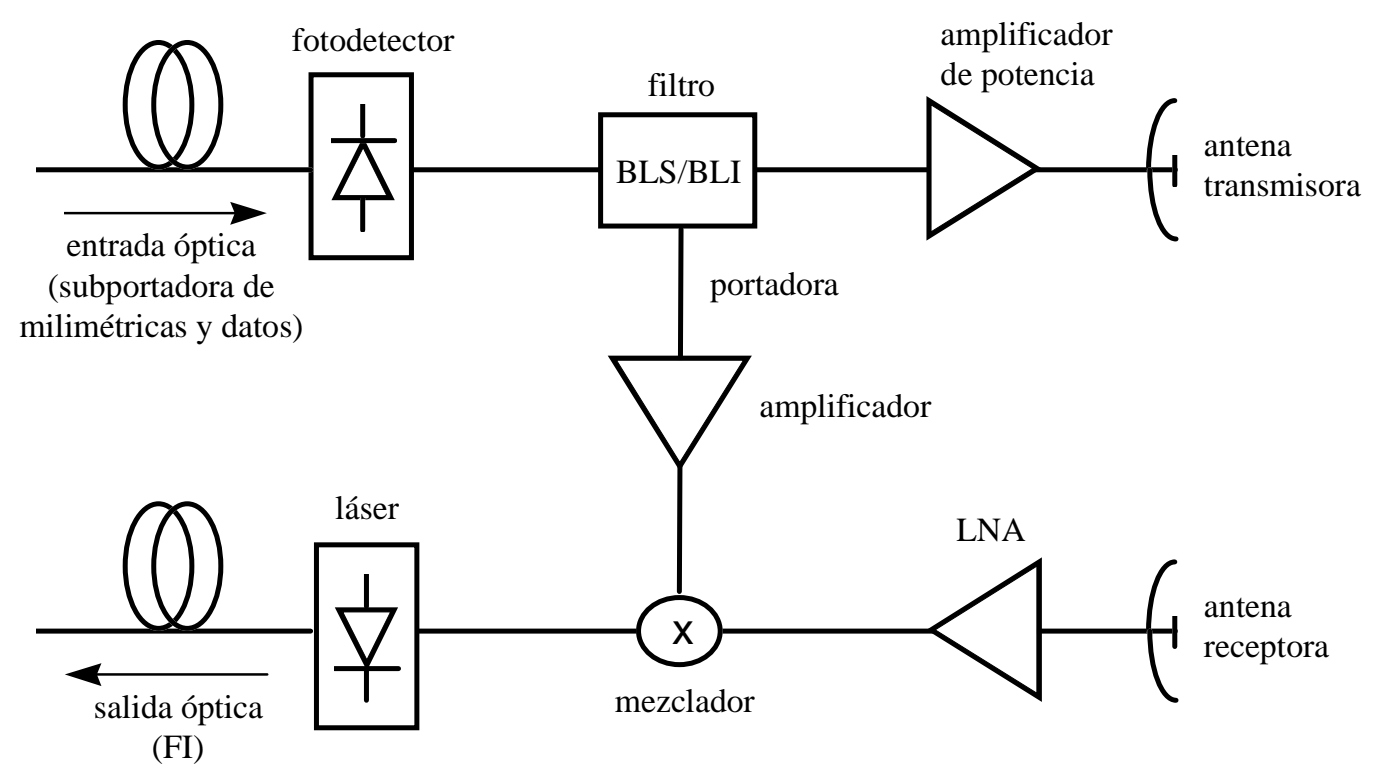

Figura 1.14 Diagrama esquemático de la configuración de un transceptor de milimétricas típico para aplicaciones de alimentación remota de antenas.

transmitirla a la estación móvil. El filtro también sirve para proporcionar la portadora de milimétricas al enlace de retorno. Por otra parte, la señal recibida del móvil se amplifica antes de convertirla a FI y se transmite de vuelta por la fibra óptica utilizando un láser. De este modo, esta configuración simplifica enormemente la optoelectrónica del enlace de retorno, puesto que se puede utilizar un láser de baja frecuencia modulado directamente.

Uno de los métodos utilizados para generar señales milimétricas hace uso de un láser modulado en frecuencia por medio de una señal de control aplicada a uno de sus terminales [Wal92]. El espectro óptico de un láser modulado en frecuencia contiene varias líneas separadas por la frecuencia de la señal de modulación, y es posible generar frecuencias milimétricas por medio de batidos de estas bandas laterales en un fotodetector. Sin embargo, una señal FM pura tiene una intensidad constante y no producirá ninguna fotocorriente a armónicos de la frecuencia de modulación. Por lo tanto, la señal se propaga a través de una fibra óptica dispersiva para que la fase relativa de las bandas laterales se altere y se puedan obtener armónicos de la señal moduladora a la salida del fotodetector. En [Wak92] se realizó un 
experimento de transmisión de vídeo a $40 \mathrm{GHz}$ (MVDS) utilizando esta misma técnica. La fuente óptica consistía en un láser BH DFB con una eficiencia de modulación de aproximadamente $1 \mathrm{GHz} / \mathrm{mA}$. La señal moduladora era un tono de $4 \mathrm{GHz}$ aplicado a la sección central del láser y la salida se inyectaba en $12,5 \mathrm{~km}$ de fibra monomodo convencional. Finalmente, como receptor óptico se empleó un fotodetector de alta velocidad, a la salida del cual se seleccionaba el décimo armónico y después se transmitía vía radio por medio de antenas.

Aunque la técnica de conversión $\mathrm{FM}$-IM anterior se ha demostrado como un método efectivo para generar señales milimétricas, son necesarios láseres con desviaciones de frecuencia grandes para conseguir buenas eficiencias. Además, esta técnica no resulta útil con longitudes de fibra reducidas o fibras de dispersión desplazada. Para evitar estas limitaciones, en [Mar99, Mar99a] se propone el uso de moduladores de fase conjuntamente con redes de difracción sobre fibra óptica (CFG, chirped fiber grating). Los resultados experimentales demuestran la generación de subportadoras a $28 \mathrm{GHz}$ con prestaciones de ruido de fase excelentes $(-87 \mathrm{dBc} / \mathrm{Hz} @ 10 \mathrm{kHz})$ y la conversión en frecuencia y transmisión libre de errores de señales QPSK.

Posibles alternativas a esta técnica incluyen el uso de moduladores externos, como por ejemplo MZMs o EAMs. La generación de armónicos de la frecuencia de modulación en un MZM se basa en la característica no lineal de su función de transferencia. En particular, la característica es sinusoidal, existiendo tres zonas típicas de funcionamiento. La zona lineal es la de funcionamiento normal, aunque utilizando excursiones de modulación elevadas es posible obtener armónicos de orden $3,5,7, \ldots$ como consecuencia de su comportamiento impar. Adicionalmente, las zonas no lineales conocidas como MITB ${ }^{i x}$ y MATB ${ }^{x}$ producen armónicos de orden 2, 4, 6 , ... como consecuencia de su comportamiento par. En [Sch94a, Sch96, Pol98, Pol99a] se realizaron diversos experimentos de generación y subida a frecuencias milimétricas empleando MZMs. Por último, los EAMs son una prometedora alternativa a los MZMs para la generación armónica debido a

\footnotetext{
ix MITB (minimum transmission bias)
} 
que su función de transferencia es más no lineal. Algunos resultados experimentales de generación armónica empleando un EAM se presentan en [Mo095], junto con un estudio comparativo de los niveles obtenidos en el caso de utilizar un MZM. El modelado de ambos se puede consultar en el apéndice I.

\subsubsection{Medida de calidad: ruido y distorsión}

Para evaluar la calidad de los sistemas SCM se suelen utilizar las relaciones portadora a ruido (CNR) y portadora a interferencia (CIR) a la salida del fotodetector, las cuales representan el cociente entre la potencia el éctrica de portadora del canal y la potencia eléctrica de ruido o distorsión, respectivamente, que cae dentro del ancho de banda del receptor. La primera de ellas tiene en cuenta el ruido introducido a lo largo del sistema, tanto en la parte óptica como eléctrica. Así, se tendrían términos de ruido debidos a RIN (Relative Intensity Noise, ruido de intensidad relativo), ASE (Amplified Spontaneous E mission, emisión espontánea amplificada), térmico o shot.

En la mayor parte de los enlaces con modulación directa, el RIN del láser predomina sobre los ruidos shot o térmico. La corriente de ruido a la salida del fotodetector debida a RIN se expresa como

$$
\sigma_{\mathrm{RIN}}^{2}=\left(\mathrm{RP}_{\mathrm{rec}}\right)^{2} \mathrm{RIN} B \text {, }
$$

donde $\mathrm{P}_{\text {rec }}$ es la potencia óptica a la entrada, $\mathrm{B}$ es el ancho de banda del receptor y RIN es el parámetro que caracteriza las fluctuaciones de potencia del láser y que se mide en $\mathrm{dB} / \mathrm{Hz}$. En (1.4) se observa que el ruido causado por el RIN del láser aumenta con el cuadrado de $\mathrm{P}_{\text {rec }}$. Dado que la potencia eléctrica de la señal recibida presenta la misma dependencia con $\mathrm{P}_{\text {rec }}$ la CNR debida al RIN se mantiene constante con la potencia óptica recibida. Como estimación, el RIN del transmisor debe mantenerse por debajo de $150 \mathrm{~dB} / \mathrm{Hz}$ para conseguir una CNR de $50 \mathrm{~dB}$, si se considera un índice de modulación $m_{i}=0,1$ y un ancho de banda $B=50 \mathrm{MHz}$ como valores

× MATB (maximum transmission bias) 
representativos. De hecho, se han desarrollado láseres DFB con valores bajos de RIN para aplicaciones CATV, donde suelen utilizarse modulaciones AM-VSB que requieren $50 \mathrm{~dB}$ de CNR.

Por otro lado, los enlaces con modulación externa pueden utilizar como fuente óptica láseres de estado sólido [Cox97], los cuales se caracterizan por poseer frecuencias de relajación de unos cientos de kHz. Como el espectro de RIN decae como $\mathrm{f}^{-2}$ por encima de esta frecuencia de relajación [Pet91 (cap. 7)], se obtienen valores de RIN despreciables en la mayoría de los sistemas una vez que se aumenta la frecuencia inferior de la banda de transmisión. Por lo tanto, el ruido dominante es ahora el ruido shot del fotodetector, el cual se expresa como

$$
\sigma_{s}^{2}=2 q\left(R P_{\text {rec }}+I_{d}\right) B
$$

siendo q la carga del electrón y $I_{d}$ la corriente de oscuridad, que suele despreciarse en la práctica.

No obstante, el ruido RIN puede llegar a ser un problema incluso empleando transmisores de bajo RIN. La razón se debe a que el RIN puede aumentar como consecuencia de la transmisión por la fibra óptica. Uno de tales mecanismos se debe a reflexiones múltiples entre dos extremos de un enlace, ya que las dos superficies reflectantes actúan como un interferómetro FP y convierten el ruido de fase en ruido de intensidad [Bet98]. Este fenómeno puede evitarse empleando láseres de ancho de línea estrecho. Adicionalmente, la dispersión cromática de la fibra también puede provocar una conversión del ruido de fase del láser en ruido de intensidad, originándose un RIN inducido por dispersión a la entrada del fotodetector que aumenta cuadráticamente con la longitud de fibra. Este efecto ha sido estudiado por varios autores [Pet90, Yam90, Sch97], y la solución consiste en emplear técnicas de compensación de dispersión [Mar96].

Por último, en el caso de enlaces de gran longitud o en los que la potencia óptica recibida sea reducida, el ruido predominante será el térmico, el cual viene dado por 


$$
\sigma_{T}^{2}=\frac{4 k_{B} T F_{n} B}{Z_{\text {rec }}},
$$

donde $\mathrm{K}_{\mathrm{B}}$ es la constante de Boltzmann, $\mathrm{T}$ es la temperatura absoluta y $\mathrm{F}_{\mathrm{n}}$ representa el factor de ruido del preamplificador eléctrico. En la figura 1.15 se representan las zonas donde predomina cada uno de los ruidos comentados.

La expresión de la CNR para un enlace de comunicaciones ópticas analógico queda final mente como

$$
\mathrm{CNR}=\frac{Z_{\text {rec }}\left(m_{i} R P_{\text {rec }}\right)^{2} / 2}{\text { Noise }}=\frac{\left(m_{i} R P_{\text {rec }}\right)^{2} / 2}{\sigma_{\mathrm{s}}^{2}+\sigma_{\mathrm{T}}^{2}+\sigma_{\mathrm{RIN}}^{2}+\sigma_{\text {ASE }}^{2}},
$$

donde se ha añadido un término de ruido adicional debido al ASE de los amplificadores ópticos que puedan existir a lo largo del trayecto de fibra [Agr97 (cap. 8)].

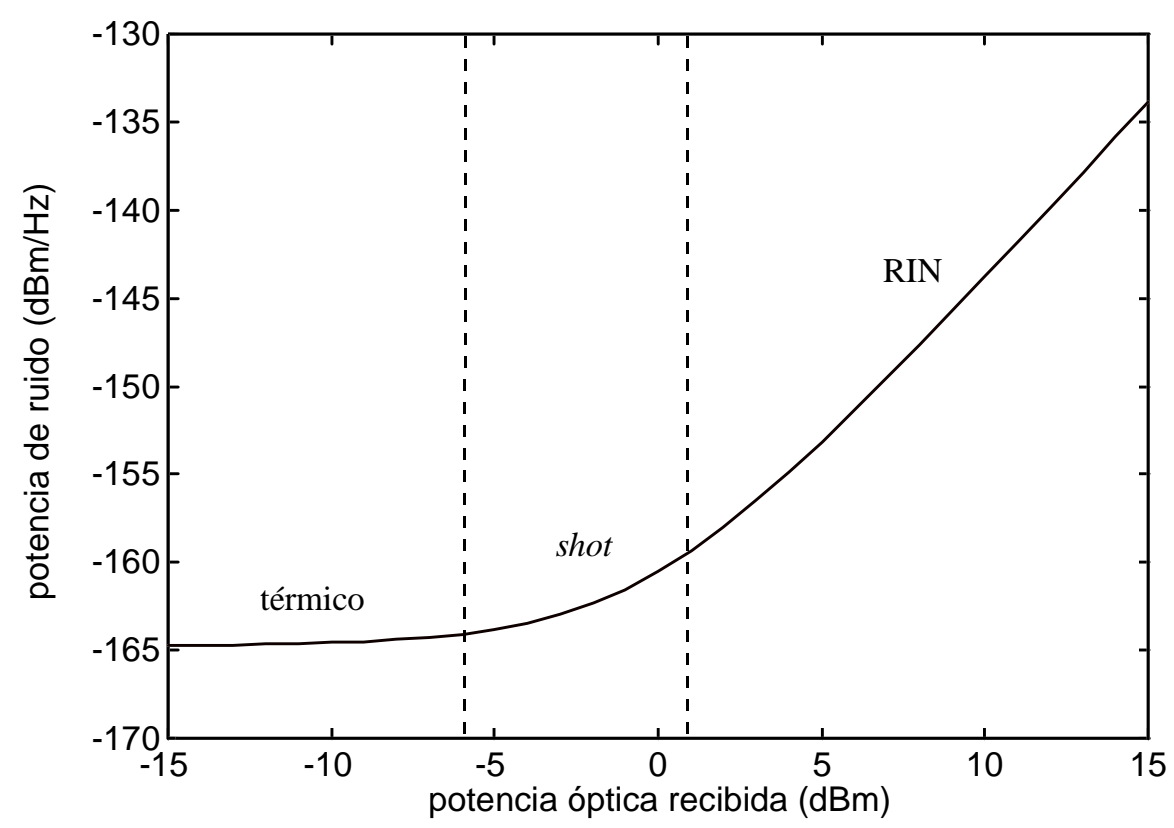

Figura 1.15 Contribuciones de las fuentes de ruido térmico, shot y RIN. Otros parámetros: $T=290 \mathrm{~K}, \mathrm{~F}_{\mathrm{n}}=3 \mathrm{~dB}, \mathrm{RIN}=-150 \mathrm{~dB} / \mathrm{Hz}$ y $Z_{\text {rec }}=50 \Omega$. 
En el caso de la distorsión no lineal que aparece a la salida del fotodetector, ésta se modela por medio de la CIR. En este término se incluye tanto la distorsión armónica como la de intermodulación. N ormalmente, la CIR suele expresarse a partir de los parámetros de CSO (composite second order) y CTB (composite triple beat), los cuales representan el cociente entre la potencia de la distorsión de segundo o tercer orden, respectivamente, y la potencia de portadora del canal. Así pues, se tiene que

$$
\mathrm{CIR}^{-1}=\mathrm{CSO}^{-1}+\mathrm{CTB}^{-1} \text {. }
$$

Usualmente, la medida de la calidad del transporte de la señal en sistemas CATV incluye las distorsiones de segundo y tercer orden. No obstante, en el caso de sistemas multicanal que emplean una banda de frecuencias inferior a una octava, sólo sería necesario considerar el CTB puesto que el CSO cae fuera de banda.

La existencia de amplificadores ópticos basados en fibra a $1300^{x i}$ y $1550^{x i i}$ $\mathrm{nm}$ es de gran relevancia para el transporte de señales AM-SCM [Miy91, Dai96]. La alta potencia disponible y el bajo nivel de ruido de los amplificadores de fibra proporciona un potencial número de usuarios que pueden servirse con la misma calidad de señal a partir de una única fuente en arquitecturas punto a multipunto. Adicionalmente, la lenta saturación de la ganancia dinámica de estos dispositivos permite mantener la linealidad del sistema en ausencia de chirp del transmisor óptico [Che91]. Para sistemas de larga distancia, es más deseable combinar el uso de láseres DF B con amplificadores de alta potencia. De este modo, se tendría una configuración consistente en un láser DFB modulado directamente, seguido de uno o más amplificadores ópticos para alcanzar el nivel de potencia necesario y, finalmente, un enlace de fibra óptica. Dado que la calidad del transporte se mide por las prestaciones del sistema, la consideración individual de cada una de las componentes de distorsión que se generan resulta insuficiente. En el caso del láser DFB, la oscilación de relajación da lugar a distorsiones dependientes de la frecuencia. Esta distorsión, a su vez,

\footnotetext{
xi amplificador de fibra dopada con praseodimio (PDFA)

xii amplificador de fibra dopada con erbio (EDFA)
} 


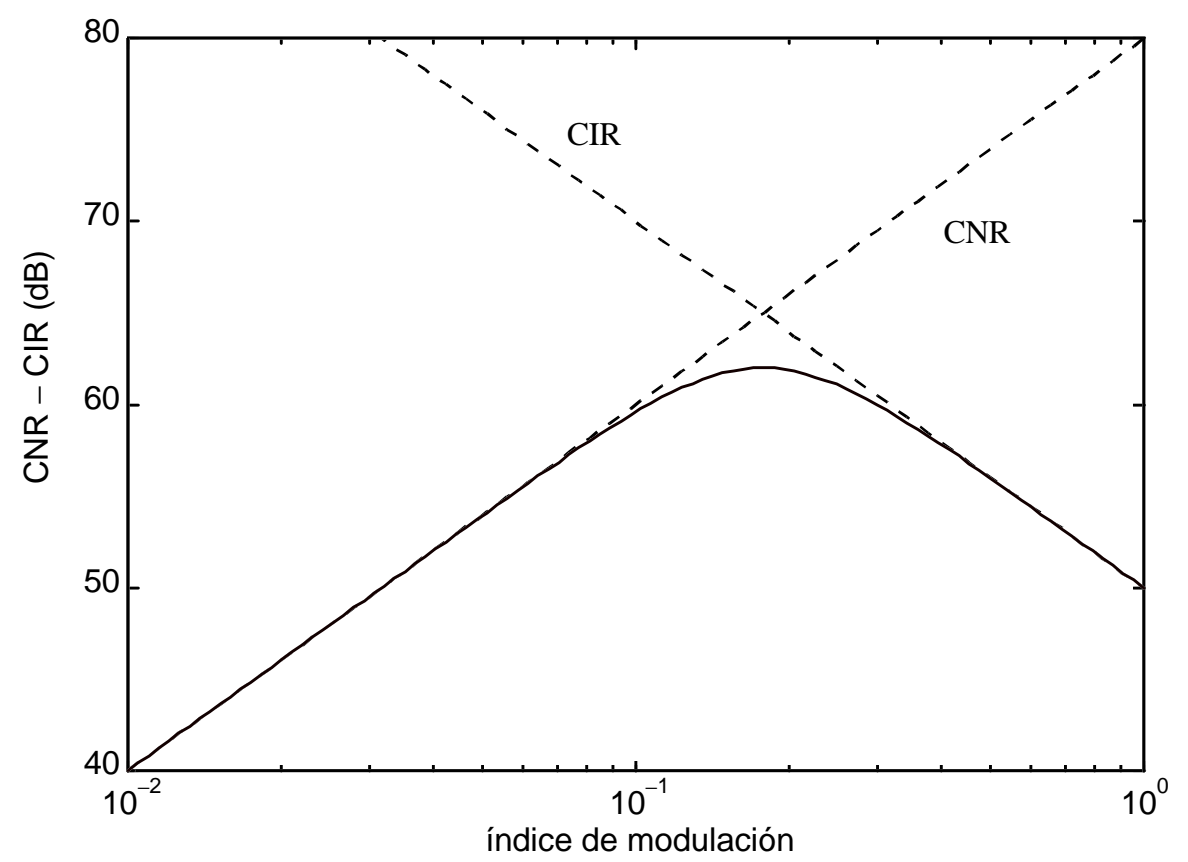

Figura 1.16 Limitaciones impuestas a la calidad del sistema SCM por la CNR y CIR en función del índice de modulación de intensidad.

se combina con la debida a la saturación de la ganancia, que es independiente de la frecuencia y proporciona un primer nivel de complejidad [Ku092].

Además de las distorsiones dinámicas, surge distorsión adicional como consecuencia de la interacción entre el perfil de ganancia del amplificador de fibra y el chirp de frecuencia inherente a la modulación del láser [Kuo91]. Incluso la combinación del chirp del transmisor óptico con la dispersión cromática de la fibra da lugar a niveles de distorsión considerables a la salida del fotodetector [Ber91, Cro94, Phi91]. Dado que el chirp del transmisor óptico afecta significativamente a los niveles de distorsión, una posible solución sería el uso de modulación externa tal y como se ha comentado con anterioridad. En el capítulo 2 analizaremos en detalle la distorsión introducida por la fibra óptica con especial atención a la influencia que tienen las no linealidades. 
En la figura 1.16 se representan las contribuciones de CNR y CIR a la calidad del sistema SCM en función del índice de modulación. Por una parte, la ec. (1.7) muestra que la CNR es proporcional a $\mathrm{m}_{\mathrm{i}}^{2}$. Por otra parte, en el capítulo 2 se verá que la $\mathrm{CIR}$ es proporcional a $1 / \mathrm{m}_{i}^{2}$. Esto significa que cuando $m_{i}$ es pequeño, el ruido limita la calidad, mientras que cuando $m_{i}$ es grande, predomina la distorsión no lineal. En especial, se observa que existe un val or óptimo de $m_{i}$ que, en el caso de la figura, se sitúa en torno a 0,2. 


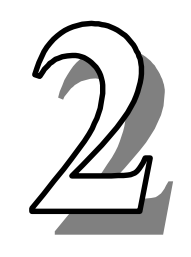

\section{Distorsión no lineal introducida por la fibra óptica}

\subsection{INTRODUCCIÓN}

La utilización de técnicas de multiplexación de subportadoras (SCM) para la transmisión de señales de vídeo multicanal sobre enlaces ópticos punto a punto ha sido estudiada en detalle [Dar86, Ols87, Ols89]. Aunque algunos sistemas operan en la región de los 1300 nm, es deseable operar en tercera ventana con el objetivo de reducir las pérdidas de la fibra y permitir el uso de amplificadores ópticos EDFA. En la región de $1550 \mathrm{~nm}$, la fibra monomodo estándar presenta un incremento considerable de la dispersión cromática, lo cual da como resultado distorsión no lineal [Mes84, Ih90]. En el caso de modulación directa, este efecto se agrava, ya que el chirp generado por el diodo láser se combina con la dispersión de la fibra dando lugar a niveles de distorsión intolerables [Yon91, Ber91, Phi91, Cro94, Bet97]. El empleo de moduladores externos es una solución ante el problema 
del chirp. No obstante, el efecto Kerr de las fibras ópticas cuando se someten a niveles de potencia óptica elevados se convierte entonces en la principal fuente de degradación [Phi91, Des94]. El fenómeno de self-phase modulation (SPM) debido a la no linealidad de la fibra interacciona con la dispersión cromática limitando la distancia de transmisión en los sistemas MI-DD con modulación externa [Wil96, Gop96].

En este capítulo se estudia en profundidad la distorsión no lineal introducida por las fibras ópticas monomodo dispersivas y no lineales. En primer lugar, en la sección 2.2 se introducen dos modelos para la fibra óptica: lineal que incluye sólo la dispersión cromática, y no lineal que tiene en cuenta además el efecto Kerr. En este último caso, se comenta un algoritmo que se utiliza para resolver la propagación a través de una fibra óptica dispersiva y no lineal y que se conoce con el nombre de split-step Fourier method [Wei86]. Este método produce muy buenos resultados y será el que utilizaremos en nuestras simulaciones. En la sección 2.3 se analiza ya en profundidad la distorsión no lineal, incluyendo expresiones del CSO (composite second order) y CTB (composite triple beat) para los casos de dispersión y efecto Kerr [Phi91, Des94]. En esta sección se presentan los primeros resultados de simulación empleando una única subportadora (distorsión armónica). A continuación, en la sección 2.4 se estudia la penalización de potencia inducida por la fibra óptica. Esta es una de las consecuencias de la distorsión no lineal y se manifiesta en un efecto de supresión de la portadora que ocurre para determinadas longitudes de fibra y frecuencias de modulación [Sch95]. El análisis del margen dinámico se lleva a cabo en la sección 2.5, donde se presentará una expresión teórica del mismo para enlaces ópticos dispersivos, así como al gunos resultados. En la sección 2.6 se realiza un completo estudio de distorsión no lineal en sistemas radio-fibra reales. Para ello, se presentan resultados de simulación relativos a CSO, CTB, penalización de potencia, margen dinámico, etc. para diversos sistemas multicanal: CATV, MMDS, LMDS y MVDS. Por último, un análisis comparativo de las técnicas de compensación de dispersión basadas en la modulación óptica en banda lateral única y en las redes de difracción sobre fibra óptica se realiza en la sección 2.7. 


\subsection{MODELADO DE LA FIBRA ÓPTICA}

\subsubsection{Modelo lineal de la fibra}

La fibra óptica se puede considerar como un dispositivo óptico lineal cuando los niveles de potencia a la entrada de la misma son reducidos y su longitud no es excesivamente elevada como para potenciar efectos no lineales. En este caso, la función de transferencia que modela la propagación del campo eléctrico a lo largo de una fibra de longitud $L$ viene dada por

$$
H(\omega)=\exp [(-\alpha / 2-j \beta(\omega)) L],
$$

donde $\alpha$ representa las pérdidas (expresadas normalmente en $\mathrm{dB} / \mathrm{km}^{\mathrm{i}}$ ) y $\beta(\omega)$ es la constante de propagación. Desarrollando en serie de Taylor este último término al rededor de la frecuencia central $\omega_{0}$ se obtiene

$$
\beta(\omega)=\beta_{0}+\beta_{1}\left(\omega-\omega_{0}\right)+\frac{1}{2} \beta_{2}\left(\omega-\omega_{0}\right)^{2}+\frac{1}{6} \beta_{3}\left(\omega-\omega_{0}\right)^{3}+\cdots
$$

con

$$
\beta_{\mathrm{m}}=\left.\frac{\mathrm{d} \beta}{\mathrm{d} \omega}\right|_{\omega=\omega_{0}}(m=0,1,2,3, \ldots)
$$

Los coeficientes $\beta_{\mathrm{m}}$ con $\mathrm{m} \geq 2$ son los responsables de la dispersión cromática, también llamada group-velocity dispersion (GVD), mientras que $\beta_{1}$ determina la velocidad a la cual se propaga la informaciónii. Normalmente, en lugar de $\beta_{2}$, suele utilizarse el parámetro $D$ para caracterizar la dispersión de primer orden de las fibras. La relación entre ambos es la siguiente [Agr97 (cap. 2)]:

$$
D=\frac{d \beta_{1}}{d \lambda}=-\frac{2 \pi c}{\lambda^{2}} \beta_{2}
$$

\footnotetext{
${ }^{\mathrm{i}} \alpha(\mathrm{dB} / \mathrm{km})=4,343 \alpha$

ii la vel ocidad de grupo viene dada por $v_{g}=1 / \beta_{1}$
} 
Fibra óptica monomodo (SMF)

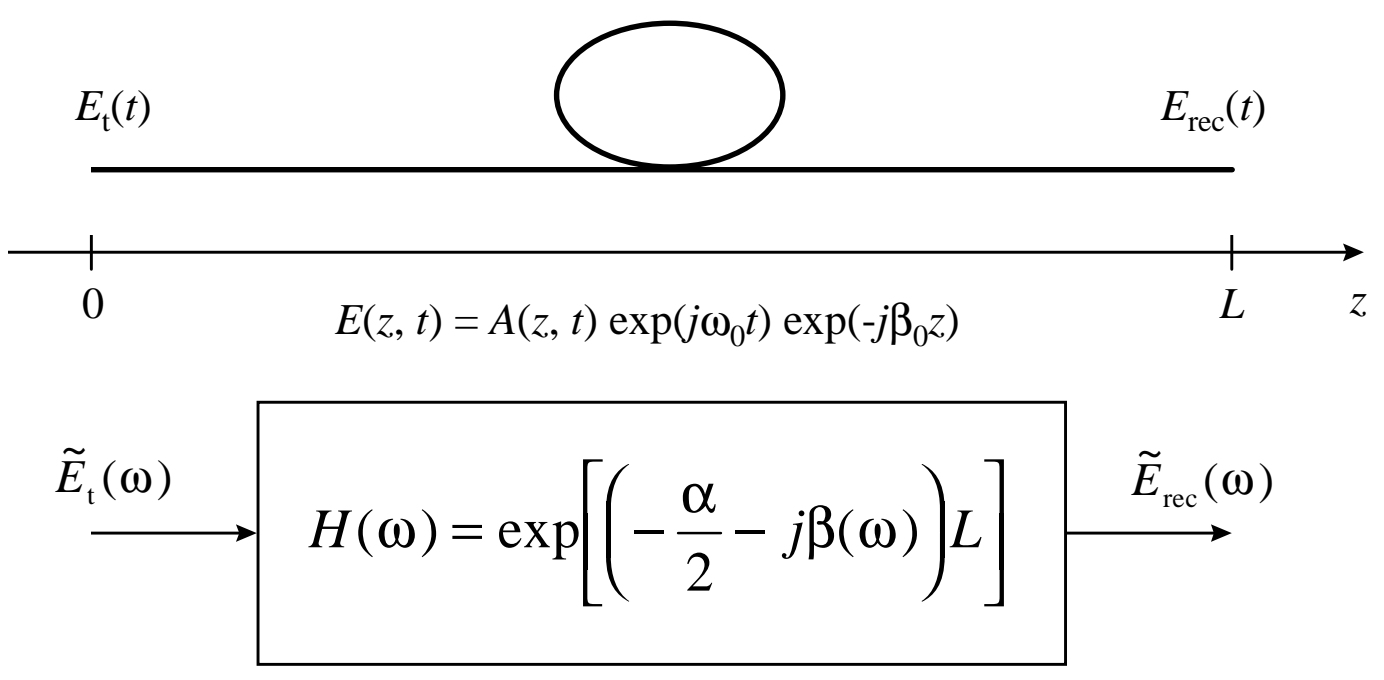

Figura 2.1 Modelo de propagación de una fibra óptica monomodo lineal. $H(\omega)$ es la función de transferencia de una fibra de longitud $L$, con pérdidas $\alpha$ y constante de propagación $\beta(\omega)$.

con $\lambda$ la longitud de onda óptica y c la velocidad de la luz en el vacío. Para las fibras estándar (SSMFs, standard singlemode fibers) operando en tercera ventana se tiene un valor típico $D=17 \mathrm{ps} /(\mathrm{km} \cdot \mathrm{nm})$.

De acuerdo con la figura 2.1, si $E_{t}(t)=E(0, t)$ es el campo eléctrico a la entrada de la fibra de longitud $L$, el campo a su salida $E_{r e c}(t)=E(L, t)$ se calculará mediante

$$
\widetilde{E_{r e c}}(\omega)=H(\omega) \widetilde{E_{t}}(\omega),
$$

donde $\widetilde{E_{r e c}}(\omega)$ y $\widetilde{E_{t}}(\omega)$ son las transformadas de Fourier de $E_{r e c}(t)$ y $E_{t}(t)$ respectivamente, definidas como

$$
\widetilde{E}(z, \omega)=\int_{-\infty}^{\infty} E(z, t) \exp (-j \omega t) d t
$$

A continuación, expresando la transformada de Fourier del campo eléctrico como

$$
\widetilde{E}(z, \omega)=\widetilde{A}\left(z, \omega-\omega_{0}\right) \exp \left(-j \beta_{0} z\right),
$$


siendo $\widetilde{A}\left(z, \omega-\omega_{0}\right)$ la transformada de Fourier de la envolvente compleja $A(z, t)$, se llega finalmente a partir de (2.1) y (2.2) a la siguiente ecuación en derivadas parciales que modela la propagación a lo largo de una fibra óptica dispersiva y con pérdidas:

$$
\frac{\partial A}{\partial z}+\beta_{1} \frac{\partial A}{\partial t}-\frac{j}{2} \beta_{2} \frac{\partial^{2} A}{\partial t^{2}}-\frac{1}{6} \beta_{3} \frac{\partial^{3} A}{\partial t^{3}}+\frac{\alpha}{2} A=0,
$$

donde se han despreciado los efectos dispersivos de orden superior.

\subsubsection{Modelo no lineal de la fibra}

Cuando los niveles de potencia óptica dejan de ser reducidos, el índice de refracción de la fibra muestra una dependencia con la intensidad de campo el éctrico en un fenómeno conocido como efecto Kerr [Mar91]. En este caso, la expresión del mismo está dada por

$$
\mathrm{n}=\mathrm{n}_{0}(\omega)+\mathrm{n}_{2}|\mathrm{E}|^{2}
$$

donde $n_{0}(\omega)=\varnothing(\omega) / \omega$ incluye los efectos dispersivos comentados en la sección anterior y $n_{2}$ es el índice de refracción no lineal que se calcula como

$$
\mathrm{n}_{2}=\frac{\mathrm{N}_{2}}{\mathrm{~A}_{\mathrm{eff}}}
$$

con el índice no linealiii, $\mathrm{N}_{2}$, y el área efectiva del núcleo de la fibra, $A_{\text {eff. }}$ Este último depende de parámetros de la fibra tales como el radio del núcleo o la diferencia de índices entre núcleo y cubierta. Los valores típicos se encuentran en el rango de 50-80 $\mu \mathrm{m}^{2}$ para la región de $1550 \mathrm{~nm}$.

En esta situación, la ec. (2.8) se ve modificada para incluir los efectos de las no linealidades, dando lugar a la que se conoce como ecuación de Schrödinger nolineal con pérdidas [Agr89]

iii $\mathrm{N}_{2}=3,2 \cdot 10^{-16} \mathrm{~cm}^{2} \mathrm{~W}$ para fibras de sílice 


$$
\frac{\partial A}{\partial z}+\beta_{1} \frac{\partial A}{\partial t}-\frac{j}{2} \beta_{2} \frac{\partial^{2} A}{\partial t^{2}}-\frac{1}{6} \beta_{3} \frac{\partial^{3} A}{\partial t^{3}}+\frac{\alpha}{2} A=-j k n_{2}|A|^{2} A,
$$

donde $k=2 \pi / \lambda$. Esta ecuación, para $\alpha=0$, ha sido extensamente estudiada en el contexto de los solitones [Dod82, Agr97 (cap. 10)].

Normalmente, la dispersión de segundo orden suele despreciarse $\left(\beta_{3}=0\right)$, y al mismo tiempo se realiza el cambio de variable $\mathrm{T}=\mathrm{t}-\beta_{1} \mathrm{z}$ para expresar (2.11) de una forma más compacta:

$$
\frac{\partial A}{\partial z}-\frac{j}{2} \beta_{2} \frac{\partial^{2} A}{\partial T^{2}}+\frac{\alpha}{2} A=-j k n_{2}|A|^{2} A .
$$

De (2.12) puede deducirse que las no linealidades de la fibra producen un chirp de frecuencia inducido por la propia señal que se propaga, fenómeno conocido como self-phase modulation (SPM). El desplazamiento de fase no lineal provocado por este chirp se puede estimar como

$$
\phi_{\mathrm{nl}}=k \mathrm{n}_{2} \mathrm{P}_{0} \mathrm{~L}_{\text {eff }},
$$

donde $\mathrm{P}_{0}$ es la potencia óptica a la entrada de la fibra y $L_{\text {eff }}$ es la longitud efectiva que está dada por

$$
\mathrm{L}_{\mathrm{eff}}=\frac{1-\exp (-\alpha \mathrm{L})}{\alpha}
$$

La combinación del SPM con la dispersión cromática de la fibra veremos posteriormente que da lugar a la aparición de distorsión no lineal a la salida del fotodetector [Phi91, Des94].

\subsubsection{Split-step Fourier method}

La ecuación de propagación (2.12) es una ecuación en derivadas parciales no lineal que generalmente no conduce a soluciones analíticas salvo para casos muy concretos en que el Inverse Scattering method puede ser aplicado [Zak72]. Por lo tanto, es necesario aplicar algún método numérico para poder estudiar los fenómenos no lineales que ocurren en las fibras ópticas. 
EI más ampliamente utilizado para resolver el problema de propagación en un medio dispersivo y no lineal ha sido el split-step Fourier method [Wei86, Agr89 (cap. 2)]. La velocidad relativa de este método con respecto a otros basados en diferencias finitas se debe a la aplicación de la transformada rápida de Fourier (FFT, fast Fourier transform). A continuación describiremos detalladamente en qué consiste este método.

En primer lugar, la ecuación de propagación (2.12) se escribe de la forma

$$
\frac{\partial A}{\partial z}=(\hat{D}+\hat{N}) A ，
$$

donde $\hat{D}$ es un operador diferencial que tiene en cuenta la dispersión y la atenuación en un medio lineal y $\hat{N}$ es un operador no lineal que gobierna el efecto de las no linealidades de la fibra. Estos operadores vienen dados por

$$
\begin{aligned}
& \hat{D}=\frac{j}{2} \beta_{2} \frac{\partial^{2}}{\partial T^{2}}-\frac{\alpha}{2}, \\
& \hat{N}=-j k n_{2}|A|^{2} .
\end{aligned}
$$

En general, la dispersión y las no linealidades actúan de manera conjunta a lo largo de la fibra. Sin embargo, el split-step Fourier method obtiene una solución aproximada suponiendo que en la propagación del campo óptico sobre una pequeña distancia $h$, los efectos dispersivos y no lineales pueden calcularse independientemente. De manera más específica, la propagación desde $z$ hasta $z+h$ se lleva a cabo en dos pasos. En un primer paso, sólo actúan las no linealidades, y $\hat{D}=0$ en la ec. (2.15). En un segundo paso, sólo existe dispersión, y $\hat{N}=0$ en la ec. (2.15). Matemáticamente,

$$
A(z+h, T) \approx \exp (h \hat{D}) \exp (h \hat{N}) A(z, T) .
$$

La aplicación del operador exponencial $\exp (\mathrm{h} \hat{\mathrm{D}})$ se realiza en el dominio de Fourier, de acuerdo a la expresión general

$$
\exp (h \hat{D}) B(z, T)=\left\{F^{-1} \exp [h \hat{D}(\omega)] F\right\} B(z, T),
$$


donde $\mathrm{F}$ denota la transformada de Fourier, $\hat{\mathrm{D}}(\omega)$ se obtiene a partir de la ec. (2.16) sustituyendo el operador diferencial $\partial / \partial T$ por $-\mathrm{j} \omega$, y $\omega$ es la frecuencia en el dominio de Fourier. El uso del algoritmo de la FFT permite evaluar la ec. (2.19) de forma relativamente rápida.

La precisión del split-step Fourier method puede mejorarse adoptando un procedimiento diferente para la propagación de la señal óptica sobre un segmento desde $z$ hasta $z+h$. Con este cambio, la ec. (2.18) se reemplaza por

$$
A(z+h, T) \approx \exp \left(\frac{h}{2} \hat{D}\right) \exp \left[\int_{z}^{z+h} \hat{N}\left(z^{\prime}\right) d z^{\prime}\right] \exp \left(\frac{h}{2} \hat{D}\right) A(z, T) .
$$

La principal diferencia es que el efecto de las no linealidades se incluye en la mitad del segmento. Debido a la simetría de los operadores en la ec. (2.20), a este método se le conoce como symmetrized split-step Fourier method. La ventaja de emplear esta forma simétrica es que el término de error resultante es del orden de $\mathrm{h}^{3}$ frente $a \mathrm{~h}^{2}$ para la ec. (2.18).

A continuación, la precisión puede mejorarse todavía más evaluando la integral de (2.20) con una fórmula más precisa que $\mathrm{hN}(\mathrm{z})$, utilizada en (2.18). Una solución simple consiste en emplear la regla de los trapecios para aproximar la integral por

$$
\int_{z}^{z+h} \hat{N}\left(z^{\prime}\right) d z^{\prime} \approx \frac{h}{2}[\hat{N}(z)+\hat{N}(z+h)] .
$$

No obstante, la aplicación de la ecuación anterior no es sencilla, ya que $\hat{N}(z+h)$ se desconoce en el punto medio del segmento situado en $z+h / 2$. Es necesario, pues, seguir un procedimiento iterativo que se inicia reemplazando $\hat{N}(z+h)$ por $\hat{N}(z)$. La ec. (2.20) se utiliza entonces para estimar $A(z+h, T)$, que a su vez sirve para calcular el nuevo valor de $\hat{N}(z+h)$. Aunque este procedimiento iterativo consume tiempo de cálculo, la mejora en la precisión del algoritmo numérico permite tomar valores mayores para el tamaño del paso $\mathrm{h}$. 
Para finalizar, el algoritmo en pseudocódigo del symmetrized split-step Fourier method se muestra a continuación:

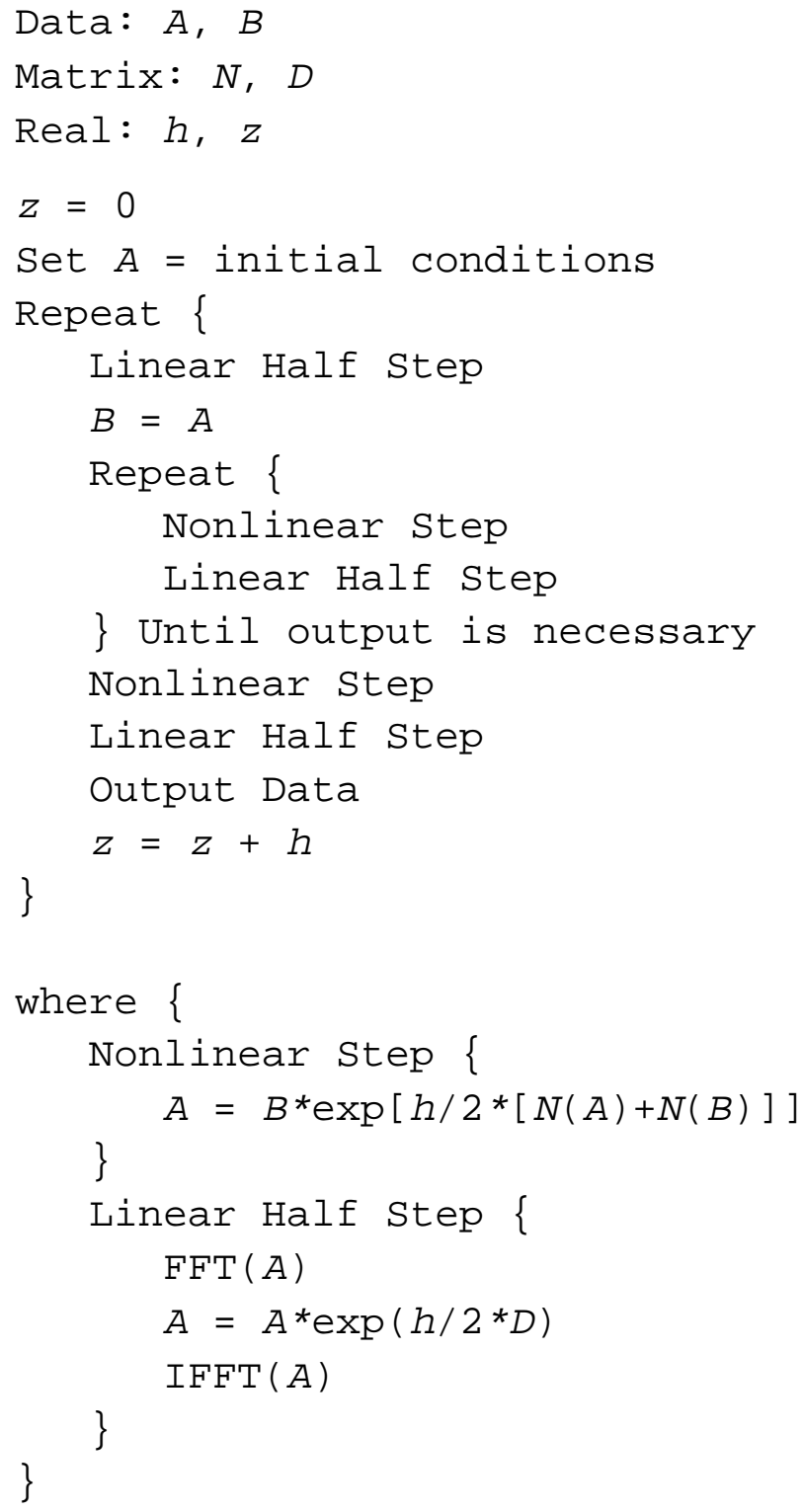




\subsection{DISTORSIÓN NO LINEAL}

La transmisión de señales multicanal a través de fibras dispersivas y no lineales da lugar a la aparición de múltiples términos de distorsión a la salida del fotodetector. Éstos se pueden clasificar en base a distintos criterios que se resumen en la tabla I. La distorsión armónica se genera a frecuencias múltiplos de la subportadora, mientras que la de intermodulación lo hace para una combinación lineal de frecuencias de subportadora. Finalmente, los términos de distorsión de orden superior a tres suelen despreciarse.

TABLA I Clasificación de los términos de distorsión en función de la frecuencia a la cual se generan.

\begin{tabular}{|l|c|c|}
\cline { 2 - 3 } \multicolumn{1}{c|}{} & segundo orden & tercer orden \\
\hline armónicos & $2 \omega_{i}$ & $3 \omega_{i}$ \\
\hline intermodulación & $\omega_{i} \pm \omega_{j}$ & $\omega_{i}, 2 \omega_{i} \pm \omega_{j}, \omega_{i} \pm \omega_{j} \pm \omega_{k}$ \\
\hline
\end{tabular}

Para caracterizar las distorsiones no lineales de segundo y tercer orden a la salida del fotodetector se emplean los parámetros CSO (composite second order) y CTB (composite triple beat), respectivamente. Éstos se definen como el cociente entre los niveles de potencia eléctrica de los términos de distorsión de segundo o tercer orden y el nivel de potencia eléctrica de la subportadora. Considerando el caso más simple en el que transmitimos una única subportadora a frecuencia $\omega_{m}$, la corriente eléctrica a la salida del fotodetector vendrá dada por

$$
I(t)=I_{P}+\sum_{k=1}^{\infty} A_{k} \cos \left(k \omega_{m} t+\theta_{k}\right)
$$

donde existe un término de continua, $I_{p}, y$ aparecen una serie de armónicos a frecuencias múltiplos de $\omega_{\mathrm{m}}$ como consecuencia de la distorsión no lineal. Obsérvese que cada armónico puede tener un desfase arbitrario, $\theta_{\mathrm{k}}$. 
Las expresiones para el CSO y el CTB en este caso particular serían las siguientes:

$$
\begin{aligned}
& \text { CSO }=20 \log _{10}\left(\frac{A_{2}}{A_{1}}\right), \\
& C T B=20 \log _{10}\left(\frac{A_{3}}{A_{1}}\right),
\end{aligned}
$$

donde ambos se miden en $\mathrm{dB} \mathrm{c}^{\mathrm{i}}$. Por último, la amplitud de la subportadora recibida se calcularía como

$$
A_{1}=m_{i} I_{p}=m_{i} R P_{\text {rec }},
$$

siendo $m_{i}$ el índice de modulación, $R$ la responsividad del fotodiodo y $\mathrm{P}_{\text {rec }}$ la potencia óptica recibida.

\subsubsection{Distorsión no lineal producida por dispersión}

Para comenzar con el análisis de la distorsión no lineal introducida por la fibra óptica supondremos inicialmente una situación en la que únicamente exista dispersión cromática, por lo que se considerará $\mathrm{n}_{2}=0$. En este caso particular, para modelar la fibra puede utilizarse la función de transferencia (2.1). Despreciando los efectos dispersivos de segundo orden caracterizados por el coeficiente $\beta_{3}$, así como el retardo constante $\beta_{1} L$ que introduce la fibra y que no distorsiona la señal, se llega finalmente a la expresión

$$
H\left(\omega_{\mathrm{e}}\right)=\exp \left[\left(-\frac{\alpha}{2}+\mathrm{j} \frac{\lambda^{2} \mathrm{D}}{4 \pi \mathrm{c}} \omega_{\mathrm{e}}^{2}\right) \mathrm{L}\right]
$$

donde además se ha realizado el cambio de variable $\omega_{\mathrm{e}}=\omega-\omega_{0}$ para desplazar el origen de la función de transferencia hasta la frecuencia de la portadora óptica.

\footnotetext{
iv $\mathrm{dB}$ per carrier (respecto al nivel de subportadora)
} 
Suponiendo un espectro de campo óptico $\widetilde{E_{t}}(\omega)$ a la entrada de la fibra, la corriente eléctrica a la salida del fotodetector puede calcularse de acuerdo a

$$
I(t)=R\left|F^{-1}\left\{H\left(\omega_{e}\right) \widetilde{E_{t}}\left(\omega_{e}+\omega_{0}\right)\right\}\right|^{2} .
$$

Como se desprende de la ecuación anterior, la distorsión no lineal no se genera en la fibra óptica, pues ésta es un sistema lineal. La distorsión surge tras el fotodetector, donde al aplicar la operación $|\cdot|^{2}$, las distintas componentes frecuenciales del campo no se suman coherentemente para formar la señal a la entrada de la fibra como consecuencia de los distintos retardos producidos por la dispersión cromática de la fibra. En la ec. (2.26) se observa que el retardo de grupo depende con la frecuencia de la forma $\tau\left(\omega_{\mathrm{e}}\right)=-\lambda^{2} \mathrm{DL} \omega_{\mathrm{e}} / 4 \pi \mathrm{c}$. Esto ocasiona que la señal multicanal recuperada no sea exactamente igual a la señal transmitida, ya que se generan productos de intermodulación que degradan la calidad del sistema.

Considerando que $\tilde{\Gamma}\left(\omega_{\mathrm{e}}\right)$ es el espectro de potencia de la fotocorriente (2.27), la distorsión no lineal a la frecuencia $\omega_{\mathrm{e}}=\omega_{\mathrm{d}}$, bien sea CSO ó CTB, puede calcularse comov

$$
\mathrm{CSO}, \mathrm{CTB}=10 \log _{10}\left|\frac{2 \widetilde{\Gamma}\left(\omega_{\mathrm{d}}\right)}{\mathrm{m}_{\mathrm{i}}^{2} \widetilde{\Gamma}(0)}\right| \quad(\mathrm{dBc})
$$

Phillips et al. [Phi91] obtuvieron expresiones teóricas para las distorsiones armónicas de segundo y tercer orden considerando modulación directa con un solo tono de un láser DFB con chirp. En este caso, el campo a la entrada de la fibra viene dado porvi

$$
E_{t}(t)=\sqrt{P_{0}} \sqrt{1+m_{i} \cos \left(2 \pi f_{m} t\right)} \exp \left[j\left(\omega_{0} t+\frac{\vartheta}{f_{m}} m_{i} \operatorname{sen}\left(2 \pi f_{m} t\right)\right)\right] .
$$

\footnotetext{
$\checkmark$ el factor 2 del numerador de la ecuación aparece como resultado de las relaciones entre val ores de pico y valores eficaces (la potencia de continua es precisamente $\mathrm{RP}_{\mathrm{rec}}$ )

vi véase sección I.3 del apéndice, donde se modela el chirp en láseres de semiconductor ( $\vartheta$ se mide en $\mathrm{GHz}$ )
} 
Suponiendo únicamente el efecto de la dispersión, las expresiones del CSO y del CTB en dBc son las siguientes [Phi91]:

$$
\begin{aligned}
& \text { CSO }=20 \log _{10}\left[4 \pi^{2} m_{i} \beta_{2} f_{m} z \sqrt{\vartheta^{2}+\left(\pi^{2} \beta_{2} f_{m}^{3} z\right)^{2}}\right], \\
& C T B=20 \log _{10}\left[\frac{9 \pi^{4}}{2}\left(m_{i} \beta_{2} f_{m} z\right)^{2}\left(4 \vartheta^{2}+f_{m}^{2}\right)\right] .
\end{aligned}
$$

Estas expresiones son válidas mientras la cantidad $2 \pi \beta_{2} \vartheta \mathrm{f}_{\mathrm{m}} \mathrm{z}$ sea mucho menor que 1. Para valores mayores, es necesario incluir términos de orden superior en $z$.

En (2.30) y (2.31) pueden verse dos términos claramente diferenciados. Por una parte, el término de distorsión debido a la dispersión cromática de la fibra. Por otra parte, la distorsión debida a la combinación del chirp del transmisor óptico con la dispersión. En general, un valor $\vartheta \neq 0$ provoca niveles más elevados de CSO y CTB.

Otros resultados de distorsión no lineal inducida por la fibra óptica se presentan en [Ber91] y [Cro94]. En el primero, Bergmann et al. calculan una expresión teórica del CSO generado por la acción conjunta de dispersión cromática y chirp del transmisor en sistemas multicanal. La expresión del mismo a la frecuencia $f_{d}$ es la siguiente [Ber91]:

$$
\mathrm{CSO}=\frac{1}{4} N_{\mathrm{CSO}} m_{i}^{2} P_{0}^{2}\left(\frac{d P}{d l}\right)^{-2} \frac{A^{2}+\left(2 \pi f_{d}\right)^{2} E^{2}}{G^{2}+\left(2 \pi f_{c}\right)^{2} H^{2}}
$$

con $\mathrm{f}_{\mathrm{c}}$ la frecuencia de la subportadora deseada, $\mathrm{dP} / \mathrm{dl}$ la pendiente de la curva potencia óptica-corriente eléctrica del modulador o diodo láser y $\mathrm{N}_{\mathrm{CSO}}$ el product count. Los coeficientes A, E, G y H vienen dados porvii

$$
A=\frac{1}{2} \frac{d^{2} P}{d l^{2}}
$$

vii nótese que en la ec. (14) de [Ber91] falta el término $\alpha_{s}=\exp (-\alpha L)$ en la expresión de $h$ 


$$
\begin{aligned}
& E=D L \frac{d v}{d l} \frac{d P}{d l} \frac{\lambda^{2}}{c}, \\
& G=\frac{d P}{d l}, \\
& H=P_{0} D L \frac{d v}{d l} \frac{\lambda^{2}}{c} .
\end{aligned}
$$

$d^{2} P / d l^{2}$ es el parámetro de superlinealidad óptica, mientras que $d v / d l$ es el parámetro de chirp. Si suponemos un funcionamiento lineal alrededor del punto de polarización $I_{b}$, entonces se tiene $d v / d l=\vartheta /\left(I_{b}-I_{\text {th }}\right)$ y $d P / d l=P /\left(I_{b}\right.$ - $\left.I_{\text {th }}\right)$, con la corriente umbral $I_{\text {th }}$. U sando estos datos y asumiendo $A=0$ se Ilega finalmente a una expresión simplificada para el CSO que concuerda perfectamente con la distorsión armónica de la ec. (2.30):

$$
\operatorname{cSO}=N_{\operatorname{cso}}\left(\pi m_{i} D L \vartheta f_{d} \frac{\lambda^{2}}{c}\right)^{2} .
$$

Por otro lado, Crosby et al. [Cro94] presentan resultados similares pero para la distorsión de tercer orden. La expresión del CTB para sistemas multicanal en los que predominan los productos de intermodulación del tipo $f_{i} \pm f_{j} \pm f_{k}$ se puede aproximar por [Cro94]

$$
\mathrm{CTB}=\mathrm{N}_{\text {СтB }}\left(\frac{1}{\sqrt{2}} \pi \mathrm{m}_{\mathrm{i}} \mathrm{DL} \vartheta \mathrm{f}_{\mathrm{d}} \frac{\lambda^{2}}{\mathrm{c}}\right)^{4},
$$

donde $\mathrm{N}_{\text {Ств }}$ es el product count. Esta última expresión también coincide con la ec. (2.31) anterior.

\subsubsection{Distorsión no lineal producida por efecto Kerr}

Como ya comentamos en el apartado 2.2.2, cuando los niveles de potencia comienzan a ser elevados ya no es posible emplear un modelo lineal para estudiar la propagación por la fibra óptica. En este caso, el análisis de distorsión no lineal se realiza resolviendo numéricamente la ecuación en derivadas parciales (2.12) por medio del split-step Fourier method. No 
obstante, bajo ciertas aproximaciones es posible obtener resultados teóricos. En [Phi91], por ejemplo, se emplea un método iterativo mediante el cual, partiendo de la envolvente compleja a la entrada de la fibra, se obtiene una solución en forma de serie de potencias en z. Considerando únicamente los efectos de las no linealidades y una señal a la entrada de la fibra dada por (2.29), las expresiones del CSO y del CTB en $\mathrm{dBc}$ a la salida del fotodetector son las siguientes viii [Phi91]:

$$
\begin{aligned}
& \text { CSO }=20 \log _{10}\left[2 \pi^{2} m_{i} \beta_{2} k n_{2} P_{0} f_{m}^{2} z^{2}\right], \\
& C T B=20 \log _{10}\left[6 \pi^{4}\left(m_{i} \beta_{2} k n_{2} P_{0} f_{m}^{2} z^{2}\right)^{2}\right] .
\end{aligned}
$$

Obsérvese que la presencia de dispersión cromática $\left(\beta_{2} \neq 0\right)$ es necesaria para que el efecto Kerr genere distorsión no lineal. EI SPM induce un chirp de frecuencia sobre la señal que se propaga, pero no afecta a su amplitud. Es precisamente la combinación de este chirp con la dispersión cromática de la fibra la que produce distorsión no lineal. A diferencia de la situación en la que sólo existe dispersión, aquí sí que se generan productos de intermodulación en la propia fibra, antes de alcanzar el fotodetector.

Si queremos una situación más general que incluya las pérdidas de la fibra y la presencia de amplificadores ópticos a lo largo del trayecto, es necesario acudir a los resultados de Desem [Des94]. En este caso, partiendo de una modulación multitono conjunta de amplitud y fase (AM-PM) a la entrada de la fibra dada por

$$
E_{t}(t)=\sqrt{P_{0}} \sqrt{1+m_{i} \sum_{k=1}^{M} \cos \left(\omega_{k} t+\phi_{k}\right)} \exp \left[j\left(\omega_{0} t+m_{f} \sum_{k=1}^{M} \cos \left(\omega_{k} t+\phi_{k}\right)\right)\right],
$$

donde los $\phi_{\mathrm{k}}$ representan desfases aleatorios de las subportadoras, se llega a una expresión teórica del CSO que se genera a la salida del fotodetector para una frecuencia $f_{d} y$ en función de la distancia $z$. Así, la expresión del mismo en $\mathrm{dBc}$ es precisamente

\footnotetext{
viii se considera una situación ideal sin pérdidas $(\alpha=0)$
} 


$$
\mathrm{CSO}=20 \log _{10}\left|\pi^{2} \beta_{2} f_{d}^{2}\left(m_{i} g(z)-m_{f} z\right)\right|+10 \log _{10}\left(2 N_{i \pm j}+N_{2 i}\right)
$$

donde

$$
g(z)=\frac{P_{0} k n_{2}}{\alpha^{2}} \sum_{i=0}^{N_{a m p}} u\left(z-z_{i}\right) \Delta P_{i}\left[\alpha\left(z-z_{i}\right)+\exp \left(-\alpha\left(z-z_{i}\right)\right)-1\right],
$$

con

$$
u(z)=\left\{\begin{array}{l}
1, z \geq 0 \\
0, z<0
\end{array} \quad \Delta P_{i}=1-\exp \left(-\alpha\left(z_{i}-z_{i-1}\right)\right) \quad \Delta P_{0}=1\right.
$$

donde $\mathrm{z}_{\mathrm{i}}$ es la posición del i-ésimo amplificador $\left(\mathrm{z}_{0}=0\right), \mathrm{N}_{\mathrm{i} \pm \mathrm{j}}$ es el número de términos de distorsión que contribuyen como $f_{d}=f_{i}+f_{j}$ con $i \neq j$, y $N_{2 i}=1$ si existe un armónico de segundo orden a dicha frecuencia, ó $\mathrm{N}_{2 i}=0$ en caso contrario. La expresión (2.42) coincide con la ec. (2.39) de la distorsión armónica si tomamos $\alpha \rightarrow 0$ (sin pérdidas), $\mathrm{N}_{\mathrm{amp}}=0$ (sin amplificadores), $\mathrm{N}_{\mathrm{i} \pm \mathrm{j}}=0, \mathrm{~N}_{2 \mathrm{i}}=1$ y $\mathrm{m}_{\mathrm{f}}=0$. Nótese que de la ec. (2.42) se deduce que la distorsión puede cancelarse de forma exacta tomando un índice de modulación de fase dado por

$$
m_{f}=\frac{m_{i} g(z)}{z},
$$

aunque esto ocurre únicamente para una determinada distancia de propagación. Esta técnica de ecualización es la que se propone en [22] para compensar el efecto del SPM en sistemas analógicos AM-SCM con modulación externa. Su principal inconveniente es la gran sensibilidad que posee con la longitud óptima de fibra. Posteriormente, en el capítulo 4 presentaremos la técnica de inversión espectral, con la cual se obtienen mejores resultados.

\subsubsection{Resultados de distorsión no lineal}

En este apartado se realizará un estudio de la distorsión no lineal introducida por la fibra óptica empleando el algoritmo del split-step Fourier method. Para las simulaciones, consideraremos una SMF con unos 
parámetros de atenuación $\alpha=0,2 \mathrm{~dB} / \mathrm{km}$, dispersión $\mathrm{D}=17 \mathrm{ps} /(\mathrm{km} \cdot \mathrm{nm})$ y no linealidades $\gamma=\mathrm{kn}_{2}=2,6 \mathrm{~W}^{-1} \mathrm{~km}^{-1}$. El campo a la entrada de la fibra estará dado por la expresión (2.29), es decir, modulación directa o externa $(\vartheta=0)$ con un solo tono de frecuencia $\mathrm{f}_{\mathrm{m}}$.

Con estos parámetros, en la figura 2.2(a) se representa la distorsión armónica de segundo orden inducida por una fibra óptica dispersiva de 20 $\mathrm{km}$ de longitud en función de la frecuencia de modulación. Se observa que, tanto para el caso de modulación directa como externa, ésta crece con la frecuencia hasta un valor máximo, a partir del cual presenta una serie de lobulaciones de amplitud similar. Para modulación directa, el máximo es de $-23 \mathrm{dBc}$ y se produce para una frecuencia de modulación de $7 \mathrm{GHz}$, mientras que para modulación externa, se tienen $-36 \mathrm{dBc}$ para una frecuencia de 10 GHz. Por debajo de este máximo, se obtiene una mejora significativa empleando un modulador externo, pues evitamos el efecto indeseado del chirp introducido por el diodo láser. En esta zona, son válidas las expresiones calculadas en [Phi91] y podemos aplicar la ec. (2.30) para estimar los valores de distorsión. Suele fijarse como criterio de calidad un valor límite de distorsión de $-60 \mathrm{dBc}$, por lo que el sistema sería válido para frecuencias de modulación no superiores a $4 \mathrm{GHz}$, y todo esto considerando modulación externa.

Por lo que respecta a la distorsión de tercer orden, en la figura 2.2(b) se muestran los valores de distorsión armónica en función de la frecuencia de modulación. La forma de las curvas es similar a las de la figura anterior, si bien, los valores de distorsión son algo inferiores. En este caso, con modulación directa el máximo es de $-47 \mathrm{dBc}$ para una frecuencia de $6 \mathrm{GHz}$, y con modulación externa de $-82 \mathrm{dBc}$ para la misma frecuencia. Aun así, en este último caso aparecen lóbulos de amplitud superior que llegan a alcanzar incluso los $-65 \mathrm{dBc}$. Al igual que antes, empleando modulación externa se consigue una mejora significativa para frecuencias de modulación inferiores a $6 \mathrm{GHz}$. 


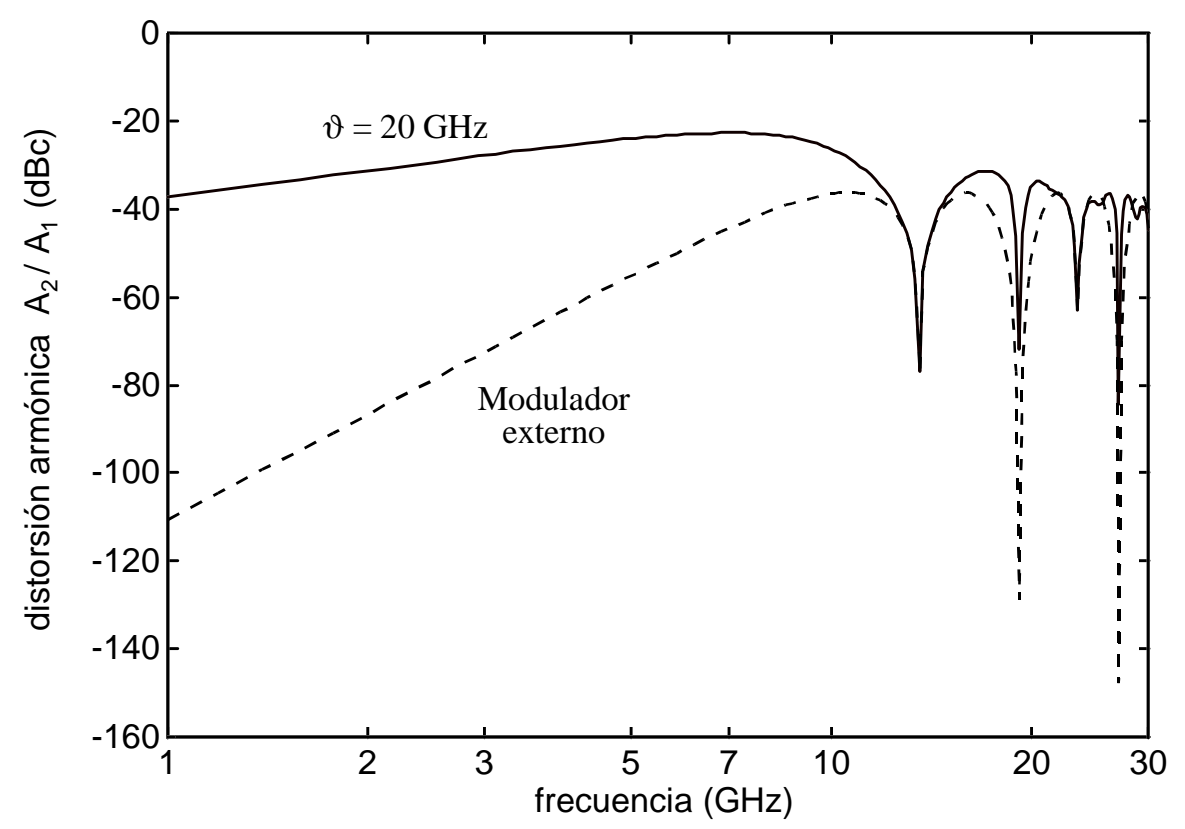

(a)

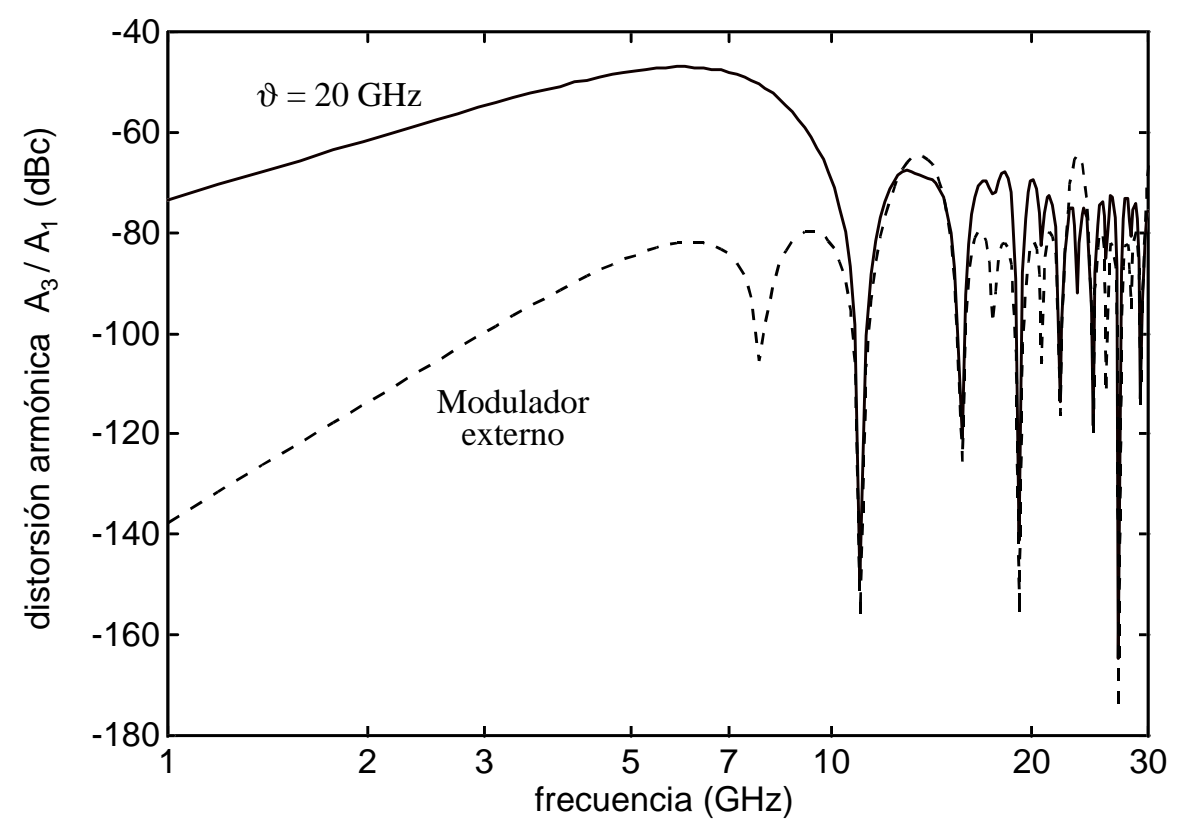

(b)

Figura 2.2 Distorsiones armónicas de (a) segundo y (b) tercer orden en función de la frecuencia de modulación $\mathrm{f}_{\mathrm{m}}$ para una fibra de $20 \mathrm{~km}$ de longitud. La curva continua es suponiendo modulación directa de un láser con chirp $\vartheta=20 \mathrm{GHz}$, mientras que la de trazos es empleando un modulador externo $(\vartheta=0)$. Los parámetros de modulación son: $\mathrm{m}_{\mathrm{i}}=0,04$ y $\mathrm{P}_{0}$ reducida (no afectan las no linealidades de la fibra: $\mathrm{n}_{2}=0$ ). 
Conviene resaltar que, aunque en la figura 2.2 se consideran frecuencias de modulación de hasta $30 \mathrm{GHz}$, en el caso de modulación directa no tiene demasiado sentido. El objetivo de estas curvas es presentar los resultados simplemente de una forma teórica, a pesar de que a nivel de laboratorio se ha conseguido modular láseres a frecuencias de hasta $33 \mathrm{GHz}$ [Ral94].

Si los valores de potencia óptica del transmisor comienzan a ser elevados, entonces es necesario considerar los efectos de las no linealidades de la fibra, tal y como se comentó en el apartado 2.2.2. En la figura 2.3 se representan las distorsiones armónicas de segundo y tercer orden en función de la frecuencia de modulación del láser y para un valor de potencia óptica de $P_{0}=20 \mathrm{~mW}$. Aquí se ha considerado modulación directa del láser y una longitud de fibra de $10 \mathrm{~km}$. Se puede ver que entre las lobulaciones de la distorsión de segundo orden destaca un pico de bastante amplitud. Este fenómeno es típico del efecto Kerr de la fibra, y ocurre periódicamente. En este caso la longitud de fibra no es muy elevada, ya que de lo contrario

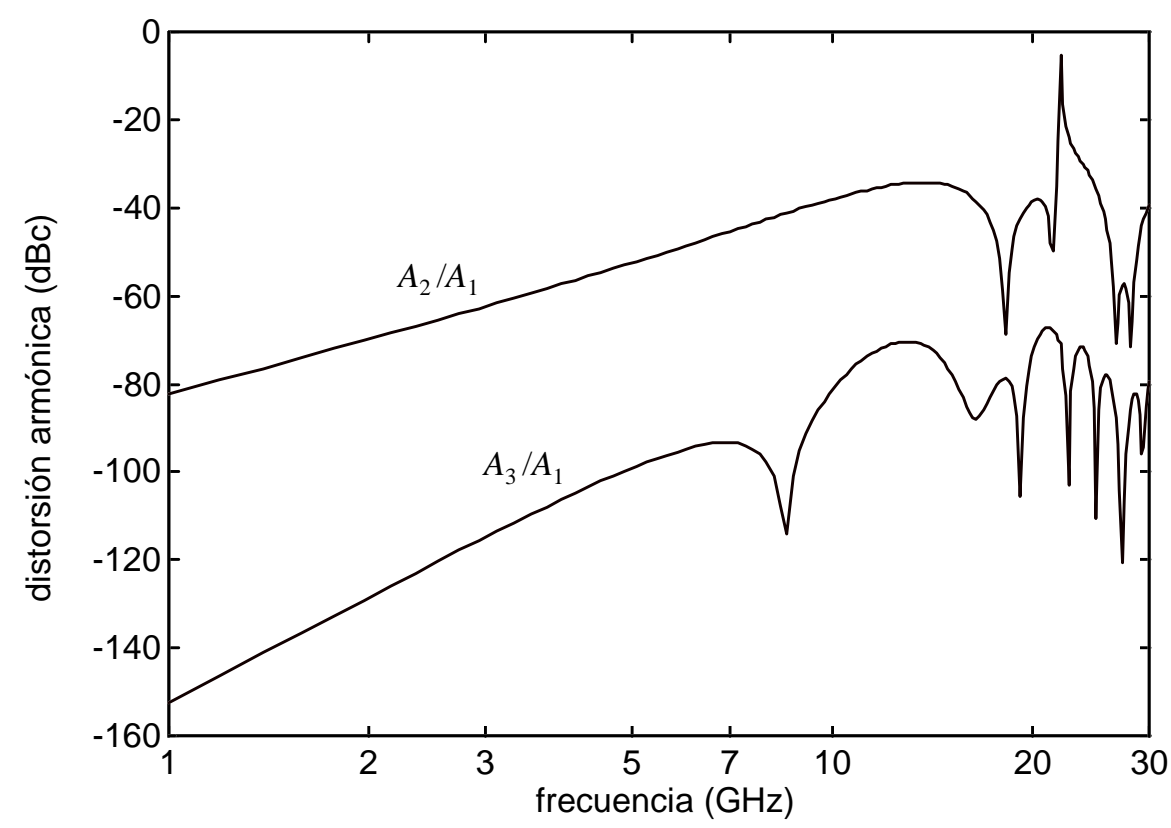

Figura 2.3 Distorsiones armónicas de segundo y tercer orden en función de la frecuencia de modulación $\mathrm{f}_{\mathrm{m}}$ para una fibra de $20 \mathrm{~km}$ de longitud. Los parámetros de modulación son: $m_{i}=0,04, \vartheta=20$ $\mathrm{GHz}$ y $\mathrm{P}_{0}=20 \mathrm{~mW}$. 
también podría observarse en la distorsión de tercer orden. Su presencia, obliga a operar a frecuencias no excesivamente elevadas, o emplear trayectos de fibra más reducidos.

Es importante indicar que en esta gráfica y las anteriores se representa el nivel real de distorsión armónica $A_{k} / A_{1}$, y no el teórico en el cual se supone que la amplitud $A_{1}$ se ve afectada únicamente por las pérdidas del trayecto de propagación. En la sección posterior dedicada al estudio de la penalización de potencia inducida por la fibra óptica podrán aclararse un poco más estos conceptos.



(a)

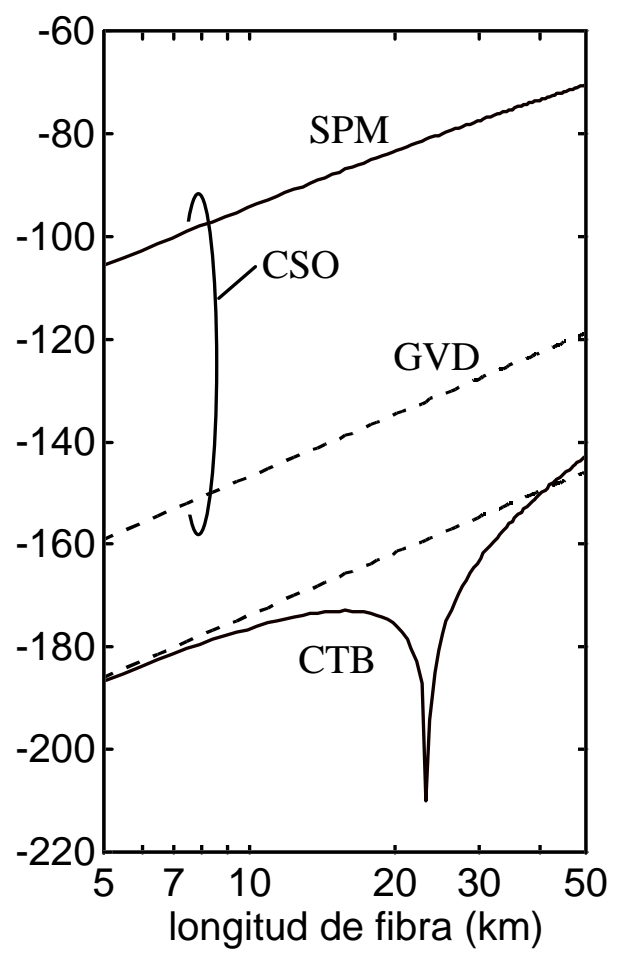

(b)

Figura 2.4 Distorsiones armónicas de segundo y tercer orden (CSO y CTB) en función de la longitud de fibra óptica para dos situaciones distintas: (a) modulación directa y (b) modulación externa. En el primer caso se supone un parámetro de chirp $\vartheta=20 \mathrm{GHz}$ y además se presentan los resultados empleando una DSF con $\mathrm{D}=0,5 \mathrm{ps} /(\mathrm{km} \cdot \mathrm{nm}$ ) (curvas de trazos y puntos). En el segundo caso $(\vartheta=0)$, las curvas de trazos son para sólo dispersión (GVD), mientras que las continuas son para dispersión + no linealidades (SPM). Se han supuesto unos parámetros de modulación: $\mathrm{f}_{\mathrm{m}}=500 \mathrm{MHz}, \mathrm{m}_{\mathrm{i}}=0,04$ y $\mathrm{P}_{0}=$ $20 \mathrm{~mW}$. 
Tras haber analizado la dependencia de la distorsión no lineal con la frecuencia de modulación, ahora conviene estudiar el caso más típico de dependencia con la longitud de fibra óptica. La figura 2.4 muestra las distorsiones armónicas de segundo y tercer orden en función de la longitud de fibra y para dos situaciones: (a) modulación directa y (b) externa. Se incorporan además los resultados empleando fibra de dispersión desplazada (DSF, dispersion-shifted fiber) con un valor $\mathrm{D}=0,5 \mathrm{ps} /(\mathrm{km} \cdot \mathrm{nm})$. Como era de esperar, utilizando DSF se consiguen reducir los niveles de distorsión, pues éstos dependen proporcionalmente del parámetro $\beta_{2}$ tanto en el caso de sólo dispersión (GVD) como de dispersión + no linealidades (SPM). Se puede observar que la distorsión crece con la longitud de fibra, aunque en el caso de modulación externa se produce un nulo en el CTB para una determinada distancia de propagación. Este fenómeno ocurre como consecuencia del distinto signo que poseen los términos de distorsión debidos a dispersión y a no linealidades (efecto Kerr).

Los valores de CSO empleando modulación directa del diodo láser son intolerables, pues para una longitud de fibra de $30 \mathrm{~km}$ se superan los -40 $\mathrm{dBc}$. No obstante, si sustituimos el enlace por una DSF o utilizamos un modulador externo, se obtiene una importante mejora que permite desplazarse hasta distancias superiores a los $50 \mathrm{~km}$ fijando como límite un valor máximo de $-60 \mathrm{dBc}$. Téngase en cuenta que la frecuencia de modulación es de tan sólo $500 \mathrm{MHz}$ (CATV) y transmitimos una única subportadora, por lo que las distancias alcanzables deberían ser grandes.

Los resultados de estas gráficas, si exceptuamos la DSF, son equivalentes a los obtenidos por Phillips et al. [Phi91] pero considerando un valor $\alpha \neq 0$. Las pérdidas de la fibra sí que afectan a la distorsión producida por SPM, ya que disminuye la amplitud de la señal y a su vez el valor del chirp inducido por efecto Kerr. De este modo, incluso resultan beneficiosas en el caso de emplear modulación externa. 


\subsection{PENALIZACIÓN DE POTENCIA INDUCIDA POR LA FIBRA ÓPTICA}

\subsubsection{Análisis teórico}

La distorsión no lineal inducida por fibras dispersivas y no lineales provoca la aparición de armónicos y productos de intermodulación. La potencia de estos términos surge de la propia señal, por lo que la amplitud de las subportadoras recibidas se ve atenuada con respecto al nivel teórico $m_{i} R P_{0} \exp (-\alpha z)$. Estas pérdidas caracterizadas en $d B$ es lo que se conoce como penalización de potencia inducida por la fibra óptica. El valor de la misma para una subportadora de frecuencia $\omega_{m}$ se calcularía como

$$
\delta_{\text {dist }}=-10 \log _{10}\left|\frac{2 \tilde{\Gamma}\left(\omega_{m}\right)}{m_{i}^{2} \tilde{\Gamma}(0)}\right| \quad(d B)
$$

Anteriormente, en la sección 2.3 hemos presentado las expresiones teóricas para evaluar el CSO y el CTB. Si queremos ser rigurosos, en el denominador de las mismas debería figurar la amplitud real de la subportadora. Asumiendo este hecho, se puede llegar a unas nuevas expresiones para el CSO y CTB reales:

$$
\mathrm{CSO}^{\prime}, \mathrm{CTB}^{\prime}=10 \log _{10}\left|\frac{\tilde{\Gamma}\left(\omega_{\mathrm{d}}\right)}{\tilde{\Gamma}\left(\omega_{\mathrm{m}}\right)}\right|=\mathrm{CSO}, \mathrm{CTB}+\delta_{\text {dist }},
$$

donde se ha utilizado la definición (2.46) de la penalización de potencia.

Utilizando los resultados de Meslener [Mes84], es posible obtener una expresión teórica de la penalización de potencia introducida por una fibra dispersiva. Suponiendo una señal sin chirp a la entrada de la fibra compuesta por un único tono de frecuencia $\omega_{\mathrm{m}}$, la penalización de potencia a la salida del fotodetector puede calcularse como

$$
\delta_{\text {dist }}=20 \log _{10}\left[\frac{1}{m_{i}} \sum_{n=0}^{\infty} K_{n} K_{n+1} \cos \left(\frac{(2 n+1) D L \lambda \omega_{m}^{2}}{2 \omega_{0}}\right)\right] \text {, }
$$


donde los coeficientes $K_{n}$ se corresponden con los del desarrollo en serie de Fourier de la función $\sqrt{1+m_{i} \cos \omega_{m} t}$. Para el caso general $m_{i}<1$, estos coeficientes no pueden expresarse de forma cerrada y es necesario utilizar métodos numéricos de integración.

La ec. (2.48) coincide perfectamente con el resultado de [Sch95] si nos quedamos únicamente con el primer término del sumatorio y despreciamos el resto. Esto es válido para valores del índice de modulación pequeños. En este caso, se puede ver que la potencia de señal recibida es proporcional a $\cos ^{2}(\cdot)$, por lo que para determinados valores del argumento se producen nulos. Este fenómeno se conoce con el nombre de supresión de la portadoraix y ocurre habitualmente al variar lo longitud de fibra o la frecuencia de modulación.

Para evaluar la penalización inducida por la dispersión de la fibra óptica supondremos una señal a la entrada compuesta por una única subportadora de frecuencia $\mathrm{f}_{\mathrm{m}}$. En la figura 2.5 se representa la penalización de potencia en función de la longitud de la fibra para dos casos particulares: subportadoras de 30 y $60 \mathrm{GHz}$. De la gráfica se deduce que la subportadora de milimétricas se extingue completamente para determinas distancias. EI primero de los nulos se obtiene para aquella longitud de fibra tal que la función de transferencia de la misma ha introducido un cambio de fase de $\pi / 2$ respecto de la portadora óptica en cada una de las bandas laterales. Esta distancia de transmisión se puede obtener a partir de (2.26) como

$$
\mathrm{L}_{\delta}=\frac{\mathrm{C}}{2 \mathrm{D} \lambda^{2} \mathrm{f}_{\mathrm{m}}^{2}}
$$

Para una subportadora de $30 \mathrm{GHz}$ transmitida con una portadora óptica de $1550 \mathrm{~nm}$ sobre una fibra con dispersión cromática $17 \mathrm{ps} /(\mathrm{km} \cdot \mathrm{nm})$, este mínimo ocurre para una distancia de 4,08 km. En el caso de $\mathrm{f}_{\mathrm{m}}=60 \mathrm{GHz}$, el nulo se desplaza hasta una distancia de tan sólo 1,02 km. Esta periodicidad

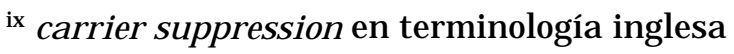






Figura 2.5 Penalización de potencia en subportadoras de milimétricas en función de la longitud de fibra. Los resultados se presentan para unas subportadoras de 30 y $60 \mathrm{GHz}$, y los parámetros de modulación son: $m_{i}=0,04, \vartheta=0$ (modulación externa) y $\mathrm{P}_{0}=20 \mathrm{~mW}$.

que exhibe la penalización puede usarse para medir el parámetro de dispersión $D$ de la fibra [Dev93] de una forma simple pero bastante precisa.

Si a continación añadimos el efecto del SPM, las curvas de penalización son similares, si bien sufren un ligero desplazamiento. En este caso, el mínimo ocurre para una longitud mayor como consecuencia del desfase adicional introducido por el efecto Kerr. Estos resultados se pueden observar en la misma gráfica anterior (curvas a trazos) para las dos subportadoras. En el caso de la subportadora de menor frecuencia, el efecto del SPM es más significativo. Posteriormente, se mostrarán resultados experimentales en función de la frecuencia de modulación donde se observa el desplazamiento que sufren las curvas en función de la potencia óptica de entrada a la fibra.

Normalmente, suele fijarse un valor de penalización de potencia máximo de $1 \mathrm{~dB}$, lo cual permite definir una distancia de transmisión máxima. La dependencia de esta distancia con la frecuencia de la subportadora puede 




Figura 2.6 Distancia de transmisión alcanzable para una penalización de potencia de $1 \mathrm{~dB}$ en función de la frecuencia de la subportadora [Gli96]. Los resultados se presentan para dos tipos de fibra: SSMF ( $D=17 \mathrm{ps} / \mathrm{km} \cdot \mathrm{nm})$ y DSF ( $D=4 \mathrm{ps} / \mathrm{km} \cdot \mathrm{nm})$. Los parámetros de modulación son: $\mathrm{m}_{\mathrm{i}}=1$ y $\vartheta=0$.

observarse en la figura 2.6 para dos valores de dispersión cromática: 4 y 17 $\mathrm{ps} /(\mathrm{km} \cdot \mathrm{nm})$. Un incremento en la dispersión de la fibra o en la frecuencia de la subportadora limita la máxima distancia alcanzable, ya que la dependencia con estos parámetros es del tipo $1 / \mathrm{Df}_{\mathrm{m}}^{2}$. Por lo tanto, las prestaciones del sistema pueden mejorarse considerablemente empleando fibras de dispersión desplazada. No obstante, para valores pequeños de dispersión es muy importante la tolerancia del parámetro $D$, ya que un cambio de unos pocos ps/(km.nm) puede resultar en una disminución drástica de la distancia de transmisión.

\subsubsection{Resultados experimentales}

Para medir experimentalmente la penalización de potencia inducida por la fibra óptica se preparó un montaje como el que se muestra en la figura 2.7. La salida eléctrica del analizador de componentes HP8703A se inyecta en la entrada de RF de un modulador de intensidad Mach-Zehnder UTP 150 


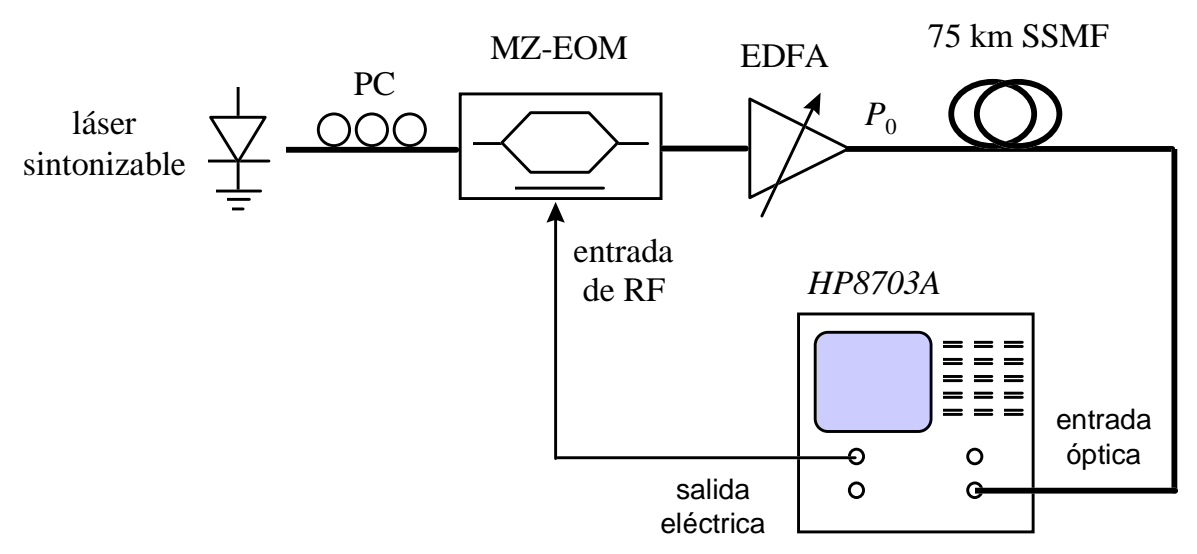

Figura 2.7 Montaje experimental utilizado para medir los efectos de la potencia óptica sobre la penalización de potencia inducida por la fibra óptica.

polarizado en régimen lineal (QB). La potencia eléctrica de modulación se fijó en $0 \mathrm{dBm}$, lo cual conduce a un índice de modulación de amplitud lo suficientemente pequeño como para despreciar las no linealidades del modulador electro-óptico (MZ-EOM). La portadora óptica se obtiene por medio de un láser sintonizable TUNICS PR-1550 operando a $1550 \mathrm{~nm}$. A la salida del MZ-EOM se coloca un EDFA con el cual controlamos la potencia óptica que se inyecta a la entrada de la fibra. Por último, la salida de los 75 km de SSMF se conecta a la entrada óptica del analizador de componentes para recuperar la subportadora transmitida. El barrido en frecuencia que realiza el analizador de componentes nos permite observar el fenómeno de supresión de la portadora al operarlo en el modo de funcionamiento eléctrico-óptico (E/O). Para eliminar la influencia de la respuesta del MZEOM y del fotodetector del HP8703A sobre las medidas realizadas es necesario calibrar previamente el equipo y corregir las curvas con la respuesta obtenida sin fibra óptica.

EI principal factor de limitación en estas medidas lo constituye el scattering estimulado de Brillouin (SBS) [I pp72, Fis93a], debido a las altas potencias ópticas y al reducido ancho de línea como consecuencia de la modulación externa. Por ello, para evitar este efecto y permitir la máxima influencia del SPM, el láser fue operado en el modo de baja coherencia (ancho de línea 
superior a $30 \mathrm{MHz}$ ) alcanzándose una potencia umbral de SBS cercana a los $+7 \mathrm{dBm}$ [Aok88].

Las curvas obtenidas se pueden ver en la figura 2.8 para una longitud de fibra de $75 \mathrm{~km}$ y dos valores de potencia óptica: -10 y $+7 \mathrm{dBm}$. Las líneas continuas representan los resultados experimentales, mientras que los símbolos son los resultados de simulación empleando el split-step Fourier method ${ }^{x}$. Como se puede ver, la analogía entre experimento y simulación es muy buena. Además, se han incluido también simulaciones con +10 y +13 $\mathrm{dBm}$ para poder observar la tendencia. De la gráfica se deduce que, aumentando la potencia óptica a la entrada de la fibra, conseguimos

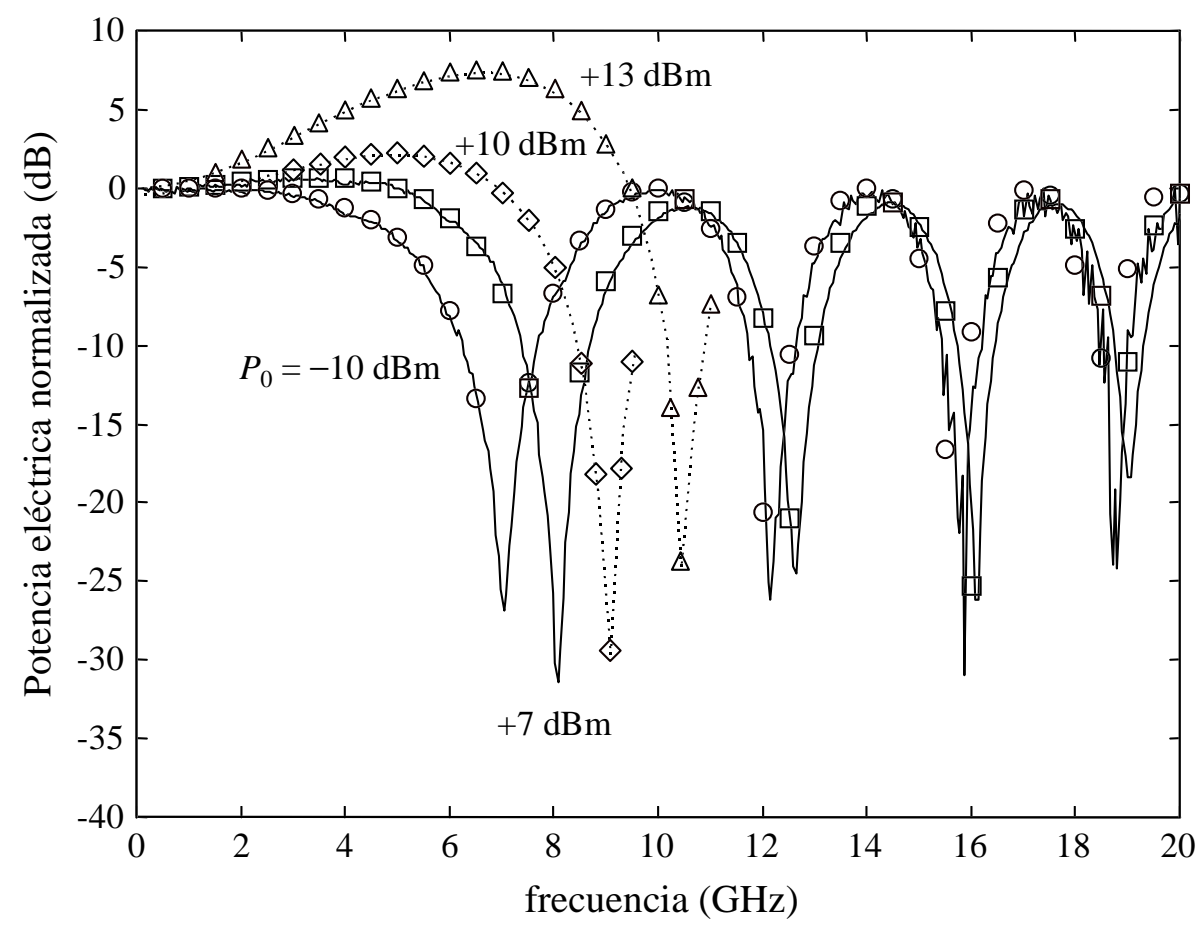

Figura 2.8 Potencia eléctrica normalizada a la salida del fotodetector en función de la frecuencia de modulación y para varios valores de potencia óptica a la entrada de la fibra. Las curvas continuas son los resultados experimentales obtenidos con el montaje de la figura 2.7, mientras que los símbol os representan la simulación empleando el split-step Fourier method.

\footnotetext{
× para una expresión teórica de la función de transferencia de una fibra óptica dispersiva y no lineal, véase capítulo 3
} 
desplazar el nulo de las curvas hacia frecuencias mayores. El principio se basa en el chirp de frecuencia inducido por SPM, el cual, al combinarse con la dispersión cromática de la fibra genera un término de distorsión de signo opuesto al término convencional debido sólo a dispersión. Este efecto es similar al de un chirp negativo en el transmisor óptico [Smi97, Pol99], aunque distribuido a lo largo de la fibra. De este modo, como refleja la ec. (2.13), por medio de la longitud de la fibra y de la potencia óptica es posible controlar la influencia de este chirp y, por lo tanto, aumentar el producto frecuencia-longitud de fibra del enlace óptico.

TABLA II Frecuencia a $-3 \mathrm{~dB}$ y ganancia de pico de los $75 \mathrm{~km}$ de SSMF en función de la potencia óptica inyectada a la entrada de la fibra.

\begin{tabular}{|c|c|c|}
\hline $\begin{array}{c}\text { potencia óptica } \\
\mathbf{( d B m )}\end{array}$ & $\begin{array}{c}\text { frecuencia -3 dB } \\
\mathbf{( G H z )}\end{array}$ & $\begin{array}{c}\text { ganancia de pico } \\
\mathbf{( d B )}\end{array}$ \\
\hline-10 & 5 & 0 \\
\hline 7 & 6,2 & 0,7 \\
\hline 10 & 7,5 & 2,5 \\
\hline 13 & 9,7 & 7,3 \\
\hline 15 & 11,3 & 12,9 \\
\hline 18 & 13,2 & 25,5 \\
\hline 20 & 12,2 & 30,4 \\
\hline
\end{tabular}

A modo de comparación, en la tabla II se presenta la frecuencia a -3 dB en función de la potencia óptica a la entrada de la fibra. Obsérvese que aumentando la potencia se consigue mejorar la respuesta de la fibra, aunque existe un valor máximo a partir del cual se producen peores resultados. Para potencias ópticas superiores a este valor (alrededor de +18 $\mathrm{dBm}$ en nuestro caso), las no linealidades de la fibra se convierten entonces en el principal factor de degradación del sistema por encima de la dispersión cromática. Otro parámetro interesante es la ganancia que introduce el SPM sobre la señal que se propaga por la fibra. Este resultado se incluye también en Ia tabla II, y se define como el valor de pico de la curva de potencia 
eléctrica normalizada. Por ejemplo, para potencias de $+15 \mathrm{dBm}$ se consiguen recibir casi $13 \mathrm{~dB}$ más de potencia eléctrica de la que se debería recibir considerando únicamente las pérdidas de la fibra. Así pues, en presencia de no linealidades, la fibra óptica puede actuar como amplificador para determinadas frecuencias de modulación. En el capítulo 3 se estudiará más en detalle este fenómeno y se demostrará la transmisión de señales empleando la técnica SPM. 


\subsection{MARGEN DINÁMICO}

EI margen dinámico libre de espúreas (SFDR, spurious-free dynamic range) es un importante parámetro de calidad que es crucial en la determinación de la utilidad de un enlace analógico en la mayor parte de las aplicaciones. El SFDR se define como la relación señal a ruido, CNR en nuestro caso, para la cual el nivel de distorsión de intermodulación coincide con el nivel de ruido [Gao95]. Por defecto, se toma la distorsión de intermodulación de orden tres del tipo $f_{d}=2 f_{1}-f_{2}$ generada por dos tonos.

\subsubsection{Cálculo de la expresión del SFDR}

Si consideramos las expresiones (2.31) y (2.40) [Phi91] que determinan la distorsión armónica de orden 3 generada en una fibra óptica, la distorsión a la frecuencia $f_{d}=2 f_{1}-f_{2}$ puede expresarse como

$$
\mathrm{CTB}=\mathrm{Km}_{\mathrm{i}}^{4},
$$

donde $\mathrm{K}$ es una constante que se calcula a partir de (2.31) y (2.40) con $\mathrm{f}_{\mathrm{m}}=$ $\mathrm{f}_{\mathrm{d}} / 3$. Esta constante viene dada por ${ }^{\mathrm{xi}}$

$$
\begin{aligned}
& K_{G V D}=\frac{9}{4} \pi^{8}\left(\beta_{2} f_{d} L\right)^{4}\left(4 \vartheta^{2}+\left(\frac{f_{d}}{3}\right)^{2}\right)^{2}, \\
& K_{S P M}=\frac{4 \pi^{8}}{81}\left(k n_{2} P_{0} \beta_{2} f_{d}^{2} L^{2}\right)^{4},
\end{aligned}
$$

dependiendo de si existe sólo dispersión (GVD) o dispersión + no linealidades (SPM) respectivamente.

De acuerdo con la definición del SF DR, la distorsión (2.50) debe coincidir con el nivel de ruido del sistema, luego despejando se obtiene

\footnotetext{
${ }^{x i}$ se incluye el product count $N_{\text {Ств }}=9$ para $2 f_{1}-f_{2}$
} 


$$
\mathrm{m}_{\mathrm{i}}^{6}=\frac{2 \text { Noise }}{\mathrm{Z}_{\text {rec }}\left(\mathrm{RP}_{\mathrm{rec}}\right)^{2}} \mathrm{~K}^{-1},
$$

siendo $Z_{\text {rec }}$ la impedancia de entrada del receptor y Noise la potencia eléctrica de ruido en dicho punto.

De este modo, el SFDR será igual a la CNR para el índice de modulación (2.53), es decir

$$
\mathrm{SFDR}=\left.\mathrm{CNR}\right|_{\text {IM3 }=\text { Noise }}=\left[\frac{Z_{\text {rec }}\left(R P_{\text {rec }}\right)^{2} / 2}{\text { Noise }}\right]^{2 / 3} \mathrm{~K}^{-1 / 3} .
$$

A continuación, operando con la ec. (2.54) y particularizándola para el caso GVD, es posible obtener por primera vez, una expresión del SFDR de la fibra en $\mathrm{dB} \cdot \mathrm{Hz}^{2 / 3}$ donde se desglosen cada uno de los términos ${ }^{\mathrm{xii}}$ :

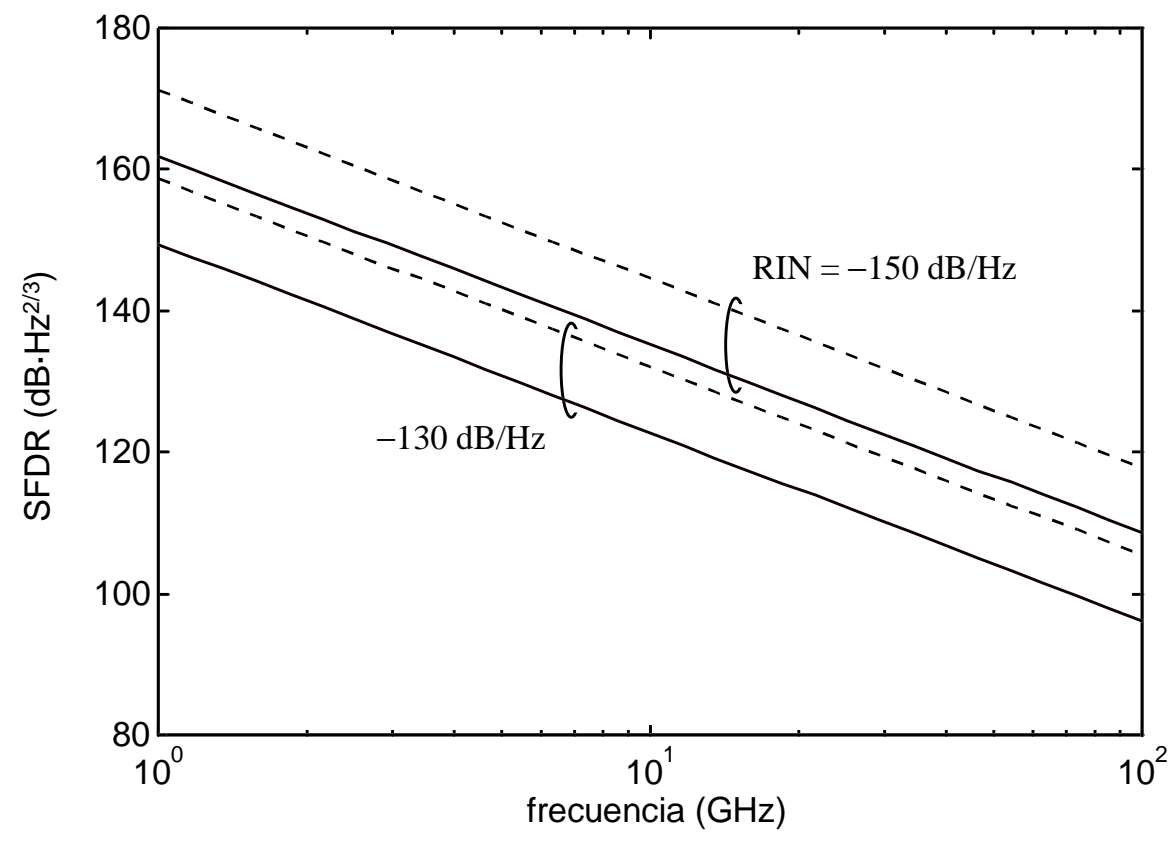

Figura 2.9 SFDR en función de la frecuencia de trabajo del sistema y parametrizado para dos valores de ruido RIN : -130 y $150 \mathrm{~dB} / \mathrm{Hz}$. Las líneas de trazos son para una longitud de fibra de $1 \mathrm{~km}$ y las continuas para $5 \mathrm{~km}$. En ambos casos se supone una potencia óptica recibida de $0 \mathrm{dBm}$ y un valor $\vartheta=0$.

xii se ha tomado $R=0,9$ y $Z_{\text {rec }}=50 \Omega$ 
$\operatorname{SFDR}=\frac{2}{3}\left\{2 \mathrm{P}_{\text {rec }}(\mathrm{dBm})-\right.$ Noise $\left.(\mathrm{dBm} / \mathrm{Hz})-10 \log _{10}\left[\mathrm{~L}^{2} \mathrm{f}_{\mathrm{d}}^{4}\left(1+\left(\frac{6 \vartheta}{f_{d}}\right)^{2}\right)\right]+484,2\right\}$

En la figura 2.9 se representa el SFDR en función de la frecuencia y para dos valores de ruido RIN. Las líneas continuas son para una longitud de fibra de $5 \mathrm{~km}$ y las de trazos para $1 \mathrm{~km}$. En general, a frecuencias y distancias mayores se obtiene un valor de SFDR menor, pues los niveles de distorsión no lineal son más elevados. Lo mismo ocurre si el ruido del sistema aumenta. Para una situación sin chirp como la que se supone en la gráfica, a partir de (2.55) se obtiene que el SFDR es proporcional a $f_{d}{ }^{-8 / 3}$, luego se produce una disminución de $8 \mathrm{~dB}$ cada vez que se duplica la frecuencia de trabajo del sistema. Por ejemplo, con una frecuencia cercana a los $28 \mathrm{GHz}$ (LMDS) se puede conseguir un SFDR de $130 \mathrm{~dB} \cdot \mathrm{Hz}^{2 / 3}$ en distancias inferiores a $1 \mathrm{~km}$.

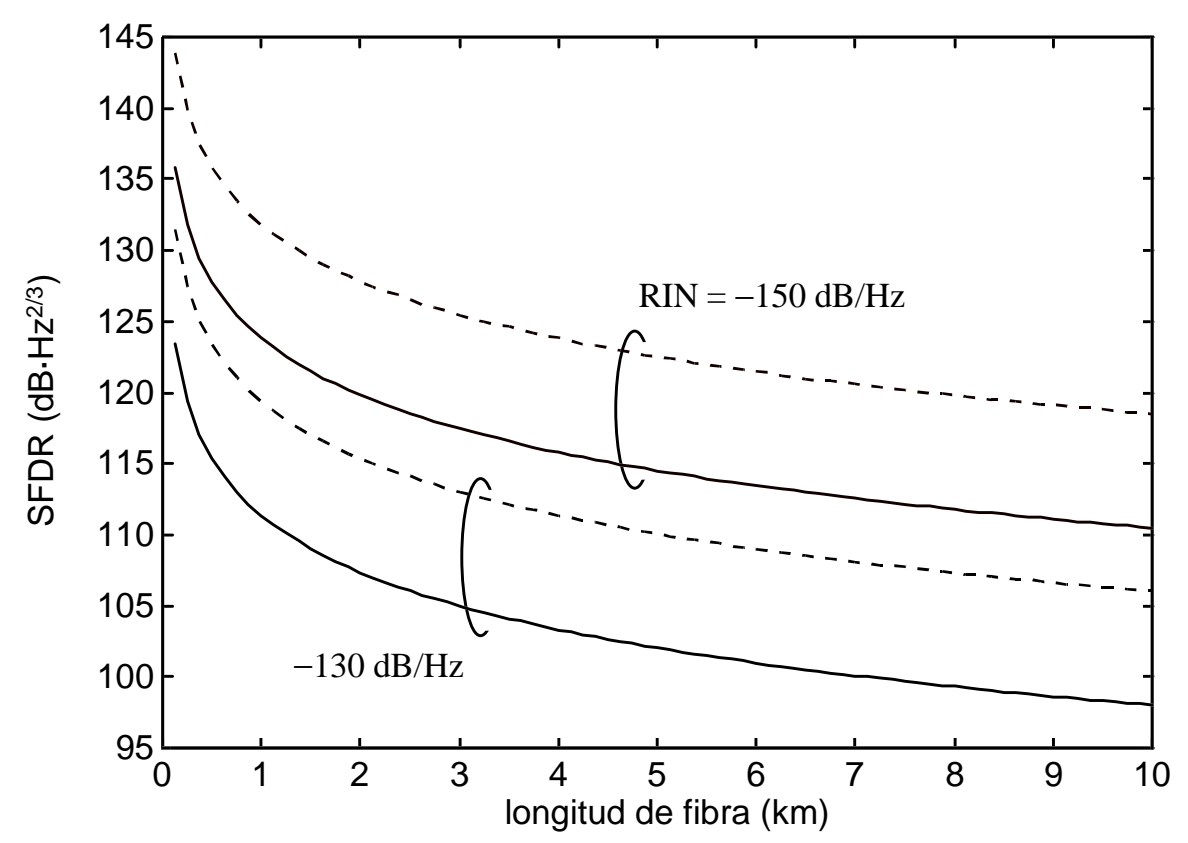

Figura 2.10 SFDR en función de la longitud de fibra y parametrizado para dos valores de ruido RIN : -130 y $-150 \mathrm{~dB} / \mathrm{Hz}$. Las líneas de trazos son para una frecuencia de $30 \mathrm{GHz}$ y las continuas para $60 \mathrm{GHz}$. En ambos casos se supone una potencia óptica recibida de $0 \mathrm{dBm}$ y un valor $\vartheta=0$. 
Por otro lado, las curvas del SFDR en función de la longitud de fibra óptica se muestran en la figura 2.10, también para dos valores de ruido RIN. Las líneas de trazos son para una frecuencia de $30 \mathrm{GHz}$ y las continuas para 60 GHz. En este caso, la dependencia del SFDR con la longitud de fibra es del tipo $L^{-4 / 3}$, por lo que duplicar la distancia significa una reducción de $4 \mathrm{~dB}$. Como ejemplo, en un sistema MBS funcionando a $60 \mathrm{GHz}$ se tendría un SFDR de $110 \mathrm{~dB} \cdot \mathrm{Hz}^{2 / 3}$ para una longitud de fibra de $10 \mathrm{~km}$ y un parámetro de RIN de $-150 \mathrm{~dB} / \mathrm{Hz}$.

Conviene resaltar que en todo este análisis no se ha tenido en cuenta las distorsiones no lineales introducidas por el láser, modulador externo o amplificadores ópticos [Kuo92], las cuales limitan el margen dinámico que se puede alcanzar en el enlace óptico. Valores típicos de SFDR empleando modulación externa se encuentran por encima de los $100 \mathrm{~dB} \cdot \mathrm{Hz}^{2 / 3}[$ Bet90, Fon94], los cuales pueden incluso mejorarse empleando técnicas de linealización del modulador [Ala95]. 


\subsection{ESTUDIO DE DISTORSIÓN EN SISTEMAS RADIO-FIBRA}

En esta sección se analizará la distorsión no lineal introducida por la fibra óptica en sistemas multicanal radio sobre fibra típicos. Todos los resultados se han obtenido por medio de simulación numérica, empleando el split-step Fourier method para modelar la propagación por la fibra. Los diferentes sistemas a analizar serán: CATV, MMDS, LMDS y MVDS.

\subsubsection{Sistema CATV}

EI primer sistema que estudiaremos será un sistema CATV de distribución de canales de vídeo analógico con modulación AM-VSB. EI plan de frecuencias será idéntico al propuesto en [Des94], donde se consideran 70 subportadoras espaciadas en interval os de $6 \mathrm{MHz}$ y comenzando en $60 \mathrm{MHz}$, es decir, cubriendo la banda 60-474 MHz. Dado que los canales ocupan más



Figura 2.11 Distorsión de segundo orden en función de la longitud de fibra para un sistema CATV de 70 canales. EI CSO se ha medido a una frecuencia de $480 \mathrm{MHz}$. Se muestran resultados tanto de modulación directa $(\vartheta=20 \mathrm{GHz})$, como empleando un modulador externo. Las líneas de trazos representan la ec. (2.42) y la de trazos y puntos la ec. (2.39) para una situación sin pérdidas. Los parámetros de modulación son: $\mathrm{m}_{\mathrm{i}}=0,04$ y $\mathrm{P}_{0}=40 \mathrm{~mW}$. 
de una octava de frecuencia, la distorsión de segundo orden caerá dentro de la banda y será la que limite la calidad del sistema. La distorsión suele medirse por encima del último canal de la banda $\left(f_{d}=480 \mathrm{MHz}\right.$ ), donde en este caso coincide un armónico de segundo orden (canal 31) y 30 productos de intermodulación del tipo $\mathrm{f}_{\mathrm{i}}+\mathrm{f}_{\mathrm{j}}$, proporcionando un valor $\mathrm{N}_{\mathrm{CSO}}=61$.

En la figura 2.11 se representan los niveles de CSO en función de la longitud de fibra y para dos situaciones distintas: modulación directa con un parámetro de chirp $\vartheta=20 \mathrm{GHz}$ y modulación externa. En el primer caso, los niveles de distorsión son intolerables, pues para una distancia de $5 \mathrm{~km}$ el CSO se encuentra cercano a los $-40 \mathrm{dBc}$. Por otro lado, empleando modulación externa conseguimos que el efecto predominante sea el del SPM y podemos alcanzar unos $50 \mathrm{~km}$ fijando como límite un valor de CSO de -60 $\mathrm{dBc}$. Téngase en cuenta que en este tipo de sistemas suelen emplearse potencias ópticas el evadas, del orden de $+16 \mathrm{dBm}(40 \mathrm{~mW})$ a la entrada de la fibra. No obstante, es posible reducir aún más los niveles de distorsión

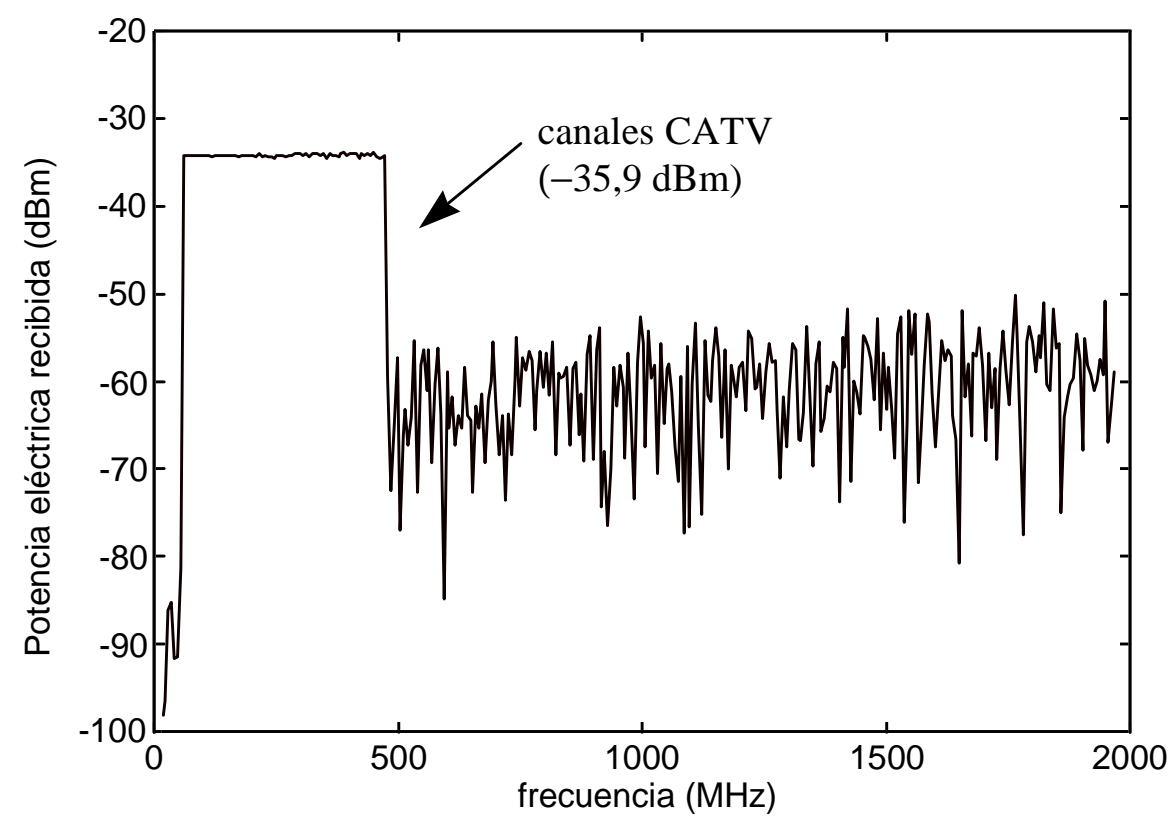

Figura 2.12 Espectro de la señal multicanal recibida tras $50 \mathrm{~km}$ de fibra óptica dispersiva y no lineal. La potencia eléctrica teórica de los canales recibidos es de $-35,9 \mathrm{dBm}$. Los parámetros del sistema son: $\mathrm{m}_{\mathrm{i}}=0,04, \vartheta=20 \mathrm{GHz}, \mathrm{P}_{0}=40 \mathrm{~mW}, \mathrm{R}=0,9$ y $\mathrm{Z}_{\mathrm{rec}}=$ $50 \Omega$. 
empleando técnicas de ecualización que veremos posteriormente, como por ejemplo OPC. En la misma figura se presentan también los resultados de Desem [Des94] (líneas de trazos) utilizando la fórmula (2.42) pero sin emplear amplificadores en el trayecto, y los resultados de Phillips [Phi91] (línea de trazos y puntos) utilizando la fórmula (2.39) y suponiendo una situación ideal sin pérdidas. Se observa que la similitud entre teoría y simulación es muy buena.

En cuanto a la señal recibida, su espectro puede verse en la figura 2.12 para una longitud de fibra de $50 \mathrm{~km}$. La potencia eléctrica de cada una de las subportadoras a la salida del fotodetector es de $-35,9 \mathrm{dBm}$ sobre una impedancia de $50 \Omega$, mientras que el nivel de distorsión se mantiene en torno a los $-30 \mathrm{dBc}$. Este nivel tan elevado se debe a que se está considerando una situación con chirp en el transmisor óptico. Por otra parte,

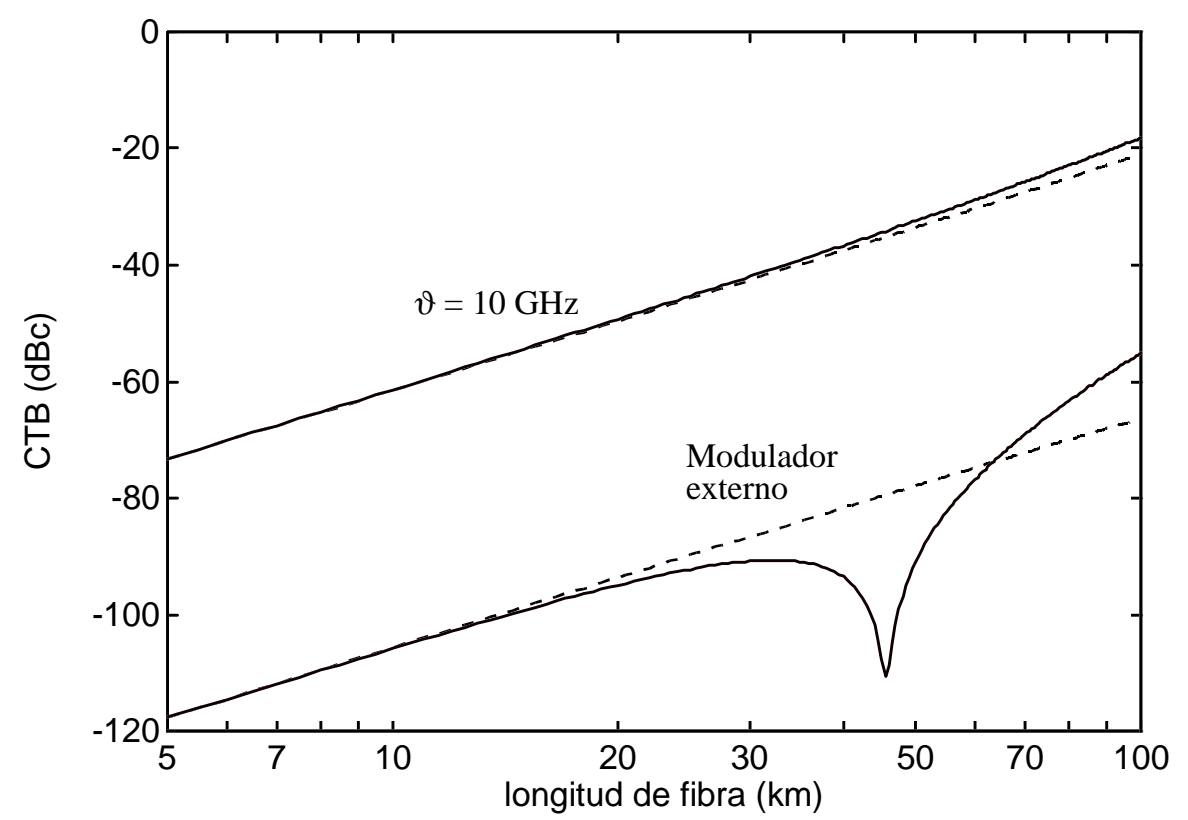

Figura 2.13 Distorsión de tercer orden en función de la longitud de fibra para un sistema MMDS con 33 canales. EI CTB se ha medido a una frecuencia de $2,7 \mathrm{GHz}$. Se muestran resultados tanto de modulación directa $(\vartheta=10 \mathrm{GHz})$, como empleando un modulador externo. Las líneas de trazos son para GVD y las continuas para SPM. Los parámetros de modulación son: $\mathrm{m}_{\mathrm{i}}=$ 0,04 y $\mathrm{P}_{0}=20 \mathrm{~mW}$. 


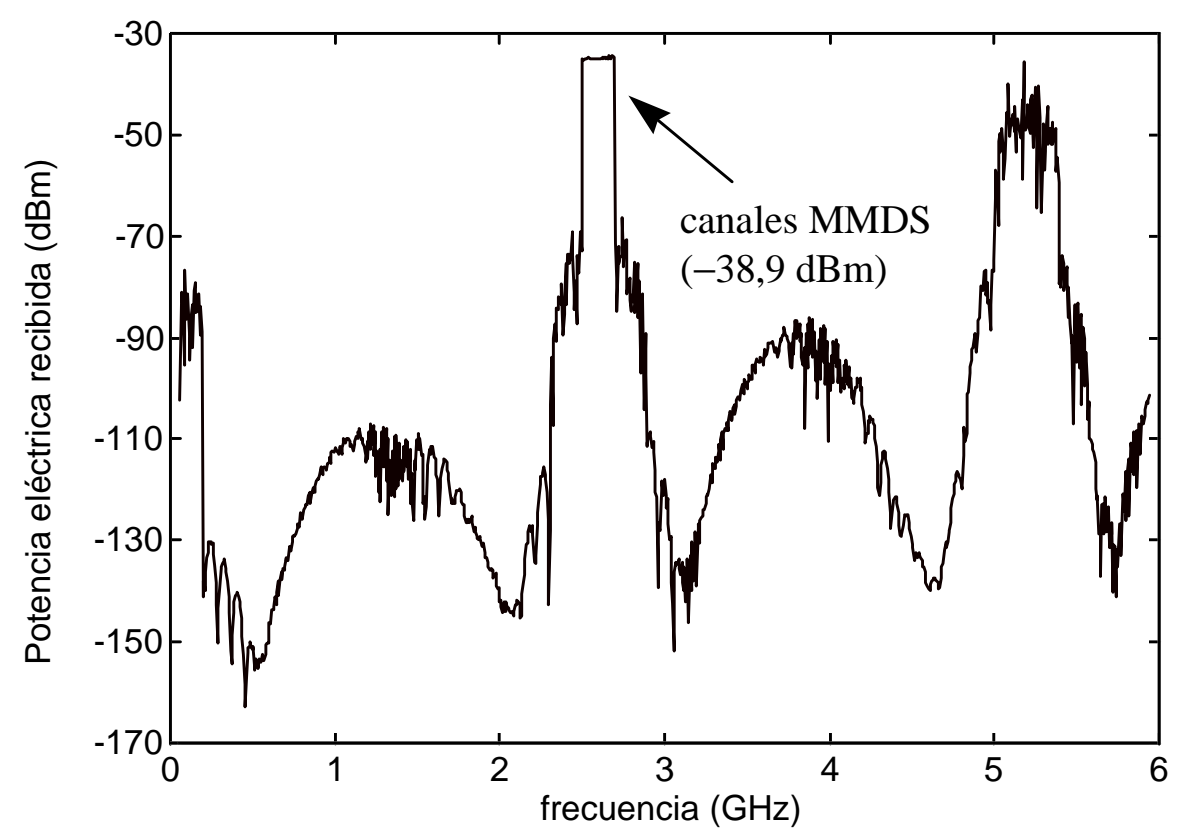

Figura 2.14 Espectro de la señal multicanal recibida tras $50 \mathrm{~km}$ de fibra óptica dispersiva y no lineal. La potencia eléctrica teórica de los canales recibidos es de $-38,9 \mathrm{dBm}$. Los parámetros del sistema son: $\mathrm{m}_{\mathrm{i}}=0,04, \vartheta=10 \mathrm{GHz}, \mathrm{P}_{0}=20 \mathrm{~mW}, \mathrm{R}=0,9$ y $\mathrm{Z}_{\text {rec }}=$ $50 \Omega$.

las fluctuaciones que se observan en los niveles de distorsión son debidas a que las subportadoras presentan desfases aleatorios, y por este motivo los productos de intermodulación no se suman coherentemente. En cualquier caso, sí que se observa un ligero aumento con la frecuencia.

\subsubsection{Sistema MMDS}

El plan de frecuencias del sistema consiste en 33 canales espaciados $6 \mathrm{MHz}$ y cubriendo el rango de frecuencias desde 2,502 hasta 2,694 GHz. En este caso, la banda ocupa menos de una octava de frecuencia, y por lo tanto la degradación vendrá determinada por la distorsión de tercer orden: $2 f_{1}-f_{2}$ y $f_{1} \pm f_{2} \pm f_{3}$.

Para comenzar con el análisis, los niveles de CTB medidos a una frecuencia de $2,7 \mathrm{GHz}$ se muestran en la figura 2.13. Las líneas de trazos representan los casos de sólo dispersión (potencia óptica reducida), mientras que las 


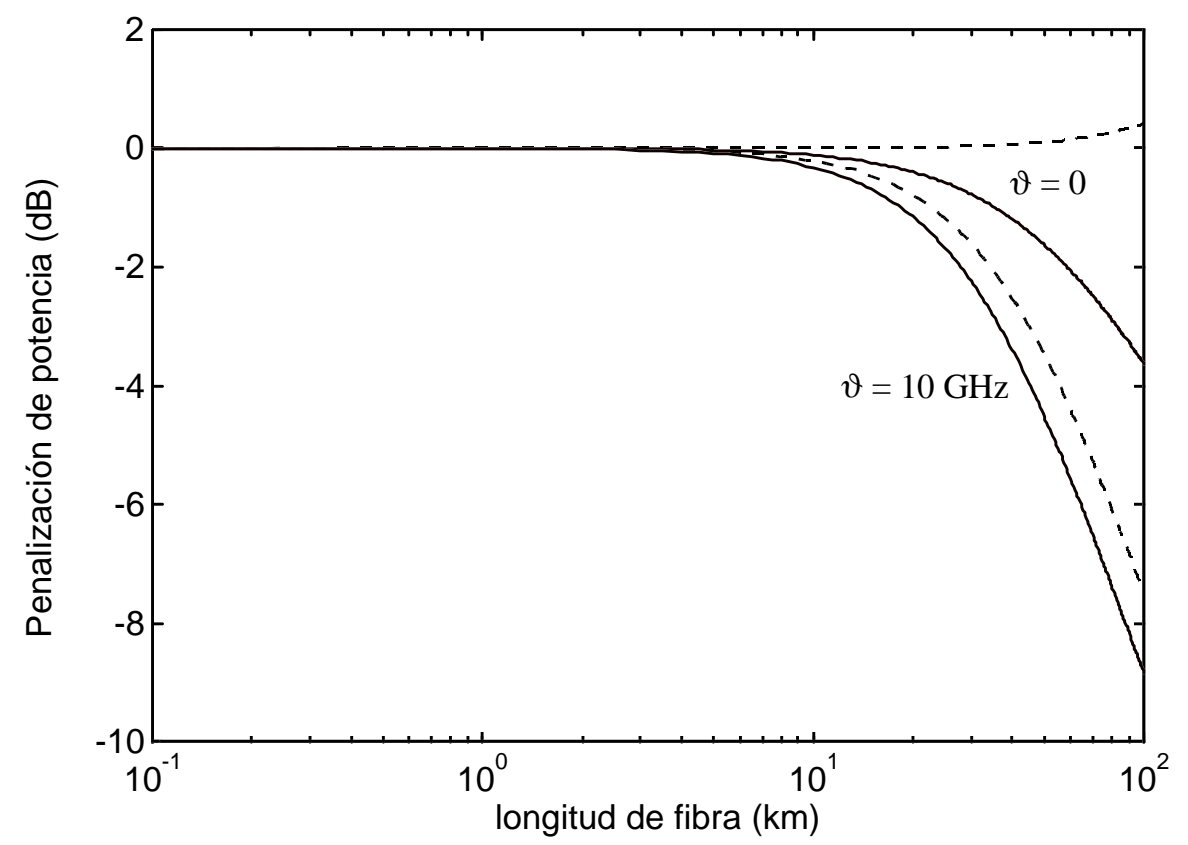

Figura 2.15 Penalización de potencia en función de la longitud de fibra óptica para el último canal de la banda $(2,694 \mathrm{GHz})$. Se muestran resultados tanto de modulación directa $(\vartheta=10 \mathrm{GHz})$, como empleando un modulador externo $(\vartheta=0)$. Las líneas de trazos son para GVD y las continuas para SPM. Los parámetros de modulación son: $\mathrm{m}_{\mathrm{i}}=0,04$ y $\mathrm{P}_{0}=20 \mathrm{~mW}$.

contínuas incluyen el efecto del SPM. Con modulación directa ( $\vartheta=10 \mathrm{GHz}$ ) se obtienen unos 40 dB más de distorsión que empleando modulación externa, lo cual hace que fijando el límite de $-60 \mathrm{dBc}$ debamos restringir la longitud del enlace a $10 \mathrm{~km}$ como máximo. Además, con modulador externo es posible anular la distorsión para una distancia especifica, pues los efectos de dispersión y SPM se cancelan debido al distinto signo de los mismos. Este fenómeno es idéntico al comentado en la figura 2.4 del apartado 2.3.3. Para los datos de la figura, en torno a los $45 \mathrm{~km}$ se consiguen valores de CTB inferiores a los $-100 \mathrm{dBc}$, y se alcanzan distancias de aproximadamente unos $90 \mathrm{~km}$.

El espectro de la señal multicanal recibida se puede ver en la figura 2.14 para una longitud de fibra de $50 \mathrm{~km}$ y un parámetro de chirp de $\vartheta=10 \mathrm{GHz}$. En este caso, se han representado valores de frecuencia hasta los $6 \mathrm{GHz}$, Io cual permite observar la presencia de productos de intermodulación de 
segundo orden (CSO) a partir de los $5 \mathrm{GHz}$. EI nivel de éstos es muy elevado $y$, de no caer fuera de la banda de canales, degradaría considerablemente la calidad del sistema. La potencia eléctrica teórica recibida es de -38,9 dBm para cada una de las subportadoras, y el CTB se puede ver que se encuentra en torno a los $-30 \mathrm{dBc}$.

Como vimos en la sección 2.4, los niveles de potencia de las subportadoras se ven afectados por la dispersión de la fibra en un fenómeno conocido como supresión de la portadora, y los sistemas MMDS no son una excepción. Las curvas de penalización de potencia en función de la longitud de fibra óptica y para distintas situaciones se muestran en la figura 2.15. En general, la presencia de chirp en el transmisor óptico produce un efecto beneficioso, pues el nivel de potencia de las subportadoras recibidas aumenta respecto al valor teórico como consecuencia de la dispersión de la fibra. Este efecto ya ha sido comprobado teórica y experimentalmente [Wed94]. Las líneas de

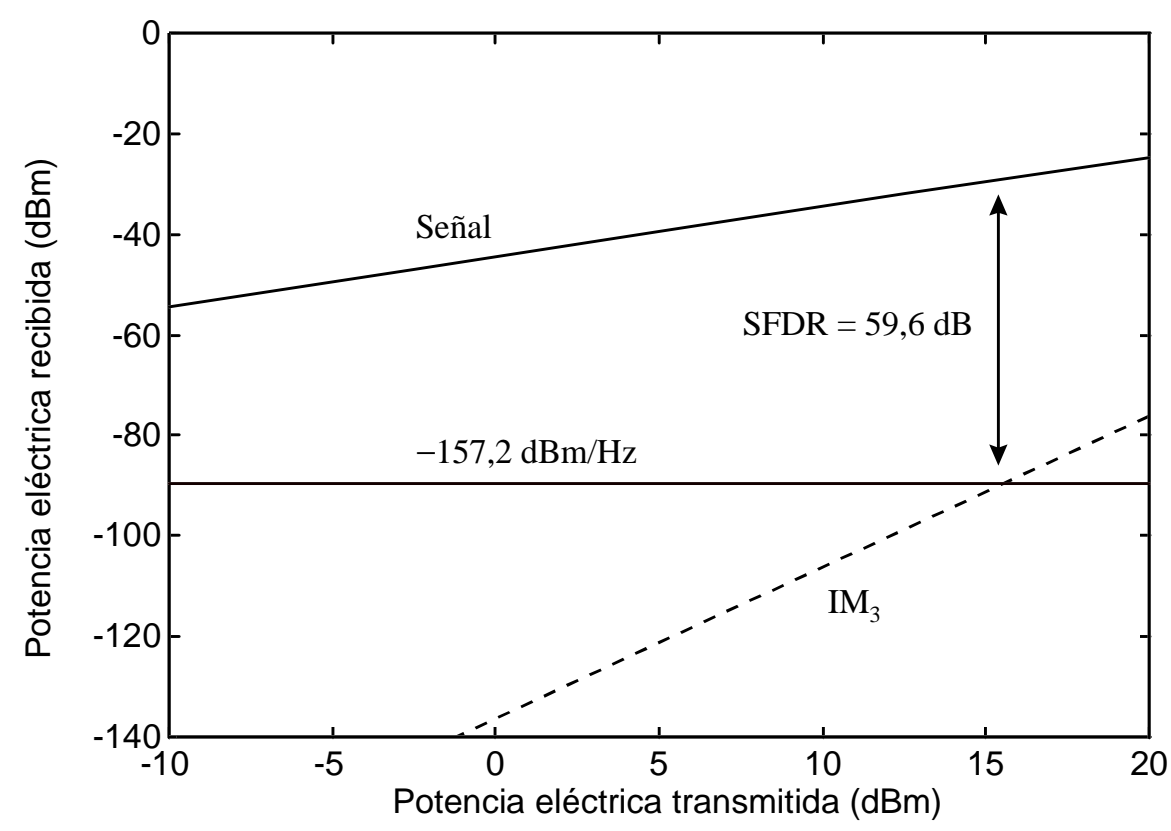

Figura 2.16 Potencias eléctricas de señal y de intermodulación $\left(\mathrm{IM}_{3}\right)$ a la salida del fotodetector en función de la potencia eléctrica de entrada al modulador óptico. Los parámetros del sistema son: $\vartheta=10 \mathrm{GHz}, \mathrm{L}=20 \mathrm{~km}, \mathrm{R}=0,9, \mathrm{P}_{\text {rec }}=+3 \mathrm{dBm}$ y $\mathrm{RIN}$ $=-150 \mathrm{~dB} / \mathrm{Hz}$, los cuales proporcionan un nivel de ruido de $-157,2$ $\mathrm{dBm} / \mathrm{Hz}$ y un SFDR de 59,6 dB para un ancho de banda de $6 \mathrm{MHz}$. 
trazos representan el caso GVD, mientras que las continuas incluyen el SPM. En el mejor de los casos, para una longitud de fibra de $50 \mathrm{~km}$ se pueden recibir más de $4 \mathrm{~dB}$ de potencia respecto al valor teórico de la subportadora. No obstante, a distancias mayores a las que se observan en la gráfica se produce un cambio de signo en la pendiente de las curvas y aparecen puntos en los que se anula la subportadora. En la situación convencional de GVD y $\vartheta=0$ se tiene siempre una diminución en los niveles de las subportadoras.

Por último, en la figura 2.16 se representan las potencias de señal y de distorsión $\left(\mathrm{IM}_{3}\right)$ a la salida del fotodetector en función de la potencia el éctrica de entrada al modulador óptico y tras $20 \mathrm{~km}$ de fibra. La pendiente de la recta del producto de intermodulación es el triple que la de la señal, lo cual significa que la $\mathrm{CIR}$ disminuye progresivamente al aumentar la potencia eléctrica de modulación, o lo que es lo mismo, el índice de modulación $m_{i}$. Para una potencia óptica recibida de $+3 \mathrm{dBm}$ se tiene una densidad espectral de potencia de ruido de $-157,2 \mathrm{dBm} / \mathrm{Hz}$, la cual proporciona un SFDR de 59,6 dB para un ancho de banda de $6 \mathrm{MHz}$.

\subsubsection{Sistema LMDS}

Para estudiar la distorsión no lineal en los sistemas LMDS consideraremos un plan de frecuencias formado por 40 canales espaciados $40 \mathrm{MHz}$ y extendiéndose desde 27,52 hasta 29,08 GHz. En esta situación, el nivel de distorsión se medirá para una frecuencia $\mathrm{f}_{d}=29,12 \mathrm{GHz}$ situada por encima de la banda de canales. Dado que la frecuencia de estos sistemas es bastante elevada $(\sim 28 \mathrm{GHz})$, las distancias de propagación se encontrarán mucho más limitadas que en los sistemas CATV o MMDS. Por ejemplo, en la figura 2.17 se representa el CTB en función de la longitud de fibra para las dos situaciones posibles: GVD y SPM. Fijando un valor de $-60 \mathrm{dBc}$ se consiguen distancias de tan sólo $1 \mathrm{~km}$. En general, en presencia de SPM se obtienen unos mayores niveles de distorsión. Sin embargo, para las longitudes típicas de trabajo $(L<3 \mathrm{~km})$ los valores son muy similares. Esto es debido a que el efecto predominante es el de la dispersión de la fibra, pues el SPM necesita de unas longitudes de fibra algo mayores para que sea significativo. De este 


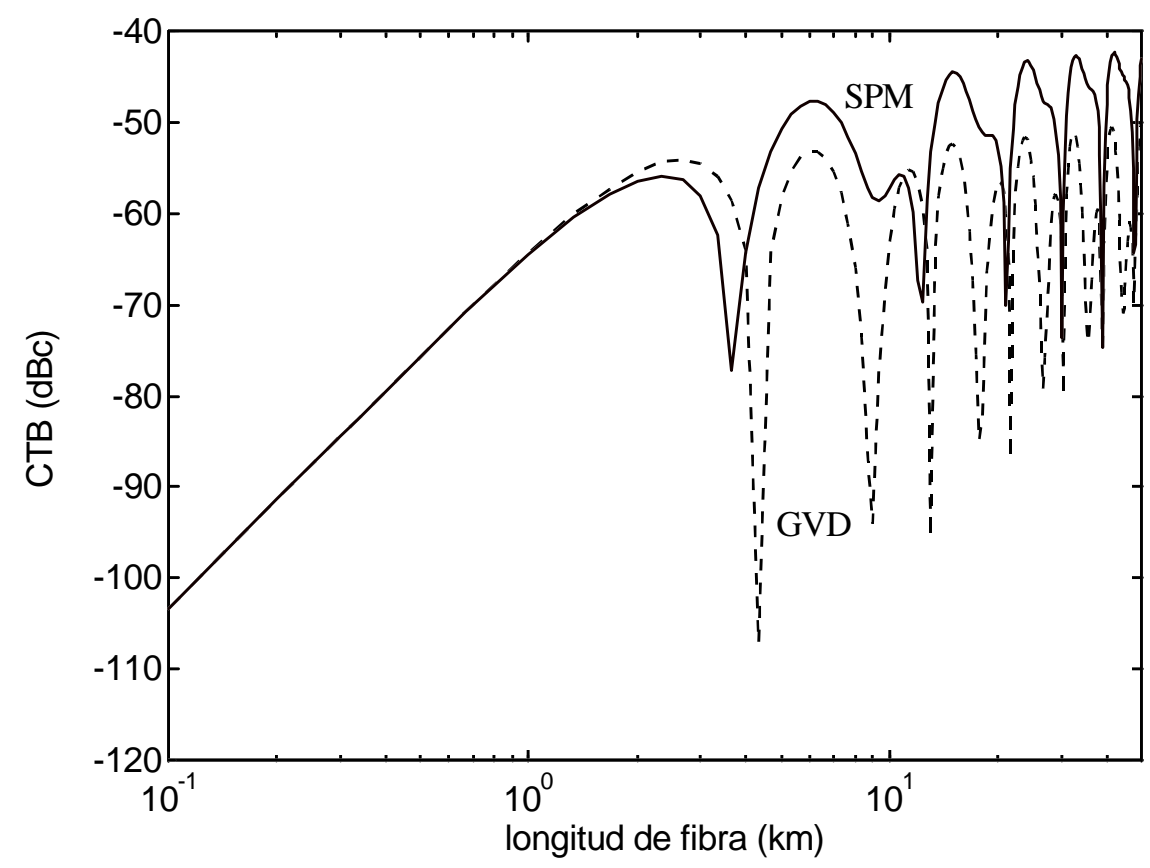

Figura 2.17 Distorsión de tercer orden en función de la longitud de fibra para un sistema LMDS de 40 canales. EI CTB se ha medido a una frecuencia de $29,12 \mathrm{GHz}$. Las líneas de trazos son para GVD y las continuas para SPM. Los parámetros de modulación son: $m_{i}=0,04, \vartheta=0$ (modulación externa) y $P_{0}=20$ $\mathrm{mW}$.

modo, podemos utilizar potencias ópticas elevadas sin que ello afecte a las prestaciones del sistema, aunque para las longitudes de fibra típicas no sería necesario.

El espectro de la señal recibida tras $6 \mathrm{~km}$ de fibra óptica se puede ver en la figura 2.18. La potencia eléctrica teórica de las subportadoras es de $-21,3$ $\mathrm{dBm}$, sin embargo, se observa que la amplitud de las mismas no es uniforme a consecuencia de la distorsión no lineal. Para los canales situados a mayor frecuencia se detecta una mayor potencia eléctrica. En cuanto a los niveles de distorsión, éstos se mantienen por debajo de los $-30 \mathrm{dBc}$ y decrecen al alejarse de la banda donde están situadas las subportadoras, ya que el número de productos de intermodulación es cada vez menor. 


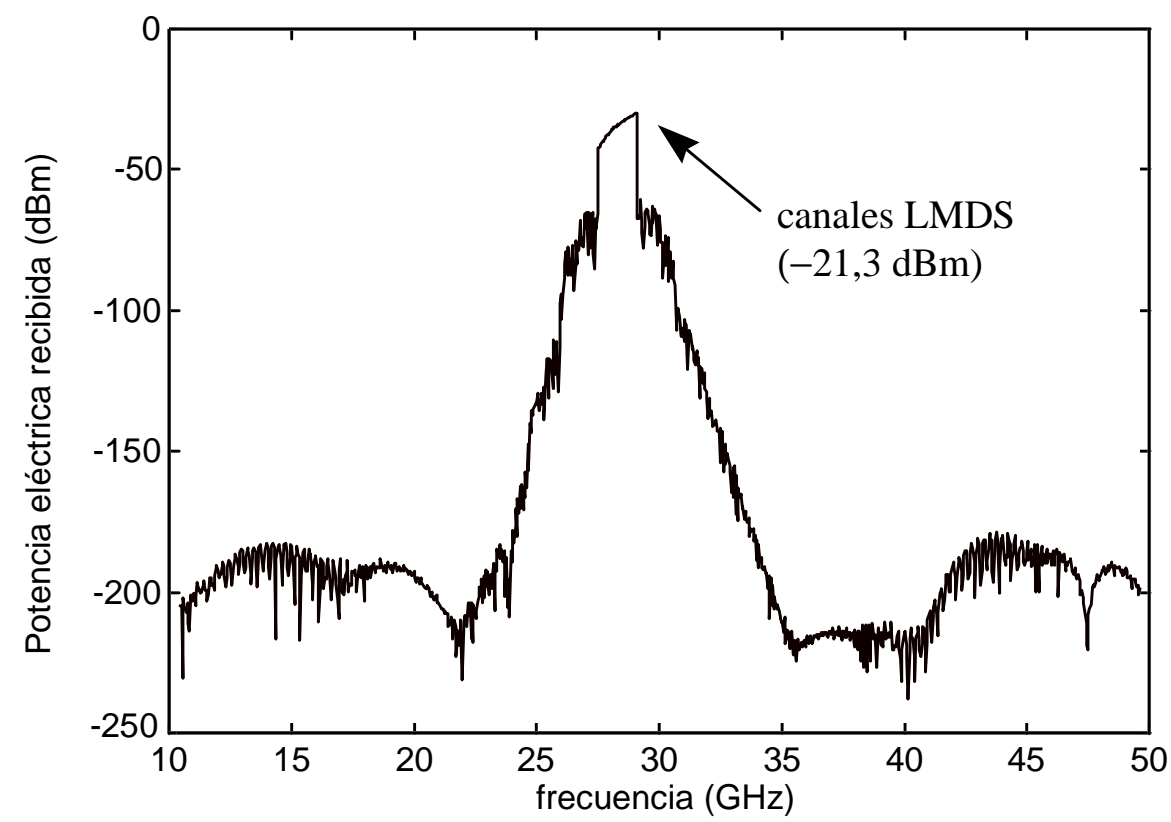

Figura 2.18 Espectro de la señal multicanal recibida tras $6 \mathrm{~km}$ de fibra óptica dispersiva y no lineal. La potencia eléctrica teórica de los canales recibidos es de $-21,3 \mathrm{dBm}$. Los parámetros del sistema son: $\mathrm{m}_{\mathrm{i}}=0,04, \vartheta=0, \mathrm{P}_{0}=20 \mathrm{~mW}, \mathrm{R}=0,9$ y $\mathrm{Z}_{\text {rec }}=50 \Omega$.

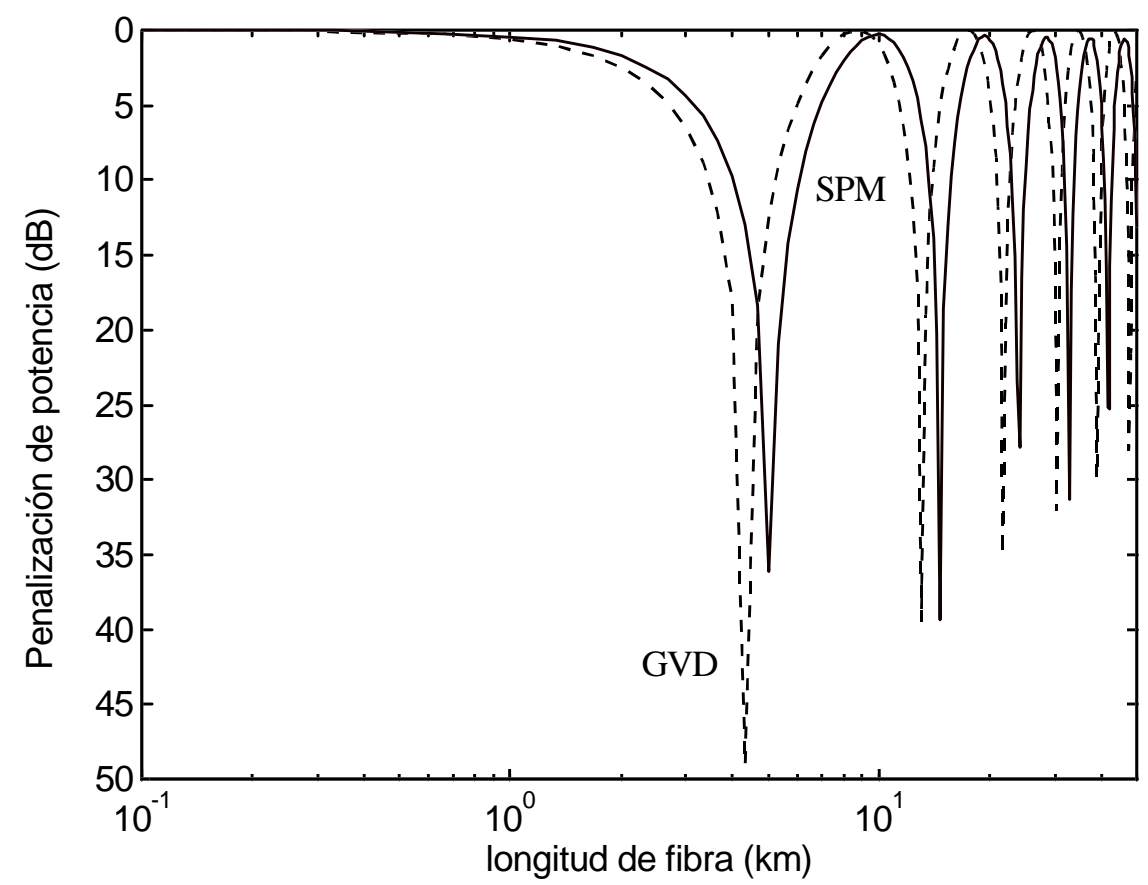

Figura 2.19 Penalización de potencia en función de la longitud de fibra óptica para el último canal de la banda $(29,08 \mathrm{GHz})$. Las líneas de trazos son para GVD y las continuas para SPM. LoS parámetros de modulación son: $\mathrm{m}_{\mathrm{i}}=0,04, \vartheta=0$ y $\mathrm{P}_{0}=20 \mathrm{~mW}$. 


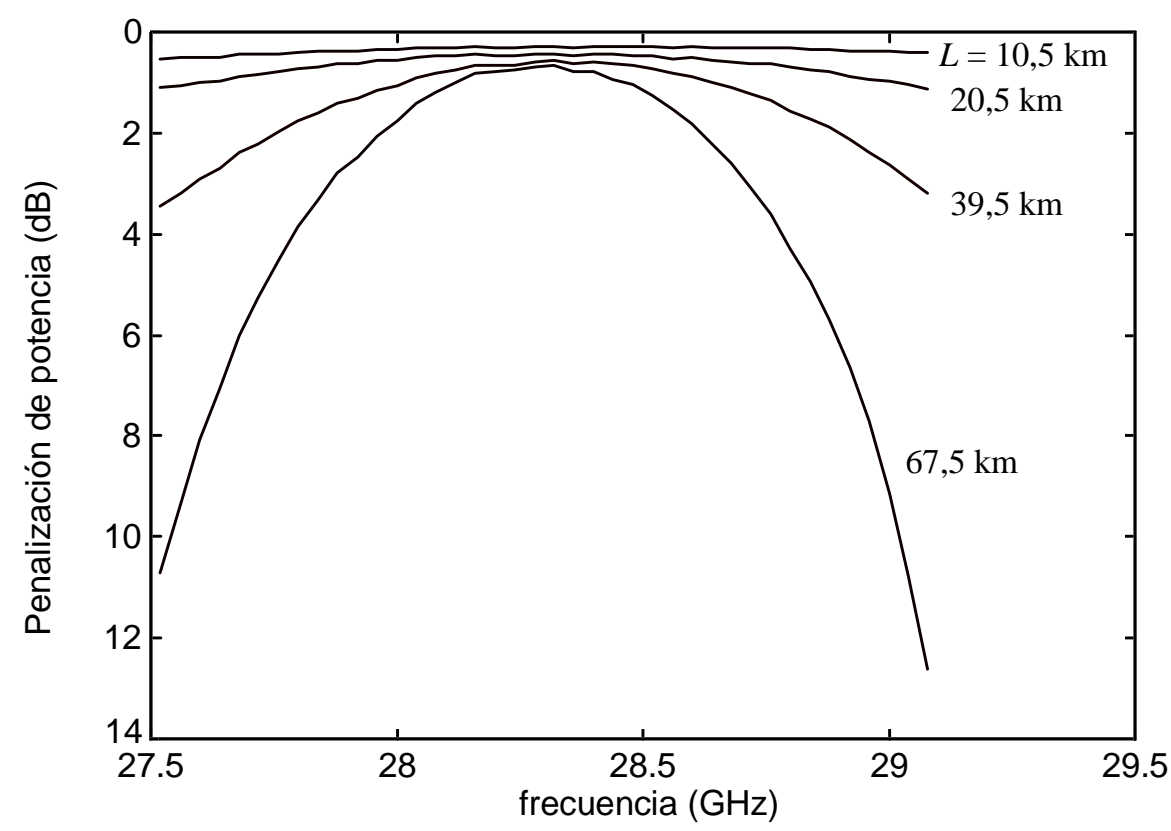

Figura 2.20 Penalización de potencia inducida por la fibra óptica en función de la frecuencia del sistema LMDS para varios valores de longitud de fibra. Estas longitudes se han escogido de tal forma que aparezca un máximo en el centro de la banda de canales. Los parámetros de modulación son idénticos a los de la figura anterior.

Un resultado interesante lo constituye la penalización de potencia de las subportadoras. En particular, en la figura 2.19 aparece la penalización de potencia en función de la longitud de fibra para la subportadora situada a $29,08 \mathrm{GHz}$. Al igual que se pudo ver en la figura 2.5, el efecto del SPM se refleja en un ligero desplazamiento de los notch de las curvas hacia longitudes de fibra mayores. Aquí también, a diferencia de los sistemas CATV y MMDS se produce una rápida caída en las amplitudes de las subportadoras que limita considerablemente las distancias alcanzables. Por ejemplo, para los $3 \mathrm{~km}$ se tienen prácticamente $5 \mathrm{~dB}$ de penalización de potencia. De todos modos, es posible aprovechar el comportamiento periódico de las lobulaciones de una forma beneficiosa. Si representamos la penalización de potencia en función de las frecuencias de la banda donde están situados los canales y parametrizándola con la longitud de fibra, es posible colocar las subportadoras dentro de alguno de estos lóbulos. Esto mismo es lo que se realiza en [Par96, Par97a] y se muestra en la figura 2.20 para nuestro sistema en particular. Fijando una penalización de potencia 




Figura 2.21 Potencias eléctricas de señal y de intermodulación $\left(\mathrm{IM}_{3}\right)$ a la salida del fotodetector en función de la potencia eléctrica de entrada al modulador óptico. Los parámetros del sistema son: $\vartheta=0, L=2 \mathrm{~km}, \mathrm{R}=0,9, \mathrm{P}_{\text {rec }}=+3 \mathrm{dBm}$ y $\mathrm{RIN}=-150$ $\mathrm{dB} / \mathrm{Hz}$, los cuales proporcionan un nivel de ruido de $-157,2$ $\mathrm{dBm} / \mathrm{Hz}$ y un SFDR de 57,9 dB para un ancho de banda de 40 $\mathrm{MHz}$.

inferior a $1 \mathrm{~dB}$ se pueden alcanzar distancias de transmisión de hasta 20 $\mathrm{km}$, las cuales son muy superiores a las que se deducían de la figura 2.19. Además, para $67,5 \mathrm{~km}$ de fibra se tiene un ancho de banda a $-3 \mathrm{~dB}$ de unos $800 \mathrm{MHz}$ que es suficiente para transmitir un buen número de canales. No obstante, el principal problema lo constituye la precisión con la que debe calcularse la longitud de fibra del enlace, pues la curva de penalización debe tener su máximo lo más próximo al centro de la banda de canales a transmitir. Posteriormente, los canales situados en los extremos de la banda serán los que limiten la máxima distancia alcanzable.

Por último, en la figura 2.21 se pueden ver los niveles de potencia eléctrica de señal y de intermodulación a la salida del fotodetector en función de la potencia eléctrica de entrada al modulador externo. Para este sistema se tiene un SFDR de 57,9 dB para un ancho de banda de $40 \mathrm{MHz}$. Este valor es mejor que el del sistema MMDS teniendo en cuenta que el ancho de banda 
es superior, sin embargo, aquí se considera una longitud de fibra de tan sólo $2 \mathrm{~km}$. La potencia óptica recibida se está manteniendo constante en todos los sistemas $(+3 \mathrm{dBm})$ para evitar que, al realizar comparaciones entre los mismos, influya el ruido del sistema sobre el val or final de SFDR.

\subsubsection{Sistema MVDS}

En este sistema se supondrá una canalización que abarca desde 40,8 hasta $41,67 \mathrm{GHz}$ y que está formada por 30 canales de vídeo con modulación FM en intervalos de $30 \mathrm{MHz}$. La distorsión no lineal vendrá determinada por el CTB, el cual se representa en la figura 2.22 para una frecuencia de 41,7 GHz. Como se puede ver, los niveles de distorsión son más elevados que en cualquiera de los sistemas anteriores, por lo que las distancias que se alcanzan en estos sistemas se encuentran bastantes limitadas $(\sim 1 \mathrm{~km})$. Para estas longitudes de fibra no es demasiado importante el efecto Kerr,



Figura 2.22 Distorsión de tercer orden en función de la longitud de fibra para un sistema MVDS de 30 canales. El CTB se ha medido a una frecuencia de $41,7 \mathrm{GHz}$. Las líneas de trazos son para GVD y las continuas para SPM. Los parámetros de modulación son: $\mathrm{m}_{\mathrm{i}}=0,04, \vartheta=0$ (modulación externa) y $\mathrm{P}_{0}=20$ $\mathrm{mW}$. 


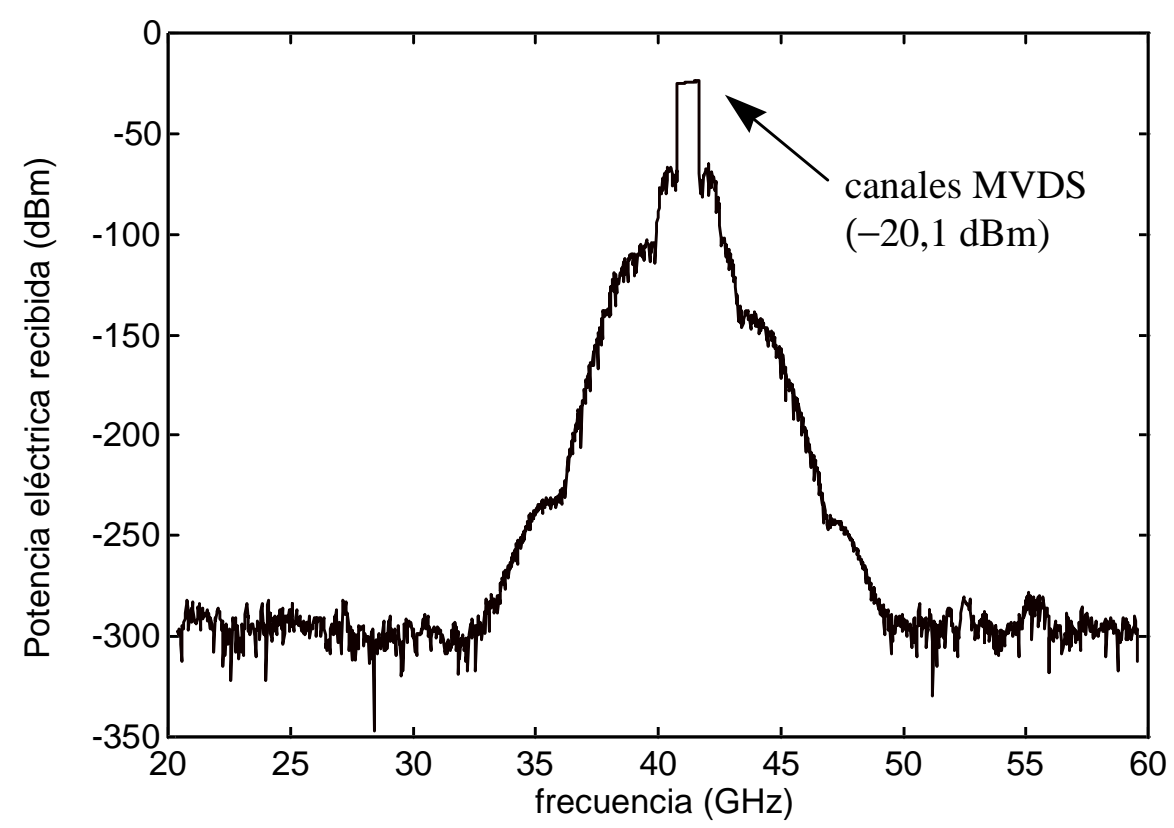

Figura 2.23 Espectro de la señal multicanal recibida tras $3 \mathrm{~km}$ de fibra óptica dispersiva y no lineal. La potencia eléctrica teórica de los canales recibidos es de $-20,1 \mathrm{dBm}$. Los parámetros del sistema son: $\mathrm{m}_{\mathrm{i}}=0,04, \vartheta=0, \mathrm{P}_{0}=20 \mathrm{~mW}, \mathrm{R}=0,9$ y $\mathrm{Z}_{\text {rec }}=50 \Omega$.

siendo el CTB prácticamente independiente de la potencia óptica. Esto mismo se desprende de la figura, pues las curvas para GVD y SPM aparecen superpuestas. En este sentido se comporta igual que el sistema LMDS.

En cuanto a la señal recibida, ésta tiene un espectro que se puede ver en la figura 2.23. El nivel de potencia eléctrica a la salida del fotodetector es de 20,1 dBm. Esta potencia tan elevada se debe a que estamos suponiendo una potencia óptica a la entrada de la fibra de $+13 \mathrm{dBm}$, pues interesa medir la distorsión producida por SPM. La distorsión se encuentra en torno a los -50 $\mathrm{dBc}$ y decrece progresivamente al alejarnos de la banda de canales en la misma proporción que lo hace el número de productos de intermodulación. No obstante, aquí se puede apreciar también la presencia de productos de intermodulación de orden $5,7,9$, etc., ya que los de tercer orden ocupan sólo la banda 39,93-42,54 GHz. En general, los términos de distorsión presentes en la gráfica son del tipo $\mathrm{mf}_{1}-\mathrm{nf}_{2}$, con $\mathrm{m}=\mathrm{n}+1$ y $\mathrm{m}+\mathrm{n}=$ orden de la distorsión. La caída se debe al aumento del orden y disminución del número de productos de intermodulación. Volviendo a la figura 2.18 del espectro del 


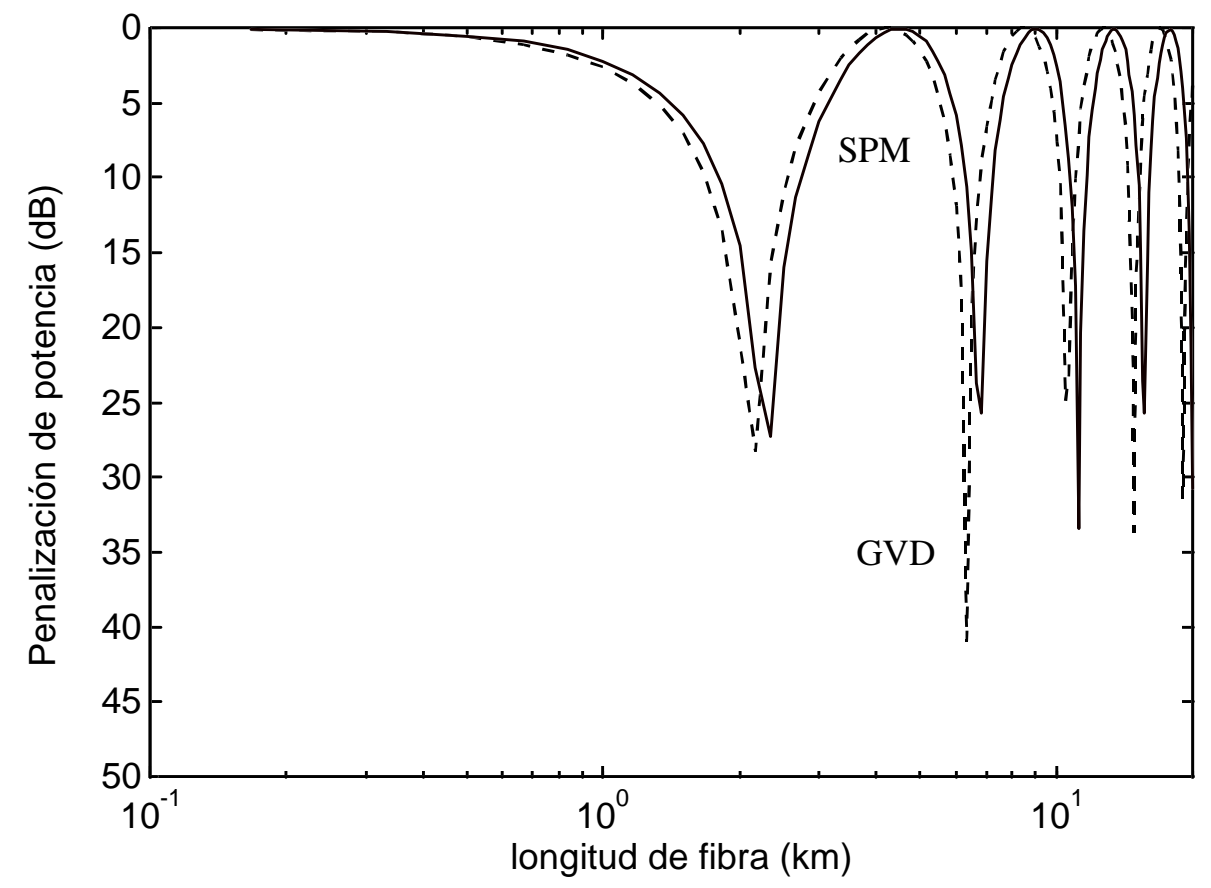

Figura 2.24 Penalización de potencia en función de la longitud de fibra óptica para el último canal de la banda $(41,67 \mathrm{GHz})$. Las líneas de trazos son para GVD y las continuas para SPM. Los parámetros de modulación son: $\mathrm{m}_{\mathrm{i}}=0,04, \vartheta=0$ y $\mathrm{P}_{0}=20 \mathrm{~mW}$.

sistema LMDS, en la misma se aprecia también este fenómeno. Conviene recalcar que en estas figuras sólo se representa la señal y su distorsión, no incluyendo en ningún caso el ruido presente a la salida del fotodetector. En este último caso, a partir de una determinada frecuencia a ambos lados de la banda de canales el espectro sería aproximadamente plano (ruido blanco).

Las curvas de penalización de potencia para GVD y SPM se representan en la figura 2.24. También en este caso son muy similares a las del sistema LMDS, si bien las distancias alcanzables son ligeramente menores. Para tan sólo $1 \mathrm{~km}$ de fibra se producen ya penalizaciones en torno a los $3 \mathrm{~dB}$. La única forma de contrarrestar este efecto es con técnicas de ecualización de distorsión no lineal o empleando métodos como el comentado en el apartado anterior para el sistema LMDS.

Para terminar, en la figura 2.25 se muestran las potencias eléctricas de señal y de intermodulación a la salida del fotodetector en función de la 
potencia eléctrica de entrada al modulador externo. Para este sistema el SFDR es de 58,6 dB sobre $30 \mathrm{MHz}$ de ancho de banda, muy similar al del sistema LMDS. La longitud de fibra considerada es de $1 \mathrm{~km}$ y la potencia óptica recibida de $+3 \mathrm{dBm}$.

\subsubsection{Comparación entre sistemas}

En los apartados anteriores se ha realizado un estudio de simulación relativo a la distorsión no lineal introducida por la fibra óptica en sistemas de comunicaciones ópticas radio-fibra. A continuación, se recogerán algunos de los resultados para resumir en una serie de gráficas comparativas las prestaciones que se pueden alcanzar con cada uno de estos sistemas.

Para comenzar, en la figura 2.26(a) se compara la CIR de cada uno de los sistemas. Todas las curvas han sido obtenidas con modulación externa y



Figura 2.25 Potencias eléctricas de señal y de intermodulación $\left(\mathrm{IM}_{3}\right)$ a la salida del fotodetector en función de la potencia eléctrica de entrada al modulador óptico. Los parámetros del sistema son: $\vartheta=0, L=1 \mathrm{~km}, \mathrm{R}=0,9, \mathrm{P}_{\mathrm{rec}}=+3 \mathrm{dBm}$ y $\mathrm{RIN}=-150$ $\mathrm{dB} / \mathrm{Hz}$, los cuales proporcionan un nivel de ruido de $-157,2$ $\mathrm{dBm} / \mathrm{Hz}$ y un SFDR de 58,6 dB para un ancho de banda de 30 $\mathrm{MHz}$. 
niveles de potencia elevados, por lo que el factor limitante es el SPM. En el caso del sistema CATV, este parámetro viene determinado por el nivel de CSO, mientras que en los sistemas MMDS, LMDS y MVDS afecta únicamente el CTB, pues la banda de canales ocupa menos de una octava de frecuencia. Como se aprecia en la gráfica, el sistema MMDS es el más favorable desde el punto de vista de la CIR, mientras que los sistemas LMDS y MVDS son los que se encuentran más limitados debido a las elevadas frecuencias a las que trabajan. El sistema CATV posee unas prestaciones intermedias entre ambos. A pesar de que utiliza frecuencias en la banda de UHF, los niveles de distorsión son más elevados que en el sistema MMDS a consecuencia del CSO y del mayor número de canales.

Los resultados de penalización de potencia introducida por la fibra óptica se resumen en la figura $2.26(\mathrm{~b})$. Las curvas de trazos son para GVD y las continuas incluyen el SPM. Para $20 \mathrm{~km}$ de fibra no se aprecia penalización en el sistema MMDS. En cambio, los sistemas LMDS y MVDS se encuentran bastante limitados, aunque todavía es posible escoger cuidadosamente la longitud del enlace para evitar este efecto.

Por último, en la tabla III se proporcionan valores del SFDR para cada uno de los sistemas. Obsérvese que en estos resultados no se incluye el sistema CATV. Esto es debido a que en la definición del SFDR se utiliza la distorsión de tercer orden (producto de intermodulación $2 f_{1}-f_{2}$ ), mientras que en este sistema predomina el CSO y los resultados no serían reales. Para este caso se podría utilizar una definición similar pero tomando el producto de intermodulación $f_{1}+f_{2}$. Los valores de la tabla se han obtenido a partir de la ec. (2.55), y por lo tanto suponen la presencia de dispersión cromática únicamente. 


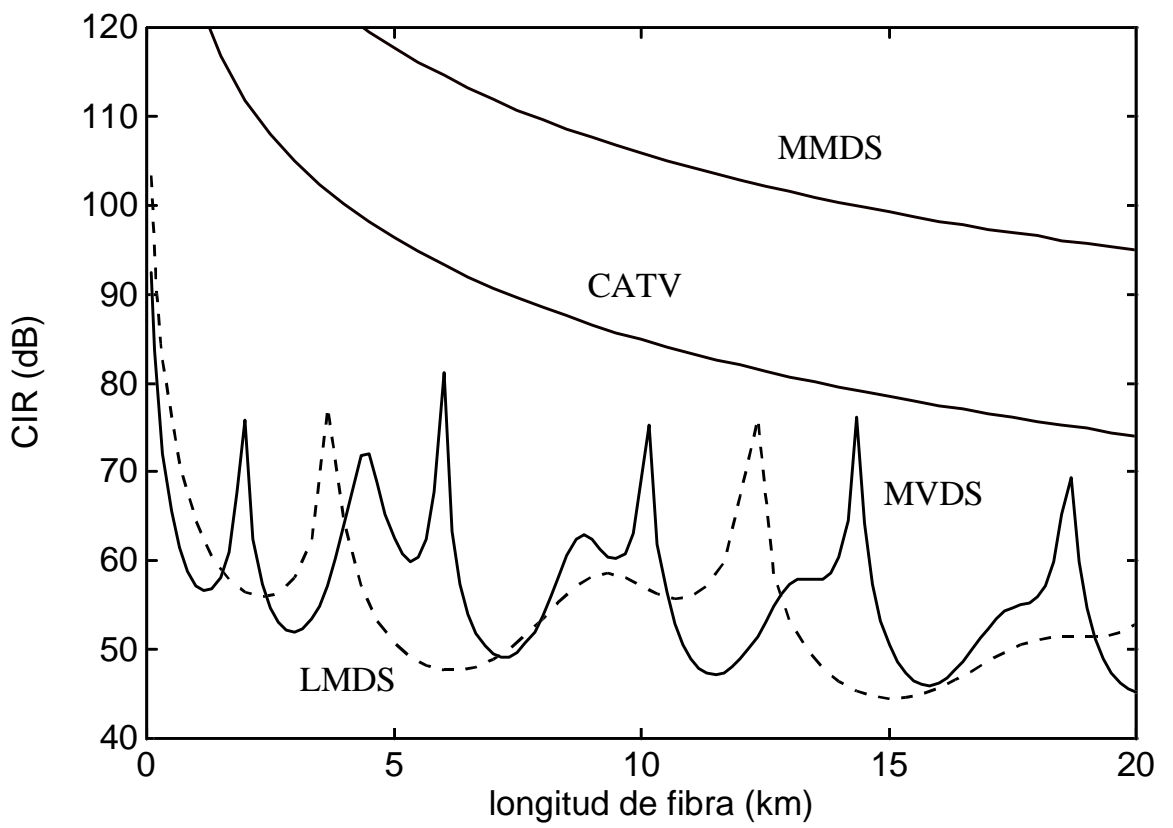

(a)

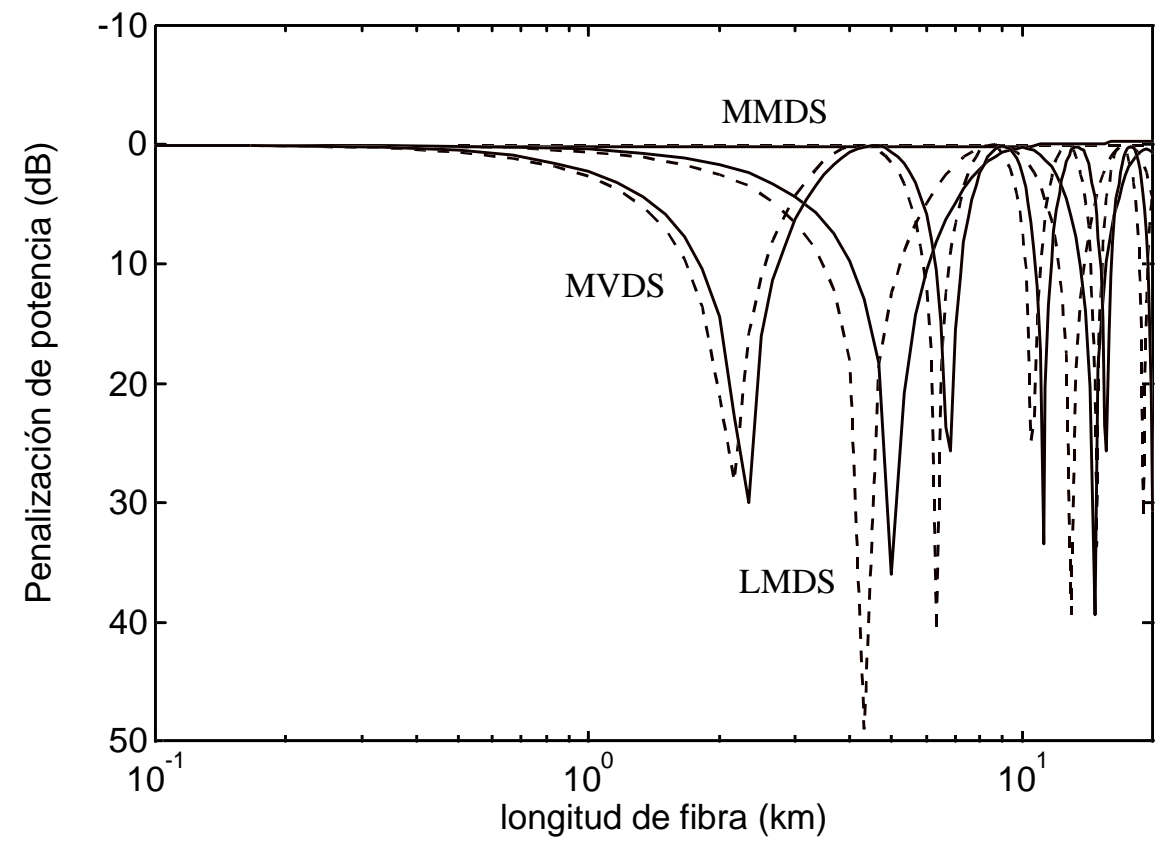

(b)

Figura 2.26 Comparación entre los distintos sistemas: (a) CIR en función de la longitud de fibra, y (b) penalización de potencia en función de la longitud de fibra para GVD (trazos) y SPM (continuas). Se considera modulación externa en todos los casos. 
TABLA III Valores de SFDR en $\mathrm{dB} \cdot \mathrm{Hz} \mathrm{z}^{2 / 3}$ para cada uno de los sistemas en función de la longitud de fibra y para dos valores de ruido RIN. En todos los casos se supone una potencia óptica recibida de $+3 \mathrm{dBm}$.

\begin{tabular}{|l|c|c|c|c|c|c|}
\cline { 2 - 7 } \multicolumn{1}{c|}{} & \multicolumn{3}{c|}{ RIN $=-\mathbf{1 5 0} \mathbf{d B} / \mathbf{H z}$} & \multicolumn{3}{c|}{ RIN =-130 dB/Hz } \\
\hline Sistema & $1 \mathrm{~km}$ & $2 \mathrm{~km}$ & $20 \mathrm{~km}$ & $1 \mathrm{~km}$ & $2 \mathrm{~km}$ & $20 \mathrm{~km}$ \\
\hline MMDS & 122,17 & 118,16 & 104,82 & 109,31 & 105,29 & 91,96 \\
\hline LMDS & 112,59 & 108,58 & - & 99,73 & 95,72 & - \\
\hline MVDS & 108,43 & - & - & 95,57 & - & - \\
\hline
\end{tabular}




\subsection{TÉCNICAS DE COMPENSACIÓN DE DISPERSIÓN}

La dipersión cromática se ha demostrado como un efecto perjudicial para la propagación de señales sobre SMFs. De entre las distintas técnicas propuestas para reducir sus efectos, destacan la modulación óptica en banda lateral única (OSSB, optical singlesideband) [Smi97a, Par97] o las redes de difracción sobre fibra ópticaxiii [Oue87, Mar97a]. En esta sección, se abordará un estudio comparativo de ambas técnicas en lo relativo a compensación de distorsión no lineal. Otra técnica como la de inversión espectral se tratará en detalle en el capítulo 4.

\subsubsection{Estudio de distorsión no lineal en la modulación SSB}

La modulación óptica SSB es una técnica tolerante a la dispersión que ha sido demostrada con múltiples experimentos de transmisión [Par97, Smi97b]. El nombre de "tolerante" viene del hecho que no compensa realmente la dispersión cromática como lo haría una DCF (dispersion

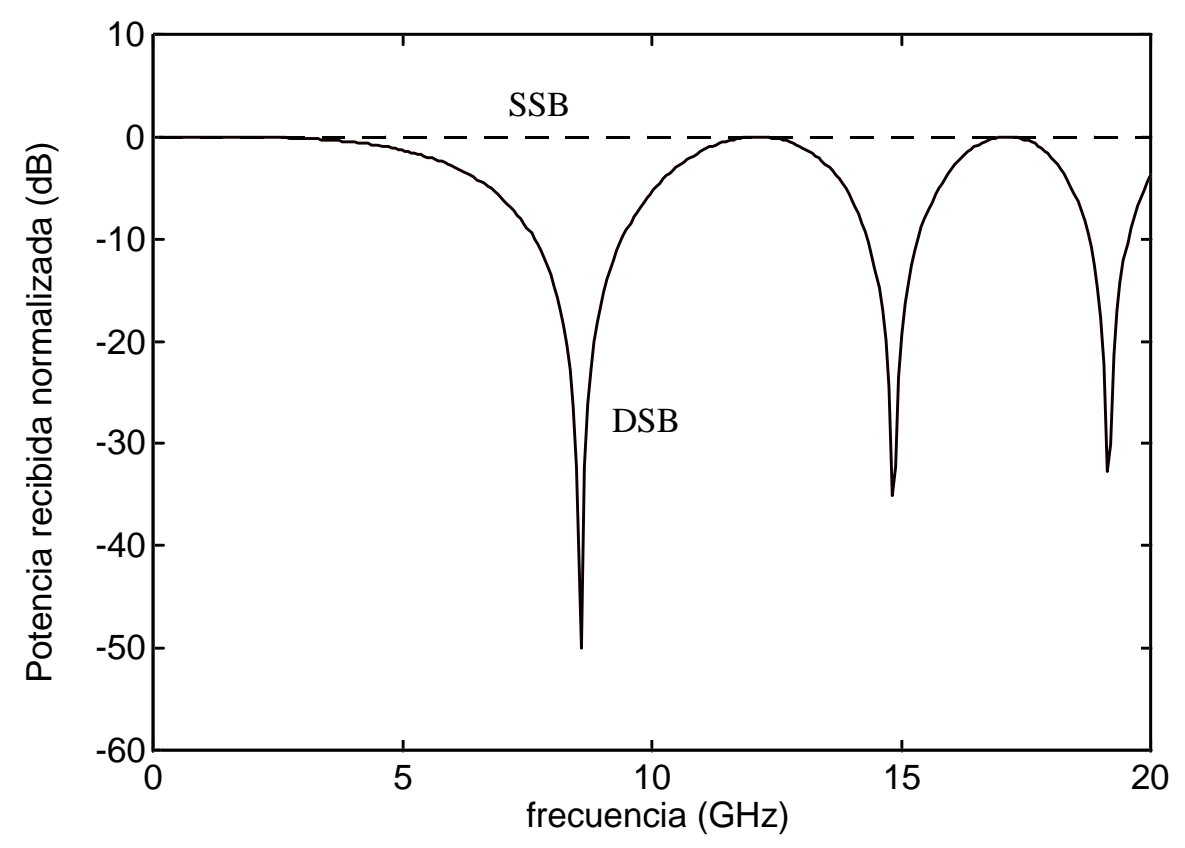

Figura 2.27 Función de transferencia de 50 km de SSMF lineal con modulaciones DSB y SSB.

\footnotetext{
xiii en realidad, se trata de redes de difracción con chirp (CFGs, chirped fiber gratings)
} 
compensating fiber) o un CFG, sino que introduce un chirp sobre la señal modulada que evita el fenómeno de supresión de la portadora generado por la dispersión de la fibra. En la figura 2.27 se representa la respuesta en frecuencia de $50 \mathrm{~km}$ de fibra con modulaciones SSB o DSB (double-sideband) convencional. Como se puede ver, con modulación DSB aparecen los ya conocidos nulos de transmisión que limitan la frecuencia o longitud de fibra máximas del sistema, mientras que con modulación SSB desaparecen completamente. Se puede demostrar que con una modulación SSB ideal y sin considerar el SPM de la fibra, la respuesta del sistema es independiente de la frecuencia ${ }^{\mathrm{xiv}}$. Sin embargo, esto no significa que la técnica SSB compense la dispersión cromática, como se verá a continuación.

En la figura 2.28 aparecen los niveles de CSO y CTB a la salida del fotodetector en función de la frecuencia de modulación para un enlace de fibra de $50 \mathrm{~km}$ de longitud. La señal modulada se ha obtenido por medio de un modulador Mach-Zehnder (MZM) polarizado en lineal con dos configuraciones distintas: DSB y SSB (apéndice I). Las curvas continuas son para una potencia eléctrica de modulación de $+3 \mathrm{dBm}$ y las de trazos para +9 dBm. Como es lógico, en el segundo caso se obtiene mayor distorsión porque el MZM también contribuye a los niveles de CSO y CTB como consecuencia de su respuesta no lineal. Lo realmente importante que se deduce de las gráficas es que la modulación SSB no compensa la dispersión cromática de la fibra, ya que si fuera así, los niveles de distorsión deberían disminuir con respecto a la modulación DSB. Además, en general los niveles de distorsión con modulación SSB son superiores. En particular, a frecuencias bajas el CSO con modulación SSB es claramente superior. Fijando un límite de $-60 \mathrm{dBc}$ y con una potencia de modulación de $+9 \mathrm{dBm}$, se obtiene una frecuencia de modulación máxima de $2,5 \mathrm{GHz}$ para SSB frente a $4 \mathrm{GHz}$ con DSB. En el caso del CTB de la figura 2.28(b), aunque se obtienen mejores resultados para frecuencias bajas, estos no son nada significativos y además con SSB se eliminan todos los nulos de distorsión de la respuesta.

xiv véase apéndice I 


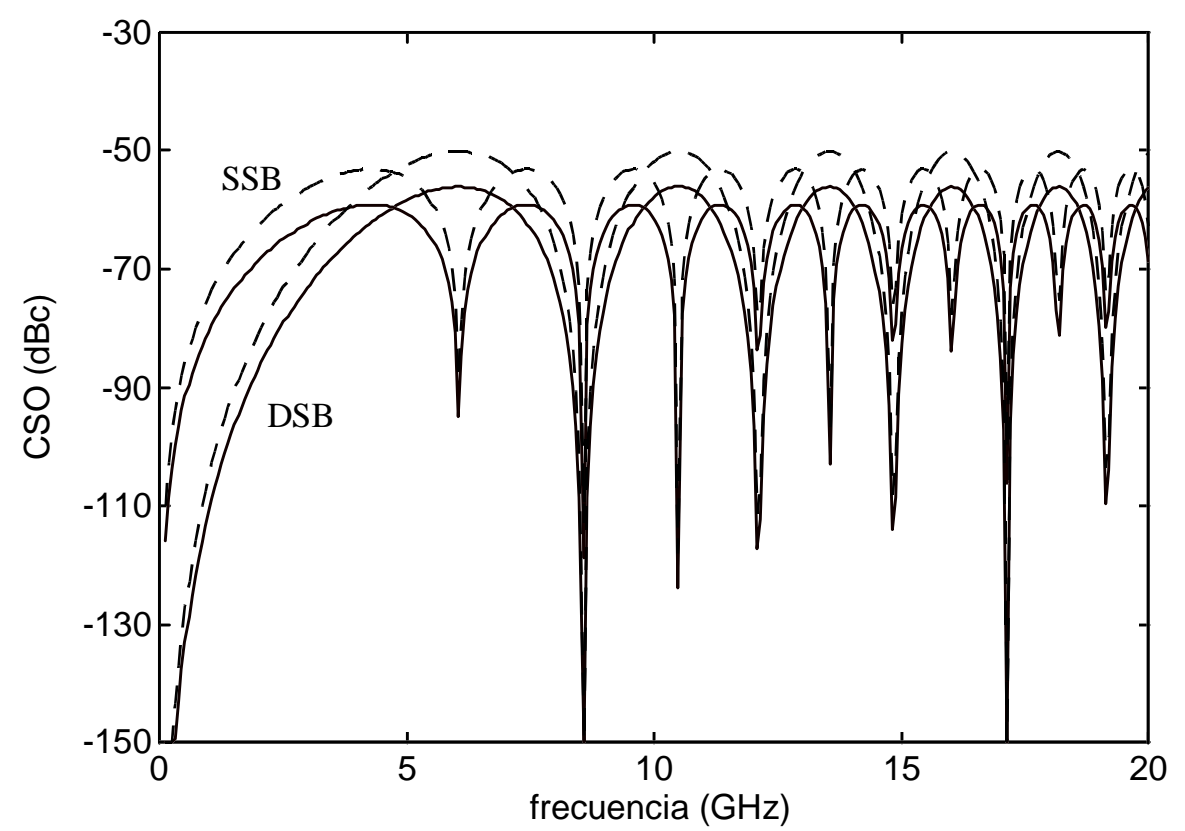

(a)

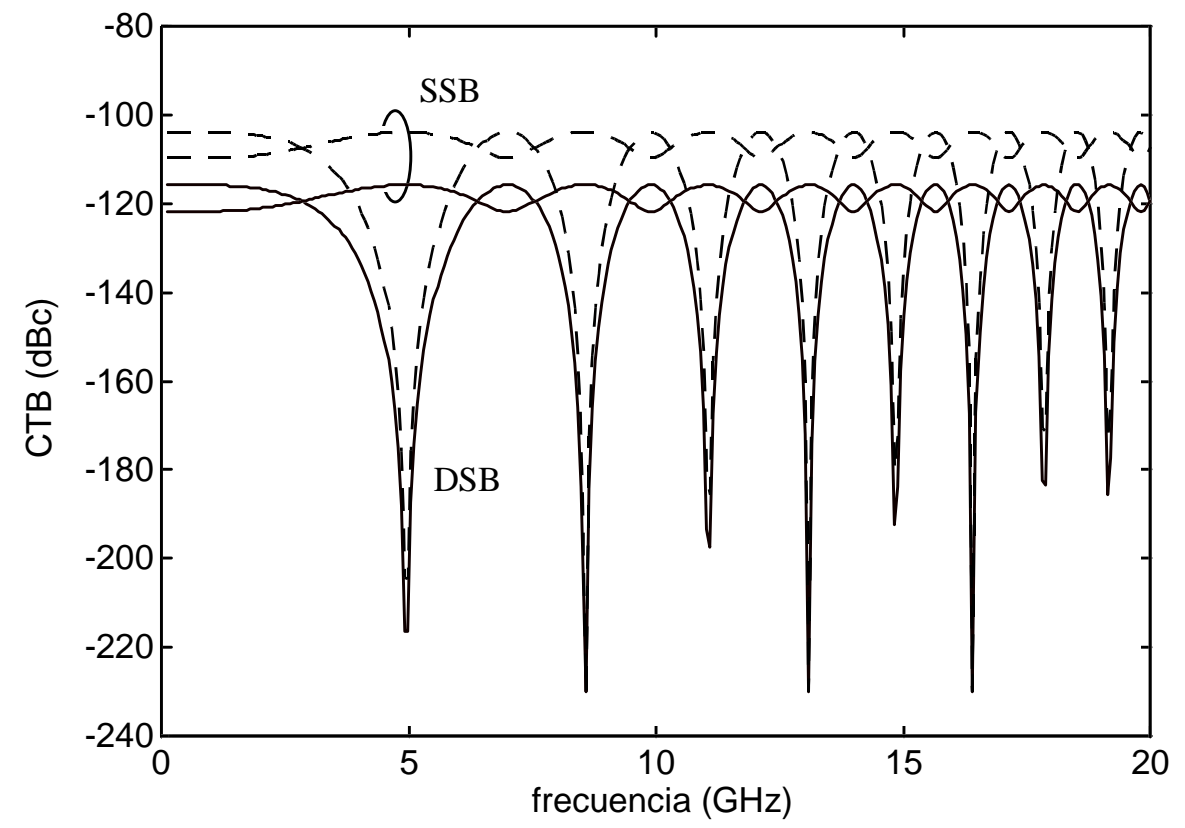

(b)

Figura 2.28 Distorsiones armónicas de (a) segundo y (b) tercer orden en función de la frecuencia de modulación para una longitud de fibra de $50 \mathrm{~km}$. La señal modulada se ha obtenido por medio de un MZM trabajando en una configuración DSB $\left(\phi_{1}=0, \phi_{2}\right.$ $=\pi) \circ \operatorname{SSB}\left(\phi_{1}=0, \phi_{2}=\pi / 2\right)$. Las curvas continuas son para una potencia eléctrica de modulación de $+3 \mathrm{dBm}$ y las de trazos para $+9 \mathrm{dBm}$. Otros parámetros: $Z_{\text {in }}=50 \Omega$ y $\mathrm{V}_{\pi}=9 \mathrm{~V}$. 
Estos mismos resultados en función de la distancia se presentan en la figura 2.29 para una frecuencia de modulación de $2 \mathrm{GHz}$. Debido a la frecuencia utilizada, se obtiene un mayor nivel de CSO y uno menor de CTB con modulación SSB. No obstante, el SPM de la fibra aumenta los niveles de distorsión y hace que en el caso del CSO se igual en y el CTB con modulación SSB supere al obtenido con modulación DSB para distancias cercanas a los $100 \mathrm{~km}$. En cualquier caso, se demuestra que la modulación SSB no compensa la distorsión no lineal generada por la dispersión cromática y el SPM de la fibra óptica.

\subsubsection{Breve introducción a las redes de difracción sobre fibra óptica}

Los CF Gs se han demostrado como efectivos compensadores de dispersión en sistemas de comunicaciones ópticas de gran distancia y velocidad [Loh96]. El éxito con respecto a otros métodos de compensación se debe a que se trata

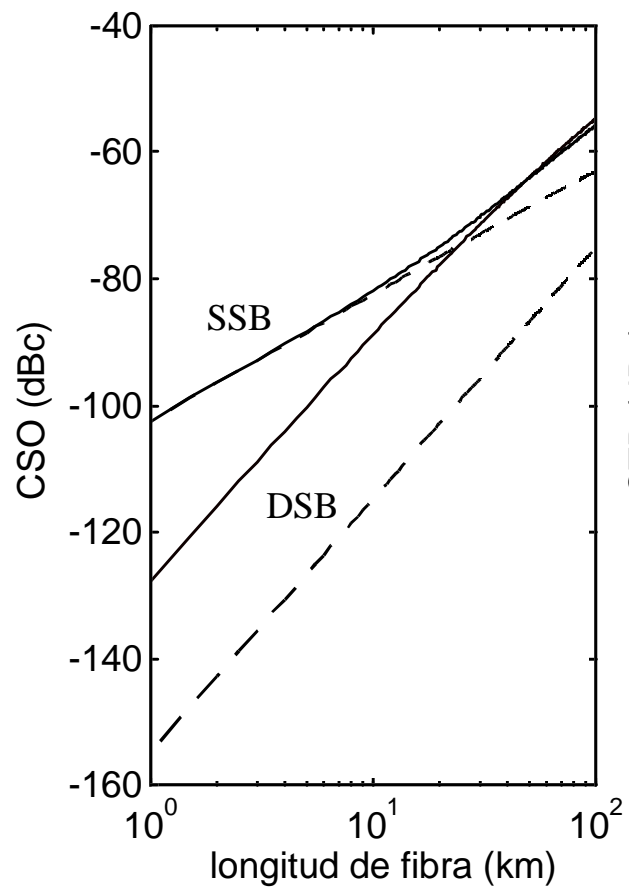

(a)

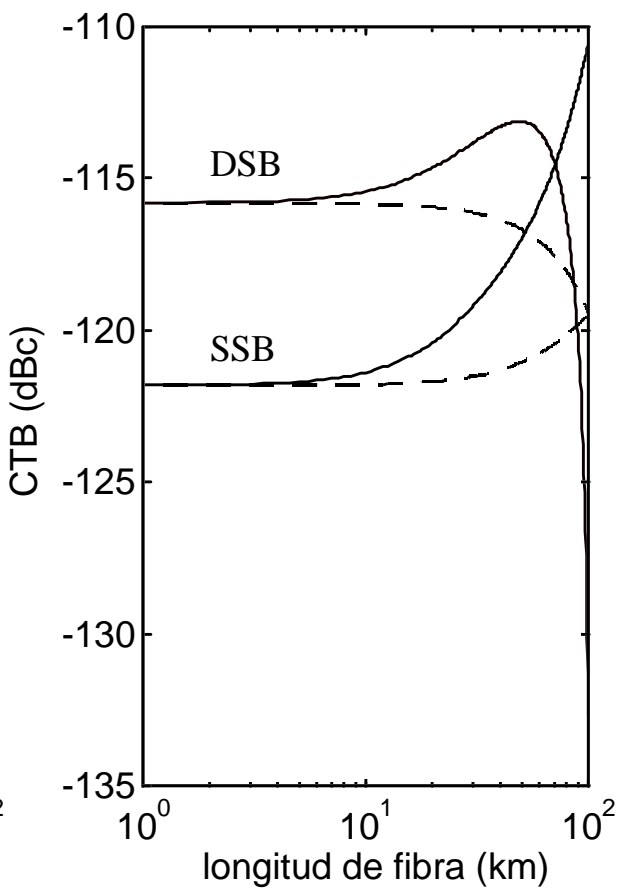

(b)

Figura 2.29 Distorsiones armónicas de (a) segundo y (b) tercer orden para una frecuencia de modulación de $2 \mathrm{GHz}$ y en función de la longitud de fibra óptica. Las curvas de trazos son para $\gamma=0$ y las continuas con SPM. Los parámetros son idénticos a los de la figura anterior. 
de dispositivos compactos, pasivos pero con bajas pérdidas, insensibles a la polarización y relativamente fáciles de fabricar [Oue87, Hil94].

La idea de compensación de dispersión se basa en variar la frecuencia de resonancia a lo largo del dispositivo por medio de un chirp en el índice de refracción, de tal forma que diferentes frecuencias se reflejan en distintos puntos $y$ con distintos retardos experimentados. Esto proporciona dispersiones positivas o negativas, dependiendo del signo de la constante de chirp. El esquema utilizado se muestra en la figura 2.30. Tras el enlace de fibra óptica y previo a la fotodetección se coloca un CFG, donde las señales incidente y reflejada se separan por medio de un circulador. La señal incidente es la que proviene del enlace de fibra y que ha sufrido los efectos de la dispersión cromática. La señal reflejada es la de salida del CFG, que tras la ecualización será fotodetectada. Existen variantes en las cuales se intercambian las posiciones del CFG y de la fibra óptica, o el CFG se coloca en el propio trayecto.

Suponiendo un modelo idealizado con chirp lineal, el retardo de grupo, $\tau$, experimentado por la señal al atravesar el CFG cumple [Gil97]

$$
\frac{\mathrm{d} \tau}{\mathrm{d} \lambda}=\frac{2 \mathrm{n}_{0}}{\mathrm{C}} \mathrm{C}^{-1}=\mathrm{D}_{\mathrm{CFG}}
$$

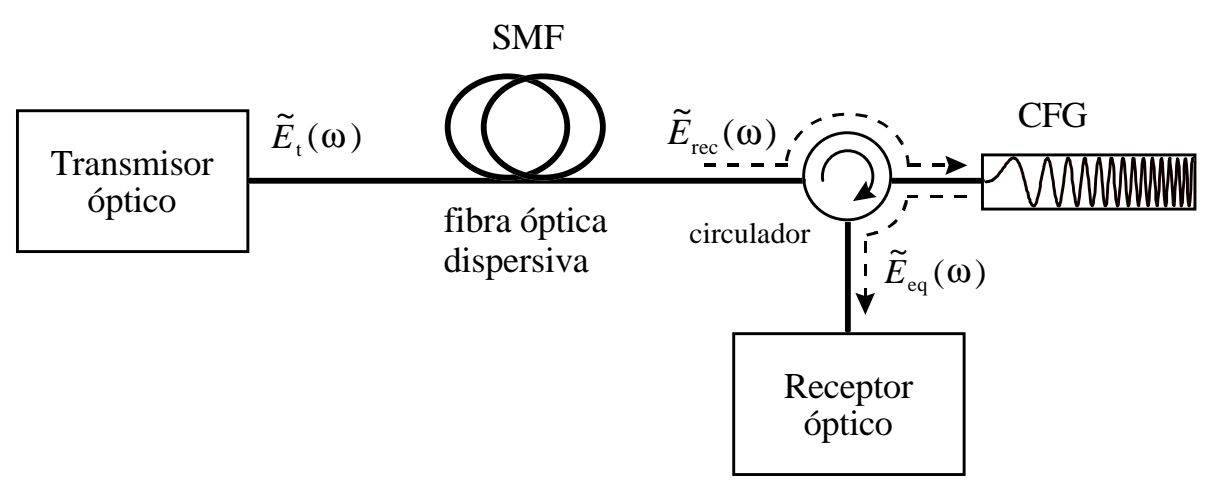

Figura 2.30 Diagrama de bloques de un sistema con compensación de dispersión mediante CFG. 
donde $\mathrm{C}$ es la constante de chirp en $\mathrm{nm} / \mathrm{m}$. De este modo, la condición para compensar dispersión vendrá impuesta por $D_{C F G}=D L$, obteniéndose una constante de chirp:

$$
\mathrm{C}=\frac{2 \mathrm{n}_{0}}{\mathrm{CDL}}
$$

Además, el CFG debe tener la suficiente longitud para asegurar que todo el espectro de la señal, $\Delta \lambda_{s}$, es acomodado en el interior del dispositivo. Esta condición se expresa por medio de

$$
\mathrm{L}_{\mathrm{CFG}}>\frac{\Delta \lambda_{\mathrm{s}}}{\mathrm{C}} \text {. }
$$

Por ejemplo, un CFG de $5 \mathrm{~cm}$ con un chirp de $0,06 \mathrm{~nm} / \mathrm{cm}$ puede compensar la dispersión cromática de una señal a $1550 \mathrm{~nm}$, con un ancho de banda de $0,3 \mathrm{~nm}$ y que atraviesa $100 \mathrm{~km}$ de SSMF .

No obstante, este análisis ha sido muy simplificado, ya que no se ha tenido en cuenta la apodización que presenta el CFG para reducir el rizado del retardo de grupo como consecuencia de reflexiones en los extremos, y que conduce a una disminución del ancho de banda. Aun así, todavía permanecen fluctuaciones en la reflectividad y retardo del grupo del dispositivo que habrá que caracterizar. La mayoría de estas fluctuaciones se deben a variaciones estocásticas como consecuencia de un proceso de fabricación imperfecto.

La respuesta de un CFG viene dada por la función compleja $R=|R| \exp (j \theta)$, donde la reflectividad y el retardo en función de la longitud de onda vienen dados por [Enn98]

$$
\begin{aligned}
& |R(\lambda)|=\left(1-r_{1}\right)+r_{1} \operatorname{sen}\left(\frac{2 \pi}{p} \lambda\right), \\
& \tau(\lambda)=a_{1} \lambda+a_{2}+b_{1} \operatorname{sen}\left(\frac{2 \pi}{p} \lambda\right),
\end{aligned}
$$


siendo $r_{1}$ y $b_{1}$ las amplitudes de las modulaciones y $p$ el período. Los coeficientes $a_{1}$ y $a_{2}$ dependen de la dispersión total del enlace.

Si integramos a continuación el retardo, se obtiene la expresión de la fase [Enn98]

$$
\begin{aligned}
\theta(\lambda) & =-2 \pi c \int\left(\frac{\tau(\lambda)}{\lambda^{2}}\right) d \lambda \\
& \cong-\frac{2 \pi c}{\lambda_{0}^{2}}\left[\frac{a_{1}}{2} \lambda^{2}+a_{2} \lambda^{2}-\frac{b_{1} p}{2 \pi} \cos \left(\frac{2 \pi}{p} \lambda\right)\right]+c_{0},
\end{aligned}
$$

donde $c_{0}$ es la constante de integración inicial. La simplificación es posible realizarla debido a que el ancho de banda del CFG es despreciable frente a la longitud de onda de trabajo, $\lambda_{0}$.

En la figura 2.31 se representan las respuestas de amplitud y de retardo medidas en el laboratorio de un CFG típico preparado para compensar 50

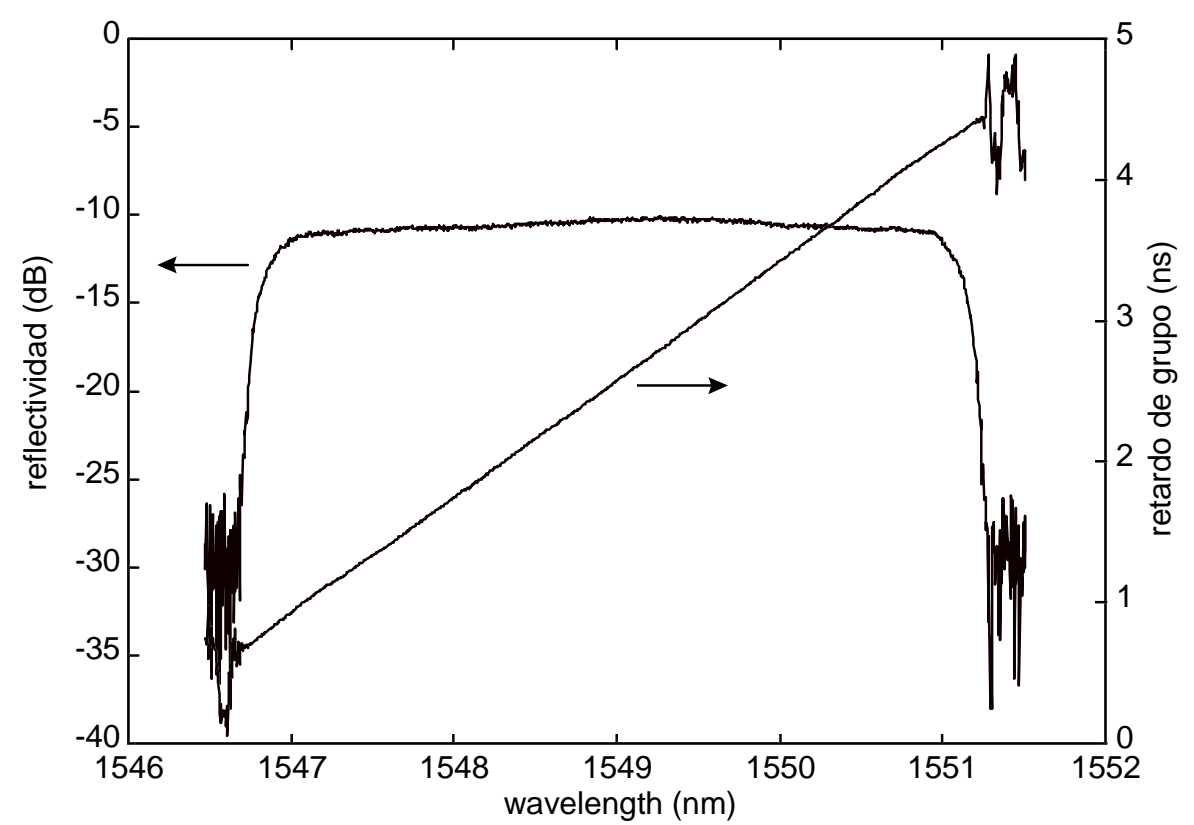

Figura 2.31 Reflectividad y retardo de grupo en función de la longitud de onda de un CFG. ancho de banda: 4 nm; dispersión: $850 \mathrm{ps} / \mathrm{nm}$. 
km de SSMF. Este dispositivo se caracteriza por un ancho de banda de $4 \mathrm{~nm}$ @ 1550 nm y una dispersión de aproximadamente 850 ps/nm. Obsérvese el rizado característico que presenta el retardo de grupo y que limita los resultados de ecualización.

\subsubsection{Comparación de las técnicas SSB y CF G}

A continuación se realizará un estudio comparativo de la técnica SSB frente al uso de CFG para compensar la distorsión no lineal que aparece en sistemas multicanal radio sobre fibra óptica. Los CFGs ya han sido propuestos para compensar la distorsión inducida por la dispersión de la fibra en sistemas multicanal radio sobre fibra [Mar97b, Pas97]. Sin embargo, hasta el momento no se había realizado un estudio de la técnica SSB.

El objetivo de las simulaciones es medir la distorsión no lineal a la salida del fotorreceptor para tres técnicas diferentes de transmisión: modulación DSB convencional, modulación SSB y modulación DSB con CFG. EI diagrama de bloques del sistema considerado se muestra en la figura 2.32.

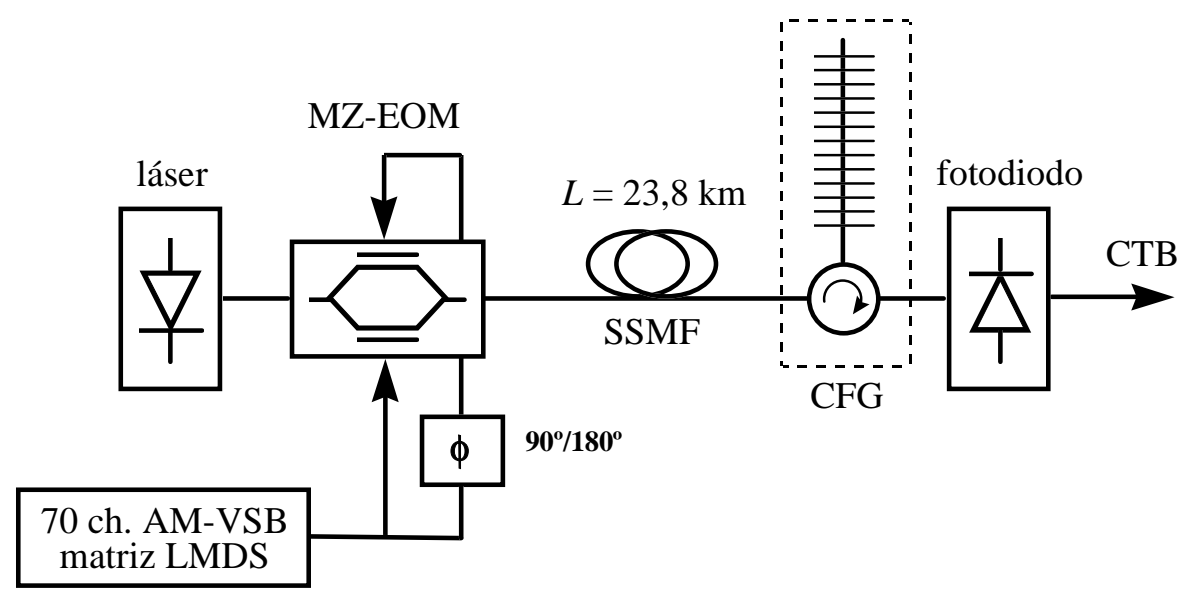

Figura 2.32 Diagrama de bloques de un sistema de distribución óptica de señales CATV subidas en frecuencia a la banda LMDS. Este sistema se utilizará para comparar las técnicas DSB, SSB y CFG en lo relativo a distorsión no lineal en sistemas multicanal a frecuencias milimétricas. 
La salida de un diodo láser operando a $1549 \mathrm{~nm}$ es modulada por medio de un modulador el ectroóptico Mach-Zehnder (MZ-EOM) dual-drive polarizado en cuadratura (QB) para operación lineal. La señal eléctrica moduladora, compuesta por 70 subportadoras equiespaciadas ( $\Delta \mathrm{f}=6 \mathrm{MHz}$ ) cubriendo la banda 27,558-27,972 GHz, alimenta los dos electrodos del MZ-EOM con un desfase diferente entre ellos dependiendo del esquema de modulación: $\phi=$ 90 o para modulación SSB o $\phi=180$ para modulación DSB. La salida del MZ-EOM se transmite a través de una SSMF de longitud $L=23,8 \mathrm{~km}$ en la cual se desprecian los efectos no lineales. En caso de ecualizar la dispersión cromática de la fibra por medio de un CFG, éste se coloca previo a la fotodetección.

Centrándonos en el CTB generado por la dispersión de la fibra, se consideró un modulador ideal linealizado en todas las simulaciones. Los campos ópticos para las modulaciones DSB y SSB a la salida de un MZ-EOM ideal linealizado son ${ }^{\times v}$ :

$$
\begin{gathered}
E_{D S B}(t)=\sqrt{1+m_{i} \sum_{k=1}^{M} \cos \left(2 \pi f_{k} t+\phi_{k}\right)} \exp \left(j \omega_{0} t\right), \\
E_{S S B}(t)=\sqrt{1+m_{i} \sum_{k=1}^{M} \cos \left(2 \pi f_{k} t+\phi_{k}\right)+\operatorname{sen}\left(2 \pi f_{k} t+\phi_{k}\right)} \\
\times \exp \left[j \omega_{0} t+j \frac{m_{i}}{2} \sum_{k=1}^{M} \cos \left(2 \pi f_{k} t+\phi_{k}\right)-\operatorname{sen}\left(2 \pi f_{k} t+\phi_{k}\right)\right],
\end{gathered}
$$

donde $f_{k}$ y $\phi_{k}$ son respectivamente la frecuencia y la fase de cada una de las $M=70$ subportadoras eléctricas. Se suponen fases aleatorias para cada una de las subportadoras y $m_{i} \ll 1$.

Para estudiar el impacto de las características no ideales del CFG sobre los resultados de ecualización de distorsión no lineal se abordarán dos estudios distintos. En el primero, se considerará el modelo teórico del CFG con rizado en las respuestas de reflectividad y retardo de grupo de acuerdo con las ecuaciones (2.59)-(2.60). El caso sin rizado sería equivalente a emplear una 


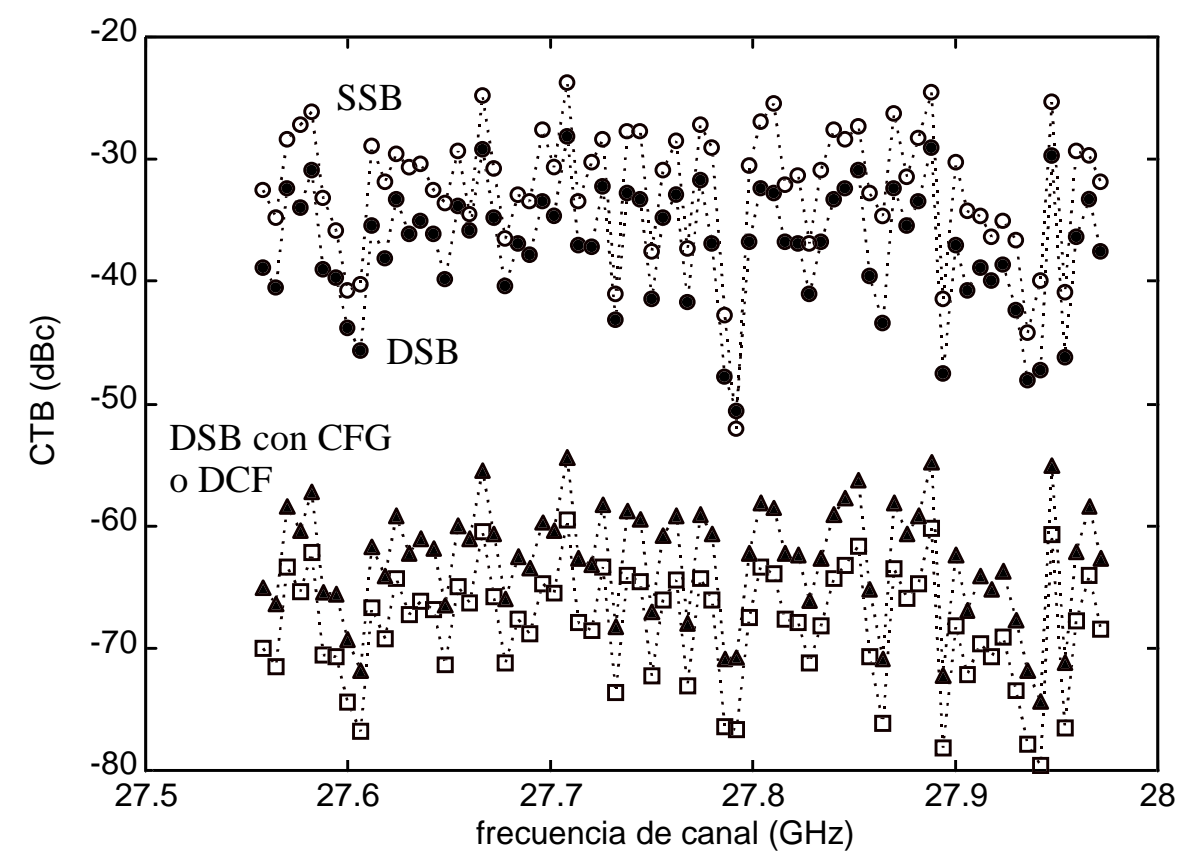

Figura 2.33 CTB en cada canal de la banda para las tres técnicas: DSB (puntos negros), SSB (puntos blancos), CFG teórico (cuadrados blancos) y CFG experimental (triángulos negros). EI índice de modulación de los canales es del $4 \%$.

DCF. El valor $D_{C F G}$ se selecciona para compensar un cierto porcentaje de la dispersión total introducida por el enlace de fibra. En cuanto al segundo, se tomará la respuesta experimental del CFG de la figura 2.31. Dado que este CFG se ha fabricado para compensar $50 \mathrm{~km}$ de SSMF, la respuesta del retardo de grupo será ponderada para compensar la dispersión cromática de una determinada longitud de fibra óptica.

EI CTB inducido por la dispersión de la fibra a la salida del fotorreceptor en cada canal del plan de frecuencias LMDS se representa en la figura 2.33 para las tres técnicas. EI CSO no se ha considerado debido a que la banda de canal es ocupa menos de una octava de frecuencia. La longitud de fibra se ha tomado 23,8 km, ya que para un parámetro de dispersión de $17 \mathrm{ps} /(\mathrm{km} \cdot \mathrm{nm})$ se produce un nulo de transmisión en mitad de la banda de canales. En la figura 2.33 se observa que, empleando la técnica de modulación DSB (puntos negros), los niveles de distorsión son claramente superiores a -50 
dBc. Los estándares CATV fijan un umbral de percepción del CTB en torno a $-57 \mathrm{dBc}$ [Tho95 (cap. 8)]. Como se ha mencionado con anterioridad, el fenómeno de supresión de la portadora puede evitarse por medio de la modulación SSB [Smi97a] o el uso de CFGs [Mar97a], por lo que se puede suprimir el nulo que aparece en mitad de la banda de canales. Sin embargo, el CTB inducido por la dispersión de la fibra no puede compensarse por medio de la modulación SSB (puntos blancos), como se muestra en la figura 2.33. Esto se debe al chirp introducido por el transmisor óptico SSB de (2.63) que es útil para compensar la supresión de la portadora pero que, de hecho, aumenta el nivel de distorsión con respecto a la modulación DSB convencional. Utilizando un CFG, no obstante, sí que es posible reducir significativamente los niveles de distorsión y cumplir los requerimientos de Ias señales AM-VSB. Con este objetivo, se han simulado los resultados para los dos modelos de CFG considerados. En el modelo teórico (cuadrados blancos), el CFG se ha seleccionado para compensar el $98 \%$ de la dispersión cromática de la fibra, es decir, $D_{\mathrm{CFG}}=0,98 \cdot \mathrm{DL}=396,51 \mathrm{ps} / \mathrm{nm}$. En un principio, se ha supuesto un CFG ideal sin rizado (DCF). En cuanto al modelo experimental (triángulos negros), el retardo de grupo medido se ha ponderado por un factor $23,8 / 50$.

La dependencia del CTB para el canal 26 de la banda con la longitud de fibra se representa en la figura 2.34 también para las tres técnicas. El comportamiento para las modulaciones DSB y SSB es prácticamente el mismo, aunque los niveles de distorsión son ligeramente superiores en el caso SSB. A partir de una cierta longitud de fibra (sobre $7 \mathrm{~km}$ ), aparecen los típicos lóbulos en la distorsión. Empleando un CFG, se observa que estos lóbulos pueden suprimirse junto con una reducción significativa del CTB para distancias cortas. No obstante, cada modelo de CFG proporciona resultados diferentes. EI modelo teórico compensa el $98 \%$ de la dispersión total del enlace y es el que produce los mejores resultados. El modelo experimental considera el rizado medido en las respuestas de reflectividad y retardo de grupo del CFG. Para una longitud de fibra de $23,8 \mathrm{~km}$, la reducción en el CTB es aproximadamente de $25 \mathrm{~dB}$ para ambos model os de CFG. 


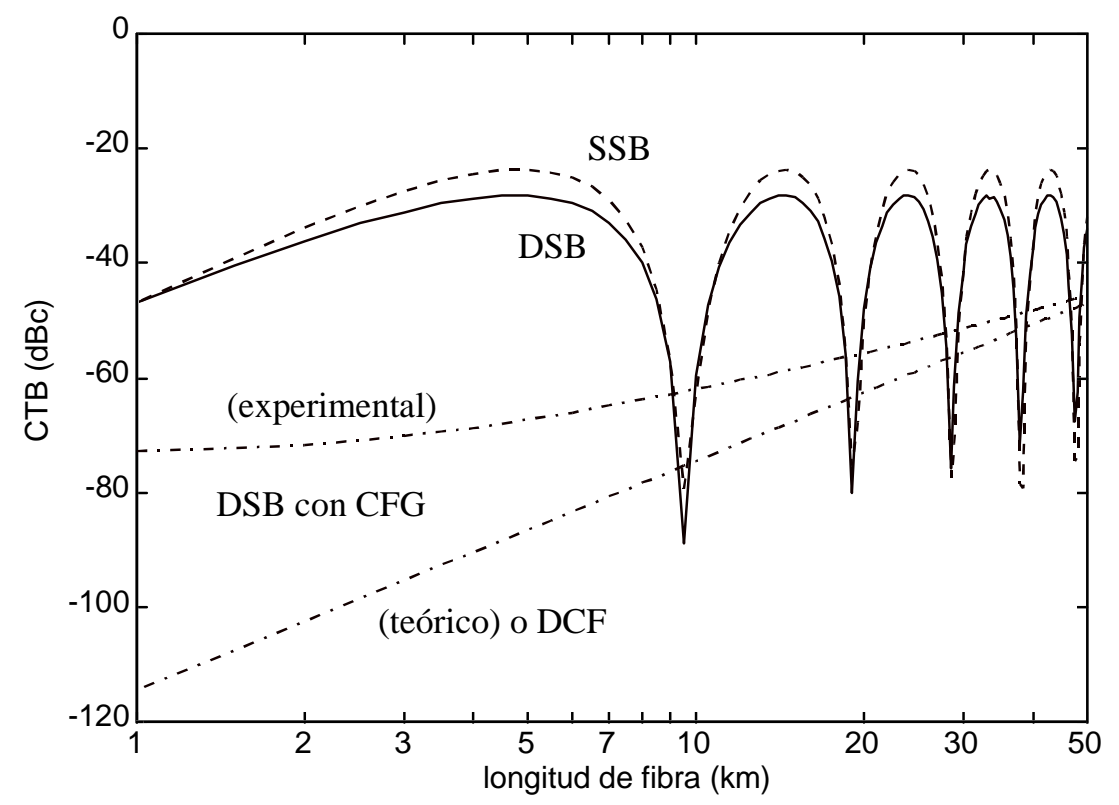

Figura 2.34 CTB para el canal 26 en función de la longitud del enlace y para las tres técnicas: DSB (continua), SSB (trazos) y CFG (trazos y puntos).

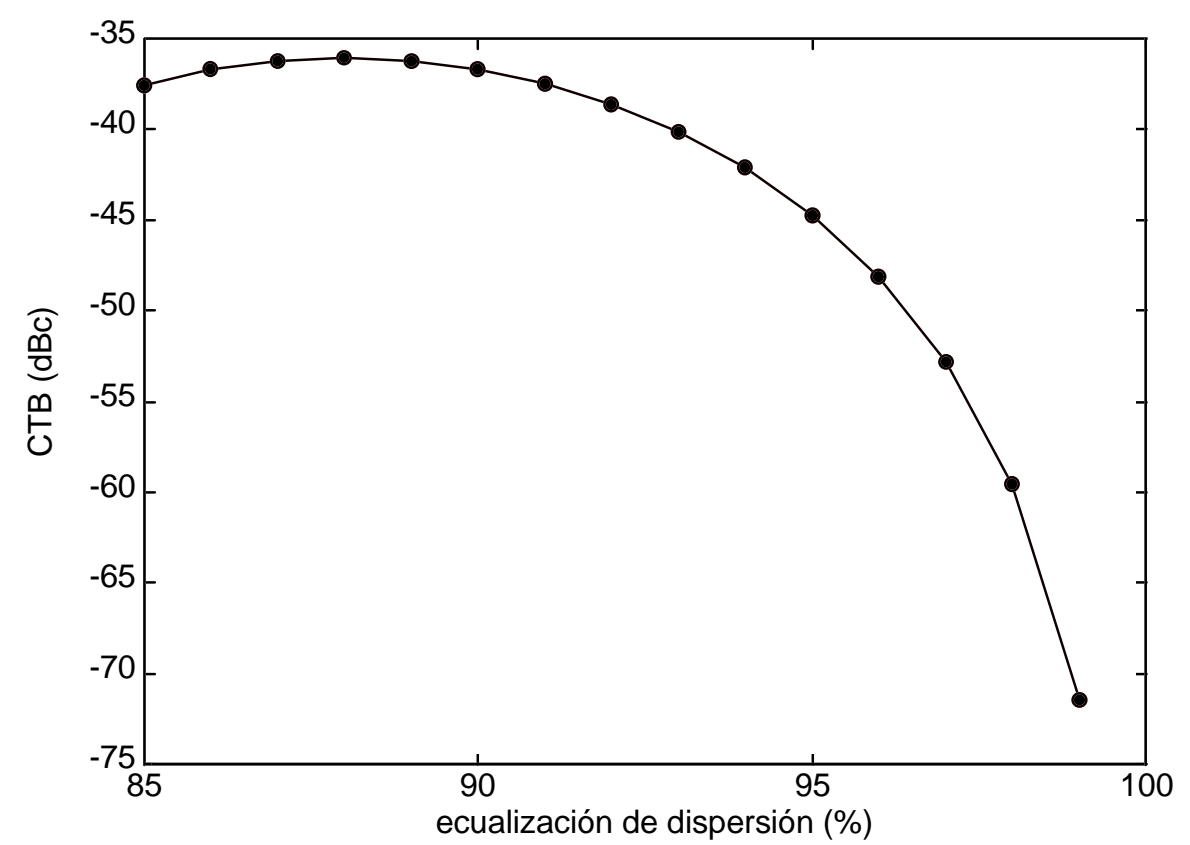

Figura 2.35 CTB ecualizado para el canal 26 en función del porcentaje de dispersión cromática compensado mediante el CFG. Se supone un CFG ideal sin rizado, por lo que los resultados también son válidos para una DCF. 
EI CTB para el canal 26 de la banda en función del porcentaje de dispersión ecualizado con el CFG se muestra en la figura 2.35. Dado que el método de compensación de dispersión basado en CFG depende de la longitud de fibra, en la figura se observa que la distorsión es muy sensible frente a variaciones en el valor de $D_{C F G}$. Este aspecto debe tenerse en cuenta en sistemas reales, donde suelen modificarse los enlaces de fibra para operaciones de mantenimiento. De hecho, ésta es la principal limitación de la técnica que obliga a un diseño cuidadoso del CFG y de los tramos de fibra.

Por último, se estudiarán los efectos del rizado en la respuesta del CFG sobre los resultados de ecualización. La figura 2.36 representa el nivel de CTB ecualizado en función del período de modulación del rizado del retardo de grupo para una amplitud $b_{1}=30 \mathrm{ps}$. Inicialmente, se ha supuesto que no existe rizado en la reflectividad. Se observa que el CTB ecualizado oscila muy rápidamente para períodos de modulación pequeños con una envolvente que presenta un máximo para $p=80$ pm (10 GHz @ 1549 nm). Para valores mayores del período de modulación, el CTB comienza a

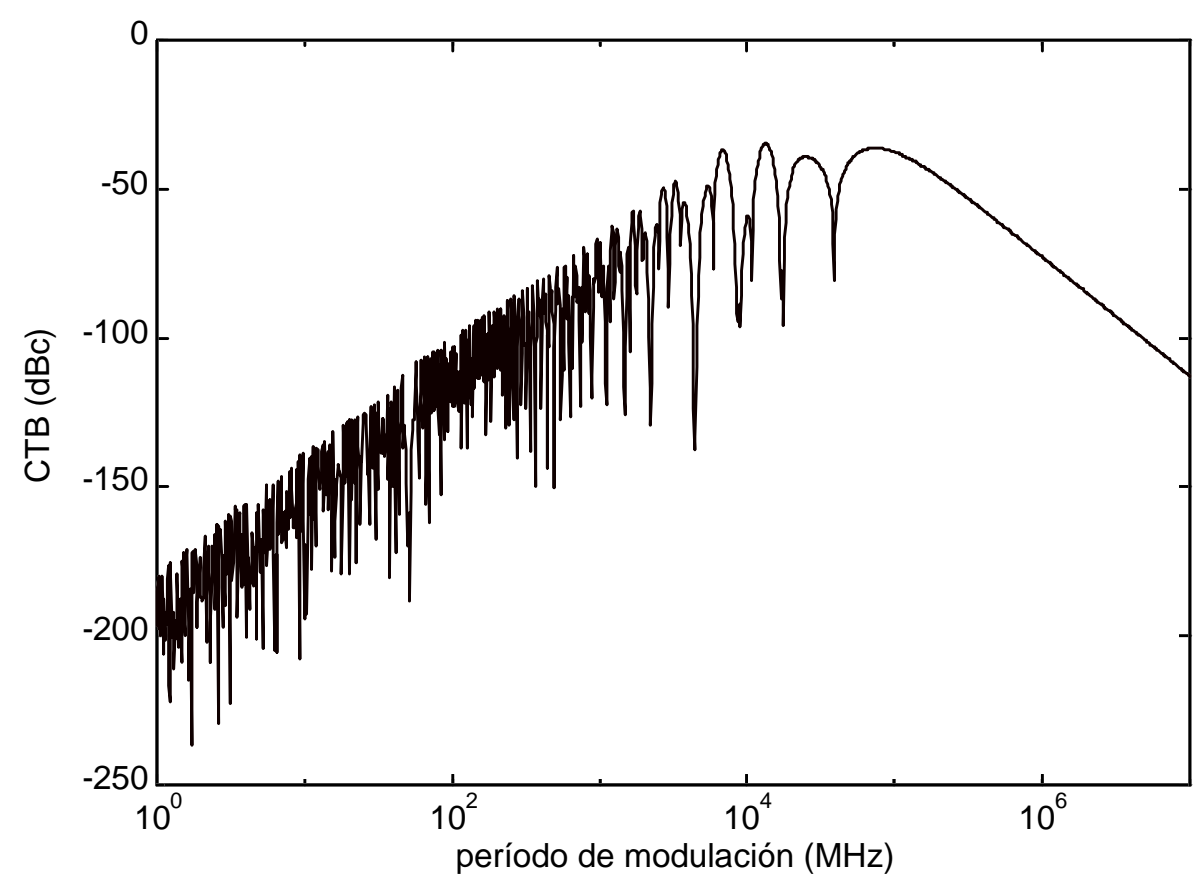

Figura 2.36 CTB ecualizado para el canal 26 en función del período de modulación del rizado del CFG. Otros parámetros: $b_{1}=30$ ps y $r_{1}=0$. 
decrecer. Esto se debe al aplanamiento del rizado como consecuencia de grandes períodos de modulación. De este modo, el efecto del rizado supone simplemente una ligera desviación de la pendiente del retardo de grupo.

Durante las simulaciones se comprobó que mayores amplitudes de modulación producen peores resultados, como es lógico. Además, el rizado de amplitud fija un límite en el nivel de CTB que puede ser ecualizado. Por ejemplo, para $\mathrm{p}=8$ pm ( $1 \mathrm{GHz} @ 1549 \mathrm{~nm}$ ) y un límite típico de CTB de -60 $\mathrm{dBc}$, se obtiene un valor máximo de $\mathrm{b}_{1}=40$ ps y un rizado máximo en la reflectividad de $\pm 2,5 \mathrm{~dB}$. 


\subsection{CONCLUSIONES Y CONTRIBUCIONES}

En este capítulo se ha analizado en profundidad la problemática de la distorsión no lineal introducida por la fibra óptica en sistemas multicanal radio sobre fibra. Esta es debida principalmente a dos factores: por una parte la dispersión cromática, y por otra el SPM como consecuencia de las no linealidades. Para obtener los resultados de simulación del capítulo se ha utilizado el algoritmo del split-step Fourier method, el cual proporciona muy buenos resultados con la suficiente velocidad y permite tratar el problema del efecto conjunto GVD + SPM. Los resultados teóricos de propagación de señales de RF sobre fibras ópticas dispersivas y no lineales se describen en el apéndice 1 , donde se presenta una expresión de la función de transferencia de la fibra a partir de las funciones de conversión faseintensidad. Las principales conclusiones y resultados obtenidos se comentan a continuación:

- Se han obtenido resultados de CSO, CTB y penalización de potencia inducidos por la fibra en sistemas multicanal típicos: CATV, MMDS, LMDS y MVDS. Debido a la modulación AM-VSB típica de los sistemas CATV o MMDS, éstos sistemas se encuentran principalmente limitados por la distorsión. Fijando un valor límite típico de $-60 \mathrm{dBc}$, se obtienen unas longitudes de enlace de $50 \mathrm{~km}$ para el sistema CATV y de $10 \mathrm{~km}$ para el sistema MMDS. Considerando modulación externa, el SPM contribuye de forma significativa a los niveles de CSO y CTB como consecuencia de las potencias ópticas de estos sistemas ( $+13 \mathrm{dBm})$. Sin embargo, el efecto del chirp del transmisor óptico supera claramente al del SPM en el caso de modulación directa. En el caso de los sistemas LMDS y MVDS, la principal limitación viene impuesta por la penalización de potencia como consecuencia del efecto de supresión de la portadora debido a las altas frecuencias de modulación: $28-40 \mathrm{GHz}$. EI efecto del SPM se manifiesta simplemente en un ligero desplazamiento de las curvas de penalización de potencia. Debido en parte a la modulación externa, y a los esquemas de modulación empleados, los niveles de CTB no suponen prácticamente ninguna limitación en estos sistemas (CTB <$40 \mathrm{dBc}$ ). Las longitudes típicas que se obtienen son de $2 \mathrm{~km}$ para el 
sistema LMDS y de $1 \mathrm{~km}$ para MVDS. Así pues, queda claro que es necesaria alguna técnica de ecualización del efecto de supresión de la portadora para extender el alcance de estos sistemas. Debemos indicar que el análisis de la distorsión no lineal introducida por la fibra en sistemas multicanal a frecuencias milimétricas constituye una aportación original del autor.

- Se ha derivado una expresión teórica del SFDR para enlaces ópticos dispersivos (ec. (2.55)) a partir de las expresiones teóricas de distorsión armónica de [Phi91]. Mediante esta expresión, se ha observado que los resultados de margen dinámico de la fibra óptica no suponen gran limitación en sistemas ópticos multicanal típicos, ya que se obtienen valores superiores a los $100 \mathrm{~dB} \cdot \mathrm{Hz}^{2 / 3}$. En este caso, el margen dinámico del enlace viene limitado típicamente por las prestaciones del modulador óptico, el cual debe ser extremadamente lineal.

- La respuesta en frecuencia de una SSMF para niveles altos de potencia óptica inyectados a la entrada de la misma se ha medido experimentalmente. En especial, se ha demostrado que el chirp inducido por el SPM de la fibra produce un alejamiento de los nulos de la respuesta, mejorando el ancho de banda del sistema. Estos resultados se han publicado en [Ram98], aunque en el capítulo 3 se realizará un estudio más completo junto con resultados de transmisión de datos.

- Se han obtenido resultados de ecualización de distorsión no lineal comparando la técnica SSB frente al uso de CFGs. Se ha demostrado que la técnica SSB no es válida para compensar la distorsión no lineal inducida por la dispersión de la fibra en sistemas ópticos multicanal LMDS como consecuencia del chirp generado en el transmisor óptico por la propia modulación SSB. EI CFG, sin embargo, sí que reduce los niveles de distorsión $(\sim 25 \mathrm{~dB})$, aunque existe una limitación impuesta por el rizado de las respuestas de reflectividad y retardo de grupo del dispositivo. Esta contribución ha sido publicada en [Ram99a]. 


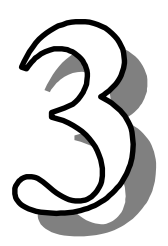

\section{Aplicación del SPM inducido por la fibra óptica para reducir los efectos de la dispersión cromática}

\subsection{INTRODUCCIÓN}

Los sistemas de comunicaciones radio sobre fibra operando a $1550 \mathrm{~nm}$ se benefician de las bajas pérdidas de la SSMF y del uso de los amplificadores ópticos EDFA para conseguir transparencia a las señales de RF. No obstante, las SSMFs presentan valores de dispersión cromática considerables a estas longitudes de onda además de diversos efectos no lineales. La respuesta de una fibra óptica bajo propagación dispersiva ha sido analizada en profundidad [Mes84, Wan92], observándose un fenómeno de desvanecimiento de la potencia eléctrica de RF recibida a la salida del fotodetector [Sch95]. Este desvanecimiento se produce de forma periódica con la variación de la frecuencia de modulación o la longitud de fibra óptica 
y se conoce con el nombre de efecto de supresión de la portadora. Su explicación se debe al diferente desfase que introduce la dispersión cromática sobre cada una de las bandas de modulación de la señal óptica, lo cual conduce a una cancelación de ambas al realizar la fotodetección para ciertas frecuencias o longitudes de fibra. Previamente se han demostrado diversas técnicas para compensar este efecto, tales como la modulación óptica en banda lateral única [Smi97a], el uso de fibras compensadoras de dispersión [I br93] o redes de difracción sobre fibra óptica [Mar97a], técnicas de modulación con portadora suprimida [Fus97] o inversión espectral [Ram99]. Sin embargo, una técnica muy simple consiste en utilizar un transmisor óptico de gran potencia para inducir efectos no lineales, tales como SPM, en el enlace de fibra [Ram98]. EI chirp inducido por el SPM de la fibra durante la propagación conduce a un aumento del producto ancho de banda-longitud de fibra del sistema. Su efecto es similar al producido por los transmisores ópticos con chirp negativo [Smi97, Pol99], de tal forma que se produce un alejamiento de los nulos de transmisión de la función de transferencia del sistema al aumentar la potencia óptica inyectada a la entrada de la fibra. En [Ram98, Car99a] se proporcionan resultados experimentales y de simulación de la respuesta de la fibra con dispersión y SPM en sistemas de comunicaciones ópticas analógicos. No obstante, todavía no se ha obtenido una expresión teórica cerrada para caracterizar la propagación de señales en sistemas radio sobre fibra con potencias ópticas no despreciables.

En la sección 3.2 de este capítulo se calculan, por primera vez, expresiones teóricas para modelar la propagación de señales de modulación a través de SSMFs dispersivas y no lineales en sistemas analógi cos MI-DD resolviendo la ecuación de Schrödinger no lineal con pérdidas. Aunque previamente se han obtenido soluciones de esta ecuación para modelar el fenómeno no lineal de ganancia paramétrica [Kar95, Car97], estos resultados se han aplicado principalmente al análisis del ruido de intensidad y son de difícil particularización para sistemas ópticos analógicos. Recientemente, se ha publicado un completo análisis teórico que describe la propagación de las componentes en fase y cuadratura de la modulación a través de SMFs sin pérdidas para modelar el desvanecimiento de señal producido por el efecto 
conjunto de dispersión y SPM [Sot99a]. En nuestro caso, obtendremos expresiones teóricas basadas en funciones de Bessel de orden imaginario puro para modelar la propagación a través de fibras dispersivas y no lineales, incluyendo pérdidas, en sistemas radio sobre fibra con diferentes formatos de modulación. Posteriormente, a partir de estas expresiones calcularemos la función de transferencia de la SSMF bajo los efectos de dispersión cromática y SPM. En la sección 3.3 se presentan medidas experimentales de esta función de transferencia para distintas potencias ópticas a la entrada de la fibra. A partir de estos resultados, se calculará la influencia del SPM sobre diversos parámetros de la respuesta: ancho de banda, ganancia de pico, etc. Por último, en la sección 3.4 se calcula un model o del chirp inducido por SPM y se analiza su aplicación para reducir la penalización de potencia debida a la dispersión cromática, lo que se conoce como técnica SPM. Asimismo, se demuestra la transmisión de una señal de $8 \mathrm{Mbit} / \mathrm{s}$ a $7 \mathrm{GHz}$ sobre $75 \mathrm{~km}$ de SSMF empleando esta misma técnica. 


\subsection{FUNCIÓN DE TRANSFERENCIA DE FIBRAS ÓPTICAS MONOMODO}

Conforme aumenta la potencia óptica inyectada en la fibra, su comportamiento se aleja del modelo teórico dispersivo presentado en [Wan92], siendo necesario considerar también los efectos no lineales [Car98, Ram98, Car99a]. En el caso de sistemas SCM operando sobre fibra monomodo estándar (SSMF), la degradación principal viene impuesta por el SPM tal y como se ha visto en el capítulo 2 . A continuación, analizaremos la respuesta de una SSMF frente a señales de RF considerando el efecto conjunto de dispersión cromática y SPM.

\subsubsection{Expresión teórica de la función de transferencia de fibras ópticas dispersivas y no lineales}

La propagación de señales a través de una SMF dispersiva y no lineal se rige por medio de la ecuación de Schrödinger no lineal [Agr89]. Bajo condiciones de pequeña señal [Wan92], esta propagación puede modelarse con el siguiente sistema de ecuaciones diferenciales lineal es [ [Car98]:

$$
\begin{aligned}
& \frac{\partial \widetilde{\mathrm{p}}_{\mathrm{N}}\left(z, \omega_{\mathrm{e}}\right)}{\partial z}=\beta_{2} \omega_{\mathrm{e}}^{2} \mathrm{P}_{0} \widetilde{\phi}\left(z, \omega_{\mathrm{e}}\right), \\
& \frac{\partial \widetilde{\phi}\left(z, \omega_{\mathrm{e}}\right)}{\partial z}=-\left[\frac{\beta_{2} \omega_{\mathrm{e}}^{2}}{4 \mathrm{P}_{0}}+\gamma \exp (-\alpha z)\right] \widetilde{\mathrm{p}}_{\mathrm{N}}\left(z, \omega_{\mathrm{e}}\right),
\end{aligned}
$$

donde $z$ es la distancia de propagación, $\omega_{e}$ es la pulsación de RF, $\widetilde{p}_{N}\left(z, \omega_{e}\right)$ y $\widetilde{\phi}\left(z, \omega_{\mathrm{e}}\right)$ son respectivamente las transformadas de Fourier de las señales de modulación de intensidad y de fase, $\mathrm{P}_{0}$ es la potencia óptica media inyectada en la fibra, $\beta_{2}$ es el coeficiente de dispersión cromática y $\gamma$ es el coeficiente no lineal. En el caso de transmisión lineal $(\gamma=0)$, el sistema (3.1)-(3.2) se puede resolver fácilmente para llegar a las soluciones de [Wan92]. No obstante, la solución analítica bajo propagación no lineal necesita de un

\footnotetext{
' en el apéndice I se explica cómo llegar a estas expresiones
} 
proceso más elaboradoii. Dado que en los sistemas MI-DD se recupera la información de la amplitud de la señal óptica recibida, nos centraremos en calcular la evolución de la modulación de intensidad.

Diferenciando (3.1) y sustituyendo (3.2) se obtiene la siguiente ecuación diferencial de segundo orden:

$$
\frac{\partial^{2} \widetilde{p}_{N}\left(z, \omega_{e}\right)}{\partial z^{2}}=-\left[\frac{\beta_{2}^{2} \omega_{e}^{4}}{4}-\beta_{2} \omega_{e}^{2} \gamma P_{0} \exp (-\alpha z)\right] \widetilde{p}_{N}\left(z, \omega_{e}\right),
$$

cuyas condiciones iniciales son

$$
\begin{aligned}
& \widetilde{\rho}_{N}\left(0, \omega_{e}\right)=\widetilde{p}\left(\omega_{e}\right),(3.4) \\
& \left.\frac{\partial \widetilde{\Gamma}_{N}}{\partial z}\right|_{z=0}=\beta_{2} \omega_{e}^{2} P_{0} \widetilde{\phi}\left(0, \omega_{e}\right)=\beta_{2} \omega_{e}^{2} P_{0} \widetilde{\phi}\left(\omega_{e}\right) .
\end{aligned}
$$

Realizando a continuación el cambio de variable $x=2 \sqrt{R} \exp (-\alpha z / 2)$ con $\mathrm{R}=-\beta_{2} \omega_{\mathrm{e}}^{2} \gamma \mathrm{P}_{0} / \alpha^{2}$, (3.3) se puede expresar como

$$
x^{2} \frac{\partial^{2} \widetilde{p}_{N}}{\partial x^{2}}+x \frac{\partial \widetilde{p}_{N}}{\partial x}-\left(x^{2}-v^{2}\right) \widetilde{p}_{N}=0
$$

donde $v=-\beta_{2} \omega_{\mathrm{e}}^{2} / \alpha$. Las soluciones de (3.6) son las funciones modificadas de Bessel de orden imaginario puro, $L_{i v}(x)$ y $K_{i v}(x)$ [Dun90]. En el apéndice II se proporcionan fórmulas cerradas para estas funciones.

Finalmente, considerando las condiciones iniciales para las modulaciones de intensidad y de fase a la entrada de la fibra, $\widetilde{p}\left(\omega_{\mathrm{e}}\right)$ y $\widetilde{\phi}\left(\omega_{\mathrm{e}}\right)$, respectivamente, se puede obtener la solución de la evolución de la modulación de intensidad a lo largo de la fibra óptica como

\footnotetext{
ii los resultados que se obtienen al resolver el sistema de ecuaciones por Runge-Kutta se muestran en el apéndice I
} 


$$
\begin{aligned}
\widetilde{p}_{N}\left(z, \omega_{e}\right)= & \frac{2 \operatorname{senh}(\pi v)}{\pi}\left\{\sqrt{R} \widetilde{p}\left(\omega_{e}\right)\left[L_{i v}^{\prime}(2 \sqrt{R}) K_{i v}(x)-K_{i v}^{\prime}(2 \sqrt{R}) L_{i v}(x)\right]\right. \\
& \left.+\frac{\alpha R}{\gamma} \widetilde{\phi}\left(\omega_{e}\right)\left[K_{i v}(2 \sqrt{R}) L_{i v}(x)-L_{i v}(2 \sqrt{R}) K_{i v}(x)\right]\right\},
\end{aligned}
$$

donde las tildes representan las derivadas de las funciones de Bessel y se ha utilizado la expresión (II.17) del Wronskiano.

Así pues, la ec. (3.7) es una expresión general para caracterizar la propagación de señales moduladas en intensidad a través de fibras ópticas monomodo dispersivas y no lineales, teniendo en cuenta las modulaciones de intensidad y de fase a la entrada de la fibra. La señal de información a la entrada de la fibra viene transportada por el término de modulación de intensidad, $\widetilde{\rho}\left(\omega_{\mathrm{e}}\right)$, mientras que el chirp del transmisor óptico se considera por medio del término de modulación de fase, $\widetilde{\phi}\left(\omega_{\mathrm{e}}\right)$. La relación entre ambos términos para un determinado transmisor óptico se expresa con la función de transferencia de pequeña señaliii $H_{P M}\left(\omega_{e}\right)$ [Bje96]. Por ejemplo, en láseres con modulación directa $\mathrm{H}_{\mathrm{PM}}\left(\omega_{\mathrm{e}}\right)$ viene dada por (I.24). En el caso de moduladores externos, $\mathrm{H}_{\mathrm{PM}}\left(\omega_{\mathrm{e}}\right)=\alpha_{\mathrm{C}}$, siendo $\alpha_{\mathrm{c}}$ el parámetro de chirp (I.30).

Para caracterizar la respuesta de una SMF dispersiva y no lineal frente a las señales RF de modulación, resulta útil definir dos funciones de conversión entre las modulaciones de intensidad (IM) y de fase (PM). Éstas son las funciones de conversión IM-IM y PM-IM, las cuales se calculan a partir de (3.7) comoiv

$$
\begin{aligned}
& C_{I M-I M}\left(z, \omega_{e}\right)=\frac{2 \sqrt{R} \operatorname{senh}(\pi v)}{\pi}\left[L_{i v}^{\prime}(2 \sqrt{R}) K_{i v}(x)-K_{i v}^{\prime}(2 \sqrt{R}) L_{i v}(x)\right], \\
& C_{P M-I M}\left(z, \omega_{e}\right)=\frac{2 \alpha R \operatorname{senh}(\pi v)}{\pi \gamma P_{0}}\left[K_{i v}(2 \sqrt{R}) L_{i v}(x)-L_{i v}(2 \sqrt{R}) K_{i v}(x)\right] .
\end{aligned}
$$

De este modo, quedan completamente caracterizadas las funciones de conversión IM-IM y PM-IM que ocurren en SMFs bajo los efectos de

iii consultar apéndice I, ec. (I.18) 
dispersión cromática y SPM. La función de transferencia de la fibra en sistemas MI-DD puede calcularse finalmente como [Car99a]

$$
H_{F}\left(z, \omega_{e}\right)=C_{I M-I M}\left(z, \omega_{e}\right)+\frac{H_{P M}\left(\omega_{e}\right)}{2} C_{P M-I M}\left(z, \omega_{e}\right)
$$

\subsubsection{Resultados teóricos de la función de transferencia}

La función de conversión IM-IM calculada en el apartado anterior se representa en la figura 3.1 para $100 \mathrm{~km}$ de SSMF y unos niveles de potencia óptica a la entrada de +10 y $+13 \mathrm{dBm}$ (curvas continuas). La SSMF se caracteriza por poseer unos parámetros: $\alpha=0,2 \mathrm{~dB} / \mathrm{km}, \mathrm{D}=17 \mathrm{ps} /(\mathrm{km} \cdot \mathrm{nm})$, $\mathrm{N}_{2}=3,2 \cdot 10^{-20} \mathrm{~m}^{2} / \mathrm{W}$ y $A_{\text {eff }}=70 \mu \mathrm{m}^{2}$ para $\lambda=1550 \mathrm{~nm}$. En la figura $3.1 \mathrm{se}$ representa también el resultado de propagación lineal $(\gamma=0)$ [Wan92]. En este caso, a partir de (3.8) y utilizando las expresiones (II.18) y (II.19) de $\mathrm{L}_{\text {iv }}(\mathrm{x})$ y $\mathrm{K}_{\text {iv }}(\mathrm{x})$ para $\mathrm{x} \rightarrow 0^{+}$, se obtiene

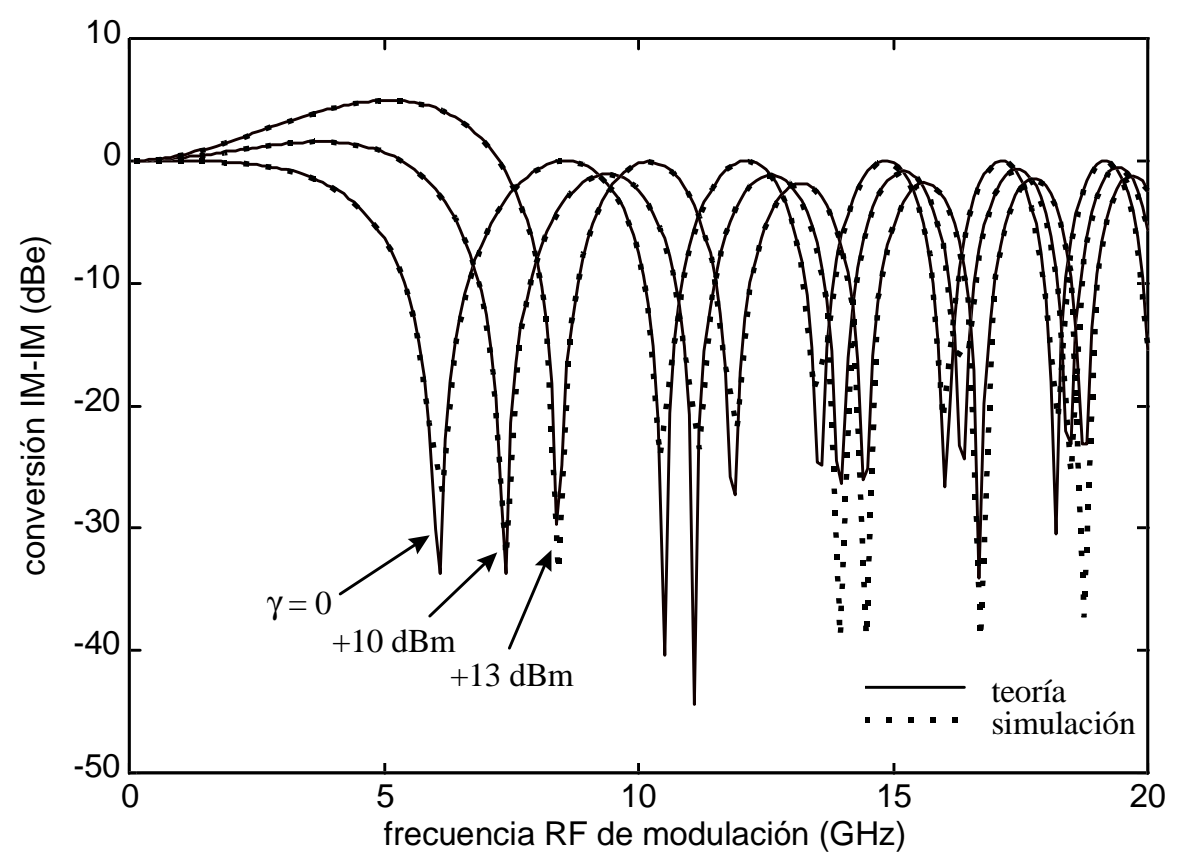

Figura 3.1 Conversión IM-IM en función de la frecuencia de modulación para varias potencias ópticas a la entrada de $100 \mathrm{~km}$ de SSMF $(\gamma=0$ : propagación lineal).

\footnotetext{
iv consultar apéndice I, ecuaciones (I.10) y (I.11)
} 


$$
C_{I M-I M}\left(Z, \omega_{e}\right)=\cos \left(\frac{\alpha v z}{2}\right)=\cos \left(\frac{\beta_{2} \omega_{e}^{2} z}{2}\right)
$$

que es idéntica a la expresión calculada en [Wan92]. Por último, los resultados de simulación empleando el split-step Fourier method muestran un ajuste excelente a los resultados teóricos.

De los resultados de la figura 3.1 se deduce que para potencias ópticas de $+10 \mathrm{dBm}$ o superiores, el modelo lineal dispersivo (3.11) difiere significativamente de la respuesta real de la fibra. Se observa que los nulos de la función de conversión IM-IM se desplazan hacia frecuencias mayores conforme aumenta la potencia óptica, apareciendo una ligera ganancia para frecuencias bajas. Este efecto ya fue demostrado experimentalmente en [Ram98] y en el capítulo 2.

Por otro lado, la función de conversión PM-IM se representa en la figura 3.2 para los mismos parámetros de fibra que los utilizados en la figura 3.1. En

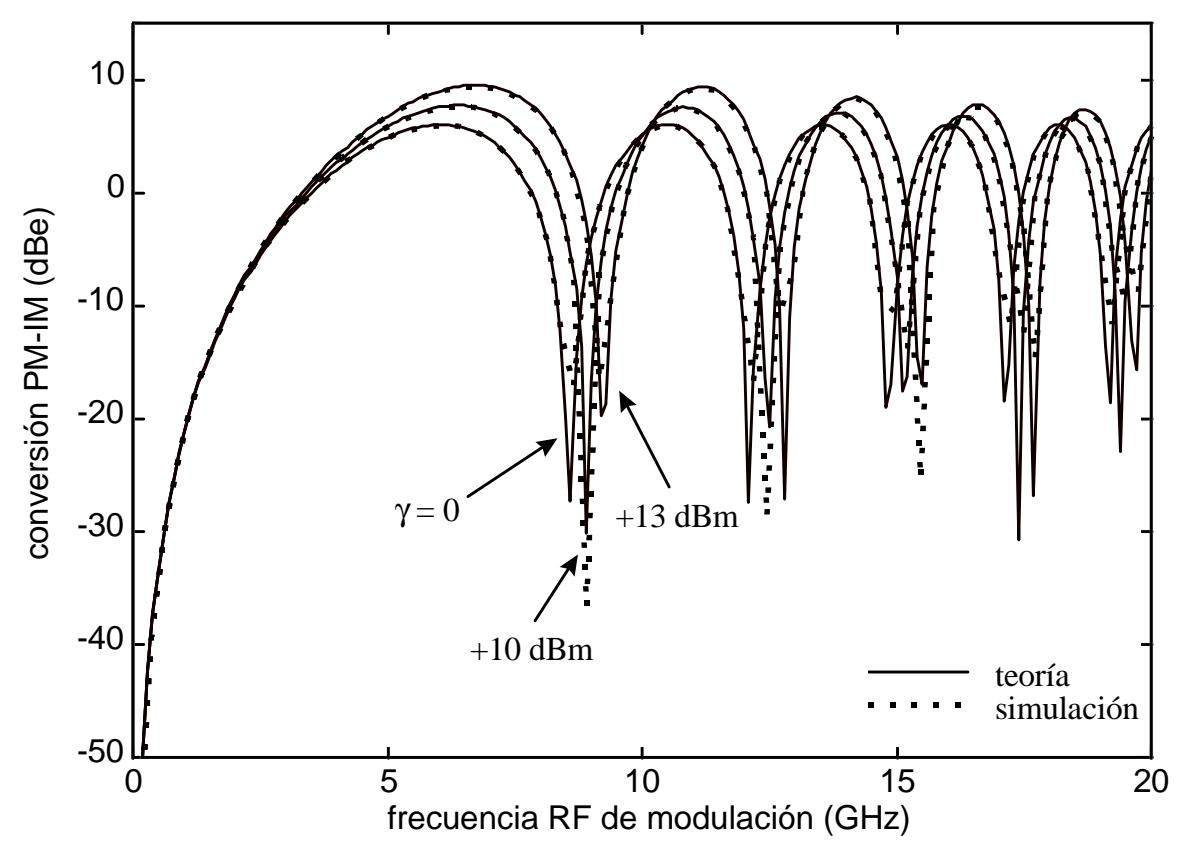

Figura 3.2 Conversión PM-IM en función de la frecuencia de modulación para varias potencias ópticas a la entrada de $100 \mathrm{~km}$ de SSMF ( $\gamma=0$ : propagación lineal). 


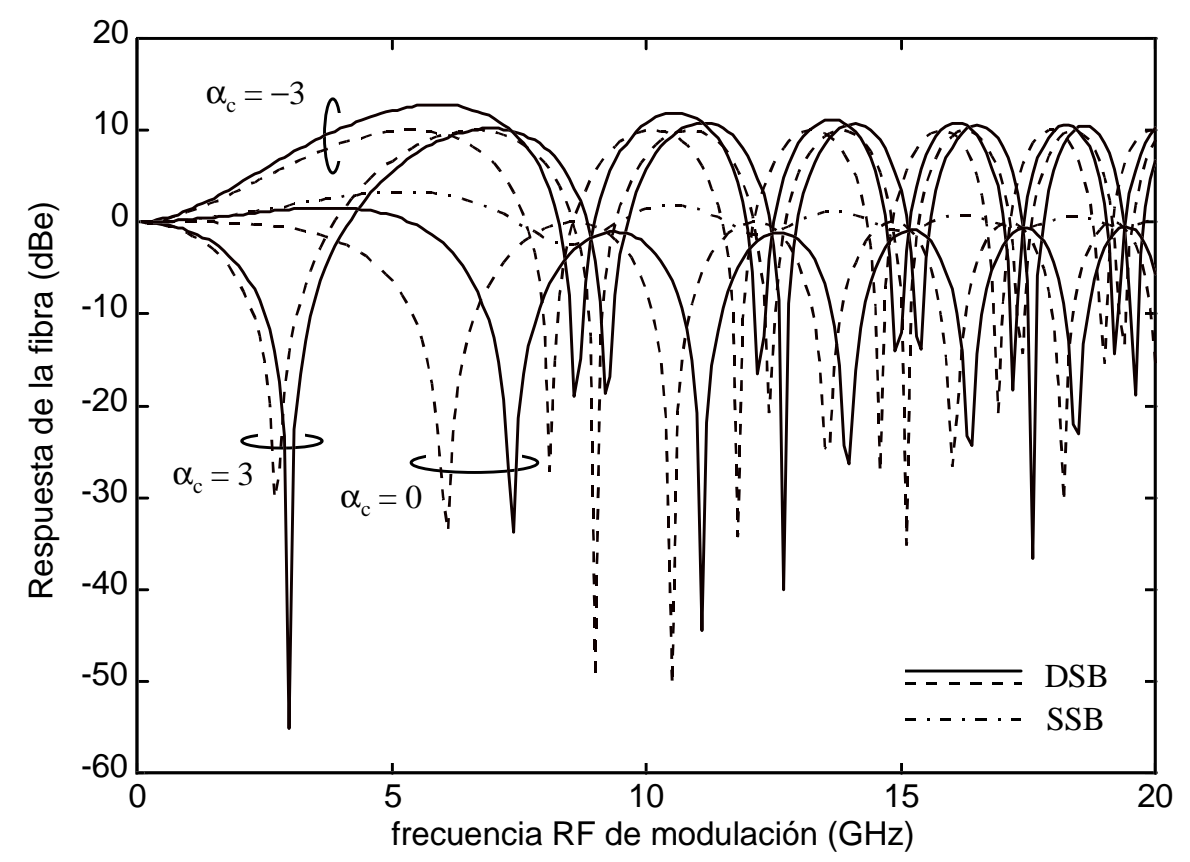

Figura 3.3 Respuesta de $100 \mathrm{~km}$ de SSMF para varios tipos de transmisores ópticos y esquemas de modulación. La potencia óptica es de $+10 \mathrm{dBm}$ (línea de trazos: $\gamma=0$ ).

este caso, la respuesta de la fibra es menos sensible a la potencia óptica y se produce un ligero desplazamiento y aumento de nivel de las curvas. Siguiendo un procedimiento similar al anterior, la función de conversión PM-IM para el caso de propagación lineal puede calcularse como

$$
C_{P M-I M}\left(Z, \omega_{e}\right)=-\frac{2 \alpha R}{v \gamma P_{0}} \operatorname{sen}\left(\frac{\alpha v Z}{2}\right)=2 \operatorname{sen}\left(\frac{\beta_{2} \omega_{e}^{2} z}{2}\right),
$$

que es idéntica a la expresión presentada en [Wan92].

En la figura 3.3 se representa la función de transferencia de $100 \mathrm{~km}$ de SSMF para dos valores distintos de chirp del transmisor y una potencia óptica de $+10 \mathrm{dBm}$ (curvas continuas). Comparando los resultados con el caso de referencia sin chirp $\left(\alpha_{c}=0\right)$, para $\alpha_{c}>0$ el ancho de banda del sistema se reduce, mientras que para $\alpha_{c}<0$ el ancho de banda alcanzable aumenta de un modo similar al producido por el SPM (figura 3.1). Por lo tanto, los transmisores ópticos con chirp negativo proporcionan una mayor tolerancia a la dispersión [Smi97]. Los resultados de propagación lineal se 
representan con línea de trazos, de tal forma que se puede observar el desplazamiento que se produce en los nulos como consecuencia del SPM incluso en las curvas con chirp.

La respuesta de la fibra con modulación óptica en banda lateral única (SSB) también se representa en la figura 3.3 con línea de trazos y puntos. Este resultado corresponde a la expresión (3.10) con $\mathrm{H}_{\mathrm{PM}}\left(\omega_{\mathrm{e}}\right)=\mathrm{j}$. Puede verse que este técnica de modulación compensa el fenómeno de supresión de la portadora y elimina completamente los nulos de la respuesta incluso en presencia de no linealidades ${ }^{\vee}$. No obstante, esta compensación depende del SPM y aparece un cierto rizado de la respuesta con respecto al caso de transmisión SSB lineal. La amplitud de este rizado es proporcional a la potencia óptica y puede controlarse fácilmente. De este modo, se deduce que el SPM inducido por la fibra óptica debe tenerse en cuenta en otros esquemas de modulación como SSB para caracterizar con detalle la propagación a través de SMFs dispersivas y no lineales.

\subsubsection{Resultados experimentales de la función de transferencia}

La figura 2.7 describe el montaje experimental utilizado para medir la función de transferencia del sistema. Un analizador de componentes ópticos HP8703 operando en el modo electroóptico (E/O) se utiliza para medir la respuesta de un enlace de $75 \mathrm{~km}$ de SSMF con modulación externa. La señal RF de barrido en frecuencia del analizador se aplica al puerto de RF de entrada de un modulador Mach-Zehnder UTP-150 con un ancho de banda a $-3 \mathrm{~dB}$ de $12 \mathrm{GHz}$, un voltaje de media onda de 8,9 $\vee$ y sin chirp. En particular, se utiliza una potencia eléctrica de $0 \mathrm{dBm}$ para minimizar el comportamiento no lineal del modulador. La portadora óptica la proporciona un láser sintonizable TUNICS PR-1550 y la salida del modulador se aplica a un EDFA de ganancia variable para controlar la potencia óptica que se aplica a la entrada de la fibra. Para compensar las respuestas del generador de RF, modulador y fotodetector se realiza una calibración previa del sistema sin el enlace de fibra. El principal factor limitante de estas medidas lo constituye el scattering estimulado de Brillouin (SBS) [Ipp72], como 


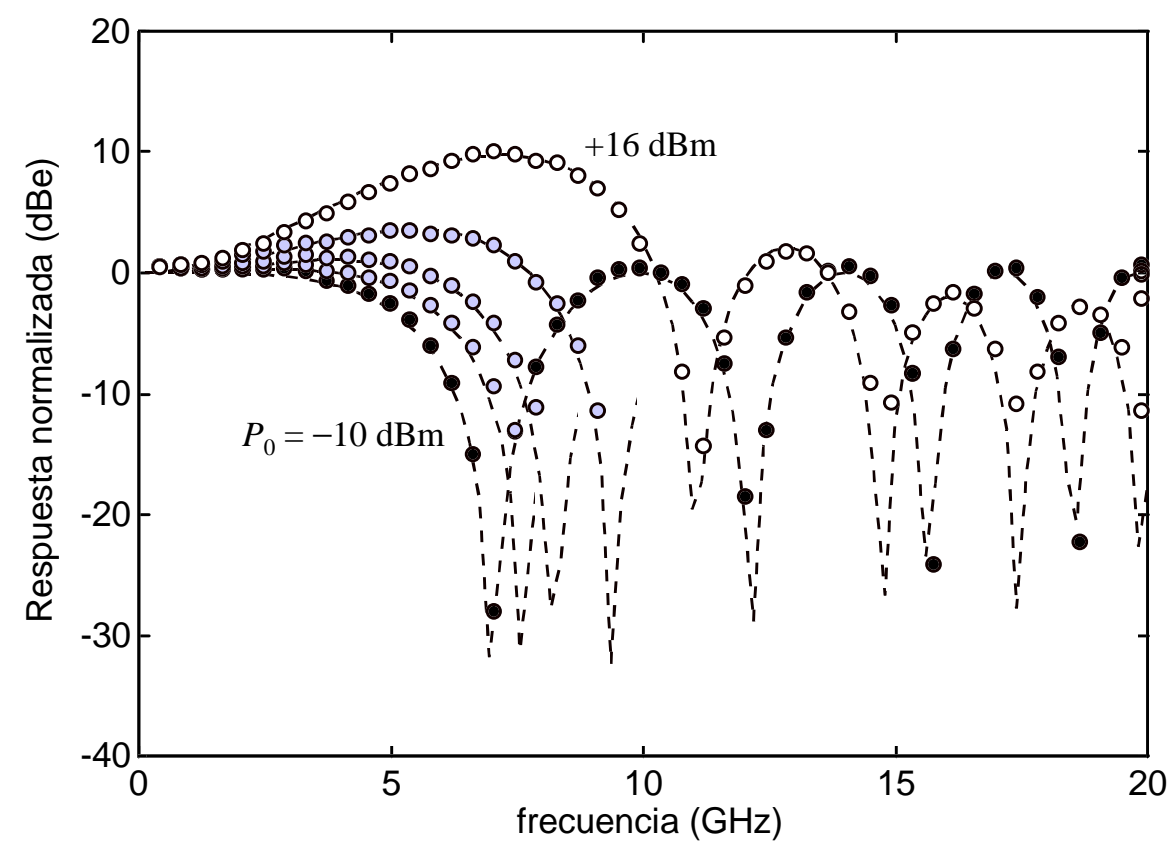

Figura 3.4 Respuesta en frecuencia de $75 \mathrm{~km}$ de SSMF para varias potencias ópticas a la entrada: $-10,+7,+10,+13$ y $+16 \mathrm{dBm}$ (curvas crecientes). Las medidas experimentales se representan con símbolos.

consecuencia de las altas potencias ópticas y reducido ancho de línea de la fuente. En los experimentos se utilizaron técnicas para aumentar el ancho de línea de la fuente [Wil94] y, de este modo, conseguir aumentar el nivel de potencia óptica que puede inyectarse en la fibra sin que se produzcan reflexiones no deseadas. En un apartado posterior se comenta con más detalle este fenómeno.

Los resultados experimentales de la respuesta del sistema se muestran en la figura 3.4 (símbolos) para diferentes valores de potencia óptica inyectados a la entrada de la fibra: $-10,+7,+10,+13$ y $+16 \mathrm{dBm}$. Para $-10 \mathrm{dBm}$, la degradación se debe únicamente a la dispersión cromática, mientras que para potencias mayores comienzan a producirse efectos debidos al SPM. Puede observarse claramente que aumentando la potencia óptica se produce el desplazamiento de los nulos hacia frecuencias mayores y aparece una cierta ganancia a frecuencias bajas como se vio en los resultados teóricos de

` véase sección I.4 del apéndice I 
la figura 3.1. En la misma gráfica 3.4 se representan también las curvas teóricas de la ec. (3.8) (curvas de trazos) mostrando un ajuste excel ente a los resultados experimentales. De esta forma, el modelo teórico queda perfectamente validado. Solamente la no linealidad del modulador podría afectar a las medidas experimentales, lo cual se evitó utilizando una potencia eléctrica reducida en la entrada RF de modulación.

A continuación, para evaluar la capacidad del SPM para reducir los efectos de la dispersión cromática se midió la frecuencia a $-3 \mathrm{~dB}$ del sistema en función de la potencia óptica inyectada, como se muestra en la figura 3.5. De la figura se deduce que esta frecuencia aumenta rápidamente con la potencia óptica, aunque aparece un máximo cercano a los $+18 \mathrm{dBm}$ para 75 $\mathrm{km}$ de SSMF. A partir de este punto, el efecto del SPM es predominante y produce una degradación de la respuesta que empeora los resultados. Así pues, existe un valor máximo de potencia que puede aplicarse al sistema, por encima del cual comienzan a degradarse las prestaciones.

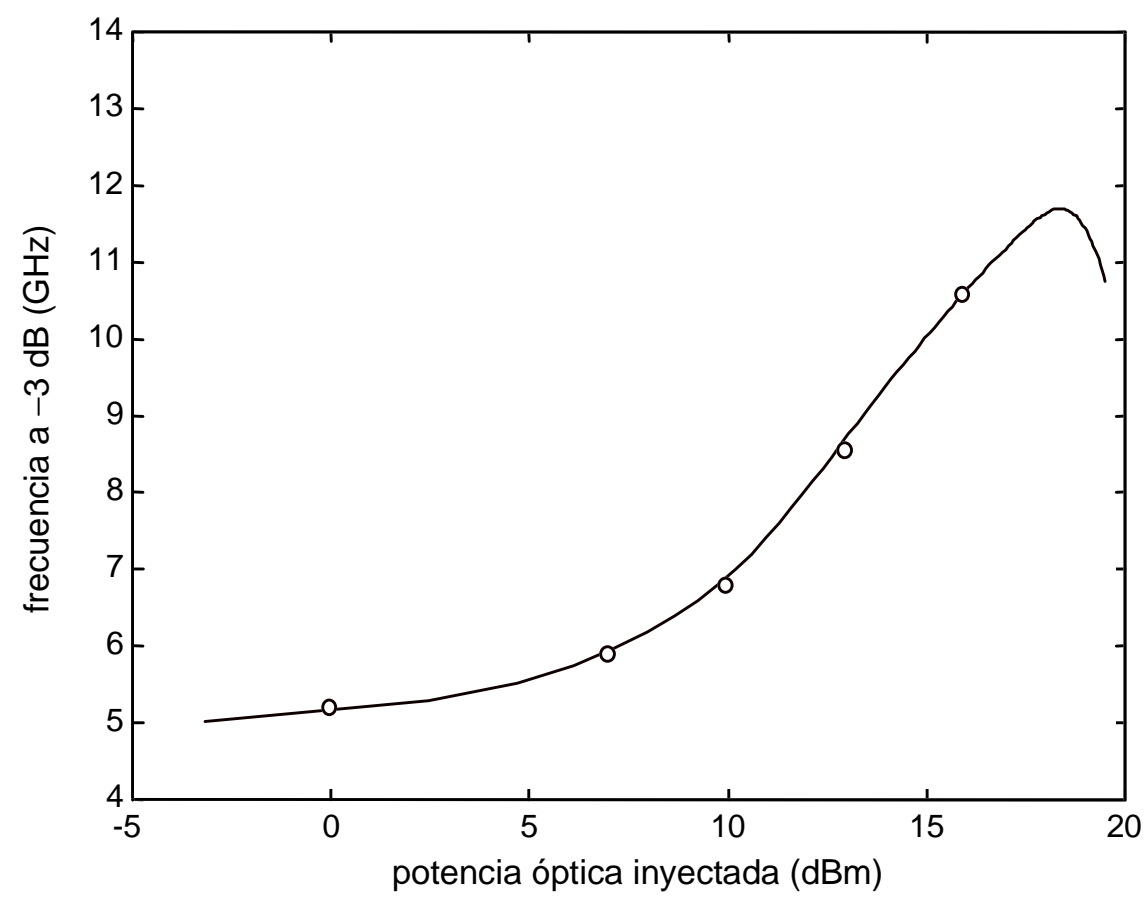

Figura 3.5 Frecuencia a $-3 \mathrm{~dB}$ de $75 \mathrm{~km}$ de SSMF en función de la potencia óptica inyectada. Los símbolos representan las medidas experimentales. 


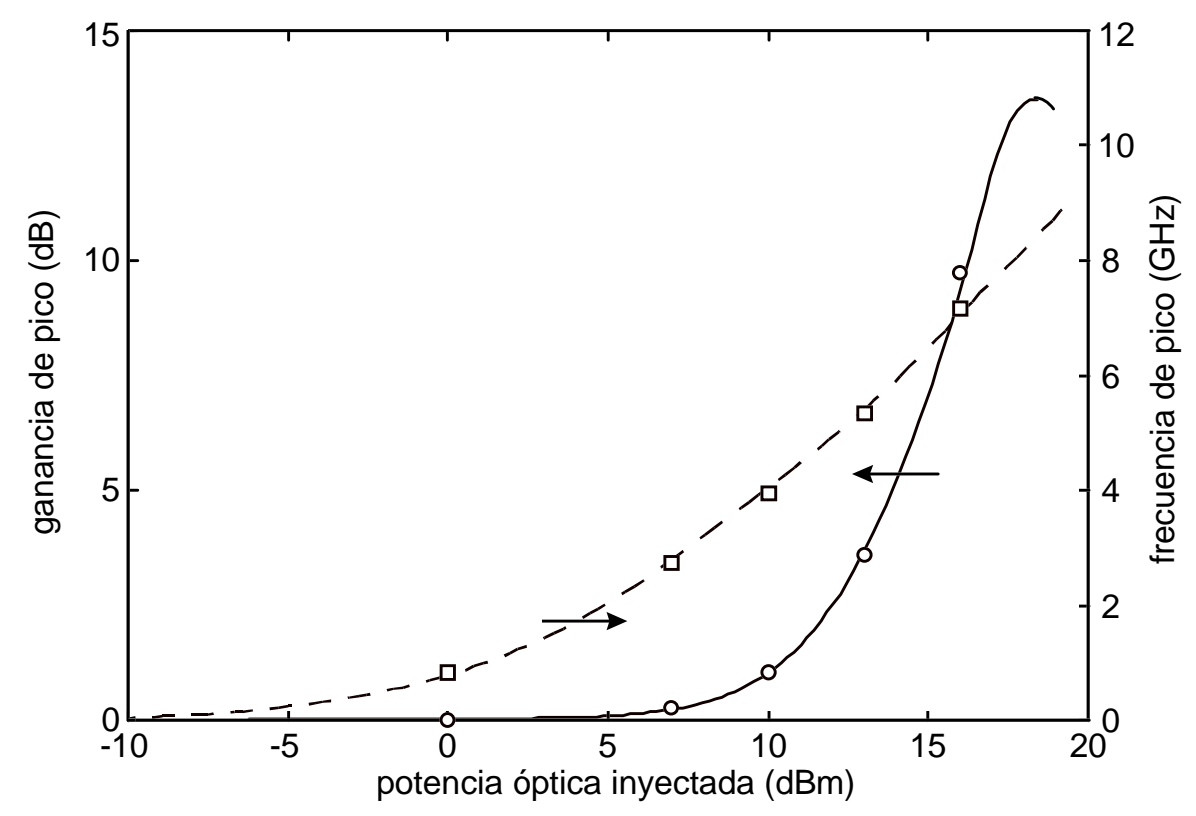

Figura 3.6 Ganancia y frecuencia de pico de la respuesta de 75 $\mathrm{km}$ de SSMF en función de la potencia óptica inyectada. LoS símbolos representan las medidas experimentales.

De los resultados de la figura 3.4, también pueden obtenerse dos parámetros adicionales que caracterizan las prestaciones del sistema. Éstos son la ganancia y frecuencia de pico, definidos como el valor máximo de la función de transferencia normalizada y la frecuencia a la que se produce este máximo. Ambos parámetros se representan en la figura 3.6 en función de la potencia óptica. Se puede observar que la ganancia de pico crece rápidamente para potencias mayores que $+5 \mathrm{dBm}$, alcanzando valores por encima de $10 \mathrm{~dB}$. Esta ganancia de pico representa el aumento relativo que se producirá en el nivel de señal recibido a la salida del fotodetector adicionalmente al producido por el propio aumento de la potencia óptica. Con respecto a la frecuencia de pico, presenta un comportamiento similar al de la frecuencia a $-3 \mathrm{~dB}$, presentado un máximo para una potencia óptica superior a los $+20 \mathrm{dBm}$. 


\subsection{APLICACIÓN DEL SPM INDUCIDO POR LA FIBRA}

En esta sección analizaremos la aplicación del SPM de la fibra para reducir los efectos de la dispersión cromática en sistemas radio sobre fibra operando a frecuencias de microondas, en lo que desde este momento se conocerá como técnica SPM.

\subsubsection{Modelo de chirp del SPM}

El montaje experimental utilizado en la técnica SPM se muestra en la figura 3.7. La potencia óptica inyectada a la entrada de la fibra debe seleccionarse de acuerdo con la longitud de fibra, aunque generalmente potencias altas producen mejores prestaciones. EI SPM inducido por la fibra cuando se inyectan niveles altos de potencia óptica genera un chirp de frecuencia. Este chirp inducido por el SPM depende de la potencia óptica y de la longitud de la fibra [Ram98], en lugar de lo que ocurre con el chirp del transmisor óptico que depende de las amplitudes de señal o del voltaje de polarización del modulador. Sin embargo, los resultados que se obtienen



Figura 3.7 Montaje para la medida de la técnica SPM. Se representan simultáneamente dos tipos de medida: subportadora de frecuencia fija configurable o barrido en frecuencia para caracterizar la respuesta del sistema. LCA: analizador de componentes óptico; EOM: modulador electróptico. 
mediante esta técnica son similares a los presentados en [Smi97, Pol99] utilizando moduladores Mach-Zehnder o de electroabsorción.

La función de transferencia de una fibra dispersiva alimentada por un transmisor óptico con chirp puede expresarse como

$$
\mathrm{H}_{\mathrm{GVD}}\left(\omega_{\mathrm{e}}\right)=\cos \left(\frac{\beta_{2} \omega_{\mathrm{e}}^{2} \mathrm{~L}}{2}\right)+\alpha_{c} \operatorname{sen}\left(\frac{\beta_{2} \omega_{\mathrm{e}}^{2} \mathrm{~L}}{2}\right) \text {, }
$$

donde $L$ es la longitud del enlace de fibra. Como se ha visto, parámetros de chirp $\alpha_{c}$ negativos, desplazan los nulos hacia frecuencias mayores.

Resulta interesante comparar el chirp inducido por SPM con aquel generado por los transmisores ópticos, de tal forma que puede calcularse un parámetro de chirp efectivo, $\alpha_{\mathrm{SPM}}$. A partir de (3.13) y considerando la
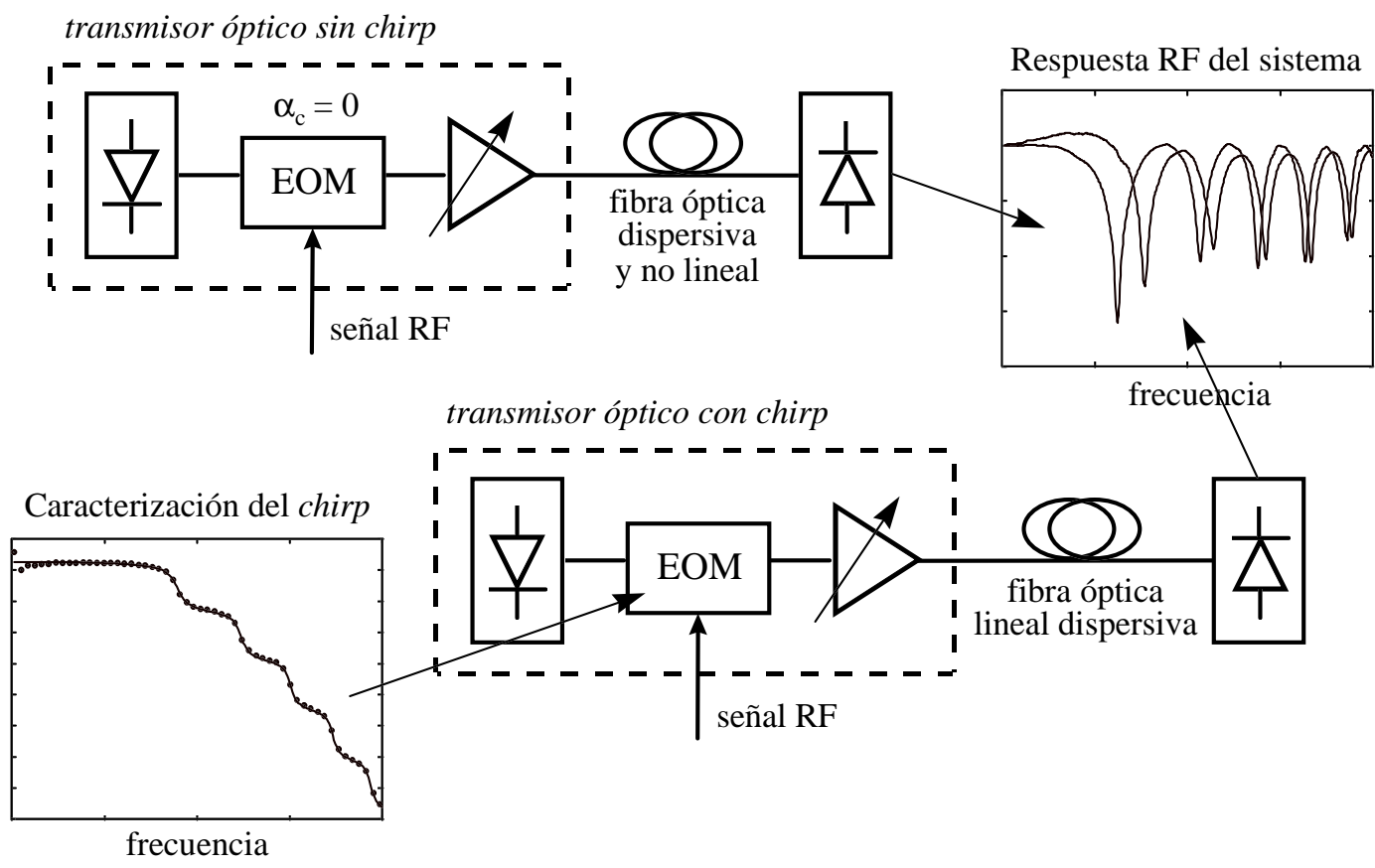

Figura 3.8 Modelo equivalente de la respuesta de un enlace dispersivo y no lineal empleando un enlace lineal dispersivo y un transmisor óptico modificado con chirp. Obsérvese que el factor de chirp equivalente depende de la frecuencia. 




Figura 3.9 Factor de chirp equivalente en función de la frecuencia de modulación debido al SPM inducido por $96 \mathrm{~km}$ de SSMF con una potencia óptica de $+11 \mathrm{dBm}$. Interior: Respuestas medidas en el laboratorio de $96 \mathrm{~km}$ de SSMF para unas potencias ópticas de 0 y +11 dBm (curva de trazos: simulación).

función de transferencia de la fibra con no linealidades, $\mathrm{H}_{\mathrm{SPM}}\left(\omega_{\mathrm{e}}\right)$, este chirp efectivo se puede escribir como

$$
\alpha_{\mathrm{SPM}}\left(\omega_{\mathrm{e}}\right)=\frac{\mathrm{H}_{\mathrm{SPM}}\left(\omega_{\mathrm{e}}\right)-\cos \left(\frac{\beta_{2} \omega_{\mathrm{e}}^{2} \mathrm{~L}}{2}\right)}{\operatorname{sen}\left(\frac{\beta_{2} \omega_{\mathrm{e}}^{2} \mathrm{~L}}{2}\right)} .
$$

No obstante, para este estudio resulta más conveniente introducir un nuevo parámetro llamado factor de chirp, $\delta$, y definido como $\alpha=\operatorname{tg}(\delta)$. De este modo, el sistema no lineal completo puede modelarse mediante una fibra lineal dispersiva y un transmisor óptico modificado con chirp. De hecho, el parámetro de chirp efectivo de este transmisor es la función de transferencia de pequeña señal entre las modulaciones de intensidad y de fase, $\mathrm{H}_{\mathrm{PM}}\left(\omega_{\mathrm{e}}\right)=$ $\alpha_{\text {SPM }}\left(\omega_{\mathrm{e}}\right)$, la cual depende de la frecuencia en este caso. Esto mismo se resume esquemáticamente en la figura 3.8. 
Empleando el montaje de la figura 3.7 se midió en el laboratorio la respuesta de $96 \mathrm{~km}$ de SSMF en presencia de SPM. Las funciones de transferencia medidas para dos valores distintos de potencia óptica a la entrada de la fibra $(0 \mathrm{dBm}$ y $+11 \mathrm{dBm}$ ) se representan en el interior de la figura 3.9. Los resultados de simulación (curvas de trazos) se obtuvieron empleando el split-step Fourier method. Se observa claramente que al aumentar la potencia óptica los nulos de la respuesta se desplazan mejorando el producto ancho de banda-longitud del enlace de fibra.

Del resultado para $+11 \mathrm{dBm}$ se puede calcular un parámetro de chirp efectivo debido a SPM. De la ec. (3.14), y considerando $\delta_{\text {SPM }}=\operatorname{arctg}\left(\alpha_{\text {SPM }}\right)$, el factor de chirp efectivo experimental debido a SPM para una potencia de $+11 \mathrm{dBm}$ y una longitud de fibra de $96 \mathrm{~km}$ se representa con puntos en la figura 3.9. La coincidencia entre los resultados experimentales y los de simulación (curva continua) es excelente, excepto para frecuencias cercanas al origen que son mucho más sensibles a imprecisiones debido al término del denominador de (3.14). Puede observarse que el factor de chirp equivalente al SPM tiene un comportamiento similar a una escalera pero suavizada, con saltos a frecuencias coincidentes con los nulos de la función de transferencia. Para frecuencias bajas, los resultados pueden aproximarse por un parámetro $\alpha$ constante e independiente de la frecuencia, que en este caso es igual a $-0,77$. Por lo tanto, a frecuencias bajas la propagación dispersiva y no lineal sin chirp puede modelarse mediante propagación lineal dispersiva con chirp negativo.

\subsubsection{Reducción del efecto de supresión de la portadora}

Como se ha visto en los apartados 3.2.2 y 3.2.3, el SPM produce un alejamiento del primer nulo de la función de transferencia, lo cual equivale a un aumento del ancho de banda del enlace de comunicaciones. Lógicamente, esto mismo puede extrapolarse en términos de longitudes de fibra, por lo que el empleo de la técnica SPM también conducirá a un aumento en el alcance del sistema. En definitiva, una reducción del efecto de supresión de la portadora y de la penalización de potencia. 




Figura 3.10 Potencia eléctrica recibida normalizada en función de la distancia para varias frecuencias de modulación y potencias ópticas. Los símbolos representan los resultados experimentales, mientras que las curvas corresponden a la simulación (curvas de trazos y puntos: propagación lineal).

Utilizando el montaje de la figura 3.7, se realizaron medidas experimentales de la potencia de RF recibida normalizada en función de la longitud de fibra y para varias frecuencias y potencias ópticas, las cuales se representan en la figura 3.10. Los círculos corresponden a una frecuencia de modulación de 7 $\mathrm{GHz}$, mientras que los cuadrados son para $10 \mathrm{GHz}$. Para cada frecuencia, los símbolos negros representan una potencia inyectada a la entrada de la fibra de $0 \mathrm{dBm}$, mientras que los símbolos blancos son para $+11 \mathrm{dBm}$. Adicionalmente, se muestran también los resultados de simulación con curvas continuas y de trazos, y el resultado ideal sin SPM con línea de trazos y puntos. Se observa que para potencias ópticas inferiores a los 0 $\mathrm{dBm}$, la degradación en la potencia recibida es debida principalmente a la dispersión cromática. Sin embargo, inyectando una potencia óptica de +11 $\mathrm{dBm}$ el alcance máximo de fibra puede aumentarse considerablemente como consecuencia del efecto SPM. Nótese que el nulo que aparece en torno a los $75 \mathrm{~km}$ de distancia para una frecuencia de $7 \mathrm{GHz}$ puede eliminarse completamente empleando una potencia óptica de $+11 \mathrm{dBm}$. De este modo, 
el punto de penalización $1 \mathrm{~dB}$ se extiende desde los $20 \mathrm{~km}$ hasta una distancia por encima de los $70 \mathrm{~km}$.

Para demostrar la transmisión tolerante a la dispersión utilizando las no linealidades de la fibra, se transmitió una PRBS de $8 \mathrm{Mbit} / \mathrm{s}$ subida a una frecuencia de $7 \mathrm{GHz}$ sobre $75 \mathrm{~km}$ de SSMF (tres bobinas de fibra de $25 \mathrm{~km}$ cada una) tal y como muestra la figura 3.11. Debemos indicar que la frecuencia de subportadora coincide con el primer nulo de la función de transferencia de los $75 \mathrm{~km}$ de SSMF lineal dispersiva. Por lo tanto, la comparación de los resultados del sistema con y sin SPM proporcionará la viabilidad de la técnica para reducir los efectos de la dispersión cromática. Tras la fotodetección, la señal recibida se baja a banda base y se realizan las medidas de diagramas de ojos de la PRBS vi para comprobar las prestaciones del sistema completo. En particular, se realizaron dos tipos de medidas con y sin SPM que se describen en la figura 3.11. Para el caso no lineal, se colocó un EDFA de $+11 \mathrm{dBm}$ de potencia a la entrada de la primera bobina de fibra para inducir SPM de forma significativa. Por otro lado, en el caso de propagación lineal el EDFA se cambió a la entrada de la tercera bobina de fibra, ajustando la potencia de salida para recibir la misma potencia óptica a



Figura 3.11 Montaje experimental utilizado con el fin de demostrar la validez de la técnica SPM para reducir los efectos de la dispersión cromática.

vi secuencia de bits pseudoaleatoria 


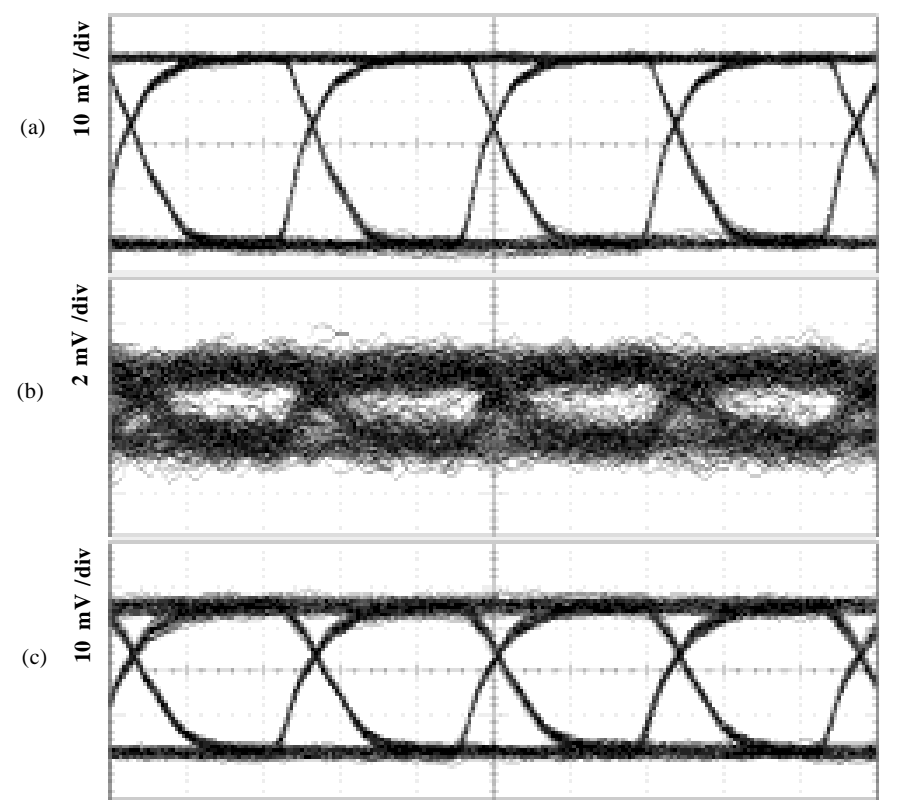

Figura 3.12 Diagramas de ojos de la señal de $8 \mathrm{Mbit} / \mathrm{s}$ recibida a una frecuencia de $7 \mathrm{GHz}$ y después de $75 \mathrm{~km}$ de SSMF. (a) sin sistema, (b) $\mathrm{P}_{0}=0 \mathrm{dBm}$ y (c) $\mathrm{P}_{0}=+11 \mathrm{dBm}$. Otros parámetros: $\mathrm{P}_{\text {rec }}$ $=-4,5 \mathrm{dBm}$.

la entrada del fotodetector que en el caso no lineal $\left(P_{\text {rec }}=-4,5 \mathrm{dBm}\right)^{\text {vii. En }}$ este caso, la potencia inyectada a la entrada del enlace era de $0 \mathrm{dBm}$ para que los efectos del SPM pudieran despreciarse y suponer transmisión dispersiva solamente.

Los diagramas de ojos medidos se muestran en la figura 3.12 para los casos: sin sistema, propagación lineal dispersiva y propagación dispersiva con SPM. Puede observarse que se consigue transmisión tolerante a la dispersión empleando la técnica SPM si comparamos los resultados con el caso de propagación lineal convencional, para el que el nivel de señal es mucho menor y el diagrama de ojos aparece prácticamente cerrado. Conviene resaltar que la potencia óptica recibida en ambos casos es exactamente la misma, por lo que las prestaciones se deben únicamente a los efectos del SPM. Así pues, queda demostrada la validez de esta técnica para reducir la degradación producida por el efecto de supresión de la vii obsérvese que se produce una variación de $0,5 \mathrm{~dB}$ debida a pérdidas en conectores o a
desviaciones de las longitudes de fibra de las bobinas. 
portadora en sistemas de comunicaciones radio sobre fibra óptica operando a frecuencias de microondas.

\subsubsection{Limitaciones de la técnica}

La principal limitación de esta técnica viene impuesta por el SBS [Ipp72, Aok88, Fis93a] debido a las el evadas potencias ópticas y longitudes de fibra utilizadas en los experimentos. Este fenómeno fija una potencia óptica máxima que se puede inyectar a la entrada de la fibra, la cual depende principalmente de la longitud de ésta y del ancho de línea de la fuente óptica de acuerdo a [Aok88]:

$$
\mathrm{P}_{\mathrm{SBS}} \approx 42 \frac{\mathrm{A}_{\text {eff }}}{\mathrm{g}_{0} \mathrm{~L}_{\text {eff }}}\left(\frac{\Delta v_{\mathrm{B}}+\Delta v_{0}}{\Delta \mathrm{v}_{\mathrm{B}}}\right),
$$

siendo $\Delta v_{0}$ y $\Delta v_{B}$ los anchos de línea de la fuente láser y del proceso de Brillouin espontáneo, respectivamente, y $g_{0}$ una constante que para fibras monomodo típicas es igual a $4 \cdot 10^{-9} \mathrm{~cm} / \mathrm{W}$. Se deduce que el hecho de utilizar modulación externa $\left(\Delta v_{0} / \Delta v_{B} \ll 1\right)$ provoca que el umbral de potencia del SBS sea menor. En especial, para $50 \mathrm{~km}$ de SSMF se obtiene una potencia $\mathrm{P}_{\mathrm{SBS}} \approx+6 \mathrm{dBm}$.

Para intentar evitar en lo posible el SBS y aumentar el nivel de potencia que se puede aplicar a la entrada de la fibra en sistemas con modulación externa se han propuesto diferentes técnicas [Wil94, Ada98]. En general, todas ellas se basan en aumentar el ancho de línea de la fuente óptica por algún medio distinto al que se consigue con la modulación directa. Por ejemplo, en la figura 3.13 se representa el aumento que se produce en la potencia umbral de SBS en función del ancho de línea de la fuente óptica. Obsérvese que es posible conseguir valores de $\mathrm{P}_{\mathrm{SBS}}$ cercanos a los $+20 \mathrm{dBm}$ empleando un ancho de línea $\Delta v_{0}=1 \mathrm{GHz}$.

Otra de las limitaciones de la técnica viene impuesta por la cantidad de distorsión de intermodulación introducida por el SPM cuando se transmiten 


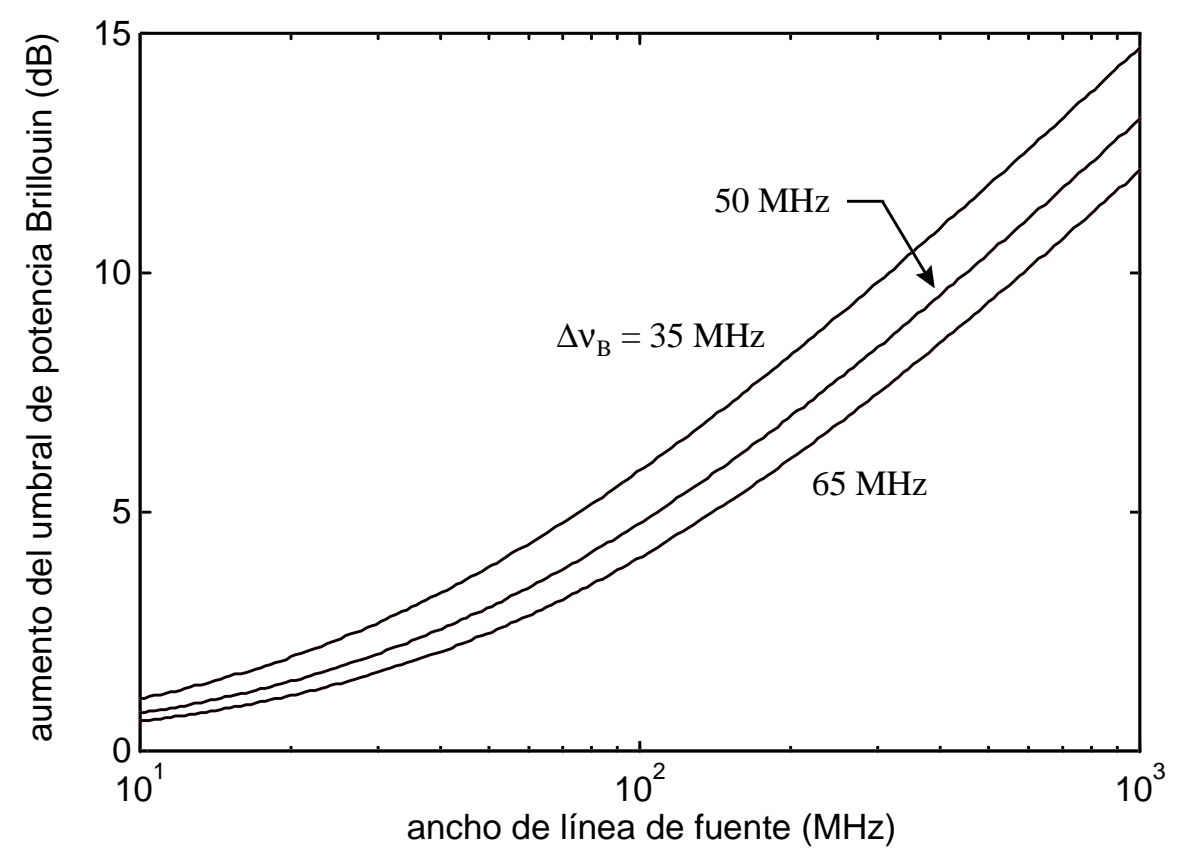

Figura 3.13 Aumento del nivel de potencia umbral de Brillouin en función del ancho de línea de la fuente óptica utilizada y para varios valores de ancho de línea Brillouin. Este último parámetro depende del tipo de fibra utilizado.

señales multicanal a través del sistemaviii. Como ejemplo, en la tabla I se resumen algunos resultados de simulación de CTB para diferentes valores de potencia óptica a la entrada de $75 \mathrm{~km}$ de SSMF. Se ha considerado una señal con dos tonos de índice de modulación $4 \%$ y se ha medido el CTB a la frecuencia $2 f_{1}-f_{2}$. Para una potencia óptica dada, se ha escogido como frecuencia a la cual medir la distorsión aquella que corresponde al punto óptimo comentado en la figura 3.6. Así pues, los requerimientos de CTB del sistema fijan un máximo de potencia óptica que puede inyectarse a la entrada de la fibra. La distorsión de CSO se ha despreciado dado que a frecuencias de microondas las señales multicanal suelen estar confinadas en el interior de un octava de frecuencia.

Por último, otros efectos no lineales como pudieran ser la modulación de fase cruzada (cross-gain modulation) o el mezclado de cuatro ondas (four-wave mixing, FWM) no afectan sobre la técnica SPM. El primero de ellos debido a

viii esto ya se pudo ver con detalle en el capítulo 2 
que estamos considerando un sistema con una única portadora óptica, mientras que el segundo debido a que la eficiencia de este proceso es extremadamente pequeña como consecuencia de trabajar a una longitud de onda muy alejada de la región de dispersión nula para fibras estándar que se encuentra en torno a los $1310 \mathrm{~nm}$. Téngase en cuenta que para inducir FWM a 1550 nm se utilizan fibras de dispersión desplazada [I no92].

TABLA I Resultados de simulación de distorsión CTB para una señal de modulación compuesta por dos tonos $\left(m_{i}=0,04\right)$ [Ram98].

\begin{tabular}{|c|c|c|}
\hline Potencia óptica (dBm) & frecuencia: $\mathbf{2 f}_{\mathbf{1}}-\mathbf{f}_{\mathbf{2}} \mathbf{( G H z )}$ & CTB (dBC) \\
\hline 0 & 0,75 & $-147,2$ \\
\hline 10 & 4,6 & $-86,1$ \\
\hline 13 & 6,3 & $-57,4$ \\
\hline 16 & 8,3 & $-29,5$ \\
\hline
\end{tabular}




\subsection{CONCLUSIONES Y CONTRIBUCIONES}

Este capítulo se ha destinado a estudiar la técnica SPM propuesta para reducir los efectos de la dispersión cromática en sistemas radio sobre fibra operando a $1550 \mathrm{~nm}$. Entre las conclusiones y resultados obtenidos destacan:

- Se han derivado unas expresiones cerradas, a partir de funciones de Bessel modificadas de orden imaginario puro, que caracterizan perfectamente las conversiones IM-IM y PM-IM que ocurren como consecuencia de la propagación de señales de RF a través de fibras ópticas dispersivas y no lineales. A su vez, estas expresiones sirven para calcular la función de transferencia de una SMF teniendo en cuenta los efectos conjuntos de dispersión cromática, SPM y chirp del transmisor óptico, y además son válidas tanto para modulaciones ópticas DSB como SSB. Todos estos resultados se presentan en la publicación [Ram00].

- La función de transferencia de una SSMF se ha medido en el laboratorio para frecuencias de RF hasta $20 \mathrm{GHz}$ y con distintos valores de potencia óptica a su entrada. Estos resultados han servido para validar el modelo teórico y, en particular, para comprobar la influencia que tiene el SPM sobre la respuesta de la fibra. EI chirp inducido por el SPM de la fibra conduce a un alejamiento de los nulos de la función de transferencia y, en consecuencia, a un aumento del ancho de banda de transmisión. Este efecto es similar al que se obtiene empleando transmisores con chirp negativo. Adicionalmente, se produce cierta ganancia a frecuencias bajas con respecto al caso convencional. Algunos de estos resultados se han publicado en [Ram98], coincidiendo temporalmente con otros estudios similares realizados por Cartaxo et. al. [Car98, Car99a]

- Como contribución, se ha demostrado experimentalmente la validez de la técnica SPM para reducir los efectos de la dispersión cromática de la fibra en sistemas MI-DD operando a $1550 \mathrm{~nm}$ sobre SSMF. Se han realizado medidas de la penalización de potencia utilizando la técnica SPM y se ha conseguido extender el punto de penalización a $1 \mathrm{~dB}$ del sistema 
significativamente con respecto al caso de transmisión convencional: desde los $20 \mathrm{~km}$ hasta más de $70 \mathrm{~km}$. Además, la técnica se ha validado realizando la transmisión de una PRBS de $8 \mathrm{Mbit} / \mathrm{s}$ sobre una subportadora de $7 \mathrm{GHz}$ y a través de $75 \mathrm{~km}$ de SSMF. En el caso de no emplear la técnica, los resultados demostraron que no era posible recuperar la señal de información a la salida del fotodetector, ya que ésta se había desvanecido total mente como consecuencia de que la frecuencia de portadora coincidía con el primero de los nulos de la función de transferencia. Por el contrario, empleando la técnica SPM se consiguió transmisión libre de errores con una potencia óptica recibida de $-4,5$ $\mathrm{dBm}$. 




\section{Compensación de distorsión no lineal mediante la técnica de inversión espectral}

\subsection{INTRODUCCIÓN}

En sistemas de comunicaciones ópticas de gran distancia, velocidad y potencia, la dispersión cromática y las no linealidades (efecto Kerr) en fibras monomodo (SMF) limitan la máxima distancia y capacidad alcanzables [Agr89]. Las propiedades dispersivas de la fibra, junto con el SPM debido al efecto Kerr, introducen distorsión sobre las señales que se propagan a lo largo del enlace de fibra óptica. En sistemas ópticos analógicos, las principales limitaciones vienen impuestas por el fenómeno de supresión de la portadora [Sch95] o por la distorsión no lineal o de intermodulación en sistemas multicanal [Ber91, Phi91, Cro94, Gop96, Wil96]. 
Hasta el momento, se han propuesto y demostrado múltiples técnicas de compensación de dispersión. Éstas incluyen, por ejemplo, el uso de fibras ecualizadoras [Ven93, I br93], el empleo de redes de difracción sobre fibra óptica (CF Gs) [Oue87, Mar97a] o la generación de un chirp de determinadas características en el transmisor óptico [Koc85]. Sin embargo, la distorsión causada por SPM no puede compensarse con estas técnicas. En transmisiones digitales de pulsos, la generación de solitones es un candidato para compensar el efecto del SPM [Agr97 (cap. 10)]. Los solitones se caracterizan por mantener su forma durante la propagación a través de una fibra con dispersión anómala $\left(\beta_{2}<0\right)$, debido a que el efecto Kerr cancela el ensanchamiento del pulso producido por la dispersión. Recientemente, se ha propuesto también una nueva técnica basada en solitones para la transmisión de señales analógicas [K ho99].

En particular, existe un método basado en la conjugación de la señal óptica, conocido como OPC (optical phase conjugation) y propuesto por Yariv et. al. [Yar79], que supone un acercamiento alternativo al problema de compensación de la distorsión producida por GVD y SPM. La técnica se basa en situar un dispositivo conjugador (OPC, optical phase conjugator) en mitad del trayecto de fibra. Dado que el fenómeno de conjugación supone un giro del espectro, a esta técnica también se le conoce con el nombre de inversión espectral. Debemos hacer notar que OPC compensa tanto dispersiones positivas como negativas, y además no depende del esquema de modulación empleado, lo cual permite aplicarlo en un gran número de configuraciones. Además, bajo ciertas condiciones, también es posible compensar la distorsión no lineal causada por el SPM [Wat96].

Hasta el momento, la práctica totalidad de los resultados se han obtenido para sistemas de comunicaciones ópticas digitales, mientras que relativamente pocos estudios se han centrado en sistemas analógicos, salvo algunos experimentos de conjugadores y convertidores de longitud de onda [Shi96, Fre97]. Sólo recientemente, Kitayama demostró la propagación de señales a $60 \mathrm{GHz}$ sobre un enlace de fibra empleando la técnica OPC [Kit99]. 
El objetivo de este capítulo es demostrar la viabilidad de la técnica de inversión espectral para compensar la dispersión cromática y el SPM introducidos por la fibra en sistemas de comunicaciones ópticas analógicos. Los fundamentos de la técnica OPC, así como las distintas configuraciones y arquitecturas empleadas o la generación de la señal conjugada se analizan en la sección 4.2. La sección 4.3 presenta los primeros resultados de compensación de distorsión no lineal introducida por la fibra óptica empleando la técnica de inversión espectral. En particular, se incluyen resultados de ecualización de CSO, CTB y penalización de potencia en sistemas radio-fibra típicos: CATV, MMDS, LMDS y MVDS, además de la respuesta en frecuencia del sistema OPC (véase apéndice I). Finalmente, en la sección 4.4 se tratan algunos aspectos adicionales sobre la técnica OPC. Básicamente, se estudia la sensibilidad de los resultados frente a diversos parámetros de la técnica: longitudes de onda, potencias ópticas, dispersión de segundo orden, etc. Además, se presenta una modificación de la técnica para mejorar los resultados de ecualización. La compensación del SPM viene limitada por la asimetría del efecto Kerr a lo largo del enlace con respecto a la posición del bloque OPC. Esto se debe a los cambios que las pérdidas de la fibra y los amplificadores introducen sobre la potencia de señal. Los estudios realizados en [Pep80, Fis83] se basaron en una situación sin pérdidas. Nosotros, analizaremos el caso en el que existan pérdidas, empleando la aproximación propuesta en [Wat96] y que se basa en minimizar el efecto de los cambios en la intensidad de la señal. El empleo de esta técnica viene limitado a situaciones en que las variaciones de SPM a lo largo de la fibra sean pequeñas. También se comentarán algunas soluciones para abordar el problema de la compensación exacta de dispersión y SPM. 


\subsection{ANÁLISIS DE LA TÉCNICA OPC}

En esta sección se estudiarán los fundamentos de la técnica de inversión espectral utilizada para compensar la distorsión no lineal introducida por la fibra óptica, incluyendo el proceso de conjugación de la señal, así como las distintas arquitecturas empleadas en un sistema de comunicaciones ópticas que implementa OPC.

\subsubsection{Compensación de dispersión cromática y SPM}

El modelo de compensación de distorsión no lineal en fibras monomodo mediante OPC se muestra en la figura 4.1(a). La señal transmitida, $E_{t}(t)$, con potencia óptica $P_{1}(t)$ y frecuencia $\omega_{\mathrm{S}}$,

$$
E_{t}(t)=A_{t}(t) \exp \left(j \omega_{s} t\right)
$$

incide a la entrada de una fibra óptica monomodo (SMF1) con constante de propagación dependiente de la frecuencia $\beta_{1}(\omega)$, índice de refracción no lineal $\mathrm{n}_{21}$ y longitud $\mathrm{L}_{1}$.

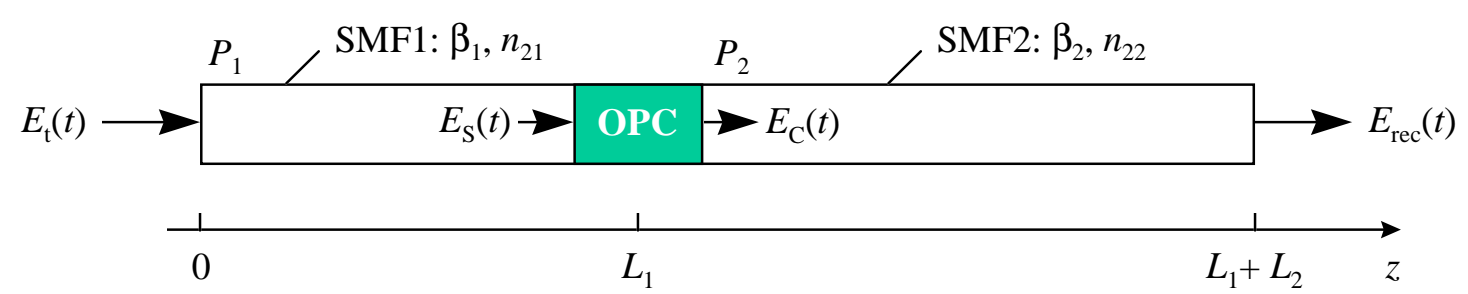

(a)

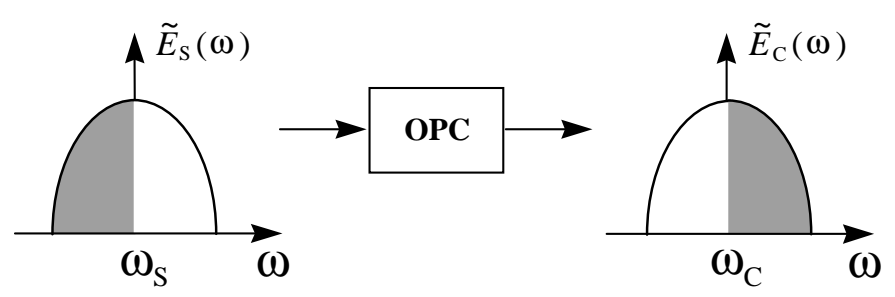

(b)

Figura 4.1 Compensación de distorsión no lineal mediante OPC. (a) Modelo de sistema de comunicaciones ópticas que emplea la técnica OPC. (b) Diagrama esquemático del proceso de conjugación de la señal óptica (inversión espectral). 
Durante la propagación de la señal a través de la fibra, ésta sufre de dispersión cromática (GVD) y SPM. Posteriormente, un conjugador óptico convierte la señal de entrada $E_{S}(t)$ en una réplica conjugada $E_{C}(t)$ a frecuencia óptica $\omega_{C}$, de tal forma que una componente de Fourier a frecuencia $\omega_{\mathrm{S}}+\omega_{\mathrm{e}}$ se convierte a $\omega_{\mathrm{C}}-\omega_{\mathrm{e}}$. Es decir,

$$
\begin{aligned}
& A_{C}(t)=\sqrt{G_{O P C}} A_{s}^{*}(t), \\
& \tilde{A}_{C}(\omega)=\sqrt{G_{O P C}} \widetilde{A}_{s}^{*}(-\omega) .
\end{aligned}
$$

siendo $\widetilde{A}(\omega)$ la transformada de Fourier de la envolvente compleja $A(t)$ y $\mathrm{G}_{\mathrm{OPC}}$ la ganancia en potencia del conjugador. Este proceso invierte el espectro de la señal, y de ahí viene precisamente el nombre de inversión espectral: figura 4.1(b). A continuación, esta señal $E_{C}(t)$ con potencia óptica $\mathrm{P}_{2}(\mathrm{t})$ se transmite a través de una segunda fibra óptica monomodo (SMF2) con constante de propagación $\beta_{2}(\omega)$, índice de refracción no lineal $n_{22}$ y longitud $L_{2}$. La señal recibida resultante $E_{\text {rec }}(t)$ es entonces detectada. Así pues, la longitud global del enlace óptico es $L=L_{1}+L_{2}$.

Desarrollando $\beta_{1}(\omega)$ y $\beta_{2}(\omega)$ en serie de Taylor alrededor de $\omega_{\mathrm{S}}$ y $\omega_{\mathrm{C}}$ respectivamente, y considerando que la eficiencia del conjugador es constante sobre el rango espectral de la señal de entrada, la señal a la salida puede expresarse comoi [Wat94]

$$
\begin{aligned}
E_{C}(t) & \propto \exp \left[j\left(\omega_{C} t+\beta_{1}\left(\omega_{S}\right) L_{1}-\beta_{2}\left(\omega_{C}\right) L_{2}\right)\right] \\
& \times \int_{-\infty}^{+\infty} d \omega_{e} A_{t}^{*}\left(\omega_{e}\right) \\
& \times \exp \left\{-j \omega_{e}\left[t-\frac{\partial \beta_{1}\left(\omega_{S}\right)}{\partial \omega} L_{1}-\frac{\partial \beta_{2}\left(\omega_{C}\right)}{\partial \omega} L_{2}\right]\right\} \\
& \times \exp \left[j\left(\Delta \Phi_{1}\left(\omega_{e}, t\right)-\Delta \Phi_{2}\left(-\omega_{e}, t\right)\right)\right]
\end{aligned}
$$

donde $\Delta \Phi_{j}(j=1,2)$ es la distorsión de fase que experimenta la señal cuando atraviesa la SMFj. El término $\Delta \Phi_{\mathrm{j}}$ incluye el desplazamiento de fase debido

\footnotetext{
i en [Wat94] se considera una igualdad, ya que el autor no incluye las pérdidas de la fibra en el análisis previo de compensación de GVD
} 
a GVD y efectos dispersivos de orden superior, además del SPM, siendo el causante de la distorsión no lineal a la salida del fotodetector. La ecuación anterior muestra que cuando el desplazamiento de fase es estacionario (independiente del tiempo) e igual en ambas fibras, la propagación a través de la segunda puede compensar el desplazamiento de fase introducido por la primera. De este modo, tanto la dispersión generada por potencias pares de $\omega_{\mathrm{e}}$ como el SPM pueden compensarse empleando esta técnica.

Dejando aparte por un momento el SPM, la distorsión debida a la dispersión cromática puede compensarse si se cumple la siguiente condición [Yar79, Wat93a, Wat94]:

$$
\frac{\partial^{2} \beta_{1}\left(\omega_{S}\right)}{\partial \omega^{2}} L_{1} \equiv \beta_{21} L_{1}=\beta_{22} L_{2} \equiv \frac{\partial^{2} \beta_{2}\left(\omega_{C}\right)}{\partial \omega^{2}} L_{2}
$$

Es decir, la dispersión cromática que sufre la señal en ambas fibras debe ser idéntica. Sin embargo, la dispersión de segundo orden caracterizada por $\beta_{3}$ no puede compensarse con esta técnica salvo que tenga distinto signo en cada una de las fibras.

Cuando el efecto Kerr es significativo, la distorsión inducida por SPM se añade a la causada por la dispersión de la fibra. En este caso, la propagación de la señal viene expresada por la ecuación de Schrödinger no lineal (2.11). No obstante, todavía es posible discutir los efectos causados por el SPM de una forma cualitativa si consideramos que podemos compensar la distorsión causada por GVD y SPM independientemente [Wat93]. Dado que el desplazamiento de fase causado por el efecto Kerr en la fibra es proporcional a $\left(\omega n_{2} / c\right) P$, como pudo verse en el apartado 2.2.2, la condición para compensar SPM bajo la aproximación anterior viene dada por [Wat94]

$$
\frac{\omega_{S} n_{21}}{C} \int_{0}^{L_{1}} P_{1}(z, t) d z \approx \frac{\omega_{C} n_{22}}{C} \int_{L_{1}}^{L_{1}+L_{2}} P_{2}(z, t) d z
$$

A continuación, expresando la potencia óptica en cada una de las fibras como $P_{1}(z, t)=P_{1}(0, t) \exp \left(-\alpha_{1} z\right)$ y $P_{2}(z, t)=P_{2}\left(L_{1}, t\right) \exp \left(-\alpha_{2}\left(z-L_{1}\right)\right)$, donde $\alpha_{j}$ 
representa el coeficiente de atenuación de la fibra SMFj, e introduciendo el coeficiente no lineal $\gamma_{\mathrm{j}}$, la condición para compensar SPM queda como

$$
\gamma_{1} \bar{P}_{1}(t) L_{1} \approx \gamma_{2} \bar{P}_{2}(t) L_{2}
$$

Los términos $\overline{P_{j}}(t)$ representan un promediado de la potencia óptica a lo largo de la fibra. La ec. (4.7) indica que, si el producto de $\gamma_{j}, \overline{P_{j}}$ y $L_{j}$ es prácticamente el mismo para las dos fibras, la distorsión puede suprimirse. No obstante, para una mayor compensación, el chirp inducido por SPM en ambas fibras debería ser el mismo [Wat93, Wat94]:

$$
\gamma_{1} \int_{0}^{L_{1}} \frac{\partial P_{1}(z, t)}{\partial t} d z \approx \gamma_{2} \int_{L_{1}}^{L_{1}+L_{2}} \frac{\partial P_{2}(z, t)}{\partial t} d z
$$

Dado que este chirp depende de la propia señal a transmitir, como muestra la derivada con respecto al tiempo de $\mathrm{P}_{\mathrm{j}}$ en la ecuación anterior, la fase de $E_{C}$ no se desplaza de forma inversa con el tiempo debido a que las pérdidas de la fibra evitan que la potencia óptica se distribuya simétricamente con respecto a la posición del OPC. En una sección posterior se demostrará teóricamente cuál es la condición para compensar de una forma perfecta el efecto conjunto de GVD y SPM. La condición impuesta por (4.7) es únicamente válida, por lo tanto, cuando los cambios de intensidad a lo largo de la fibra son pequeños. De (4.7) se deduce también que, por medio de una fibra SMF2 con un valor de $\mathrm{n}_{2}$ ligeramente mayor que la SMF1 o amplificando la señal hasta una potencia mayor que $\mathrm{P}_{1}$ a la salida del OPC, podemos compensar la distorsión debida a SPM empleando una longitud de fibra SMF 2 menor que la de SMF 1.

\subsubsection{Configuraciones y arquitecturas empleadas}

A la hora de aplicar la técnica OPC para compensar distorsión no lineal existen distintas configuraciones posibles, especialmente en función de la posición en que se coloca el OPC en relación al enlace de comunicaciones ópticas. 


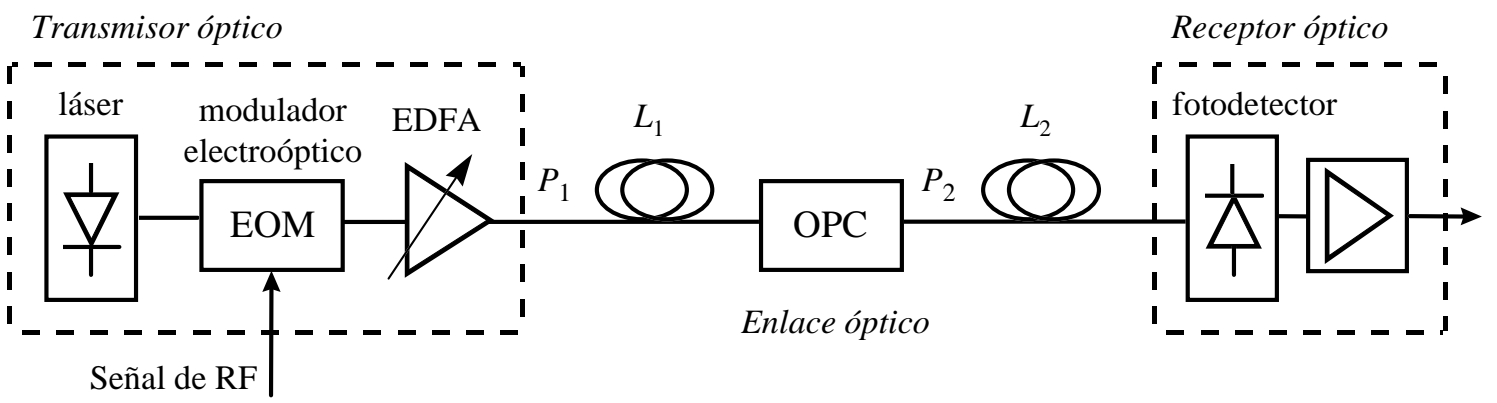

(a)

Transmisor óptico

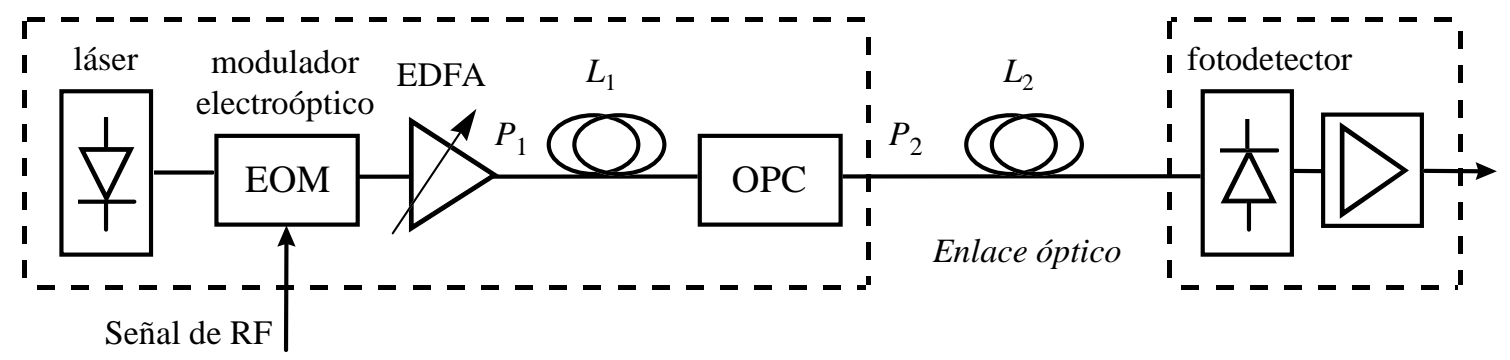

(b)

Transmisor óptico

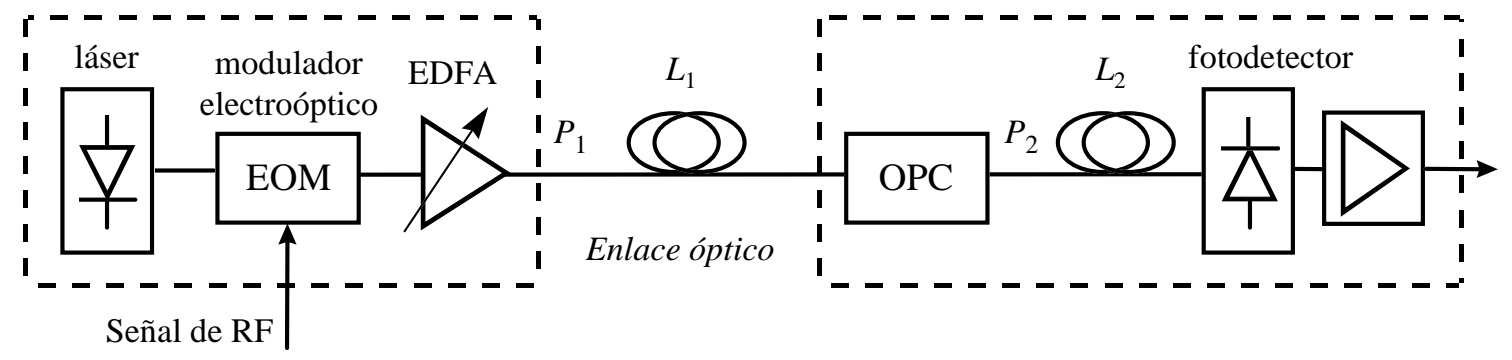

(c)

Figura 4.2 Ejemplos de sistemas de comunicaciones ópticas que emplean la técnica OPC. (a) Amplificador conjugador, (b) transmisor conjugador, y (c) receptor conjugador.

La configuración más típica consiste en colocar el bloque OPC en mitad del enlace, como se muestra en la figura 4.2(a), utilizando dos fibras con longitudes y valores de dispersión similares. En particular, el caso $L_{1}=L_{2}$ se conoce habitualmente con el nombre de mid-span spectral inversion (MSSI). Para transmisiones de larga distancia en las cuales se utilizan múltiples etapas de amplificación, es interesante el uso de fibras de dispersión desplazada [Kur93]. Como solución alternativa, también puede utilizarse una SMF con dispersión reducida como línea de transmisión y una SMF con 
dispersión el evada para realizar la compensación. Por último, cualquiera de los dos trayectos de fibra puede incluir amplificadores cuando la longitud del enlace sea elevada.

En el sistema de la figura 4.2(b) la señal se distorsiona previamente en el transmisor óptico (SMF1) para posteriormente compensar dicha distorsión por medio de la fibra óptica que forma parte del enlace (SMF2). Esta configuración es atractiva debido a que el estado de polarización de la señal óptica a la entrada del OPC puede controlarse fácilmente. Téngase en cuenta que el conjugador es un dispositivo normalmente sensible a la polarización de la señal de entrada, ya que la eficiencia del proceso de conjugación tiene una clara dependencia con ésta. Recientemente han aparecido nuevas técnicas para la construcción de conjugadores ópticos insensibles a la polarización [Set98, Eis98, Leu98].

Finalmente, en la figura 4.2(c) se muestra otro esquema posible. Aquí, la ecualización de la señal se lleva a cabo en el interior del receptor óptico por medio del OPC y de la segunda fibra (SMF2). La ventaja de esta última configuración consiste en que, en el caso de múltiples receptores, cada usuario puede configurar independientemente los parámetros de la SMF 2 para obtener los mejores resultados de ecualización. Conviene tener en cuenta que los tramos de fibra hasta cada uno de los receptores no tienen por qué ser idénticos. Ahora bien, como desventaja se necesita un OPC para cada uno de los usuarios frente al único de los esquemas (a) y (b).

En todas estas configuraciones, los parámetros deben escogerse de tal forma que satisfagan las ecuaciones (4.5) y (4.7). De este modo, si la señal se transmite a través de una DSF con $D_{1}=-0,2 \mathrm{ps} /(\mathrm{km} \cdot \mathrm{nm})$ y $\mathrm{L}_{1}=100 \mathrm{~km}$, por ejemplo, la distorsión no lineal puede compensarse por medio de una fibra con $D_{2} \sim-20 \mathrm{ps} /(\mathrm{km} \cdot \mathrm{nm})$ y $L_{2}=1 \mathrm{~km}$, además de una potencia óptica de entrada $\sim 20 \mathrm{~dB}$ superior a la de la DSF suponiendo características no lineales idénticas para ambas fibras.

En la figura 4.3 se propone una posible arquitectura para un sistema de comunicaciones ópticas radio-fibra basado en la técnica de inversión 
espectral. La cabecera de red (estación central) está unida a la estación de control por medio de un enlace de fibra óptica de longitud $L_{1}$. Desde la estación de control, donde se sitúa el dispositivo OPC, se distribuye la señal hasta los distintos nodos ópticos empleando enlaces de fibra de distinta longitud. El esquema es totalmente flexible, ya que la señal puede distribuirse a estaciones base (antenas remotas), redes de cable (HFC) o directamente hasta el propio hogar del usuario (FTTH).

Mediante esta arquitectura se optimiza el uso del bloque OPC, ya que es compartido por multitud de usuarios con el consiguiente ahorro económico. Ahora bien, el hecho de que cada uno de los enlaces tenga distinta longitud dependiendo del emplazamiento del nodo óptico, obliga a realizar un ajuste de ecualización en la estación de control. Así, considerando el enlace de



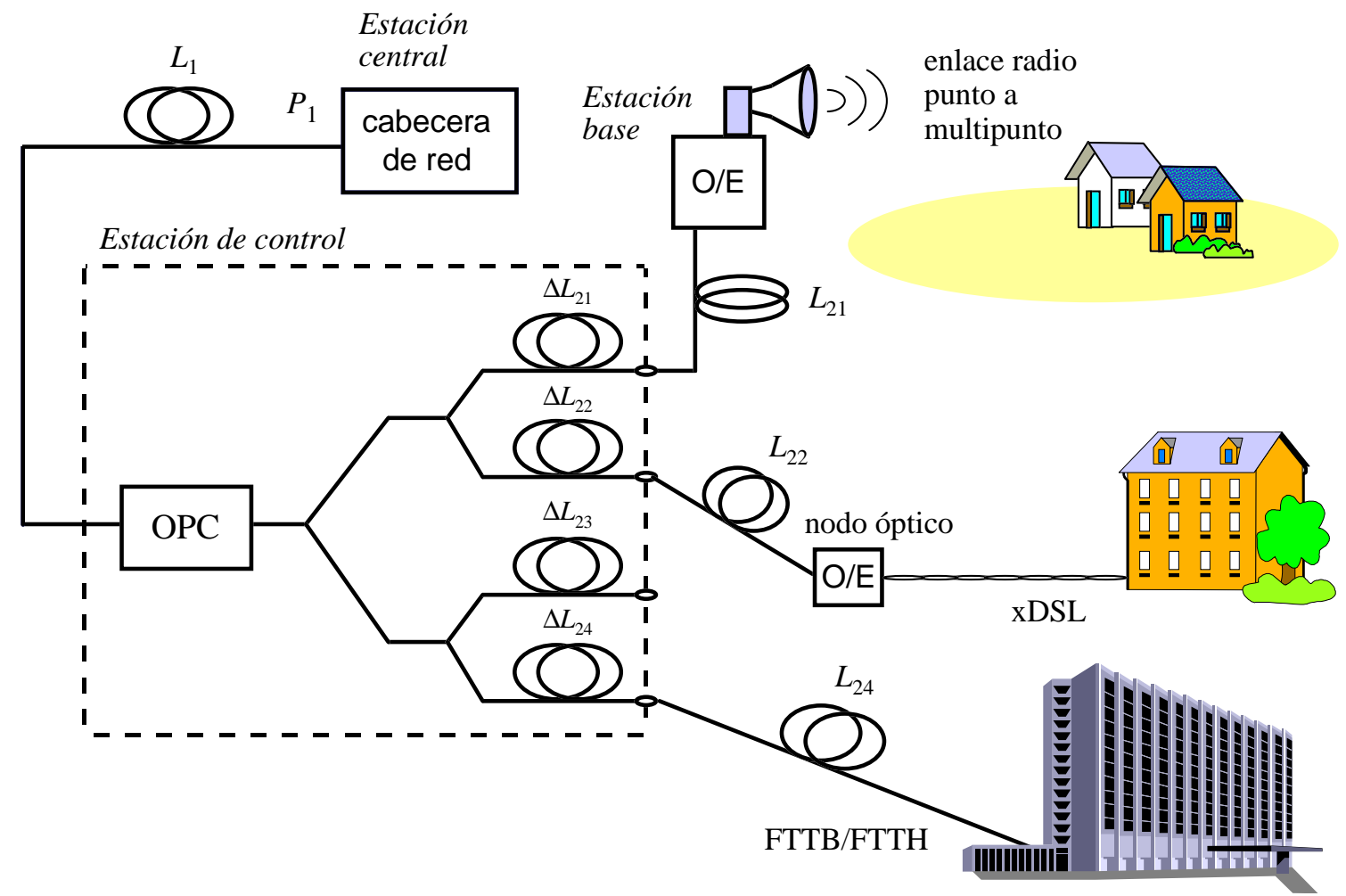

Figura 4.3 Arquitectura de un sistema radio sobre fibra óptica empleando la técnica de inversión espectral. El dispositivo OPC es compartido por todos los usuarios, con el consiguiente ahorro de costes. 
enlaces restantes para asegurar que todos poseen la misma longitud total. El tipo de fibra a utilizar, así como la potencia óptica transmitida, dependerá de las características del enlace de fibra entre la estación central y la estación de control. Hay que resaltar que esta arquitectura es unidireccional debido a la propia construcción del conjugador óptico y, por lo tanto, únicamente válida para sistemas de distribución. No obstante, el retorno de los usuarios se podría realizar en segunda ventana (1,3 $\mu \mathrm{m})$ aprovechando los mismos enlaces de fibra y empleando un esquema SCM. A esta longitud de onda, dado que no afecta la dispersión cromática, no sería necesaria la presencia de ningún dispositivo OPC.

\subsubsection{Generación de la señal conjugada}

La implementación de la técnica de inversión espectral requiere el uso de un dispositivo óptico no lineal que genere la señal conjugada. El método más utilizado se basa en FWM en un medio no lineal. Las dos configuraciones más deseables son las fibras de dispersión desplazada (DSFs) [Wu94, Zha96] y los amplificadores de semiconductor (SOAs) [Duc96, Kin97]. En cualquiera de las dos variantes, el diagrama de bloques del conjugador es como el mostrado en la figura 4.4. La señal de entrada, tras ser amplificada y filtrada para eliminar ruido ASE, se combina con una señal de onda

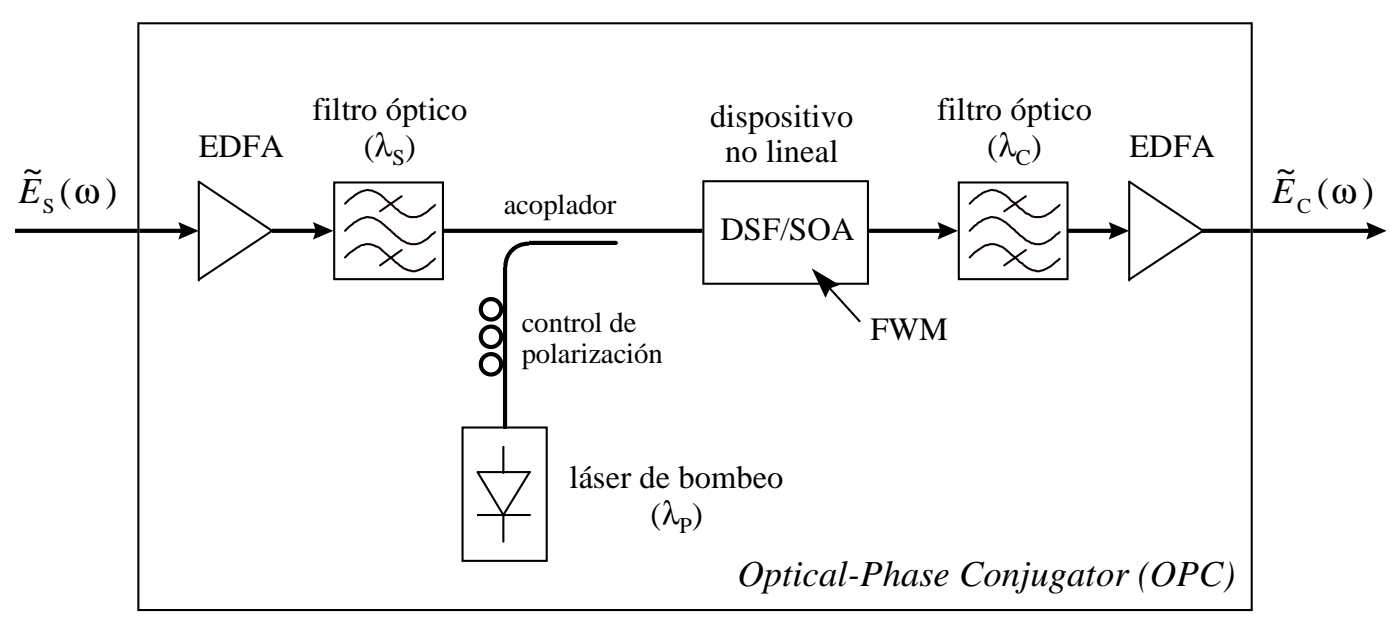

Figura 4.4 Diagrama de bloques de un conjugador óptico basado en FWM en una DSF o SOA ( $\lambda_{S}$ : señal, $\lambda_{\mathrm{P}}$ : bombeo, y $\lambda_{\mathrm{C}}$ : conjugada). 


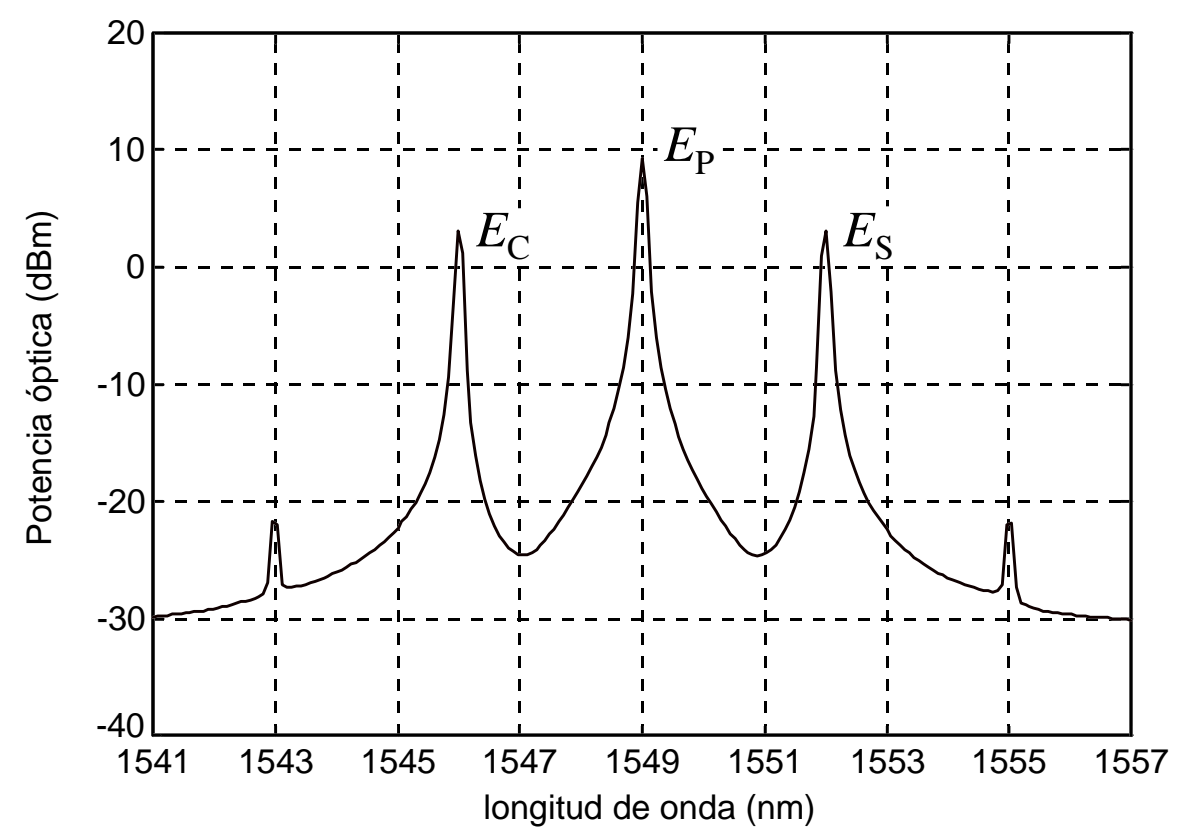

Figura 4.5 Espectro óptico a la salida del conjugador (DSF/SOA). Se observa la presencia de la señal conjugada junto con algunos productos FWM no deseados.

continua proveniente de un diodo láser de bombeo. Es importante controlar el estado de polarización de esta señal de bombeo para conseguir la máxima eficiencia en el proceso de FWM. Estas dos señales se aplican entonces a la entrada del dispositivo no lineal (DSF/SOA) donde tendrá lugar el proceso de conjugación. Finalmente, la señal conjugada se selecciona por medio de un filtro óptico y se amplifica hasta el nivel de potencia deseado. Opcionalmente, se puede emplear un nuevo filtro a la salida para eliminar ruido ASE.

El proceso de FWM es una interacción no lineal que tiene lugar entre tres ondas ópticas de frecuencias $\omega_{\mathrm{k}},(\mathrm{k}=\mathrm{P} 1, \mathrm{P} 2, \mathrm{~S})$, y que da lugar a la generación de una cuarta onda a frecuencia $\omega_{\mathrm{C}}=\omega_{\mathrm{P} 1}+\omega_{\mathrm{P} 2}-\omega_{\mathrm{S}}$ [Hil78]. Cuando las frecuencias de las dos ondas de bombeo, P1 y P2, coinciden, el proceso se denomina FWM parcialmente degenerado. En el capítulo 5 se analizará en detalle el proceso de FWM aplicado a la elaboración de conjugadores ópticos. Por el momento, en la figura 4.5 se representa el espectro óptico de salida de un conjugador. Se observa la señal de bombeo central y las dos bandas laterales que representan la señal de entrada y su 
conjugada. Adicionalmente, aparecen algunas nuevas frecuencias como resultado de productos no deseados. En cualquier caso, la eficiencia de éstos es muy reducida y desaparecen completamente tras el filtrado de la señal conjugada. 


\subsection{RESULTADOS DE COMPENSACIÓN DE DISTORSIÓN NO LINEAL}

En la sección anterior se ha esbozado la posibilidad de compensar la dispersión cromática y el SPM empleando la técnica de inversión espectral. A continuación, presentaremos resultados de compensación de distorsión no lineal en sistemas de comunicaciones ópticas analógicos. Algunos de estos resultados han sido publicados en [Mar97, Ram98a]. Mientras no se diga lo contrario, se supondrá que se aplica la técnica OPC en su configuración básica con $L_{1}=L_{2}=L / 2$, y los tramos de fibra empleados son idénticos y vienen caracterizados por unos parámetros: $D_{1}=D_{2}=17 \mathrm{ps} /(\mathrm{km} \cdot \mathrm{nm}), \gamma_{1}=\gamma_{2}$ $=2,6 \mathrm{~W}^{-1} \mathrm{~km}^{-1}$ y $\alpha_{1}=\alpha_{2}=0,2 \mathrm{~dB} / \mathrm{km}$. Además, el dispositivo OPC compensa las pérdidas del tramo de fibra previo, de tal forma que $P_{2}=P_{1}=P_{0}$, es decir $\mathrm{G}_{\mathrm{OPC}}=\exp \left(\alpha \mathrm{L}_{1}\right)$.

\subsubsection{Ecualización de los efectos de GVD y SPM}

Un primer resultado relativo a la ecualización de la distorsión no lineal introducida por la fibra óptica lo constituye la reducción en los niveles de CSO y CTB. En la figura 4.6 se muestra este resultado para una frecuencia de modulación de $500 \mathrm{MHz}$ y considerando modulación directa con un parámetro de chirp $\vartheta=20 \mathrm{GHz}$. Se observa que empleando la técnica OPC se consigue una reducción considerable en los nivel es de distorsión no lineal. Así, es posible extender la longitud de fibra por encima de los $100 \mathrm{~km}$ con valores de CSO y CTB inferiores a $-70 \mathrm{dBc}$ y $-100 \mathrm{dBc}$, respectivamente. Compárese esto con el límite de $2 \mathrm{~km}$ que se consigue para un valor de CSO de-60 dBc empleando modulación directa.

Los resultados de ecualización empleando modulación externa son prácticamente idénticos, ya que la técnica OPC compensa perfectamente los efectos dispersivos de la fibra, aun incluyendo el chirp del transmisor óptico. $\mathrm{Si}$ bien, existen ligeras diferencias debidas a efectos conjuntos GVD-SPM que no pueden compensarse. EI nivel último de ecualización viene fijado por la potencia óptica y la simetría del efecto Kerr a ambos lados del elemento conjugador. Como se verá posteriormente, existe la posibilidad de una 


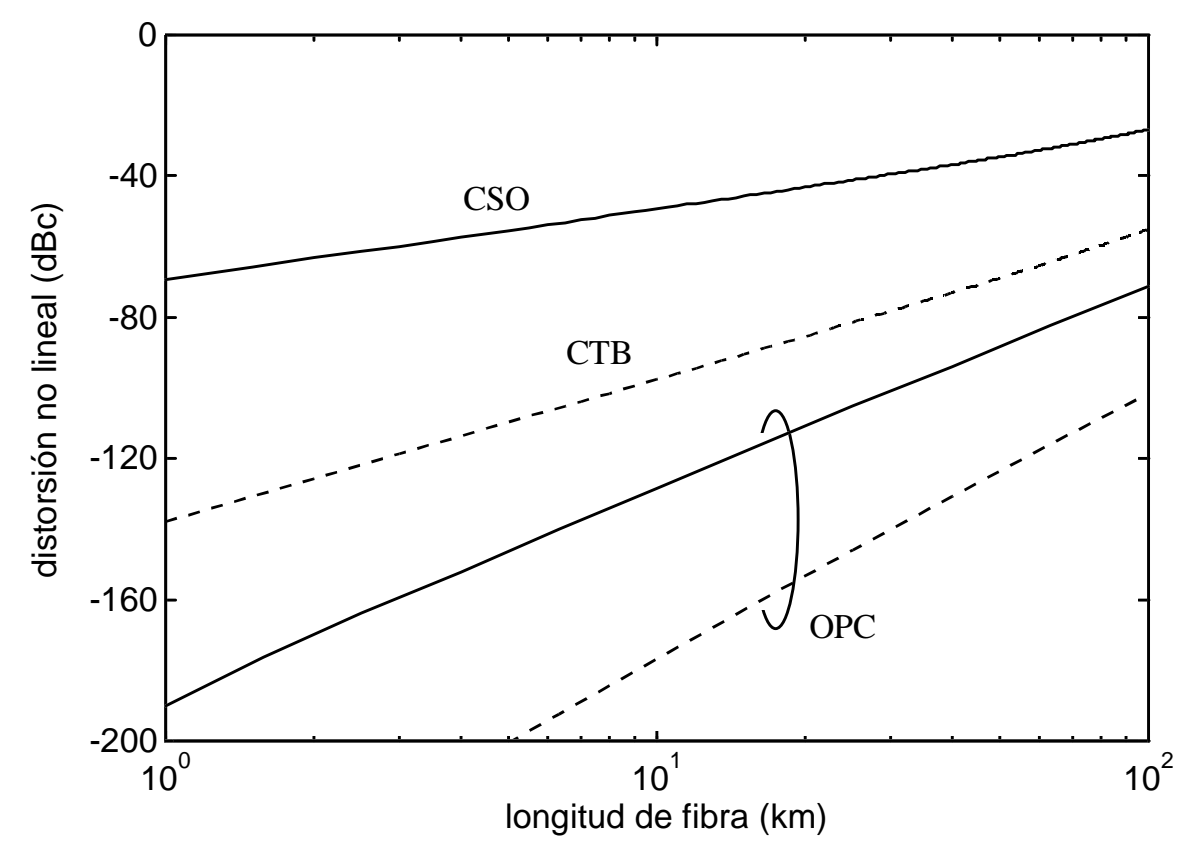

Figura 4.6 Ecualización de distorsión no lineal introducida por la fibra óptica mediante inversión espectral: CSO en línea continua y CTB en línea de trazos. Los parámetros de modulación son: $\mathrm{f}=$ $500 \mathrm{MHz}, \mathrm{m}_{\mathrm{i}}=0,04, \vartheta=20 \mathrm{GHz}$ y $\mathrm{P}_{0}=20 \mathrm{~mW}$.

compensación perfecta fijando ciertas condiciones sobre el parámetro de dispersión de la fibra óptica. Esta falta de simetría provoca que la reducción relativa en los niveles de CSO y CTB sea menor conforme aumenta la longitud del enlace, ya que debido a las pérdidas de la fibra se rompe cada vez más la simetría de la potencia óptica [Wat96].

Esto mismo se puede deducir también de la figura 4.7, donde se representan los niveles de CSO con y sin ecualización en función de la potencia óptica transmitida y para distintas frecuencias de modulación. Al tratarse de modulación externa, el CSO aumenta proporcionalmente con la potencia óptica debido al efecto Kerr de la fibra. A menor frecuencia existe menos distorsión, aunque la reducción que se consigue ecualizando también suele ser menor. Por otra parte, la reducción disminuye al aumentar la potencia óptica y además es más acusado para frecuencias de subportadora mayores. Todos estos factores se explican por la falta de simetría del efecto Kerr a ambos lados del OPC. Debe tenerse en cuenta que aunque las pérdidas de la 




Figura 4.7 CSO en función de la potencia óptica transmitida con y sin ecualización para $50 \mathrm{~km}$ de fibra. Los resultados se muestran para modulación externa con tres frecuencias de subportadora distintas: $500 \mathrm{MHz}, 2 \mathrm{GHz}$ y $5 \mathrm{GHz}$. Las curvas de trazos corresponden a la ecualización con OPC. Los parámetros de modulación son: $m_{i}=0,04$ y $\vartheta=0$.

fibra son de tan sólo $0,2 \mathrm{~dB} / \mathrm{km}$, para $\mathrm{L} / 2=25 \mathrm{~km}$ se obtiene una variación de potencia óptica de $5 \mathrm{~dB}$.

Por el momento se han obtenido resultados de ecualización suponiendo trayectos de fibra de la misma longitud. Sin embargo, resulta interesante probar otras configuraciones distintas como las que se muestran en la figura 4.8 para dos casos: (a) sólo dispersión o (b) dispersión + SPM. Las curvas continuas son para la configuración MSSI $\left(\mathrm{L}_{1}=50 \mathrm{~km}\right)$, mientras que las de trazos son para $L_{1}=70 \mathrm{~km}$ y las de trazos y puntos para $L_{1}=30 \mathrm{~km}$. En todos los casos, los parámetros se han optimizado utilizando las ecuaciones (4.5) y (4.7) para obtener el mínimo de distorsión a una distancia de $100 \mathrm{~km}$. En el caso de GVD, el parámetro de dispersión del segundo trayecto de fibra se ha fijado de acuerdo a: 


$$
D_{2}=\frac{L_{1}}{L_{2}} D_{1}
$$

condición típica utilizada en las fibras compensadoras de dispersión. De esta forma se obtiene $D_{2}=7,3 \mathrm{ps} /(\mathrm{km} \cdot \mathrm{nm})$ para $L_{1}=30 \mathrm{~km}$ y $D_{2}=39,7$ $\mathrm{ps} /(\mathrm{km} \cdot \mathrm{nm})$ para $\mathrm{L}_{1}=70 \mathrm{~km}$. Además, en el caso de GVD +SPM, la potencia a la salida del bloque conjugador viene dada por (4.7),

$$
P_{2}=\frac{1-\exp \left(-\alpha_{1} L_{1}\right)}{1-\exp \left(-\alpha_{2} L_{2}\right)} P_{1}
$$

obteniéndose $P_{2}=15,6 \mathrm{~mW}$ para $L_{1}=30 \mathrm{~km}$ y $P_{2}=25,7 \mathrm{~mW}$ para $L_{1}=70$ $\mathrm{km}$. Considerando la atenuación introducida por el primer trayecto de fibra, la ganancia del dispositivo OPC vendrá dada por

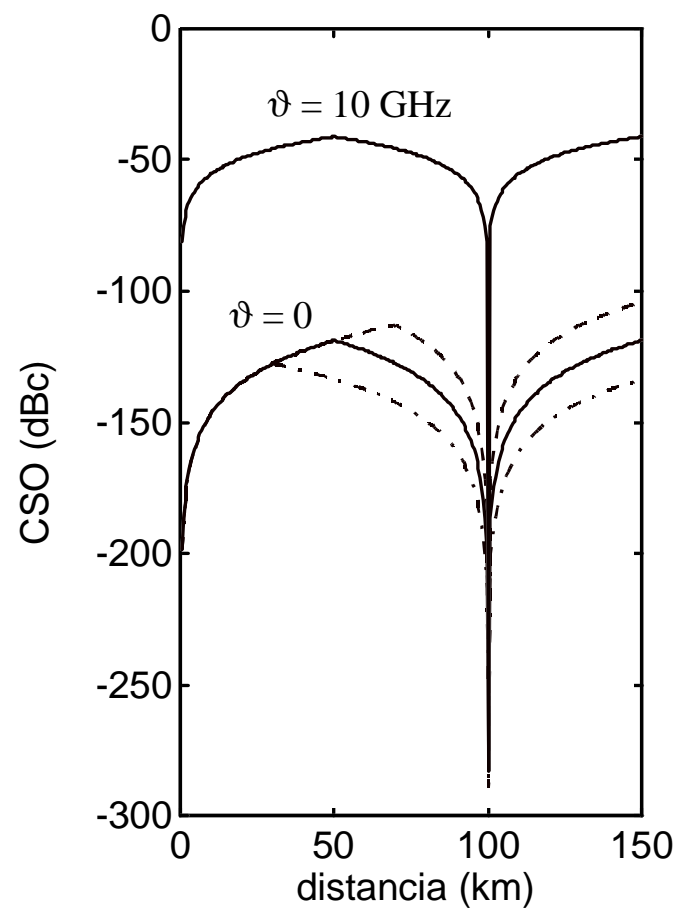

(a)

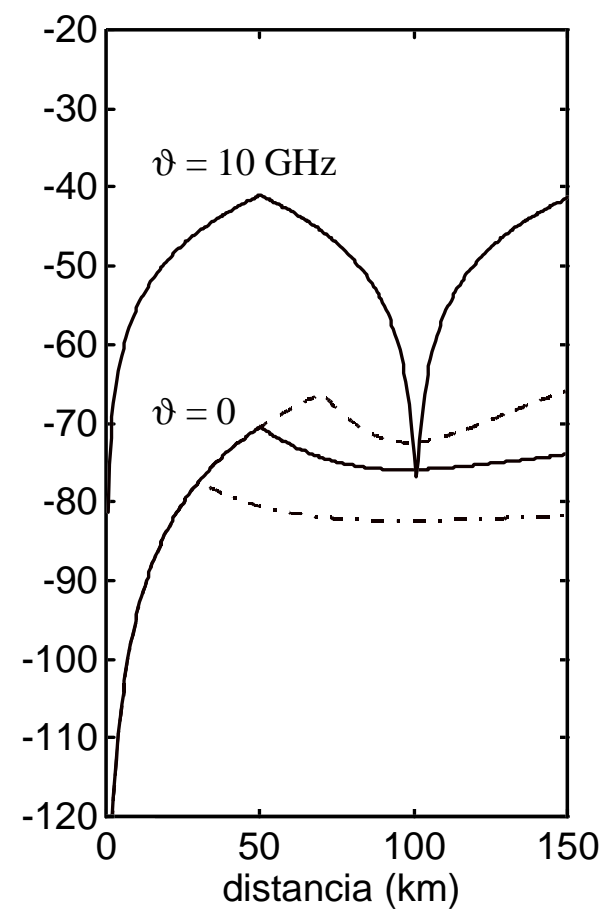

(b)

Figura 4.8 Ecualización de CSO mediante inversión espectral: (a) GVD y (b) GVD +SPM. Las curvas continuas son para la configuración básica $\left(\mathrm{L}_{1}\right.$ $=\mathrm{L}_{2}=\mathrm{L} / 2$ ), mientras que las de trazos son para $\mathrm{L}_{1}=70 \mathrm{~km}$ y las de trazos y puntos para $L_{1}=30 \mathrm{~km}$. Los parámetros de modulación son: $\mathrm{fm}_{\mathrm{m}}=500$ $\mathrm{MHz}, \mathrm{m}_{\mathrm{i}}=0,04$ y $\mathrm{P}_{0}=20 \mathrm{~mW}$. 


$$
\mathrm{G}_{\mathrm{OPC}}=\frac{\mathrm{P}_{2}}{\mathrm{P}_{0}} \exp \left(\alpha \mathrm{L}_{1}\right)
$$

lo cual proporciona una ganancia $\mathrm{G}_{\mathrm{OPC}}=4,91 \mathrm{~dB}$ para $\mathrm{L}_{1}=30 \mathrm{~km}$ y $\mathrm{G}_{\mathrm{OPC}}=$ $15,07 \mathrm{~dB}$ para $\mathrm{L}_{1}=70 \mathrm{~km}$.

En la figura 4.8(a) se puede observar que el CSO aumenta con la distancia hasta llegar al punto donde se encuentra situado el bloque OPC. A partir de este punto, la propagación por el segundo tramo de fibra compensa la distorsión acumulada en el primer tramo, obteniéndose un mínimo para aquella longitud de fibra a la que se ha optimizado el enlace. Tanto con modulación directa como externa se produce un nulo a los 100 km, ya que el chirp no afecta a la hora de compensar perfectamente la distorsión causada por la dispersión cromática de la fibra. Por otro lado, para longitudes $L_{1}$ mayores se obtienen niveles de distorsión superiores al alejarse ligeramente de la distancia óptima de ecualización, ya que la distorsión acumulada durante el primer tramo de fibra es superior.

En cuanto a la figura 4.8(b), en ella se observa que no es posible compensar perfectamente el CSO en presencia de SPM. De todos modos, todavía es posible optimizar el enlace para obtener el mínimo de distorsión a una distancia de $100 \mathrm{~km}$. Como ocurría en la figura 4.8(a), la presencia de chirp no influye prácticamente en los resultados de ecualización, si bien los niveles de distorsión acumulados a lo largo del trayecto son superiores y la sensibilidad al alejarse del punto óptimo es mucho mayor con respecto al caso de modulación externa. Precisamente para $\vartheta=0$, se deduce que emplear longitudes $L_{1}$ menores produce niveles de CSO más reducidos y además los resultados son menos sensibles frente a desviaciones de la longitud de enlace óptima. Esta es una de las ventajas más significativas de la técnica de inversión espectral frente a otros métodos de ecualización, como por ejemplo la técnica de modulación de amplitud y fase conjunta en el transmisor óptico propuesta por Desem [Des94].

La gráfica de la sensibilidad, definida como la diferencia en dB entre el valor de CSO a una determinada distancia y el valor de CSO óptimo 




Figura 4.9 Sensibilidad de la técnica OPC frente a variaciones en la distancia óptima de ecualización para tres configuraciones de enlace. Esta gráfica se ha obtenido a partir de los resultados de la figura 4.8(b).

obtenido para $100 \mathrm{~km}$, se muestra en la figura 4.9. Tomando una penalización máxima en el CSO de $1 \mathrm{~dB}$ se consigue un margen de distancias superior a los $20 \mathrm{~km}$ para el caso peor de $L_{1}=70 \mathrm{~km}$. Esto permite escoger la longitud del segundo enlace de fibra de forma flexible en sistemas de distribución multipunto con gran número de usuarios (arquitectura de la figura 4.3), principal ventaja de esta técnica de ecualización.

Otro de los efectos perjudiciales introducido por la fibra óptica es el de la penalización de potencia, el cual ya fue comentado en el capítulo 2 . Al igual que el CSO y el CTB, es posible reducir sus efectos empleando la técnica de inversión espectral. En la figura 4.10 se representa la penalización de potencia causada por SPM y su ecualización mediante OPC para dos longitudes de fibra distintas: 50 y $75 \mathrm{~km}$. Como se comentó en el capítulo 3, Ias no linealidades de la fibra producen un desplazamiento del primer nulo de las curvas hacia frecuencias mayores e introducen una cierta ganancia sobre las frecuencias inferiores a dicho nulo. Este efecto beneficioso respecto 


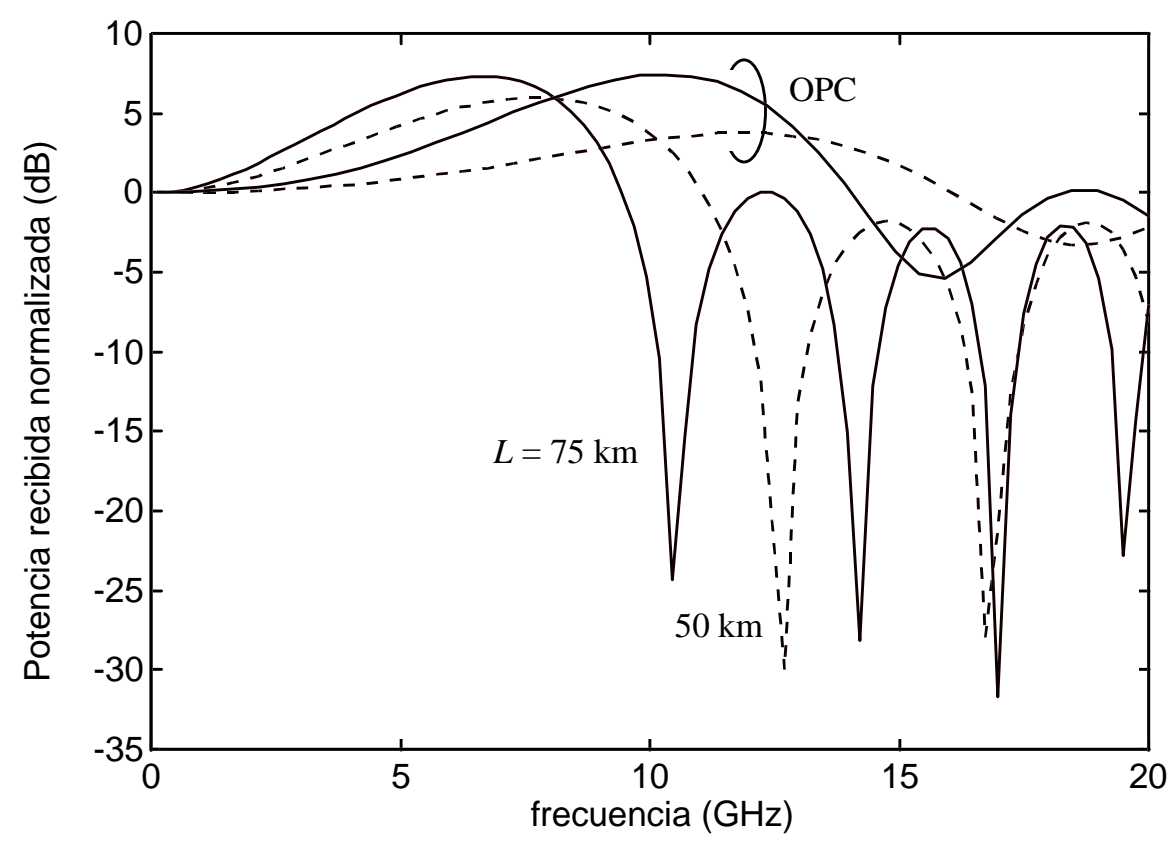

Figura 4.10 Ecualización de la penalización de potencia introducida por la fibra óptica mediante inversión espectral. Las curvas continuas son para $75 \mathrm{~km}$ y las de trazos para $50 \mathrm{~km}$. Los parámetros de modulación son: $\mathrm{m}_{\mathrm{i}}=0,04, \vartheta=0$ y $\mathrm{P}_{0}=20 \mathrm{~mW}$.

al caso de sólo dispersión es posible mejorarlo todavía más si empleamos la técnica OPC. En este último caso las curvas se suavizan, desapareciendo completamente los nulos y manteniéndose en un margen de unos $\pm 5 \mathrm{~dB}$, dependiendo de la longitud de fibra. Obsérvese que para una longitud de fibra de $50 \mathrm{~km}$ se obtiene un ancho de banda a $-3 \mathrm{~dB}$ cercano a los $20 \mathrm{GHz}$. En el caso ideal de que no exista SPM (válido para potencias ópticas reducidas) la ecualización es completa, obteniéndose una respuesta en frecuencia del sistema totalmente plana aun en presencia de chirpii. El efecto Kerr, no obstante, da lugar a un rizado en los resultados de ecualización como se muestra en la figura 4.10, tanto mayor conforme aumenta la potencia óptica o la longitud total del enlace.

En el caso de subportadoras de milimétricas también se mejoran notablemente los resultados. Por ejemplo, en la figura 4.11 se representa la penalización de potencia ecualizada en función de la longitud de fibra para 


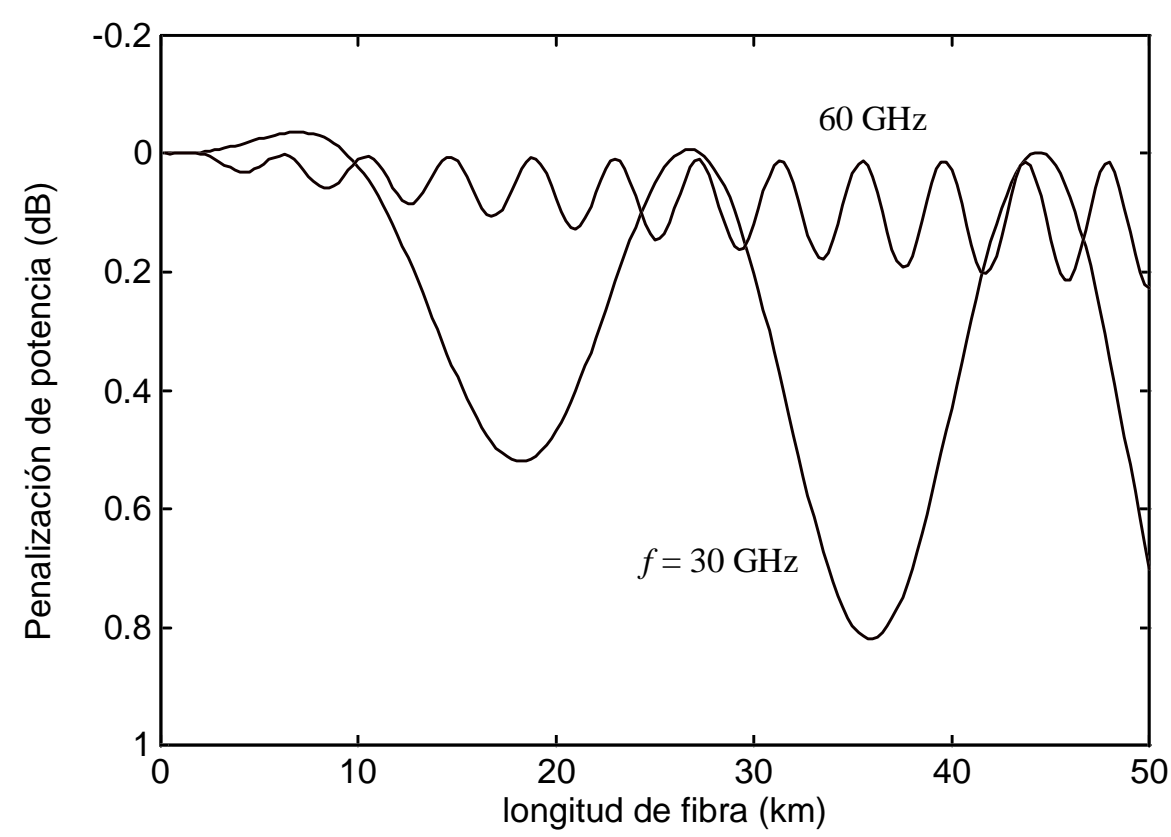

Figura 4.11 Penalización de potencia ecualizada mediante OPC para dos subportadoras de milimétricas de frecuencias 30 y 60 $\mathrm{GHz}$. Los parámetros de modulación son idénticos a los de la figura anterior.

unas frecuencias de subportadora de 30 y $60 \mathrm{GHz}$. Compárense estas curvas con las de la figura 2.5. El punto de penalización $1 \mathrm{~dB}$ se encuentra con ecualización por encima de los $50 \mathrm{~km}$ frente a menos de $2 \mathrm{~km}$ en el caso de no emplear ecualización. Al igual que ocurría en la figura 4.10, los nulos de las curvas de penalización desaparecen y se genera un cierto rizadoiii. Obsérvese como fenómeno curioso que la penalización en media es mayor para la subportadora de menor frecuencia. Esto es debido al propio rizado con la frecuencia que tienen las curvas ecualizadas.

En las figuras 4.12 y 4.13 se representa la penalización de potencia en función de la potencia óptica transmitida con y sin ecualización para dos longitudes de fibra: 50 y $75 \mathrm{~km}$, y dos frecuencias de modulación: 5 y 10 $\mathrm{GHz}$, respectivamente. En el primer caso (figura 4.12) la frecuencia se encuentra por debajo del primer nulo de GVD. Como se puede observar,

\footnotetext{
ii véase apéndice I, donde se analiza la respuesta en frecuencia de un sistema que emplea la técnica de inversión espectral

iii aunque en este caso en función de la longitud de fibra
} 
aumentando la potencia óptica se obtienen mejores resultados, pues la penalización de potencia disminuye. Existe un punto situado en torno a los +7 dBm a partir del cual se consiguen mejores resultados sin ecualizar, ya que el efecto del SPM induce una cierta ganancia sobre el enlace de fibra óptica tal y como se explicó en la sección 3.2. No obstante, esto es una situación local, ya que en general interesa aplicar la técnica OPC para suavizar las curvas y eliminar los nulos. En el segundo caso (figura 4.13) la frecuencia de modulación se encuentra por encima del primer nulo de GVD. De este modo, al aumentar la potencia óptica el nulo se desplaza hacia frecuencias mayores y llega un momento en el cual coincide a la frecuencia de $10 \mathrm{GHz}$, obteniéndose un máximo en la penalización de potencia para unas potencias ópticas de $+8 \mathrm{dBm}$ para $50 \mathrm{~km}$ y $+12 \mathrm{dBm}$ para $75 \mathrm{~km}$ de fibra. Empleando OPC se consigue pues una mejora considerable.

Finalmente, los resultados de ecualización de la distorsión armónica de la figura 2.3 en función de la frecuencia de modulación se representan en la

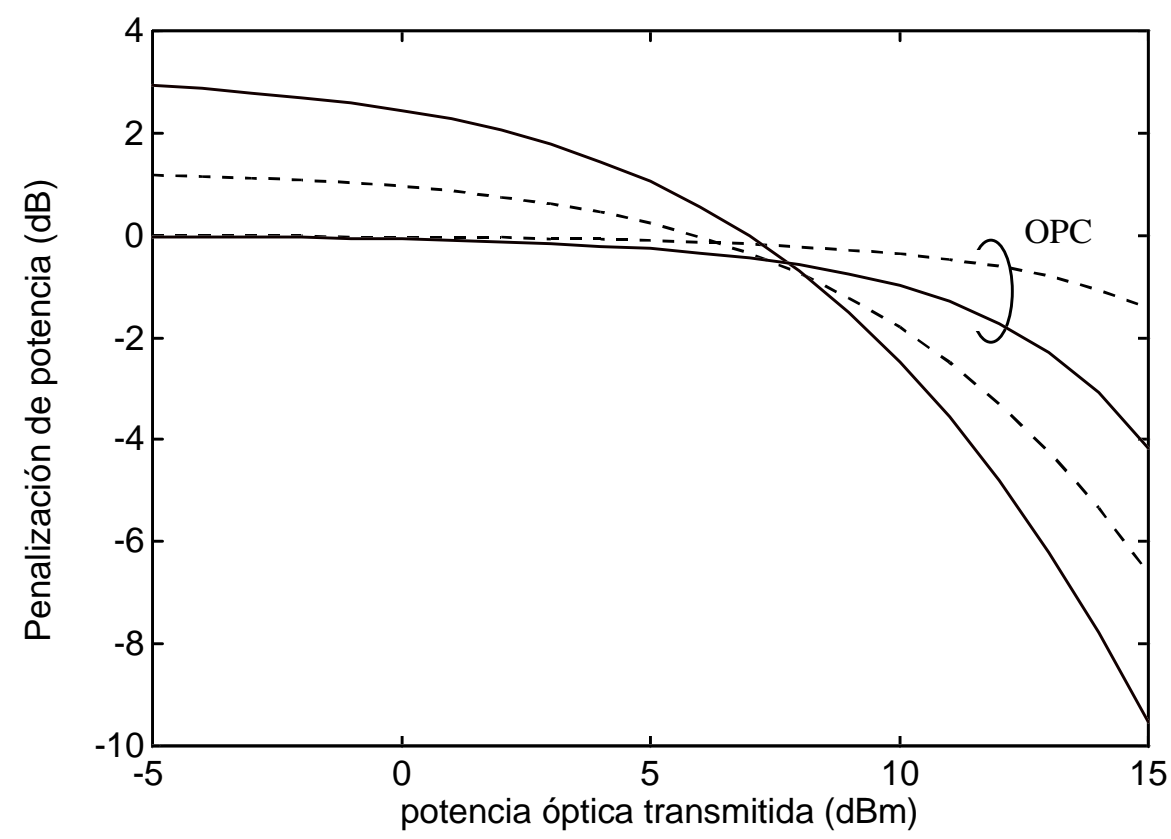

Figura 4.12 Penalización de potencia con y sin ecualización en función de la potencia óptica transmitida. Las curvas continuas son para una longitud de fibra de $75 \mathrm{~km}$, mientras que las de trazos son para $50 \mathrm{~km}$. Los parámetros de modulación son: $\mathrm{f}_{\mathrm{m}}=5$ $\mathrm{GHz}, \mathrm{m}_{\mathrm{i}}=0,04$ y $\vartheta=0$. 


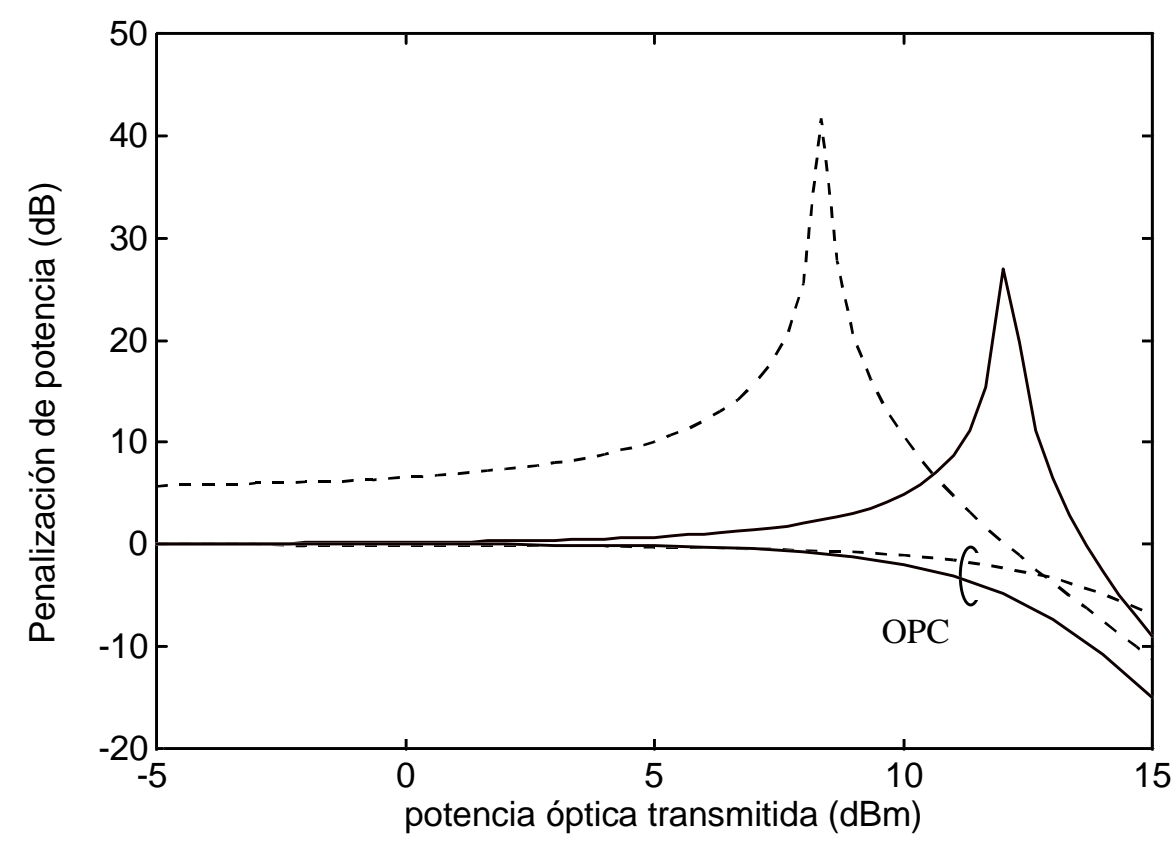

Figura 4.13 Penalización de potencia con y sin ecualización en función de la potencia óptica transmitida. Las curvas continuas son para una longitud de fibra de $75 \mathrm{~km}$, mientras que las de trazos son para $50 \mathrm{~km}$. Los parámetros de modulación son: $\mathrm{f}_{\mathrm{m}}=10$ $\mathrm{GHz}, \mathrm{m}_{\mathrm{i}}=0,04$ y $\vartheta=0$.

figura 4.14. En este caso, se deduce que la mejora obtenida en la distorsión de segundo orden es muy superior a la de tercer orden. Unos $40 \mathrm{~dB}$ en el término $A_{2} / A_{1}$ frente a tan sólo unos $10 \mathrm{~dB}$ para el término $A_{3} / A_{1}$. En cualquier caso, los niveles de la distorsión de orden tres son suficientemente pequeños. Fijando como límite un valor de $-60 \mathrm{dBc}$ para $A_{2} / A_{1}$, se consigue extender la frecuencia máxima de modulación desde los $3 \mathrm{GHz}$ hasta valores por encima de $10 \mathrm{GHz}$ empleando ecualización.

\subsubsection{Compensación de distorsión no lineal en sistemas radio-fibra}

En la sección 2.6 se obtuvieron resultados de distorsión no lineal introducida por la fibra óptica en sistemas radio sobre fibra típicos. Ahora que se ha estudiado la técnica OPC para compensar los efectos de la dispersión cromática y del SPM, es el momento de aplicarla en estos sistemas. En las simulaciones, los parámetros de cada sistema se considerarán idénticos a los 


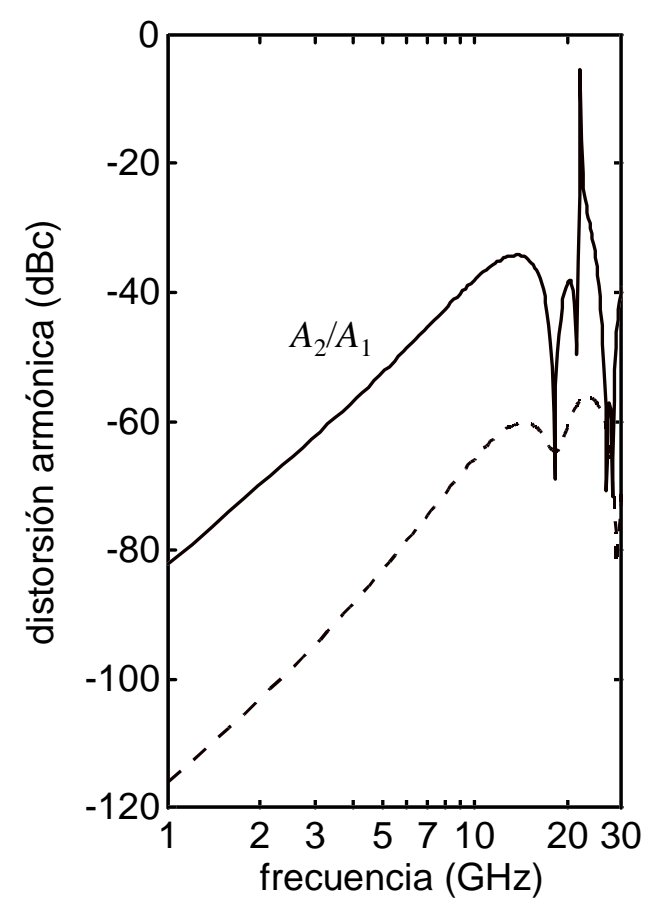

(a)

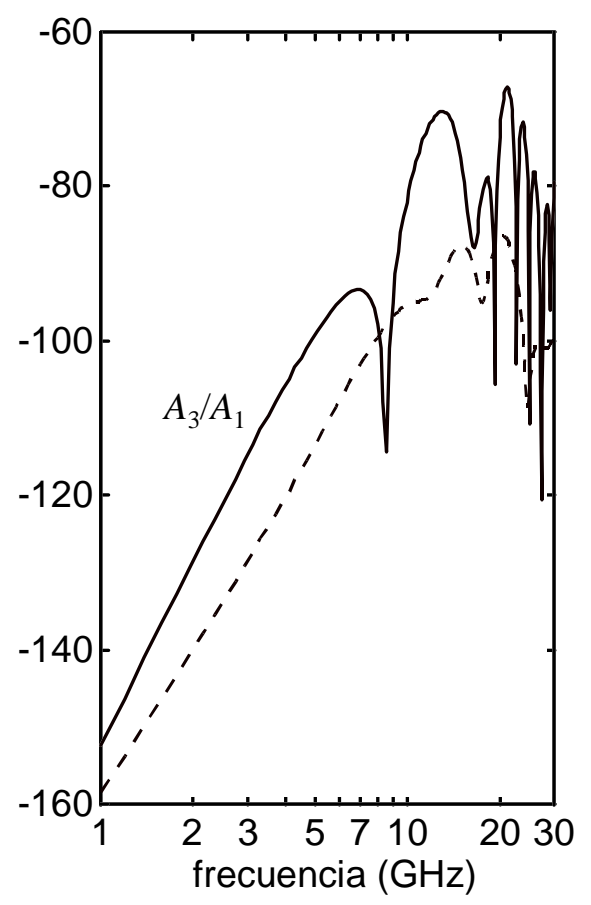

(b)

Figura 4.14 Distorsión armónica en función de la frecuencia de modulación con y sin ecualización: (a) distorsión de segundo orden y (b) distorsión de tercer orden. Las curvas de trazos representan la ecualización mediante OPC para una longitud de fibra de $10 \mathrm{~km}$. Los parámetros de modulación son: $m_{i}=0,04, \vartheta=10 \mathrm{GHz}$ y $\mathrm{P}_{0}=20 \mathrm{~mW}$.

de la sección 2.6 y para realizar la ecualización tomaremos la configuración básica de OPC con $L_{1}=L_{2}=L / 2$ (MSSI) y tramos de fibra idénticos.

Para empezar, en la figura 4.15 se muestran los nivel es de CSO del sistema CATV con y sin ecualización mediante OPC en función de la longitud de fibra óptica. Como se puede apreciar, la mejora obtenida en el caso de emplear modulación directa es considerablemente superior debido a que el principal factor limitante lo constituye el SPM y no la combinación del chirp del transmisor con la dispersión de la fibra. Aun así, los niveles finales de CSO ecualizado son unos $10 \mathrm{~dB}$ inferiores en el caso de emplear modulación externa. Fijando el valor límite de $-60 \mathrm{dBc}$, empleando ecualización se consigue incrementar la longitud del enlace de fibra desde los $50 \mathrm{~km}$ hasta distancias por encima de $100 \mathrm{~km}$. 
Por otro lado, los resultados de ecualización del sistema MMDS se representan en la figura 4.16. En este caso sirve lo mismo dicho para el sistema CATV, sin embargo, a diferencia de este último la distorsión viene determinada por el CTB. Como se observa, en este sistema no resulta interesante emplear la técnica OPC con modulación externa por encima de los $30 \mathrm{~km}$, donde predominan las no linealidades. En el caso de modulación directa, sí que se obtienen resultados significativos extendiéndose la longitud máxima de fibra desde los $10 \mathrm{~km}$ hasta los $50 \mathrm{~km}$. Por último, los resultados finales de ecualización difieren en unos $20 \mathrm{~dB}$ en función de la existencia o no de chirp en el transmisor óptico.

También es posible mediante la técnica OPC extender las longitudes de enlace de los sistemas radio sobre fibra que trabajan en la banda de milimétricas. Como ejemplo, en la figura 4.17 se representan los resultados de ecualización de distorsión no lineal y penalización de potencia del sistema LMDS que opera a $28 \mathrm{GHz}$. La reducción que se consigue en los niveles de CTB es significativa, con valores más de 40 dB inferiores. De este



Figura 4.15 CSO con y sin ecualización para el sistema CATV de 70 canales. Las curvas continuas son con modulación directa, mientras que las de trazos con modulación externa $(\vartheta=0)$. Los parámetros del sistema se consideran por defecto. 




Figura 4.16 CTB con y sin ecualización para el sistema MMDS de 33 canales. Las curvas continuas son con modulación directa, mientras que las de trazos con modulación externa $(\vartheta=0)$. Los parámetros del sistema se consideran por defecto.

modo, la longitud de fibra puede aumentar desde los $2 \mathrm{~km}$ hasta los $40 \mathrm{~km}$. Obsérvese que para distancias inferiores a los $5 \mathrm{~km}$ el CTB se mantiene por debajo de $-100 \mathrm{dBc}$.

En cuanto a la penalización de potencia, los resultados obtenidos con ecualización son también muy satisfactorios como muestra la figura 4.17(b). La técnica OPC elimina completamente los nulos de potencia y suaviza la respuesta, alejando el punto de penalización $1 \mathrm{~dB}$ hasta distancias por encima de los $50 \mathrm{~km}$ frente a los $2 \mathrm{~km}$ que se conseguían sin ecualizar. Este es real mente el punto fuerte de la técnica OPC, ya que en los sistema LMDS suelen emplearse modulaciones digitales (QPSK, QAM, ...) y los requerimientos de CTB, al contrario que en los sistemas AM-VSB, no son tan estrictos. En cualquier caso, la técnica OPC permite extender el alcance del sistema LMDS empleando longitudes de fibra de hasta $40 \mathrm{~km}$. Finalmente, los resultados de distorsión no lineal y penalización de potencia del sistema MVDS que opera a $42 \mathrm{GHz}$ aparecen en la figura 4.18. Al igual que se dijo anteriormente, la técnica OPC produce una mejora considerable 
tanto en el caso del CTB como de la penalización de potencia. La longitud máxima de fibra es claramente superior a los $20 \mathrm{~km}$ frente a tan sólo $1 \mathrm{~km}$ que se consigue sin ecualización.

En la tabla I se muestran, a modo de resumen, los resultados de ecualización de CIR y de penalización de potencia en los distintos sistemas radio-fibra y para determinados valores de longitud de fibra donde cada uno de los sistemas puede operar correctamente. Destacan en particular los 0,82 dB de penalización de potencia para $40 \mathrm{~km}$ de fibra en el sistema LMDS y los 0,12 dB para $20 \mathrm{~km}$ de fibra en el sistema MVDS.

TABLA I Resultados de ecualización de CIR y penalización de potencia mediante OPC en sistemas radio-fibra típicos.

\begin{tabular}{|l|c|c|c|c|}
\hline Sistema & $\begin{array}{c}\text { Lenlace } \\
(\mathbf{k m})\end{array}$ & $\begin{array}{c}\text { CIR modulación } \\
\text { directa (dB) }\end{array}$ & $\begin{array}{c}\text { CIR modulación } \\
\text { externa (dB) }\end{array}$ & $\begin{array}{c}\text { Penalización } \\
(\mathbf{d B})\end{array}$ \\
\hline CATV & 100 & 56,6 & 66,7 & - \\
\hline MMDS & 50 & 65,8 & 83,8 & - \\
\hline LMDS & 40 & - & 59,5 & 0,82 \\
\hline MVDS & 20 & - & 73,2 & 0,12 \\
\hline
\end{tabular}




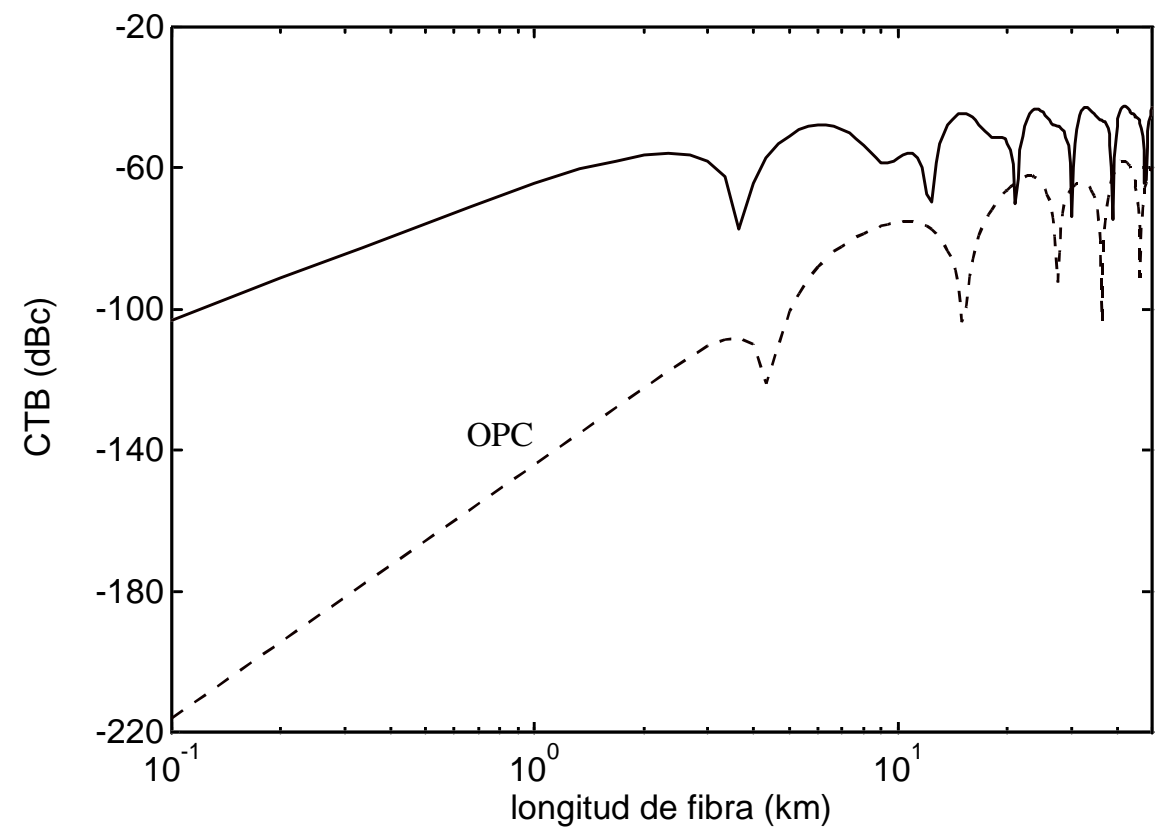

(a)

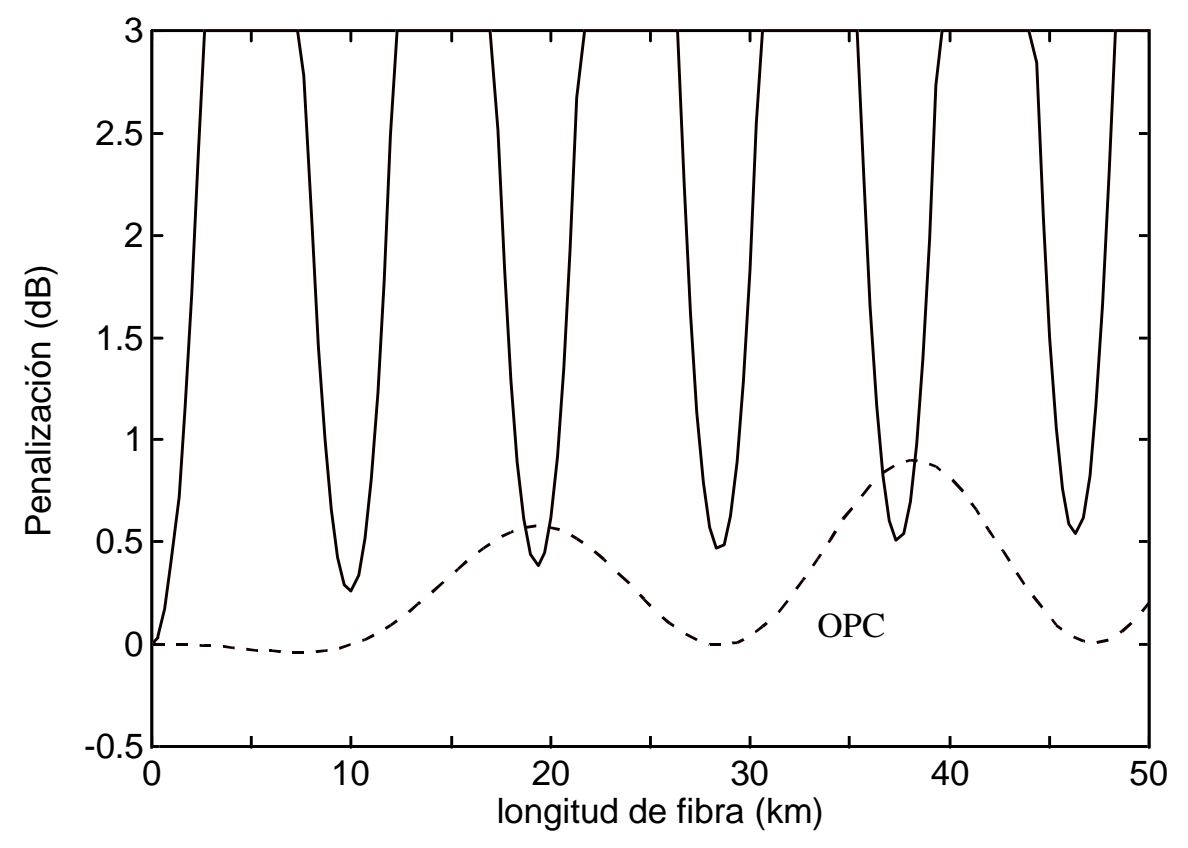

(b)

Figura 4.17 Ecualización de (a) CTB y (b) penalización de potencia del sistema LMDS de 40 canales. Los parámetros del sistema se consideran por defecto. 


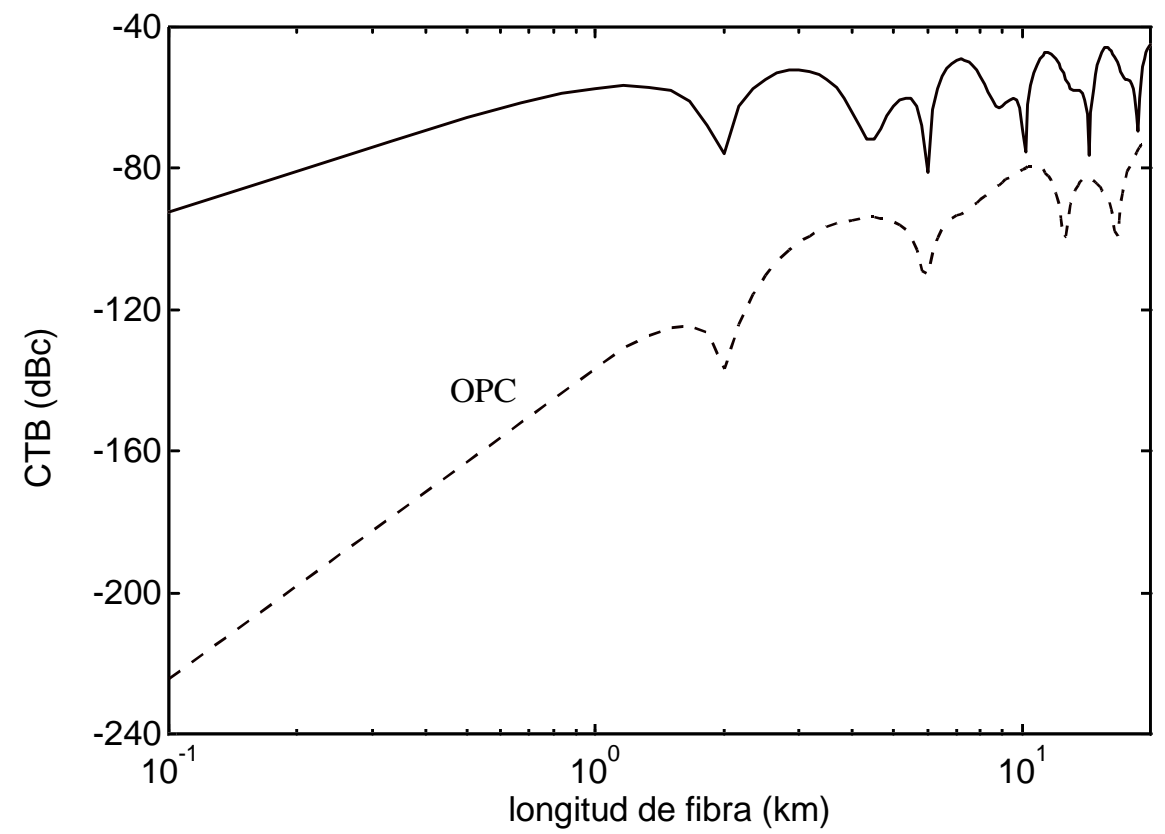

(a)

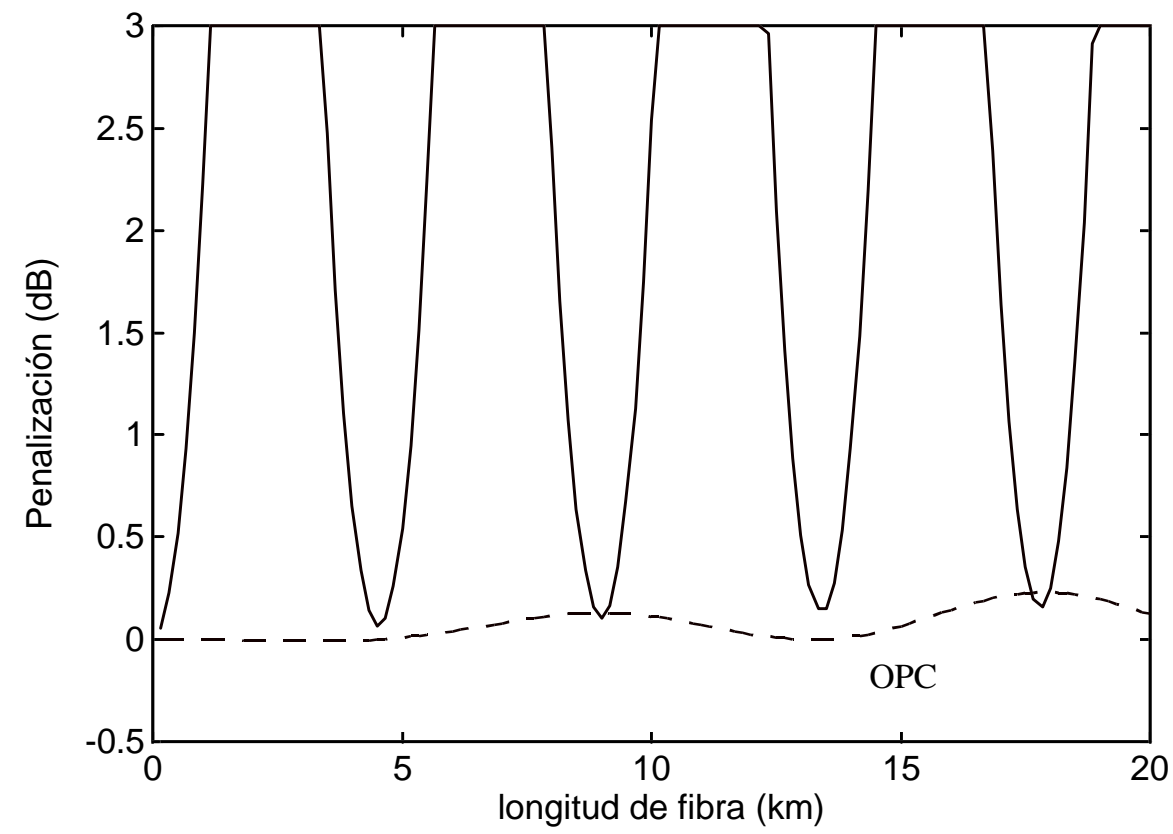

(b)

Figura 4.18 Ecualización de (a) CTB y (b) penalización de potencia del sistema MVDS de 30 canales. Los parámetros del sistema se consideran por defecto. 


\subsection{CONSIDERACIONES ADICIONALES SOBRE LA TÉCNICA OPC}

A continuación se analizarán algunos aspectos adicionales de la técnica de inversión espectral que no han sido considerados hasta el momento. En primer lugar, se realizará un estudio de sensibilidad de los resultados de ecualización frente a diversos parámetros del sistema, como pueden ser la longitud de fibra, la potencia óptica o la dispersión cromática. Posteriormente, se estudiará la posibilidad de compensar dispersión y SPM de una forma exacta. Finalmente, se analizará la influencia del parámetro de dispersión de segundo orden en los resultados de ecualización.

\subsubsection{Sensibilidad de la técnica OPC}

En el apartado 4.3.1 ya se comentó que una de las principales ventajas de la técnica OPC era su reducida sensibilidad frente a variaciones en la longitud óptima de eculización. A continuación, realizaremos un estudio más

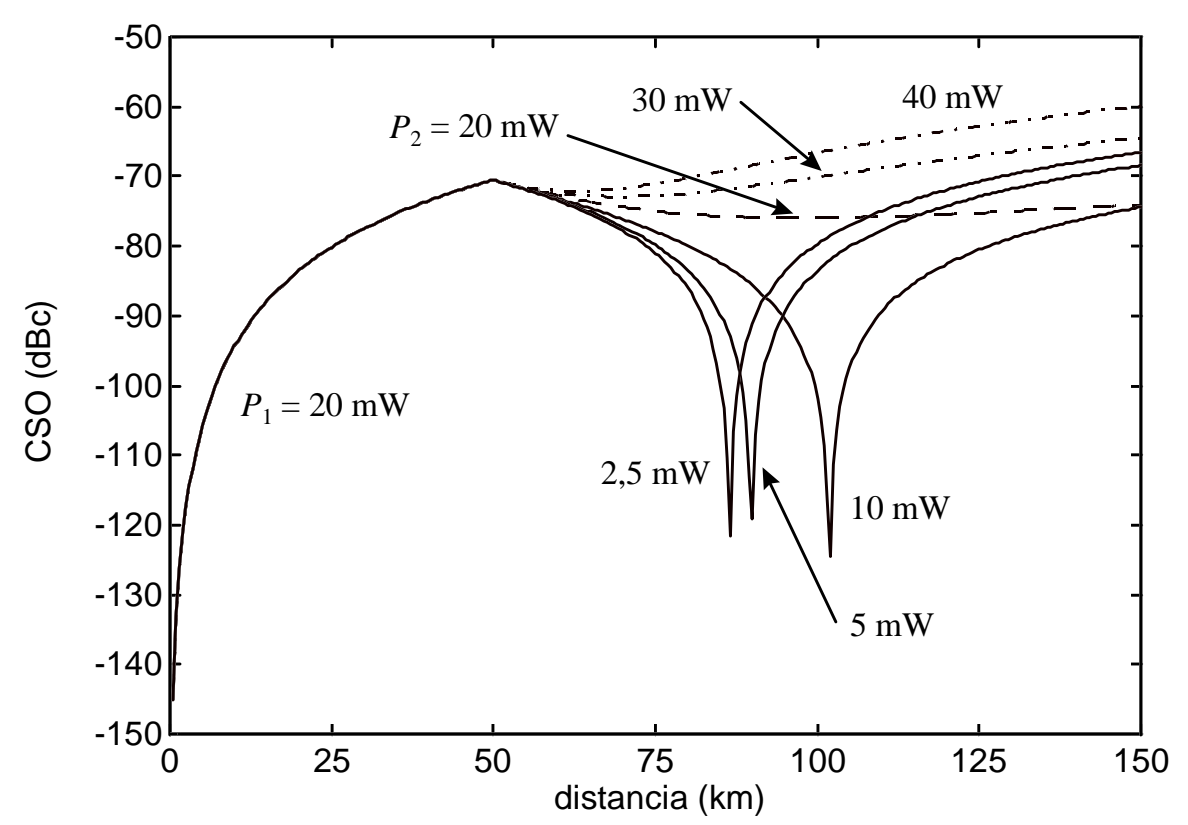

Figura 4.19 CSO en función de la distancia y para varios valores de potencia óptica a la salida del dispositivo OPC. Otros parámetros del sistema son: $\mathrm{f}_{\mathrm{m}}=500 \mathrm{MHz}, \mathrm{m}_{\mathrm{i}}=0,04, \vartheta=0$ y $\mathrm{L}_{1}=$ $50 \mathrm{~km}$. 


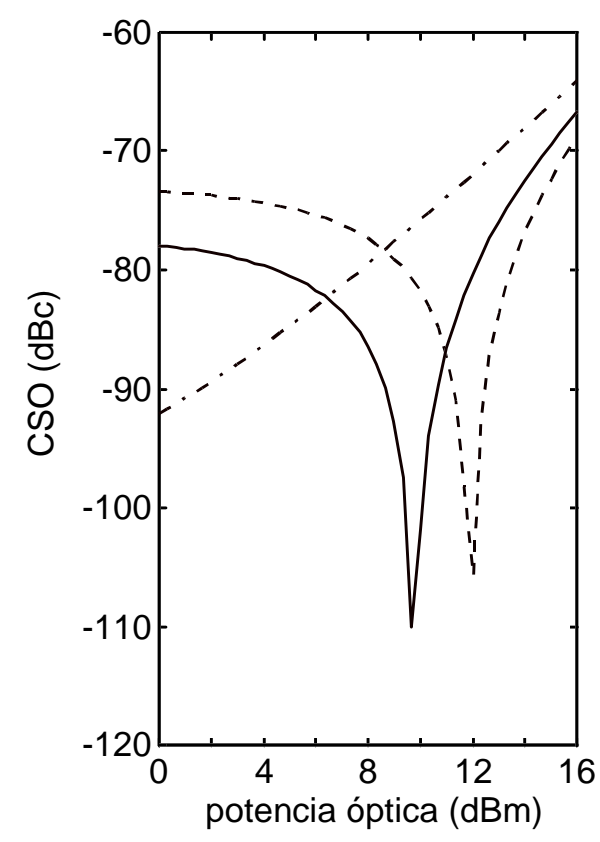

(a)

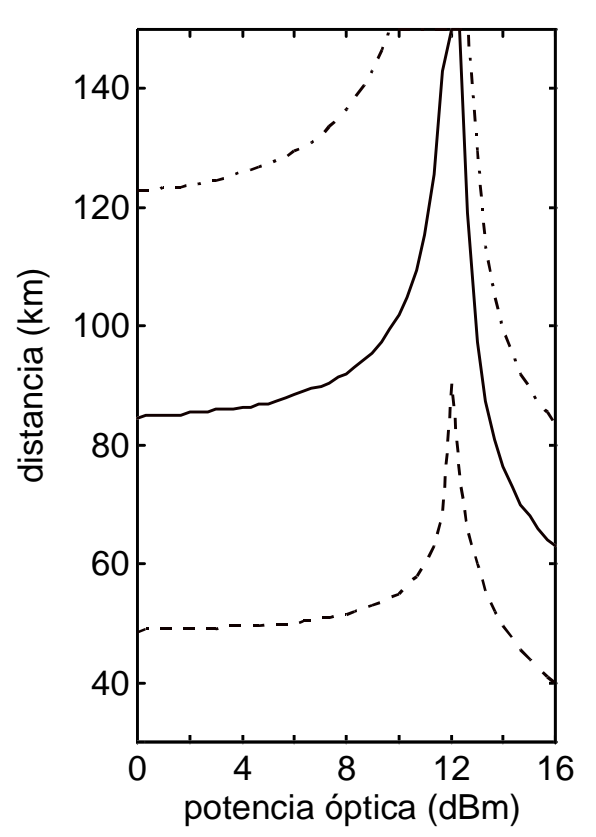

(b)

Figura 4.20 (a) CSO para una distancia de $100 \mathrm{~km}$ y (b) distancia a la cual se produce un mínimo de distorsión en función de la potencia óptica a la salida del conjugador. Los resultados están parametrizados en función de la posición del OPC: $L_{1}=30$ $\mathrm{km}$ (trazos), $\mathrm{L}_{1}=50 \mathrm{~km}$ (continua) y $\mathrm{L}_{1}=70 \mathrm{~km}$ (trazos y puntos).

compl eto sobre la influencia que tienen diversos parámetros del sistema en los resultados de ecualización. Estos parámetros incluyen, por ejemplo, la longitud de los tramos de fibra, la potencia óptica a la salida del conjugador, o la separación entre las longitudes de onda de señal y de bombeo.

En la figura 4.19 se representan los niveles de CSO en función de la distancia para un sistema que emplea la técnica OPC. Los resultados se han obtenido para distintos valores de potencia óptica a la entrada del segundo trayecto de fibra tras el bloque OPC. Como se puede observar, para una potencia óptica $\mathrm{P}_{2}=20 \mathrm{~mW}$ (curva de trazos), idéntica a la del transmisor, se consigue un mínimo de distorsión para una distancia $L=2 L_{1}=100 \mathrm{~km}$ (MSSI). Si por el contrario se aumenta la potencia óptica (curvas de trazos y puntos), los niveles de CSO son superiores. No obstante, en el caso de emplear potencias ópticas menores (curvas continuas) es posible obtener nulos de distorsión y mejorar considerablemente los resultados de 
ecualización con respecto a la situación estándar $\mathrm{P}_{1}=\mathrm{P}_{2}$. Obsérvese que para un valor de potencia óptica $\mathrm{P}_{2}=10 \mathrm{~mW}$ se consigue un nulo cerca de los $100 \mathrm{~km}$. Conforme la potencia óptica disminuye, este nulo se desplaza hacia distancias menores.

Estos efectos se muestran en las figuras 4.20 (a) y (b), donde se representan el nivel de CSO ecualizado para $100 \mathrm{~km}$ y la distancia donde ocurre el mínimo de distorsión, respectivamente, para distintas posiciones del conjugador óptico: $L_{1}=30,50$ ó $70 \mathrm{~km}$. De la figura 4.20(a) se deduce que, en el caso de $L_{1}=70 \mathrm{~km}$, no es posible obtener un nulo de distorsión para $100 \mathrm{~km}$. En cambio, con $L_{1}=50 \mathrm{~km}$ se obtiene un nulo para una potencia óptica de $+9,7 \mathrm{dBm}$ y con $\mathrm{L}_{1}=30 \mathrm{~km}$ para una potencia de $+12 \mathrm{dBm}$. Luego se deduce que emplear potencias $\mathrm{P}_{2}<\mathrm{P}_{1}$ produce en general mejores resultados.

Por otro lado, en la figura 4.20(b) se observa que el nulo con $L_{1}=70 \mathrm{~km}$ se consigue para distancias superiores a los $120 \mathrm{~km}$. No obstante, existe un punto de inflexión a partir del cual aumentando la potencia óptica el mínimo se desplaza hacia distancias menores. Este fenómeno es el representado en la figura 4.19 por las potencias ópticas de 30 y $40 \mathrm{~mW}$, el cual no es deseable debido a que los niveles de distorsión son superiores. A grandes rasgos, para potencias ópticas inferiores al punto de inflexión, la ecualización se produce principalmente por efectos dispersivos, mientras que para potencias por encima de este punto se combinan dispersión y no linealidades. En el primer caso es posible obtener incluso un nulo de distorsión, lo cual proporciona resultados claramente mejores. Para $\mathrm{P}_{1}=\mathrm{P}_{2}$ $=+13 \mathrm{dBm}$ nos encontramos a la derecha de este punto de inflexión y, aunque se produce un mínimo para $100 \mathrm{~km}$ en el caso de $L_{1}=50 \mathrm{~km}$, los resultados no son tan buenos como empleando una potencia óptica de +10 $\mathrm{dBm}$. En cualquier caso, debe tenerse en cuenta que la situación óptima no se mantiene para cualquier frecuencia. De este modo, un nulo en el CSO no lleva consigo igualmente un mínimo en el CTB o en la penalización de potencia. Para la frecuencia de modulación utilizada $\left(f_{m}=500 \mathrm{MHz}\right)$, este nulo se produce para una potencia óptica de $+9,7 \mathrm{dBm}$, pero si cambiamos la 
frecuencia a $600 \mathrm{MHz}$, el nulo se consigue para una potencia óptica distinta. Sin embargo, empleando $\mathrm{P}_{1}=\mathrm{P}_{2}$ sí que se obtiene siempre un mínimo de distorsión para una distancia $L=2 L_{1}$ (MSSI), independientemente de la frecuencia de modulación utilizada. Además, los resultados ecualizados también son menos sensibles a variaciones en la distancia óptima de ecualización (figura 4.9). Esto se debe a que la técnica compensa realmente la distorsión no lineal, de acuerdo con la teoría de la sección 4.2, mientras que en el otro caso se anula el CSO sólo para determinadas frecuencias de modulación. Posteriormente veremos en el dominio frecuencial cómo son mejores, en general, los resultados obtenidos con $\mathrm{P}_{1}=\mathrm{P}_{2}$.

EI proceso no lineal de FWM que ocurre en el conjugador óptico lleva consigo un cambio de la longitud de onda de la señal de entrada de acuerdo a $\lambda_{C}=$ $2 \lambda_{P}-\lambda_{s}$. Aunque se han propuesto configuraciones de OPC donde no se produce desplazamiento en longitud de onda [Ino97, Cor99], no suele ser el caso habitual, por lo que habrá que estudiar la influencia que tiene este efecto en los resultados de ecualización. Téngase en cuenta que los parámetros de la fibra óptica tienen una dependencia con la longitud de onda de la señal, en particular la dispersión cromática.

Considerando la separación entre las longitudes de onda de la señal de entrada al dispositivo OPC y su conjugada, $\Delta \lambda=\lambda_{S}-\lambda_{C}$, los parámetros de dispersión de cada una de las fibras están relacionados mediante:

$$
\mathrm{D}_{2}=\mathrm{D}\left(\lambda_{\mathrm{C}}\right)=\mathrm{D}\left(\lambda_{\mathrm{S}}\right)-\left.\frac{\mathrm{dD}}{\mathrm{d} \lambda}\right|_{\lambda_{\mathrm{P}}} \Delta \lambda=\mathrm{D}_{1}-\mathrm{S} \Delta \lambda
$$

donde en el caso de fibras estándar se tiene $\mathrm{S}=0,06 \mathrm{ps} /\left(\mathrm{km} \cdot \mathrm{nm} \mathrm{m}^{2}\right)$.

En la figura 4.21 se representa la respuesta en frecuencia del sistema OPC para varios valores de potencia óptica y con distintas separaciones $\Delta \lambda$. Las curvas continuas son para la situación ideal $\Delta \lambda=0$, mientras que las de trazos son para $\Delta \lambda=2 \mathrm{~nm}$ y las de trazos y puntos para $\Delta \lambda=-2 \mathrm{~nm}$. Obsérvese que para GVD coinciden estos dos últimos casos, como es lógico, mientras que para GVD + SPM se reciben potencias ligeramente mayores 


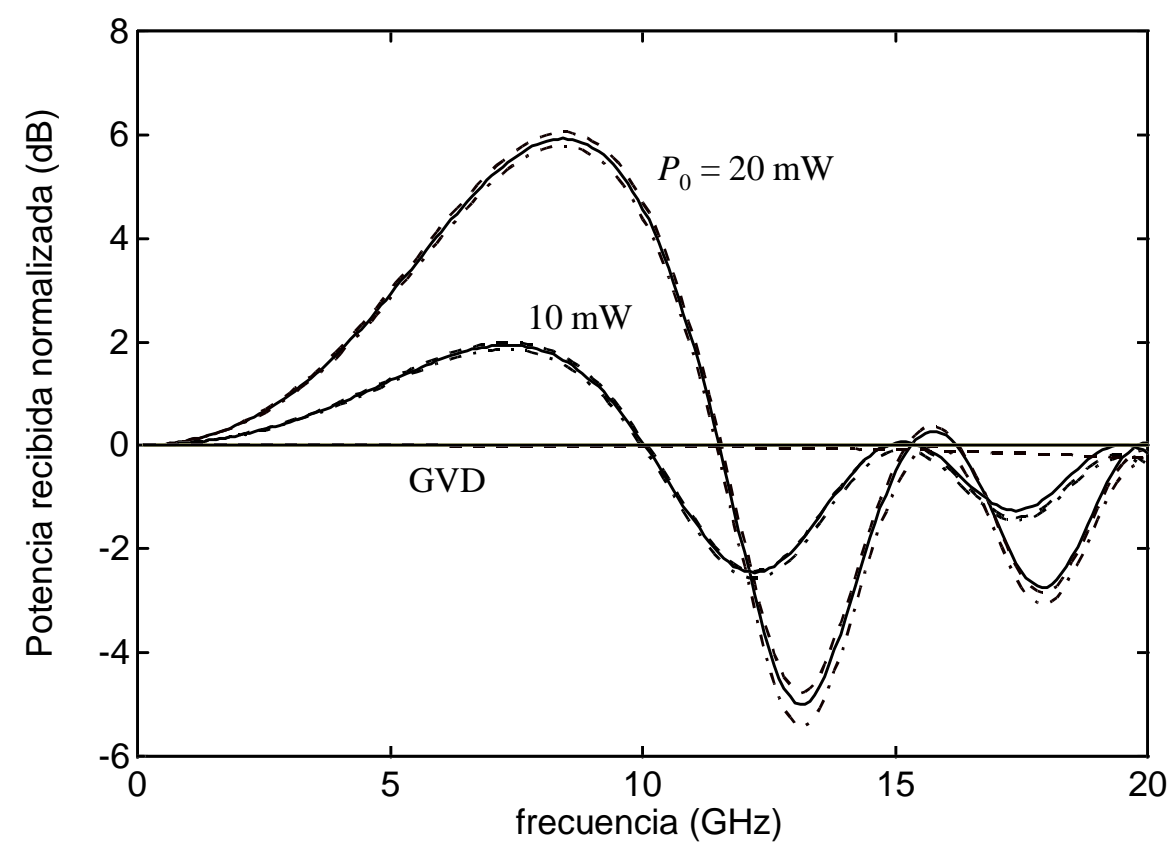

Figura 4.21 Respuesta del sistema OPC en función de $\Delta \lambda$ y para varias potencias ópticas. Las curvas continuas son para $\Delta \lambda=0$, mientras que las de trazos son para $\Delta \lambda=2 \mathrm{~nm}$ y las de trazos y puntos para $\Delta \lambda=-2 \mathrm{~nm}$. Otros parámetros son: $\mathrm{D}\left(\lambda_{\mathrm{P}}\right)=17$ $\mathrm{ps} /(\mathrm{km} \cdot \mathrm{nm}), \mathrm{S}=0,06 \mathrm{ps} /\left(\mathrm{km} \cdot \mathrm{nm}^{2}\right)$ y $\mathrm{L}=100 \mathrm{~km}$.

con $\Delta \lambda>0$. De todas formas, para una separación entre portadoras ópticas de $\pm 2 \mathrm{~nm}$ (valor típico), el efecto sobre la ecualización es prácticamente nulo. En el caso GVD es posible obtener una expresión cerrada para caracterizar el empeoramiento que se produce en los resultados de ecualización como consecuencia de este efecto.

La respuesta en frecuencia de un sistema OPC viene dada poriv

$$
H_{O P C}\left(\omega_{e}\right)=C_{I M-I M}^{(1)}\left(L_{1}, \omega_{e}\right) C_{I M-I M}^{(2)}\left(L_{2}, \omega_{e}\right)-\frac{P_{2}}{P_{1}} C_{I M-P M}^{(1)}\left(L_{1}, \omega_{e}\right) C_{P M-I M}^{(2)}\left(L_{2}, \omega_{e}\right),
$$

donde $\mathrm{C}_{\mathrm{x}}^{(\mathrm{j})}$ son las funciones de conversión fase-intensidad de la fibra óptica SMFj y se ha supuesto que no introduce chirp el transmisor óptico.

\footnotetext{
iv para un análisis más detallado, consúltese el apéndice I
} 
Suponiendo que $P_{2}=P_{1}$ y el SPM es despreciable, las funciones de conversión de la fibra óptica vienen dadas por las ecuaciones (I.14)-(I.16), obteniéndose

$$
\begin{aligned}
\mathrm{H}_{\mathrm{OPC}}\left(\omega_{\mathrm{e}}\right) & =\cos \left(\frac{\beta_{21} \omega_{\mathrm{e}}^{2} \mathrm{~L}_{1}}{2}\right) \cos \left(\frac{\beta_{22} \omega_{\mathrm{e}}^{2} \mathrm{~L}_{2}}{2}\right)+\operatorname{sen}\left(\frac{\beta_{21} \omega_{\mathrm{e}}^{2} \mathrm{~L}_{1}}{2}\right) \operatorname{sen}\left(\frac{\beta_{22} \omega_{\mathrm{e}}^{2} \mathrm{~L}_{2}}{2}\right) \\
& =\cos \left[\frac{\left(\beta_{21} \mathrm{~L}_{1}-\beta_{22} \mathrm{~L}_{2}\right) \omega_{\mathrm{e}}^{2}}{2}\right] .
\end{aligned}
$$

En realidad, esta expresión es idéntica a la de un sistema que emplea fibras compensadoras de dispersión. En el caso ideal de que $\beta_{21} \mathrm{~L}_{1}=\beta_{22} \mathrm{~L}_{2}$ (condición de compensación de dispersión), la respuesta del sistema es independiente de la frecuencia.

Como se ha visto, el desplazamiento en longitud de onda introducido por el conjugador conduce a un cambio en el valor de dispersión cromática de la fibra. A partir de las ecuaciones (4.12) y (4.14) se puede llegar a la siguiente expresión del ancho de banda a $-3 \mathrm{~dB}$ del sistema:



Figura 4.22 Ancho de banda a $-3 \mathrm{~dB}$ del sistema OPC en función de $\Delta \lambda$ y para varios valores de longitud del enlace $\left(\lambda_{P}=1550 \mathrm{~nm}\right)$. 


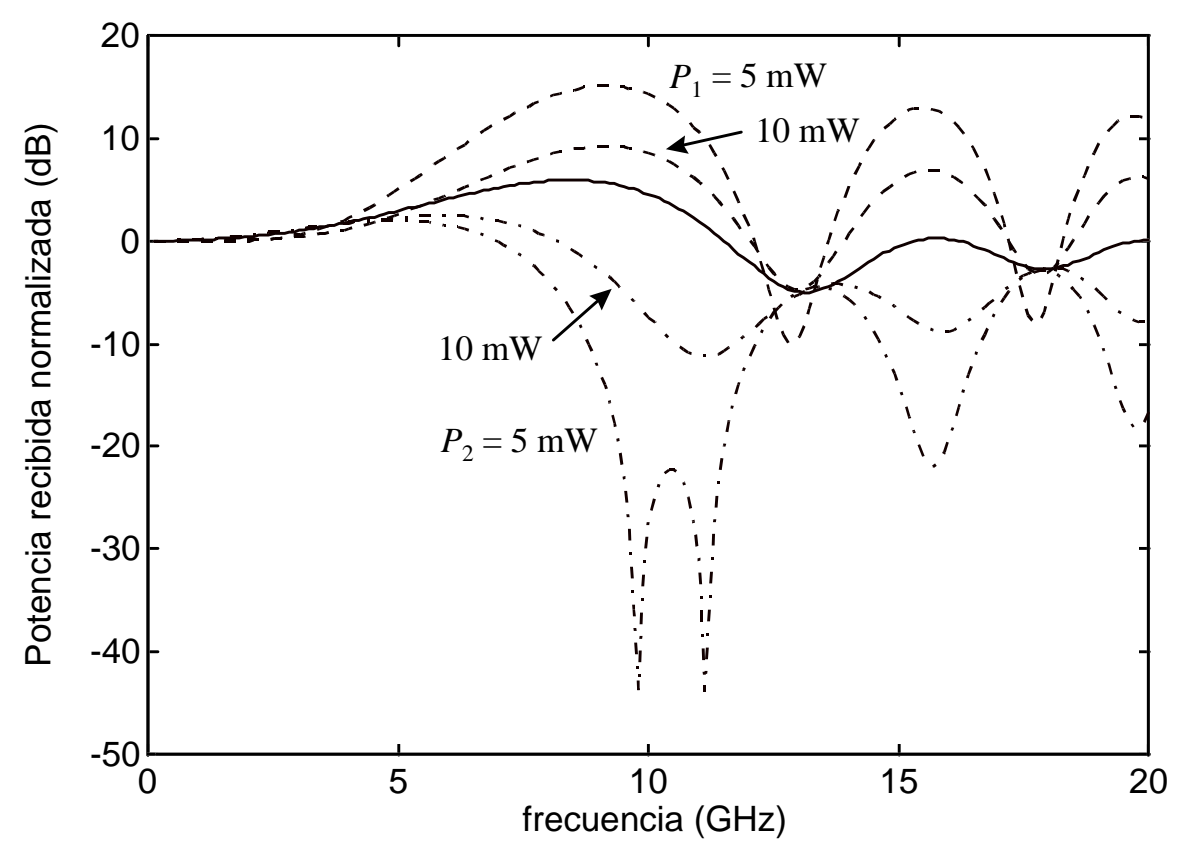

Figura 4.23 Respuesta del sistema OPC para varios valores de potencia óptica y una longitud de enlace de $100 \mathrm{~km}$. La curva continua es para $P_{1}=P_{2}=20 \mathrm{~mW}$, mientras que las de trazos son variando $P_{1}$ y las de trazos y puntos variando $P_{2}$.

$$
f_{-3 \mathrm{~dB}}=\frac{1}{\lambda_{\mathrm{P}} \sqrt{2\left|\frac{\mathrm{S} \Delta \lambda}{\mathrm{C}}\right| \mathrm{L}}}
$$

suponiendo MSSI. En la figura 4.22 se representa el ancho de banda del sistema en función de la separación entre las portadoras ópticas, $\Delta \lambda$, y para algunos valores de longitud de enlace: 50, 100 y 200 km. Como se observa, este efecto no es significativo en sistemas radio-fibra típicos, ya que para valores $\Delta \lambda \sim 1-3 \mathrm{~nm}$ y longitudes de enlace de $100 \mathrm{~km}$ se obtienen anchos de banda superiores a los $60 \mathrm{GHz}$. No obstante, puede influir en el caso de sistemas de comunicaciones ópticas digitales de elevadas velocidades [Ian94], lo cual se resuelve modificando la longitud del segundo trayecto de fibra de acuerdo a

$$
\mathrm{L}_{2}=\left[1+\mathrm{S} \frac{\Delta \lambda}{\mathrm{D}_{2}}\right] \mathrm{L}_{1} .
$$


Uno de los parámetros que tiene gran influencia en la técnica de inversión espectral es la potencia óptica. EI SPM tiene una clara dependencia con ésta y se convierte en la principal limitación en los resultados de ecualización debido a la falta de simetría del efecto Kerr anteriormente comentada. En las figuras 4.19 y 4.20 se presentaron resultados de ecualización de CSO, donde se pudo ver que trabajar con valores de potencia óptica $\mathrm{P}_{2}<\mathrm{P}_{1}$ producía niveles de distorsión inferiores. Sin embargo, la figura 4.23 contradice este hecho. En esta figura se representa la respuesta en frecuencia del sistema OPC para varios valores de potencia óptica a la entrada de cada una de las fibras. La curva continua es para la situación por defecto: $P_{1}=P_{2}=20 \mathrm{~mW}$, mientras que las curvas de trazos son para $P_{2}=20$ $m W$ y variando $P_{1}$, y las de trazos y puntos para $P_{1}=20 \mathrm{~mW}$ y variando $P_{2}$. Se observa claramente que el mejor resultado se produce para $\mathrm{P}_{1}=\mathrm{P}_{2}$, dado que el rizado tiene una amplitud menor. Incluso para una potencia $\mathrm{P}_{1}=5$ mW Ilegan a aparecer nulos en la respuesta del sistema. Luego en esta figura es donde se demuestra realmente que la condición (4.7) de compensación de SPM es la que produce un resultado óptimo en general. EI resto de situaciones pueden conducir a mejores resultados pero sólo para un sistema o parámetro en particular.

Finalmente, la influencia que tiene la variación de la longitud de los tramos de fibra se muestra en la figura 4.24. Las curvas continuas son para $L_{1}=L_{2}$ $=50 \mathrm{~km}$, las de trazos para $\mathrm{L}_{1}=50 \mathrm{~km}$ y $\mathrm{L}_{2}=45 \mathrm{~km}$, y las de trazos $\mathrm{y}$ puntos para $L_{1}=45 \mathrm{~km} \mathrm{y} L_{2}=50 \mathrm{~km}$. Al igual que en la figura anterior, el mejor resultado de ecualización se consigue para $L_{1}=L_{2}=L / 2$ (MSSI). A frecuencias altas, se observa que la pérdida de simetría conduce a una caída en la potencia recibida, debida principalmente a que la dispersión cromática no se compensa completamente. En este caso, la expresión del ancho de banda a $-3 \mathrm{~dB}$ para GVD viene dada por

$$
f_{-3 \mathrm{~dB}}=\frac{1}{\sqrt{8 \pi\left|\beta_{2} \Delta \mathrm{L}\right|}},
$$

siendo $\Delta L=L_{1}-L_{2}$. Para fibras estándar a $1550 \mathrm{~nm}$ se tiene $f_{-3 \mathrm{~dB}} \cdot \Delta \mathrm{L}^{1 / 2}=$ $42,85 \mathrm{GHz} \cdot \mathrm{km}^{1 / 2}$. 


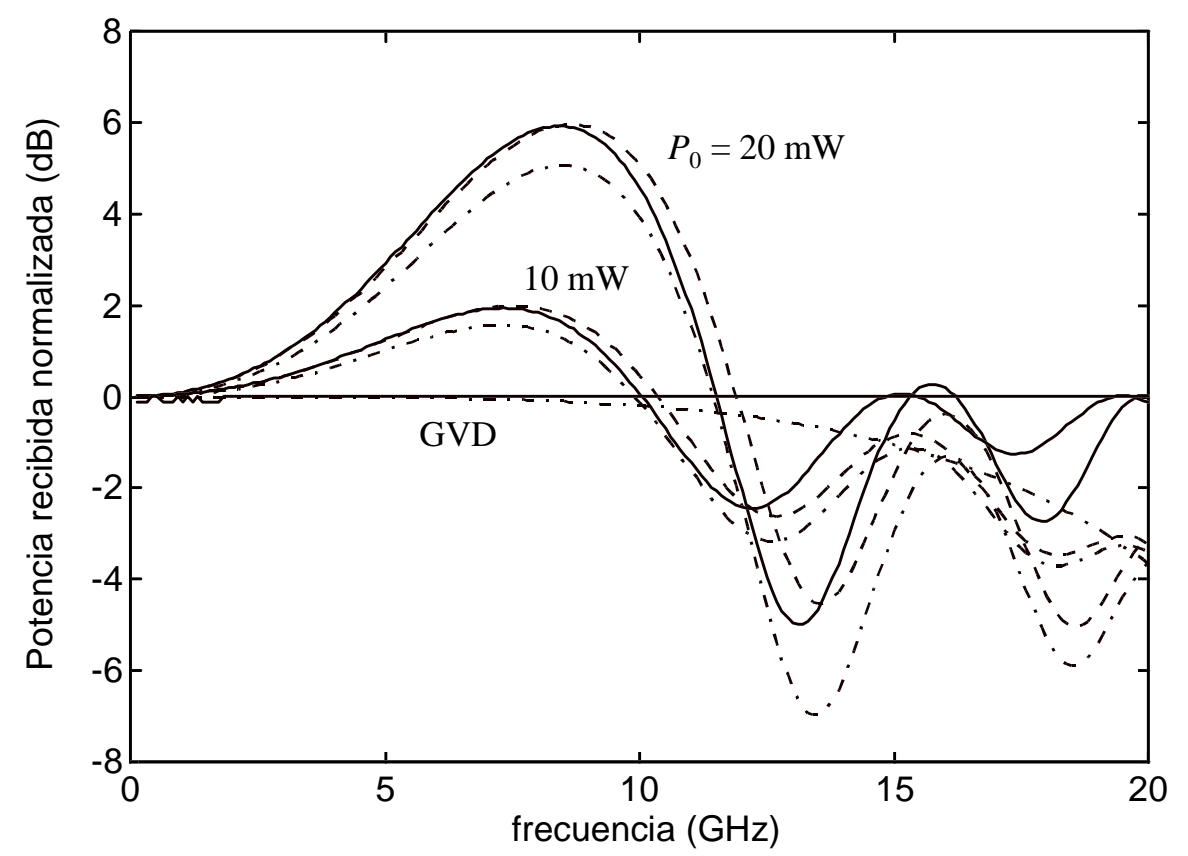

Figura 4.24 Respuesta del sistema OPC con distintas configuraciones de fibra y potencias ópticas. Las curvas continuas son para MSSI $(\mathrm{L}=100 \mathrm{~km})$, mientras que las de trazos son para $\mathrm{L}_{1}=50 \mathrm{~km}$ y $\mathrm{L}_{2}=45 \mathrm{~km}$, y las de trazos y puntos para $\mathrm{L}_{1}=45 \mathrm{~km}$ $y L_{2}=50 \mathrm{~km}$.

En la figura 4.25 se representa la penalización de potencia para una frecuencia de modulación de $10 \mathrm{GHz}$ y en función de la longitud, dispersión cromática o potencia óptica aplicada a la entrada del segundo tramo de fibra. En cierto modo, estas gráficas resumen algunos de los resultados ya vistos con anterioridad. Se observa en la figura 4.25(a) que el punto óptimo de trabajo se encuentra en torno a $P_{2}=P_{1}=10 \mathrm{~mW}$ y $L_{2}=L_{1}=50 \mathrm{~km}$, lo cual es lógico. Conforme disminuye la potencia óptica o aumenta la longitud de fibra, los resultados empeoran. Así, para compensar un aumento en la longitud del enlace de $10 \mathrm{~km}\left(\mathrm{~L}_{2}=60 \mathrm{~km}\right)$, habría que aumentar la potencia óptica a $\mathrm{P}_{2}=12 \mathrm{~mW}$. Se demuestra, pues, que es posible compensar la influencia de determinado parámetro por medio de variaciones en el resto. Esto mismo se puede ver en la figura 4.25(b). El punto óptimo se consigue para $D_{2}=D_{1}=17 \mathrm{ps} /(\mathrm{km} \cdot \mathrm{nm})$ y $\mathrm{L}_{2}=\mathrm{L}_{1}=50 \mathrm{~km}$. Si la dispersión cromática aumenta, será necesario disminuir la longitud del segundo tramo de fibra para mantener la misma dispersión acumulada y viceversa. No obstante, el efecto de la dispersión es menos influyente que el de la potencia óptica. 


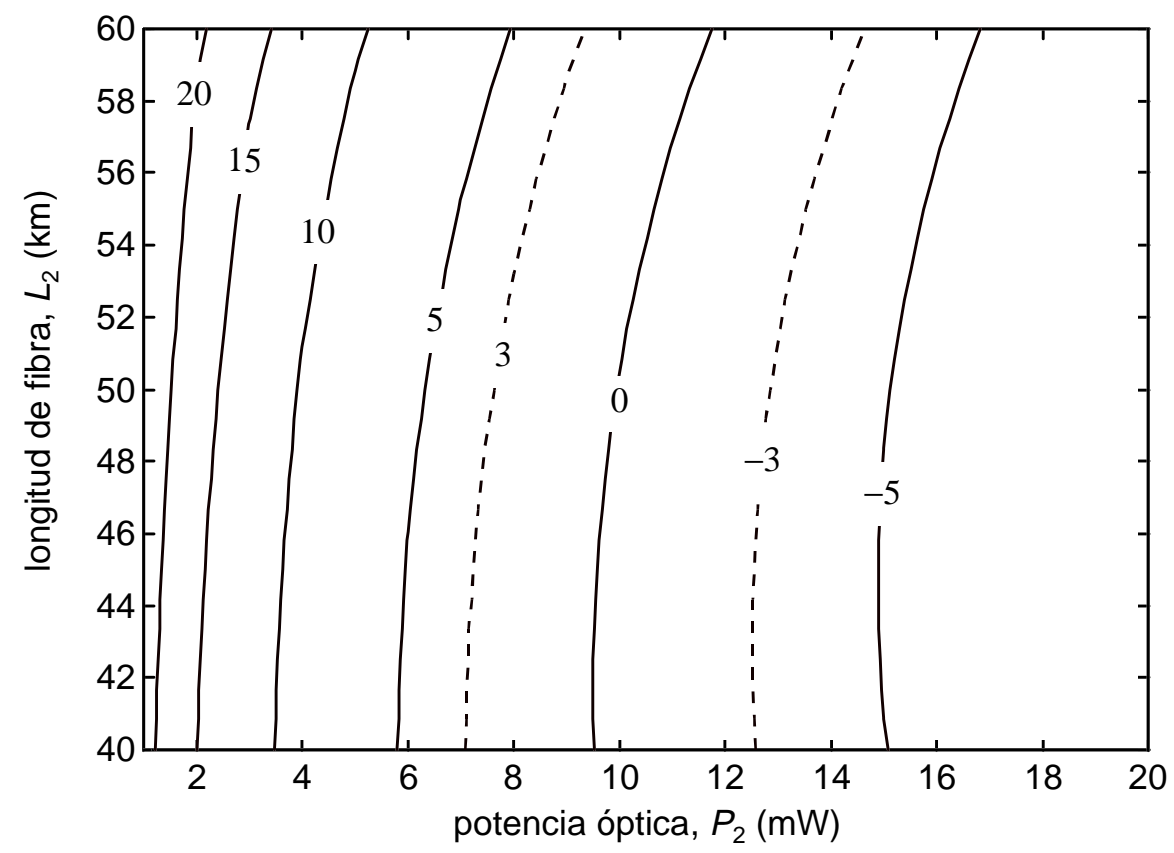

(a)

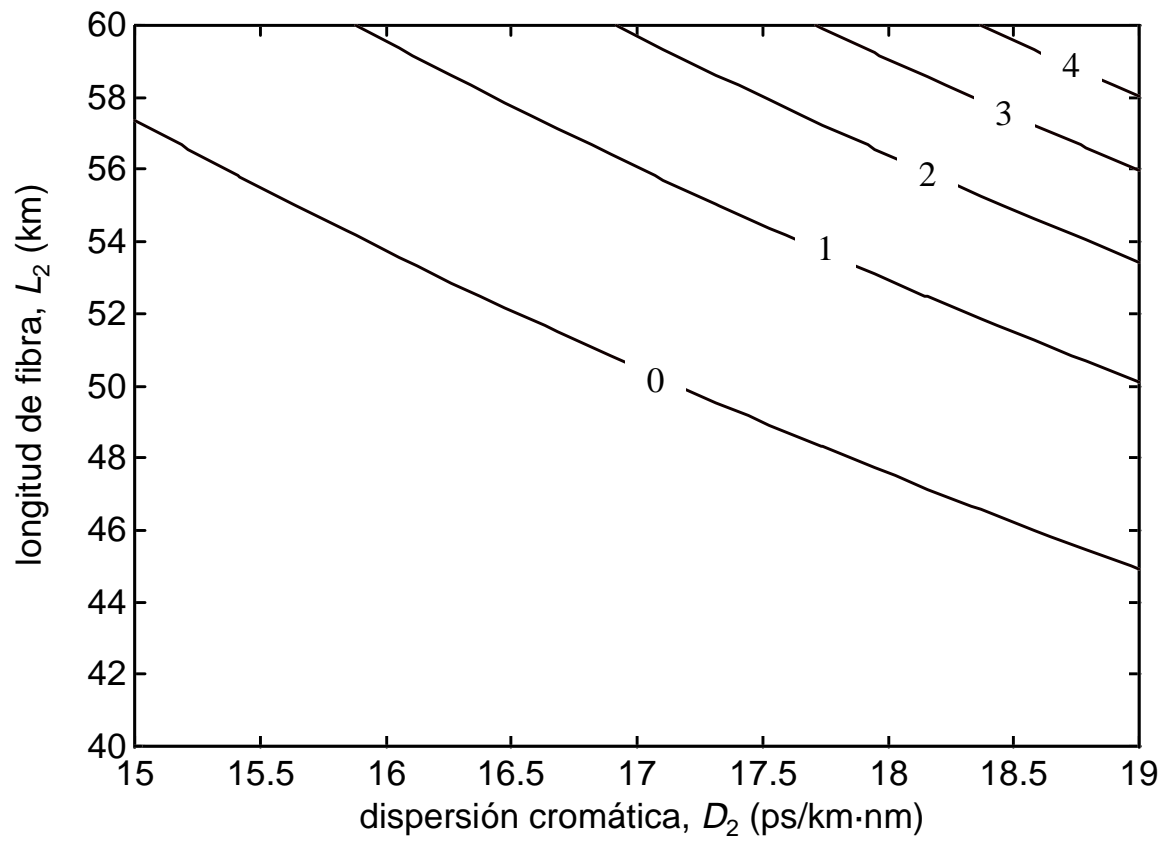

(b)

Figura 4.25 Penalización de potencia en función de la longitud del segundo tramo de fibra, la potencia óptica a la salida del conjugador o la dispersión cromática. Otros parámetros del sistema son: $\mathrm{f}_{\mathrm{m}}=10 \mathrm{GHz}, \mathrm{P}_{1}=10 \mathrm{~mW}$ y $\mathrm{L}_{1}=50 \mathrm{~km}$. 


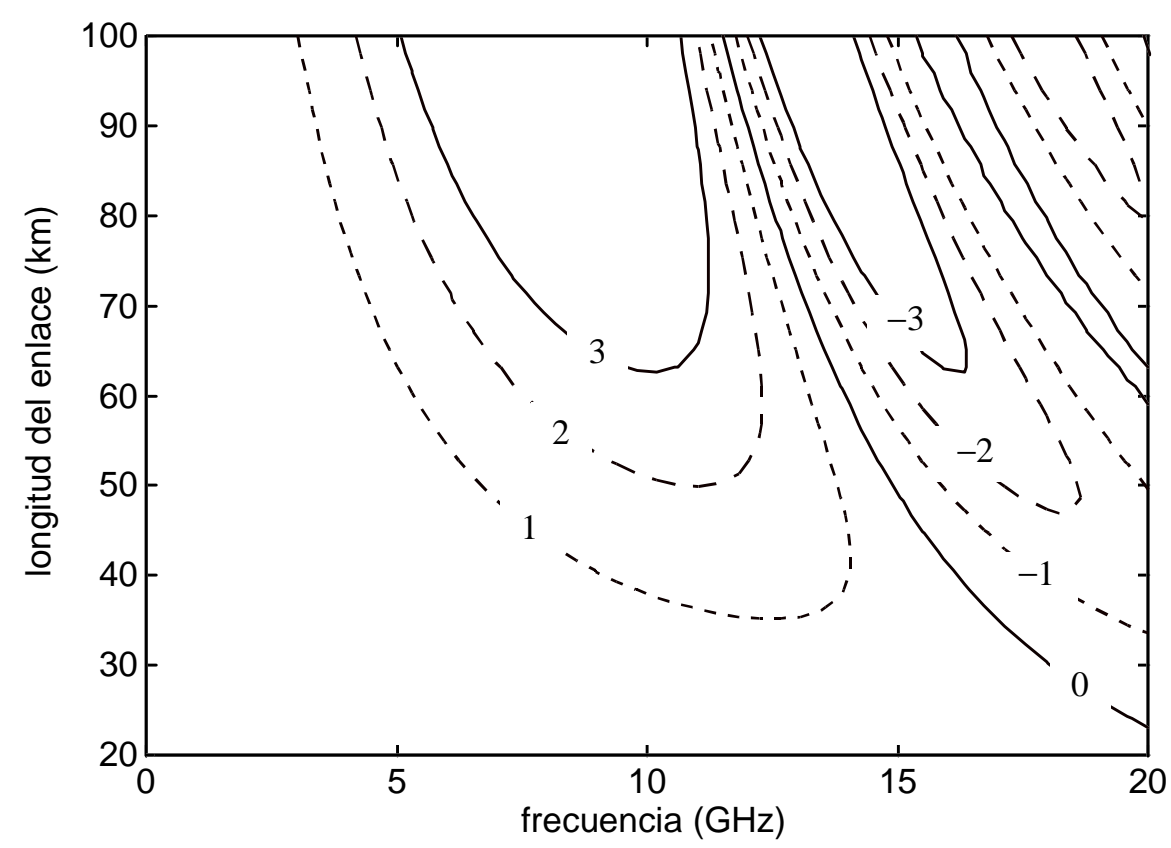

Figura 4.26 Respuesta en frecuencia del sistema OPC en función de la longitud del enlace y para una potencia $P_{0}=20 \mathrm{~mW}$. Se supone una configuración MSSI.

Por último, la figura 4.26 muestra la variación de la respuesta frecuencial de un sistema MSSI con la longitud del enlace. Conforme aumenta la longitud de fibra, las oscilaciones de la respuesta ecualizada son de mayor amplitud y se van desplazando progresivamente hacia frecuencias menores. A partir de los $60 \mathrm{~km}$, el ancho de banda a $-3 \mathrm{~dB}$ del sistema se reduce bruscamente a valores en torno a los 5-10 GHz. Estos resultados son para una potencia óptica de $20 \mathrm{~mW}$, pero empleando niveles de potencia más reducidos es posi ble atenuar la amplitud de las oscilaciones e incrementar la longitud máxima del enlace.

\subsubsection{Compensación exacta de dispersión y SPM}

El esquema considerado en este apartado se muestra en la figura 4.27, idéntico al de un sistema OPC, salvo que se han añadido unas nuevas variables para analizar el problema de la compensación perfecta de los efectos dispersivos y no lineales. Para transmisiones a grandes distancias se pueden incluir amplificadores ópticos en el trayecto, aunque esto será comentado más tarde. La señal óptica a la salida del láser se propaga en la 


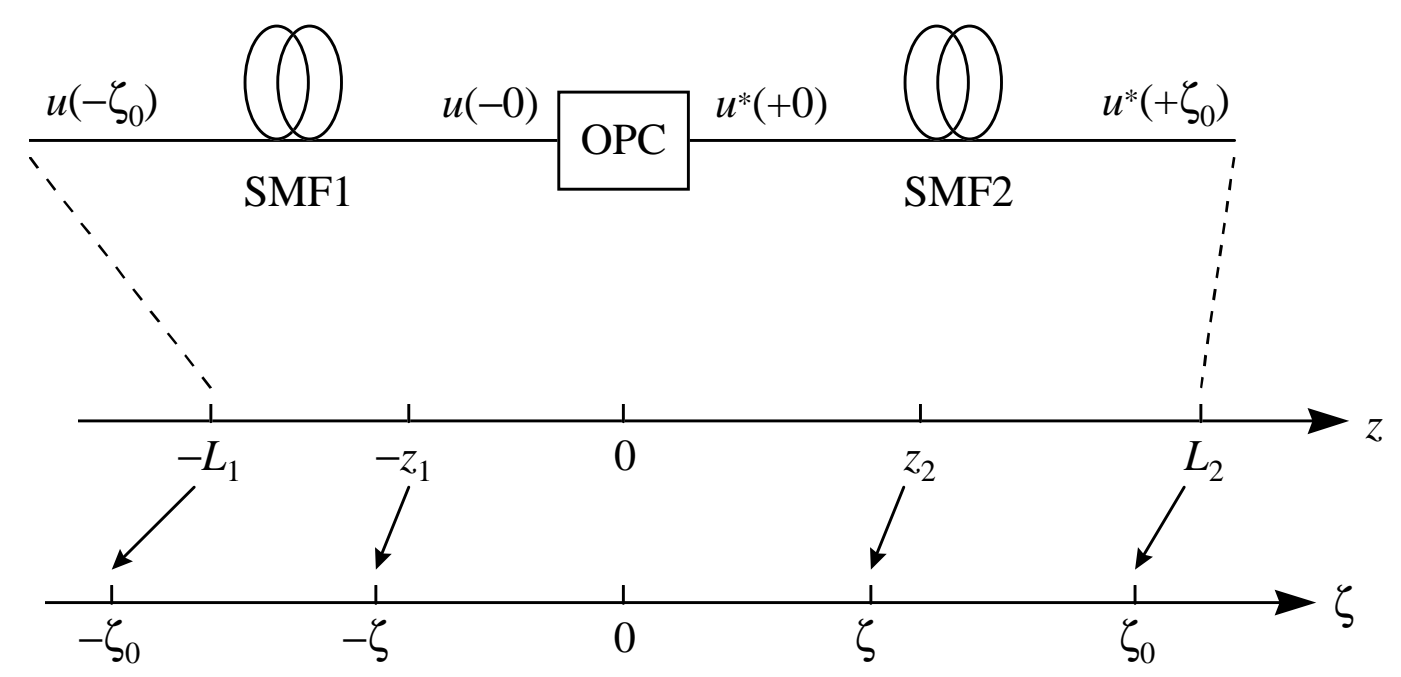

Figura 4.27 Configuración esquemática de compensación de GVD + SPM mediante inversión espectral (z: distancia real; $\zeta$ : distancia normalizada).

dirección $+z$ a través de la primera fibra (SMF 1 ) de longitud $L_{1}$. Luego, un bloque conjugador invierte la fase de la señal, lo cual es equivalente a invertir el espectro respecto a la frecuencia central $\omega_{0}$. Tras este procesado, la señal se propaga a través de la segunda fibra (SMF2) de longitud $L_{2}$ y finalmente llega hasta el fotodetector. Para el análisis se supondrá que el punto $z=0$ está situado en el OPC.

Cuando una señal óptica con portadora $\exp \left(\mathrm{j} \omega_{0} \mathrm{t}\right)$ se propaga a través de una fibra en dirección $+z$, la envolvente compleja del campo, $A(z, T)$, suponiendo la aproximación de pequeñas variaciones en su amplitud, obedece la ecuación de Schrödinger no lineal

$$
\frac{\partial A}{\partial z}-\frac{j}{2} \beta_{2} \frac{\partial^{2} A}{\partial T^{2}}=-\frac{\alpha}{2} A-j \gamma|A|^{2} A,
$$

donde $\beta_{2}=\beta_{2}(z), \alpha=\alpha(z)$ y

$$
\gamma=\gamma(z)=\frac{\omega_{0} N_{2}(z)}{c A_{\text {eff }}(z)}
$$

considerando que los distintos parámetros pueden sufrir ligeras variaciones a lo largo de la fibra. Por el momento, se considerará únicamente la 
dispersión de primer orden. En un apartado posterior analizaremos la dispersión de segundo orden para comprobar cómo afecta a las prestaciones del sistema. A continuación, introduciremos una envolvente $u(z, T)$ normalizada $\left(|u|^{2}=1\right)$, de tal modo que

$$
A(z, T)=a(z) u(z, T),
$$

donde

$$
a(z)=a(0) \exp \left[-\frac{1}{2} \int_{0}^{z} \alpha\left(z^{\prime}\right) d z^{\prime}\right]
$$

es una función de amplitud con pérdidas o ganancia dependiendo del signo de $\alpha(z)$. $a(z) \equiv a(0)$ (cte.) representa el caso sin pérdidas y $a^{2}(z)$ corresponde a la potencia óptica $\mathrm{P}(\mathrm{z})$. Si sustituimos (4.20) y (4.21) en (4.18) resulta

$$
\frac{\partial u}{\partial z}-\frac{j}{2} \beta_{2}(z) \frac{\partial^{2} u}{\partial T^{2}}=-\left.j \gamma(z) a^{2}(z) u\right|^{2} u,
$$

y realizando la transformación (figura 4.27)

$$
\zeta=\int_{0}^{z} \gamma\left(z^{\prime}\right) a^{2}\left(z^{\prime}\right) d z
$$

se obtiene final mente la siguiente ecuación normalizada:

$$
j \frac{\partial u}{\partial \zeta}=-B(\zeta) \frac{\partial^{2} u}{\partial T^{2}}+|u|^{2} u
$$

donde

$$
B(\zeta)=\frac{\beta_{2}(\zeta)}{2 \gamma(\zeta) \mathrm{a}^{2}(\zeta)}
$$

Calculando el complejo conjugado de cada uno de los términos de (4.24) se obtiene

$$
-j \frac{\partial u^{*}}{\partial \zeta}=-B(\zeta) \frac{\partial^{2} u^{*}}{\partial T^{2}}+\left|u^{*}\right|^{2} u^{*}
$$


de tal forma que si $u(-\zeta)$ obedece la evolución impuesta por $(4.24), u^{*}(\zeta)$ también obedece la ec. (4.26) si se cumple la siguiente condición:

$$
B(-\zeta)=B(\zeta)
$$

Así pues, una señal inicial $u\left(-\zeta_{0}\right)$ obedece (4.24) desde $\zeta=-\zeta_{0}$ hasta 0 , donde la señal es igual a $u(-0)$. Luego, se calcula su conjugada $u^{*}(+0)$ y se propaga desde $\zeta=0$ hasta $\zeta_{0}$ de acuerdo a (4.26), obteniéndose a la salida $u^{*}\left(+\zeta_{0}\right)$. Bajo la condición impuesta en (4.27), la señal de salida es exactamente la compleja conjugada de la señal de entrada a la primera fibra. El signo del desplazamiento de fase producido por GVD y SPM en cualquier posición $-\zeta$ de la primera fibra se invierte por medio del OPC. De este modo, la distorsión generada por un desplazamiento de fase en cualquier punto $-\zeta$, puede compensarse perfectamente con el mismo desplazamiento de fase sobre la señal conjugada en el punto $+\zeta$ de la segunda fibra.

Para que la técnica basada en OPC permita compensar los efectos de GVD y SPM es necesario que se cumpla la condición impuesta por la ec. (4.27). Introduciendo dos distancias $-z_{1}$ y $z_{2}$ en la coordenada $z$, correspondientes a $-\zeta$ y $\zeta$ respectivamente, y que por lo tanto cumplen la siguiente relación:

$$
\int_{0}^{-z_{1}} \gamma(z) a^{2}(z) d z=-\int_{0}^{z_{2}} \gamma(z) a^{2}(z) d z,
$$

la condición de compensación de (4.27) se reescribe como

$$
B\left(-z_{1}\right)=B\left(z_{2}\right),
$$

o más desarrollada

$$
\frac{\beta_{2}\left(-z_{1}\right)}{\gamma\left(-z_{1}\right) P\left(-z_{1}\right)}=\frac{\beta_{2}\left(z_{2}\right)}{\gamma\left(z_{2}\right) P\left(z_{2}\right)}
$$

Es decir, proporcionando el mismo cociente entre dispersión y no linealidades en las correspondientes posiciones, se satisface la condición para una perfecta compensación. Esto es relativamente sencillo si consideramos una situación en la que no existen pérdidas [Pep80, Fis83]. En caso contrario, suponiendo que la primera fibra presentara pérdidas, el 
medio de transmisión tras el OPC debería presentar una ganancia distribuida para poder compensar los efectos del SPM (considerando por el momento valores constantes de $\beta_{2}$ y $\gamma$ ). Este es el resultado de la simetría impuesta a ambos lados del bloque OPC, ya comentada en la sección 4.2. La señal de entrada a la primera fibra se ve más influenciada por el SPM al comienzo de la propagación, puesto que la potencia óptica de señal es mayor. Así pues, tras el OPC debe ocurrir lo contrario, es decir, el desplazamiento de fase no lineal producido por SPM debe ser progresivamente mayor (aumento de la amplitud de la señal).

Sin embargo, también existe otra solución para compensar perfectamente la distorsión sin necesidad de emplear un medio con ganancia. Ésta se basa en introducir en los parámetros $\beta_{2}(z)$ y $\gamma(z)$ una dependencia con la atenuación de la fibra, de tal forma que el medio se pueda considerar sin pérdidas en lo que respecta a la distorsión. De este modo, para cancelar la variación que se produce en $\mathrm{B}(\mathrm{z})$ a lo largo de un medio con pérdidas, deberíamos disminuir la dispersión o aumentar las no linealidades (efecto Kerr) con la distancia. En el caso de existir amplificadores, éstos también deberán tenerse en cuenta en la expresión de $B(z)$. Considerando la dispersión, $\beta_{2}(z)$ puede variarse a lo largo de la fibra modificando parámetros tales como la longitud de onda de dispersión nula, $\lambda_{\mathrm{ZD}}$, de una DSF, el índice de refracción o el diámetro del núcleo. Una fibra para compensar dispersión (DCF) es una buena alternativa para modificar $\beta_{2}(\mathrm{z})$. Es posible obtener DCF s con valores de dispersión de hasta $-150 \mathrm{ps} /(\mathrm{km} \cdot \mathrm{nm})$ [Ven93]. Por otra parte, para aumentar el efecto Kerr, debemos controlar $\mathrm{N}_{2}(\mathrm{z}) \circ \mathrm{A}_{\text {eff }}(\mathrm{z})$. Disminuyendo $A_{\text {eff }}(z)$ o el diámetro de un modo (MFD), aumenta efectivamente la intensidad a lo largo de la fibra. No obstante, una disminución excesiva en el MFD conduce a pérdidas adicionales. Por tanto, existe un límite inferior para el MFD.

Combinando estos dos efectos, podemos realizar una distribución simétrica de $\mathrm{B}(\mathrm{z})$ con respecto a la posición del OPC, incluso teniendo en cuenta las pérdidas de la fibra y la ganancia de los amplificadores. Esto conduce a la posibilidad de una perfecta compensación de la distorsión no lineal producida por GVD y SPM utilizando la técnica OPC. En particular, existen 
un tipo de fibras conocidas como fibras de dispersión decreciente (DDFs, dispersion-decreasing fibers) que constituyen la solución a este problema. Se caracterizan por poseer una distribución de GVD dada por

$$
\left|\beta_{2}(z)\right|=\left|\beta_{2}(0)\right| \exp (-\alpha z)
$$

Aunque se han fabricado fibras con precisiones mejores que 0,1 $\mathrm{ps}^{2} / \mathrm{km}$ [Ric95], no siempre están disponibles y existen soluciones más simples, como por ejemplo aproximar el perfil exponencial por medio de una función escalonada, empalmando varias fibras con diferentes valores de $\beta_{2}$ [Wat96].

La configuración general empleada se muestra en la figura 4.28. En primer lugar, cada uno de los tramos de fibra está dividido en $k_{1}$ y $k_{2}$ trayectos respectivamente, tras los cuales existe un amplificador que restaura el nivel de señal. El objetivo de estos amplificadores es obtener una distribución de potencia lo más simétrica posible respecto a la posición del OPC. No obstante, si la longitud del enlace de fibra no es muy elevada podemos

(1)

(2)

$\left(k_{1}\right)$

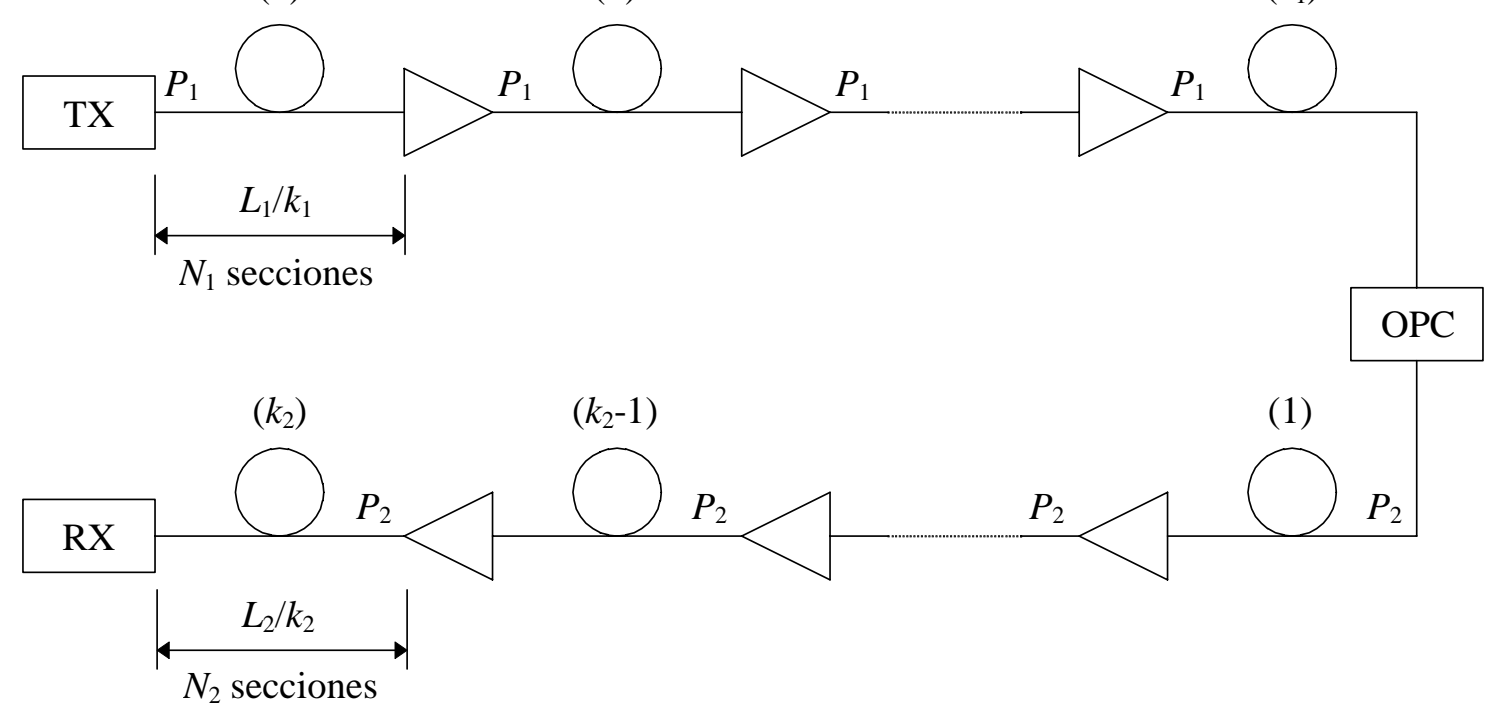

Figura 4.28 Configuración general utilizada en la técnica OPC. Los amplificadores compensan las pérdidas del tramo de fibra previo. Por otra parte, cada uno de los $k_{\mathrm{j}}$ trayectos de fibra entre amplificadores se implementa mediante $N_{j}$ secciones de DCF con dispersión $D_{j}(m)$, para $m=1,2, \ldots, N_{j}$ y $j=1,2$. 


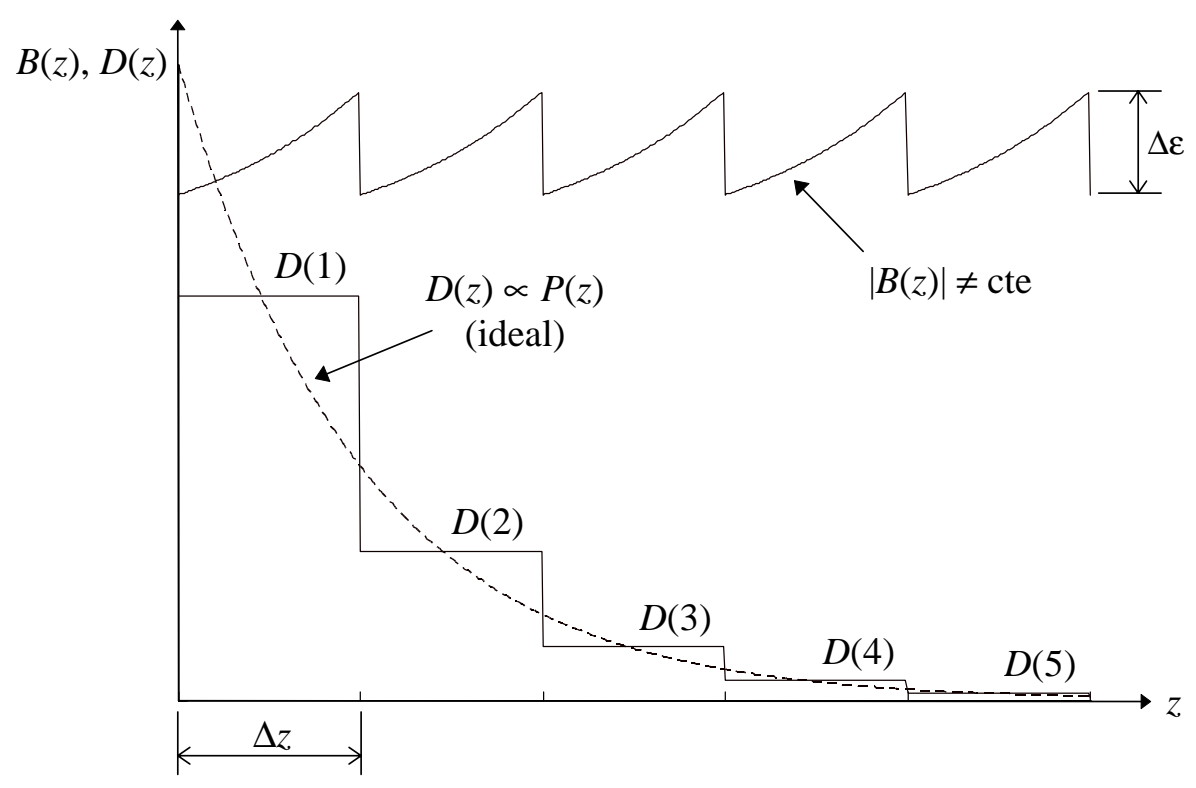

Figura 4.29 Aproximación escalonada de la distribución exponencial ideal, $\mathrm{D}(\mathrm{z})$, con una $\mathrm{DCF}$ de $\mathrm{N}=5$ secciones. Para $\mathrm{B}(\mathrm{z})$ se obtiene un comportamiento similar a un diente de sierra con una amplitud pico a pico $\Delta \varepsilon$.

evitarlos. Suelen utilizarse cuando la longitud de los trayectos es superior a los $50 \mathrm{~km}$, considerando valores típicos para el coeficiente no lineal $\gamma \sim 2,6$ $\mathrm{W}^{-1} \mathrm{~km}^{-1}$. Al mismo tiempo, en cada uno de estos trayectos $(j=1$ ó 2$)$ de longitud $L_{j} / k_{j}$ realizaremos cambios en el coeficiente de dispersión $\beta_{2 j}(z)$. La distribución que conseguiría una compensación perfecta de dispersión y SPM debería tener forma exponencial como se puede deducir de (4.30), pues éste es el comportamiento del término de potencia $a^{2}(z)=P(z)$. De este modo, forzaríamos a que el término $B(z)$ fuese constante para cualquier punto de ambas fibras, y por lo tanto simétrico respecto del OPC. Sin embargo, esta solución es compleja y queda descartada como ya explicamos con anterioridad, a que obliga al uso de DDFs.

Una solución más sencilla consiste en aproximar esta distribución exponencial por medio de una función escalonada. Así, cada uno de estos trayectos se dividiría en $N_{j}$ secciones de longitud $\Delta \mathrm{z}_{\mathrm{j}}=\mathrm{L}_{\mathrm{j}} /\left(\mathrm{N}_{\mathrm{j}} \mathrm{k}_{\mathrm{j}}\right)$ y dispersión $D_{j}(m)\left(m=1,2, \ldots, N_{j}\right)$. Para calcular el valor de este parámetro de dispersión, $D_{j}(m)$, se trabaja con valores de potencia promediados [Wat96]: 


$$
D_{j}(m)=D_{j} \frac{\bar{P}_{j}(m)}{\bar{P}_{j}}=D_{j} \frac{P_{j}(m)\left[1-\exp \left(-\alpha_{j} \Delta z_{j}\right)\right] / \alpha_{j} \Delta z_{j}}{\bar{P}_{j}}
$$

siendo $\overline{P_{j}}(m)$ la potencia media promediada a lo largo de la sección mésima, $P_{j}(m)$ la potencia a la entrada de esta misma sección y $D_{j}$ el valor medio del parámetro de dispersión. Este proceso puede verse gráficamente en la figura 4.29 para $\mathrm{N}_{\mathrm{j}}=5$. Al aproximar el parámetro de dispersión por una función escalonada, se obtiene una distribución de $\mathrm{B}(\mathrm{z})$ exponencial a trozos con un valor de pico a pico $\Delta \varepsilon$. Para obtener una buena ecualización, se debe forzar a que sea lo más constante posible, lo cual equivale a hacer tender este término de error a cero $(\Delta \varepsilon \rightarrow 0)$. Un modo de conseguirlo puede ser aumentando el número de secciones, o disminuyendo la distancia entre amplificadores. Ambas equivalen a una disminución en el valor de $\Delta z$.

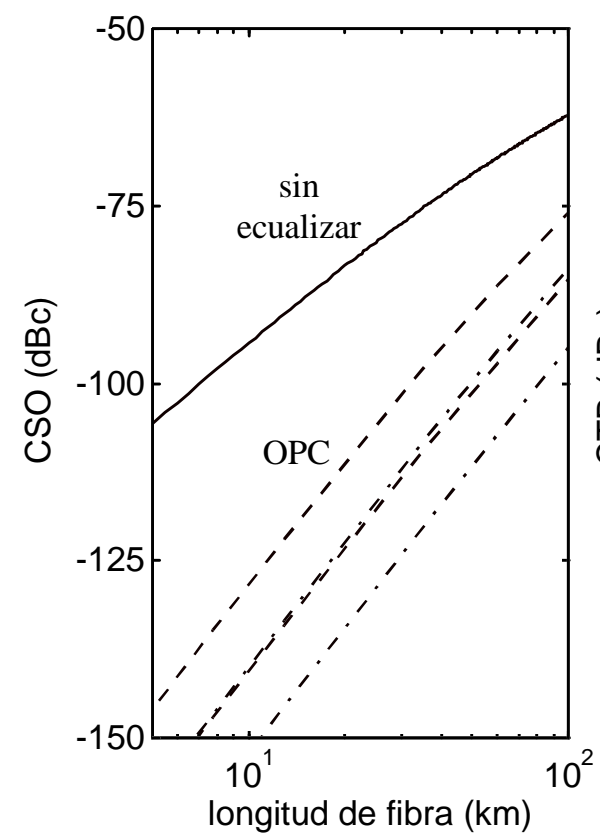

(a)

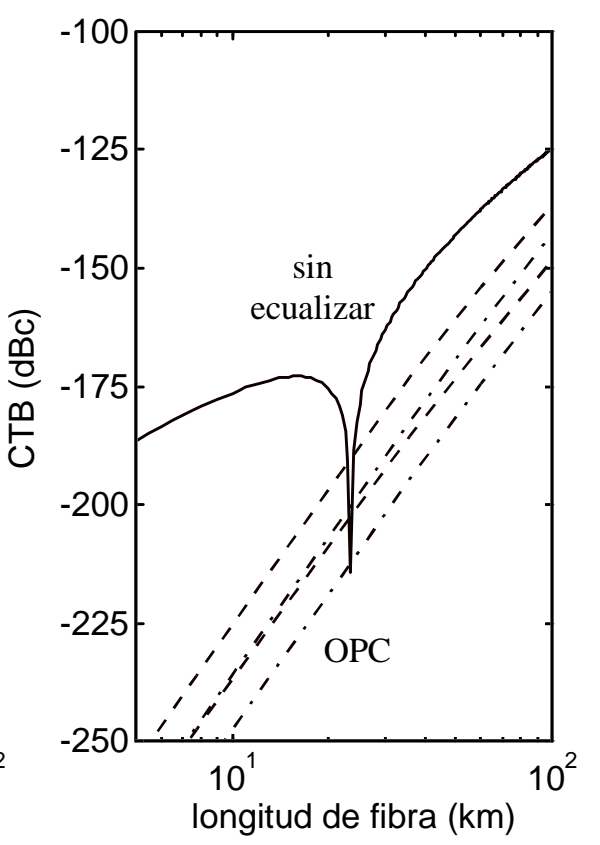

(b)

Figura 4.30 Compensación de (a) CSO y (b) CTB mediante OPC para distintos valores de $\mathrm{N}=\mathrm{N}_{1}=\mathrm{N}_{2}$ y $\mathrm{k}=\mathrm{k}_{1}=\mathrm{k}_{2}$. Las curvas de trazos son para $\mathrm{N}=1,2$ y $\mathrm{k}=1$, mientras que las de trazos y puntos son para $\mathrm{N}=1,2$ y $\mathrm{k}=2$. Los parámetros de modulación son: $\mathrm{f}_{\mathrm{m}}=500 \mathrm{MHz}, \mathrm{m}_{\mathrm{i}}=0,04, \vartheta=0$ y $\mathrm{P}_{0}=20 \mathrm{~mW}$. 


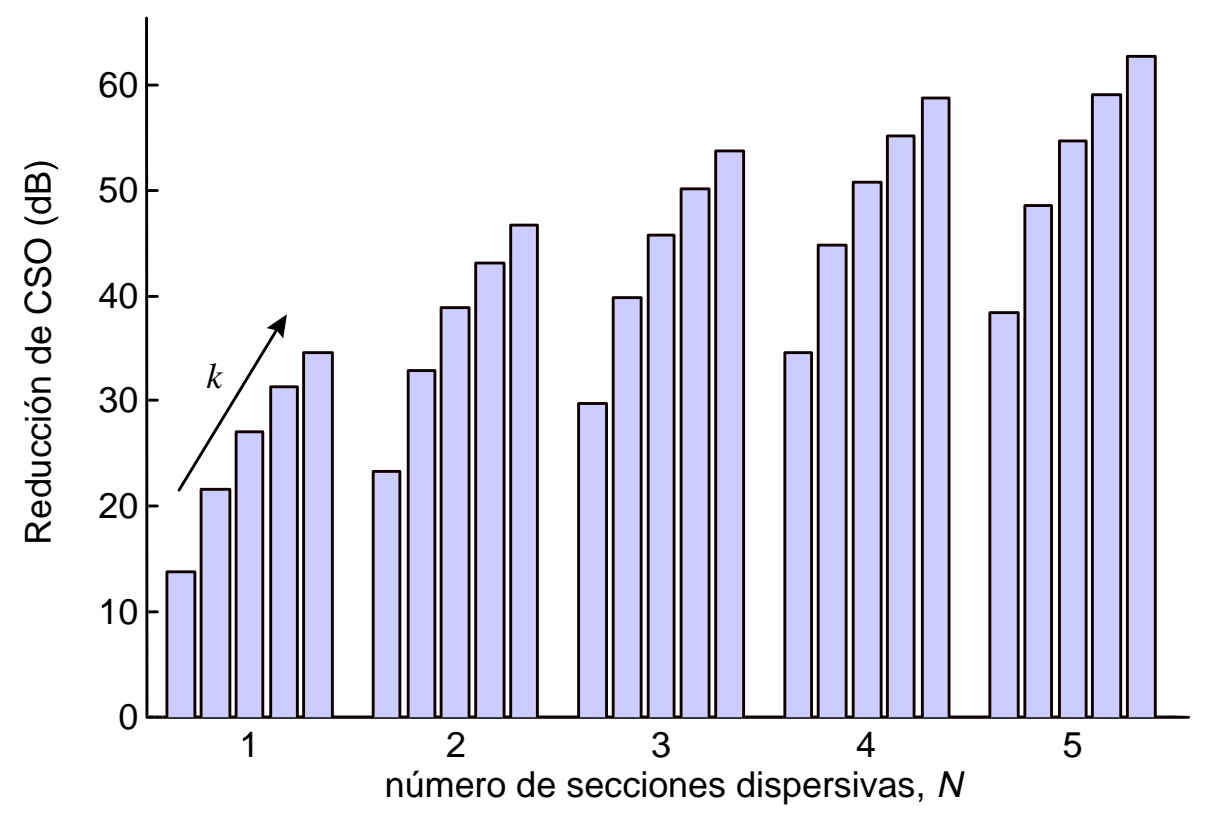

Figura 4.31 Reducción de los niveles de CSO de la figura 4.30 para una longitud de fibra de $100 \mathrm{~km}$ y en función de $\mathrm{N}$ y $\mathrm{k}$.

En la figura 4.30 se presentan los resultados de ecualización de CSO y CTB empleando distintos valores de $\mathrm{N}$ y $\mathrm{k}$. Las curvas de trazos son para $\mathrm{k}=1$ y las de trazos y puntos para $k=2$, en ambos casos variando $N=1$, 2 . Se observa que, como es lógico, aumentando el valor de $\mathrm{N}$ ó $\mathrm{k}$ se obtienen progresivamente mejores resultados como consecuencia de una mayor simetría del efecto GVD + SPM. La reducción que se obtiene en los niveles de CSO respecto a la situación sin ecualización se resumen en la figura 4.31 en función de $\mathrm{N}$ y k. En general, se obtiene mejor resultado aumentado $\mathrm{N}$ que $k$, lo cual suele ser también tecnológicamente más simple y económico. De este modo, empleando fibras de 3 secciones, se consiguen reducciones en el CSO de hasta $30 \mathrm{~dB}$.

Por último, también es posible ecualizar las oscilaciones que aparecen en la respuesta en frecuencia del sistema OPC empleando esta técnica. En la figura 4.32 aparecen los resultados para una subportadora de $10 \mathrm{GHz}$ en función de la longitud del enlace y para varios valores de N. Nuevamente se observa que los resultados son cada vez mejores conforme aumenta el número de secciones dispersivas, aplanándose las curvas y desplazándose hacia distancias mayores el punto de penalización a $1 \mathrm{~dB}$. Por ejemplo, con 


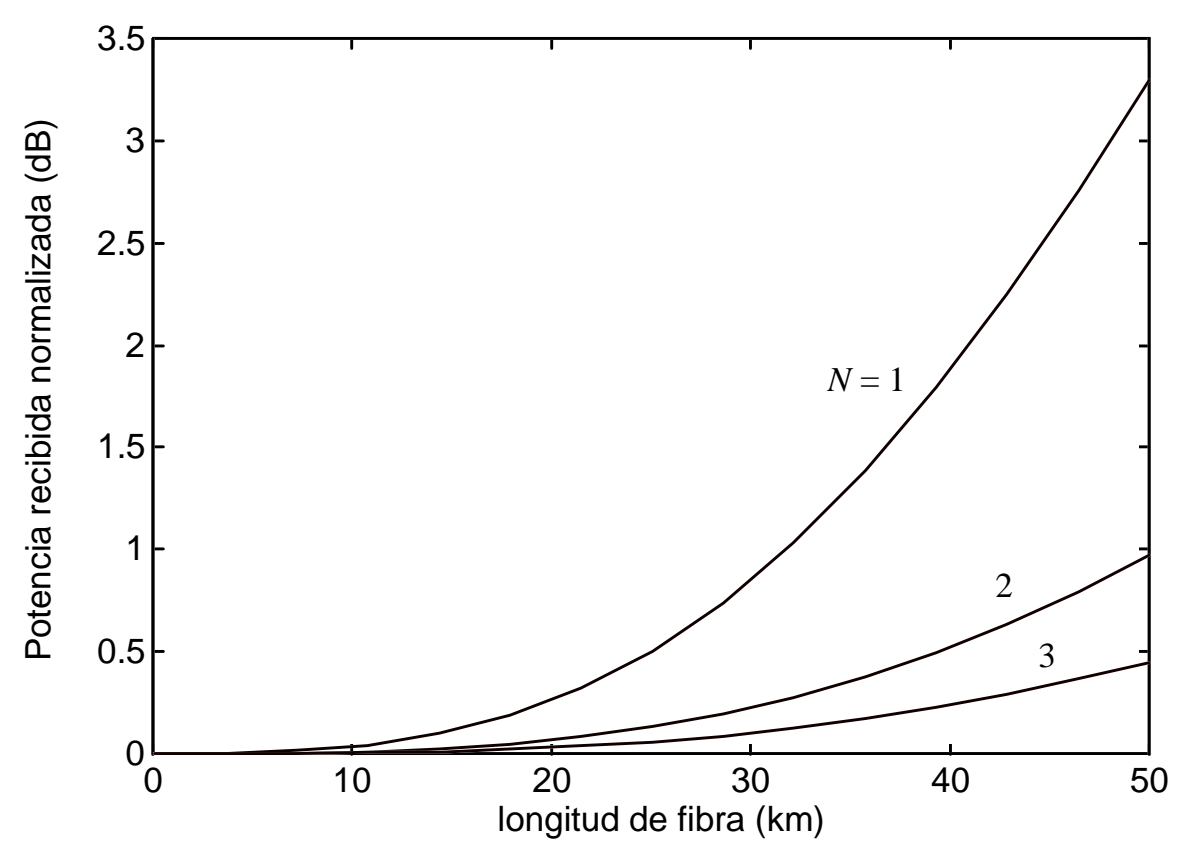

Figura 4.32 Potencia recibida normalizada para una subportadora de $10 \mathrm{GHz}$ en función de la longitud de fibra y para varios valores de $\mathrm{N}$. Otros parámetros son: $\mathrm{k}=1, \mathrm{P}_{0}=20 \mathrm{~mW}$ y $\vartheta$ $=0$.

$\mathrm{N}=2$ se aumenta la distancia de los 30 a los $50 \mathrm{~km}$. El caso límite $\mathrm{N} \rightarrow \infty$ correspondería a emplear una DDF.

\subsubsection{Influencia de la dispersión de segundo orden}

Por el momento, se ha tenido en cuenta en el análisis únicamente la dispersión de primer orden representada por el coeficiente $\beta_{2}$. La explicación se debe a que el transmisor óptico opera a $1550 \mathrm{~nm}$ y el coeficiente $\beta_{3}$ es despreciable frente al anterior a esta longitud de onda en el caso de fibras estándar. Ahora bien, al aplicar la técnica OPC para ecualizar, se obtendrán unos resultados que estarán limitados por la dispersión de segundo orden. Debe tenerse en cuenta que con esta técnica no se puede compensar la dispersión de segundo orden salvo que tenga distinto signo para cada uno de los tramos de fibra a ambos lados del conjugador, lo que no suele ocurrir en la práctica. A continuación, veremos cómo afecta a las prestaciones del sistema un coeficiente de dispersión $\beta_{3}$ distinto de cero. 


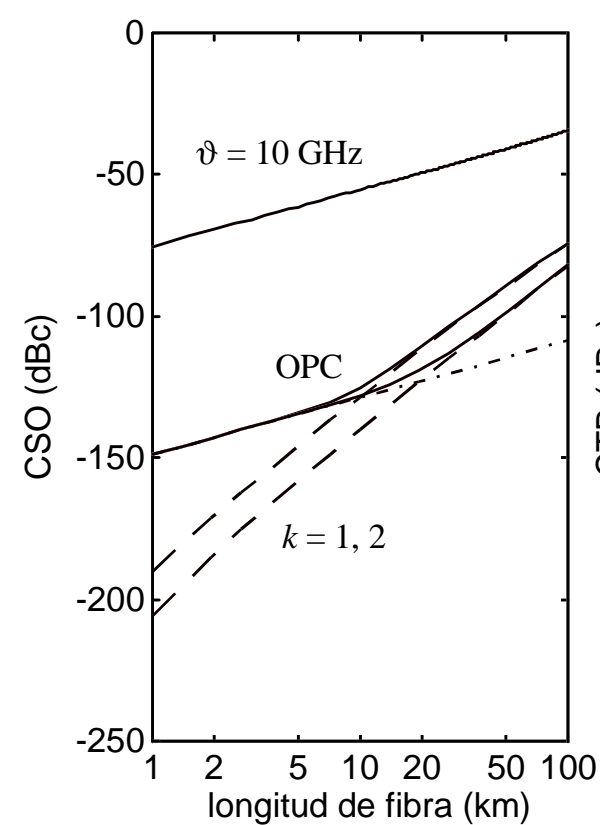

(a)



(b)

Figura 4.33 Ecualización de (a) CSO y (b) CTB en presencia de dispersión de segundo orden. Las curvas de trazos son para $\beta_{3}=0$ y variando $k$. La curva de trazos y puntos representa el límite impuesto por la dispersión de segundo orden: $S=0,07$ $\mathrm{ps} /\left(\mathrm{km} \cdot \mathrm{nm}^{2}\right)$. Otros parámetros son: $\mathrm{N}=1, \mathrm{fm}_{\mathrm{m}}=500 \mathrm{MHz}, \mathrm{m}_{\mathrm{i}}=$ 0,04 y $\mathrm{P}_{0}=20 \mathrm{~mW}$.

Dado que la dispersión de segundo orden no afecta a la respuesta en frecuencia de la fibra ópticav , nos centraremos en estudiar sus efectos sobre la distorsión no lineal. En la figura 4.33 se representan los niveles de CSO y CTB con y sin ecualización mediante OPC para una subportadora de 500 $\mathrm{MHz}$ con chirp $(\vartheta=10 \mathrm{GHz}$ ). Las curvas de trazos son para una situación ideal con $\beta_{3}=0$ y variando $k$. No obstante, se observa que para longitudes de fibra reducidas ( $L<10 \mathrm{~km}$ para el CSO y $L<20 \mathrm{~km}$ para el CTB) existe un límite en la ecualización. Este límite viene impuesto por la dispersión de segundo orden que no puede compensarse y se caracteriza por la curva de trazos y puntos. Depende de parámetros tales como la frecuencia de subportadora o el chirp del transmisor óptico. En cualquier caso, si empleamos fibras estándar con $\mathrm{S}<0,1 \mathrm{ps} /\left(\mathrm{km} \cdot \mathrm{nm}^{2}\right)$ su efecto no es

\footnotetext{
v la función de transferencia de la fibra óptica se ve modificada por un término de fase, pero no afecta a su amplitud (véase apéndice I)
} 
excesivamente significativo, pues en la figura 4.33(a) se mantiene unos 75 $\mathrm{dB}$ por debajo del nivel de CSO sin ecualizar. 


\subsection{CONCLUSIONES Y CONTRIBUCIONES}

La técnica de inversión espectral se ha demostrado como un método eficiente para compensar la distorsión no lineal inducida por la dispersión cromática y las no linealidades de las fibras ópticas monomodo. Las principales conclusiones y resultados obtenidos en este estudio se resumen a continuación:

- La técnica OPC compensa perfectamente la dispersión de primer orden suponiendo que se cumple la condición impuesta por (4.5). No obstante, la dispersión de segundo orden no puede compensarse salvo que tenga distinto signo en ambas fibras a cada lado del conjugador. Aun así, en el caso de fibras estándar no constituye ninguna limitación para las longitudes de enlace típicas de los sistemas radio-fibra. Por otro lado, para que el SPM pueda compensarse, es necesario que exista simetría del efecto Kerr con respecto a la posición del conjugador. En caso de longitudes de enlace reducidas, puede utilizarse la aproximación (4.7). Si se desean obtener mejores resultados, entonces es necesario utilizar amplificadores ópticos o dividir los tramos de fibra en distintas secciones con valores de dispersión escogi dos adecuadamente según la ec. (4.32). En especial, el uso de DDFs constituye una solución para el problema de la compensación perfecta de dispersión y SPM. EI hecho de que el SPM no se compense perfectamente da lugar a la aparición de oscilaciones dependientes de la frecuencia o de la longitud de fibra en la respuesta del sistema OPC, las cuales se han analizado por primera vez en el contexto de los sistemas ópticos analógicos.

- Como contribución, en este capítulo se han obtenido resultados de ecualización del CSO y del CTB introducidos por la fibra óptica en sistemas multicanal radio sobre fibra empleando la técnica OPC. Los resultados han sido muy satisfactorios, obteniéndose reducciones de unos $40 \mathrm{~dB}$ en los niveles de CSO y CTB de los sistemas CATV y MMDS, respectivamente. En los sistemas LMDS y MVDS, sin embargo, la distorsión no se convierte en la principal limitación, ya que estos sistemas emplean modulaciones digitales QPSK o QAM que no son tan restrictivas 
como la modulación AM-VSB empleada habitualmente en los sistemas CATV y MMDS. El principal problema lo constituye la penalización de potencia, la cual limita la máxima distancia del sistema a 1-2 km como consecuencia de las altas frecuencias de modulación: 28 y $40 \mathrm{GHz}$. Los resultados finales de ecualización obtenidos posibilitan longitudes de fibra de $100 \mathrm{~km}$ para el sistema CATV, $50 \mathrm{~km}$ para MMDS, $40 \mathrm{~km}$ para LMDS o $20 \mathrm{~km}$ para MVDS, con valores de CIR superiores a los $60 \mathrm{~dB}$ y de penalización de potencia inferiores a $1 \mathrm{~dB}$. Algunos de estos resultados de ecualización en sistemas de comunicaciones ópticas analógicos han sido publicados en [Mar97, Ram98a].

- Se ha obtenido, por primera vez, una expresión teórica para caracterizar la respuesta en frecuencia de un sistema OPC a partir de las funciones de conversión fase-intensidad de la fibra óptica (véase apéndice I). Esta expresión es válida aun en presencia de chirp en el transmisor óptico y tiene en cuenta tanto la dispersión cromática como el SPM. Los resultados teóricos y de simulación concuerdan perfectamente.

- Una de las ventajas de la técnica OPC es su reducida sensibilidad frente a variaciones en la longitud de fibra óptima de ecualización. Esto permite emplear arquitecturas donde el dispositivo OPC se coloca en un lugar centralizado, y desde aquí se distribuye la señal por medio de una red óptica pasiva hasta los distintos usuarios. De este modo, el conjugador se comparte con el consiguiente ahorro económico. En particular, se han estudiado configuraciones distintas de MSSI y se ha demostrado que en general interesa $L_{1}<L_{2}$, ya que la distorsión acumulada durante el primer trayecto de fibra es menor.

- Adicionalmente, se han obtenido resultados de sensibilidad de la respuesta del sistema OPC frente a diversos parámetros: dispersión cromática, potencia óptica, longitudes de fibra, etc. El parámetro más influyente ha resultado ser la potencia óptica, debido a que la simetría del efecto Kerr se convierte en el principal factor limitante [Sot99]. Otros efectos, como por ejemplo el cambio de longitud de onda que ocurre en el conjugador, son muy poco significativos en sistemas típicos. 
- Conviene resaltar que en todo el análisis realizado se ha supuesto que el bloque OPC es un dispositivo ideal, no introduciendo ninguna degradación adicional sobre el proceso de conjugación óptico. Esto se debe a que en este capítulo se ha pretendido enfocar el estudio solamente en la técnica de inversión espectral, dejando para el capítulo siguiente un análisis detallado del conjugador $y$, en particular, del proceso de FWM que ocurre en el mismo. 


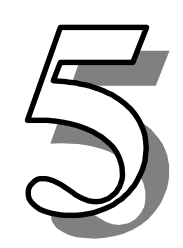

\section{Construcción de conjugadores ópticos}

\subsection{INTRODUCCIÓN}

El proceso de mezclado de cuatro ondas (FWM) es uno de los fenómenos no lineales más interesantes que ocurre en dispositivos fotónicos. Se caracteriza por la aparición de nuevas frecuencias del tipo $\omega_{i}+\omega_{j}-\omega_{k}$ a la salida del dispositivo. Aunque este fenómeno produce degradaciones en los sistemas ópticos multicanal [Shi90], encuentra un gran número de aplicaciones como la generación de frecuencias [Lin82], o la elaboración de convertidores de longitud de onda y conjugadores ópticos [I no92a, Wu94, Zho94, Zha96, Shi96, Duc96]. Debe tenerse en cuenta que la nueva onda generada mediante FWM es la conjugada compleja de la onda de entrada a frecuencia $\omega_{k}$. Actualmente, los principales dispositivos fotónicos utilizados 
para inducir FWM son las fibras ópticas y los amplificadores de semiconductor (SOAs).

El fenómeno de FWM en fibras ópticas ha sido extensamente estudiado [Hil78, Shi87, Agr89, Cap91, Mar91, Ino92, Yam97]. Para inducirlo requiere la inyección de una señal de bombeo a una frecuencia $\omega_{\mathrm{p}}$ desplazada de la frecuencia de señal $\omega_{S}$ en una pequeña cantidad $(\sim 0,5 \mathrm{THz})$. Las no linealidades de la fibra generan una señal conjugada a una frecuencia $\omega_{C}=$ $2 \omega_{\mathrm{p}}-\omega_{\mathrm{s}}$ considerando que se cumple la condición de fasei. En fibras ópticas monomodo, la condición de fase se satisface aproximadamente si se escoge la longitud de onda de dispersión nula de la fibra coincidente con la longitud de onda de bombeo. Luego si se desea trabajar en tercera ventana son necesarias fibras de dispersión desplazada (DSFs). Este método fue el utilizado en varios experimentos realizados en 1993 [Wat93, Wat93a] que demostraron por primera vez el potencial de la técnica OPC para compensar los efectos de la dispersión cromática.

EI proceso de FWM en SOAs ha sido también utilizado para generar la señal conjugada en la técnica OPC. Por ejemplo, en un experimento realizado en 1997 se demuestra la transmisión de una señal de $40 \mathrm{Gbit} / \mathrm{s}$ sobre 406 km de fibra estándar [Mar97c]. EI fenómeno de FWM no degenerado en SOAs fue propuesto en 1987 [Agr87] y ha sido estudiado extensamente desde entonces para la elaboración de convertidores de longitud de onda [Zho94, Mec95, Shi96, Ian96, Lin98]. Su principal ventaja se debe a que la señal conjugada puede generarse utilizando un dispositivo de $1 \mathrm{~mm}$ de longitud o inferior. Además, la eficiencia de conversión es típicamente superior a la que se obtiene mediante FWM en fibras ópticas [Agr97].

En este capítulo se estudia la construcción de conjugadores ópticos basados en FWM en configuraciones DSF o SOA. Y todo ello como aplicación de la técnica OPC en sistemas ópticos analógicos para la compensación de los efectos de dispersión cromática y no linealidades. En la sección 5.2 se analiza el fenómeno de FWM en fibras ópticas monomodo. Se estudia su

' phase-matching condition 
origen, las ecuaciones que lo modelan, y se presentan resultados de la eficiencia de conversión. Adicionalmente, se observa la influencia de la condición de fase, así como de distintos efectos no lineales como el scattering estimulado de Brillouin. EI FWM en SOAs se estudia en la sección 5.3, donde se realiza un análisis similar al de la sección 5.2. En particular, se observa la influencia de la separación entre las longitudes de onda de señal y de bombeo, y se obtienen resultados de eficiencia de conversión y de relación señal a ruido. Por último, en la sección 5.4 se aborda la construcción de conjugadores ópticos basados en FWM en DSF/SOA. A diferencia de otros estudios previos [Gei97], el análisis se efectúa en el contexto de los sistemas radio sobre fibra óptica, por lo que se realizan medidas de prestaciones típicas en estos sistemas (linealidad, CNR y diagrama de ojos de un canal digital de microondas) comparando ambas configuraciones. Finalmente, se demuestra la compensación del efecto de supresión de la portadora en un enlace de $50 \mathrm{~km}$ de SSMF mediante FWM en una DSF de $12,7 \mathrm{~km}$ de longitud [Ram99]. 


\subsection{FWM EN FIBRAS ÓPTICAS MONOMODO}

De entre los distintos dispositivos fotónicos utilizados para inducir FWM, las fibras ópticas son uno de los más estudiados. Como se ha comentado en la introducción, para que el proceso de generación sea eficiente, es necesario trabajar cerca de la longitud de onda de dispersión nula. Por ello, las fibras de dispersión desplazada se convierten en el principal candidato. A continuación pasaremos a estudiar las características del FWM en estos dispositivos.

\subsubsection{Origen del FWM en fibras ópticas}

El proceso de FWM en fibras ópticas ha sido extensamente estudiado [Hil78, Shi87, Agr89, Cap91, Mar91, Ino92, Yam97]. Su origen se debe a que la polarización inducida en el medio no es lineal con respecto al campo aplicado, sino que contiene un término no lineal cuya magnitud depende de la susceptibilidad no lineal de tercer orden, $\chi^{(3)}$, de acuerdo a [Agr89]

$$
\mathbf{P}_{\mathrm{NL}}=\varepsilon_{0} \chi^{(3)}: \text { EEE , }
$$

donde $\mathbf{E}$ es el campo eléctrico, $\mathbf{P}_{\mathrm{NL}}$ es la polarización no lineal inducida y $\varepsilon_{0}$ es la permitividad del vacío.

Considerando cuatro ondas ópticas oscilando a frecuencias $\omega_{1}, \omega_{2}, \omega_{3}$ y $\omega_{4}$ linealmente polarizadas a lo largo del mismo eje $x$, el vector campo eléctrico total puede escribirse como

$$
\mathbf{E}=\hat{x} \frac{1}{2} \sum_{i=1}^{4} E_{i} \exp \left[j\left(\omega_{i} t-k_{i} z\right)\right]+c . c .,
$$

donde

$$
\mathrm{k}_{\mathrm{i}}=\frac{\widetilde{\mathrm{n}_{\mathrm{i}}} \omega_{\mathrm{i}}}{\mathrm{C}}
$$

siendo $\widetilde{n_{\mathrm{i}}}$ el índice de refracción de la onda i-ésima, y se supone que todas las ondas se propagan en la misma dirección z. 
Si a continuación sustituimos (5.2) en la ec. (5.1) y expresamos $\mathbf{P}_{\mathrm{NL}}$ de la forma

$$
\mathbf{P}_{\mathrm{NL}}=\hat{\mathrm{x}} \frac{1}{2} \sum_{\mathrm{i}=1}^{4} \mathrm{P}_{\mathrm{i}} \exp \left[\mathrm{j}\left(\omega_{\mathrm{i}} \mathrm{t}-\mathrm{k}_{\mathrm{i}} \mathrm{z}\right)\right]+\mathrm{c} . \mathrm{C} .
$$

se encuentra que $\mathrm{P}_{\mathrm{i}}$ para $\mathrm{i}=1-4$, consiste en un gran número de términos que involucran productos de tres campos eléctricos. Por ejemplo, $\mathrm{P}_{4}$ puede expresarse como

$$
\begin{aligned}
P_{4} & =\frac{3 \varepsilon_{0}}{4} \chi\left\{\left[\left|E_{4}\right|^{2}+2\left(\left|E_{1}\right|^{2}+\left|E_{2}\right|^{2}+\left|E_{3}\right|^{2}\right)\right] E_{4}\right. \\
& \left.+2 E_{1} E_{2} E_{3} \exp \left(j \theta_{+}\right)+2 E_{1} E_{2} E_{3}^{*} \exp \left(j \theta_{-}\right)+\cdots\right\},
\end{aligned}
$$

donde $\mathrm{e}^{\mathrm{i}}$

$$
\chi=\frac{3 \mathrm{n}_{0}}{8} \mathrm{~N}_{2}
$$

y las fases se calculan como

$$
\begin{aligned}
& \theta_{+}=\left(\omega_{1}+\omega_{2}+\omega_{3}-\omega_{4}\right) t-\left(k_{1}+k_{2}+k_{3}-k_{4}\right) z, \\
& \theta_{-}=\left(\omega_{1}+\omega_{2}-\omega_{3}-\omega_{4}\right) t-\left(k_{1}+k_{2}-k_{3}-k_{4}\right) z .
\end{aligned}
$$

El término proporcional $a E_{4}$ es responsable de los fenómenos de SPM y $X P M^{i i i}$, mientras que los restantes términos son responsables del FWM. La eficiencia de estos términos depende de la fase relativa entre $E_{4}$ y $P_{4}$, dada por $\theta_{+}, \theta_{-}$, o un ángulo similar. Un FWM significativo ocurre si la fase relativa se anula prácticamente, lo cual requiere la coincidencia de las frecuencias además de los vectores de onda. Este requerimiento se le conoce habitualmente con el nombre de coincidencia de fase (phase matching).

\footnotetext{
ii dado que el campo eléctrico se encuentra linealmente polarizado, sólo una componente $\chi$ del tensor de cuarto rango contribuye al índice no lineal $\mathrm{N}_{2}$

iii modulación de fase cruzada (cross-phase modulation)
} 
En términos de mecánica cuántica, el FWM ocurre cuando los fotones de dos o más ondas son destruidos y se crean nuevos fotones a frecuencias diferentes, de tal forma que la energía y momento netos se conservan durante la interacción paramétricaiv. La principal diferencia entre los procesos paramétricos y los procesos de scattering estimulado es que la condición de fase se satisface automáticamente en el caso del scattering estimulado de Raman o de Brillouin, como consecuencia de la participación activa del medio no lineal. Por el contrario, la condición de fase requiere una elección adecuada de las frecuencias e índices de refracción para que los procesos paramétricos ocurran.

En la expresión (5.5) existen dos tipos de términos de FWM. El primero de ellos corresponde al caso en el cual tres fotones transfieren su energía a un único fotón de frecuencia $\omega_{4}=\omega_{1}+\omega_{2}+\omega_{3}$. Este término es responsable del fenómeno conocido como generación de tercer armónico cuando $\omega_{1}=\omega_{2}=\omega_{3}$, o conversión de frecuencia a la onda $2 \omega_{1}+\omega_{3}$ cuando $\omega_{1}=\omega_{2} \neq \omega_{3}$. En general, resulta difícil satisfacer la condición de fase para que estos procesos ocurran con altas eficiencias en fibras ópticas, por lo que desde este momento nos referiremos con FWM al segundo de los términos. Este último término corresponde al caso en que dos fotones de frecuencias $\omega_{1}$ y $\omega_{2}$ desaparecen junto con la creación simultánea de dos fotones a frecuencias $\omega_{3}$ y $\omega_{4}$ tales que

$$
\omega_{3}+\omega_{4}=\omega_{1}+\omega_{2}
$$

La condición de fase para que este proceso ocurra es $\Delta \mathrm{K}=0$, donde

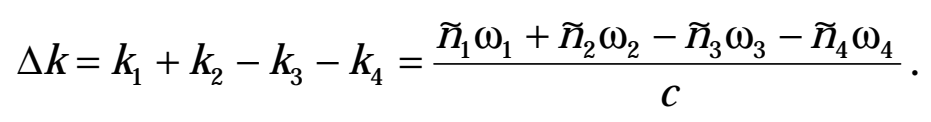

Sin embargo, resulta relativamente fácil de satisfacer $\Delta k=0$ en el caso particular en que $\omega_{1}=\omega_{2}$, en lo que se conoce como FWM parcialmente degenerado (PDFWM, partially degenerate FWM). Físicamente, se manifiesta de forma similar al scattering estimulado de Raman. Una fuerte

\footnotetext{
iv el FWM se incluye dentro de los Ilamados procesos paramétricos
} 
onda de bombeo a frecuencia $\omega_{1}$ crea dos bandas laterales localizadas de forma simétrica a las frecuencias $\omega_{3}$ y $\omega_{4}$, con un desplazamiento de frecuencia dado por

$$
\Omega_{\mathrm{S}}=\omega_{1}-\omega_{3}=\omega_{4}-\omega_{1}
$$

donde se ha supuesto por definición $\omega_{3}<\omega_{4}$. De hecho, a la banda inferior de frecuencia $\omega_{3}$ y a la superior de frecuencia $\omega_{4}$ se les conoce como bandas de Stokes y anti-Stokes, respectivamente, en analogía con el scattering Raman.

Así pues, el PDFWM transfiere la energía desde una fuerte onda de bombeo a dos ondas desplazadas $\Omega_{\mathrm{S}}$ de la frecuencia de bombeo $\omega_{1}$. Si únicamente incide la onda de bombeo en la fibra y se satisface la condición de fase, entonces las ondas de Stokes y anti-Stokes a frecuencias $\omega_{3}$ y $\omega_{4}$ pueden generarse a partir de ruido de forma similar a los procesos de scattering estimulado. Por otra parte, si también se inyecta una señal débil a $\omega_{3}$ junto con el bombeo, la señal se amplifica y una nueva onda a $\omega_{4}$ se genera simultáneamente. La ganancia responsable de dicha amplificación se conoce con el nombre de ganancia paramétrica.

\subsubsection{Ecuaciones del FWM en fibras ópticas monomodo}

Consideremos la propagación del proceso de FWM en una SMF. Utilizando notaciones similares a las de [Agr89], los campos eléctricos de las cuatro ondas pueden escribirse como

$$
E_{i}=A_{i}(z) F_{i}(x, y) \exp \left[j\left(\omega_{i} t-k_{i} z\right)\right], \quad i=1,2,3,4,
$$

donde $F_{i}(x, y)$ son funciones de onda que describen la distribución transversal de cada onda en la fibra. $A_{1}$ y $A_{2}$ representan las ondas de bombeo, mientras que $A_{3}$ es la señal de entrada y $A_{4}$ la señal conjugada generada por FWM. Obsérvese que $A_{i}$ no depende del tiempo, dado que se está analizando el caso de onda continua (sin modulación).

En el análisis se supondrá que las cuatro frecuencias se encuentran cerca del punto de dispersión nula de la SMF. No obstante, el espaciado entre las 
cuatro ondas se supone lo suficientemente grande como para que sólo se induzca FWM, mientras que procesos de mezclado de orden superior entre las nuevas ondas generadas y las de entrada puedan despreciarse. Además, el análisis se realiza sin considerar el SPM o XPM. Esto se justifica en el caso de potencias de entrada moderadas, lo cual es cierto para diodos láser operando cerca de 1300 ó $1500 \mathrm{~nm}$.

En primer lugar, se analizará el caso de FWM no degenerado, donde las cuatro frecuencias se consideran distintas. Este proceso está gobernado por el siguiente sistema de ecuaciones de modos acopladas [Agr89, Wu94]:

$$
\begin{aligned}
& \frac{d A_{1}}{d z}=-j \frac{N_{2} \omega_{1}}{c} 2 f_{1234} A_{2}^{*} A_{3} A_{4} \exp (j \Delta k z)-\frac{\alpha}{2} A_{1}, \\
& \frac{d A_{2}}{d z}=-j \frac{N_{2} \omega_{2}}{c} 2 f_{2134} A_{1}^{*} A_{3} A_{4} \exp (j \Delta k z)-\frac{\alpha}{2} A_{2}, \\
& \frac{d A_{3}}{d z}=-j \frac{N_{2} \omega_{3}}{c} 2 f_{3412} A_{1} A_{2} A_{4}^{*} \exp (-j \Delta k z)-\frac{\alpha}{2} A_{3}, \\
& \frac{d A_{4}}{d z}=-j \frac{N_{2} \omega_{4}}{c} 2 f_{4312} A_{1} A_{2} A_{3}^{*} \exp (-j \Delta k z)-\frac{\alpha}{2} A_{4},
\end{aligned}
$$

donde las $f_{i j k l}$ son constantes que describen el solapamiento de los modos en la SMF. Como se observa, los términos responsables del SPM y XPM se han descartado en (5.13)-(5.16), al igual que los efectos dispersivos puesto que no existe modulación de las portadoras.

Para resolver (5.13)-(5.16), se supondrá que $\omega_{1} \approx \omega_{2} \approx \omega_{3} \approx \omega_{4}$ y que las constantes $\mathrm{f}_{\mathrm{ijkl}}$ son iguales a $1 / \mathrm{A}_{\text {eff }}$ [Agr89]. Además, se analizará el caso en el que se satisfaga la condición de fase, es decir, $\Delta \mathrm{k}=0$. Esto puede conseguirse fijando $\omega_{1}-\omega_{\mathrm{ZD}}=\omega_{\mathrm{ZD}}-\omega_{2}$, donde $\omega_{\mathrm{ZD}}$ es la frecuencia de dispersión nula de la fibra. De ahí la importancia de trabajar cerca del punto de dispersión nula de la fibra, lo que conlleva en la práctica el uso de DSF s. Si a continuación sustituimos $A_{i}$ por

$$
A_{i}=\sqrt{P_{i}} \exp \left(j \phi_{i}\right)=\sqrt{Q_{i}} \exp (-\alpha z / 2) \exp \left(j \phi_{i}\right),
$$


donde $P_{\mathrm{i}}$ es la potencia óptica de cada una de las ondas, (5.13)-(5.16) puede escribirse como

$$
\begin{aligned}
& \frac{d Q_{1}}{d z}=-4 \gamma \sqrt{Q_{1} Q_{2} Q_{3} Q_{4}} \exp (-\alpha z) \operatorname{sen}(\Delta \phi), \\
& \frac{d Q_{2}}{d z}=-4 \gamma \sqrt{Q_{1} Q_{2} Q_{3} Q_{4}} \exp (-\alpha z) \operatorname{sen}(\Delta \phi), \\
& \frac{d Q_{3}}{d z}=+4 \gamma \sqrt{Q_{1} Q_{2} Q_{3} Q_{4}} \exp (-\alpha z) \operatorname{sen}(\Delta \phi), \\
& \frac{d Q_{4}}{d z}=+4 \gamma \sqrt{Q_{1} Q_{2} Q_{3} Q_{4}} \exp (-\alpha z) \operatorname{sen}(\Delta \phi),
\end{aligned}
$$

y

$$
\frac{\mathrm{d} \Delta \phi}{\mathrm{d} z}=2\left(\frac{1}{\mathrm{Q}_{3}}+\frac{1}{\mathrm{Q}_{4}}-\frac{1}{\mathrm{Q}_{1}}-\frac{1}{\mathrm{Q}_{2}}\right) \gamma \sqrt{\mathrm{Q}_{1} \mathrm{Q}_{2} \mathrm{Q}_{3} \mathrm{Q}_{4}} \exp (-\alpha \mathrm{z}) \cos (\Delta \phi),
$$

donde $\gamma=N_{2} \omega / \mathrm{CA}_{\text {eff }}$ y $\Delta \phi=\phi_{1}+\phi_{2}-\phi_{3}-\phi_{4}$. De las ecuaciones anteriores se puede deducir que existen cuatro invariantes independientes: $\mathrm{Q}_{1}+\mathrm{Q}_{2}+\mathrm{Q}_{3}+$ $\mathrm{Q}_{4}, \mathrm{Q}_{3}-\mathrm{Q}_{4}-\mathrm{Q}_{1}-\mathrm{Q}_{2}, \mathrm{y} \sqrt{\mathrm{Q}_{1} \mathrm{Q}_{2} \mathrm{Q}_{3} \mathrm{Q}_{4}} \cos (\Delta \phi)[\mathrm{Wu} 94]$.

Las soluciones de (5.22) pueden obtenerse inmediatamente como $\Delta \phi= \pm \pi / 2$. Esta solución también puede calcularse mediante el uso de los invariantes [Cap91]. Utilizando la condición de contorno $\mathrm{P}_{4}(0)=0$, se tiene que $\sqrt{\mathrm{Q}_{1} \mathrm{Q}_{2} \mathrm{Q}_{3} \mathrm{Q}_{4}} \cos (\Delta \phi)=0$ para todo $z$. Dado que las cuatro ondas son finitas cuando $z \neq 0$, se concluye que $\cos (\Delta \phi)=0$, por lo que $\Delta \phi= \pm \pi / 2$. De acuerdo con las condiciones de contorno y (5.18)-(5.21), se encuentra que $\Delta \phi=\pi / 2$ es una solución apropiada [Wu94]. Nótese que se ha supuesto que la cuarta onda de entrada (conjugada) está ausente, lo cual es válido en sistemas de comunicaciones ópticas donde se emplea la técnica de inversión espectral o conversión de longitud de onda.

Si sustituimos ahora la solución en (5.18)-(5.21), las ecuaciones acopladas pueden integrarse en el caso de idénticas potencias de bombeo, $P_{1}(0)=P_{2}(0)$ 
$=\mathrm{P}_{\mathrm{p}}(0)$. Con la ayuda de los invariantes y de las condiciones de contorno, la solución de las ecuaciones acopladas toma la forma [Wu94]:

$$
\begin{aligned}
& \mathrm{Q}_{1}=\mathrm{Q}_{2}=\mathrm{P}_{\mathrm{p}}(0) \sigma, \\
& \mathrm{Q}_{3}=\mathrm{P}_{\mathrm{p}}(0)(1-\sigma)+\mathrm{P}_{3}(0), \\
& \mathrm{Q}_{4}=\mathrm{P}_{\mathrm{p}}(0)(1-\sigma),
\end{aligned}
$$

donde

$$
\sigma=\frac{r+1}{r \cosh ^{2}(\sqrt{r+1} p)+1}
$$

con $r=P_{3}(0) / P_{p}(0)$ y $\rho=2 \gamma P_{p}(0)[1-\exp (-\alpha z)] / \alpha$.

En el caso de PDFWM donde $\omega_{1}=\omega_{2}$, únicamente están presentes tres ondas distintas. Así, (5.13)-(5.16) debe modificarse como

$$
\begin{aligned}
& \frac{d A_{1}}{d z}=-j \frac{N_{2} \omega_{1}}{c} 2 f_{1134} A_{1}^{*} A_{3} A_{4} \exp (j \Delta k z)-\frac{\alpha}{2} A_{1}, \\
& \frac{d A_{3}}{d z}=-j \frac{N_{2} \omega_{3}}{c} f_{3411} A_{1} A_{1} A_{4}^{*} \exp (-j \Delta k z)-\frac{\alpha}{2} A_{3}, \\
& \frac{d A_{4}}{d z}=-j \frac{N_{2} \omega_{4}}{c} f_{4311} A_{1} A_{1} A_{3}^{*} \exp (-j \Delta k z)-\frac{\alpha}{2} A_{4},
\end{aligned}
$$

donde $\Delta \mathrm{k}=2 \mathrm{k}_{1}-\mathrm{k}_{3}-\mathrm{k}_{4}$. Con condiciones de contorno $\mathrm{P}_{1}(0)=\mathrm{P}_{\mathrm{p}}(0)$ y $\mathrm{P}_{4}(0)=$ 0 , la solución de (5.27)-(5.29), suponiendo de nuevo $\Delta k=0$, puede obtenerse de forma similar [Wu94]:

$$
\begin{aligned}
& \mathrm{Q}_{1}=\mathrm{P}_{\mathrm{p}}(0) \sigma, \\
& \mathrm{Q}_{3}=\frac{1}{2} \mathrm{P}_{\mathrm{p}}(0)(1-\sigma)+\mathrm{P}_{3}(0), \\
& \mathrm{Q}_{4}=\frac{1}{2} \mathrm{P}_{\mathrm{p}}(0)(1-\sigma),
\end{aligned}
$$




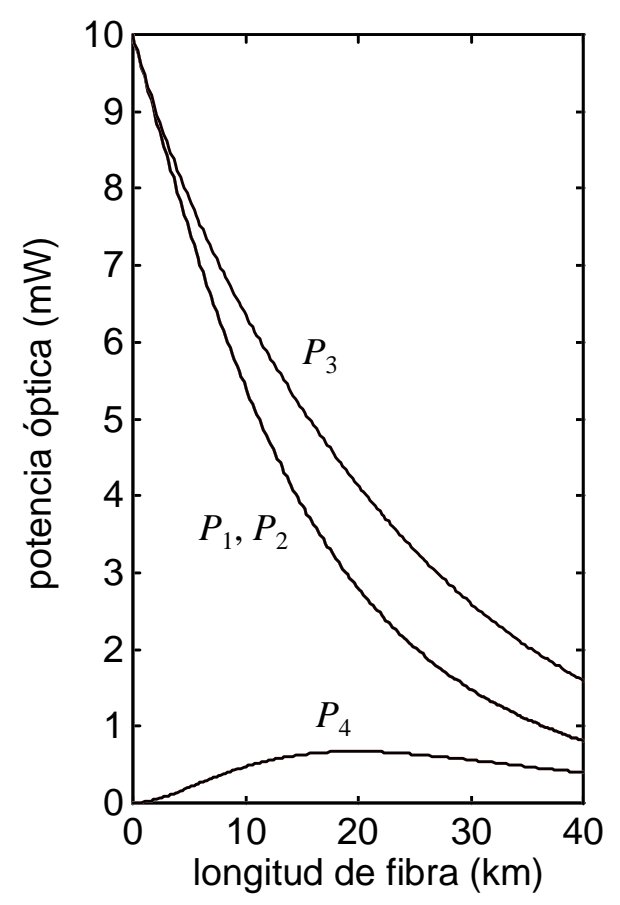

(a)

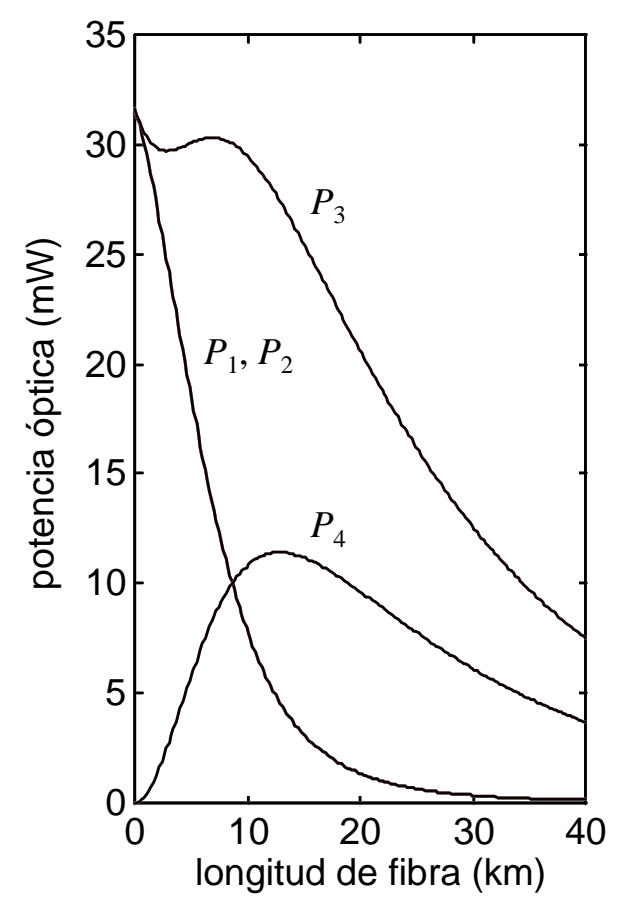

(b)

Figura 5.1 Acoplamiento de potencia debido a FWM no degenerado en una DSF en función de la longitud de fibra. Las potencias ópticas de entrada son de (a) $+10 \mathrm{dBm}$ y (b) $+15 \mathrm{dBm}$. Otros parámetros: $\lambda=$ $1550 \mathrm{~nm}, \alpha=0,23 \mathrm{~dB} / \mathrm{km}$ y $\mathrm{A}_{\text {eff }}=70 \mu \mathrm{m}^{2}$.

donde $\sigma$ viene de nuevo dado por (5.26), con los parámetros $r$ y $\rho$ reemplazados por $2 \mathrm{P}_{3}(0) / \mathrm{P}_{\mathrm{p}}(0)$ y $\gamma \mathrm{P}_{\mathrm{p}}(0)[1-\exp (-\alpha z)] / \alpha$, respectivamente.

La magnitud de $\sigma$ es una medida de la transferencia de potencia entre las ondas de bombeo y la nueva onda generada. En ausencia de atenuación $\alpha$, la función $\rho$ se reduce a $2 \gamma \mathrm{P}_{\mathrm{p}}(0) z$. Con el aumento de la longitud de fibra $z, \sigma$ tiende a cero, lo que significa una completa transferencia de potencia a la nueva onda conjugada. No obstante, la absorción de la fibra no puede despreciarse desde un punto de vista práctico, por lo que $\sigma$ es función de $[1-\exp (-\alpha z)] / \alpha$. Se puede concluir, entonces, que el acoplamiento de potencia desde la onda de bombeo a la conjugada cesará si $z \gg$ 1/a. La evolución de las potencias ópticas de cada una de las ondas se representa en la figura 5.1 para una DSF. Se observa que cuando $z$ es mayor que un cierto valor (en torno a $13 \mathrm{~km}$ para $\mathrm{P}_{\mathrm{p}}(0)=\mathrm{P}_{3}(0)=+15 \mathrm{dBm}$ ), la atenuación de la fibra prevalece. Así pues, la potencia de la onda conjugada comienza a 


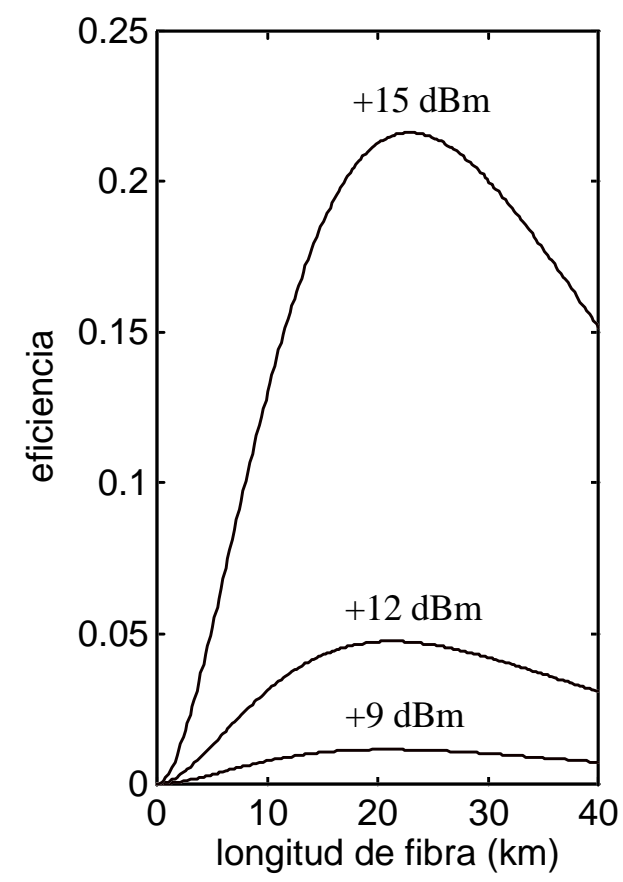

(a)

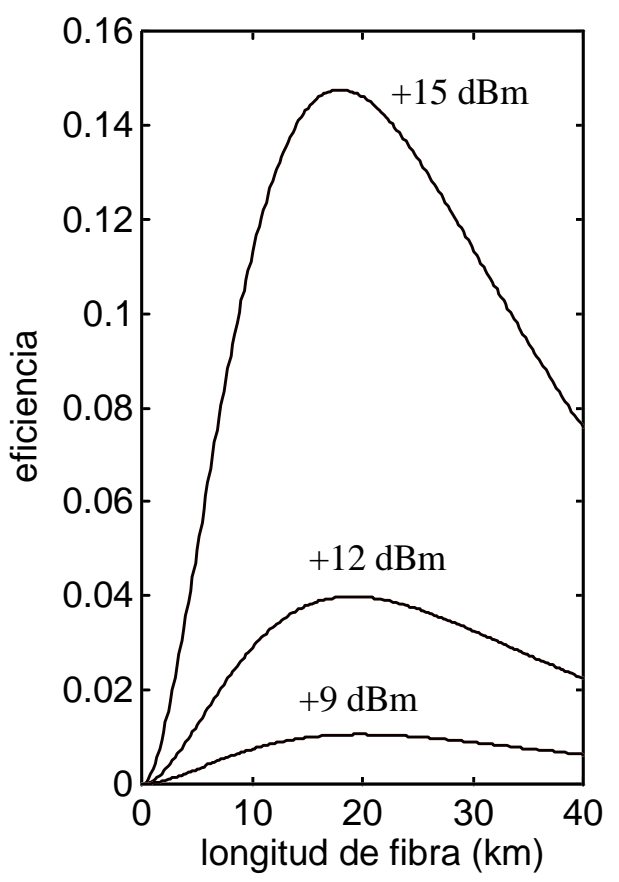

(b)

Figura 5.2 Eficiencia de la conjugación mediante PDFWM en una DSF. La potencia óptica de entrada es: (a) $P_{3}(0)=-6 \mathrm{dBm}$ y (b) $P_{3}(0)$ $=+12 \mathrm{dBm}$.

decrecer, existiendo una longitud de fibra para la cual se obtiene un máximo. Precisamente, esta longitud de fibra óptima puede calcularse fijando $\mathrm{dP}_{4} / \mathrm{dz}=0$, llegándose a la siguiente relación ${ }^{\mathrm{v}}$ :

$$
2 \sigma \sqrt{r+1} \frac{d \rho}{d z}=\alpha \operatorname{tgh}(\sqrt{r+1} \rho)
$$

Si se cumple que $\gamma \mathrm{P}_{\mathrm{p}}(0) / \alpha \ll 1$, entonces $\sigma \approx 1$ y la tangente hiperbólica puede aproximarse por su argumento. La longitud de fibra óptima, tras operar, viene dada entonces por (In 3)/ $\alpha$, lo cual equivale a 20,74 km para una atenuación de $0,23 \mathrm{~dB} / \mathrm{km}$.

La eficiencia del proceso de conjugación, definida como $\mathrm{P}_{4}(\mathrm{z}) / \mathrm{P}_{3}(0)$, puede escribirse como [Wu94]

\footnotetext{
${ }^{\vee}$ obsérvese que en la ec. (9) de [Wu94] falta el término $(r+1)^{1 / 2}$
} 


$$
\eta=\frac{\operatorname{senh}^{2}(\sqrt{r+1} \rho)}{r \cosh ^{2}(\sqrt{r+1} \rho)+1} \exp (-\alpha z)
$$

La dependencia de la eficiencia con la longitud de fibra se representa en la figura 5.2 para distintos valores de potencia de bombeo y en el caso de PDFWM. $\mathrm{P}_{3}(0)$ se ha considerado $-6 \mathrm{dBm}$ y $+12 \mathrm{dBm}$ en 5.2(a) y 5.2(b), respectivamente. Nótese que para potencias $\mathrm{P}_{3}$ inferiores se consigue una mayor eficiencia, aunque la potencia de salida $\mathrm{P}_{4}$ es menor. $\mathrm{Para} \mathrm{P}_{\mathrm{p}}(0)=+15$ $\mathrm{dBm}$ y $\mathrm{P}_{3}(0)=-6 \mathrm{dBm}$ se obtiene un máximo de eficiencia del $22 \%(-6,6$ $d B)$.

\subsubsection{Influencia de la condición de fase}

En el apartado anterior se ha realizado un estudio teórico del proceso de FWM considerando que se cumplía perfectamente la condición de fase, es decir, $\Delta \mathrm{k}=0$. A continuación, analizaremos la influencia que tiene este parámetro en la eficiencia del proceso no lineal.

La potencia de salida de FWM puede escribirse como [Hil78]

$$
P_{4}(z)=\frac{1024 \pi^{6}}{n_{0}^{4} \lambda^{2} c^{2}}\left(D_{F} \chi\right)^{2} \frac{P_{1}(0) P_{2}(0) P_{3}(0)}{A_{\text {eff }}^{2}} \frac{[1-\exp (-\alpha z)]^{2}}{\alpha^{2}} \exp (-\alpha z) \eta_{\beta}
$$

donde $D_{F}$ es el factor de degeneración y $\eta_{\beta}$ representa la dependencia de la eficiencia del FWM con la condición de fase $\Delta \beta$, la cual viene dada por

$$
\eta_{\beta}=\frac{\alpha^{2}}{\alpha^{2}+\Delta \beta^{2}}\left\{1+\frac{4 \exp (-\alpha z) \operatorname{sen}^{2}(\Delta \beta z / 2)}{[1-\exp (-\alpha z)]^{2}}\right\}
$$

con

$$
\Delta \beta=\beta\left(\omega_{1}\right)+\beta\left(\omega_{2}\right)-\beta\left(\omega_{3}\right)-\beta\left(\omega_{4}\right),
$$

donde $\beta$ es la constante de propagación. Al igual que antes, la eficiencia toma el valor máximo de 1 para $\Delta \beta=0$ (condición de fase). 
Si a continuación desarrollamos $\Delta \beta$ al rededor de la frecuencia de dispersión nula de la fibra, se obtiene [I no92]

$$
\Delta \beta=-\frac{\lambda_{\mathrm{ZD}}^{4}}{8 \pi^{2} \mathrm{c}^{2}} \frac{\mathrm{dD}}{\mathrm{d} \lambda}\left[\left(\omega_{1}-\omega_{\mathrm{ZD}}\right)+\left(\omega_{2}-\omega_{\mathrm{ZD}}\right)\right]\left(\omega_{1}-\omega_{3}\right)\left(\omega_{2}-\omega_{3}\right) .
$$

Obsérvese que la condición de fase se satisface cuando la frecuencia de dispersión nula se sitúa en el punto medio entre las dos ondas de frecuencias $\omega_{1}$ y $\omega_{2}$. De este modo, la onda generada por FWM se sitúa simétricamente a $\omega_{3}$ con respecto al punto $\omega_{\mathrm{ZD}}$. Esto mismo se ilustra en la figura 5.3(a).

Cuando dos de las tres ondas son degeneradas, $\omega_{1}=\omega_{2}$, la ecuación anterior se reescribe como

$$
\Delta \beta=-\frac{\lambda_{\mathrm{ZD}}^{4}}{4 \pi^{2} \mathrm{c}^{2}} \frac{\mathrm{dD}}{\mathrm{d} \lambda}\left(\omega_{1}-\omega_{3}\right)^{2}\left(\omega_{1}-\omega_{\mathrm{ZD}}\right)
$$

Nótese que ahora la condición de fase se satisface cuando $\omega_{1}$ coincide con la frecuencia de dispersión nula. Esto mismo se ilustra en la figura 5.3(b).

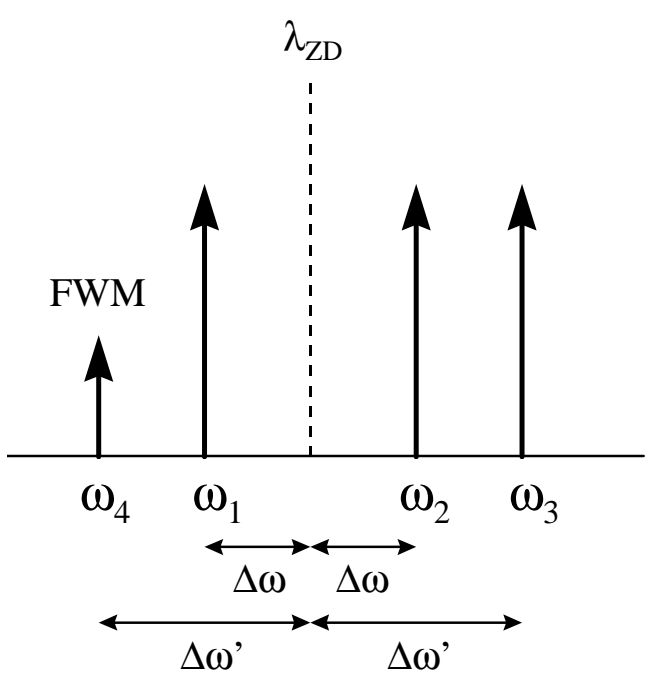

(a)

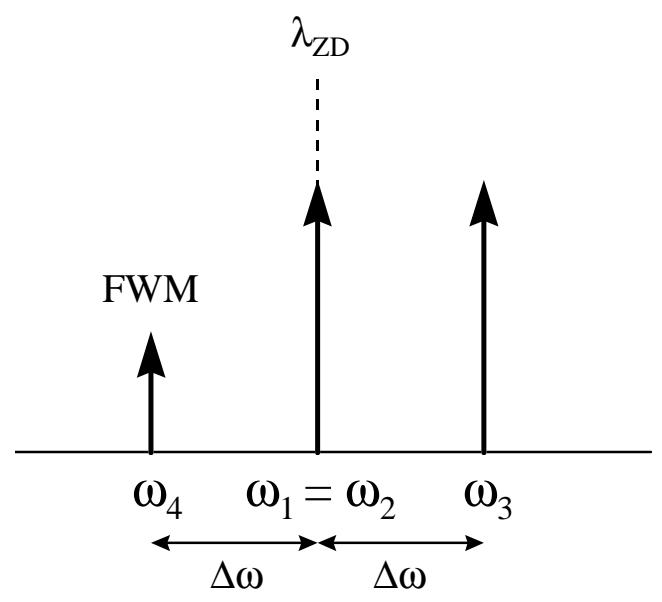

(b)

Figura 5.3 Disposición de portadoras ópticas para satisfacer la condición de fase en la región de dispersión nula de la fibra: (a) caso no degenerado, (b) caso parcial mente degenerado. 


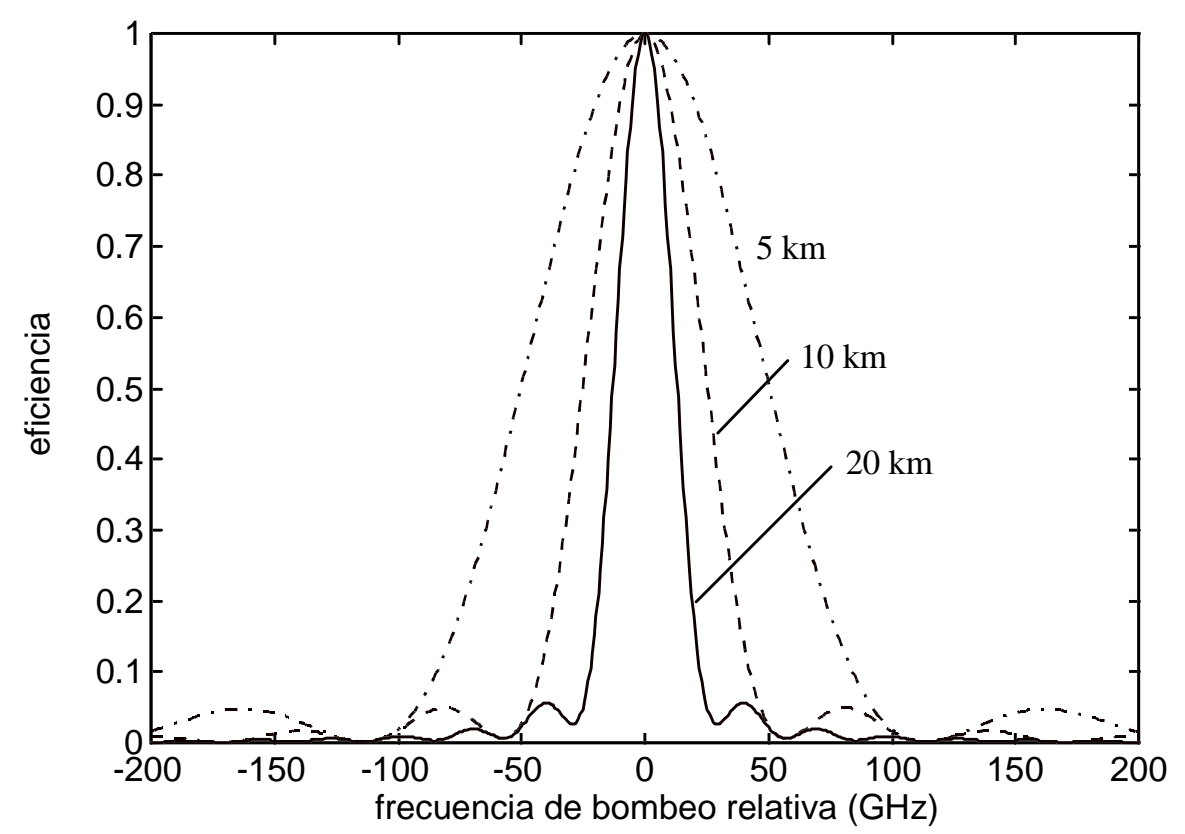

(a)



(b)

Figura 5.4 Eficiencia del PDFWM en función de la frecuencia de bombeo relativa $\left(\omega_{1}-\omega_{\mathrm{ZD}}\right) / 2 \pi$ para (a) varias longitudes de DSF y (b) varias separaciones entre la longitud de onda de señal y de bombeo $\lambda_{1}-\lambda_{3}$. Parámetros de la DSF : $\alpha=0,23 \mathrm{~dB} / \mathrm{km}, \lambda_{\text {ZD }}=1550$ $\mathrm{nm}$ y $\mathrm{S}=0,07 \mathrm{ps} /\left(\mathrm{km} \cdot \mathrm{nm}^{2}\right)$. En (a) $\lambda_{3}-\lambda_{1}=5 \mathrm{~nm}$ y en (b) $\mathrm{z}=10$ $\mathrm{km}$. 
La dependencia de la eficiencia del FWM con la condición de fase alrededor de la longitud de onda de dispersión nula se calcula, en el caso de PDFWM, a partir de (5.36) y (5.39). Los resultados se muestran en la figura 5.4 en función de la diferencia de frecuencias $\omega_{1}-\omega_{Z D}$, y variando la longitud de fibra o la separación en longitud de onda entre la señal de bombeo y la de entrada. Se observa que la eficiencia es máxima cuando la señal de bombeo se encuentra en la longitud de onda de dispersión nula, y decrece rápidamente conforme se separa de este punto. Además, el ancho de banda del proceso depende de la separación entre las ondas de entrada y de la longitud de DSF. Así, el ancho de banda es inferior cuando las ondas se encuentran más alejadas entre sí o la longitud de fibra es mayor.

\subsubsection{Influencia de otros efectos no lineales}

Anteriormente se ha estudiado teóricamente el proceso de FWM en SMFs sin tener en cuenta posibles efectos no lineales como el SPM, XPM o el scattering estimulado de Brillouin (SBS, stimulated Brillouin scattering). A continuación veremos la influencia que pueden tener estos efectos.

Ya se ha visto que la eficiencia del FWM tiene una clara dependencia con la condición de fase $\Delta \beta$. En particular, la ec. (5.39) puede expresarse en función de las longitudes de onda en lugar de las frecuencias como

$$
\Delta \beta=2 \beta\left(\omega_{\mathrm{P}}\right)-\beta\left(\omega_{\mathrm{S}}\right)-\beta\left(\omega_{\mathrm{C}}\right)=-\frac{2 \pi \mathrm{C}_{\mathrm{ZD}}^{3}}{\lambda_{\mathrm{P}}^{3} \lambda_{\mathrm{S}}^{2}} \frac{\mathrm{dD}}{\mathrm{d} \lambda}\left(\lambda_{\mathrm{S}}-\lambda_{\mathrm{P}}\right)^{2}\left(\lambda_{\mathrm{ZD}}-\lambda_{\mathrm{P}}\right),
$$

donde se han utilizado los subíndices S, P y C para denotar la señal, el bombeo y la conjugada en el caso de PDFWM. Sin embargo, cuando se inyecta una intensa onda de bombeo en la fibra, la condición de fase varía con la potencia de bombeo como consecuencia del cambio en el índice de refracción causado por el efecto Kerr. En este caso, la condición de fase $\kappa$ se expresa como [Agr89, Yam97]

$$
\kappa=\Delta \beta-2 \gamma \mathrm{P}_{\mathrm{p}} .
$$




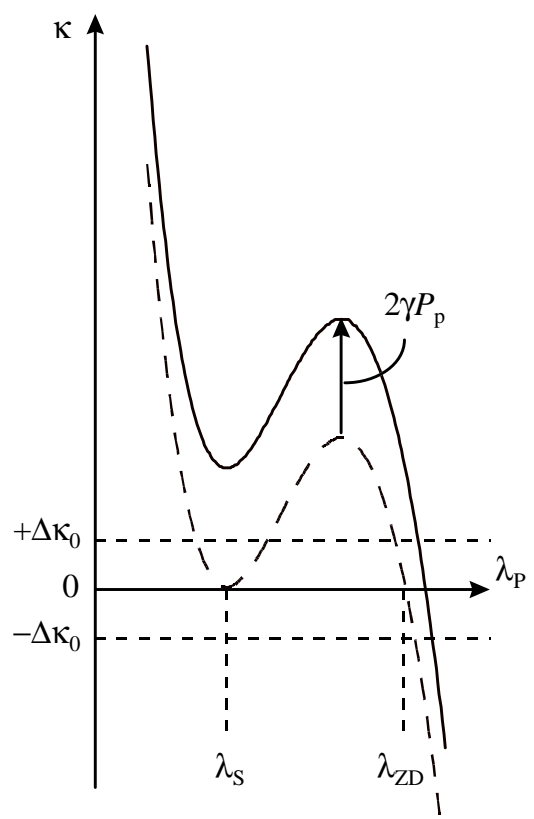

(a)



(b)

Figura 5.5 Dependencia de la condición de fase $\kappa$ con la longitud de onda de bombeo. (a) $\lambda_{s}<\lambda_{Z D}$, (b) $\lambda_{s}>\lambda_{Z D}$.

La figura 5.5 muestra la dependencia de $\kappa$ con la longitud de onda de bombeo para (a) $\lambda_{\mathrm{S}}<\lambda_{\mathrm{ZD}}$ y (b) $\lambda_{\mathrm{S}}>\lambda_{\mathrm{ZD}}$. Las líneas de trazos representan la situación en que la potencia de bombeo es pequeña y se puede ignorar la dependencia de $\kappa$ con la intensidad. Así, $\kappa=0$ para $\lambda_{P}=\lambda_{Z D} \circ \lambda_{P}=\lambda_{S}$, como se vio con anterioridad. Por otro lado, cuando la potencia de bombeo es elevada, $\kappa$ se desplaza hacia arriba la cantidad $2 \gamma \mathrm{P}_{\mathrm{p}}$. En particular, la ec. (5.41) muestra que es posible aumentar el desplazamiento utilizando una fibra con área efectiva pequeña y el evando la intensidad $\mathrm{P}_{\mathrm{p}} / \mathrm{A}_{\text {eff. }}$. Cuando la condición de fase se modifica por medio de una intensa onda de bombeo, $\kappa$ resulta nulo a una longitud de onda superior a $\lambda_{\text {ZD }}$. Además, la región de longitudes de onda de bombeo en la que $\kappa$ se acerca a cero también se modifica. Para ilustrarlo, tomemos un pequeño desplazamiento $\Delta \kappa_{0}$ de val or arbitrario. Resulta evidente que cuando $\lambda_{S}>\lambda_{Z D}$, el ancho de banda dentro de $\pm \Delta \kappa_{0}$ aumenta como consecuencia del desplazamiento de $\kappa$. La región de longitudes de onda en la que $\kappa$ se encuentra dentro de $\pm \Delta \kappa_{0}$ se ensancha cuando $\Delta \kappa_{\mathrm{pp}}$ es pequeño, donde este último se expresa como [Yam97] 




(a)



(b)

Figura 5.6 Ganancia de conversión en función de la longitud de onda de bombeo $\left(\lambda_{P}-\lambda_{Z D}\right)$ para (a) una DSF ordinaria o (b) una fibra de núcleo reducido. La línea de trazos es para $\lambda_{S}=\lambda_{\mathrm{ZD}}-8$ $\mathrm{nm}$ y la continua para $\lambda_{\mathrm{S}}=\lambda_{\mathrm{ZD}}+8 \mathrm{~nm}$. La potencia de bombeo es de $+18 \mathrm{dBm}$. 


$$
\Delta \kappa_{\mathrm{pp}}=\frac{8 \pi \mathrm{c} \lambda_{\mathrm{ZD}}\left|\lambda_{\mathrm{s}}-\lambda_{\mathrm{ZD}}\right|^{3}}{27 \lambda_{\mathrm{s}}^{3}} \frac{\mathrm{dD}}{\mathrm{d} \lambda}
$$

Por lo tanto, utilizando una fibra con área efectiva y dispersión de segundo orden inferiores a las de una DSF ordinaria, es posible aumentar la intensidad y ensanchar la región de longitudes de onda de bombeo en la que se produce FWM de forma eficiente.

La ganancia del proceso de FWM (= potencia onda generada/potencia onda señal) puede derivarse de forma analítica cuando se supone que la potencia de bombeo es mucho mayor que la de señal y permanece sin variación [Agr89]:

$$
\mathrm{G}_{\mathrm{FWM}}=\left(1+\frac{\kappa^{2}}{4 g^{2}}\right) \operatorname{senh}^{2}(\mathrm{gz})
$$

donde g se expresa como

$$
g=\sqrt{\left(\gamma P_{p}\right)^{2}-\left(\frac{\kappa}{2}\right)^{2}}
$$

A continuación, calcularemos la ganancia de conversión para dos tipos de fibra: una DSF ordinaria $\left(A_{\text {eff }}=50 \mu \mathrm{m}^{2}, \mathrm{~S}=0,07 \mathrm{ps} / \mathrm{km} / \mathrm{nm}^{2}\right.$ ) y una fibra de núcleo reducido $\left(A_{\text {eff }}=15 \mu \mathrm{m}^{2}, \mathrm{~S}=0,03 \mathrm{ps} / \mathrm{km} / \mathrm{nm}^{2}\right)$. La longitud de fibra es de $5 \mathrm{~km}$ y la longitud de onda de dispersión nula de $1550 \mathrm{~nm}$ en ambos casos. La figura 5.6(a) muestra la dependencia de la ganancia de conversión con la longitud de onda de bombeo cuando se utiliza una DSF ordinaria. La curva de trazos es para $\lambda_{\mathrm{S}}=\lambda_{\mathrm{ZD}}-8 \mathrm{~nm}$, mientras que la continua es para $\lambda_{\mathrm{S}}$ $=\lambda_{\mathrm{ZD}}+8 \mathrm{~nm}$. La potencia de bombeo es de $+18 \mathrm{dBm}$. Se observa en ambos casos que la ganancia es inferior a $0 \mathrm{~dB}$ y que la ganancia decrece rápidamente conforme la longitud de onda de bombeo se aleja de la longitud de onda de dispersión nula de la DSF. Por otro lado, en la figura 5.6(b) se representan los mismos resultados en el caso de una fibra de núcleo reducido, obteniéndose una ganancia de hasta $18 \mathrm{~dB}$. Además, cuando $\lambda_{\mathrm{S}}=$ $\lambda_{\mathrm{ZD}}+8 \mathrm{~nm}$ (línea continua) se consigue ensanchar de forma apreciable la región de longitudes de onda de bombeo donde se alcanza la condición de 


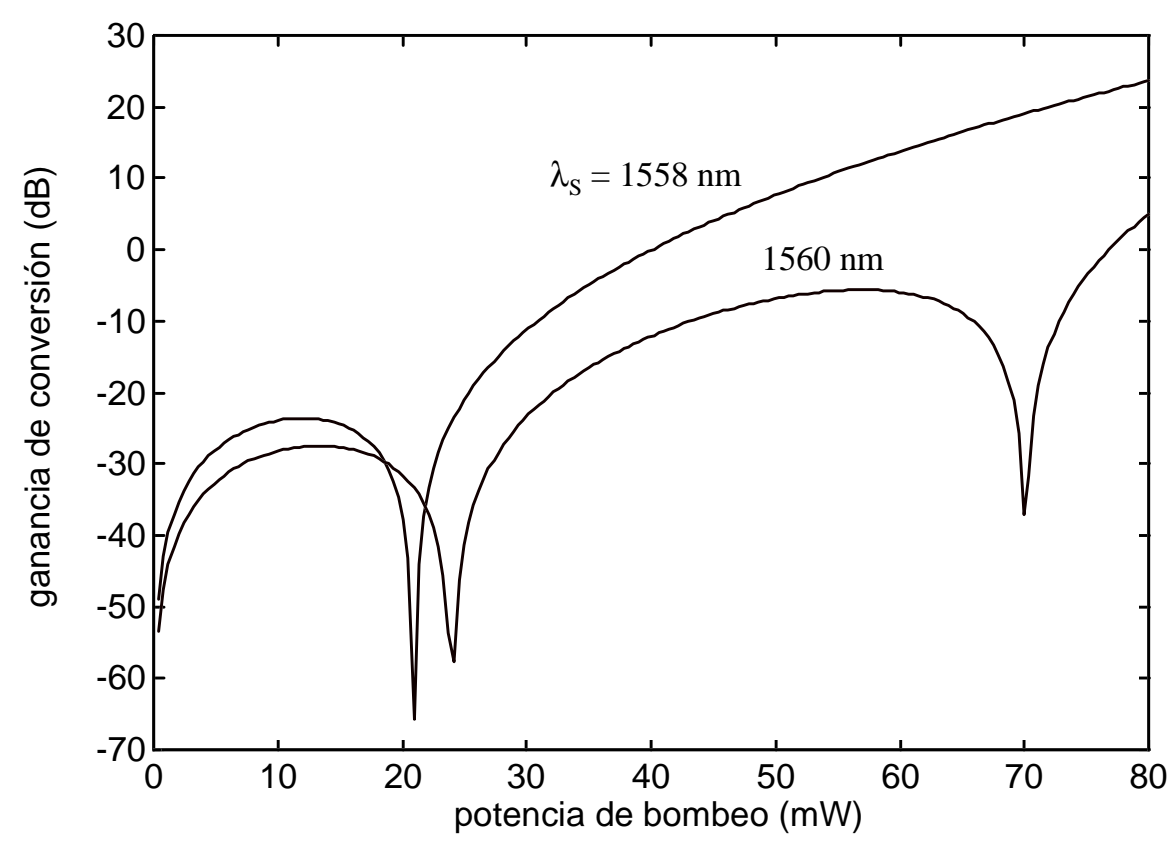

Figura 5.7 Ganancia de conversión en función de la potencia de bombeo para dos longitudes de onda de señal y $\lambda_{P}=1552 \mathrm{~nm}$. Los parámetros de la fibra son: $\lambda_{\mathrm{ZD}}=1550 \mathrm{~nm}, \mathrm{~S}=0,03 \mathrm{ps} / \mathrm{km} / \mathrm{nm}^{2}$ y $A_{\text {eff }}=15 \mu \mathrm{m} 2$.

fase. En particular, se obtiene una ganancia superior a los $10 \mathrm{~dB}$ para un margen de unos $8 \mathrm{~nm}$.

Finalmente, la dependencia de la ganancia de conversión con la potencia de bombeo se muestra en la figura 5.7. Para potencias de bombeo por encima de los $25 \mathrm{~mW}$, la ganancia crece rápidamente. En particular, se consigue un aumento de ganancia de unos $30 \mathrm{~dB}$ (de $-20 \mathrm{a}+10 \mathrm{~dB}$ ) con un incremento en la potencia de tan sólo $3 \mathrm{~dB}$ (de 25 a $50 \mathrm{~mW}$ ). Sin embargo, ésta presenta algunas lobulaciones dependiendo de la longitud de onda de señal que hacen que no se produzca FWM para determinados valores de potencia de bombeo. Esto será, pues, un factor que habrá que tener en cuenta.

En el apartado 5.2.2 analizamos de forma teórica las ecuaciones del FWM en fibras ópticas sin tener en cuenta otros efectos como el SPM, el XPM o la dispersión cromática. Para realizar un estudio más completo, ahora partiremos del sistema general de ecuaciones de onda acopladas que modela la generación y propagación del FWM a través de una fibra [Mar91, Zha96]: 


$$
\frac{\partial A_{i}}{\partial z}+\beta_{1}\left(\omega_{i}\right) \frac{\partial A_{i}}{\partial t}-\frac{j}{2} \beta_{2}\left(\omega_{i}\right) \frac{\partial^{2} A_{i}}{\partial t^{2}}-\frac{1}{6} \beta_{3}\left(\omega_{i}\right) \frac{\partial^{3} A_{i}}{\partial t^{3}}=-\frac{\alpha}{2} A_{i}-j \gamma_{i} B_{i},
$$

para $\mathrm{i}=1,2,3$ y 4 , y donde las funciones $B_{i}$ se definen como sigue:

$$
\begin{aligned}
B_{1} & =\left(\left|A_{1}\right|^{2}+2\left|A_{2}\right|^{2}+2\left|A_{3}\right|^{2}+2\left|A_{4}\right|^{2}\right) A_{1} \\
& +A_{2}^{2} A_{4}^{*} \exp \left[-j\left(2 \beta\left(\omega_{2}\right)-\beta\left(\omega_{1}\right)-\beta\left(\omega_{4}\right)\right) z\right] \\
& +2 A_{1}^{*} A_{2} A_{3} \exp \left[-j\left(\beta\left(\omega_{3}\right)+\beta\left(\omega_{2}\right)-2 \beta\left(\omega_{1}\right)\right) z\right] \\
& +2 A_{2}^{*} A_{3} A_{4} \exp \left[-j\left(\beta\left(\omega_{3}\right)+\beta\left(\omega_{4}\right)-\beta\left(\omega_{1}\right)-\beta\left(\omega_{2}\right)\right) z\right], \\
B_{2} & =\left(2\left|A_{1}\right|^{2}+\left|A_{2}\right|^{2}+2\left|A_{3}\right|^{2}+2\left|A_{4}\right|^{2}\right) A_{2} \\
& +A_{1}^{2} A_{3}^{*} \exp \left[-j\left(2 \beta\left(\omega_{1}\right)-\beta\left(\omega_{2}\right)-\beta\left(\omega_{3}\right)\right) z\right] \\
& +2 A_{1} A_{2}^{*} A_{4} \exp \left[-j\left(\beta\left(\omega_{1}\right)-2 \beta\left(\omega_{2}\right)+\beta\left(\omega_{4}\right)\right) z\right] \\
& +2 A_{1}^{*} A_{3} A_{4} \exp \left[-j\left(\beta\left(\omega_{3}\right)+\beta\left(\omega_{4}\right)-\beta\left(\omega_{1}\right)-\beta\left(\omega_{2}\right)\right) z\right], \\
B_{3} & =\left(2\left|A_{1}\right|^{2}+2\left|A_{2}\right|^{2}+\left|A_{3}\right|^{2}+2\left|A_{4}\right|^{2}\right) A_{3} \\
& +A_{1}^{2} A_{2}^{*} \exp \left[-j\left(2 \beta\left(\omega_{1}\right)-\beta\left(\omega_{2}\right)-\beta\left(\omega_{3}\right)\right) z\right] \\
& +2 A_{1} A_{2} A_{4}^{*} \exp \left[-j\left(\beta\left(\omega_{1}\right)+\beta\left(\omega_{2}\right)-\beta\left(\omega_{3}\right)-\beta\left(\omega_{4}\right)\right) z\right], \\
B_{4} & =\left(2\left|A_{1}\right|^{2}+2\left|A_{2}\right|^{2}+2\left|A_{3}\right|^{2}+\left|A_{4}\right|^{2}\right) A_{4} \\
& +A_{2}^{2} A_{1}^{*} \exp \left[-j\left(2 \beta\left(\omega_{2}\right)-\beta\left(\omega_{1}\right)-\beta\left(\omega_{4}\right)\right) z\right] \\
& +2 A_{1} A_{2} A_{3}^{*} \exp \left[-j\left(\beta\left(\omega_{1}\right)+\beta\left(\omega_{2}\right)-\beta\left(\omega_{3}\right)-\beta\left(\omega_{4}\right)\right) z\right] .
\end{aligned}
$$

Las frecuencias de las ondas se relacionan entre sí de acuerdo a

$$
\begin{aligned}
& \omega_{3}=2 \omega_{1}-\omega_{2}, \\
& \omega_{4}=2 \omega_{2}-\omega_{1} .
\end{aligned}
$$

Dado que vamos a realizar un análisis de onda continua, se despreciarán los términos con derivadas temporales, lo cual es equivalente a que la dispersión cromática afecte únicamente sobre la condición de fase puesto que no existen señales de modulación. De este modo, las principales diferencias con respecto a las ecuaciones del apartado 5.2.2 son la inclusión 


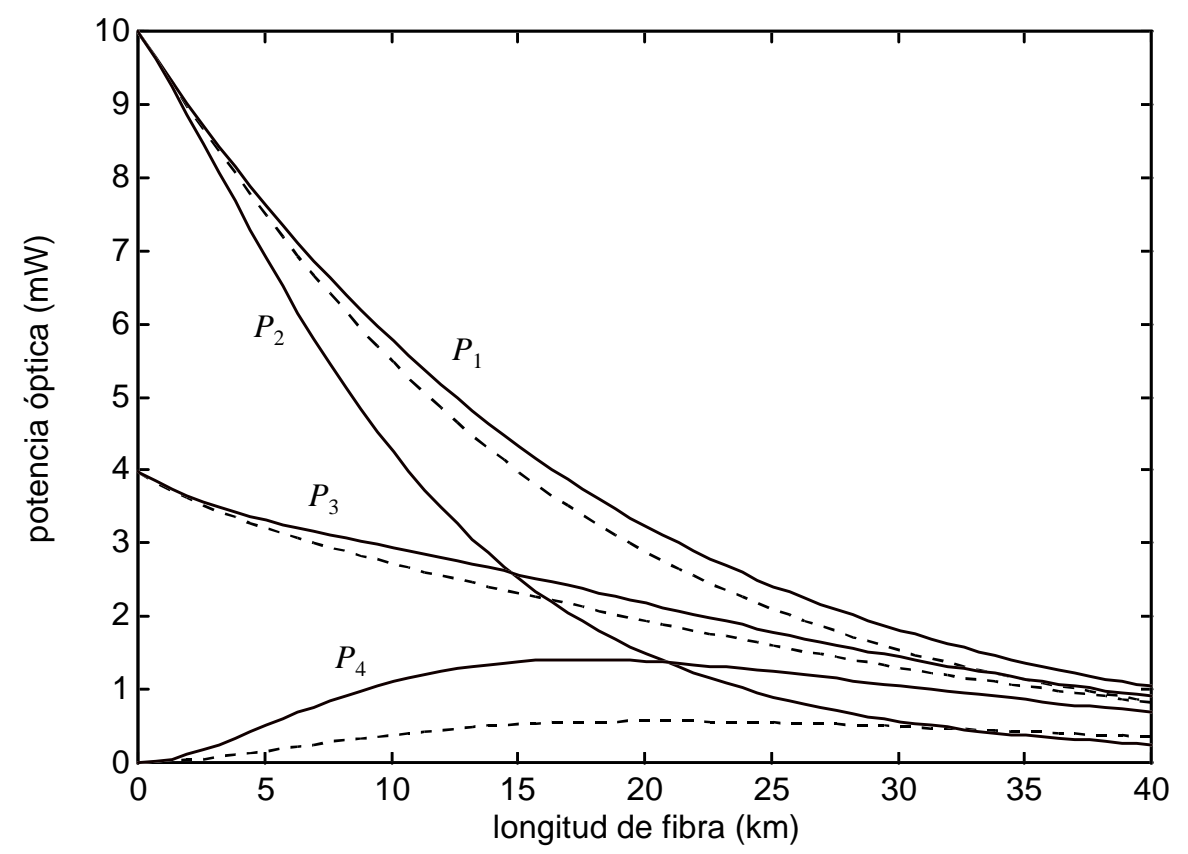

Figura 5.8 Efecto de considerar todos los productos FWM además del término dominante en una DSF. Las curvas de trazos son los resultados teóricos de [Wu94]. Otros parámetros: $\beta_{3}=0$, $A_{\text {eff }}=50 \mu \mathrm{m}^{2}, \alpha=0,23 \mathrm{~dB} / \mathrm{km}, \lambda_{\mathrm{ZD}}=1550 \mathrm{~nm}, \lambda_{1}=1550,5 \mathrm{~nm}, \lambda_{2}=$ $1549,5 \mathrm{~nm}, \lambda_{3}=1551,5 \mathrm{~nm}$ y $\lambda_{4}=1548,5 \mathrm{~nm}$.

de términos de SPM y XPM, así como la presencia de diversos productos de FWMvi.

En la figura 5.8 se muestra inicialmente la desviación que sufren los resultados teóricos de [Wu94] (líneas de trazos) al considerar todos los productos de FWM que intervienen en el proceso. Las potencias de bombeo y de señal a la entrada de la DSF son de +10 y $+6 \mathrm{dBm}$, respectivamente. En un principio, las curvas de las potencias de bombeo, $\mathrm{P}_{1}$ y $\mathrm{P}_{2}$, se desdoblan. En el caso de la portadora óptica situada junto a la nueva onda generada, la potencia óptica decrece más rápidamente con la longitud de fibra. Por otro lado, la potencia de las ondas $\mathrm{P}_{1}$ y $\mathrm{P}_{3}$ aumenta ligeramente. Sin embargo, la potencia de la onda conjugada, $\mathrm{P}_{4}$, aumenta claramente (del orden del doble) al considerar todos los términos de FWM. Así pues, resulta claro que

\footnotetext{
vi anteriormente sólo se consideraba la contribución predominante
} 


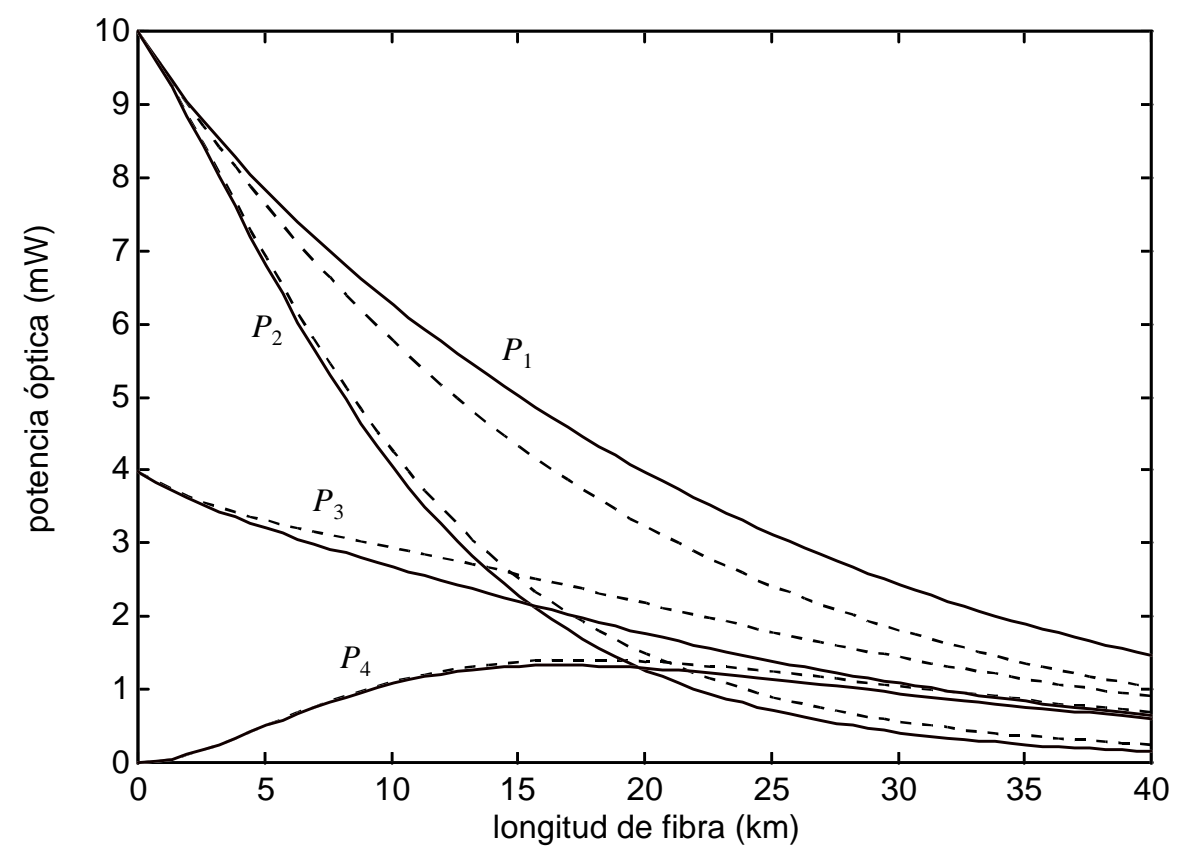

Figura 5.9 Efecto de considerar la dispersión cromática. La DSF se caracteriza por un parámetro $\mathrm{S}=0,1 \mathrm{ps} / \mathrm{km} / \mathrm{nm}^{2}$. Las curvas de trazos son las obtenidas en la figura anterior considerando los productos FWM.

debemos considerar este fenómeno para modelar de forma más correcta el proceso de conjugación en DSFs.

Si queremos mejorar el modelo de FWM todavía más, entonces es necesario considerar la dispersión cromática de la DSF. Aunque anteriormente se ha comentado que la dispersión no afecta sobre las portadoras ópticas por tratarse de ondas continuas, sí que influye sobre la condición de fase de cada uno de los productos FWM generados. Dada la disposición de las portadoras ópticas, el término de FWM predominante tendrá un valor nulo para la condición de fase, aunque esto no ocurrirá con el resto. En la figura 5.9 se representan los resultados que tiene este efecto. En este caso, se observa sól o un ligero aumento de las potencias ópticas de las ondas $P_{1}$ y $P_{3}$.

Por último, en la figura 5.10 se muestra la influencia que tienen el SPM y el XPM. Como se puede ver, su influencia es mínima, sobre todo en el caso de la onda conjugada. De hecho, en [Wu94] ya se comentó que tanto el SPM como el XPM pueden despreciarse para potencias de entrada por debajo de 


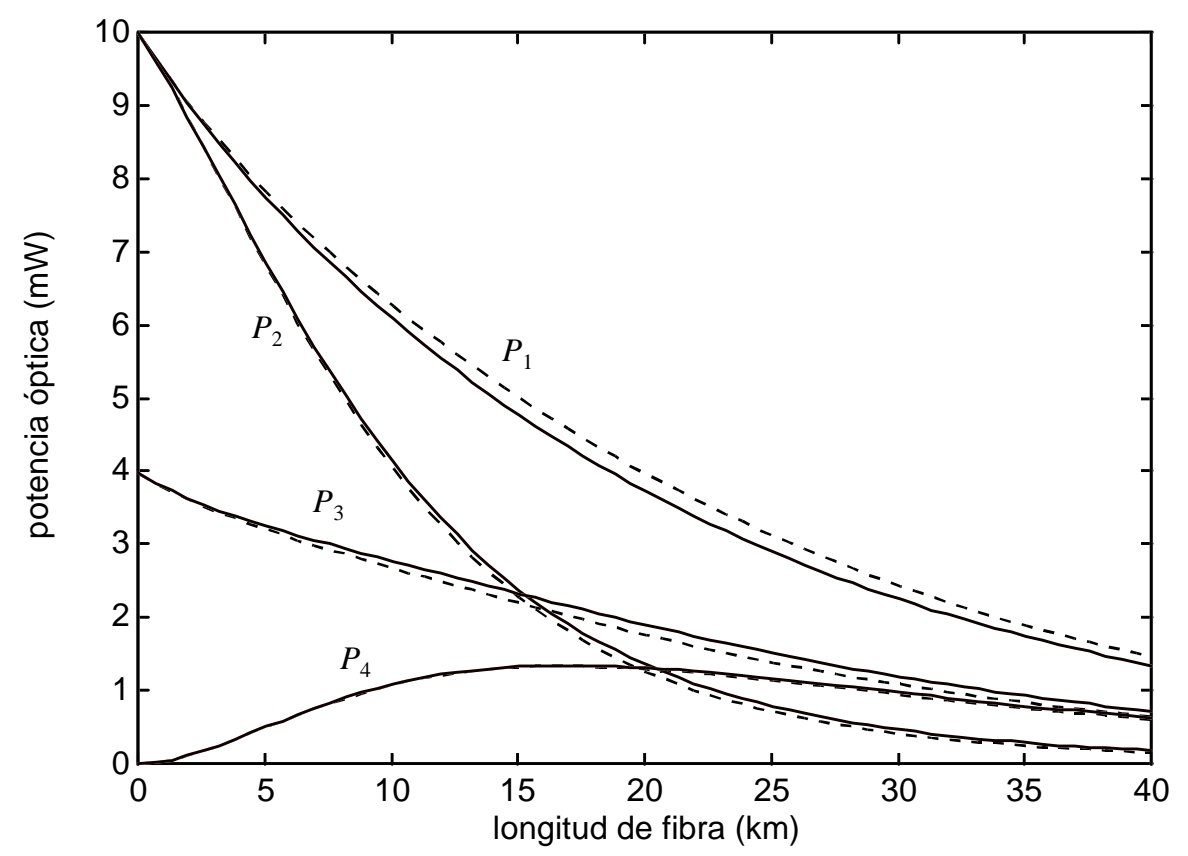

Figura 5.10 Efecto de considerar el SPM y el XPM. Las curvas de trazos son las obtenidas en la figura anterior para $\beta_{3} \neq 0$.

los $+16 \mathrm{dBm}$. Centrándonos en la nueva onda generada, en la figura 5.11 se resume la influencia del SPM y XPM para distintos valores de potencias ópticas. Por ejemplo, para unas potencias de bombeo de $+15 \mathrm{dBm}$ y de señal de $+6 \mathrm{dBm}$, la potencia de la onda conjugada aumenta tan sólo $1 \mathrm{~mW}$ para longitudes de fibra por encima del punto óptimo. Lo que sí que se observa, sin embargo, es un claro aumento de la potencia de salida al subir la potencia de bombeo de $+10 \mathrm{a}+15 \mathrm{dBm}$.

Dado que la eficiencia del proceso de conjugación es proporcional al cuadrado de la potencia óptica de la onda de bombeo, interesa utilizar potencias de bombeo el evadas para conseguir altas eficiencias. Sin embargo, la potencia de entrada a la fibra viene limitada por el SBS, que por lo tanto también limita la eficiencia de la conversión. Al mismo tiempo, la fluctuación que sufre la potencia de bombeo por encima del umbral de SBS origina ruido en el sistema, el cual se transfiere a la nueva onda generada degradando la calidad de la señal que transporta la misma [I no95]. De este modo, la potencia de bombeo debe mantenerse por debajo del umbral de 

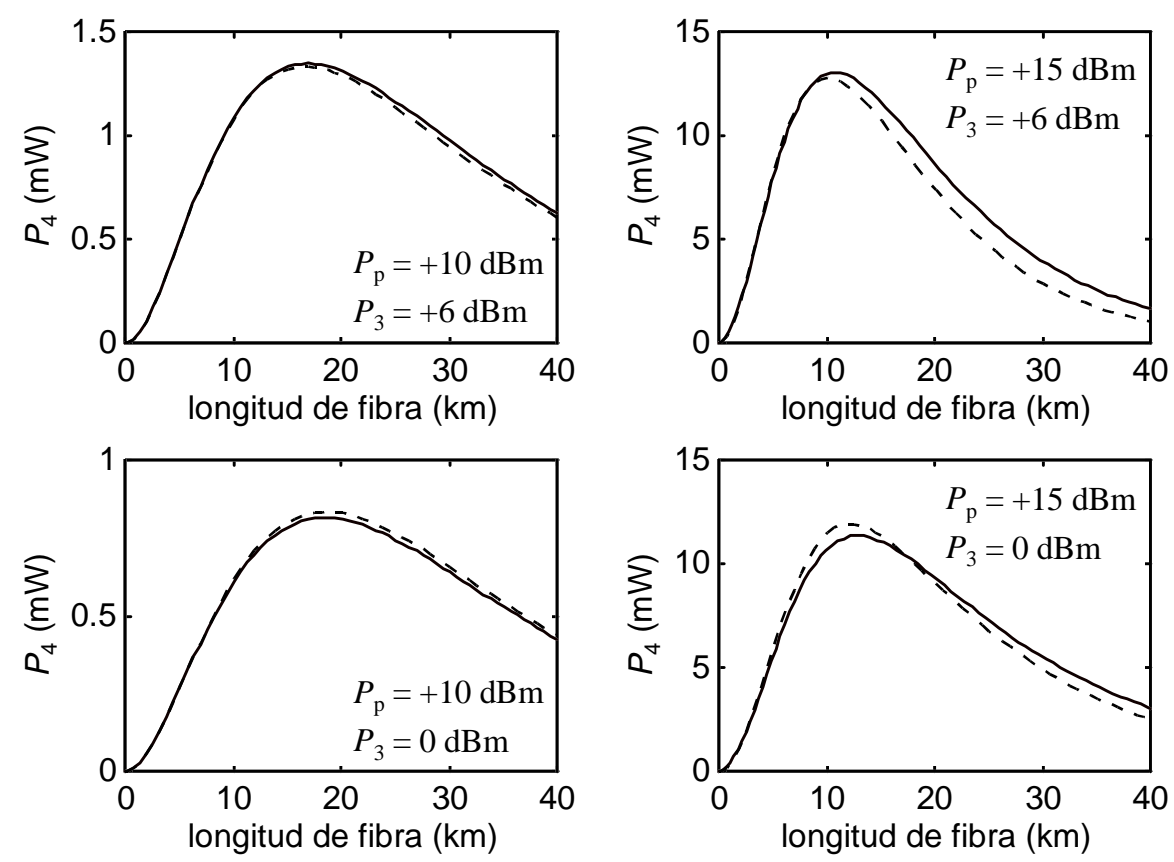

Figura 5.11 Influencia del SPM y XPM sobre la nueva onda generada mediante FWM en una DSF. Las curvas de trazos no tienen en cuenta este efecto. $P_{p}=P_{1}(0)=P_{2}(0)$.

SBS. Basándose en esta suposición, a continuación calcularemos una expresión de la eficiencia óptima.

Considerando la ec. (5.35), la potencia de la onda conjugada, $\mathrm{P}_{\mathrm{c}}$, puede expresarse a partir de las potencias de bombeo y de señal, $\mathrm{P}_{\mathrm{p}}$ y $\mathrm{P}_{\mathrm{s}}$, como

$$
P_{c}=C_{N L} P_{p}^{2} P_{s} \exp (-\alpha z) L_{\text {eff }}^{2} \eta_{\beta},
$$

siendo $\mathrm{C}_{\mathrm{NL}}$ una constante que describe la no linealidad de tercer orden y $\mathrm{L}_{\text {eff }}$ la longitud efectiva de la DSF.

Por otra parte, el umbral de SBS también depende de la longitud efectiva, lo cual se escribe como [l pp72]

$$
P_{S B S}=\frac{C_{S B S}}{L_{\text {eff }}},
$$

donde $\mathrm{C}_{\mathrm{SBS}}$ es una constante que expresa la eficiencia de SBS. 
Como se ha comentado anteriormente, la potencia de bombeo debe ser inferior al umbral de SBS. Por lo tanto, la máxima eficiencia se obtiene cuando la potencia de bombeo se fija al valor umbral de SBS. Bajo esta condición, la eficiencia de conversión viene dada por

$$
\eta=\frac{P_{C}}{P_{S}}=C_{N L} C_{S B S}^{2} \exp (-\alpha z) \eta_{\beta},
$$

donde se observa que la eficiencia final no depende de la longitud efectiva. Esta ecuación sugiere, pues, que una fibra de longitud reducida puede utilizarse para conseguir alta eficiencia, siempre que exista una alta potencia de bombeo disponible. De hecho, estas fibras son preferibles puesto que la condición de fase representada por el término de eficiencia $\eta_{\beta}$ se satisface más fácilmente [Ino92]. Por ejemplo, en [Tsu98] se presenta un nuevo tipo de fibra que incorpora supresión de SBS, además de dispersión cromática uniforme para satisfacer la condición de fase. Los resultados finales proporcionan un umbral de SBS de $+12 \mathrm{dBm}, 4 \mathrm{~dB}$ superior al que se consigue con una DSF convencional, lo cual favorece claramente el proceso de FWM. 


\subsection{FWM EN AMPLIFICADORES DE SEMICONDUCTOR}

Otra de las posibilidades para generar FWM se basa en el empleo de amplificadores de semiconductor (SOAs). Sus principales ventajas con respecto a las DSFs se refieren a una alta eficiciencia de conversión, operación sobre un mayor rango de longitudes de onda y gran capacidad de integración [Mar97c]. A continuación estudiaremos los fundamentos del proceso de FWM en SOAs para su posterior aplicación en la construcción de conjugadores ópticos.

\subsubsection{Origen del FWM en SOAs}

El proceso de FWM en SOAs posee una eficiencia de conversión extremadamente alta cuando la separación entre las ondas de bombeo y de señal es de unos pocos $\mathrm{GHz}$ [Agr87]. Esto se debe a que la densidad de portadores se modula fácilmente a la frecuencia diferencia entre ambas ondas, afectando a la ganancia dinámica y al índice de refracción de la cavidad. Particularmente, el elevado factor de ensanchamiento de línea de la cavidad favorece la eficiencia del scattering de Bragg que se produce como consecuencia de la modulación del índice de refracción. Varios trabajos en esta línea han sido ya demostrados [Agr87, Muk90].

Sin embargo, el otro mecanismo responsable del FWM es el coeficiente de ganancia no lineal, $\varepsilon$, que se conoce como el coeficiente de supresión de ganancia modal frente a un campo intenso. En el caso de FWM, la variación de la densidad de fotones a la frecuencia diferencia entre las ondas de bombeo y señal genera, directamente, unas redes de difracción en la ganancia dinámica y el índice de refracción a través del efecto de ganancia no lineal, sin acompañar al proceso de modulación de la densidad de portadores anteriormente comentado [Agr88]. No obstante, la eficiencia de este proceso es mucho menor que la producida por la modulación de portadores. Adicionalmente, el tiempo de relajación que gobierna este efecto es menor de 1 ps, y por lo tanto el FWM inducido por la ganancia no lineal llega a ser dominante sól o cuando la separación entre las ondas de bombeo y 
de señal es mucho mayor que la frecuencia de corte de la modulación de la densidad de portadores.

En el origen de la ganancia no lineal intervienen diversos factores. Por una parte, el efecto del SHBvii, el cual representa el nulo que aparece en el espectro de ganancia inducido por la reducción de la probabilidad de ocupación de los portadores del nivel de energía correspondiente a la frecuencia de este nulo como consecuencia de campos intensos [Asa85]. Precisamente, la separación entre las ondas de bombeo y de señal produce la modulación del nulo en el espectro de ganancia, lo cual resulta en la generación de las redes de difracción de la ganancia dinámica y del índice de refracción. Dado que la eficiencia del scattering de estas redes de difracción está gobernado por el tiempo de relajación intra-banda de los electrones de la banda de conducción $(\sim 0,3$ ps), la señal conjugada generada puede observarse para separaciones entre las ondas de bombeo y de señal por encima de los $100 \mathrm{GHz}$.

Por otro lado, Ippen et al. concluyeron que el calentamiento de los portadores dinámicos viii, que consiste en el aumento de temperatura de los portadores como consecuencia de la absorción de los portadores libres, es el fenómeno dominante en el efecto de ganancia no lineal [Kes87]. EI tiempo de relajación de este proceso es de 0,65 ps en amplificadores de InGaAsP operando a $1,5 \mu \mathrm{m}$.

Así pues, existen dos procesos en la saturación de la ganancia como son el SHB y el calentamiento de los portadores que pueden utilizarse para modelar el FWM en SOAs [Mec95, Mor97]. No obstante, la validez de este análisis se restringe al caso de señales con potencias relativamente débiles, dado que se basa en teoría perturbacional. Recientemente, un modelo desarrollado por Mecozzi y Mork [Mec97] extiende el análisis para el caso de pulsos intensos. Sin embargo, considerando señales intensas, no pueden despreciarse los fenómenos de deplexión de la onda de señal y de XGM. Adicionalmente, la saturación de la ganancia y el cambio de fase de las

\footnotetext{
vii spectral holeburning
} 
ondas que se mezclan inducidos por los efectos de absorción de dos fotones ${ }^{i x}$ y refracción no lineal ultra-rápida× deberían incorporarse también al modelo en el caso de potencias de pico superiores a 1 W [Tan98]. En [Tan98] se presenta un completo modelo que incluye todos estos efectos, el cual se utiliza para estudiar principalmente la influencia de la deplexión y del XPM sobre la eficiencia del FWM de pulsos ópticos de anchuras del orden de picosegundos.

\subsubsection{Ecuaciones del FWM en SOAs}

Para modelar el proceso de FWM en SOAs consideraremos amplificadores de onda progresivaxi, es decir, aquellos en que las ondas de bombeo, señal y conjugada se propagan en la misma dirección. En [Bis99] se puede encontrar un completo estudio del FWM bidireccional. Asimismo, se supondrá que la potencia acoplada a las ondas de bombeo y de señal es despreciable, lo cual es válido para FWM de banda ancha [Zho94].

Siguiendo un procedimiento similar al de [Agr88], se puede obtener el siguiente sistema de ecuaciones acopladas para el FWM [Zho94]:

$$
\begin{aligned}
& \frac{d A_{P, S}}{d z}=\frac{1}{2}\left[\frac{g_{0}}{1+\frac{P(z)}{P_{\text {sat }}}}\left(1+j \alpha_{1}\right)-\alpha\right] A_{P, S}, \\
& \frac{d A_{C}}{d z}=\frac{1}{2}\left[\frac{g_{0}}{1+\frac{P(z)}{P_{\text {sat }}}}\left(1+j \alpha_{1}\right)-\alpha\right] A_{C}-\xi(z) A_{P}^{2} A_{S}^{*} \exp (-j \Delta k z),
\end{aligned}
$$

donde $A_{p}, A_{S}$ y $A_{c}$ son, respectivamente, la envolvente de las ondas de bombeo, señal y conjugada, $\Delta \mathrm{k}=2 \mathrm{k}_{\mathrm{P}}-\mathrm{k}_{\mathrm{S}}-\mathrm{k}_{\mathrm{C}}$ representa la condición de fase del FWM y $\xi(z)$ es un coeficiente de acoplamiento. Adicional mente, $g_{0}$ es

\footnotetext{
viii dynamic carrier heating

ix two-photon absorption (TPA)

$\times$ ul trafast nonlinear refraction (UNR)
} 
la ganancia óptica no saturada por unidad de longitud, P(z) es la potencia óptica total en la posición $z$ del dispositivo, $\mathrm{P}_{\text {sat }}$ es la potencia de saturación del amplificador, $\alpha_{1}$ es el factor de ensanchamiento de línea y $\alpha$ es la atenuación no saturable por unidad de longitud del dispositivo.

En cuanto al coeficiente de acoplamiento $\xi(z)$, éste es el elemento central de todo el análisis teórico. Para simplificar el análisis, se supondrá que los diferentes mecanismos que contribuyen al FWM (modulación de la densidad de portadores, calentamiento de los portadores dinámicos y SHB) ocurren independientemente. Evaluando las polarizaciones inducidas por estos mecanismos a la longitud de onda de la señal conjugada, $\xi(z)$ resulta [Zho94]

$$
\xi(z)=\frac{1}{2} \frac{g_{0}}{1+\frac{P(z)}{P_{\text {sat }}}} \sum_{m=1}^{3} \frac{1+j \alpha_{m}}{1+j \omega_{d} \tau_{m}} \frac{1}{P_{m}},
$$

donde $\mathrm{m}=1,2,3$, para la modulación de la densidad de portadores, calentamiento de los portadores y SHB, respectivamente, $\omega_{d}=\omega_{\mathrm{p}}-\omega_{\mathrm{s}}, \tau_{\mathrm{m}}$ y $\mathrm{P}_{\mathrm{m}}$ son los tiempos de vida y potencias de saturación de cada uno de los mecanismos, y $\alpha_{m}$ es el cociente entre las partes real e imaginaria del cambio de índice de refracción inducido por dichos mecanismos.

Utilizando la condición de contorno $A_{C}(0)=0$, las ecuaciones (5.55) y (5.56) pueden resolverse bajo la condición de saturación moderada del SOA [Zho94]:

$$
A_{C}(z)=-\frac{A_{p}^{2}(z) A_{s}^{*}(z) \xi(z) z \exp (-j \Delta k z)}{0,23 G-j \Delta k z}
$$

donde $\mathrm{G}$ es la ganancia saturada en $\mathrm{dB}$. A su vez, utilizando valores típicos y una longitud de dispositivo de $0,8 \mathrm{~mm}$, la condición de fase para una separación de portadoras de $30 \mathrm{~nm}$ se estima en -0,33, que es totalmente despreciable frente al primer término para una ganancia óptica típica de $\mathrm{G}$ $\sim 15 \mathrm{~dB}$.

\footnotetext{
xi traveling-wave amplifier (TWA)
} 
Utilizando (5.58), la eficiencia de conversión mediante FWM expresada en $\mathrm{dB}$ viene dada por [Zho94]

$$
\begin{aligned}
\eta & =10 \log _{10} \frac{P_{c}(z)}{P_{s}(0)} \\
& =3 G+2 P_{p}+20 \log _{10}\left|\sum_{m=1}^{3} c_{m} \frac{1}{1+j \omega_{d} \tau_{m}}\right|
\end{aligned}
$$

donde el coeficiente de acoplamiento complejo, $c_{m}$, se define como

$$
c_{m}=\frac{g_{0} z}{0,46 G\left(1+\frac{P(z)}{P_{s a t}}\right)} \frac{1+j \alpha_{m}}{P_{m}} .
$$

Obsérvese que el término $3 G$ de la expresión de la eficiencia aparece del hecho que tanto la onda de bombeo como la de señal experimentan una ganancia de $\mathrm{G}$ (nótese que $\mathrm{G}$ afecta doblemente a la onda de bombeo por la propia estructura de la ec. (5.58)). Por lo tanto, para conseguir FWM con buena eficiencia es crucial el empleo de un SOA con ganancia elevada.

En la figura 5.12 se representa la eficiencia del FWM en un SOA caracterizado por unos parámetros: $\tau_{1}=200 \mathrm{ps}, \tau_{2}=650 \mathrm{fs}, \tau_{3}=50 \mathrm{fs}, \mathrm{c}_{1}=$ $0,24 \cdot \exp (j 1,30), c_{2}=0,0027 \cdot \exp (-j 1,30), c_{3}=0,00048 \cdot \exp (-j 1,53)$. Como se observa, la eficiencia decae conforme se separan las Iongitudes de onda de señal y conjugada, inicialmente con una pendiente de $20 \mathrm{~dB} /$ década. La asimetría que aparece con respecto a los casos $\lambda_{S}>\lambda_{P}$ y $\lambda_{S}<\lambda_{P}$ se cree que es debida a la interferencia de fases entre las contribuciones de los distintos mecanismos. En particular, colocando la longitud de onda de señal por encima de la del bombeo se consigue una mayor eficiencia. Nótese que se está representando la eficiencia relativa (sin el término $3 \mathrm{G}+2 \mathrm{P}_{\mathrm{p}}$ ), por lo que el nivel de la señal conjugada dependerá de las potencias de entrada y de la ganancia del dispositivo. Por ejemplo, para una ganancia de $15 \mathrm{~dB}$ y una potencia de bombeo a la entrada de $-10 \mathrm{dBm}$, se consigue una eficiencia de conversión de unos $-35 \mathrm{~dB}$ para $\Delta \lambda=10 \mathrm{~nm}$. Esta eficiencia podría llegar hasta $0 \mathrm{~dB}$ (100\%) aumentando la ganancia a unos $27 \mathrm{~dB}$, de donde se desprende la importancia que tiene este parámetro. 


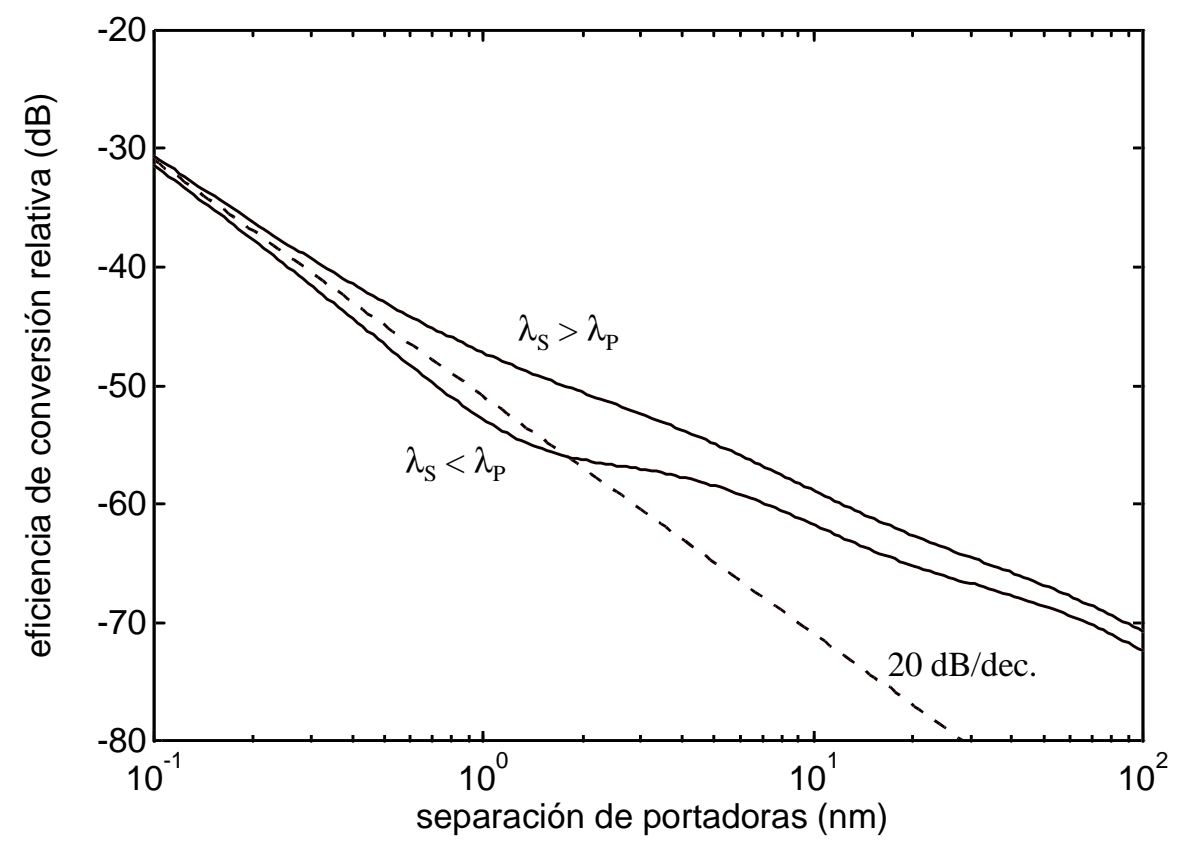

Figura 5.12 Eficiencia de conversión relativa a $3 G+2 P_{p}$ en función de la separación entre la onda conjugada y la señal: $\Delta \lambda=$ $\lambda_{c}-\lambda_{s}$. Los parámetros del dispositivo son los mismos que en [Zho94].

\subsubsection{Influencia de diferentes variables y parámetros}

La expresión de la eficiencia del FWM en SOAs suele escribirse en la práctica como [Zho94, Duc96]

$$
\eta=G_{p}^{2} G_{s} P_{p}^{2} R(\Delta \lambda),
$$

donde $R(\Delta \lambda)$ representa el último término de (5.59) y se han desglosado las ganancias a la longitud de onda de señal, $G_{s}$ y a la de bombeo, $G_{p}$. Si nos olvidamos por un momento del término $R(\Delta \lambda)$, que representa la separación espectral de las ondas y presenta una caída de $20 \mathrm{~dB} / \mathrm{dec}$. (figura 5.12), se observa que la eficiencia depende del cuadrado de la potencia de bombeo. Ahora bien, en la práctica, si vamos aumentando progresivamente la potencia de bombeo se llega a un punto en el que la eficiencia presenta un máximo y comienza a decrecer [Sum96]. Esto efecto se debe a la saturación de la ganancia del SOA [Mec95, Kol96], ya que la eficiencia tiene una dependencia cúbica con esta. En particular, en la zona de saturación la 
ganancia decrece como $1 / P_{\mathrm{p}}$. Valores de potencia de bombeo en torno a los 0 dBm pueden ser suficientes para saturar la eficiencia del proceso de FWM [Duc96]. Por la misma razón, potencias de señal bajas conducen a mejores resultados de eficiencia, aunque la potencia de la señal conjugada a la salida pueda ser inferior. En la figura 5.13 se representan las curvas de eficiencia de conversión para un SOA típico en función de la potencia de bombeo y diferentes valores de potencia de señal, donde se puede observar el comportamiento descrito anteriormente.

Adicionalmente, en [Duc96] se demuestra que la potencia de señal debe mantenerse como mínimo $5 \mathrm{~dB}$ por debajo de la potencia de bombeo para que no afecte el fenómeno de modulación cruzada de la ganancia (XGM, cross-gain modulation), el cual se manifiesta en una modulación de la ganancia del SOA inducida por la propia onda que transporta la señal y que satura el dispositivo. En [F re97], por ejemplo, se ha propuesto este método para la elaboración de convertidores de longitud de onda en sistemas SCM.

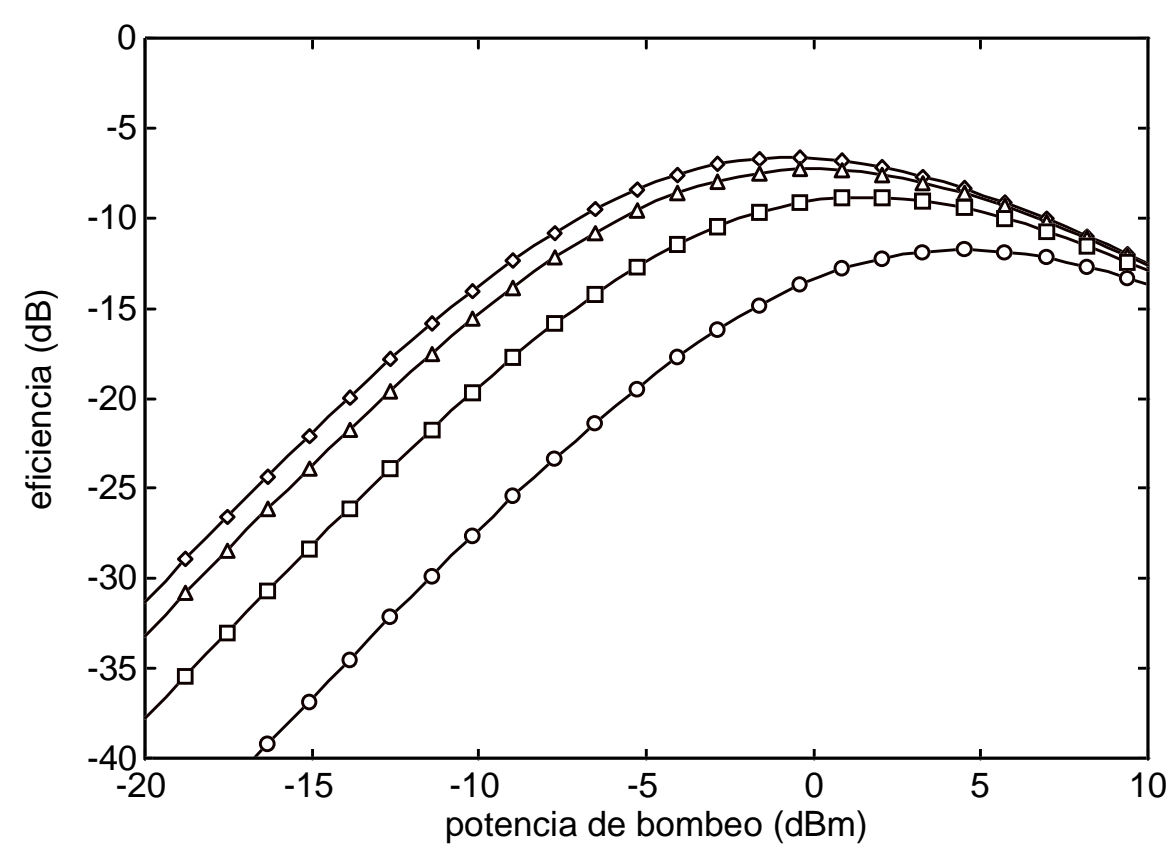

Figura 5.13 Eficiencia de conversión en función de la potencia de bombeo para diferentes valores de potencia de señal: $-15 \mathrm{dBm}$ (rombos), $-10 \mathrm{dBm}$ (triángulos), $-5 \mathrm{dBm}$ (cuadrados) y $0 \mathrm{dBm}$ (ćŕrculos). 


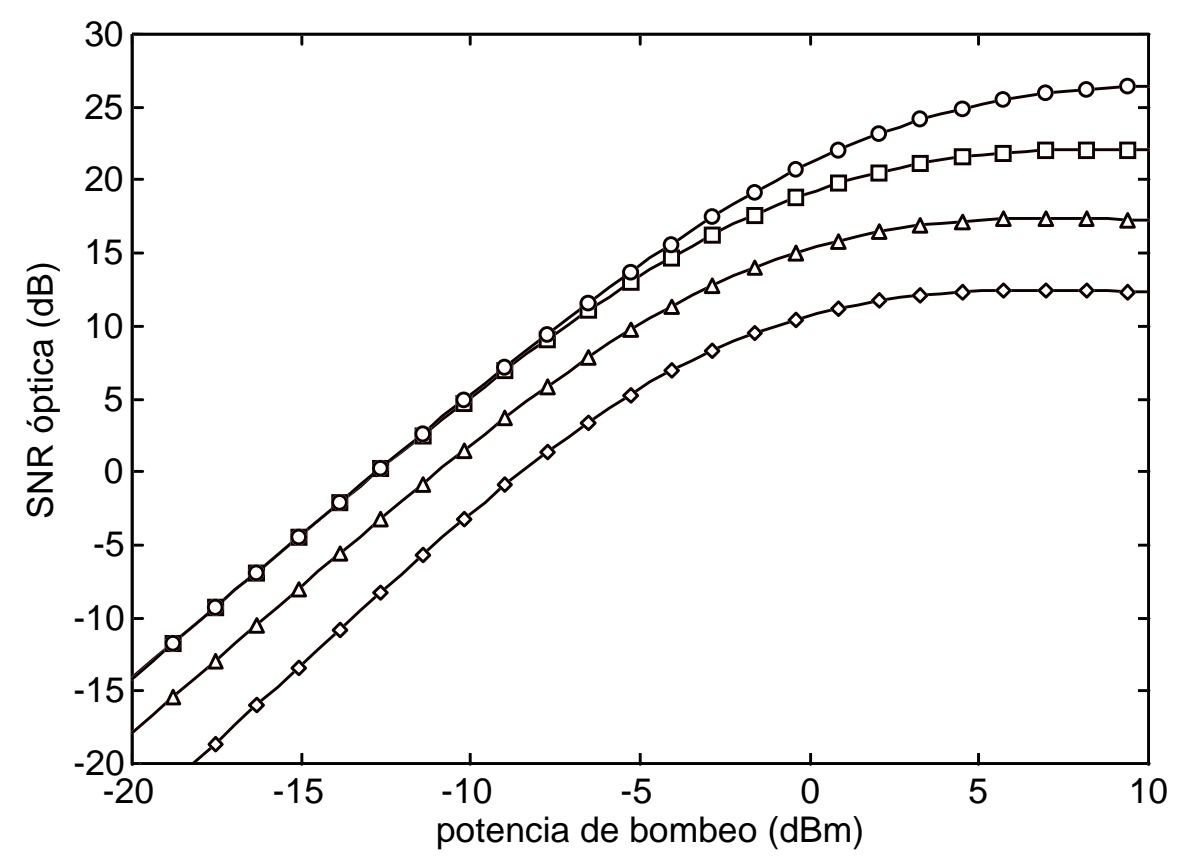

Figura 5.14 SNR óptica para la señal conjugada en función de la potencia de bombeo y para diferentes valores de potencia de señal: $-15 \mathrm{dBm}$ (rombos), $-10 \mathrm{dBm}$ (triángulos), $-5 \mathrm{dBm}$ (cuadrados) y 0 dBm (ć́rculos).

Si bien pueda parecer que interese trabajar con aquella potencia de bombeo que produce la máxima eficiencia, esto no es cierto. De hecho, cuando se obtiene el óptimo de eficiencia las tres ondas son relativamente débiles, mientras que la ganancia es alta y conduce a una importante amplificación del ruido ASExii. La expresión de la SNR óptica de la onda conjugada viene dada por [Duc96]

$$
S N R_{0}=\frac{G_{p}^{2} G_{s} P_{p}^{2} P_{s} R(\Delta \lambda)}{(G-1) h v_{0} n_{s p} B_{0}}
$$

donde $v_{0}$ es la frecuencia óptica, $\mathrm{n}_{\mathrm{sp}}$ es el parámetro de inversión de población [Agr97] y $\mathrm{B}_{0}$ es el ancho de banda del filtro óptico. Esta fórmula indica que la $S N R_{0}$ tiene una dependencia $G^{2} P_{p}^{2} / n_{s p}$ para una $P_{S}$ dada. Por debajo del punto de saturación del SOA, $\mathrm{G}$ y $\mathrm{n}_{\mathrm{sp}}$ son constantes, por lo que la $S N R_{0}$ crece como $P_{p}^{2}$. En saturación, $G$ decrece como $1 / P_{p}$, proporcionando 
un producto $\mathrm{G}^{2} \mathrm{P}_{\mathrm{p}}^{2}$ constante. Pero $\mathrm{n}_{\mathrm{sp}}$ aumenta, por lo que $\mathrm{SNR} \mathrm{R}_{0}$ disminuye. De este modo, $\mathrm{SNR}_{0}$ alcanza un máximo para un nivel de potencia de bombeo moderado [Sum96]. En la figura 5.14 se representan las curvas de SNR óptica para distintas potencias de señal, donde se observa este fenómeno. Ahora se obtiene mejor $\mathrm{SNR}_{0}$ para $\mathrm{P}_{\mathrm{S}}$ superior debido a que la potencia de la señal conjugada suele ser mayor. Luego existe un compromiso entre $\eta$ y $S N R_{0}$ a la hora de seleccionar unas potencias $P_{p}$ y $P_{s}$ adecuadas [Sum96, Duc96]. Por último, indicaremos que la dependencia de $\mathrm{SNR}_{0}$ con la longitud de onda es totalmente similar a la representada en la figura 5.12 para $\eta$ [Lin98].

Debemos considerar que las figuras 5.13 y 5.14 describen el comportamiento de un SOA típico a modo orientativo, ya que los valores de $\eta$ y $S_{N R}$ presentan una gran dependencia con diferentes parámetros geométricos del dispositivo. Los resultados experimentales demuestran que resulta

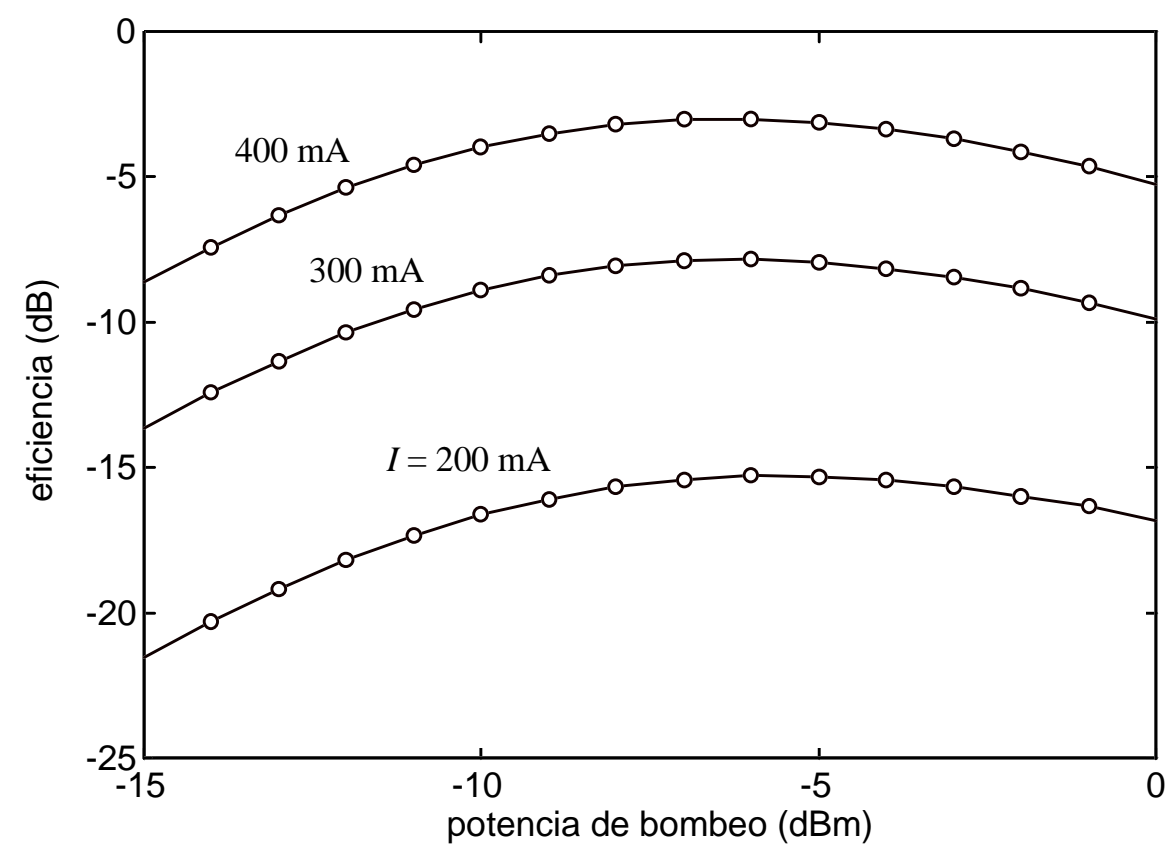

Figura 5.15 Dependencia de la eficiencia de conversión con la corriente de polarización del SOA. Otros parámetros: $P_{S}=-10$ $\mathrm{dBm}$.

xii ASE (amplified spontaneous emission) 
conveniente construir amplificadores de gran longitud para optimizar $\eta$ y $\mathrm{SNR}_{0}$ [Sco97]. No obstante, existe un límite en la longitud del dispositivo impuesto por el aumento de la asimetría entre las curvas de conversión para $\lambda_{S}>\lambda_{P}$ y $\lambda_{S}<\lambda_{P}$. En especial, esta diferencia puede reducirse cuando la longitud de onda de bombeo se desplaza desde el pico de ganancia hacia la longitud de onda de band-gap [Sco97]. Esto permite optimizar el dispositivo diseñando convenientemente la curva de ganancia.

Finalmente, dado que la eficiencia y la SNR óptica del FWM se ven favorecidas por un aumento de la ganancia del dispositivo, resulta interesante polarizar el SOA con corrientes elevadas. En la figura 5.15, por ejemplo, se observa el aumento que se produce en $\eta$ con la corriente de polarización para un SOA típico. 


\subsection{CONSTRUCCIÓN DE CONJUGADORES ÓPTICOS}

El conjugador óptico es el elemento principal de la técnica de inversión espectral estudiada en el capítulo 4. Su función consiste en invertir la fase de la señal óptica de entrada o, dicho de otro modo, invertir el espectro de modulación por medio de la conjugación de la envolvente del campo óptico.

La implementación del conjugador óptico se realiza usualmente mediante FWM en un medio no lineal como la DSF o el SOA, de ahí que haya sido estudiado en profundidad en este capítulo. No obstante, también es posible encontrar configuraciones distintas basadas en otros dispositivos y procesos no lineales. Por ejemplo, recientemente [Fur99] se ha propuesto el uso de un nuevo componente conocido como difusor óptico paramétrico que produce FWM con alta eficiencia en un ancho de banda superior al que se consigue empleando SOAs. Adicionalmente, otras nuevas configuraciones hacen uso de un interferómetro de Sagnac para conseguir una mejor eficiencia o ancho de banda de conversión [Fei99, Lim99]. En este último caso, además, se obtiene inversión espectral sin desplazamiento de longitud de onda [Lim99a]. En [lan94] se ha demostrado también la técnica OPC sobre 333 km de fibra realizando la conjugación en el interior de un láser $V$-Groove de InGaAsP. Otros procesos no lineales como el XPM o XGM han sido utilizados con éxito en la construcción de convertidores de longitud de onda para redes WDM [Pat94, Fre97], aunque estos procesos no llevan consigo la conjugación de la señal.

A continuación estudiaremos la construcción de conjugadores ópticos basados en FWM en DSFs o SOAs, particularizando los resultados y el análisis de prestaciones al caso de sistemas ópticos analógicos [Shi96, Kit99]. En especial, se demostrará la compensación del efecto de supresión de la portadora mediante FWM en una configuración DSF [Ram99].

\subsubsection{Consideraciones de un conjugador óptico basado en FWM}

La arquitectura de un conjugador óptico basado en FWM en un medio no lineal se representa esquemáticamente en la figura 5.16. Aunque suelen 
dispositivo OPC (optical phase conjugator)



Figura 5.16 Diagrama de bloques de un dispositivo OPC basado en FWM en una configuración DSF o SOA.

formar parte del OPC otros componentes como EDFAs o filtros ópticos para reducir ASE, tal y como se mostraba en la figura 4.4, en este caso nos hemos centrado en los elementos indispensables para realizar el proceso de conjugación y consideraremos que el resto de componentes son externos al dispositivo.

La señal óptica de entrada al OPC se combina con la señal de bombeo de gran potencia para inducir efectos no lineales en el SOA o en la DSF. Para que el proceso de FWM se produzca con la máxima eficiencia es indispensable que ambas ondas presenten el mismo estado de polarización a la entrada del medio no lineal. Esto se consigue por medio de un controlador de polarización sobre la onda de bombeo cuando la polarización de la onda de señal es conocida y estable. Sin embargo, la polarización del campo eléctrico durante la propagación a través de las fibras ópticas varía aleatoriamente, de tal forma que no es posible conocer de antemano su estado a la entrada del OPC en un sistema real de compensación de dispersión. Estas variaciones aleatorias afectan significativamente a la eficiencia del proceso de FWM, haciendo que la técnica de inversión espectral no sea adecuada para aplicaciones reales. Afortunadamente, se ha investigado activamente en este tema y se han encontrado configuraciones de FWM insensibles a la polarización de la señal de entrada. Entre ellas se encuentran varios experimentos realizados empleando técnicas de diversidad de polarización [Has93] o utilizando dos ondas de bombeo 
polarizadas ortogonal mente [J op93, I no94]. Recientemente se han propuesto nuevas técnicas basadas en estructuras interferométricas Mach-Zehnder y SOAs [Leu98] o interferómetros de Sagnac [Lim99a] que también consiguen este propósito. Sin embargo, una técnica que destaca por su simplicidad es la realizada en [Yam98]. Consiste en FWM en una estructura construida completamente en fibra que utiliza láseres DF $B$ con bombeos ortogonales, y consigue una dependencia con la polarización tan pequeña como 0,5 dB.

Además del problema de la polarización, el proceso de conjugación mediante FWM presenta otro efecto no deseado como es el desplazamiento de la portadora óptica. En este esquema de generación la longitud de onda de la señal conjugada aparece separada de la longitud de onda de la señal original en una cantidad dada por $2\left(\lambda_{S}-\lambda_{P}\right)$. Este es un factor a tener en cuenta en los sistemas de compensación de dispersión mediante OPC, ya que las propiedades de propagación a través del segundo trayecto de fibra serán diferentes ${ }^{\text {xiii, }}$ además de que reduce a la mitad el ancho de banda óptico de transmisión disponible [Lim99]. Para evitar este efecto se han propuesto distintas configuraciones basadas en el empleo de dos ondas de bombeo ortogonales [Ino97, Mec98, Cor99]. En este caso, la longitud de onda de la señal conjugada viene dada por

$$
\lambda_{\mathrm{C}}=\lambda_{\mathrm{P} 1}+\lambda_{\mathrm{P} 2}-\lambda_{\mathrm{S}},
$$

y situando las ondas de bombeo de forma simétrica con respecto a $\lambda_{\mathrm{S}}$ se consigue que $\lambda_{c}=\lambda_{s}$. En [Ino94a], por ejemplo, se utilizan también dos ondas de bombeo para conseguir sintonizar la longitud de onda de la señal conjugada en una cierta región.

Otros métodos de inversión espectral sin desplazamiento de longitud de onda hacen uso de un PALM (parametric loop mirror) [Mor96] o de un interferómetro de Sagnac [Lim99a], el cual además puede ser insensible a la polarización de la señal de entrada como se ha dicho con anterioridad.

xiii véase apartado 4.4.1 
La eficiencia relativamente pequeña del proceso de conjugación en fibras ópticas merece una mención especial. Típicamente, la eficiencia de conversión $\eta$ se encuentra por debajo del 1\%, siendo necesaria la amplificación posterior de la señal conjugada. Efectivamente, la pérdida de inserción del conjugador excede los $20 \mathrm{~dB}$ [Wat93a]. Sin embargo, el fenómeno de FWM no es inherentemente un proceso de baja eficiencia y puede, en principio, proporcionar ganancia [Agr89]. De hecho, el análisis de las ecuaciones de FWM muestra que $\eta$ se incrementa considerablemente aumentando la potencia de bombeo a la vez que decrece la potencia de señal. Puede incluso exceder el $100 \%$ optimizando los niveles de potencia y la diferencia entre las longitudes de onda de bombeo y de señal [Wab95]. Pero suelen evitarse las altas potencias como consecuencia del SBS que ocurre cerca de los $10 \mathrm{~mW}$. Si bien, éste puede suprimirse modulando la onda de bombeo a una frecuencia de $\sim 100 \mathrm{MHz}$. En un experimento realizado en [Wat94a] se consigue una eficiencia de conversión del $35 \%$ utilizando esta técnica.

En cuanto a los conjugadores basados en SOAs, la eficiencia de conversión es generalmente mayor que la del FWM en DSF s como consecuencia de la amplificación. Aunque esta ventaja se reduce como consecuencia de las elevadas pérdidas de acoplamiento que se producen cuando se vuelve a inyectar la señal en la fibra. La señal conjugada puede generarse empleando un dispositivo de $1 \mathrm{~mm}$ de longitud o incluso inferior. Escogiendo adecuadamente la separación entre la señal y el bombeo, es posible obtener eficiencias de conversión por encima del $100 \%$ [Dot96], es decir, ganancia neta sobre la señal conjugada. Estas prestaciones hacen muy atractiva a esta técnica en sistemas de compensación de dispersión [Kit99]. No obstante, como se ha visto la eficiencia de conversión y la relación señal a ruido del FWM en SOAs son altamente dependientes de esta separación entre las ondas de señal y de bombeo [Zho94, Mec95, Lin98]. Por ello se han propuesto diversas técnicas para intentar ecualizar esta respuesta en la banda de trabajo [Mor98, Mor98a]. Finalmente, la influencia de otros efectos como distorsión debida a modulaciones residuales de amplitud o de fase de la señal conjugada [Kin97] o ruido de fase de la señal de bombeo [Sab95] también afectan a las prestaciones de la técnica OPC. 
Así pues, considerando los distintos efectos y fenómenos que influyen en el proceso de conjugación mediante FWM en DSF s o SOAs, el dispositivo OPC puede modelarse mediante las siguientes ecuaciones:

$$
\begin{aligned}
& E_{\text {out }}(t)=A_{\text {out }}(t) \exp \left(j \omega_{C} t\right), \\
& E_{\text {in }}(t)=A_{\text {in }}(t) \exp \left(j \omega_{S} t\right), \\
& A_{\text {out }}(t)=\sqrt{G_{\text {OPC }}} D_{\text {OPC }}\left[A_{\text {in }}^{*}(t)\right]+n_{0}(t),
\end{aligned}
$$

donde $\omega_{C}=2 \omega_{p}-\omega_{s}, G_{O P C}$ es un término de ganancia que incluye la eficiencia $\eta$ y las ganancias de los EDFAs, $D_{\text {OPC }}$ es una función que representa la posible distorsión que se produce en el proceso de

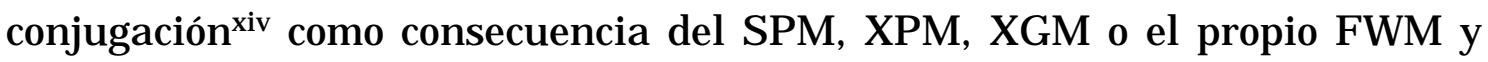
$\mathrm{n}_{0}(\mathrm{t})$ es el ruido total que aparece a la salida del bloque OPC.

\subsubsection{Estudio comparativo de conjugadores basados en DSF/SOA en sistemas ópticos analógicos}

Para realizar las medidas experimentales comparativas de ambas configuraciones de conjugador óptico se utilizó el montaje de laboratorio mostrado en la figura 5.17. La onda de señal era modulada por una subportadora de RF empleando un MZM sin chirp. Esta señal se combinaba



Figura 5.17 Montaje experimental utilizado para comparar las prestaciones de las configuraciones DSF/SOA de conjugador óptico en sistemas ópticos analógi cos. OSA: optical spectrum analyzer.

xiv idealmente $\mathrm{D}_{\mathrm{OPC}}(\mathrm{x})=\mathrm{x}$ 
posteriormente con la onda de bombeo por medio de un acoplador óptico 50:50. Ambos láseres son de cavidad externa con un ancho de línea de unos $100 \mathrm{kHz}$. A la entrada del conjugador se utilizó un EDFA de potencia de saturación de $+16 \mathrm{dBm}$ y figura de ruido $<6,5 \mathrm{~dB}$ para aumentar la potencia óptica disponible. Finalmente, la señal conjugada se extraía tras la DSF o el SOA empleando un filtro óptico y se fotodetectaba para recuperar la señal de RF. Los dispositivos utilizados para inducir FWM fueron una DSF de 13 $\mathrm{km}$ con $\lambda_{\mathrm{ZD}}=1550 \mathrm{~nm}$ y pérdidas de $0,2 \mathrm{~dB} / \mathrm{km}$ y un SOA MQW (CQF 871/6 de Philips) polarizado con una corriente de $300 \mathrm{~mA}$, con una ganancia de $16 \mathrm{~dB}$ y una potencia de saturación a la salida de $+15 \mathrm{dBm}$. Para medir las prestaciones del conjugador se realizaron medidas, tanto en el dominio eléctrico sobre la señal de RF detectada, como en el dominio óptico empleando un analizador de espectros (OSA) a la salida del conjugador.

Los espectros ópticos medidos a la salida de la DSF y del SOA para unas potencias de bombeo de $0 \mathrm{dBm}$ y de señal de $-10 \mathrm{dBm}$ se pueden ver en la figura 5.18. La longitud de onda de bombeo se hizo coincidir con la de dispersión nula de la DSF $\left(\lambda_{\mathrm{P}}=\lambda_{\mathrm{ZD}}=1550 \mathrm{~nm}\right)$ y se seleccionó en un principio $\lambda_{S}=1551 \mathrm{~nm}^{\times v}$. Sobre la misma figura se definen gráficamente las medidas de eficiencia de conversión y relación señal a ruido óptica del conjugador.

En la figura 5.19 se muestran las medidas experimentales de eficiencia de las configuraciones DSF y SOA para dos valores distintos de potencia de bombeo y en función de la potencia de señal a la entrada del EDFA. Como se puede observar, la eficiencia disminuye en ambos casos al aumentar la potencia de señal como consecuencia de la saturación que se produce en el EDFA y en el propio proceso de FWM. Al mismo tiempo, la eficiencia de la configuración SOA es claramente mejor debido a la amplificación que se produce en el dispositivo. Estos resultados concuerdan perfectamente con el estudio realizado en [Gei97], aunque en nuestro caso destacan los val ores de eficiencia obtenidos para la configuración SOA $(\eta>5 \mathrm{~dB})$.

\footnotetext{
${ }^{x v}$ a esta longitud de onda el MZM tiene una pérdida de inserción de $\sim-9,5$ dB
} 




(a)



(b)

Figura 5.18 Espectros ópticos a la salida del conjugador para las configuraciones (a) DSF y (b) SOA. Otros parámetros: $P_{\mathrm{s}}=-10$ $\mathrm{dBm}$ y $\mathrm{P}_{\mathrm{p}}=0 \mathrm{dBm}$. 




(a)

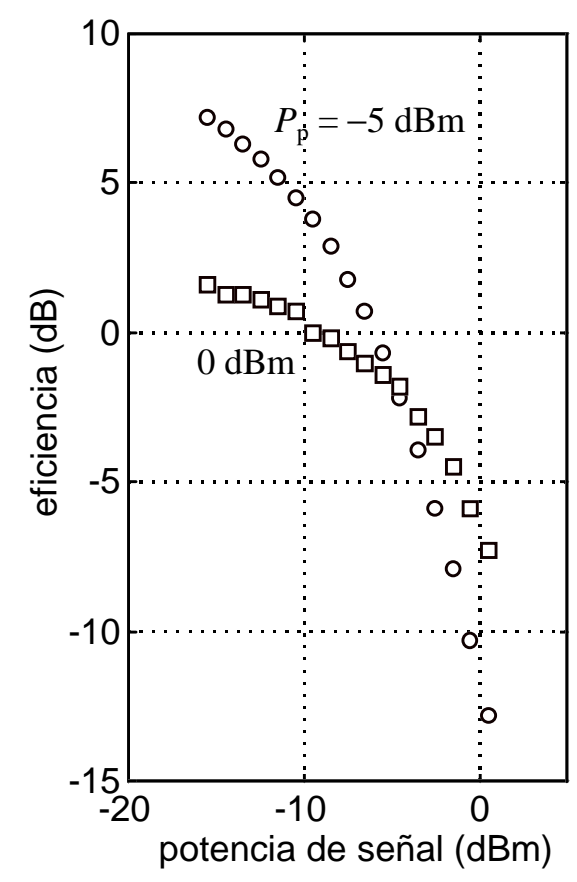

(b)

Figura 5.19 Eficiencia de las configuraciones (a) DSF y (b) SOA de OPC en función de la potencia de señal y para dos potencias de bombeo: -5 y $0 \mathrm{dBm}$. Otros parámetros: $\lambda_{\mathrm{S}}=1551 \mathrm{~nm}$ y $\lambda_{\mathrm{P}}=1550$ $\mathrm{nm}$.

Otra medida interesante es la relativa a la SNR óptica de la señal conjugada. Para comparar ambas configuraciones de OPC, se realizaron medidas de $\mathrm{SNR}_{0}$ a la salida del conjugador empleando un analizador de espectros óptico con un ancho de banda de resolución de 0,06 nm, las cuales se representan en la figura 5.20. En este caso, las curvas son crecientes con la potencia de señal hasta un valor máximo, a partir del cual comienzan a decrecer por la propia saturación del EDFA. Obsérvese que los valores de $\mathrm{SNR}_{0}$ son generalmente mejores para una potencia de bombeo superior, como ya se comentó con anterioridad, obteniéndose unos val ores máximos de $21 \mathrm{~dB}$ y $35 \mathrm{~dB}$ para las configuraciones DSF y SOA, respectivamente. En el caso del OPC basado en SOA se obtienen valores de SNR $\mathrm{R}_{0}$ superiores a los obtenidos en [Gei97], incluso sin utilizar filtro para eliminar ruido ASE a la entrada del conjugador. 


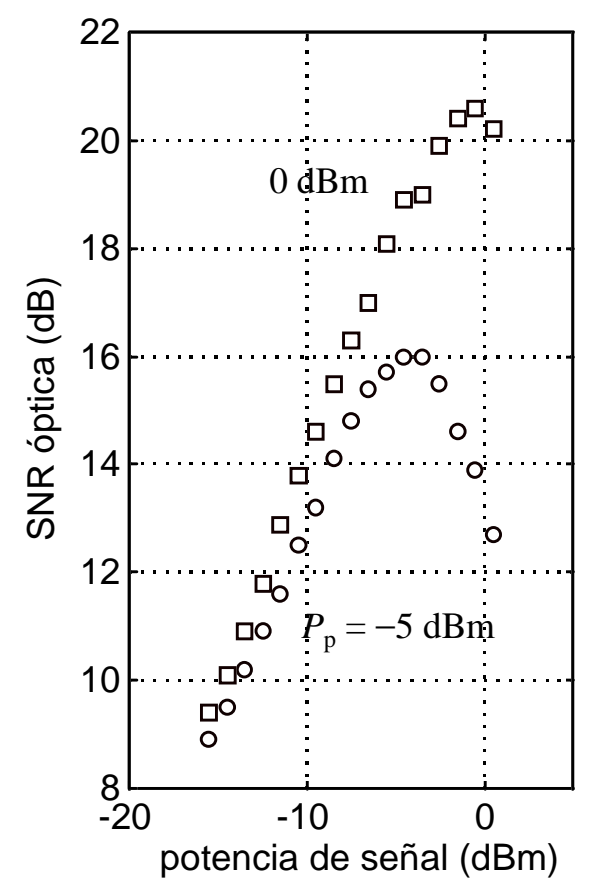

(a)



(b)

Figura 5.20 SNR óptica de la señal conjugada $\left(\lambda_{C}=1549 \mathrm{~nm}\right)$ en las configuraciones (a) DSF y (b) SOA de OPC en función de la potencia de señal y para dos potencias de bombeo: -5 y $0 \mathrm{dBm}$. Otros parámetros: $\lambda_{\mathrm{S}}=1551 \mathrm{~nm}, \lambda_{\mathrm{P}}=1550 \mathrm{~nm}$ y $B_{0}=0,06 \mathrm{~nm}$.

La $\mathrm{SNR}_{0}$ es un parámetro necesario para caracterizar el ruido ASE introducido por el conjugador. Sin embargo, en el caso de sistemas ópticos analógicos resulta mucho más útil el valor de la CNR a la salida del fotodetector. La relación entre ambas puede obtenerse fácilmente a partir de las expresiones de ruido de los amplificadores ópticos en [Agr97 (cap. 8)]. De este modo, suponiendo que el término de ruido dominante proviene del batido señal-emisión espontánea, la CNR debida a ruido ASE puede calcularse como [Ram98a]

$$
\mathrm{CNR}_{\mathrm{ASE}}=\frac{\mathrm{m}_{\mathrm{i}}^{2} \mathrm{~B}_{0}}{8 \mathrm{~B}} \mathrm{SNR}_{0}
$$

Conviene indicar que el valor de $\mathrm{CNR}_{\mathrm{ASE}}$ no depende del ancho de banda del filtro óptico utilizado para seleccionar la señal conjugada, sino que $\mathrm{B}_{0}$ se refiere únicamente al ancho de banda de resolución utilizado en el 
analizador de espectros óptico para medir la $S_{N} R_{0}$, y el producto de ambos es independiente de $B_{0}$ por la propia definición de $S N R_{0}$.

En la tabla I se resumen los valores de $\mathrm{CNR}_{\text {ASE }}$ para diferentes sistemas multicanal radio-fibra considerando los valores óptimos de $S N R_{0}$ de la figura 5.20 para cada configuración. De los resultados de la tabla se desprende que Ios sistemas CATV y MMDS están claramente limitados por ruido ASE y no alcanzan el requerimiento de CNR de $50 \mathrm{~dB}$ de la modulación AM-VSB. De hecho, para estos sistemas se necesitaría un valor de $\mathrm{SNR}_{0}$ en torno a los 55 $\mathrm{dB}$, el cual no puede ser alcanzado en la actualidad con las tecnologías de OPC existentes [Ram98a]. Por otro lado, los esquemas de modulación utilizados en los sistemas LMDS y MVDS suelen ser menos exigentes y, aunque los niveles de CNR que se consiguen con la configuración DSF no son suficientes, en el caso del SOA pueden ser válidos (CNR $>20 \mathrm{~dB}$ ).

TABLA I CNR debida a ruido ASE a la salida del conjugador para diversos sistemas multicanal radio-fibra. Otros parámetros: $S N R_{0(D S F)}=21 \mathrm{~dB}, \mathrm{SNR}_{0(\mathrm{SOA})}=$ $35 \mathrm{~dB}, \mathrm{~m}_{\mathrm{i}}=0,04$ y $\mathrm{B}_{0}=0,06 \mathrm{~nm}$.

\begin{tabular}{|c|c|c|c|}
\hline Sistema & ancho de banda (MHz) & CNR-DSF (dB) & CNR-SOA (dB) \\
\hline CATV & 6 & 14,6 & 29,1 \\
\hline MMDS & 6 & 14,6 & 29,1 \\
\hline LMDS & 40 & 6,3 & 20,8 \\
\hline MVDS & 30 & 7,6 & 22,1 \\
\hline
\end{tabular}

Así pues, parece ser que la configuración de OPC basada en DSF no resulta válida como elemento de ecualización en sistemas multicanal radio-fibra como consecuencia principalmente de su baja eficiencia de conversión. Sin embargo, existen técnicas para mejorar la $\mathrm{SNR}_{0}$, como por ejemplo la propuesta en [Gei97] y que se basa en el empleo a la entrada del conjugador de un filtro óptico elimina banda para reducir el ruido ASE sobre la longitud de onda de la señal conjugada. Adicionalmente, otra posibilidad para aumentar la CNR está directamente relacionada con un aumento del índice 


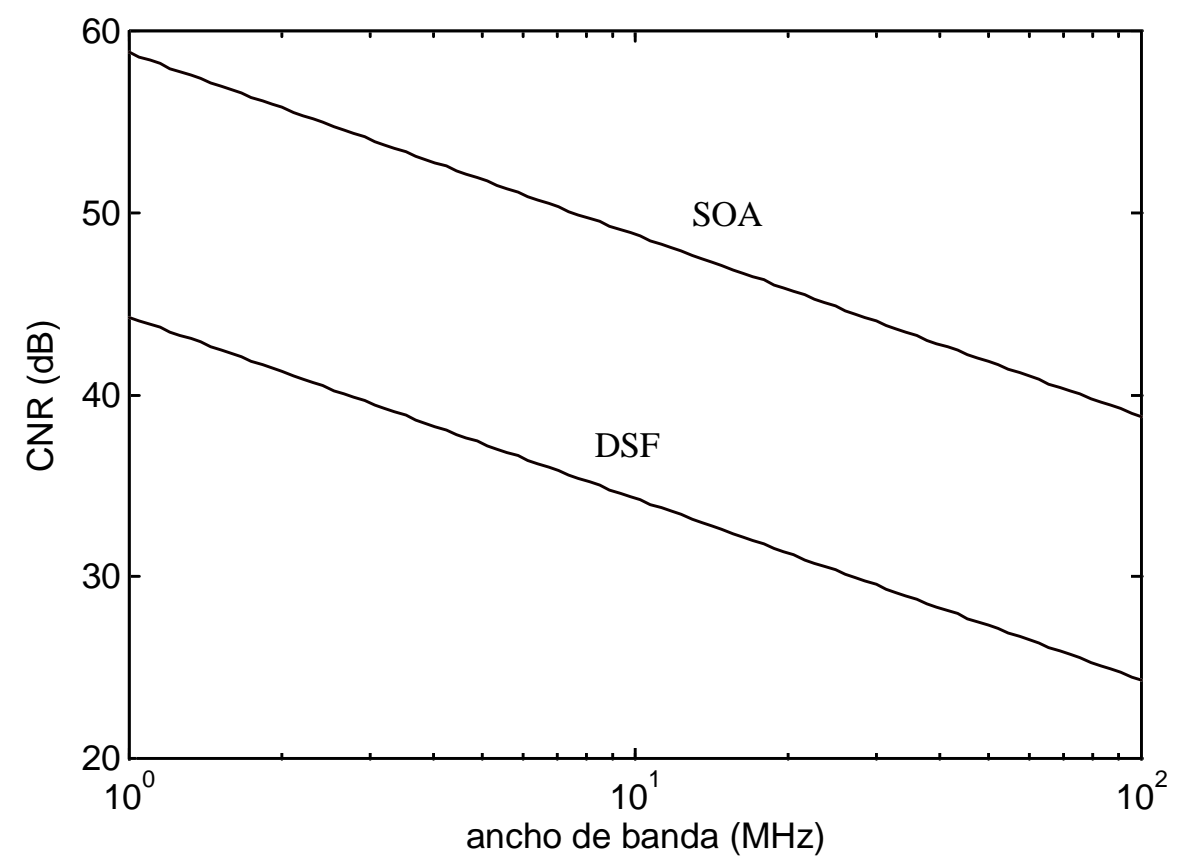

Figura 5.21 CNR $\mathrm{CSE}_{\mathrm{ASE}}$ en función del ancho de banda de canal para las configuraciones óptimas de conjugador $\left(\mathrm{SNR}_{0}=21\right.$ ó $35 \mathrm{~dB}$ con DSF o SOA, respectivamente). Otros parámetros: $\mathrm{m}_{\mathrm{i}}=0,5, \mathrm{~B}_{0}=$ $0,06 \mathrm{~nm}$.

de modulación. Precisamente en la figura 5.21 se muestran los resultados de $\mathrm{CNR}_{\mathrm{ASE}}$ en función del ancho de banda de canal para un índice de modulación del $50 \%$, donde se observa claramente la mejora obtenida en la CNR para ambas configuraciones.

Para continuar analizando las prestaciones del conjugador, se realizó la transmisión de una subportadora de RF a través del dispositivo OPC. En un principio, la señal de RF consistía en una PRBS de $2 \mathrm{Mbit} / \mathrm{s}$ sobre una frecuencia $\mathrm{f}_{\mathrm{FI}}=1 \mathrm{GHz}$ y posteriormente subida en frecuencia hasta los 28 $\mathrm{GHz}$. A la salida del fotodetector, se realizaron medidas del espectro tanto a $28 \mathrm{GHz}$ como a $1 \mathrm{GHz}$ tras la bajada en frecuencia. Los equipos utilizados para realizar estas conversiones de frecuencia fueron unos transceptores de milimétricas de Spacek Labs trabajando con una frecuencia de oscilador local $\mathrm{f}_{\mathrm{OL}}=9 \mathrm{GHz}$. Los espectros medidos a la salida de ambas configuraciones se presentan en la figura 5.22. Las potencias ópticas de bombeo y de señal se seleccionaron para trabajar en el punto óptimo de cada 



Figura 5.22 Espectros a la salida del fotorreceptor para una PRBS de $2 \mathrm{Mbit} / \mathrm{s}$ transmitida sobre una subportadora de $28 \mathrm{GHz}$ a través del conjugador óptico. (a) espectro de RF con DSF, (b) espectro de FI con DSF, (c) espectro de RF con SOA y (d) espectro de FI con SOA. Otros parámetros: $\lambda_{\mathrm{S}}=1552 \mathrm{~nm}$.

una de las configuraciones. Al igual que antes, se observa que las prestaciones de la configuración SOA son mejores, especialmente en términos de CNR. Adicionalmente, Ia potencia de subportadora recibida con la configuración SOA es unos $30 \mathrm{~dB}$ superior a la obtenida con la DSF. No obstante, en ambos casos podría llegar a recuperarse la señal de datos transmitida.

Posteriormente, se realizaron medidas del diagrama de ojos de una PRBS transmitida sobre una subportadora de $1,5 \mathrm{GHz}$ para comprobar la transparencia del proceso de conjugación óptica. En este caso, Ios resultados medidos para distintas tasas binarias empleando un osciloscopio digital se muestran en la figura 5.23. Como se puede observar nuevamente, las mejores prestaciones se producen claramente con la configuración SOA. Si bien, de la figura se deduce que es perfectamente posible conseguir 

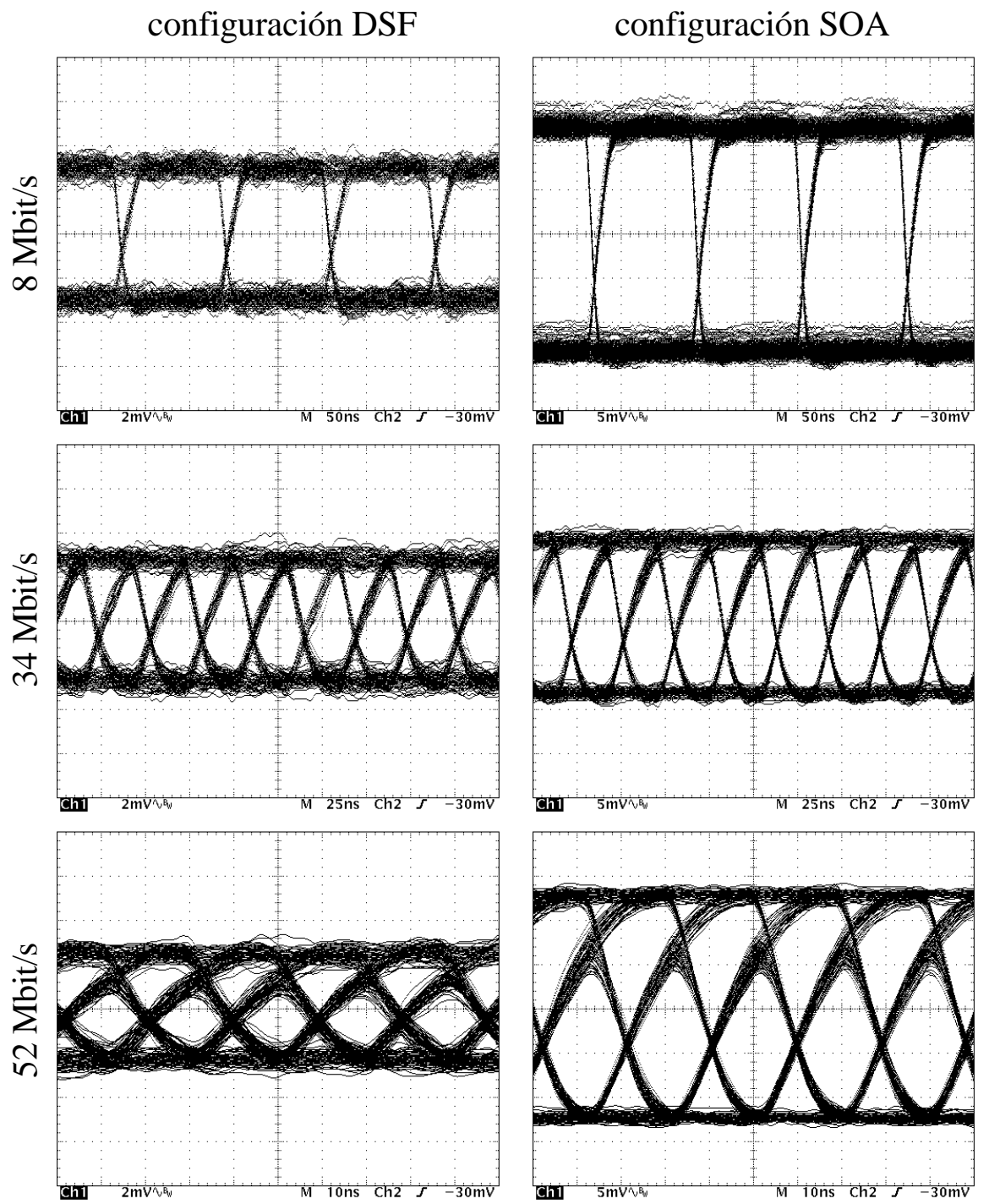

Figura 5.23 Diagramas de ojos de la señal recibida para ambas configuraciones de OPC y distintas tasas binarias. Otros parámetros: $\mathrm{f}_{\mathrm{RF}}=1,5 \mathrm{GHz}, \lambda_{\mathrm{S}}=1552 \mathrm{~nm}$ y $\lambda_{\mathrm{P}}=1550 \mathrm{~nm}$.

conversión libre de errores para ambas configuraciones. Los niveles de potencia óptica se han optimizado en cada configuración hasta obtener las mejores prestaciones.

Conviene indicar por último que, aunque en nuestro caso se han obtenido mejores prestaciones utilizando una configuración de OPC basada en SOA, esto dependerá de los dispositivos utilizados en cada caso concreto. De hecho, todavía no se ha comentado nada acerca de la distorsión introducida 
en el dispositivo OPC y que puede intuirse que será superior en el caso de la configuración SOA al tratarse de un dispositivo activo.

En la figura 5.24, por ejemplo, se representan los resultados de distorsión para ambas configuraciones obtenidos por medio de simulación mediante el programa OptSimvi. El set-up de medida utilizado es totalmente similar al de la figura 5.17. La señal de RF en este caso está compuesta por dos subportadoras de frecuencias $\mathrm{f}_{1}=3 \mathrm{GHz}$ y $\mathrm{f}_{2}=4 \mathrm{GHz}$, y la distorsión se mide a las frecuencias $2 f_{1}-f_{2}=2 \mathrm{GHz}$ y $2 f_{2}-f_{1}=5 \mathrm{GHz}$. Como se observa en la figura 5.24, en general la distorsión es menor en el caso de la configuración DSF al tratarse de un dispositivo pasivo. Esto mismo ya fue demostrado previamente en [Gei97] en el contexto de los sistemas digitales. En cualquier caso, de la figura 5.24 se deduce que para potencias de señal bajas la distorsión decrece con $\mathrm{P}_{\mathrm{s}}$, existiendo un mínimo a partir del cual comienza a crecer. En el caso de la DSF, se obtiene mayor distorsión para potencias de bombeo mayores (excepto para $\mathrm{P}_{\mathrm{s}}$ pequeña), mientras que en el SOA ocurre justamente lo contrario. La explicación puede deberse a la influencia de la potencia de bombeo en la eficiencia del FWM tal y como se representa en la figura 5.19 o a la presencia de otros fenómenos no lineales. No obstante, para conocer las causas exactas del comportamiento de los términos de distorsión de la figura 5.24 es necesario acudir a un modelo teórico. Precisamente esto es lo que se propone como línea futura de investigación del autor de la tesis en el capítulo 6.

Existe pues un compromiso entre CNR y CIR a la hora de escoger entre una configuración u otra de OPC, lo cual dependerá de las características del sistema y de los dispositivos utilizados como conjugadores en cada situación particular. A diferencia de [Gei97], en los experimentos realizados en esta tesis se ha demostrado que con la configuración SOA se obtienen mejores prestaciones que con la DSF, ya que la degradación predominante es el ruido ASE por encima de la distorsión.

\footnotetext{
xvi simulador de sistemas de comunicaciones ópticas (@ ARTIS Software Corporation)
} 


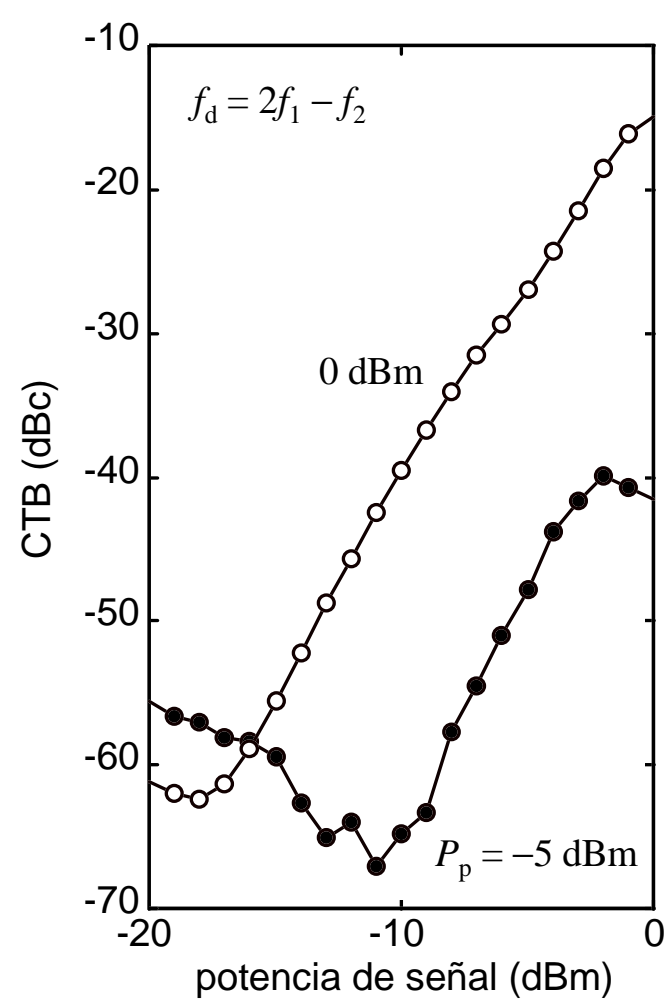

(a)



(c)

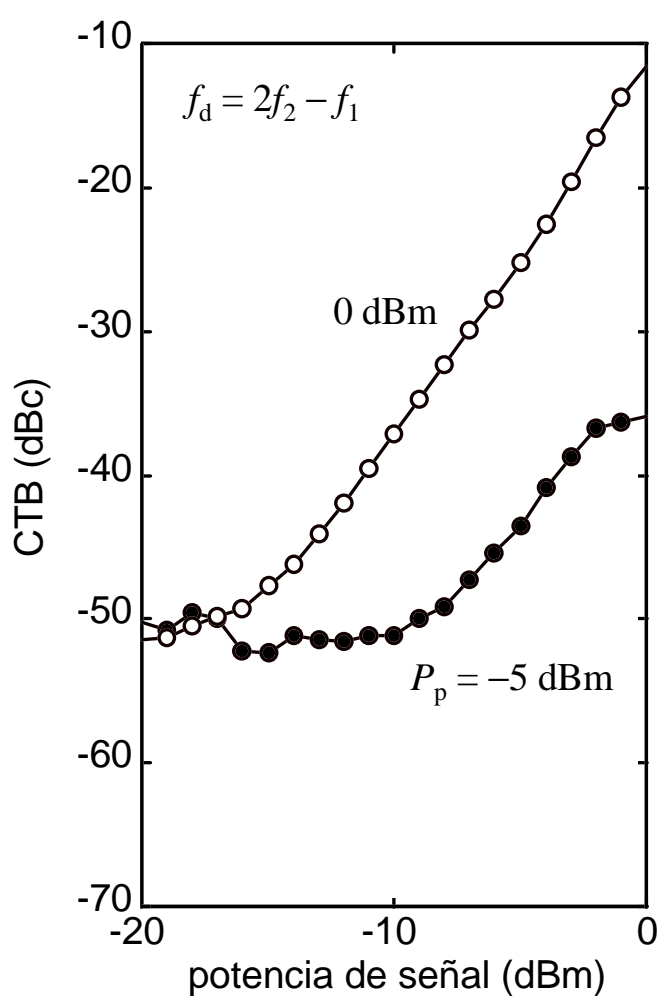

(b)

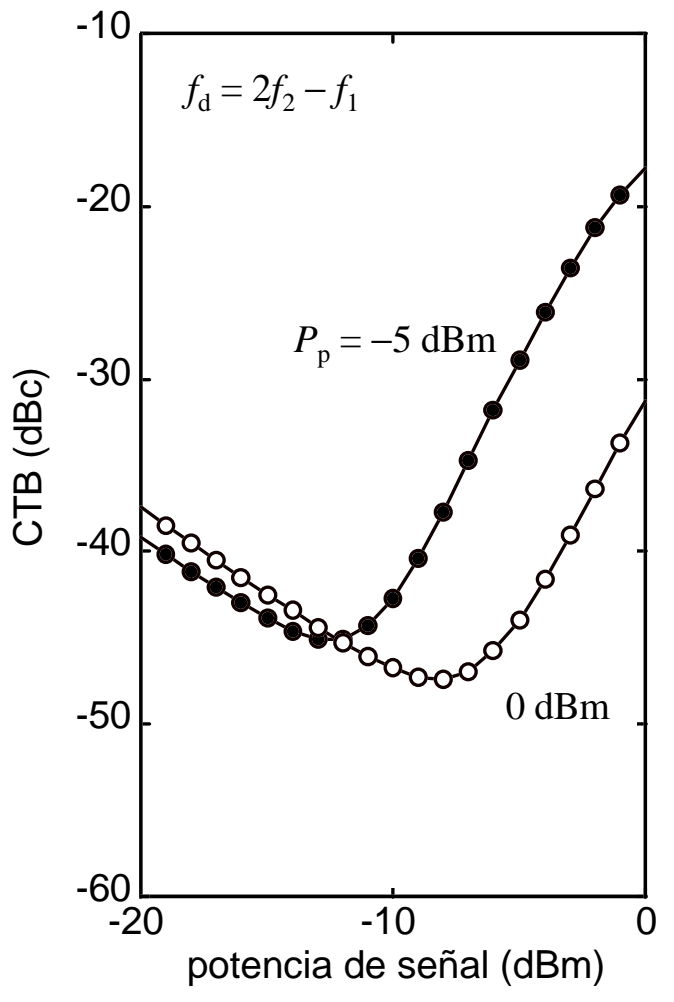

(d)

Figura 5.24 Distorsión a la salida del conjugador para las configuraciones (a)-(b) DSF y (c)-(d) SOA con distintas potencias ópticas de señal y bombeo. 


\subsubsection{Compensación del efecto de supresión de la portadora empleando un conjugador óptico basado en DSF}

El set-up experimental utilizado para demostrar la compensación del efecto de supresión de la portadora mediante FWM en una configuración DSF se muestra en la figura 5.25 [Ram99]. Un analizador de componentes ópticos (LCA) operando en el modo E/O se usa para medir la función de transferencia del sistema completo. EI puerto de RF de un MZM se alimenta con la señal eléctrica de barrido amplificada del LCA. Un láser sintonizable operando a $1551 \mathrm{~nm}$ se encarga de proporcionar la portadora óptica que será modulada en el MZM. La salida del MZM con una potencia óptica de $0 \mathrm{dBm}$ se inyecta entonces en un enlace de $25 \mathrm{~km}$ de SSMF. Para compensar los efectos de la dispersión cromática que ocurren durante la propagación se emplea un OPC basado en DSF situado en mitad del enlace completo. EI OPC convierte la señal de entrada en una réplica conjugada por medio de FWM en una DSF de 12,7 km de longitud. EI láser de bombeo posee una potencia óptica de $+3 \mathrm{dBm}$ y se encuentra sintonizado a la longitud de onda de dispersión nula de la DSF (1550 nm). Para evitar en lo posible el SBS, el láser de bombeo se opera en el modo de baja coherencia (ancho de línea de unos $30 \mathrm{MHz}$ ). A continuación, tanto la onda transmitida como la de bombeo se combinan y amplifican por medio de un EDFA de $+16 \mathrm{dBm}$ de potencia de saturación para inducir efectos no lineales en la DSF. Mediante un controlador de polarización se asegura que ambas ondas se inyectan en la DSF con idéntico estado de polarización.

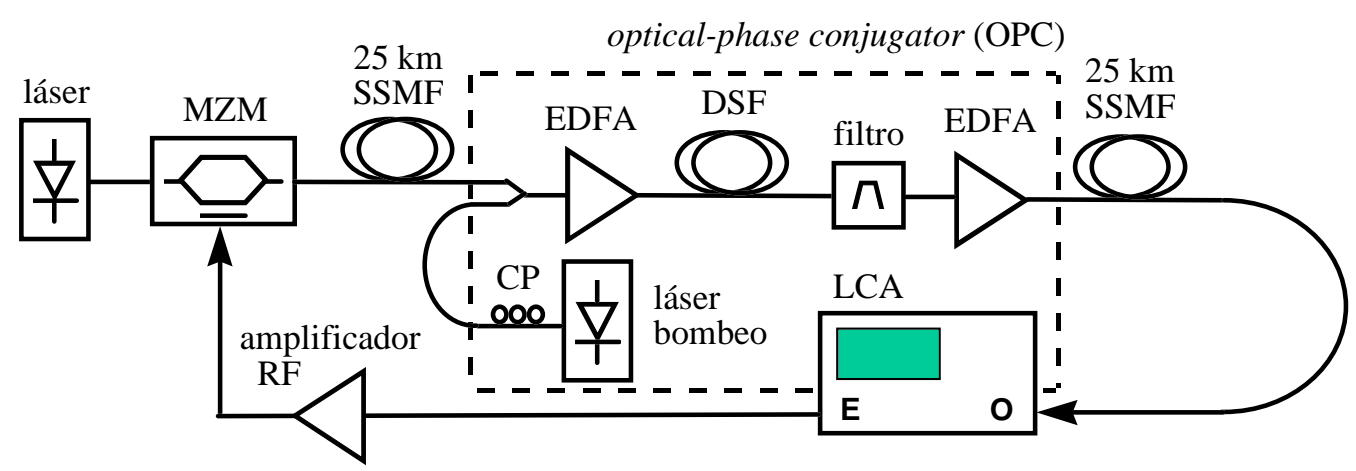

Figura 5.25 Configuración experimental de un sistema de compensación de dispersión mediante FWM en una DSF. 


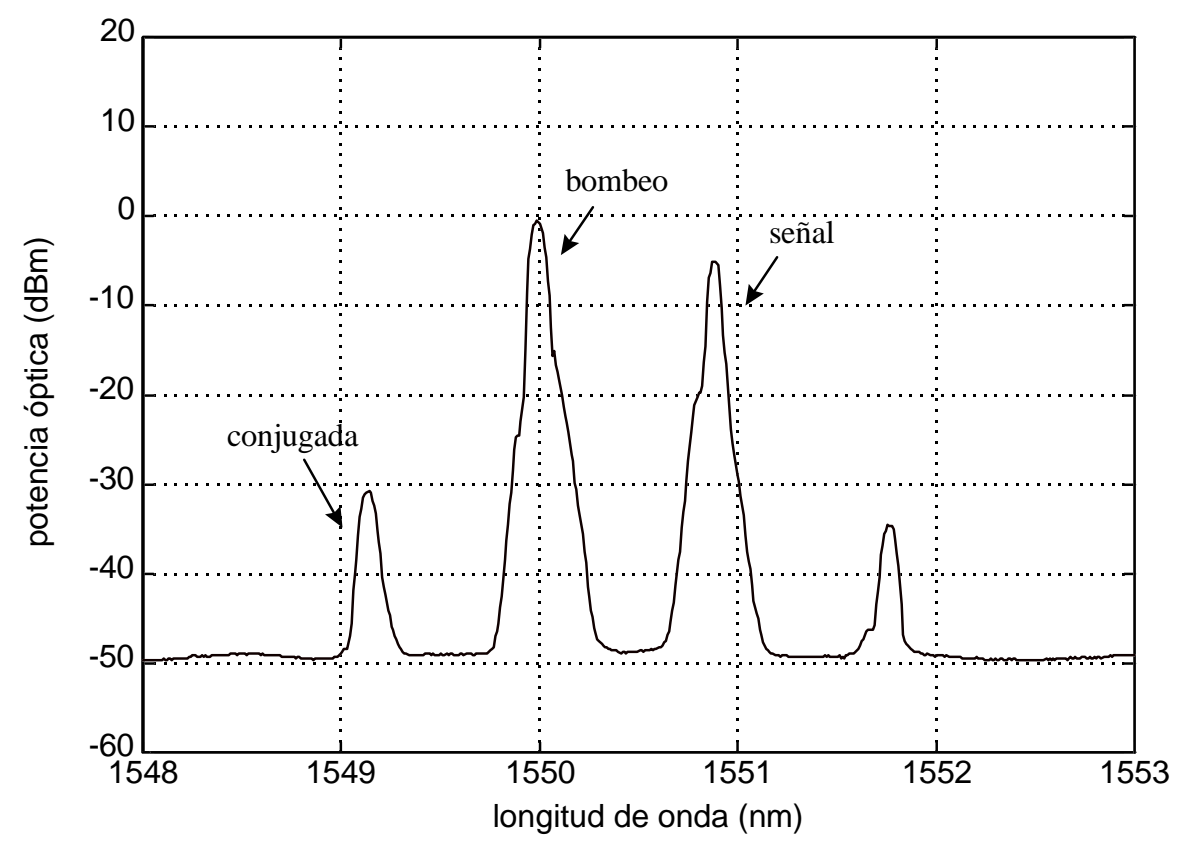

Figura 5.26 Espectro óptico a la salida de la DSF . La DSF tenía una longitud de $12,7 \mathrm{~km}$ y unos parámetros $\alpha=0,201 \mathrm{~dB} / \mathrm{km}$ y $\lambda_{\mathrm{ZD}}$ $=1550 \mathrm{~nm}$.

El espectro óptico a la salida de la DSF se midió con un analizador de espectros óptico de 0,08 $\mathrm{nm}$ de resolución, tal y como se representa en la figura 5.26. Se pueden observar las ondas de bombeo, señal y conjugada a $\lambda_{C}$ $=\lambda_{\mathrm{P}}-2 \lambda_{\mathrm{S}}=1549 \mathrm{~nm}$, además de un producto de FWM no deseado a 1552 $\mathrm{nm}$. Las pérdidas de conversión del proceso de conjugación son de unos 25 dB. De acuerdo con el análisis realizado en el apartado 5.2.2, la eficiencia del proceso de conjugación es inversamente proporcional a la potencia de bombeo y depende de la longitud DSF, presentando un máximo para una longitud de fibra de aproximadamente 1,1/ $\alpha$. Con una atenuación $\alpha=0,201$ dB/km @ 1550 nm para la DSF utilizada se obtiene una longitud óptima de unos $24 \mathrm{~km}$ que es en torno al doble de la longitud de nuestra DSF.

La señal conjugada generada se filtra ahora y se amplifica hasta $0 \mathrm{dBm}$ para transmitirla a través de otro enlace de $25 \mathrm{~km}$ de SSMF. La propagación por este segundo trayecto de fibra compensa la dispersión cromática acumulada a la salida del primer trayecto de fibra. Finalmente, la salida de la fibra se fotodetecta por medio de la entrada óptica del LCA y se mide la respuesta completa del sistema. Previamente a las medidas se 


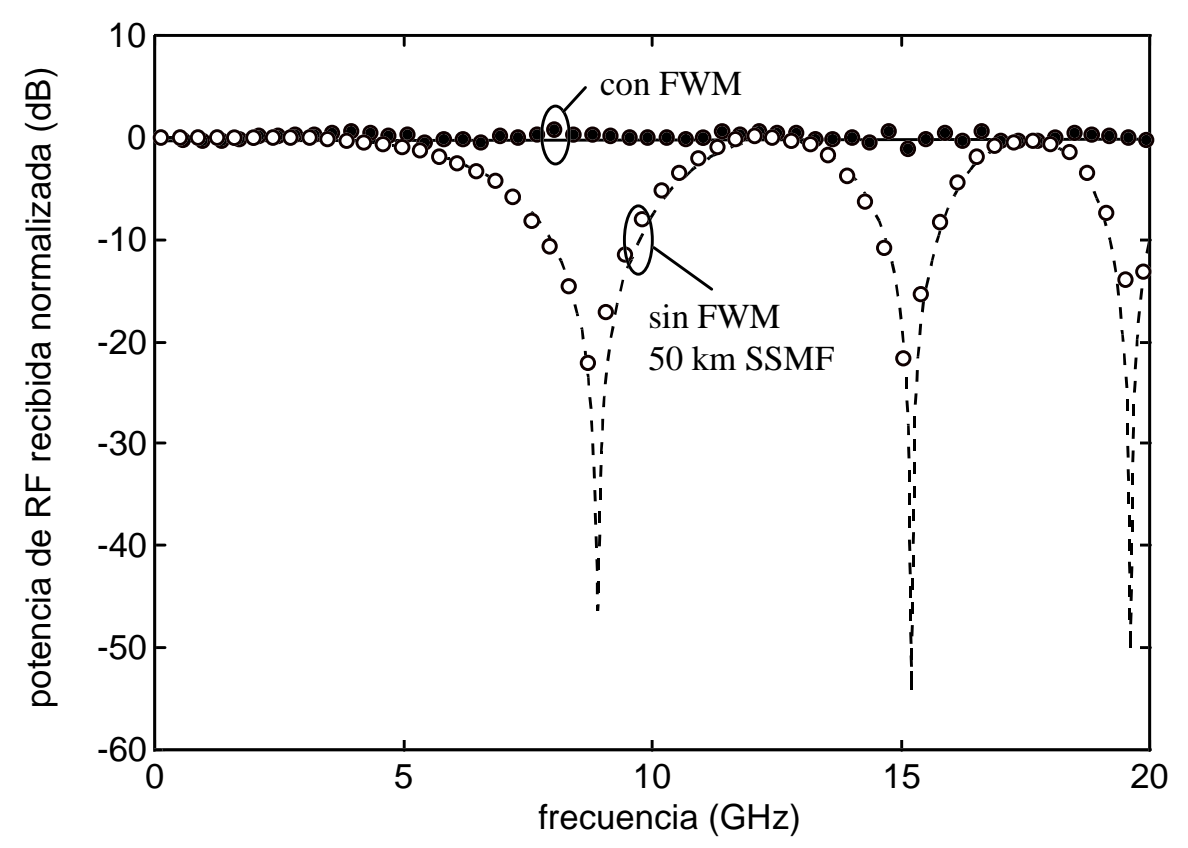

Figura 5.27 Resultados experimentales (símbolos) y de simulación (curvas) de la potencia de RF recibida normalizada para un enlace de SSMF de $50 \mathrm{~km}$ con y sin el empleo de la técnica OPC basada en FWM en una DSF.

realizó una calibración del LCA conectando la salida del MZM directamente a la entrada óptica del analizador para corregir las respuestas del MZM, del amplificador de RF y del fotodetector.

Los resultados de simulación de la potencia de RF detectada normalizada para $50 \mathrm{~km}$ de SSMF se obtuvieron empleando el split-step F ourier method y se representan en la figura 5.27 para los casos con FWM (curva continua) o sin FWM (curva de trazos). Los resultados experimentales obtenidos con FWM (ć́rculos rellenos) y sin FWM (ć́rculos vacíos) también se representan en la misma figura mostrando un ajuste excelente con los resultados de simulación. Se puede ver cómo el efecto de supresión de la portadora se mitiga completamente utilizando la técnica OPC mediante un conjugador basado en FWM en una DSF. Los nulos de transmisión desaparecen y la respuesta en frecuencia con FWM es prácticamente plana excepto una variación inferior $a \pm 2 \mathrm{~dB}$. Este ligero rizado pensamos que es debido a armónicos de la señal de RF generados por el MZM como consecuencia del elevado índice de modulación utilizado en los experimentos. La potencia 
eléctrica en el puerto de RF del MZM era de $+10 \mathrm{dBm}$. Para un MZM con un voltaje de media onda de $9 \mathrm{~V}$ y una frecuencia de modulación de $2 \mathrm{GHz}$ se midieron unos niveles de distorsión de segundo y de tercer armónico de -30 $y-43 \mathrm{dBc}$, respectivamente.

La influencia de efectos no lineales en ambos enlaces de SSMF es despreciable debido a la reducida potencia óptica a la entrada de cada uno de los trayectos de fibra: $0 \mathrm{dBm}$. Adicionalmente, en las simulaciones no se han tenido en cuenta otros efectos como el ruido ASE, SPM O XPM generados durante la propagación a través de la DSF, los cuales pueden ser importantes para altas potencias ópticas a la entrada de la DSF. Sin embargo, tal y como se vio con anterioridad en el apartado 5.2.4, los efectos no lineales del SPM y XPM pueden despreciarse en un OPC basado en DSF para potencias de entrada de hasta $+16 \mathrm{dBm}$ [Wu94]. 


\subsection{CONCLUSIONES Y CONTRIBUCIONES}

En este capítulo se ha estudiado la construcción de conjugadores ópticos basados en el proceso no lineal de mezclado de cuatro ondas en una configuración de fibra de dispersión desplazada o de amplificador de semiconductor. El conjugador óptico es el elemento clave de la técnica de inversión espectral, por lo que interesa caracterizarlo de forma precisa. En particular, el análisis se ha realizado dentro del contexto de los sistemas ópticos analógicos. Entre los principales resultados y aportaciones destacan:

- Se ha realizado un estudio comparativo de las configuraciones DSF y SOA de OPC en el marco de los sistemas ópticos analógicos. En especial, se han derivado para ambas configuraciones resultados relativos a eficiencia de conversión, CNR, distorsión no lineal, espectros y diagramas de ojos de una transmisión digital. Los resultados experimentales demuestran en general que las mejores prestaciones se consiguen empleando la configuración SOA. Esto se debe principalmente a su mayor eficiencia de conversión que permite obtener una mejor $\mathrm{SNR}_{0}$ a la salida del dispositivo y, por lo tanto, un valor de CNR superior a la salida del fotodetector. En nuestro caso, se utilizaron como conjugadores ópticos una DSF de $13 \mathrm{~km}$ de longitud y un SOA (Philips CQF 871) polarizado con una corriente de $300 \mathrm{~mA}$.

- EI ruido ASE es el principal factor limitante de las prestaciones del OPC en sistemas de comunicaciones radio sobre fibra. De hecho, las configuraciones actuales de OPC no permiten la transmisión de subportadoras de RF con modulación AM-VSB, pues los valores de CNR que se obtienen son inferiores a $50 \mathrm{~dB}$. En nuestro caso, para un índice de modulación de intensidad del $50 \%$ y un ancho de banda eléctrico de 30 $\mathrm{MHz}$, se tiene un límite de $\mathrm{CNR}_{\mathrm{ASE}}$ de unos $30 \mathrm{~dB}$ para la DSF y de unos $45 \mathrm{~dB}$ para el SOA. En cambio, los resultados teóricos de distorsión no lineal introducida por el OPC no suponen limitación en un principio, salvo en el caso de que empleemos técnicas para la reducción del ruido ASE que es la principal fuente de degradación. En este último caso, la mayor linealidad se obtendría para la configuración DSF, dado que admite 
mayores potencias ópticas a su entrada sin distorsión. Por último, se ha realizado la transmisión de una PRBS de $52 \mathrm{Mbit} / \mathrm{s}$ a una frecuencia de $1,5 \mathrm{GHz}$ y de una señal de $2 \mathrm{M}$ bit/s sobre un canal de $28 \mathrm{GHz}$ (LMDS) a través de ambas configuraciones de OPC, obteniéndose mejores resultados nuevamente en el caso del SOA.

- Finalmente, se ha demostrado experimentalmente la compensación del efecto de supresión de la portadora en un enlace de $50 \mathrm{~km}$ de SSMF empleando un conjugador óptico basado en FWM en una DSF de 12,7 km de longitud. Estos resultados se encuentran publicados en [Ram99]. 


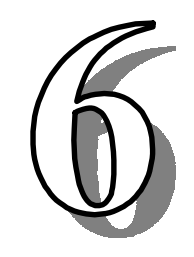

\section{Conclusiones y líneas abiertas}

\subsection{INTRODUCCIÓN}

El trabajo realizado en esta Tesis se enmarca dentro del campo de investigación de los efectos no lineales en dispositivos fotónicos. El principal objetivo de la Tesis ha sido la búsqueda de posibles aplicaciones de estos efectos no lineales en sistemas de comunicaciones ópticas analógicos, como por ejemplo los sistemas radio sobre fibra óptica. En especial, se han estudiado aplicaciones de los fenómenos no lineales de automodulación de fase y de mezclado de cuatro ondasii. El primero de ellos para reducir la degradación producida por la dispersión cromática de la fibra, mientras que el segundo para la construcción de conjugadores ópticos, elementos clave en la técnica de inversión espectral propuesta para compensar la distorsión no lineal introducida por la fibra óptica. A continuación pasaremos a comentar brevemente las principales conclusiones obtenidas en esta Tesis, así como las diversas contribuciones realizadas. Finalmente, describiremos algunas posibles líneas abiertas para continuar la investigación.

\footnotetext{
i self-phase modulation (SPM)

ii four-wave mixing (FWM)
} 


\subsection{CONCLUSIONES Y CONTRIBUCIONES ORIGINALES}

El principal objetivo de esta Tesis ha consistido en el estudio de algunos efectos no lineales que ocurren en dispositivos fotónicos y que tienen aplicación en los sistemas de comunicaciones ópticas analógicos. En relación con las líneas de trabajo planteadas al inicio de la Tesis, debemos indicar que éstas han sido cubiertas de forma satisfactoria. A continuación se resumen las conclusiones obtenidas y las contribuciones realizadas por el autor en cada uno de los capítulos.

En primer lugar, el capítulo 1 ha servido de introducción a los sistemas radio sobre fibra óptica que constituyen el marco donde se desarrolla la presente Tesis. En el mismo se han descrito las características y arquitecturas de diferentes sistemas de acceso: redes híbridas fibra-coaxial o sistemas de distribución punto a multipunto (MMDS, LMDS y MVDS), así como los parámetros que se utilizan para evaluar la calidad de los mismos.

Por otro lado, en el capítulo 2 se ha estudiado la problemática de la distorsión no lineal introducida por la fibra óptica en sistemas multicanal radio sobre fibra. Esta se debe principalmente a los efectos conjuntos de dispersión cromática y SPM, Ios cuales se han analizado por medio de simulación empleando el algoritmo del split-step Fourier method. Entre las aportaciones real izadas, se han obtenido resultados de distorsión no lineal y de penalización de potencia en sistemas multicanal LMDS y MVDS. A diferencia de los sistemas CATV, a frecuencias milimétricas la dispersión cromática se convierte en la principal fuente de degradación muy por encima del fenómeno no lineal de SPM. De este modo, se obtienen alcances de fibra de 1-2 km como consecuencia del efecto de supresión de la portadora, siendo necesario emplear alguna técnica de ecualización. La distorsión, sin embargo, no supone ninguna limitación en estos sistemas debido a los esquemas de modulación que suelen utilizarse. Los niveles de CTB alcanzados, que se mantienen por debajo de los $-40 \mathrm{dBc}$, son suficientes. En cambio, los sistemas CATV se encuentran típicamente limitados por la distorsión no lineal debido al estricto requerimiento de -60 dBc impuesto por la modulación AM-VSB. En este capítulo también se han 
calculado expresiones teóricas del SFDR para caracterizar enlaces ópticos dispersivos a frecuencias milimétricas. Adicionalmente, se han realizado medidas experimentales de la influencia del SPM inducido por la fibra en la respuesta en frecuencia del sistema, los cuales se encuentran publicados en [Ram98] y que se analizaron en detalle en el capítulo 3. Finalmente, se ha realizado un estudio comparativo entre la técnica de modulación óptica en banda lateral única (SSB) y el empleo de redes de difracción sobre fibra óptica (CFGs) propuestas para la reducción de los efectos de la dispersión cromática. El estudio se ha centrado en la ecualización de la distorsión no lineal introducida por la dispersión de la fibra en sistemas ópticos multicanal LMDS. En particular, se ha demostrado que la técnica SSB no resulta válida como consecuencia del chirp introducido en el proceso de modulación que incluso incrementa los niveles de CTB. En cambio, el empleo de CF Gs reduce considerablemente $(\sim 25 \mathrm{~dB})$ los nivel es de CTB, si bien debe realizarse un diseño cuidadoso del dispositivo porque la ecualización es muy sensible al rizado que presentan las respuestas de reflectividad y retardo de grupo del CFG. Esta contribución se ha publicado en [Ram99a].

EI capítulo 3 abordó la primera aplicación del efecto no lineal de SPM en sistemas ópticos analógicos. Ésta consistió en aprovechar el chirp negativo inducido por el SPM de la fibra para alejar el primer nulo de transmisión de la función de transferencia de una fibra óptica y, de este modo, aumentar el producto ancho de banda-longitud de fibra del sistema. Este efecto puede controlarse fácilmente por medio de la potencia óptica inyectada a la entrada de la fibra y ha sido verificado con éxito experimentalmente [Ram98]. Conviene resaltar que este estudio ha coincidido temporalmente con otros similares realizados por Cartaxo et. al. [Car98, Car99a] empleando modulación directa, en donde se referencia el trabajo del autor de esta Tesis en dicha línea de investigación. Adicionalmente, en un artículo de la Universidad Pública de Navarra presentado en la ECOC'99 [Loa99] también se referencia dicho trabajo de investigación. En especial, en este capítulo se ha derivado una expresión teórica cerrada de la función de transferencia de fibras ópticas monomodo (SMFs) dispersivas y no lineales que caracteriza con gran precisión el fenómeno del SPM comentado previamente. Esta 
expresión, basada en funciones de Bessel de orden imaginario puro, modela la propagación de señales de RF a través de SMFs sujetas a efectos no lineales y ha sido publicada en [Ram00]. Adicionalmente, la técnica SPM ha sido propuesta para reducir los efectos de la dispersión cromática en sistemas MI-DD operando a $1550 \mathrm{~nm}$ sobre SSMF. En especial, se han realizado medidas de la penalización de potencia utilizando esta técnica y se ha conseguido extender el alcance del sistema de forma significativa con respecto al caso de transmisión convencional. Finalmente, como contribución se ha demostrado la transmisión tolerante a la dispersión de una señal de $8 \mathrm{Mbit} / \mathrm{s}$ a una frecuencia de $7 \mathrm{GHz}$ sobre $75 \mathrm{~km}$ de SSMF empleando la técnica SPM con una potencia óptica transmitida de $+11 \mathrm{dBm}$.

La técnica de inversión espectral, comúnmente conocida como OPC, se analizó en detalle en el capítulo 4 dentro del contexto de los sistemas radio sobre fibra. Aunque esta técnica ya se había utilizado con éxito anteriormente en sistemas de comunicaciones ópticas digitales, no se había propuesto ninguna aplicación para sistemas ópticos analógicos. En nuestro caso, se ha demostrado por primera vez por medio de simulación la compensación de la distorsión no lineal introducida por la fibra óptica (dispersión + SPM) en sistemas SCM empleando la técnica OPC [Mar97, Ram98a]. Adicionalmente, también se ha demostrado la compensación del efecto de supresión de la portadora de forma teórica y experimental [Ram99], además de la ecualización de la penalización de potencia en sistemas radio-fibra a frecuencias milimétricas, lo cual ha coincidido temporalmente con otros estudios realizados por Kitayama y Sotobayashi en la banda de $60 \mathrm{GHz}$ [Kit99, Sot99, Sot99a]. Otra aportación ha consistido en la obtención de una expresión teórica para caracterizar la respuesta en frecuencia de un sistema OPC a partir de las funciones de conversión faseintensidad de la fibra óptica. Finalmente, también se han obtenido resultados de sensibilidad de la técnica OPC frente a diversos parámetros del sistema: longitudes de fibra, potencias ópticas, valores de dispersión cromática, etc. Dado que el efecto Kerr causante del SPM se convierte en el principal factor limitante como consecuencia de la condición de simetría impuesta por la técnica OPC, la potencia óptica es el parámetro más influyente en los resultados de sensibilidad. Esto mismo fue demostrado 
experimentalmente en [Sot99]. Debemos indicar, por último, que las publicaciones realizadas en esta línea de investigación de la Tesis han sido valoradas por otros autores mediante referencias a las mismas [Sot99, Sot99a].

En el capítulo 5 se estudió en detalle el proceso no lineal de mezclado de cuatro ondas (FWM) en configuraciones de fibra de dispersión desplazada (DSF) y de amplificador de semiconductor (SOA), como aplicación en la construcción de conjugadores ópticos utilizados en la técnica OPC. A diferencia de otros estudios previos, el análisis se realizó en el contexto de los sistemas ópticos analógicos, y se obtuvieron resultados experimentales comparativos de las prestaciones de cada una de las configuraciones: $13 \mathrm{~km}$ de DSF y un SOA (Philips CQF 871) polarizado con $300 \mathrm{~mA}$. EI ruido ASE se convirtió en el principal factor limitante en las prestaciones de ambas configuraciones de conjugador. Por ejemplo, para un índice de modulación del $50 \%$ y un ancho de banda de canal de $30 \mathrm{MHz}$, se obtuvieron unos valores de CNR por ruido ASE de 30 y $45 \mathrm{~dB}$, respectivamente, para las configuraciones DSF/SOA construidas en el laboratorio. En general, se consiguen mejores prestaciones con el OPC basado en SOA debido a su mayor eficiencia de conversión y a una mejor relación señal a ruido óptica. Para la demostración experimental se realizó la transmisión a través del conjugador de una PRBS de $52 \mathrm{Mbit} / \mathrm{s}$ a una frecuencia de $1,5 \mathrm{GHz}$ y de un canal digital sobre una subportadora de $28 \mathrm{GHz}$. Por otro lado, en el caso de la distorsión no lineal introducida por el conjugador, la configuración DSF posee mejores prestaciones, ya que se comporta de forma relativamente lineal frente a mayores potencias ópticas a su entrada que el SOA si evitamos el fenómeno de scattering de Brillouin. No obstante, esta característica sólo se observa cuando se utilizan técnicas de reducción del ruido ASE [Gei97], que es la degradación predominante. Por último, también se demostró experimentalmente la compensación del efecto de supresión de la portadora en un margen de frecuencias de $20 \mathrm{GHz}$ y para un enlace de $50 \mathrm{~km}$ de SSMF empleando un conjugador basado en FWM en $12,7 \mathrm{~km}$ de DSF. Estos resultados se encuentran publicados en [Ram99] y, como se ha dicho antes, han coincidido con otros estudios similares para el OPC basado en SOA realizados por Kitayama y Sotobayashi [Kit99]. 


\subsection{LÍNEAS ABIERTAS}

La continuación natural del trabajo presentado en esta Tesis consiste en la búsqueda de nuevas aplicaciones de efectos no lineales de dispositivos fotónicos en sistemas radio sobre fibra óptica. En especial, esta línea de investigación es muy amplia y puede dar lugar a la elaboración de otras tesis doctorales. Por otro lado y centrándonos en las aplicaciones presentadas en la Tesis, existen varias direcciones en que puede continuarse el trabajo de investigación, especialmente la verificación experimental de algunos resultados teóricos o de simulación. A continuación comentamos brevemente estas posibles líneas abiertas:

- Existen otros efectos no lineales que se conocen habitualmente por sus efectos de degradación, como pueden ser la modulación de fase cruzada, la modulación de ganancia cruzada en SOAs, o el scattering estimulado de Raman o de Brillouin, que pueden encontrar aplicación en sistemas ópticos analógicos. Como ejemplo, previamente a la elaboración de esta Tesis se han propuesto aplicaciones del SBS para realizar una modulación óptica SSB o para la construcción de mezcladores de señales de RF [Yao98, Yao98a]. El trabajo de investigación podría continuar en la búsqueda de nuevas aplicaciones de estos efectos no lineales.

- Una continuación del trabajo realizado en el modelado teórico de la propagación de señales de RF a través de SMFs dispersivas y no lineales podría consistir en la obtención de expresiones cerradas de las funciones de conversión $\mathrm{C}_{\mathrm{IM}-\mathrm{PM}}$ y $\mathrm{C}_{\mathrm{PM}-\mathrm{PM}}$, análogas a las ya calculadas para las funciones $\mathrm{C}_{\mathrm{IM}-\mathrm{IM}}$ y $\mathrm{C}_{\mathrm{PM}-\mathrm{IM}}$. Estas expresiones podrían utilizarse posteriormente para modelar teóricamente la respuesta en frecuencia de sistemas OPC.

- En relación con el proceso no lineal de FWM aplicado a la construcción de conjugadores ópticos en sistemas analógicos, resulta interesante la elaboración de un modelo teórico para evaluar la distorsión no lineal introducida sobre las señales de RF en el proceso de conjugación mediante SOAs o DSFs, junto con la posterior medida y validación experimental. 
- Una aplicación interesante de los dispositivos fotónicos es la referente a generación de subportadoras de milimétricas. Dado que los osciladores a frecuencias de milimétricas suelen ser costosos, habitualmente se suelen utilizar osciladores a submúltiplos de la frecuencia que queremos obtener y aprovechar el comportamiento no lineal de determinados dispositivos para generar armónicos de esta señal [Moo95]. En este caso, lo que se pretende es encontrar nuevas configuraciones en las cuales se aproveche el comportamiento óptico no lineal de dispositivos fotónicos para la generación y/o subida en frecuencia de subportadoras como aplicación en sistemas radio sobre fibra.

- Finalmente, en lo referente a la distorsión no lineal introducida por la fibra óptica en sistemas de comunicaciones radio sobre fibra, se han obtenido resultados de simulación en sistemas de banda ancha LMDS y MVDS operando a frecuencias milimétricas. Como posible línea de trabajo futura, sería interesante estudiar también la distorsión introducida en el trayecto de propagación vía radio y analizar bajo qué condiciones predomina sobre las degradaciones introducidas en el trayecto óptico. 


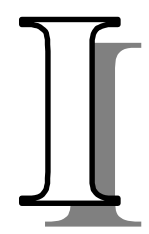

\section{Apéndice: Respuesta en frecuencia de los sistemas de comunicaciones ópticas analógicos}

\section{I.1 INTRODUCCIÓN}

La función de transferencia es un importante parámetro para caracterizar los sistemas de comunicaciones ópticas analógicos. En este apéndice se pretende calcular la respuesta en frecuencia de los sistemas que emplean modulación de intensidad y detección directa (MI-DD). El estudio comienza en la sección I. 2 con las funciones de conversión entre las modulaciones de intensidad y de fase a lo largo de fibras ópticas monomodo dispersivas y no lineales. En la sección 1.3 se analiza la influencia del chirp del transmisor óptico en la respuesta en frecuencia de la fibra óptica. En particular, se 
realiza un completo modelado del chirp en láseres de semiconductor, moduladores Mach-Zehnder y de electroabsorción. Las secciones I.4 y 1.5 están dedicadas a estudiar las técnicas de modulación óptica en banda lateral única y de inversión espectral, respectivamente. La primera es una técnica tolerante a la dispersión cromática, mientras que la segunda compensa los efectos de la dispersión y el SPM de la fibra. En ambos casos, se realizará un modelo del sistema y se calculará la respuesta en frecuencia del mismo. Por último, en la sección 1.5 se tratarán las fibras de dispersión desplazada y se analizará la influencia que tiene el coeficiente de dispersión de segundo orden en la respuesta del sistema. 


\section{I.2 FUNCIÓN DE TRANSFERENCIA DE LA FIBRA ÓPTICA}

\subsubsection{Análisis teórico de la propagación a través de la fibra}

Como se ha visto con anterioridad en el capítulo 2, la propagación de señales a través de fibras dispersivas y no lineales se rige por la ecuación de Schrödinger no lineal

$$
\frac{\partial A}{\partial z}-\frac{j}{2} \beta_{2} \frac{\partial^{2} A}{\partial T^{2}}=-\frac{\alpha}{2} A-j \gamma|A|^{2} A,
$$

donde $\beta_{2}$ denota la dispersión cromática (GVD) y $\gamma$ es el coeficiente no lineal responsable del SPM. Recordemos que $A(z, T)$ es el término de envolvente del campo óptico y puede expresarse como

$$
A(z, T)=\sqrt{p_{T}(z, T)} \exp \left[j \phi_{T}(z, T)\right],
$$

siendo $p_{T}(z, T)$ y $\phi_{T}(z, T)$ funciones reales que representan la potencia y la fase de la señal óptica, respectivamente. Si a continuación se sustituye (I.2) en la ec. de propagación (I.1), y se expresan las derivadas parciales de la envolvente en términos de las derivadas de las funciones de potencia y de fase, tras algunas manipulaciones matemáticas se puede llegar a un par de ecuaciones no lineales acopladas describiendo la propagación de la potencia y la fase ópticas a través de una fibra dispersiva y no lineal [Car98]:

$$
\begin{aligned}
& \frac{\partial p_{\mathrm{T}}}{\partial z}=-\beta_{2}\left(\frac{\partial p_{\mathrm{T}}}{\partial \mathrm{T}} \frac{\partial \phi_{\mathrm{T}}}{\partial \mathrm{T}}+\mathrm{p}_{\mathrm{T}} \frac{\partial^{2} \phi_{\mathrm{T}}}{\partial \mathrm{T}^{2}}\right)-\alpha \mathrm{p}_{\mathrm{T}}, \\
& \frac{\partial \phi_{\mathrm{T}}}{\partial z}=-\gamma \mathrm{p}_{\mathrm{T}}+\frac{\beta_{2}}{8 p_{\mathrm{T}}^{2}}\left[2 \mathrm{p}_{\mathrm{T}} \frac{\partial^{2} \mathrm{p}_{\mathrm{T}}}{\partial \mathrm{T}^{2}}-\left(\frac{\partial \mathrm{p}_{\mathrm{T}}}{\partial \mathrm{T}}\right)^{2}-4 \mathrm{p}_{\mathrm{T}}^{2}\left(\frac{\partial \phi_{\mathrm{T}}}{\partial \mathrm{T}}\right)^{2}\right] .
\end{aligned}
$$

Para continuar el análisis se supondrá que las señales ópticas de potencia y de fase se pueden separar en sus componentes dependientes e independientes (valor medio) del tiempo. De este modo, la potencia óptica total $\mathrm{p}_{\mathrm{T}}(\mathrm{z}, \mathrm{T})$ se escribe como la suma de una potencia media $\mathrm{P}(\mathrm{z})$ más un término de modulación o ruido $p(z, T)$, y la fase total $\phi_{T}(z, T)$ como la suma 
de una fase media $\Phi(z, T)$ más un término de modulación o ruido $\phi(z, T)$. Sustituyendo ahora estas componentes en las ecuaciones acopladas no lineales (I.3) y (I.4) y despreciando los productos de $p(z, T)$ por $\phi(z, T)$ y sus derivadas (válido en el contexto de pequeña señal [Wan92]) se puede llegar fácilmente a un par de ecuaciones diferenciales lineales acopladas que gobierna la propagación en función del tiempo de las modulaciones de intensidad (IM) y de fase (PM). Usando la potencia normalizada, $p_{N}(z, T)$, definida como

$$
p(z, T)=p_{N}(z, T) \exp (-\alpha z)
$$

y aplicando transformadas de Fourier respecto al tiempo a ambos lados de cada una de las ecuaciones se obtiene finalmente el siguiente sistema de ecuaciones diferenciales lineales [Car98]:

$$
\begin{aligned}
& \frac{\partial{\widetilde{\rho_{N}}}_{(}\left(z, \omega_{\mathrm{e}}\right)}{\partial z}=\beta_{2} \omega_{\mathrm{e}}^{2} \mathrm{P}_{0} \widetilde{\phi}\left(z, \omega_{\mathrm{e}}\right), \\
& \frac{\partial \widetilde{\phi}\left(z, \omega_{\mathrm{e}}\right)}{\partial z}=-\left[\frac{\beta_{2} \omega_{\mathrm{e}}^{2}}{4 \mathrm{P}_{0}}+\gamma \exp (-\alpha z)\right] \widetilde{\mathrm{p}}_{\mathrm{N}}\left(z, \omega_{\mathrm{e}}\right) .
\end{aligned}
$$

donde $\widetilde{p}_{N}\left(z, \omega_{e}\right)$ y $\widetilde{\phi}\left(z, \omega_{e}\right)$ son las transformadas de Fourier de $p_{N}(z, T)$ y $\phi(z, T)$, respectivamente, y $P_{0}=P(0)$ es la potencia óptica media a la entrada de la fibra. Las ecuaciones (I.6) y (I.7), pues, modelan la evolución a lo largo de la fibra óptica de las fluctuaciones de potencia y de fase, teniendo en cuenta la dispersión cromática, el efecto Kerr y las pérdidas de la fibra. En el caso particular de transmisión lineal $(\gamma=0)$, estas ecuaciones pueden resolverse fácilmente de forma analítica para llegar a los resultados de Wang y Petermann [Wan92]:

$$
\begin{aligned}
& \widetilde{\rho}_{N}\left(z, \omega_{e}\right)=\widetilde{p}_{N}\left(0, \omega_{e}\right) \cos \left(\frac{\beta_{2} \omega_{e}^{2} z}{2}\right)+2 P_{0} \widetilde{\phi}\left(0, \omega_{e}\right) \operatorname{sen}\left(\frac{\beta_{2} \omega_{e}^{2} z}{2}\right), \\
& \widetilde{\phi}\left(z, \omega_{e}\right)=-\frac{\widetilde{p}_{N}\left(0, \omega_{e}\right)}{2 P_{0}} \operatorname{sen}\left(\frac{\beta_{2} \omega_{e}^{2} z}{2}\right)+\widetilde{\phi}\left(0, \omega_{e}\right) \cos \left(\frac{\beta_{2} \omega_{e}^{2} z}{2}\right) .
\end{aligned}
$$




\subsubsection{Funciones de conversión y cálculo de la función de transferencia de la fibra}

A partir de las ecuaciones (I.8) y (I.9) del apartado anterior, es posible obtener unas funciones que modelan las conversiones que se producen entre las modulaciones de intensidad y de fase a la entrada de la fibra $(z=0)$ y las modulaciones de intensidad y de fase a una cierta distancia $z$. Estas funciones se conocen como conversión IM-IM, conversión PM-IM, conversión IM-PM y conversión PM-PM, y se definen como [Car98]

$$
\begin{aligned}
& C_{I M-I M}\left(z, \omega_{e}\right)=\left.\frac{\widetilde{\Gamma}_{N}\left(z, \omega_{e}\right)}{\widetilde{p}_{N}\left(0, \omega_{e}\right)}\right|_{\widetilde{\phi}\left(0, \omega_{e}\right)=0}, \\
& C_{P M-I M}\left(z, \omega_{e}\right)=\left.\frac{\widetilde{\Gamma_{N}}\left(z, \omega_{e}\right)}{P_{0} \widetilde{\phi}\left(0, \omega_{e}\right)}\right|_{\widetilde{p}_{N}\left(0, \omega_{e}\right)=0}, \\
& C_{\text {IM-PM }}\left(z, \omega_{e}\right)=\left.\frac{P_{0} \widetilde{\phi}\left(z, \omega_{e}\right)}{\widetilde{\Gamma}_{N}\left(0, \omega_{e}\right)}\right|_{\widetilde{\phi}\left(0, \omega_{e}\right)=0}, \\
& C_{P M-P M}\left(z, \omega_{e}\right)=\left.\frac{\widetilde{\phi}\left(z, \omega_{e}\right)}{\widetilde{\phi}\left(0, \omega_{e}\right)}\right|_{\widetilde{p}_{N}\left(0, \omega_{e}\right)=0} .
\end{aligned}
$$

Estas funciones de conversión se pueden calcular de forma sencilla aplicando, por ejemplo, el método de Runge-Kutta al sistema de ecuaciones diferenciales (I.6) y (I.7). No obstante, en el caso particular de transmisión lineal $(\gamma=0)$ pueden obtenerse directamente a partir de las ecuaciones (I.8) y (I.9). De este modo, suponiendo que existe sólo dispersión, se llega a las siguientes funciones:

$$
\begin{aligned}
& C_{I M-I M}\left(z, \omega_{e}\right)=C_{P M-P M}\left(z, \omega_{e}\right)=\cos \left(\frac{\beta_{2} \omega_{e}^{2} Z}{2}\right), \\
& C_{P M-I M}\left(z, \omega_{e}\right)=2 \operatorname{sen}\left(\frac{\beta_{2} \omega_{e}^{2} Z}{2}\right),
\end{aligned}
$$




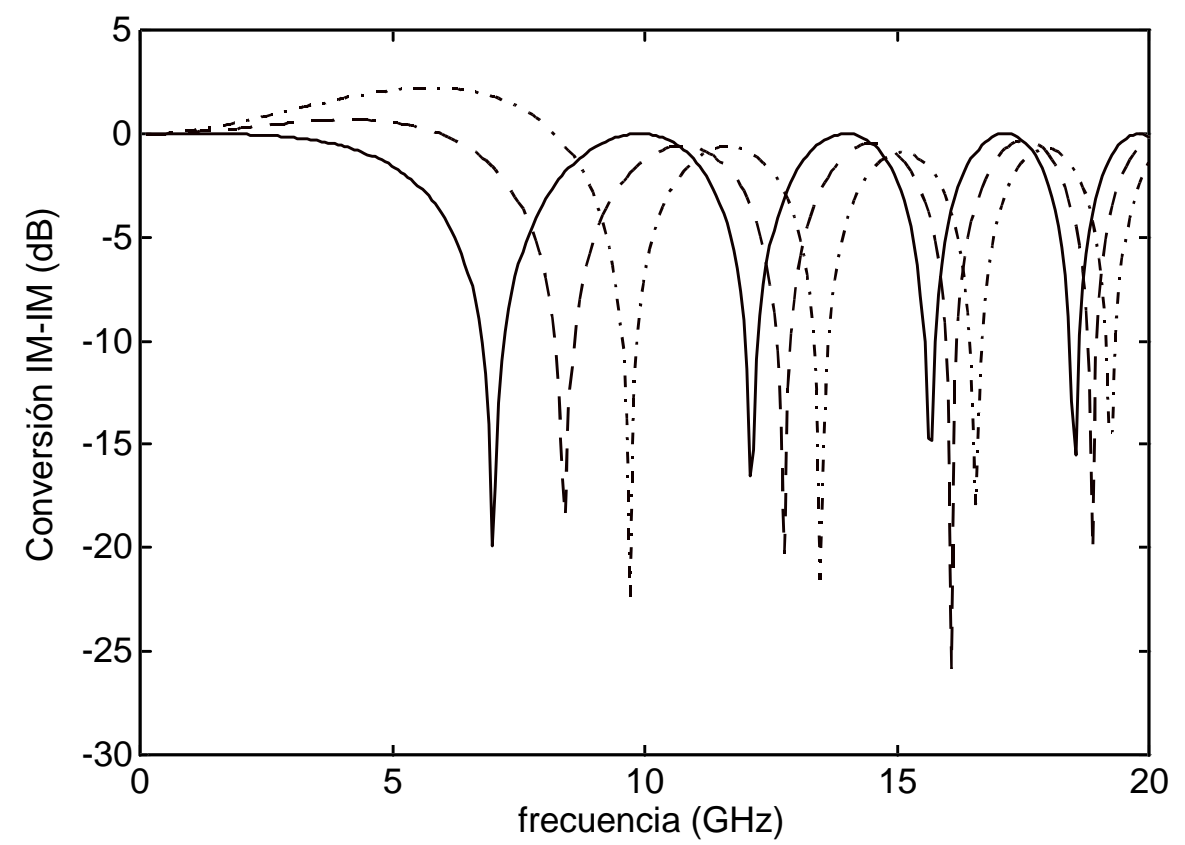

(a)

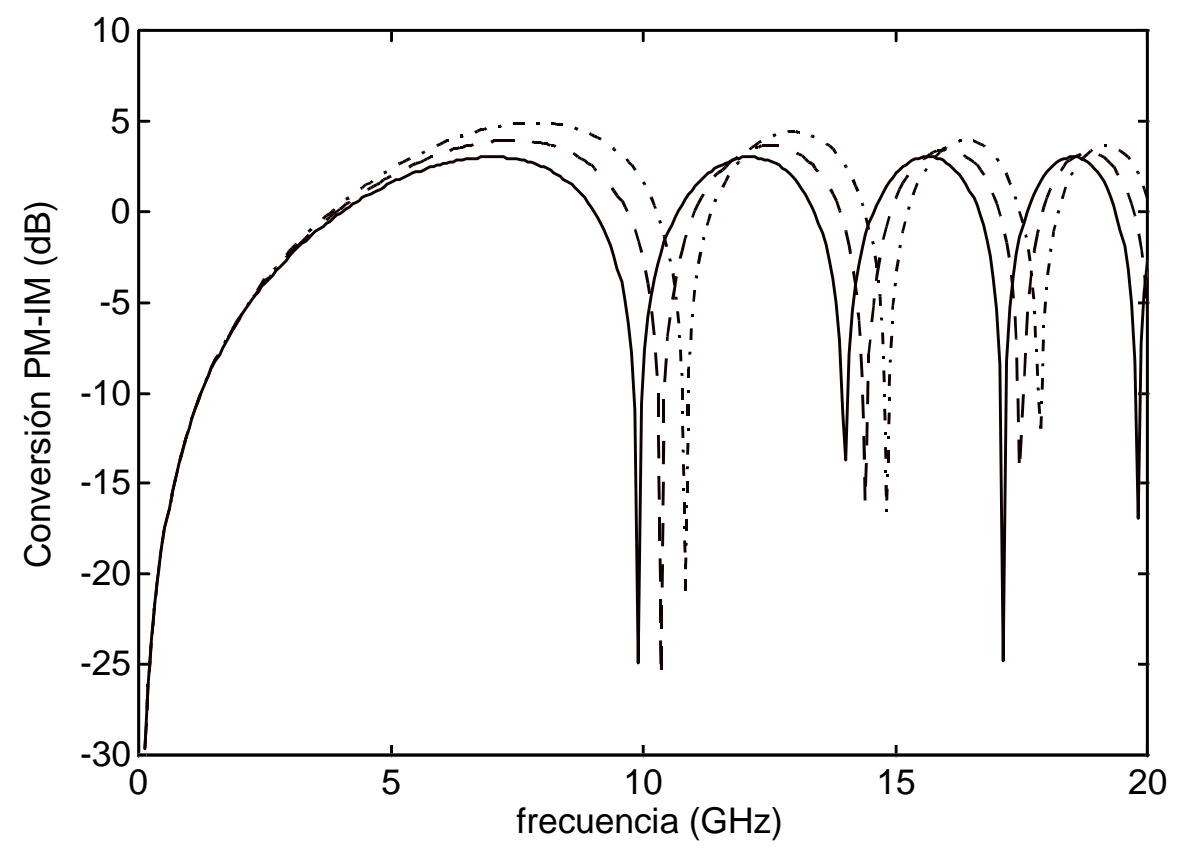

(b)

Figura I.1 Funciones de conversión (a) $\mathrm{C}_{\mathrm{IM}-\mathrm{IM}}$ y (b) $\mathrm{C}_{\mathrm{PM}-\mathrm{IM}}$ en función de la frecuencia de modulación y para una longitud de fibra de $75 \mathrm{~km}$. Las curvas continuas son para transmisión lineal $(\gamma=0)$, mientras que las de trazos son para una potencia óptica de $10 \mathrm{~mW}$ y las de trazos y puntos para $20 \mathrm{~mW}$. 


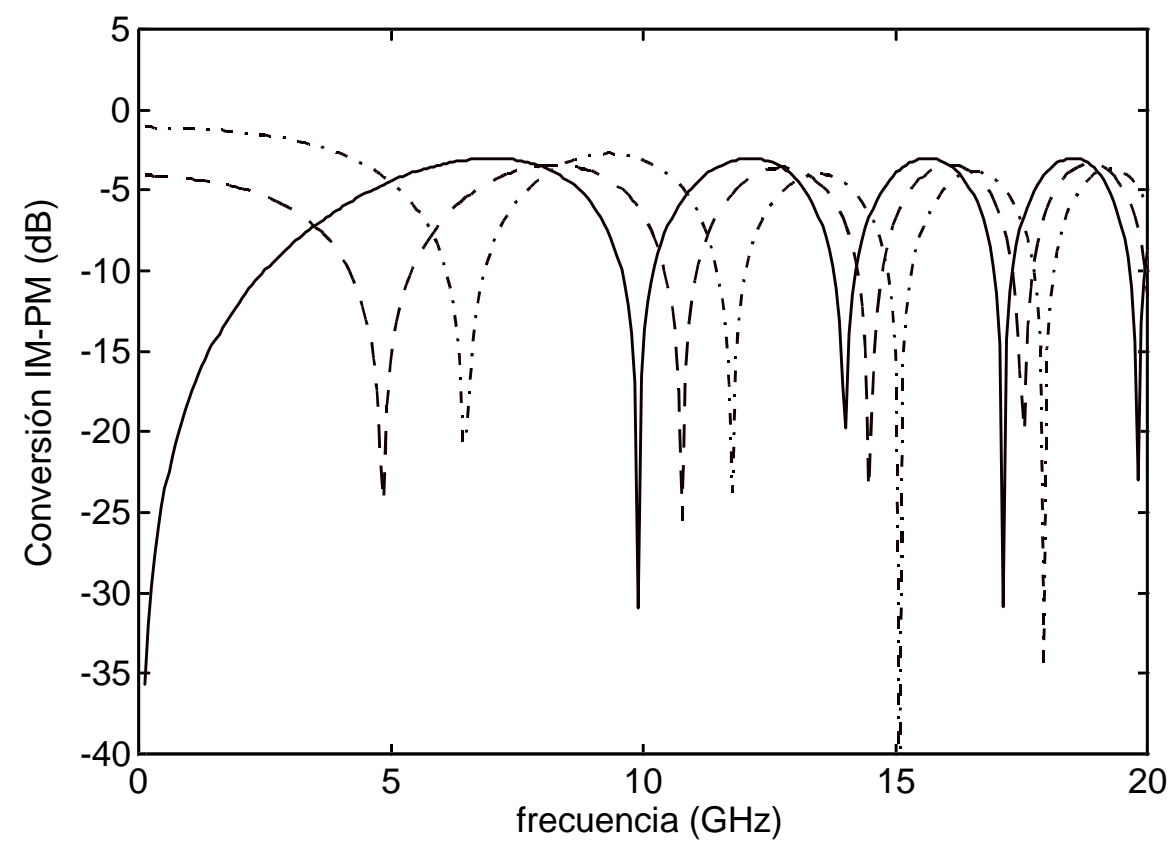

(a)

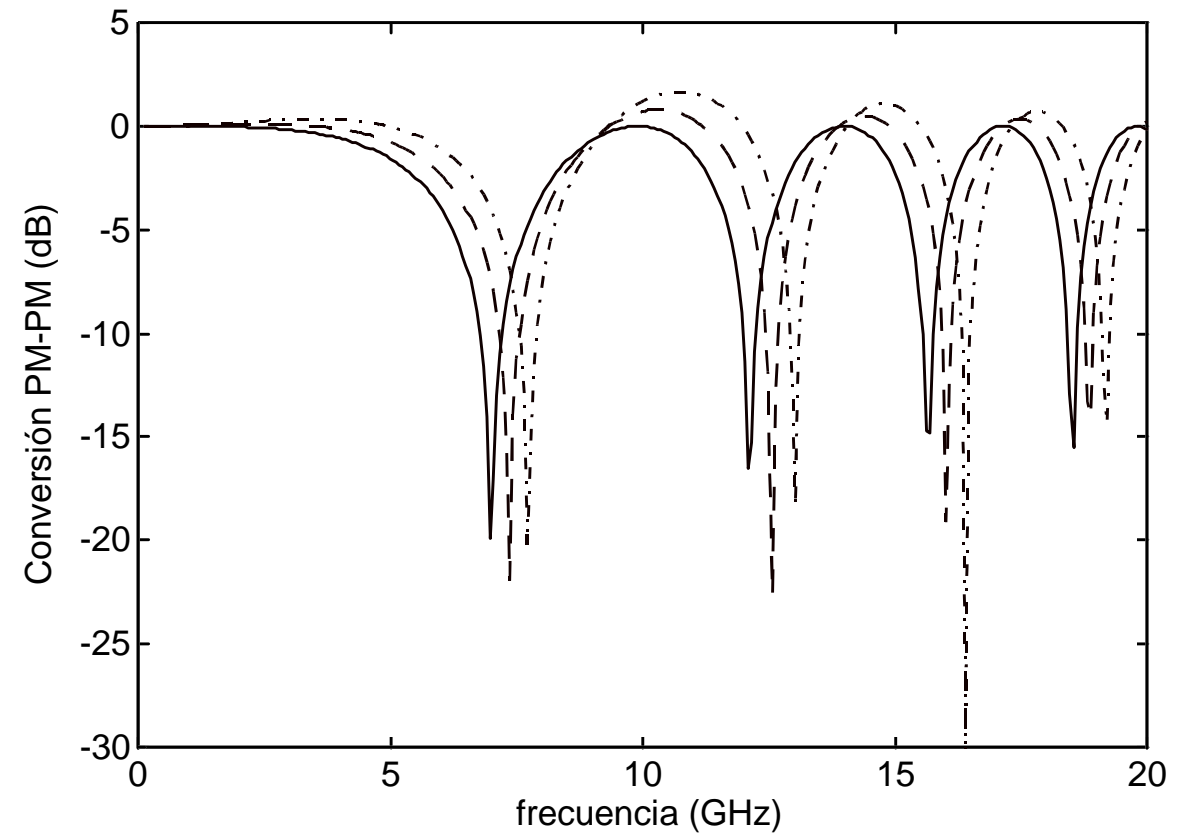

(b)

Figura I.2 Funciones de conversión (a) $C_{I M-P M}$ y (b) $C_{P M-P M}$ en función de la frecuencia de modulación y para una longitud de fibra de $75 \mathrm{~km}$. Los parámetros son idénticos a los de la figura anterior. 


$$
C_{I M-P M}\left(z, \omega_{e}\right)=-\frac{1}{2} \operatorname{sen}\left(\frac{\beta_{2} \omega_{e}^{2} z}{2}\right)
$$

En las figuras I.1 y l.2 se representan estas funciones de conversión para 75 km de fibra óptica monomodo estándar (SSMF) con parámetros de atenuación $\alpha=0,2 \mathrm{~dB} / \mathrm{km}$, dispersión cromática $\mathrm{D}=17 \mathrm{ps} /(\mathrm{km} \cdot \mathrm{nm})$ y coeficiente no lineal $\gamma=1,85 \mathrm{~W}^{-1} \mathrm{~km}^{-1}$ correspondiente a un área efectivai $A_{\text {eff }}$ $=70 \mu \mathrm{m}^{2}$. Las curvas continuas son para transmisión lineal $(\gamma=0)$, mientras que las de trazos son para una potencia óptica a la entrada de la fibra de 10 $\mathrm{mW}$, y las de trazos y puntos para $20 \mathrm{~mW}$. Dado que las funciones de conversión involucran magnitudes como la potencia y la fase ópticas, para


definiciones de $\mathrm{C}_{\mathrm{PM}-\mathrm{IM}}$ y $\mathrm{C}_{\mathrm{IM}-\mathrm{PM}}$ se ha introducido la potencia óptica $\mathrm{P}_{0}$ para transformarlas en funciones adimensionales.

En general, se puede observar que los efectos no lineales (SPM) inducen un desplazamiento en frecuencia de las funciones de conversión. Como es lógico, a mayor potencia óptica se produce un mayor desplazamiento, aunque algunas funciones son más sensibles que otras. En el caso de $C_{P M-I M}$ y $C_{P M-P M}$ este desplazamiento no es demasiado significativo, y viene acompañado de un ligero aumento en el nivel de las lobulaciones. Sin embargo, en el caso de $\mathrm{C}_{\text {IM-IM }}$ Sí que se observa un cambio apreciable respecto a la situación de transmisión lineal. A frecuencias bajas aparece ganancia respecto al caso de sólo dispersión, además de un desplazamiento del primer nulo hacia frecuencias mayores, lo cual permite aumentar el ancho de banda de transmisión de la fibra óptica. Los resultados experimentales que confirman este fenómeno, así como una explicación más detallada del mismo, se muestran en el capítulo 3 [Ram98]. Por último, en la función de conversión $\mathrm{C}_{\mathrm{IM}-\mathrm{PM}}$ se observa un efecto curioso respecto al caso de transmisión lineal. Además del desplazamiento ya comentado, a bajas frecuencias se produce conversión en el caso de transmisión no lineal que no ocurría suponiendo

\footnotetext{
' recordemos que en el capítulo 2 se utilizó una SMF con área efectiva de $50 \mu \mathrm{m}^{2}\left(\gamma=2,6 \mathrm{~W}^{-}\right.$ ${ }^{1} \mathrm{~km}^{-1}$ )

ii $\mathrm{dB}$ ópticos ( $\mathrm{dBo}$ ) en contraposición de los dB eléctricos ( $\mathrm{dBe}$ ) utilizados a la salida del fotodetector
} 
sólo dispersión. La explicación de esto se debe a que la conversión PM-IM e IM-PM producida por la dispersión cromática no es significativa para frecuencias bajas, debido a la dependencia con el cuadrado de la frecuencia, mientras que el SPM sí que induce una modulación de fase que depende proporcionalmente de la potencia óptica y de la longitud de fibra.

Las figuras I.1 y I.2 muestran las funciones de conversión para una longitud constante de fibra de $75 \mathrm{~km}$. La variación de la longitud de fibra produce un efecto de compresión-expansión en frecuencia. Este efecto puede deducirse de la figura I.3 donde se representan las funciones de conversión en el caso de transmisión lineal para dos longitudes de fibra diferentes: $50 \mathrm{~km}$ y 75 $\mathrm{km}$. Se observa que un aumento de la longitud de fibra produce en general una reducción del ancho de banda y un mayor número de lobulaciones.

Una vez definidas las funciones de conversión, la función de transferencia que model a la respuesta de la fibra óptica se puede obtener a partir de éstas aplicando superposición [Car98, Wan92]:


Figura I.3 Funciones de conversión de la fibra óptica en función de la frecuencia de modulación y para dos longitudes de fibra distintas: 50 y $75 \mathrm{~km}$. 


$$
\begin{aligned}
& H_{F}\left(z, \omega_{e}\right)=\frac{\widetilde{\Gamma}_{N}\left(z, \omega_{e}\right)}{\widetilde{\rho}_{N}\left(0, \omega_{e}\right)}
\end{aligned}
$$

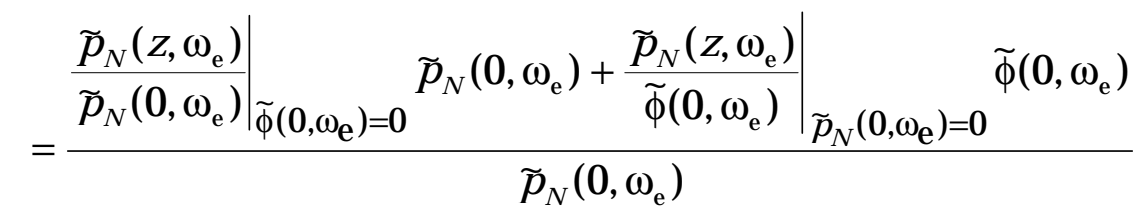

$$
\begin{aligned}
& =C_{I M-I M}\left(z, \omega_{e}\right)+\frac{1}{2} H_{P M}\left(\omega_{e}\right) C_{P M-I M}\left(z, \omega_{e}\right) \text {, }
\end{aligned}
$$

siendo $H_{P M}\left(\omega_{e}\right)$ la función de transferencia entre la modulación de intensidad y la modulación de fase en el transmisor óptico [Bje96]. A partir de la ecuación anterior, $\mathrm{H}_{\mathrm{PM}}\left(\omega_{\mathrm{e}}\right)$ se calcularía mediante

$$
H_{P M}\left(\omega_{e}\right)=2 P_{0} \frac{\widetilde{\phi}\left(0, \omega_{e}\right)}{\widetilde{P}_{N}\left(0, \omega_{e}\right)} .
$$

Como se puede observar, en la definición (I.17) no aparecen las funciones $C_{I M-P M}$ y $C_{P M-P M}$, ya que la función de transferencia de la fibra es independiente de ellas en un sistema MI-DD. Sin embargo, más tarde veremos que son indispensables al analizar la técnica OPC, puesto que en este caso existe un proceso de conjugación de la fase óptica que debe ser modelado mediante estas funciones. 


\section{I.3 INFLUENCIA DEL CHIRP DEL TRANSMISOR ÓPTICO}

Anteriormente se ha visto que la función de transferencia de la fibra óptica depende no sólo de los parámetros físicos de la fibra, sino también de los parámetros de modulación por medio de la función de transferencia $\mathrm{H}_{\mathrm{PM}}\left(\omega_{\mathrm{e}}\right)$. En sistemas MI-DD, la modulación de intensidad viene acompañada de una modulación de fase residual introducida por el transmisor óptico y que se conoce con el nombre de chirp. Si queremos pues, realizar un análisis completo de la respuesta de la fibra óptica, es necesario tener en cuenta el chirp introducido por el transmisor óptico.

\section{I.3.1 Modelado del chirp}

Si suponemos una modulación de intensidad con un tono de RF, la expresión del campo eléctrico a la salida del transmisor óptico con chirp viene dada por

$$
E(t)=\sqrt{P_{0}} \sqrt{1+m_{i} \cos \left(2 \pi f_{m} t\right)} \exp \left\{j\left[\omega_{0} t+m_{f} \cos \left(2 \pi f_{m} t+\varphi\right)\right]\right\},
$$

donde $f_{m}=\omega_{e} /(2 \pi)$ es la frecuencia de modulación, $m_{i}$ y $m_{f}$ son respectivamente los índices de modulación de intensidad y de fase, y $\varphi$ es el desfase entre ambas modulaciones. Para el análisis se supondrá $m_{i} \ll 1$.

La función de transferencia entre las modulaciones de intensidad y de fase puede entonces ser escrita como [Bje96]

$$
H_{P M}\left(\omega_{e}\right)=2 P_{0} \frac{\widetilde{\phi}\left(0, \omega_{e}\right)}{\widetilde{p}_{N}\left(0, \omega_{e}\right)}=\frac{2 m_{f}}{m_{i}} \exp (j \varphi) .
$$

Esta función es la que considera el chirp introducido por el transmisor óptico. Ahora bien, dependiendo del tipo de dispositivo empleado para realizar la modulación de intensidad, se tendrá un model o distinto del chirp del transmisor. En el estudio diferenciaremos entre los láseres de semiconductor y los moduladores externos. 
- Modelado del chirp en láseres de semiconductor

En un láser de semiconductor, el cambio en la ganancia óptica de la región activa que se produce como consecuencia de la modulación de la densidad de portadores trae consigo una modificación del índice de refracción modal. Es decir, la modulación de intensidad viene siempre acompañada de una modulación de fase indeseada y conocida con el nombre de chirp. De este modo, la frecuencia óptica de emisión instantánea $\Delta v$ se relaciona con la potencia óptica a la salida del láser por medio de [Bje96, Koc84]

$$
\Delta v=\frac{1}{2 \pi} \frac{\mathrm{d} \phi}{\mathrm{dt}}=\frac{\alpha_{c}}{4 \pi}\left(\frac{1}{\mathrm{P}} \frac{\mathrm{dP}}{\mathrm{dt}}+\kappa \mathrm{P}\right)
$$

siendo $\alpha_{c}$ el factor de ensanchamiento de línea del láser y $\kappa$ la constante de chirp adiabático. La modulación de intensidad queda expresada por

$$
P(t)=P_{L}\left(1+m_{i} \cos \left(2 \pi f_{m} t\right)\right)
$$

con $\mathrm{P}_{\mathrm{L}}$ la potencia media a la salida del láser. Desarrollando la ec. (I.21) para el caso de pequeña señal y aplicando transformadas de Fourier a ambos miembros se obtiene

$$
j \omega_{e} \widetilde{\phi}\left(0, \omega_{e}\right)=\frac{\alpha_{c}}{2}\left(j \omega_{e} \frac{\widetilde{\Gamma}_{N}\left(0, \omega_{e}\right)}{P_{L}}+\kappa \widetilde{p}_{N}\left(0, \omega_{e}\right)\right)
$$

de donde se deduce que

$$
H_{P M}\left(\omega_{e}\right)=\alpha_{c}\left(1-j \frac{2 \pi f_{c}}{\omega_{e}}\right)
$$

siendo $f_{c}=\kappa P_{L} /(2 \pi)$ la frecuencia a la cual el chirp adiabático y el chirp transitorio tienen la misma magnitud [Roy94]. Esta frecuencia tiene una relación directa con el parámetro de eficiencia FM del láser, medido en $\mathrm{MHz} / \mathrm{mA}$, mediante [Roy94, Wed94]

$$
E_{F M}=\frac{\Delta v}{\Delta l}=\frac{\alpha_{c} \kappa P_{L}}{4 \pi\left(I_{b}-I_{\text {th }}\right)}=\frac{\alpha_{c} f_{c}}{2\left(I_{b}-I_{\text {th }}\right)} .
$$


Así pues, la relación entre las modulaciones de intensidad y de fase a la salida del transmisor óptico en el caso de emplear como dispositivo modulador un láser de semiconductor, queda determinada por las siguientes ecuaciones:

$$
\begin{aligned}
& \operatorname{tg} \varphi=-\frac{f_{c}}{f_{m}}, \\
& \frac{2 m_{f}}{m_{i}}=\alpha_{c} \sqrt{1+\left(\frac{f_{c}}{f_{m}}\right)^{2}} .
\end{aligned}
$$

Para frecuencias de modulación $f_{m} \gg f_{c}$ se obtiene $2 m_{f} / m_{i}=\alpha_{c}$. Por otro lado, para frecuencias de modulación $\mathrm{f}_{\mathrm{m}}<1 \mathrm{GHz}$ (típicas de sistemas CATV) y en el caso de utilizar láseres DFB es posible realizar la aproximación

$$
\mathrm{H}_{\mathrm{PM}}\left(\omega_{\mathrm{e}}\right) \approx-\mathrm{j} \alpha_{\mathrm{c}} \frac{2 \pi \mathrm{f}_{\mathrm{c}}}{\omega_{\mathrm{e}}},
$$

para llegar de este modo a la siguiente expresión de campo a la salida del láser DFB:

$$
E_{L}(t)=\sqrt{P_{L}} \sqrt{1+m_{i} \cos \left(2 \pi f_{m} t\right)} \exp \left\{j\left[\omega_{0} t+\frac{\vartheta}{f_{m}} m_{i} \operatorname{sen}\left(2 \pi f_{m} t\right)\right]\right\}
$$

siendo $\vartheta=\alpha_{c} f_{c} / 2=E_{F M}\left(I_{b}-I_{t h}\right)$. Un valor típico de $\vartheta$ para láseres DFB puede ser $20 \mathrm{GHz}$ [Phi91].

- Modelado del chirp en moduladores externos

A diferencia de los láseres de semiconductor, el chirp introducido por los moduladores electroópticos queda completamente determinado por medio del parámetro $\alpha_{c}$, el cual representa el cociente entre las modulaciones de fase e intensidad y que se define por [Fis93, Koy88, Dju92, Sch94] 


$$
\alpha_{c}=\frac{\frac{d \phi}{d t}}{\frac{1}{2 P} \frac{d P}{d t}}=\frac{\operatorname{Im}\left(E_{\text {out }}^{*}(t) \frac{d E_{\text {out }}}{d t}\right)}{\operatorname{Re}\left(E_{\text {out }}^{*}(t) \frac{d E_{\text {out }}}{d t}\right)},
$$

donde $\phi$, $\mathrm{P}$ y $\mathrm{E}_{\text {out }}$ son la fase instantánea, la intensidad y el campo óptico a la salida del modulador, respectivamente. De esta forma, veremos que la función de transferencia $H_{P M}$ es independiente de la frecuencia de modulación e igual al parámetro de chirp $\alpha_{c}$.

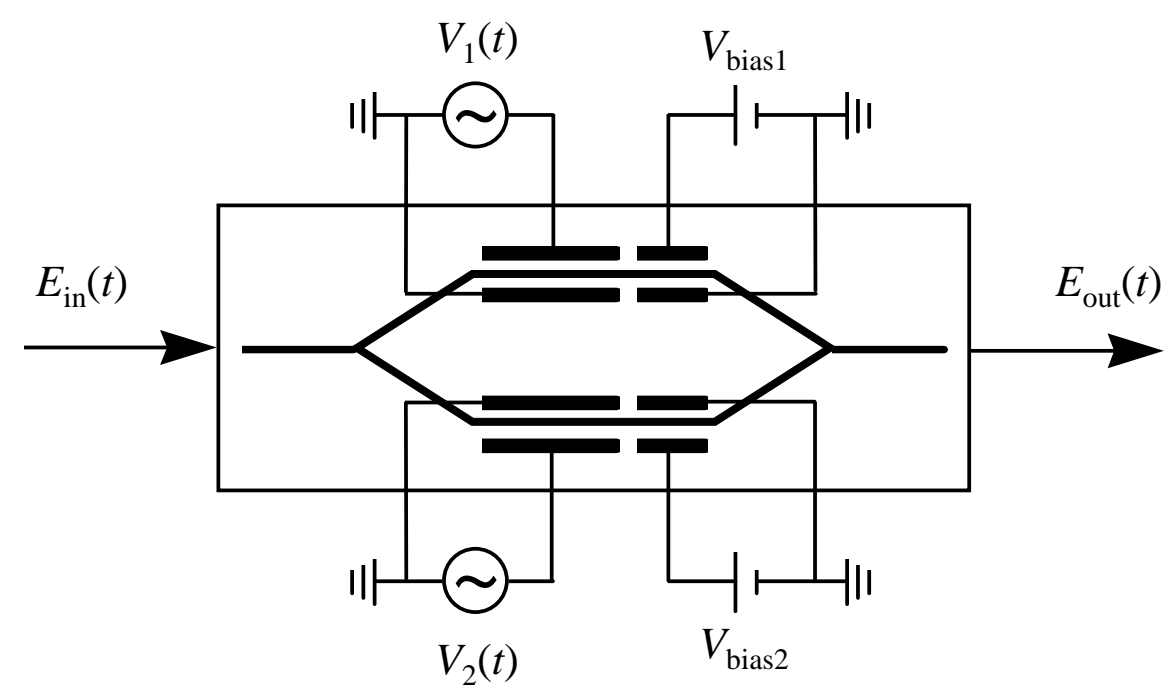

Figura I.4 Diagrama esquemático de la estructura de un MZM dual-drive A cada uno de los brazos del interferómetro se le aplican simultáneamente tensiones de polarización y señales de modulación.

Para comenzar con el estudio, en la figura I 4 se muestra la estructura de un modulador Mach-Zehnder (MZM) dual-drive. El campo óptico de entrada al modulador se divide entre los dos brazos del interferómetro. Cada uno de estos brazos se alimenta con señales el éctricas independientes que producen un cambio de fase sobre las señal es ópticas que se propagan basándose en el efecto electroóptico. Las diferencias de fase introducidas en cada uno de los brazos son proporcionales a los voltajes aplicados y vienen dadas por 


$$
\Delta \phi_{1,2}=\frac{\pi}{\mathrm{V}_{\pi}}\left(\mathrm{V}_{\text {bias } 1,2}+\mathrm{V}_{1,2}(\mathrm{t})\right),
$$

siendo $\mathrm{V}_{\pi}$ el voltaje de media onda del modulador, es decir, el voltaje necesario para inducir una diferencia de fase de $\pi$ radianes. Este es un parámetro característico de cada modulador y mide, en cierto modo, la no linealidad del mismo. Valores altos indican un comportamiento más lineal.

Finalmente, a la salida del dispositivo, las señales ópticas de cada uno de los brazos se combinan para proporcionar un campo óptico [F is93]

$$
E_{\text {out }}(t)=\frac{E_{\text {in }}}{\sqrt{L_{M Z}}} \cos \left(\frac{\Delta \phi_{1}-\Delta \phi_{2}}{2}\right) \exp \left(j \frac{\Delta \phi_{1}+\Delta \phi_{2}}{2}\right),
$$

donde $L_{M Z}$ representa las pérdidas del modulador. De esta forma, se obtienen una fase y una intensidad a la salida del modulador dadas por

$$
\begin{aligned}
& \phi=\frac{\Delta \phi_{1}+\Delta \phi_{2}}{2}=\frac{\pi}{2 \mathrm{~V}_{\pi}}\left(\mathrm{V}_{\text {bias } 1}+\mathrm{V}_{\text {bias } 2}+\mathrm{V}_{1}(\mathrm{t})+\mathrm{V}_{2}(\mathrm{t})\right) \\
& \mathrm{P}=\mathrm{P}_{0}\left[1+\cos \left(\Delta \phi_{1}-\Delta \phi_{2}\right)\right]=\mathrm{P}_{0}\left\{1+\cos \left[\frac{\pi}{\mathrm{V}_{\pi}}\left(\mathrm{V}_{\text {bias } 1}-\mathrm{V}_{\text {bias } 2}+\mathrm{V}_{1}(\mathrm{t})-\mathrm{V}_{2}(\mathrm{t})\right)\right]\right\}
\end{aligned}
$$

Las pérdidas en potencia del modulador son $2 \mathrm{~L}_{\mathrm{MZ}}$, por lo que $\mathrm{P}_{0}=\mathrm{P}_{\mathrm{in}} /\left(2 \mathrm{~L}_{\mathrm{MZ}}\right)$, siendo $P_{\text {in }}$ la intensidad a la entrada del dispositivo. Por otra parte, de la ec. (I.34) se deduce que el punto de trabajo del modulador se selecciona por medio del voltaje de polarización $\mathrm{V}_{\mathrm{dc}}=\mathrm{V}_{\text {bias1 }}-\mathrm{V}_{\text {bias2 }}$. Si deseamos trabajar en la zona lineal (modulador polarizado en cuadratura, $\mathrm{QB}$ ) entonces $\mathrm{V}_{\mathrm{dc}}=\mathrm{V}_{\pi} / 2$.

Aplicando la ec. (I.30) se obtiene un parámetro de chirp [Fis93]

$$
\alpha_{c}=\frac{\Delta \phi_{1}+\Delta \phi_{2}}{\Delta \phi_{1}-\Delta \phi_{2}} \cot \left(\pi \frac{\mathrm{V}_{1}(\mathrm{t})-\mathrm{V}_{2}(\mathrm{t})}{2 \mathrm{~V}_{\pi}}\right)
$$


Y finalmente, suponiendo modulación en pequeña señal alrededor de la zona de trabajo lineal del moduladoriii, se obtiene la conocida expresión del parámetro de chirp del MZM [Fis93, Gna91, Smi97]:

$$
\alpha_{c}=\frac{V_{1}(t)+V_{2}(t)}{V_{1}(t)-V_{2}(t)}
$$

Cuando los voltajes aplicados son de signos opuestos, el parámetro de chirp toma valores $0<\alpha_{c}<1$, mientras que si son del mismo signo, $1<\alpha_{c}<\infty$. El caso $\alpha_{c}=1$ se refiere a alimentación asimétrica $\left(V_{1}(t)=V(t)\right.$ y $\left.V_{2}(t)=0\right)$. Por último, si aplicamos alimentación simétrica $\left(\mathrm{V}_{1}(\mathrm{t})=\mathrm{V}(\mathrm{t}) / 2\right.$ y $\left.\mathrm{V}_{2}(\mathrm{t})=-\mathrm{V}(\mathrm{t}) / 2\right)$ es posible conseguir modulación sin chirp $\left(\alpha_{c}=0\right)$.

Otra prometedora alternativa para la modulación externa la constituyen los moduladores de electroabsorción (EAMs, electroabsorption modulators), ideales para transmisiones de gran velocidad y distancia utilizando EDFAs [Suz92]. Al igual que en el caso de los MZMs, presentan un chirp en la señal de salida modulada que habrá que caracterizar [Mit92, Mit94].

La curva característica V-P (voltaje eléctrico-potencia óptica) de un EAM es generalmente no lineal y se expresa como

$$
\mathrm{P}=\mathrm{P}_{0} \exp \left[-\left(\frac{\mathrm{V}}{\mathrm{V}_{0}}\right)^{\mathrm{a}}\right] \text {, }
$$

donde $\mathrm{V}_{0}$ y a son constantes y $\mathrm{V}_{0}$ es el voltaje que proporciona una relación de extinción de $\mathrm{P}_{0} / \mathrm{e}$. El parámetro a es 2-4 para moduladores MQW (multiple quantum well) [Mit92] y 1-2 para moduladores bulk [Suz92]. EI vol taje de modulación viene dado por

$$
V=V_{\text {bias }}\left[1+m_{i} \cos \left(2 \pi f_{m} t\right)\right],
$$

siendo $\bigvee_{\text {bias }}$ el voltaje $\mathrm{DC}$ de polarización.

\footnotetext{
iii en este caso, $V_{1}(t)-V_{2}(t) \sim V_{\pi} / 2$
} 
A partir de (1.37), el campo óptico a la salida del dispositivo se calcula como

$$
E_{\text {out }}(t)=P^{c} \exp \left(j \omega_{0} t\right)
$$

donde $c=\left(1+\mathrm{j} \alpha_{c}\right) / 2$. Luego si calculamos la transformada de Fourier de (I.39), lo cual es equivalente a medir la intensidad de las bandas de modulación de la potencia de salida del EAM, es posible determinar el valor del parámetro de chirp $\alpha_{c}$ [Mit92]. En particular, en [Mit94] se calculó un parámetro $\alpha_{c}$ menor que 1 para un modulador MQW de InGaAs/InAIAs empleando este método.

\section{I.3.2 Respuesta de la fibra óptica en presencia de chirp}

La modulación de fase generada por el chirp del transmisor óptico modifica la respuesta de la fibra como consecuencia de la conversión PM-IM producida por la dispersión cromática. Este efecto ha sido estudiado por varios autores [Sri95, Roy94, Wed94, Dev93] y los resultados pueden verse en la figura I.5 para distintos valores del parámetro $\alpha_{c}$. Las curvas son para una longitud de fibra de $100 \mathrm{~km}$ y la de trazos representa la situación sin chirp $\left(\alpha_{c}=0\right)$. Un primer efecto que se observa es que el nivel de los lóbulos aumenta proporcionalmente con la magnitud del parámetro $\alpha_{c}$. Además, de la figura se deduce que parámetros $\alpha_{c}$ positivos reducen el ancho de banda de transmisión, mientras que parámetros $\alpha_{c}$ negativos producen un efecto similar al del SPM. Así pues, en general interesará utilizar transmisores ópticos con $\alpha_{c}<0$ para mejorar las prestaciones del enlace de fibra óptica. No obstante, en el caso de $\alpha_{c}>0$ todavía es posible aumentar el ancho de banda si tenemos en cuenta el chirp adiabático de los láseres de semiconductor. La figura I.6 muestra la respuesta de la fibra óptica en el caso de emplear un láser de semiconductor con un factor de ensanchamiento de línea $\alpha_{c}=3$ y dos valores distintos de chirp adiabático: $\mathrm{f}_{\mathrm{c}}=5 \mathrm{GHz}$ y $\mathrm{f}_{\mathrm{c}}=$ $10 \mathrm{GHz}$. Como consecuencia del chirp adiabático, el primer nulo de la respuesta de la fibra desaparece completamente, extendiéndose la frecuencia $-3 \mathrm{~dB}$ hasta prácticamente los $9 \mathrm{GHz}$. Además, en el caso de $\mathrm{f}_{\mathrm{c}}=$ $10 \mathrm{GHz}$ incluso se observa un comportamiento paso-alto para bajas frecuencias, por lo que sería interesante ecualizar a la salida del fotodetector 


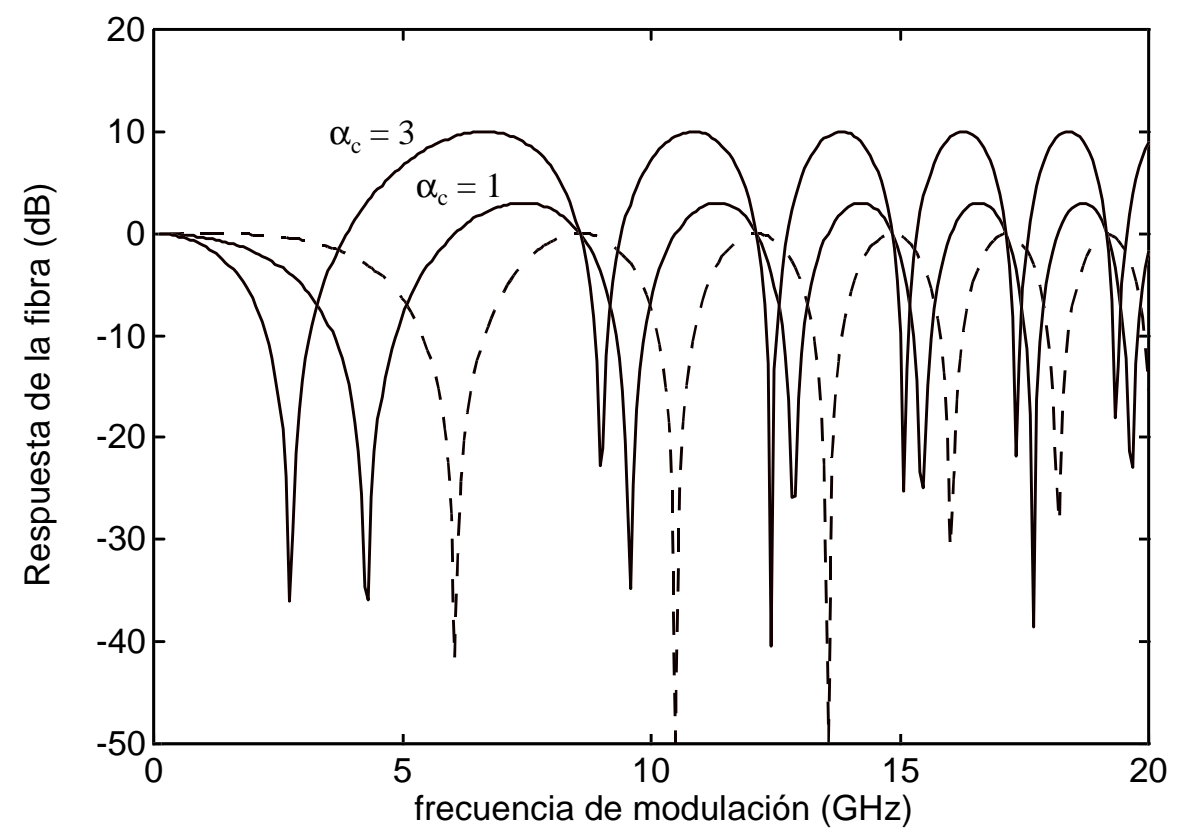

(a)

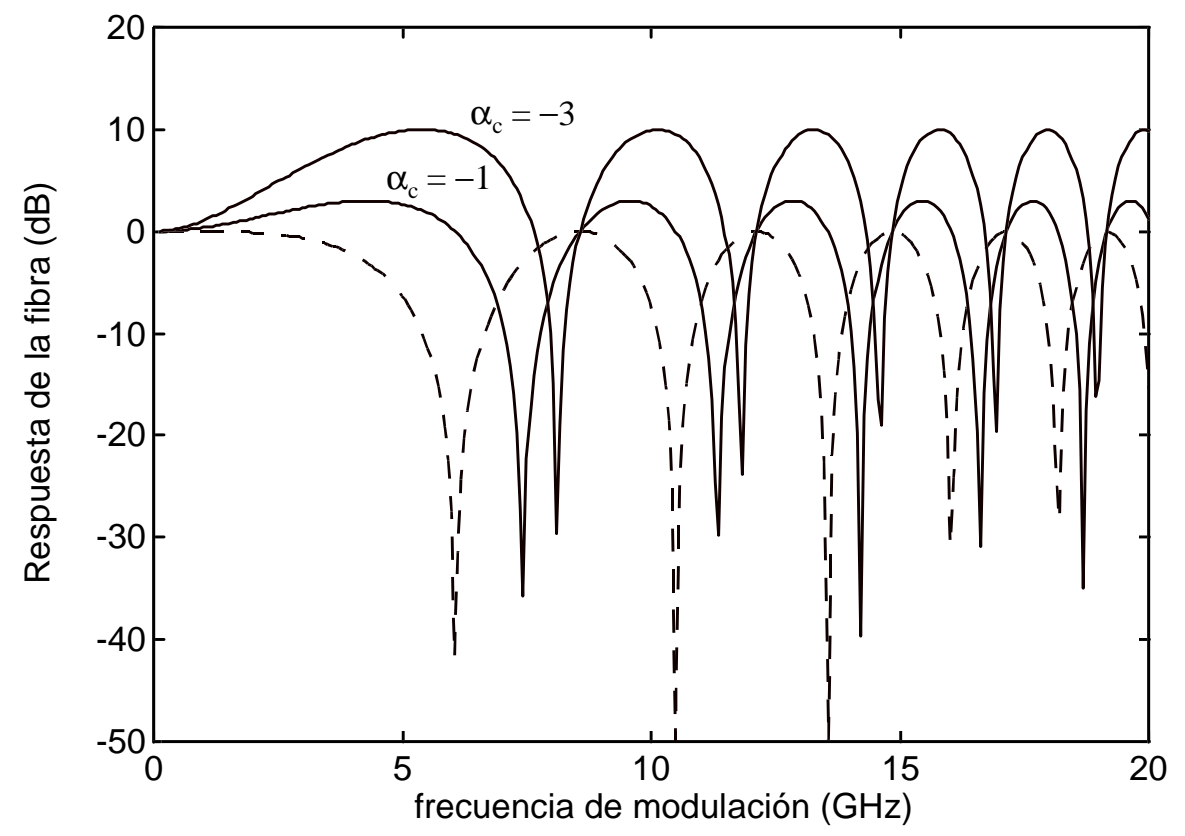

(b)

Figura I.5 Respuesta de $100 \mathrm{~km}$ de fibra óptica en presencia de chirp, caracterizado por el parámetro $\alpha_{c}$ : (a) $\alpha_{c}>0$ y (b) $\alpha_{c}<0$. La curva de trazos representa la situación sin chirp $\left(\alpha_{c}=0\right)$.

mediante un filtro paso-bajo, tal y como se propone en [Wed94]. La frecuencia de corte de este filtro paso-bajo vendría dada por la expresión 


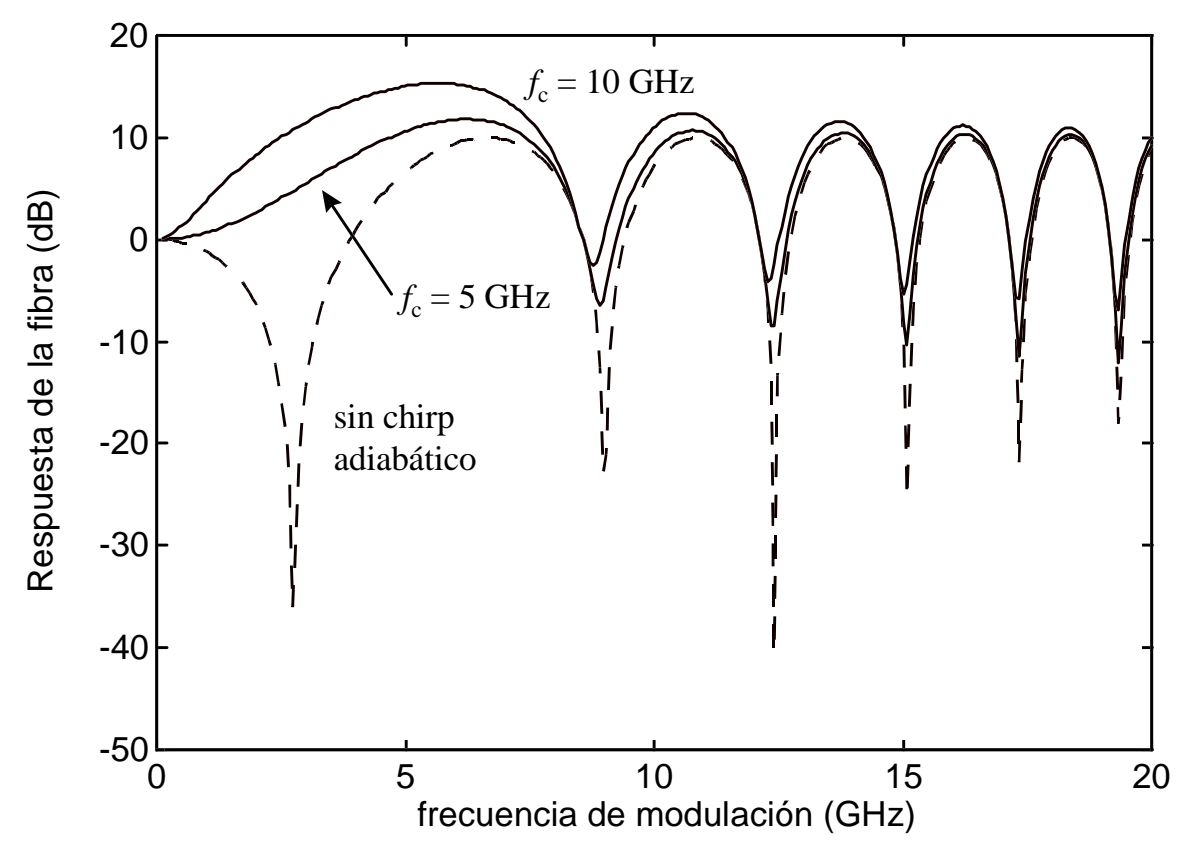

Figura I.6 Respuesta de $100 \mathrm{~km}$ de fibra empleando un transmi sor óptico caracterizado por un parámetro de chirp $\alpha_{c}=3$ y dos valores distintos de chirp adiabático: $\mathrm{f}_{\mathrm{c}}=5 \mathrm{GHz}$ y $10 \mathrm{GHz}$.

$$
\Omega_{\mathrm{g}}=\frac{\mathrm{C}}{\mathrm{E}_{\mathrm{FM}}\left(\mathrm{I}_{\mathrm{b}}-\mathrm{I}_{\mathrm{th}}\right) \lambda^{2} \mathrm{DL}} .
$$

EI objetivo de ecualizar la respuesta mediante un filtro eléctrico paso-bajo se debe a que el ancho de banda de transmisión se define como el margen de frecuencias donde la respuesta tiene una variación inferior a $3 \mathrm{~dB}$. Por lo tanto, en este caso particular el ancho de banda vendría limitado por el punto donde la respuesta crece $3 \mathrm{~dB}$ respecto al origen. Es decir, el alcance del sistema se puede caracterizar por medio de la frecuencia $-3 \mathrm{~dB}$, ya que valores de la respuesta positivos tienen un sentido de ganancia en potencia. Sin embargo, a la hora de definir la frecuencia de corte es necesario considerar cualquier variación tanto positiva como negativa [Dev94]. Esta explicación es también extrapolable a la respuesta de la fibra con SPM o parámetros $\alpha_{c}<0$.

Suponiendo transmisión lineal, la respuesta de la fibra óptica en presencia de chirp se podría expresar a partir de (I.14), (I.15) y (I.24) como 




(a)

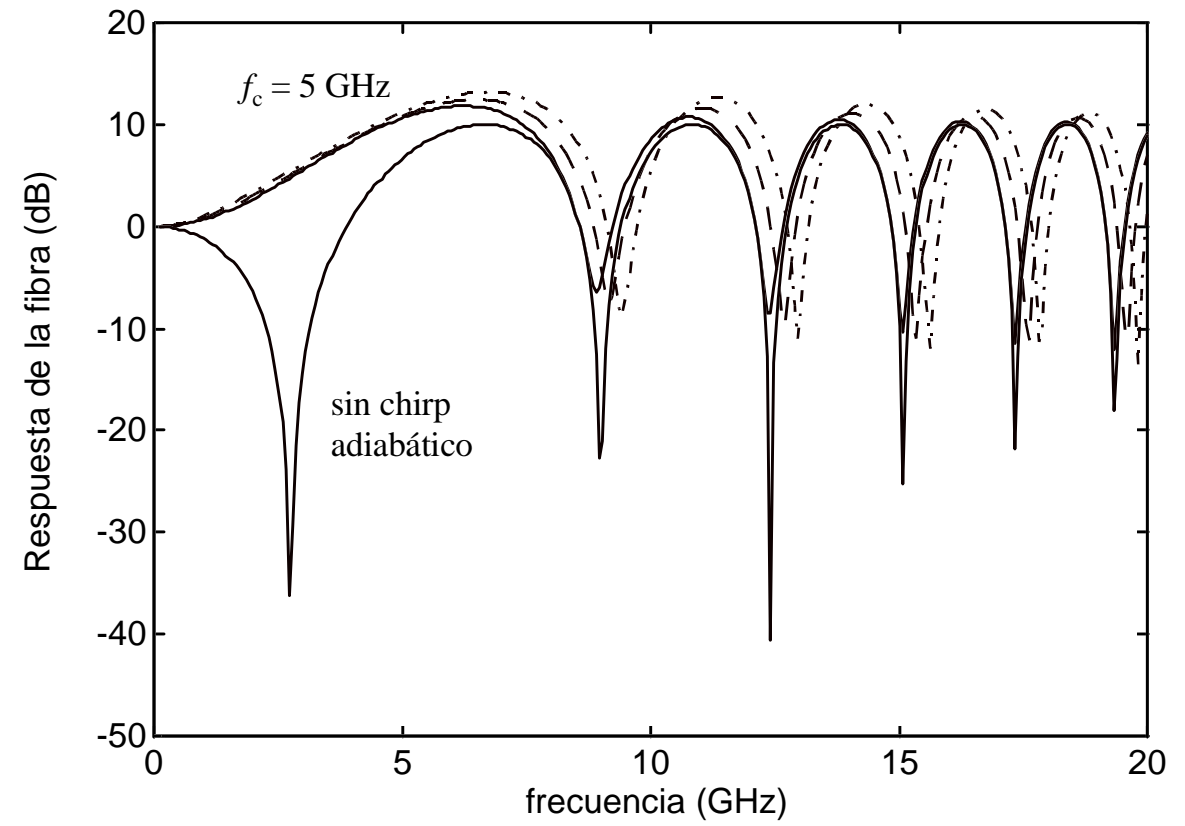

(b)

Figura I.7 Respuesta de $100 \mathrm{~km}$ de fibra óptica dispersiva y no lineal en presencia de chirp: (a) sin chirp adiabático y (b) chirp adiabático con $\alpha_{c}=3$. Las curvas continuas son suponiendo transmisión lineal, mientras que las de trazos son para una potencia óptica de $10 \mathrm{~mW}$ y las de trazos y puntos para $20 \mathrm{~mW}$. 


$$
H_{F}\left(z, \omega_{e}\right)=\cos \left(\frac{\beta_{2} \omega_{e}^{2} z}{2}\right)+\alpha_{c}\left(1-j \frac{2 \pi f_{c}}{\omega_{e}}\right) \operatorname{sen}\left(\frac{\beta_{2} \omega_{e}^{2} z}{2}\right)
$$

de donde se obtiene un módulo y una fase de la función de transferencia dados por

$$
\begin{gathered}
\left|H_{F}\left(z, \omega_{e}\right)\right|=\mid \cos \left(\frac{\beta_{2} \omega_{e}^{2} z}{2}\right) \times \sqrt{\left[1+\alpha_{c} \operatorname{tg}\left(\frac{\beta_{2} \omega_{e}^{2} z}{2}\right)\right]^{2}+\left[\frac{2 \pi \alpha_{c} f_{c}}{\omega_{e}} \operatorname{tg}\left(\frac{\beta_{2} \omega_{e}^{2} z}{2}\right)\right]^{2}}, \\
\arg _{F}\left(z, \omega_{e}\right)=\operatorname{arctg}\left[\frac{-\frac{2 \pi \alpha_{c} f_{c}}{\omega_{e}} \operatorname{tg}\left(\frac{\beta_{2} \omega_{e}^{2} z}{2}\right)}{1+\alpha_{c} \operatorname{tg}\left(\frac{\beta_{2} \omega_{e}^{2} z}{2}\right)}\right]
\end{gathered}
$$

La ec. (I.42) es precisamente la representada en las figuras I.5 y I.6. A diferencia de las funciones de conversión, en este caso se aplica $20 \log _{10}(\cdot)$, pues lo que interesa es la respuesta de la fibra óptica a la salida del fotodetector (dBe).

Finalmente, la influencia de la potencia óptica sobre la respuesta de la fibra con chirp se manifiesta también en un ligero desplazamiento de los nulos (figura I.7(a)). Dado que el efecto del SPM es similar al de un chirp negativo pero inducido por la fibra óptica, se produce un alejamiento del primer nulo (mejora de la respuesta). Es decir, el resultado final es equivalente a una ligera disminución del parámetro de chirp [J eo98]. Esto mismo también se puede apreciar en la figura I.7(b) en el caso del chirp adiabático. 


\section{I.4 MODULACIÓN ÓPTICA EN BANDA LATERAL ÚNICA}

La modulación óptica en banda lateral única, habitualmente conocida como OSSB (optical singlesideband), se ha demostrado como una técnica tolerante a la dispersión cromática de la fibra [Smi97, Con97, Par97]. En el capítulo 2 de esta tesis ya fue introducida para analizar sus efectos sobre la distorsión no lineal causada por la fibra óptica. A continuación, estudiaremos la función de transferencia de la fibra en sistemas que emplean modulación OSSB, así como su implementación práctica utilizando moduladores MZMs dual-drive.

\subsubsection{Generación de la modulación SSB empleando un MZM dual- drive}

La estructura utilizada para conseguir modulación SSB se basa en el dispositivo MZM de la figura I.4 (figura I.8). En este caso, el modulador se polariza en cuadratura con unos voltajes $V_{\text {bias } 1}=V_{\pi} / 2$ y $V_{\text {bias } 2}=0\left(V_{d c}=V_{\pi} / 2\right)$, mientras que la señal moduladora (se supondrá un tono) se aplica a ambos brazos del interferómetro con un desfase de $\pm 900\left(\phi_{1}=0\right.$ y $\left.\phi_{2}= \pm \pi / 2\right)$. Es decir, los desfases producidos como consecuencia del efecto el ectroóptico son

$$
\Delta \phi_{1}=\frac{\pi}{2}+\frac{\pi \mathrm{V}_{\mathrm{m}}}{\sqrt{2} \mathrm{~V}_{\pi}} \cos \left(2 \pi \mathrm{f}_{\mathrm{m}} \mathrm{t}\right),
$$

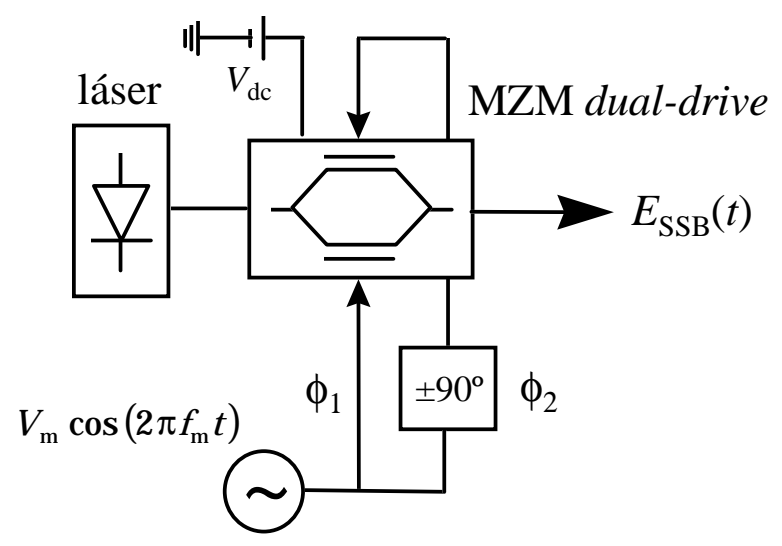

Figura I.8 Modulador óptico SSB utilizando un MZM dual-drive. 


$$
\Delta \phi_{1}= \pm \frac{\pi \mathrm{V}_{\mathrm{m}}}{\sqrt{2} \mathrm{~V}_{\pi}} \operatorname{sen}\left(2 \pi \mathrm{f}_{\mathrm{m}} \mathrm{t}\right)
$$

donde $f_{m}$ y $V_{m}$ son la frecuencia y amplitud de la señal moduladora, respectivamente. Sustituyendo (I.44) y (I.45) en la expresión (I.32), se obtiene un campo óptico a la salida del MZM dado por [Smi97]

$$
\begin{aligned}
E_{S S B}(t) & =E_{0}\left[J_{0}\left(\frac{\pi V_{m}}{\sqrt{2} V_{\pi}}\right) \cos \left(\omega_{0} t\right)-J_{0}\left(\frac{\pi V_{m}}{\sqrt{2} V_{\pi}}\right) \operatorname{sen}\left(\omega_{0} t\right)\right. \\
& \left. \pm 2 J_{1}\left(\frac{\pi V_{m}}{\sqrt{2} V_{\pi}}\right) \cos \left(\omega_{0} t \pm 2 \pi f_{m} t\right)\right]
\end{aligned}
$$

tras quedarse con los términos más significativos. $\mathrm{J} n(\cdot)$ se refiere a la función de Bessel de orden $n$.

Por último, la densidad espectral de potencia es igual a [Smi97]

$$
\mathrm{S}_{\mathrm{E}}(\omega)=\mathrm{E}_{0}^{2} \pi\left[\mathrm { J } _ { 0 } ^ { 2 } \left(\frac{\pi \mathrm{V}_{\mathrm{m}}}{\sqrt{2} \mathrm{~V}_{\pi}} \delta\left(\omega-\omega_{0}\right)+\mathrm{J}_{1}^{2}\left(\frac{\pi \mathrm{V}_{\mathrm{m}}}{\sqrt{2} \mathrm{~V}_{\pi}} \delta\left(\omega-\omega_{0} \pm 2 \pi \mathrm{f}_{\mathrm{m}} \mathrm{t}\right)\right]\right.\right.
$$

EI primer término es la portadora óptica a una longitud de onda $\lambda=2 \pi c / \omega_{0}$, mientras que el segundo término representa una banda lateral inferior o superior de modulación, dependiendo del signo escogido.

En la figura I.9 se representan los espectros de campo y de potencia óptica a la salida de un MZM polarizado con una tensión $\mathrm{V}_{\mathrm{dc}}=\mathrm{V}_{\pi} / 2=3,75 \mathrm{~V}$. Las tres primeras gráficas son para modulación SSB, $\phi_{1}=0$ y $\phi_{2}= \pm \pi / 2$, mientras que la última gráfica es para modulación en doble banda lateral (double sideband, DSB), $\phi_{1}=0$ y $\phi_{2}=\pi$. Obsérvese que en todos los casos aparecen armónicos de la frecuencia de modulación, la magnitud de los cuales depende de la relación entre el voltaje de modulación, $V_{m}=632,5 \mathrm{mV}$, y el valor de $\mathrm{V}_{\pi}=7,5 \mathrm{~V}$. Dejando aparte los armónicos que aparecen, se observa que en el espectro de campo de la modulación DSB aparecen dos bandas, mientras que en el espectro de campo de la modulación SSB aparece una única banda. Dependiendo de la diferencia de fases, esta banda será 

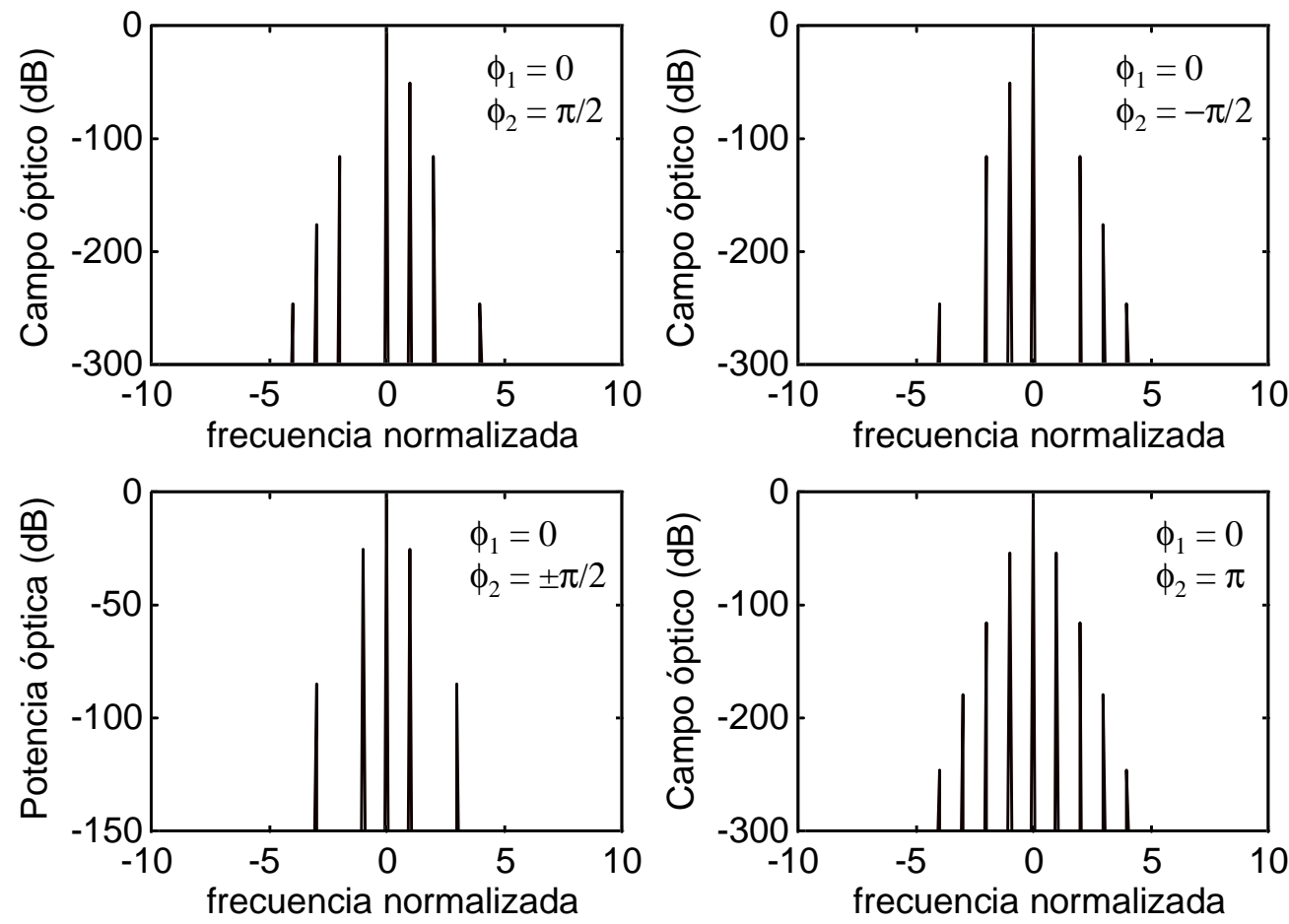

Figura I.9 Espectros de campo y de potencia a la salida de un MZM con modulación SSB y DSB. Los parámetros del modulador son: $\mathrm{V}_{\pi}=7,5 \mathrm{~V}$ y $Z_{\text {in }}=$ $50 \Omega$ (impedancia de los puertos de entrada). La potencia de la señal moduladora es de $+6 \mathrm{dBm}$.

superior o inferior. Sin embargo, en el espectro de potencia aparecen ambas bandas junto con armónicos de orden impar, al igual que ocurre con el espectro de potencia de la modulación DSB.

Centrándonos de nuevo en el espectro de campo SSB, el hecho de que exista una única banda de modulación es precisamente lo que posibilita que sea una técnica tolerante a la dispersión cromática. La propagación por fibras ópticas dispersivas induce desfases distintos sobre cada una de las frecuencias. Posteriormente, al fotodetectar se producen batidos tal y como se comentó en el capítulo 2. En el caso de la modulación DSB, los dos términos dominantes son los batidos de la portadora óptica con las dos bandas de modulación, lo cual provoca el fenómeno del carrier suppression como consecuencia de los distintos desfases. Sin embargo, en el caso de la modulación SSB existe un único término dominante (batido entre portadora 
y banda de modulación superior o inferior), por lo que se consigue evitar el efecto de la dispersión cromática.

Suponiendo un MZM perfectamente lineal, los campos ópticos a su salida para modulaciones DSB o SSB con un tono de RF vendrían dados por

$$
\begin{aligned}
E_{D S B}(t) & =\sqrt{1+m_{i} \cos \left(2 \pi f_{m} t\right)} \exp \left(j \omega_{0} t\right), \\
E_{S S B}(t) & =\sqrt{1+m_{i}\left[\cos \left(2 \pi f_{m} t\right) \pm \operatorname{sen}\left(2 \pi f_{m} t\right)\right]} \\
& \times \exp \left\{j \omega_{0} t+j \frac{m_{i}}{2}\left[\cos \left(2 \pi f_{m} t\right) \mp \operatorname{sen}\left(2 \pi f_{m} t\right)\right]\right\},
\end{aligned}
$$

donde se observa que en el caso de SSB, aparece una modulación de fase con un índice de modulación $\mathrm{m}_{\mathrm{f}}=\mathrm{m}_{\mathrm{i}} / 2$ y desfasada 90 o respecto a la modulación de intensidad. Luego se obtiene que $H_{P M}= \pm j$.

\section{4.2 Modelado y respuesta de la fibra con modulación SSB}

Las estructuras de modulador SSB presentadas en [Smi97a, Ols94, Izu81], y en particular la de la figura I.8, se basan en el concepto de Hartley, es decir, la señal moduladora y su transformada de Hilbert se aplican a uno o más MZM cuyas portadoras ópticas han sido desfasadas $90^{\circ}$ en el punto donde se combinan las señales. Un método alternativo para conseguir modulación óptica SSB consiste en la estructura de modulador SSB híbrido presentada en [Kah61]. Esta estructura se compone de un modulador de amplitud/ intensidad y otro de fase, colocados en cascada y modulados con la señal de información y su transformada de Hilbert respectivamente, tal y como muestra la figura I.10. En el caso de que la señal moduladora sea de banda estrecha, la transformada de Hilbert se reduce a un desfase de $90 \circ$ de la subportadora. En particular, si $s(t)=\cos \left(2 \pi \mathrm{f}_{\mathrm{m}} \mathrm{t}\right)$, entonces su transformada de Hilbert sería $\hat{s}(t)=\operatorname{sen}\left(2 \pi f_{m} t\right)$.

De este modo, de la figura I.10 se puede deducir que la función de transferencia del transmisor óptico SSB se calcula como $\mathrm{H}_{\mathrm{PM}}= \pm \mathrm{j}$. Obsérvese que el índice de modulación de fase es la mitad del de intensidad, y que la 


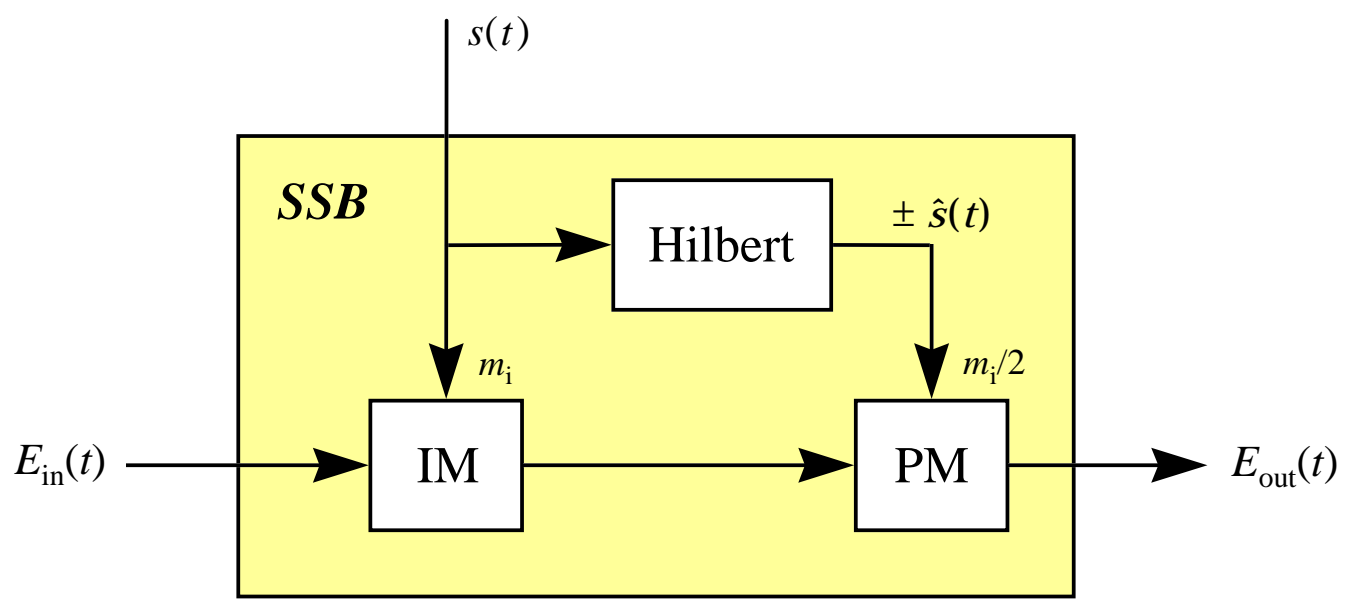

Figura I.10 Diagrama de bloques de un modulador SSB híbrido.

transformada de Hilbert puede desfasarse 180 opcionalmente. Luego la respuesta de la fibra óptica en sistemas con modulación OSSB vendrá dada por

$$
H_{F}\left(z, \omega_{e}\right)=C_{I M-I M}\left(z, \omega_{e}\right) \pm \frac{j}{2} C_{P M-I M}\left(z, \omega_{e}\right) .
$$

En el caso particular de fibra dispersiva lineal, a partir de las ecuaciones I.14 y l.15 se obtiene la siguiente función de transferencia de la fibra óptica con modulación SSB:

$$
H_{F}\left(z, \omega_{e}\right)=H_{S S B}\left(z, \omega_{e}\right)=\cos \left(\frac{\beta_{2} \omega_{e}^{2} z}{2}\right) \pm j \operatorname{sen}\left(\frac{\beta_{2} \omega_{e}^{2} z}{2}\right) .
$$

Luego se obtienen un módulo y una fase de la función de transferencia:

$$
\begin{gathered}
\left|\mathrm{H}_{\mathrm{SSB}}\left(\mathrm{z}, \omega_{\mathrm{e}}\right)\right|=1, \\
\arg \mathrm{H}_{\mathrm{SSB}}\left(\mathrm{z}, \omega_{\mathrm{e}}\right)= \pm \frac{\beta_{2} \omega_{\mathrm{e}}^{2} \mathrm{z}}{2} .
\end{gathered}
$$

El módulo de la función de transferencia es constante e independiente de la frecuencia de modulación, lo cual demuestra que la modulación SSB es tolerante a la dispersión cromática de la fibra. Sin embargo, aparece una 




Figura I.11 Respuesta de $50 \mathrm{~km}$ de fibra óptica con modulaciones DSB (continuas) y SSB (trazos) empleando un MZM. Los parámetros de modulación son: $\mathrm{V}_{\mathrm{dc}}=\mathrm{V}_{\pi} / 2=4,5 \mathrm{~V}, \mathrm{~V}_{\mathrm{m}}=$ $632,5 \mathrm{mV}$ y $\mathrm{P}_{0}=20 \mathrm{~mW}$.

fase con una dependencia cuadrática de la frecuencia de modulación que introducirá distorsión sobre la señal moduladora. El fenómeno de carrier suppression consigue eliminarse empleando modulación SSB, pero todavía queda una fase residual que deberá corregirse por medio de algún tipo de filtrado eléctrico. Es decir, SSB no compensa la dispersión cromática, sino que únicamente reduce los efectos negativos causados por ésta.

La respuesta de la fibra óptica con modulación SSB se muestra en la figura I.11 para los casos de propagación lineal (GVD) y no lineal (SPM). Para la modulación se ha empleado un MZM polarizado en lineal, con un voltaje de media onda $\mathrm{V}_{\pi}=9 \mathrm{~V}$ y alimentado con una señal de modulación de $+6 \mathrm{dBm}$. Para transmisión lineal, se puede ver que la respuesta de los $50 \mathrm{~km}$ de fibra es constante, desapareciendo completamente los nul os de la respuesta DSB. No obstante, suponiendo transmisión no lineal aparece un cierto rizado sobre la función de transferencia como consecuencia del desplazamiento de los nulos que se produce en las funciones $C_{I M-I M}$ y $C_{P M-I M}$. Aun así, el comportamiento de la fibra con SSB sigue siendo relativamente bueno en 
comparación con la modulación DSB. Como es lógico, la amplitud de este rizado es proporcional a la potencia óptica existente a la entrada de la fibra, y puede mantenerse controlada. 


\section{I.5 INVERSIÓN ESPECTRAL}

La técnica de inversión espectral, también conocida como OPC (optical phase conjugation), se ha demostrado como una técnica eficiente para compensar los efectos causados por la dispersión cromática y el SPM en fibras ópticas monomodo [Wat94, Mar97]. En el capítulo 4 ya se estudió esta técnica en detalle. Aquí analizaremos teóricamente y por medio de simulación la función de transferencia de los sistemas OPC. El esquema del sistema se resume en la figura I.12, así como las distintas variables utilizadas.

\section{I.5.1 Modelado del conjugador de fase}

El elemento clave de los sistemas de comunicaciones ópticas que emplean la técnica de inversión espectral es el conjugador óptico (optical phase conjugator, OPC). Su función es invertir la fase de la envolvente del campo óptico. Es decir, el OPC se modela mediante las siguientes ecuaciones del campo a la entrada y a la salida del dispositivo:

$$
\begin{gathered}
A_{\text {out }}(t)=\sqrt{G_{\text {OPC }}} A_{\text {in }}^{*}(t), \\
\widetilde{A}_{\text {out }}(\omega)=\sqrt{G_{\text {OPC }}} \widetilde{A}_{\text {in }}^{*}(-\omega),
\end{gathered}
$$

siendo $\mathrm{G}_{\mathrm{OPC}}$ la ganancia en potencia que presenta el dispositivo. Normalmente, se selecciona una ganancia tal que compense las pérdidas del primer tramo de fibra óptica, es decir, $\mathrm{G}_{\mathrm{OPC}}=\exp \left(\alpha_{1} \mathrm{~L}_{1}\right)$. De la ec. (I.55) se

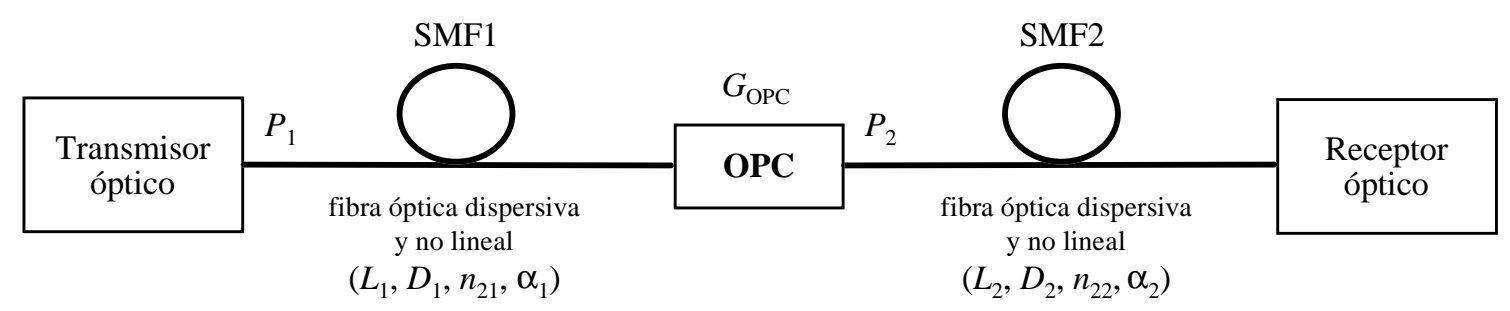

Figura I.12 Esquema de sistema de comunicaciones ópticas basado en la técnica de inversión espectral. 
deduce que el OPC invierte el espectro del campo óptico, de ahí el nombre de inversión espectral.

Si trabajamos con las señales de potencia y de fase, el dispositivo OPC se puede modelar de forma sencilla mediante,

$$
\begin{gathered}
\widetilde{\rho}_{\mathrm{N}, \text { out }}={\widetilde{\phi_{N}, \text { in }}}, \\
\widetilde{\phi}_{\text {out }}=-\widetilde{\phi}_{\text {in }} .
\end{gathered}
$$

Obsérvese que no aparece el término de ganancia del conjugador en la ec. (I.56) debido a que se trabaja con potencias ópticas normalizadas.

\section{I.5.2 Respuesta en frecuencia de sistemas con inversión espectral}

La respuesta de un sistema que emplea la técnica de inversión espectral puede obtenerse fácilmente a partir de las funciones que modelan cada uno de los dispositivos fotónicos: funciones de conversión de intensidad y de fase de la fibra óptica y del OPC.

Para el análisis, se supondrá un sistema formado por un primer tramo de fibra óptica de longitud $L_{1}$, un elemento conjugador situado a continuación, y finalmente un segundo tramo de fibra de longitud $L_{2}$. La longitud total del enlace será $L=L_{1}+L_{2}$. De esta forma, las señales de potencia y de fase a la salida del primer tramo de fibra se calculan como

$$
\begin{aligned}
& \widetilde{p}_{N, 1}\left(L_{1}, \omega_{e}\right)=C_{I M-I M}^{(1)}\left(L_{1}, \omega_{e}\right) \widetilde{p}_{N, 1}\left(0, \omega_{e}\right)+P_{1} C_{P M-I M}^{(2)}\left(L_{1}, \omega_{e}\right) \widetilde{\phi}_{1}\left(0, \omega_{e}\right), \\
& \widetilde{\phi}_{1}\left(L_{1}, \omega_{e}\right)=\frac{1}{P_{1}} C_{I M-P M}^{(1)}\left(L_{1}, \omega_{e}\right) \widetilde{p}_{N, 1}\left(0, \omega_{e}\right)+C_{P M-P M}^{(1)}\left(L_{1}, \omega_{e}\right) \widetilde{\phi}_{1}\left(0, \omega_{e}\right) .
\end{aligned}
$$

Teniendo en cuenta la respuesta del OPC, las señales a la entrada del segundo tramo de fibra serán

$$
\tilde{\rho}_{\mathrm{N}, 2}\left(0, \omega_{\mathrm{e}}\right)={\widetilde{\sigma_{N}, 1}}_{1}\left(\mathrm{~L}_{1}, \omega_{\mathrm{e}}\right)
$$




$$
\widetilde{\phi_{2}}\left(0, \omega_{\mathrm{e}}\right)=-\widetilde{\phi}_{1}\left(\mathrm{~L}_{1}, \omega_{\mathrm{e}}\right)
$$

por lo que se obtienen una potencia y una fase a la salida del sistema determinadas por

$$
\begin{aligned}
& {\widetilde{p_{N}, 2}}_{2}\left(L_{2}, \omega_{\mathrm{e}}\right)=C_{I M-I M}^{(2)}\left(L_{2}, \omega_{\mathrm{e}}\right){\widetilde{T_{N}, 1}}_{\mathrm{I}}\left(\mathrm{L}_{1}, \omega_{\mathrm{e}}\right)-\mathrm{P}_{2} \mathrm{C}_{\mathrm{PM}-\mathrm{IM}}^{(2)}\left(\mathrm{L}_{2}, \omega_{\mathrm{e}}\right) \widetilde{\phi}_{1}\left(\mathrm{~L}_{1}, \omega_{\mathrm{e}}\right) \\
& =C_{I M-I M}^{(1)}\left(L_{1}, \omega_{e}\right) C_{I M-I M}^{(2)}\left(L_{2}, \omega_{e}\right) \widetilde{\Gamma}_{N, 1}\left(0, \omega_{e}\right) \\
& +\mathrm{P}_{1} \mathrm{C}_{\mathrm{PM}-\mathrm{IM}}^{(1)}\left(\mathrm{L}_{1}, \omega_{\mathrm{e}}\right) \mathrm{C}_{\mathrm{IM}-\mathrm{IM}}^{(2)}\left(\mathrm{L}_{2}, \omega_{\mathrm{e}}\right) \widetilde{\phi}_{1}\left(0, \omega_{\mathrm{e}}\right) \\
& -\frac{P_{2}}{P_{1}} C_{I M-P M}^{(1)}\left(L_{1}, \omega_{e}\right) C_{P M-I M}^{(2)}\left(L_{2}, \omega_{e}\right) \widetilde{P}_{N, 1}\left(0, \omega_{e}\right) \\
& -\mathrm{P}_{2} \mathrm{C}_{\mathrm{PM}-\mathrm{PM}}^{(1)}\left(\mathrm{L}_{1}, \omega_{\mathrm{e}}\right) \mathrm{C}_{\mathrm{PM}-\mathrm{IM}}^{(2)}\left(\mathrm{L}_{2}, \omega_{\mathrm{e}}\right) \widetilde{\phi}_{1}\left(0, \omega_{\mathrm{e}}\right) \text {, } \\
& \widetilde{\phi}_{2}\left(L_{2}, \omega_{e}\right)=\frac{1}{P_{2}} C_{\text {IM-PM }}^{(2)}\left(L_{2}, \omega_{e}\right){\widetilde{p_{N}, 1}}_{(}\left(L_{1}, \omega_{e}\right)-C_{P M-P M}^{(2)}\left(L_{2}, \omega_{e}\right) \widetilde{\phi}_{1}\left(L_{1}, \omega_{e}\right) \\
& =\frac{1}{P_{2}} C_{I M-I M}^{(1)}\left(L_{1}, \omega_{e}\right) C_{I M-P M}^{(2)}\left(L_{2}, \omega_{e}\right) \widetilde{P}_{N, 1}\left(0, \omega_{e}\right) \\
& +\frac{P_{1}}{P_{2}} C_{P M-I M}^{(1)}\left(L_{1}, \omega_{e}\right) C_{I M-P M}^{(2)}\left(L_{2}, \omega_{e}\right) \widetilde{\phi}_{1}\left(0, \omega_{e}\right) \\
& -\frac{1}{P_{1}} C_{I M-P M}^{(1)}\left(L_{1}, \omega_{e}\right) C_{P M-P M}^{(2)}\left(L_{2}, \omega_{e}\right) \widetilde{P}_{N, 1}\left(0, \omega_{e}\right) \\
& -C_{P M-P M}^{(1)}\left(L_{1}, \omega_{e}\right) C_{P M-P M}^{(2)}\left(L_{2}, \omega_{e}\right) \widetilde{\phi}_{1}\left(0, \omega_{e}\right) \text {, }
\end{aligned}
$$

Luego la respuesta del sistema con inversión espectral se obtiene como

$$
\begin{aligned}
& H_{\mathrm{OPC}}\left(\mathrm{L}, \omega_{\mathrm{e}}\right)=\frac{{\widetilde{\mathrm{F}_{N}, 2}}_{2}\left(\mathrm{~L}_{2}, \omega_{\mathrm{e}}\right)}{\tilde{\sigma}_{\mathrm{N}, 1}\left(0, \omega_{\mathrm{e}}\right)} \\
& =\mathrm{C}_{\mathrm{IM}-\mathrm{IM}}^{(1)}\left(\mathrm{L}_{1}, \omega_{\mathrm{e}}\right) \mathrm{C}_{\mathrm{IM}-\mathrm{IM}}^{(2)}\left(\mathrm{L}_{2}, \omega_{\mathrm{e}}\right)-\frac{\mathrm{P}_{2}}{\mathrm{P}_{1}} \mathrm{C}_{\mathrm{IM}-\mathrm{PM}}^{(1)}\left(\mathrm{L}_{1}, \omega_{\mathrm{e}}\right) \mathrm{C}_{\mathrm{PM}-\mathrm{IM}}^{(2)}\left(\mathrm{L}_{2}, \omega_{\mathrm{e}}\right) \\
& +\frac{1}{2} \mathrm{H}_{\mathrm{PM}}\left(\omega_{\mathrm{e}}\right)\left[\mathrm{C}_{\mathrm{PM}-\mathrm{IM}}^{(1)}\left(\mathrm{L}_{1}, \omega_{\mathrm{e}}\right) \mathrm{C}_{\mathrm{IM}-\mathrm{IM}}^{(2)}\left(\mathrm{L}_{2}, \omega_{\mathrm{e}}\right)-\frac{\mathrm{P}_{2}}{\mathrm{P}_{1}} \mathrm{C}_{\mathrm{PM}-\mathrm{PM}}^{(1)}\left(\mathrm{L}_{1}, \omega_{\mathrm{e}}\right) \mathrm{C}_{\mathrm{PM}-\mathrm{IM}}^{(2)}\left(\mathrm{L}_{2}, \omega_{\mathrm{e}}\right)\right],
\end{aligned}
$$

Normalmente, los dos tramos de fibra son idénticos, de la misma longitud $\left(\mathrm{L}_{1}=\mathrm{L}_{2}=\mathrm{L} / 2\right)$ y además $\mathrm{P}_{2}=\mathrm{P}_{1}=\mathrm{P}_{0}$, por lo que la expresión anterior se puede escribir de forma simplificada: 


$$
\begin{aligned}
H_{O P C}\left(\omega_{e}\right)= & C_{I M-I M}^{2}\left(\omega_{e}\right)-C_{I M-P M}\left(\omega_{e}\right) C_{P M-I M}\left(\omega_{e}\right) \\
& +\frac{1}{2} H_{P M}\left(\omega_{e}\right) C_{P M-I M}\left(\omega_{e}\right)\left[C_{I M-I M}\left(\omega_{e}\right)-C_{P M-P M}\left(\omega_{e}\right)\right],
\end{aligned}
$$

siendo todas las funciones de conversión para una longitud de fibra de L/2.

Obsérvese que en la función de transferencia (I.65) aparecen dos términos, el segundo de los cuales depende de las características de chirp del transmisor óptico. En el caso de transmisión lineal, sustituyendo las expresiones (I.14)-(I.16) en la ec. (I.65) se obtiene $H_{\text {OPC }}\left(\omega_{e}\right)=1$. Es decir, la técnica de inversión espectral compensa perfectamente los efectos de la dispersión cromática (aun en presencia de chirp) suponiendo que el OPC se sitúa en mitad del enlace de fibra óptica. En el caso de transmisión no lineal, el SPM empeorará ligeramente la respuesta de forma similar a como ocurría con la modulación SSB.



Figura I.13 Función de transferencia de sistemas con inversión espectral para dos longitudes de enlace: $50 \mathrm{~km}$ (puntos blancos) y $100 \mathrm{~km}$ (puntos negros). Las curvas teóricas se representan con líneas contínuas. La línea de trazos es el caso de transmisión lineal. Los parámetros del sistema son: $\mathrm{L}_{1}=\mathrm{L}_{2}=\mathrm{L} / 2, \mathrm{P}_{0}=20 \mathrm{~mW}$ y $\alpha_{c}=0$ (sin chirp). 
En la figura I.13 se representan los resultados teóricos y de simulación de la respuesta en frecuencia de sistemas que emplean la técnica de inversión espectral. Los resultados se presentan para dos longitudes de enlace distintas: 50 y $100 \mathrm{~km}$, y se tiene en cuenta en ambos casos el SPM inducido por la fibra. Si se compara con el caso de transmisión lineal (línea de trazos), se puede ver que el SPM provoca un rizado en la respuesta, el cual depende principalmente de la potencia óptica y de la longitud de fibra. En este caso, se ha supuesto una potencia óptica de $20 \mathrm{~mW}$ para mostrar dicho efecto, pero en sistemas reales el rizado será mucho menor. En cual quier caso, aun en presencia de SPM, la técnica de inversión espectral compensa el fenómeno de supresión de la portadora, aumentando considerablemente el valor del producto ancho de banda $x$ longitud de fibra.

\section{I.5.3 Influencia del chirp en sistemas con inversión espectral}

En el apartado anterior se ha visto la función de transferencia de los sistemas que emplean la técnica de inversión espectral. A continuación, se analizará la influencia que tiene el chirp en estos sistemas. Dado que el segundo término de la ec. (1.65) que incluye la función de transferencia $H_{P M}\left(\omega_{e}\right)$ se anula en el caso de transmisión lineal, el chirp sólo va a afectar en estos sistemas si actúa conjuntamente con el SPM. Así pues, se supondrá propagación no lineal.

La respuesta del sistema en presencia de chirp se representa en la figura I.14. Básicamente, el efecto del chirp es el de aumentar la amplitud de las oscilaciones causadas por el SPM. En particular, en la figura I.14(a) se observa que para $\alpha_{c}=3$ aparecen nulos de transmisión, por lo que se degrada completamente el proceso de ecualización. En general, valores $\alpha_{c}>$ 0 son más perjudiciales. Como curiosidad, para una frecuencia de unos 18 $\mathrm{GHz}$ coinciden todas las curvas. Esta frecuencia es justamente aquella que hace que se anule el término $\mathrm{C}_{\mathrm{IM}-\mathrm{IM}}(\cdot)-\mathrm{C}_{\mathrm{PM}-\mathrm{PM}}(\cdot)$. En el caso de transmisión lineal, este término es siempre nulo, por lo que los resultados son independientes del chirp. 




(a)



(b)

Figura I.14 Respuesta de un sistema que emplea la técnica de inversión espectral en presencia de chirp. (a) Resultados para varios valores de $\alpha_{c}$ (curva de trazos para $\alpha_{c}=0$ ). (b) Influencia del chirp adiabático con $\alpha_{c}=3$. Los parámetros del sistema son: $\mathrm{L}=$ $50 \mathrm{~km}$ y $\mathrm{P}_{0}=20 \mathrm{~mW}$. 
Por último, en la figura I.14(b) se representa el efecto causado por el chirp adiabático, el cual es muy similar al ya comentado en la sección I.3. En particular, el chirp adiabático puede ser una solución para suavizar los nulos que aparecen en la respuesta como consecuencia del chirp. Se observa que aumentando el valor del parámetro $\mathrm{f}_{\mathrm{c}}$ se consiguen mejores resultados. 


\section{I.6 FIBRAS DE DISPERSIÓN DESPLAZADA}

En la sección I.2 se ha analizado la función de transferencia de fibras dispersivas y no lineales, donde se consideraba únicamente el término de dispersión de primer orden. A continuación, se generalizará este mismo estudio para fibras de dispersión desplazada (dispersion-shifted fiber, DSF), donde sí es importante considerar la dispersión de segundo orden.

\subsubsection{Análisis teórico de la propagación a través de DSFs}

La propagación de señales a través de una DSF no lineal se modela por medio de la ecuación de Schrödinger no lineal

$$
\frac{\partial A}{\partial z}-\frac{j}{2} \beta_{2} \frac{\partial^{2} A}{\partial T^{2}}-\frac{1}{6} \beta_{3} \frac{\partial^{3} A}{\partial T^{3}}=-\frac{\alpha}{2} A-j \gamma|A|^{2} A,
$$

donde se ha introducido el coeficiente de dispersión de segundo orden, $\beta_{3}$, para modelar de forma más exacta la dispersión cromática en torno a la longitud de onda de trabajo.

La dispersión de orden superior está gobernada por el parámetro $\mathrm{S}=\mathrm{dD} / \mathrm{d} \lambda$, conocido como parámetro de dispersión diferencial, y que se puede expresar como [Agr97]

$$
S=\left(\frac{2 \pi c}{\lambda^{2}}\right)^{2} \beta_{3}+\left(\frac{4 \pi c}{\lambda^{3}}\right) \beta_{2}
$$

Para $\lambda=\lambda_{\text {ZD }}$ (longitud de onda de dispersión nula), $\beta_{2}=0$, y $\mathrm{S}$ es proporcional a $\beta_{3}$. Un valor típico de S para una DSF con $\lambda_{\mathrm{ZD}}=1550 \mathrm{~nm}$ puede ser $0,05 \mathrm{ps} /\left(\mathrm{km} \cdot \mathrm{nm}^{2}\right)$.

Sustituyendo la envolvente $A(z, T)$ en (I.66), y siguiendo el mismo proceso que en la sección 1.2, se llega al siguiente sistema de ecuaciones diferenciales que rige la propagación a través de una DSF no lineal: 


$$
\begin{aligned}
& \frac{\partial \widetilde{p}_{N}\left(z, \omega_{e}\right)}{\partial z}=\beta_{2} \omega_{e}^{2} P_{0} \widetilde{\phi}\left(z, \omega_{e}\right)-\frac{j}{6} \beta_{3} \omega_{e}^{3} \widetilde{P}_{N}\left(z, \omega_{e}\right), \\
& \frac{\partial \widetilde{\phi}\left(z, \omega_{e}\right)}{\partial z}=-\left[\frac{\beta_{2} \omega_{e}^{2}}{4 P_{0}}+\gamma \exp (-\alpha z)\right] \widetilde{p}_{N}\left(z, \omega_{e}\right)-\frac{j}{6} \beta_{3} \omega_{e}^{3} \widetilde{\phi}\left(z, \omega_{e}\right) .
\end{aligned}
$$

Si comparamos este sistema de ecuaciones diferenciales con el que se obtuvo en la sección 1.2, se deduce de forma inmediata que las soluciones son exactamente las mismas salvo por un término de fase. De este modo, las soluciones vendrán dadas por

$$
\begin{aligned}
& \widetilde{p}\left(z, \omega_{\mathrm{e}}\right)=\left.\exp \left(-\frac{j}{6} \beta_{3} \omega_{\mathrm{e}}^{3} z\right) \widetilde{p}\left(z, \omega_{\mathrm{e}}\right)\right|_{\beta_{3}=0^{\prime}} \\
& \widetilde{\phi}\left(z, \omega_{\mathrm{e}}\right)=\left.\exp \left(-\frac{j}{6} \beta_{3} \omega_{\mathrm{e}}^{3} z\right) \widetilde{\phi}\left(z, \omega_{\mathrm{e}}\right)\right|_{\beta_{3}=0} .
\end{aligned}
$$

Particularizando para el caso de transmisión lineal $(\gamma=0)$, se tiene [Car99]

$$
\begin{aligned}
& C_{I M-I M}\left(z, \omega_{e}\right)=C_{P M-P M}\left(z, \omega_{e}\right)=\exp \left(-j \frac{\beta_{3}}{6} \omega_{e}^{3} z\right) \cos \left(\frac{\beta_{2} \omega_{e}^{2} Z}{2}\right), \\
& C_{P M-I M}\left(z, \omega_{e}\right)=2 \exp \left(-j \frac{\beta_{3}}{6} \omega_{e}^{3} Z\right) \operatorname{sen}\left(\frac{\beta_{2} \omega_{e}^{2} Z}{2}\right), \\
& C_{I M-P M}\left(z, \omega_{e}\right)=-\frac{1}{2} \exp \left(-j \frac{\beta_{3}}{6} \omega_{e}^{3} Z\right) \operatorname{sen}\left(\frac{\beta_{2} \omega_{e}^{2} Z}{2}\right) .
\end{aligned}
$$

Si despreciamos los efectos dispersivos de segundo orden $\left(\beta_{3}=0\right)$, las funciones de conversión son idénticas a las calculadas en la sección I.2. En caso de operar a $\lambda_{\mathrm{ZD}}\left(\beta_{2}=0\right)$, entonces se obtienen las soluciones de [Car98a]. Finalmente, la matriz de transferencia presentada en [Cro97] se puede obtener de (I.72)-(I.74) considerando únicamente los dos primeros términos en el desarrollo de Taylor de la función exponencial. En este caso, la fórmula aproximada es válida si se cumple que $\left|\beta_{3} \omega_{\mathrm{e}}^{3} z / 6\right|<<1$. La dispersión de segundo orden afecta sólo a la fase de la función de transferencia [Car99], pero la solución calculada en [Cro97] establece que 
tanto la magnitud como la fase se ven modificadas. Este hecho debe tenerse en cuenta, ya que la magnitud de la función de transferencia tiene gran importancia en los sistemas de comunicaciones ópticas.

\section{I.6.2 Respuesta de una DSF}

Como se ha visto en el apartado anterior, el coeficiente de dispersión $\beta_{3}$ introduce únicamente como distorsión un retardo dependiente de la frecuencia. Así pues, para caracterizar la respuesta de una DSF habrá que medir tanto el módulo como la fase de la función de transferencia.

Suponiendo transmisión lineal, la respuesta de una DSF es:

$$
H_{D S F}\left(z, \omega_{e}\right)=\exp \left(-\frac{j}{6} \beta_{3} \omega_{e}^{3} z\right)\left[\cos \left(\frac{\beta_{2} \omega_{e}^{2} z}{2}\right)+H_{P M}\left(\omega_{e}\right) \operatorname{sen}\left(\frac{\beta_{2} \omega_{e}^{2} z}{2}\right)\right] .
$$

Dado que cerca de la longitud de onda de dispersión nula, $D \sim 0$, no tiene excesivo interés analizar el módulo de la función de transferencia, ya que será prácticamente constante. En cambio, la fase de la función de transferencia sí que posee información. Hasta este momento no se había analizado la fase de la función de transferencia de la fibra salvo en el caso de modulación SSB. En la figura I.15 se representa la fase de esta función de transferencia para $100 \mathrm{~km}$ de DSF caracterizada por unos parámetros D $=8 \cdot 10^{-3} \mathrm{ps} /(\mathrm{km} \cdot \mathrm{nm})$ y $\mathrm{S}=0,085 \mathrm{ps} /\left(\mathrm{km} \cdot \mathrm{nm}^{2}\right)$. La curva de trazos es para modulación DSB, mientras que las continuas son para modulación SSB. La expresión teórica de esta fase es precisamente:

$$
\arg H_{\mathrm{SSB}}\left(\mathrm{z}, \omega_{\mathrm{e}}\right)= \pm \frac{\beta_{2} \omega_{\mathrm{e}}^{2} \mathrm{z}}{2}-\frac{\beta_{3} \omega_{\mathrm{e}}^{3} \mathrm{z}}{6} \text {. }
$$

En el caso de modulación DSB, existe un salto de fase de $180^{\circ}$ cada vez que se produce un nulo en el módulo de la función de transferencia. Con los parámetros anteriores, se puede ver que el primer nulo aparecería para una frecuencia de modulación de $280 \mathrm{GHz}$. En particular, para $\mathrm{f}_{\mathrm{m}}<60 \mathrm{GHz}$, la respuesta de amplitud cae menos de 0,025 dB. Se observa, pues, que la DSF elimina completamente el fenómeno de supresión de la portadora, ya que 


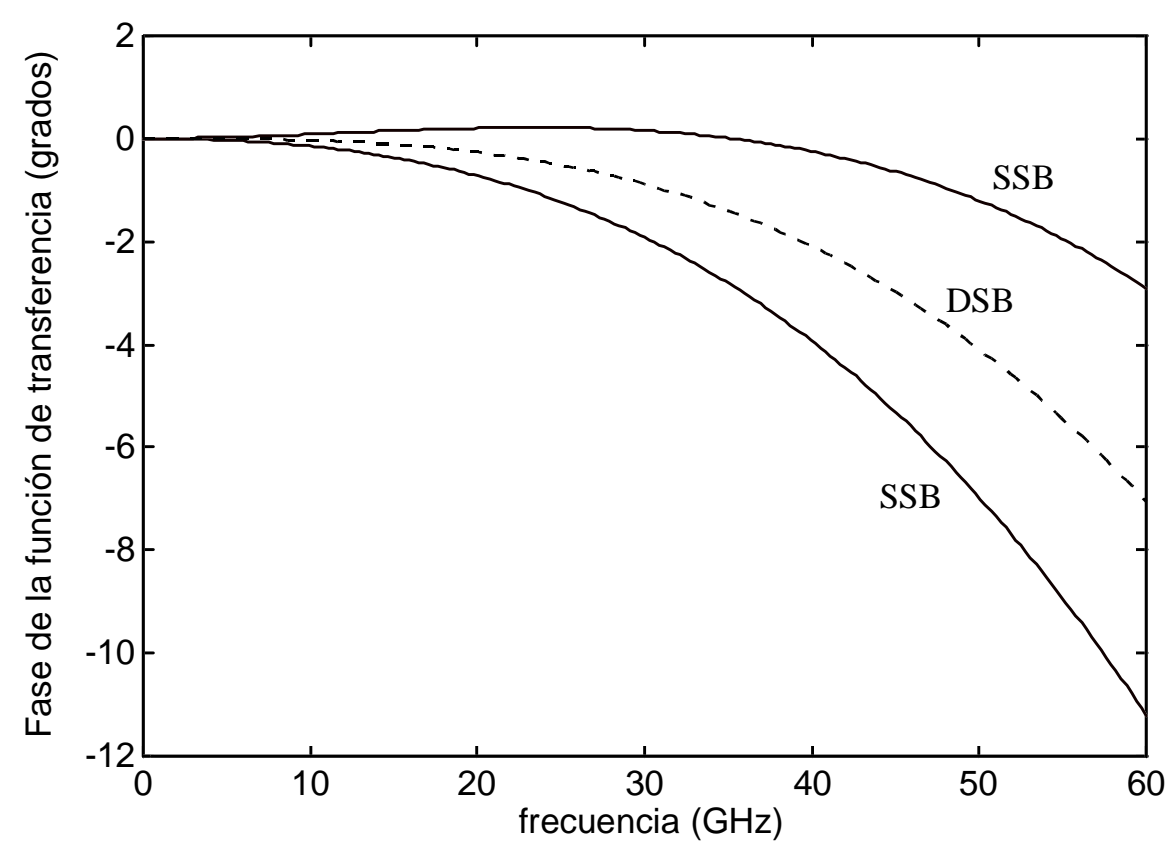

Figura I.15 Fase de la función de transferencia de $100 \mathrm{~km}$ de DSF con unos parámetros $\mathrm{D}=8 \cdot 10^{-3} \mathrm{ps} /(\mathrm{km} \cdot \mathrm{nm})$ y $\mathrm{S}=0,085$ $\mathrm{ps} /\left(\mathrm{km} \cdot \mathrm{nm}^{2}\right)$. En el caso de modulación SSB existen dos resultados dependiendo del signo de la función $\mathrm{H}_{\mathrm{PM}}\left(\omega_{\mathrm{e}}\right)= \pm \mathrm{j}$.

éste viene causado por la dispersión de primer orden que es prácticamente nula trabajando en torno a $\lambda_{\mathrm{ZD}}$. Por lo tanto, se puede deducir que el chirp del transmisor óptico tampoco será excesivamente perjudicial.

En el caso de transmisión no lineal sirve lo mencionado en la sección I.2. No obstante, hay que tener en cuenta que las DSFs suelen ser más no lineales $\left(A_{\text {eff }}>50 \mu \mathrm{m}^{2}\right)$, por lo que el SPM será más efectivo. De todos modos, en ausencia de dispersión de primer orden no se convierte en un efecto perjudicial. Adicionalmente, Ias DSF s son el medio ideal para la generación de otros efectos no lineales como el mezclado de cuatro ondas, el cual se analiza en el capítulo 5. 


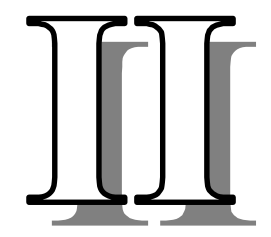

\section{Apéndice: Funciones de Bessel de orden imaginario puro}

\section{II.1 INTRODUCCIÓN}

En este apéndice se examinan las funciones de Bessel modificadas, con especial atención al caso en el cual el orden es imaginario puro y el argumento es un número real positivo. Surgen así las funciones $\mathrm{K}_{\mathrm{iv}}(\mathrm{x})$ y $\mathrm{L}_{\text {iv }}(\mathrm{x})$, que son un par de soluciones numéricamente satisfactorias de la ecuación diferencial de Bessel de orden imaginario puro. Al mismo tiempo, se proporcionan una serie de expresiones y de propiedades de las mismas que serán útiles para desarrollar la teoría del capítulo 3, donde se analiza la función de transferencia de fibras ópticas dispersivas y no lineales. 


\section{II.2 FUNCIONES DE BESSEL MODIFICADAS DE ORDEN IMAGINARIO}

\section{II.2.1 Ecuación diferencial de Bessel modificada}

La ecuación diferencial de Bessel de orden v,

$$
\frac{d^{2} w}{d z^{2}}+\frac{1}{z} \frac{d w}{d z}-\left(1+\frac{v^{2}}{z^{2}}\right) w=0
$$

tiene como soluciones las funciones de Bessel modificadas de orden v. La de primera especie y orden $v$ es la función definida por [Abr65 (cap. 9)]

$$
\mathrm{I}_{v}(\mathrm{z})=\left(\frac{\mathrm{z}}{2}\right)^{v} \sum_{\mathrm{k}=0}^{\infty} \frac{1}{\mathrm{k} ! \Gamma(v+k+1)}\left(\frac{\mathrm{z}}{2}\right)^{2 \mathrm{k}}
$$

cuando $v \notin\{-\mathrm{n}: \mathrm{n}=1,2,3, \ldots\}$, y por

$$
I_{v}(z)=I_{-v}(z),
$$

cuando $v \in\{-\mathrm{n}: \mathrm{n}=1,2,3, \ldots\}$. $\Gamma(\mathrm{z})$ es la función de gamma definida como [Abr65 (cap. 6)]

$$
\Gamma(z)=\int_{0}^{\infty} \exp (-t) t^{-1} d t
$$

La función de Bessel modificada de segunda especie y orden $v$ es la función definida por [Abr65 (cap. 9)]

$$
K_{v}(z)=\frac{\pi}{2} \frac{I_{-v}(z)-I_{v}(z)}{\operatorname{sen}(\pi v)},
$$

cuando $v \notin \mathbb{Z}$, por

$$
\begin{aligned}
K_{v}(z)= & (-1)^{v+1} \ln \left(\frac{z}{2}\right) I_{v}(z)+\frac{(-1)^{v}}{2} \sum_{k=0}^{\infty} \frac{1}{k !(v+k) !}[\Psi(k+1)+\Psi(v+k+1)]\left(\frac{z}{2}\right)^{2 k+v} \\
& +\frac{1}{2} \sum_{k=0}^{\infty}(-1)^{k} \frac{(v-k-1) !}{k !}\left(\frac{z}{2}\right)^{2 k-v},
\end{aligned}
$$




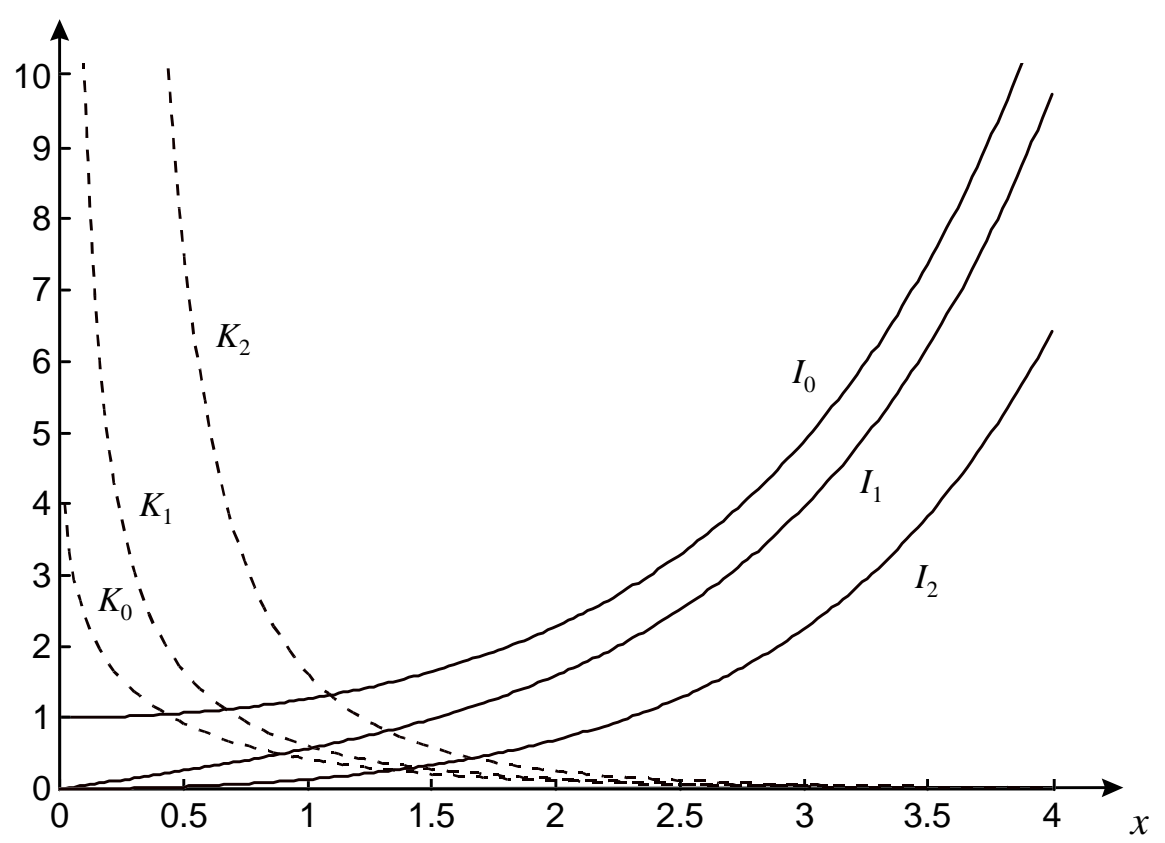

Figura II.1 Funciones de Bessel modificadas de primera y segunda especie de órdenes $v=0,1$ y 2 , y argumento real.

si $v \in \mathbb{N}, y$ por

$$
K_{-v}(z)=K_{v}(z)
$$

si $v \in\{-n: n=1,2,3, \ldots\}$. $\Psi(z)$ es la función digamma definida como [Abr65 (cap. 6)]

$$
\Psi(z)=\frac{\Gamma^{\prime}(z)}{\Gamma(z)}
$$

En la figura II.1 se representan las funciones $I_{v}(x)$ y $K_{v}(x)$ hasta orden 2 y para argumento real positivo. Se observa que $K_{v}(x)$ no está acotada conforme $x \rightarrow 0$. 


\section{II.2.2 Soluciones de la ecuación de Bessel modificada de orden imaginario puro}

Las funciones de Bessel modificadas, $I_{\mu}(z)$ y $K_{\mu}(z)$, componen un par de soluciones numéricamente satisfactoriasi de la ecuación de Bessel modificada

$$
\frac{d^{2} w}{d z^{2}}+\frac{1}{z} \frac{d w}{d z}-\left(1+\frac{\mu^{2}}{z^{2}}\right) w=0
$$

en el semiplano $|\arg z| \leq \pi / 2$, para todos los valores complejos de $\mu$ tales que $\operatorname{Re} \mu \geq 0$. Cuando $\mu$ es imaginario puro, no obstante, la función $I_{\mu}(z)$ tiene la propiedad no deseada de ser compleja en el eje real positivo $z=x>0$. Por lo tanto, resulta conveniente introducir la siguiente función ${ }^{i i}$ [Dun90]:

$$
\mathrm{L}_{\mu}(z)=\frac{\pi \mathrm{i}}{2 \operatorname{sen}(\mu \pi)}\left\{\mathrm{I}_{\mu}(z)+\mathrm{I}_{-\mu}(z)\right\} \quad(\mu \neq 0),
$$

la cual se verá que es una compañera numéricamente satisfactoria de $\mathrm{K}_{\mathrm{iv}}(\mathrm{x})$, donde $v$ es real y no negativo, y $x$ es real y positivo. Nótese que $L_{\mu}(z)$ no está definida cuando $\mu=0$. No es posible definir una compañera numéricamente satisfactoria de $\mathrm{K}_{\mathrm{iv}}(\mathrm{x})$ que permanezca finita conforme $v \rightarrow 0$.

La definición de $L_{\mu}(z)$ debe compararse con la de $K_{\mu}(z)$ :

$$
K_{\mu}(z)=\frac{\pi}{2 \operatorname{sen}(\mu \pi)}\left\{I_{-\mu}(z)-I_{\mu}(z)\right\}
$$

El desarrollo en serie de potencias de $L_{\mu}(z)$ puede derivarse directamente a partir de (II.10) junto con la conocida expresión de la serie de potencias de $\mathrm{I}_{ \pm \mu}(\mathrm{z})$ del apartado anterior. Cuando $\mu=\mathrm{i} v$ y $\mathrm{z}=\mathrm{x}$ puede expresarse como [Dun90]

\footnotetext{
' según el criterio de [Mil50]

ii en este apéndice consideraremos que i es la unidad imaginaria
} 


$$
L_{i v}(x)=\left(\frac{v \pi}{\operatorname{senh}(v \pi)}\right)^{1 / 2} \sum_{s=0}^{\infty} \frac{\left(x^{2} / 4\right)^{s} \cos \left(v \ln (x / 2)-\phi_{v, s}\right)}{s !\left[\left(v^{2}\right)\left(1^{2}+v^{2}\right) \ldots\left(s^{2}+v^{2}\right)\right]^{1 / 2}}
$$

donde

$$
\phi_{v, s}=\arg \{\Gamma(1+s+i v)\} .
$$

Para cada s definiremos la rama de (II.13) de tal forma que $\phi_{v, s}$ sea continuo para $0<v<\infty$, con $\lim _{v \rightarrow 0} \phi_{v, s}=0$.

En el caso de $K_{\mu}(z)$, se obtiene de forma similar [Dun90]

$$
K_{i v}(x)=-\left(\frac{v \pi}{\operatorname{senh}(v \pi)}\right)^{1 / 2} \sum_{s=0}^{\infty} \frac{\left(x^{2} / 4\right)^{s} \operatorname{sen}\left(v \ln (x / 2)-\phi_{v, s}\right)}{\left.s !\left(v^{2}\right)\left(1^{2}+v^{2}\right) \ldots\left(s^{2}+v^{2}\right)\right]^{1 / 2}} .
$$

En las figuras 11.2 y 11.3 se representan las funciones $L_{i v}(x)$ y $K_{i v}(x)$ para distintos órdenes y argumentos. En la figura 11.2 se observa que las



Figura II.2 Funciones de Bessel modificadas de orden imaginario puro y sus derivadas en función de $x$ y para $v=1$. 


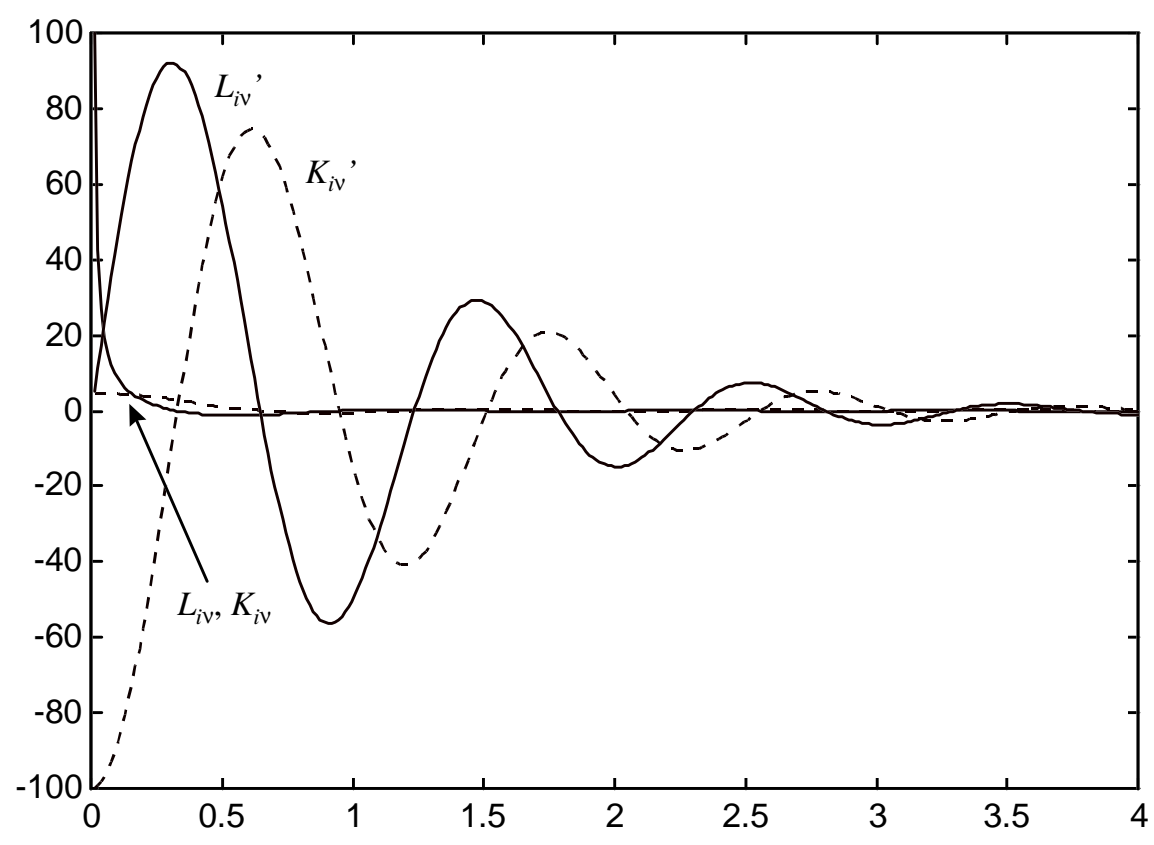

Figura II.3 Funciones de Bessel modificadas de orden imaginario puro y sus derivadas en función de $v$ y para $x=10^{-2}$.

funciones oscilan cada vez más rápidamente conforme $x \rightarrow 0$ (obsérvese que el eje de abcisas es logarítmico), por lo que las derivadas de éstas también oscilan pero con una gran amplitud. Al mismo tiempo, las oscilaciones de $\mathrm{L}_{\mathrm{iv}}$ y $\mathrm{K}_{\text {iv }}$ están desfasadas 90 on esta región. Finalmente, en la figura II.3 se representa la variación con el orden $v$. Como ya se comentó anteriormente y se puede observar, la función $L_{\text {iv }}$ deja de estar acotada conforme $v \rightarrow 0$.

\section{II.2.3 Propiedades de las funciones de Bessel modificadas}

En este apartado resumiremos algunas de las propiedades más relevantes de las funciones $L_{\mu}(z)$ y $K_{\mu}(z)$, con especial énfasis en el caso en el que $\mu$ es imaginario puro. En todas las definiciones, $\mu$ denota un parámetro complejo y $v$ un parámetro positivo no nulo. Cuando la variable independiente $z$ sea real y positiva, la denotaremos por $\mathrm{x}$.

- Relaciones de recurrencia. 
Las funciones $\exp (\mu \pi \mathrm{i}) \mathrm{K}_{\mu}(\mathrm{z})$ y $\exp (\mu \pi \mathrm{i}) \mathrm{L}_{\mu}(\mathrm{z})$ satisfacen las mismas relaciones de recurrencia que $I_{\mu}(z)$, es decir [Abr65 (cap. 9)]

$$
\begin{aligned}
& I_{\mu-1}(z)-I_{\mu+1}(z)=\frac{2 \mu}{z} I_{\mu}(z), \\
& I_{\mu-1}(z)+I_{\mu+1}(z)=2 I_{\mu}^{\prime}(z), \\
& I_{\mu+1}(z)=-\frac{\mu}{z} I_{\mu}(z)+I_{\mu}^{\prime}(z), \\
& I_{\mu-1}(z)=\frac{\mu}{z} I_{\mu}(z)+I_{\mu}^{\prime}(z) .
\end{aligned}
$$

- Fórmulas de conexión.

$$
\mathrm{L}_{-\mu}(\mathrm{z})=-\mathrm{L}_{\mu}(\mathrm{z}), \quad \mathrm{K}_{-\mu}(\mathrm{z})=\mathrm{K}_{\mu}(\mathrm{z})
$$

- Wronskiano[Dun90].

$$
\mathcal{W}\left\{\mathrm{K}_{\mu}(\mathrm{z}), \mathrm{L}_{\mu}(\mathrm{z})\right\}=\frac{\pi \mathrm{i}}{\operatorname{sen}(\mu \pi) \mathrm{z}}
$$

- Comportamiento en las singularidades $z=0, \infty$ [Dun90].

Si $v(>0)$ es fijo $y x \rightarrow 0^{+}$, entonces

$$
\begin{aligned}
& \mathrm{K}_{\mathrm{iv}}(\mathrm{x})=-\left(\frac{\pi}{v \operatorname{senh}(v \pi)}\right)^{1 / 2}\left\{\operatorname{sen}\left(v \ln (\mathrm{x} / 2)-\phi_{v, 0}\right)+O\left(x^{2}\right)\right\}, \\
& \mathrm{L}_{\mathrm{iv}}(\mathrm{x})=\left(\frac{\pi}{v \operatorname{senh}(v \pi)}\right)^{1 / 2}\left\{\cos \left(v \ln (\mathrm{x} / 2)-\phi_{v, 0}\right)+O\left(x^{2}\right)\right\} .
\end{aligned}
$$

Nótese que las amplitudes de oscilación de $\mathrm{L}_{\mathrm{iv}}(\mathrm{x})$ y $\mathrm{K}_{\text {iv }}(\mathrm{x})$ en la vecindad del origen dejan de estar acotadas conforme $v \rightarrow 0$. Por otro lado, para $z \rightarrow \infty$ 


$$
\begin{aligned}
& \mathrm{K}_{\mathrm{iv}}(\mathrm{z})=\left(\frac{\pi}{2 \mathrm{z}}\right)^{1 / 2} \exp (-\mathrm{z})\left\{1+\mathrm{O}\left(\frac{1}{\mathrm{z}}\right)\right\}, \quad|\arg \mathrm{z}| \leq 3 \pi / 2-\delta, \\
& \mathrm{L}_{\mathrm{iv}}(\mathrm{z})=\frac{1}{\operatorname{senh}(v \pi)}\left(\frac{\pi}{2 z}\right)^{1 / 2} \exp (\mathrm{z})\left\{1+\mathrm{O}\left(\frac{1}{\mathrm{z}}\right)\right\}, \quad|\arg \mathrm{z}| \leq \pi / 2-\delta,
\end{aligned}
$$

donde $\delta$ es una pequeña constante positiva arbitraria. 


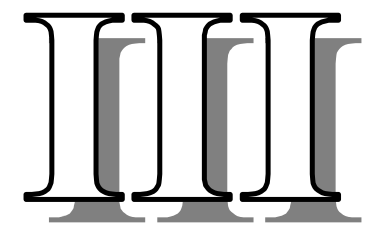

\section{Apéndice: Publicaciones internacionales del autor}

\section{III.1 PUBLICACIONES RELACIONADAS CON LA TESIS}

1. J . Marti, and F. Ramos, "Compensation for dispersion-induced nonlinear distortion in subcarrier systems using optical-phase conjugation", IEE Electronics Letters, vol. 33, no. 9, pp. 792-794, April 1997.

2. F. Ramos, and J. Marti, "Compensation for fiber-induced composite second-order distortion in externally modulated lightwave AM-SCM systems using optical-phase conjugation", IEEE J ournal of Lightwave Technology, vol. 16, no. 8, pp. 1387-1392, August 1998.

3. F. Ramos, J. Marti, V. Polo, and J. M. Fuster, "On the use of fiberinduced self-phase modulation to reduce chromatic dispersion effects in microwave/millimeter-wave optical systems", IEEE Photonics Technol ogy Letters, vol. 10, no. 10, pp. 1473-1475, October 1998. 
4. F. Ramos, J. Marti, and V. Polo, "Compensation of chromatic dispersion effects in microwave/millimeter-wave optical systems using four-wave mixing induced in dispersion-shifted fibers", IEEE Photonics Technology Letters, vol. 11, no. 9, pp. 1171-1173, September 1999.

5. F. Ramos, and J. Marti, "Comparison of optical single-sideband modulation and chirped fiber gratings as dispersion mitigating techniques in optical millimeter-wave multichannel systems", IEEE Photonics Technology Letters, vol. 11, no. 11, pp. 1479-1481, November 1999.

6. F. Ramos, and J . Marti, "Frequency transfer function of dispersive and nonlinear single-mode optical fibers in microwave optical systems", IEEE Photonics Technology Letters, vol. 12, no. 5, pp. 549-551, May 2000.

7. F. Ramos, J. Marti, V. Polo, J. M. Fuster, and F. J. Martinez, "Performance comparison of DSF- and SOA-based conjugators in ASElimited digital microwave optical links", accepted in 26th European Conference on Optical Communication (ECOC'2000), Munich, 2000.

\section{III.2 OTRAS PUBLICACIONES DEL AUTOR}

\section{III.2.1 Publicaciones en revista internacional}

1. J . Marti, A. Montero, D. Pastor, J . M. Fuster, J . Capmany, V. Polo, and F. Ramos, "Impact of apodised linearly chirped fibre gratings on the performance of dispersion-limited subcarrier systems", IE E ProceedingsOptoel ectronics, vol. 145, no. 2, pp. 117-123, April 1998.

2. J. Marti, F. Ramos, and R. I. Laming, "Photonic microwave filter employing multimode optical sources and wideband chirped fibre gratings", IEE Electronics Letters, vol. 34, no. 18, pp. 1760-1761, September 1998.

3. J . M. Fuster, J . Marti, V. Polo, F. Ramos, and J . L. Corral, "Mitigation of dispersion-induced power penalty in millimetre-wave fibre optic links", 
IEE Electronics Letters, vol. 34, no. 19, pp. 1869-1870, September 1998.

4. J . M. Fuster, J . Marti, J . L. Corral, V. Polo, and F. Ramos, "Optimisation of dispersion-induced power penalty mitigation in millimetre-wave fibre optic links", IEE Electronics Letters, vol. 35, no. 1, pp. 69-70, J anuary 1999.

5. J. Marti, V. Polo, F. Ramos, and D. Moodie, "Photonic tunable microwave filters employing electroabsorption modulators and wideband chirped fibre gratings", IEE Electronics Letters, vol. 35, no. 4, pp. 305306, February 1999.

6. J . Marti, J . L. Corral, F. Ramos, V. Polo, and J . M. Fuster, "Millimetrewave optical beamforming network for phased-array antennas employing optical upconversion and wideband chirped fibre gratings", IEE Electronics Letters, vol. 35, no. 7, pp. 517-518, April 1999.

7. V. Polo, J. Marti, F. Ramos, and D. Moodie, "Mitigation of chromatic dispersion effects employing electroabsorption modulator-based transmitters", IEEE Photonics Technology Letters, vol. 11, no. 7, pp. 883-885, J uly 1999.

8. J . Marti, F. Ramos, V. Polo, J . M. Fuster, and J. L. Corral, "Millimetrewave generation and harmonic upconversion through PM-IM conversion in chirped fibre gratings", IEE Electronics Letters, vol. 35, no. 15, pp. 1265-1266, J uly 1999.

9. J . M. Fuster, J . Marti, J . L. Corral, F. Ramos, and V. Polo, "Analysis of hybrid modulation techniques in MZ-EOM-based photonic mixers to overcome dispersion-induced power penalty in up-converting millimeterwave fiber-optic links", Microwave and Optical Technology Letters, vol. 23, no. 2, pp. 127-129, October 1999.

10. V. Polo, F. Ramos, J. Marti, D. Moodie, and D. Wake, "Synthesis of photonic microwave filters based on external optical modulators and wide-band chirped fiber gratings", IEEE Journal of Lightwave Technol ogy, vol. 18, no. 2, pp. 213-220, February 2000. 


\section{III.2.2 Actas de congresos internacionales}

1. V. Polo, J. Marti, F. Ramos, and J . M. Fuster, "Millimetre-wave optical harmonic mixer employing a single Mach-Zehnder electro-optic modulator", 28th European Microwave Conference (EuMC'98), paper MD-4, pp. 81-86, Amsterdam, 1998.

2. J . M. Fuster, J. Marti, V. Polo, F. Ramos, and J . L. Corral, "Nonlinear biasing of MZ-EOM devices to experimentally reduce chromatic dispersion effects in antenna remoting up-converting fiber-optic links", International Topical Meeting on Microwave Photonics (MWP'98), paper P15, pp. 99-102 (poster session), Princeton, 1998.

3. J . Marti, J . L. Corral, F. Ramos, V. Polo, and J . M. Fuster, "Millimeterwave optical beamforming network for phased-array antennas employing optical up-conversion and wideband chirped fiber gratings", Optical Fiber Communication Conference (OFC'99), paper WM30, pp. 283-285, San Diego, 1999.

4. M. Chavero, V. Polo, F. Ramos, and J. Marti, "Impact of vegetation on the performance of $28 \mathrm{GHz}$ LMDS transmission", IEEE MTT-S International Microwave Symposium, paper WE2D-5, Anaheim, 1999.

5. V. Polo, F. Ramos, J. Marti, and J. M. Fuster, "Demonstration of dispersion-tolerant $34 \mathrm{Mbit} / \mathrm{s}$ data transmission in electro-optically upconverted $28 \mathrm{GHz}$ LMDS fiber-optic link", IEEE MTT-S International Microwave Symposium, paper WE4B-2, Anaheim, 1999.

6. J . Marti, F. Ramos, V. Polo, J . M. Fuster, and J . L. Corral, "Millimetrewave signal generation and harmonic upconversion through PM-IM conversion in chirped fibre gratings", International Topical Meeting on Microwave Photonics (MWP'99), paper T-8.12, pp. 181-184 (poster session), Melbourne, 1999. 


\section{Bibliografía de la Tesis}

[Abr65] M. Abramowitz, I. A. Stegun, "Handbook of mathematical functions", New York: Dover, 1965.

[Ada98] L. E. Adams, G. Nykolak, T. Tanbun-Ek, A. J . Stentz, A. M. Sergent, P. F. Scortino, L. Eskildsen, "SBS suppression using a multichannel tunable laser with data encoding capability", presented at OFC 98, pp. 408-410, 1998.

[Agr87] G. P. Agrawal, "Four-wave mixing and phase conjugation in semiconductor laser media", Opt. Lett., vol. 12, no. 4, pp. 260-262, 1987.

[Agr88] G. P. Agrawal, "Population pulsations and nondegenerate four-wave mixing in semiconductor lasers and amplifiers", J. Opt. Soc. Am. B, vol. 5, no. 1, pp. 147-158, 1988.

[Agr89] G. P. Agrawal, "N onlinear fiber optics", New York: Academic, 1989.

[Agr97] G. P. Agrawal, "Fiber-optic communication systems", New York: J ohn Wiley, 1997. 
[Ahm97] Z. Ahmed, D. Novak, H. F. Liu, "SCM millimeter-wave (37 GHz) optical transport system for distribution of video and data signals", presented at OFC 97, pp. 337-338, 1997.

[Ala95] K. E. Alameh, R. A. Minasian, N. Fourikis, "High capacity optical interconnects for phased array beamformers", J. Lightwave Technol., vol. 13, no. 6, pp. 1116-1120, 1995.

[Aok88] Y. Aoki, K. Tajima, I. Mito, "Input power limits of single-mode optical fibers due to stimulated Brillouin scattering in optical communication systems", J . Lightwave Technol., vol. 6, no. 5, pp. 710, 1988.

[Asa85] M. Asada, Y. Suematsu, "Density-matrix theory of semiconductor lasers with relaxation broadening model - Gain and gain-supression in semiconductor lasers", IEEE J. Quantum Electron., vol. 21, pp. 434442, 1985.

[Ber91] E. E. Bergmann, C. Y. Kuo, S. Y. Huang, "Dispersion-induced composite second-order distortion at 1.5 um", IEEE Photon. Technol. Lett., vol. 3, no. 1, pp. 59-61, 1991.

[Bet90] G. E. Betts, C. H. Cox III, K. G. Ray, "20 GHz optical analog link using an external modulator", IEEE Photon. Technol. Lett., vol. 2, no. 12, pp. 923-925, 1990.

[Bet97] S. Betti, E. Bravi, M. Giaconi, "Nonlinear distortions due to the 'dispersive' transmission of SCM optical signals in the presence of chirping effect: an accurate analysis", IEEE Photon. Technol. Lett., vol. 9, no. 12, pp. 1640-1642, 1997.

[Bet98] S. Betti, E. Bravi, M. Giaconi, "Analysis of the distortion noise due to optical fiber reflections in subcarrier-multiplexed (SCM) lightwave systems", IEEE Photon. Technol. Lett., vol. 10, no. 11, pp. 1656-1658, 1998.

[Bis99] S. Bischoff, A. Buxens, H. N. Poulsen, A. T. Clausen, J. Mork, "Bidirectional four-wave mixing in semiconductor optical amplifiers: theory and experiment", J . Lightwave Technol., vol. 17, no. 9, pp. 16171625, 1999.

[Bit99] R. Sánchez, F. Bernues, V. Quilez, F. García, J. Pérez, J. L. García, "Nuevos sistemas de acceso vía radio en banda ancha", Revista BIT, no. 115, pp. 27-53, 1999.

[Bje96] L. Bjerkan, A. Royset, L. Hafskjaer, D. Myhre, "Measurement of laser parameters for simulation of high-speed fiberoptic systems", J . Lightwave Technol., vol. 14, no. 5, pp. 839-850, 1996.

[Bra98] R.-P. Braun, et. al., "Optical microwave generation and transmission experiments in the $12-$ and $60-\mathrm{GHz}$ region for wireless 
communications", IEEE Trans. Microwave Theory Tech., vol. 46, no. 4, pp. 320-330, 1998.

[Bra98a] R.-P. Braun, "Tutorial: Fibre radio systems, applications and devices", presented at ECOC 98, vol. 2, pp. 87-119, 1998.

[Cap91] G. Cappellini, S. Trillo, "Third-order three-wave mixing in single-mode fibers: exact solutions and spatial instability effects", J . Opt. Soc. Am. B, vol. 8, no. 4, pp. 824-838, 1991.

[Car97] A. Carena, V. Curri, R. Gaudino, P. Poggiolini, S. Benedetto, "New analytical results on fiber parametric gain and its effects on ASE noise", IEEE Photon. Technol. Lett., vol. 9, no. 4, pp. 535-537, 1997.

[Car98] A. V. T. Cartaxo, B. Wedding, W. Idler, "Influence of fiber nonlinearity on the phase noise to intensity noise conversion in fiber transmission: theoretical and experimental analysis", J . Lightwave Technol., vol. 16, no. 7, pp. 1187-1194, 1998.

[Car98a] A. Cartaxo, J. Morgado, "Intensity and frequency noise transmission along single-mode fiber at zero-dispersion wavelength", IEE Proc.Optoelectron., vol. 145, no. 4, pp. 211-216, 1998.

[Car98b] A. V. T. Cartaxo, "Impact of modulation frequency on cross-phase modulation effect in intensity modulation-direct detection WDM systems", IEEE Photon. Technol. Lett., vol. 10, no. 9, pp. 1268-1270, 1998.

[Car99] A. V. T. Cartaxo, J. A. P. Morgado, "Rigorous assessment of smallsignal analysis for linear and dispersive optical communication systems operating near zero-dispersion wavelength", J . Lightwave Technol., vol. 17, no. 1, pp. 86-94, 1999.

[Car99a] A. V. T. Cartaxo, B. Wedding, W. I dler, "I nfluence of fiber nonlinearity on the fiber transfer function: theoretical and experimental analysis", J . Lightwave Technol., vol. 17, no. 10, pp. 1806-1813, 1999.

[Con97] J . Conradi, B. Davies, M. Sieben, D. Dodds, S. Walklin, "Optical single sideband (OSSB) transmission for dispersion avoidance and electrical dispersion compensation in microwave subcarrier and baseband digital sytems", presented at OF C 97, postdeadline paper, 1997.

[Coo90] A. J . Cooper, "Fibre/radio for the provision of cordless/mobile telephony services in the access network", Electron. Lett., vol. 26, pp. 2054-2056, 1990.

[Cor99] A. Corchia, C. Antonini, A. D'Ottavi, A. Mecozzi, F. Martelli, P. Spano, G. Guekos, R. Dall'Ara, "Mid-span spectral inversion without frequency shift for fiber dispersion compensation: a system demonstration", IEEE Photon. Technol. Lett., vol. 11, no. 2, pp. 275-277, 1999. 
[Cox97] C. Cox III, E. Ackerman, R. Helkey, G. E. Betts, "Techniques and performance of intensity-modulation direct-detection analog optical links", IEEE Trans. Microwave Theory Tech., vol. 45, no. 8, pp. 13751383, 1997.

[Cro94] D. B. Crosby, G. J . Lampard, "Dispersion-induced limit on the range of octave confined optical SCM transmission systems", IEEE Photon. Technol. Lett., vol. 6, no. 8, pp. 1043-1045, 1994.

[Cro97] C. Crognale, "Small signal frequency response of a linear dispersive single-mode fiber near zero first-order dispersion wavelength", J . Lightwave Technol., vol. 15, no. 3, pp. 482-489, 1997.

[Che91] L. K. Chen, K. Y. Lau, D. R. Huber, "Fundamental distortion characteristics of the erbium fiber amplifier", presented at OFC 91, paper WL-6, 1991.

[Chr90] A. R. Chraplyvy, "Limitations on lightwave communications imposed by optical-fiber nonlinearities", J. Lightwave Technol., vol. 8, no. 10, pp. 1548-1557, 1990.

[Dai96] H. Dai, S. Ovadia, C. Lin, "Hybrid AM-VSB/M-QAM multichannel video transmission over $120 \mathrm{~km}$ of standard single-mode fiber with cascaded erbium-doped fiber amplifiers", IEEE Photon. Technol. Lett., vol. 8, no. 12, pp. 1713-1715, 1996.

[Dar86] T. E. Darcie, M. E. Dixon, B. L. Kasper, C. A. 30, "Lightwave system using microwave subcarrier multiplexing", Electron. Lett., vol. 22, pp. 774-775, 1986.

[Dar90] T. E. Darcie, "Subcarrier multiplexing for lightwave networks and video distribution systems", IEEE J. Sel. Areas Commun., vol. 8, no. 7, pp. 1240-1258, 1990.

[Dav98] B. Davies, J. Conradi, "Hybrid modulator structures for subcarrier and harmonic subcarrier optical single sideband", IEEE Photon. Technol. Lett., vol. 10, no. 4, pp. 600-602, 1998.

[Des90] C. Desem, "Optical interference in subcarrier multiplexed systems with multiple optical carriers", IEEE J. Sel. Areas Commun., vol. 8, no. 7, pp. 1290-1295, 1990.

[Des94] C. Desem, "Composite second order distortion due to self-phase modulation in externally modulated optical AM-SCM systems operating at 1550 nm", Electron. Lett., vol. 30, no. 24, pp. 2055-2056, 1994.

[Dev93] F. Devaux, Y. Sorel, J. F. Kerdiles, "Simple measurement of fiber dispersion and of chirp parameter of intensity modulated light emitter", J . Lightwave Technol., vol. 11, no. 12, pp. 1937-1940, 1993. 
[Dev94] F. Devaux, "Optimum prechirping conditions of externally modulated lasers for transmission on standard fibre", IEE Proc.-Optoel ectron., vol. 141, no. 6, pp. 363-366, 1994.

[Dju92] A. Djupsjöbacka, "Residual chirp in integrated-optic modulators", IEEE Photon. Technol. Lett., vol. 4, no. 1, pp. 41-43, 1992.

[Dod82] R. K. Dodd, J. C. Eilbeck, J. D. Gibbon, H. C. Morris, "Solitons and nonlinear wave equations", New York: Academic, 1982.

[Dot96] A. D'Ottavi, F. Martelli, P. Spano, A. Mecozzi, S. Scotti, R. Dallara, J . Eckner, G. Guekos, "Very high-efficiency four-wave mixing in a single semiconductor traveling-wave amplifier", Appl. Phys. Lett., vol. 68, no. 16, pp. 2186-2188, 1996.

[Duc96] T. Ducellier, M. B. Bibey, "Study of optical phase conjugation in bulk traveling wave semiconductor optical amplifier", IEEE Photon. Technol. Lett., vol. 8, no. 4, pp. 530-532, 1996.

[Dun90] T. M. Dunster, "Bessel functions of purely imaginary order, with an application to second-order linear differential equations having a large parameter", SI AM J . Math. Anal., vol. 21, no. 4, pp. 995-1018, 1990.

[Eis98] M. Eiselt, R. Schnabel, E. Dietrich, "Polarization insensitive frequency converter with the capability of chirp removal", IEEE Photon. Technol. Lett., vol. 10, no. 1, pp. 63-65, 1998.

[Enn98] K. Ennser, M. Ibsen, M. Durkin, M. N. Zervas, R. I. Laming, "Influence of nonideal chirped fiber grating characteristics on dispersion cancellation", IEEE Photon. Technol. Lett., vol. 10, no. 10, pp. 14761478, 1998.

[Fei99] U. Feiste, R. Ludwig, C. Schmidt, E. Dietrich, S. Diez, H. J . Ehrke, E. Patzak, H. G. Weber, "80 Gbit/s transmission over 106 km standardfiber using optical phase conjugation in a Sagnac-interferometer", presented at OFC 99, pp. 68-70, 1999.

[Fis83] R. A. Fisher, B. R. Suydam, D. Yevick, "Optical phase conjugation for time-domain undoing of dispersive self-phase-modulation effects", Opt. Lett., vol. 8, pp. 611-613, 1983.

[Fis93] D. A. Fishman, "Design and performance of externally modulated 1.5um laser transmitter in the presence of chromatic dispersion", J . Lightwave Technol., vol. 11, no. 4, pp. 624-632, 1993.

[Fis93a] D. A. Fishman, J. A. Nagel, "Degradations due to stimulated Brillouin scattering in multigigabit intensity-modulated fiber-optic systems", J . Lightwave Technol., vol. 11, no. 11, pp. 1721-1728, 1993.

[Fon94] T. K. Fong, M. Tabara, D. J. M. Sabido IX, L. G. Kazovsky, "Dynamic 
range of externally modulated analog optical links: optical amplification versus coherent detection", IEEE Photon. Technol. Lett., vol. 6, no. 2, pp. 270-272, 1994.

[Fre97] P. N. Freeman, N. K. Dutta, J. Lopata, "Semiconductor optical amplifier for wavelength conversion in subcarrier multiplexed systems", IEEE Photon. Technol. Lett., vol. 9, no. 1, pp. 46-48, 1997.

[Fur99] H. Furukawa, H. Yoshidaya, H. Takakura, K. Kuroda, "A novel optical device with wide bandwidth wavelength conversion and amplification", presented at OFC 99, pp. 368-370, 1999.

[Fus97] J. M. Fuster, J. Marti, J . L. Corral, "Chromatic dispersion effects in el ectro-optical upconverted millimetre-wave fibre optic links", Electron. Lett., vol. 33, no. 23, pp. 1969-1970, 1997.

[Ga095] Y. Gao, "Linear fiber-optical transmission systems for cellular telephone signals with wide dynamic range", presented at EFOC\&N 95, pp. 128-131, 1995.

[Gei97] H. Geiger, S. Y. Set, R. I. Laming, M. J . Cole, L. Reekie, "Comparison of DSF - and SOA-based phase conjugators employing noise-suppressing fiber", presented at OFC 97, pp. 150-151, 1997.

[Geo94] J. B. Georges, M-H. Kiang, K. Heppell, M. Sayed, K. Lau, "Optical transmission of narrowband millimetre-wave signals by resonant modulation of monolithic semiconductor lasers", IEEE Photon. Technol. Lett., vol. 6, pp. 568-570, 1994.

[Gil97] C. R. Giles, "Lightwave applications of fiber Bragg gratings", J . Lightwave Technol., vol. 15, no. 8, pp. 1391-1404, 1997.

[Gli96] U. Gliese, S. Norskov, T. N. Nielsen, "Chromatic dispersion in fiberoptic microwave and millimeter-wave links", IEEE Trans. Microwave Theory Tech., vol. 44, no. 10, pp. 1716-1724, 1996.

[Gna91] A. H. Gnauck, S. K. Korotky, J . J . Veselka, J. Nagel, C. T. Kemmerer, W. J. Minford, D. T. Moser, "Dispersion penalty reduction using an optical modulator with adjustable chirp", IEEE Photon. Technol. Lett., vol. 3, no. 10, pp. 916-918, 1991.

[Gop96] G. K. Gopalakrishnan, T. J. Brophy, C. Breverman, "Experimental study of fibre induced distortions in externally modulated $1550 \mathrm{~nm}$ analogue CATV links", Electron. Lett., vol. 32, no. 14, pp. 1309-1310, 1996.

[Har73] R. H. Hardin, F. D. Tappert, "Applications of the split-step Fourier method to the numerical solution of nonlinear and variable coefficient wave equations", SIAM Rev., vol. 15, pp. 423, 1973. 
[Has93] T. Hasegawa, K. Inoue, K. Oda, "Polarization independent frequency conversion by fiber four-wave mixing with a polarization diversity technique", IEEE Photon. Technol. Lett., vol. 5, no. 8, pp. 947-949, 1993.

[Hed99] P. O. Hedekvist, P. A. Andrekson, "Noise characteristics of fiber-based optical phase conjugators", J . Lightwave Technol., vol. 17, no. 1, pp. 7479, 1999.

[Hil78] K. O. Hill, D. C. J ohnson, B. S. Kawasaki, I. R. MacDonald, "CW threewave mixing in single-mode optical fibers", J . Appl. Phys., vol. 49, pp. 5098-5106, 1978.

[Hil94] K. O. Hill, F. Bilodeau, B. Malo, T. Kitagawa, S. Theriault, D. C. J ohnson, J . Albert, "Chirped in-fiber Bragg gratings for compensation of optical-fiber dispersion", Opt. Lett., vol. 19, pp. 1314-1316, 1994.

[Hon97] W. Honcharenko, J. P. Kruys, D. Y. Lee, N. J. Shah, "Broadband wireless access", IEEE Commun. Magazine, pp. 20-26, 1997.

[Ian94] P. P. Iannone, A. H. Gnauck, P. R. Prucnal, "Dispersion-compensated $333 \mathrm{~km} 10 \mathrm{~Gb} / \mathrm{s}$ transmission using mid-span spectral inversion in an injection-locked I nGaAsP V-Groove laser", IEEE Photon. Technol. Lett., vol. 6, no. 8, pp. 1046-1049, 1994.

[Ian96] E. Iannone, R. Sabella, L. de Stefano, F. Valeri, "All-optical wavelength conversion in optical multicarrier networks", IEEE Trans. Commun., vol. 44, no. 6, pp. 716-724, 1996.

[lbr93] H. Ibrahim, J. F. Bayon, A. Madani, J. Moalic, L. Rivoallan, D. Ronarch, E. le Coquil, "Fibre-equaliser second order distortion compensation in 1.55 um lightwave CATV transmission system", Electron. Lett., vol. 29, no. 3, pp. 315-317, 1993.

[Ih90] C. S. Ih, W. Gu, "Fibre induced distortions in a subcarrier multiplexed lightwave system", IEEE J. Sel. Areas Commun., vol. 8, no. 7, pp. 12961303, 1990.

[Ino92] K. Inoue, "Four-wave mixing in an optical fiber in the zero-dispersion wavelength region", J. Lightwave Technol., vol. 10, no. 11, pp. 15531561, 1992.

[I no92a] K. Inoue, H. Toba, "Wavelength conversion experiment using fiber fourwave mixing", IEEE Photon. Technol. Lett., vol. 4, no. 1, pp. 69-72, 1992.

[Ino94] K. Inoue, "Polarization independent wavelength conversion using fiber four-wave mixing with two orthogonal pump lights of different frequencies", J. Lightwave Technol., vol. 12, no. 11, pp. 1916-1920, 1994. 
[Ino94a] K. Inoue, "Tunable and selective wavelength conversion using fiber four-wave mixing with two pump lights", IEEE Photon. Technol. Lett., vol. 6, no. 12, pp. 1451-1453, 1994.

[Ino95] K. Inoue, T. Hasegawa, H. Toba, "Influence of stimulated Brillouin scattering and optimum length in fiber four-wave mixing wavelength conversion", IEEE Photon. Technol. Lett., vol. 7, no. 3, pp. 327-329, 1995.

[Ino97] K. Inoue, "Spectral inversion with no wavelength shift based on fourwave mixing with orthogonal pump beams", Opt. Lett., vol. 22, no. 23, pp. 1772-1774, 1997.

[Ipp72] E. P. Ippen, R. H. Stolen, "Stimulated Brillouin scattering in optical fiber", Appl. Phys. Lett., vol. 21, pp. 539-541, 1972.

[Izu81] M. Izutsu, S. Shikama, T. Sueta, "Integrated optical SSB modulator/frequency shifter", IEEE J. Quantum Electron., vol. 17, pp. 2225-2227, 1981.

[J e098] J . J eong, Y. K. Park, S. K. Kim, T. V. Nguyen, O. Mizuhara, T.-W. Oh, "10-Gb/s transmission performance for positive- and negative-chirped transmitters with the self-phase modulation effect", IEEE Photon. Technol. Lett., vol. 10, no. 9, pp. 1307-1309, 1998.

[J op93] R. M. J opson, R. E. Tench, "Polarization-independent phase conjugation of lightwave signals", Electron. Lett., vol. 29, no. 25, pp. 2216-2217, 1993.

[Kah61] L. R. Kahn, "Compatible single sideband", Proc. IRE, vol. 49, pp. 15031527, 1961.

[Kar95] M. Karlsson, "Modulational instability in lossy optical fibers", J. Opt. Soc. Am. B, vol. 12, no. 11, pp. 2071-2077, 1995.

[Kes87] M. P. Kesler, E. P. I ppen, "Subpicosecond spectral gain dynamics in AIGaAs laser diodes", Electron. Lett., vol. 24, pp. 1102-1103, 1987.

[Kho99] R. Khosravani, S. Lee, M. I. Hayee, H. Sun, A. E. Willner, "Soliton sampling for subcarrier multiplexed transmission to eliminate dispersion-induced RF power degradation", presented at OFC 99, pp. 341-343, 1999.

[Kin97] S. Kindt, K. Obermann, K. Petermann, "Characteristics of optical-phase conjugation by four-wave mixing in semiconductor-laser amplifiers", presented at ECOC 97, pp. 111-114, 1997.

[Kit99] K. Kitayama, H. Sotobayashi, "Fading-free fiber-optic transport of 60 GHz-optical DSB signal by using in-line phase conjugator", presented at OF C 99, pp. 64-66, 1999. 
[Koc84] T. L. Koch, J. E. Bowers, "Nature of wavelength chirping in directly modulated semiconductor lasers", Electron. Lett., vol. 20, pp. 10381039, 1984.

[Koc85] T. L. Koch, R. C. Alferness, "Dispersion compensation by active predistorted signal synthesis", J. Lightwave Technol., vol. 3, pp. 800805, 1985.

[Kol96] I. Koltchanov, S. Kindt, K. Petermann, S. Diez, R. Ludwig, R. Schnabel, H. G. Weber, "Gain dispersion and saturation effects in four-wave mixing in semiconductor laser amplifiers", IEEE J . Quantum Electron., vol. 32, no. 4, pp. 712-720, 1996.

[Koy88] F. Koyama, K. Iga, "Frequency chirping in external modulators", J . Lightwave Technol., vol. 6, no. 1, pp. 87-93, 1988.

[Ku091] C. Y. Kuo, E. E. Bergmann, "E rbium-doped fiber amplifier second-order distortion in analog links and electronic compensation", IEEE Photon. Technol. Lett., vol. 3, pp. 829, 1991.

[Kuo92] C. Y. Kuo, E. E. Bergmann, "Second-order distortion and electronic compensation in analog links containing fiber amplifiers", J . Lightwave Technol., vol. 10, no. 11, pp. 1751-1759, 1992.

[Ku092a] C. Y. Kuo, "Fundamental second order nonlinear distortions in analog AM CATV transport systems based on single frequency semiconductor lasers", J . Lightwave Technol., vol. 10, pp. 235, 1992.

[Kur93] C. Kurtzke, A. Gnauck, "H ow to increase capacity beyond 200 Tbit/s km without solitons", presented at ECOC 93, pp. paper ThC 12.12, 1993.

[Leu98] J. Leuthold, F. Girardin, P. A. Besse, E. Gamper, G. Guekos, H. Melchior, "Polarization independent optical phase conjugation with pump-signal filtering in a monolithically integrated Mach-Zehnder interferometer semiconductor optical amplifier configuration", IEEE Photon. Technol. Lett., vol. 10, no. 11, pp. 1569-1571, 1998.

[Lim99] H. C. Lim, F. Futami, K. Taira, K. Kikuchi, "Broad-band mid-span spectral inversion without wavelength shift of 1.7-ps optical pulses using a highly nonlinear fiber Sagnac interferometer", IEEE Photon. Technol. Lett., vol. 11, no. 11, pp. 1405-1407, 1999.

[Lim99a] H. C. Lim, F. Futami, K. Kikuchi, "Polarization-independent, wavelength-shift-free optical phase conjugator using a nonlinear fiber Sagnac interferometer", IEEE Photon. Technol. Lett., vol. 11, pp. 578580, 1999.

[Lin82] C. Lin, W. A. Reed, A. D. Pearson, H.-T. Shang, "Designing single-mode fibers for near-IR (1.1 um-1.7 um) frequency generation by phasematched four-wave mixing in the minimum chromatic dispersion 
region", Electron. Lett., vol. 18, pp. 87-88, 1982.

[Lin98] L. Y. Lin, J. M. Wiesenfeld, J. S. Perino, A. H. Gnauck, "Polarizationinsensitive wavelength conversion up to $10 \mathrm{~Gb} / \mathrm{s}$ based on four-wave mixing in a semiconductor optical amplifier", IEEE Photon. Technol. Lett., vol. 10, no. 7, pp. 955-957, 1998.

[Loa99] A. Loayssa, E. Galbete, D. Benito, M. J. Garde, "Carrier suppression compensation by SBS-induced phase shift", presented at ECOC 99, pp. 416-417, 1999.

[Loh96] W. H. Loh, R. I. Laming, N. Robinson, A. Cavaciuti, F. Vaninetti, C. Anderson, M. N. Zervas, M. Cole, "Dispersion compensation over distances in excess of $500 \mathrm{~km}$ for $10 \mathrm{~Gb} / \mathrm{s}$ systems using chirped fiber gratings", IEEE Photon. Technol. Lett., vol. 8, no. 7, pp. 944-946, 1996.

[Mao92] X. P. Mao, G. E. Bodeep, R. W. Tkach, A. R. Chraplyvy, T. E. Darcie, R. M. Derosier, "Brillouin scattering in externally modulated lightwave AM-VSB CATV transmission systems", IEEE Photon. Technol. Lett., vol. 4, no. 3, pp. 287-289, 1992.

[Mar91] D. Marcuse, A. R. Chraplyvy, R. W. Tkach, "Effect of fiber nonlinearity on long-distance transmission", J . Lightwave Technol., vol. 9, no. 1, pp. 121-128, 1991.

[Mar96] J. Marti, A. Montero, J. Capmany, J . M. Fuster, D. Pastor, "Reduction of dispersion-induced intensity noise in subcarrier systems by using tapered linearly chirped gratings", Electron. Lett., vol. 32, no. 17, pp. 1605-1606, 1996.

[Mar97] J. Marti, F. Ramos, "Compensation for dispersion-induced nonlinear distortion in subcarrier systems using optical-phase conjugation", Electron. Lett., vol. 33, no. 9, pp. 792-794, 1997.

[Mar97a] J. Marti, J. M. Fuster, R. I. Laming, "Experimental reduction of chromatic dispersion effects in lightwave microwave/millimetre-wave transmissions using tapered linearly chirped fibre gratings", Electron. Lett., vol. 33, no. 13, pp. 1170-1171, 1997.

[Mar97b] J . Marti, D. Pastor, M. Tortola, J . Capmany, A. Montero, "On the use of tapered linearly chirped gratings as dispersion-induced distortion equalizers in SCM systems", J. Lightwave Technol., vol. 15, no. 2, pp. 179-187, 1997.

[Mar97c] D. D. Marcenac, D. Nesset, A. E. Kelly, M. Brierley, A. D. Ellis, D. G. Moodie, C. W. Ford, "40 Gbit/s transmission over $406 \mathrm{~km}$ of NDSF using mid-span spectral inversion by four-wave-mixing in a $2 \mathrm{~mm}$ long semiconductor optical amplifier", Electron. Lett., vol. 33, no. 10, pp. 879-880, 1997. 
[Mar99] J . Marti, F. Ramos, V. Polo, J . M. Fuster, J . L. Corral, "Millimetre-wave generation and harmonic upconversion through PM-IM conversion in chirped fibre gratings", Electron. Lett., vol. 35, no. 15, pp. 1265-1266, 1999.

[Mar99a] J . Marti, F. Ramos, V. Polo, J . M. Fuster, J . L. Corral, "Millimetre-wave signal generation and harmonic upconversion through PM-IM conversion in chirped fibre gratings", presented at MWP 99, pp. 181184, 1999.

[Mec95] A. Mecozzi, S. Scotti, A. D'Ottavi, E. Iannone, P. Spano, "Four-wave mixing in travelling-wave semiconductor amplifiers", IEEE J . Quantum Electron., vol. 31, no. 4, pp. 689-699, 1995.

[Mec97] A. Mecozzi, J. Mork, "Saturation effects in nondegenerate four-wave mixing between short optical pulses in semi conductor laser amplifiers", IEEE J . Select. Topics Quantum Electron., vol. 3, pp. 1190-1207, 1997.

[Mec98] A. Mecozzi, G. Contestabile, F. Martelli, L. Graziani, A. D'Ottavi, P. Spano, R. Dall'Ara, J . Eckner, F. Girardin, G. Guekos, "Optical spectral inversion without frequency shift by four-wave mixing using two pumps with orthogonal polarization", IEEE Photon. Technol. Lett., vol. 10, no. 3, pp. 355-357, 1998.

[Mes84] G. J. Meslener, "Chromatic dispersion induced distortion of modulated monochromatic light employing direct detection", IEEE J. Quantum Electron., vol. 20, no. 10, pp. 1208-1216, 1984.

[Mil50] J . C. P. Miller, "On the choice of standard solutions for a homogeneous linear differential equation of the second order", Quart. J. Mech. Appl. Math., vol. 3, pp. 225-235, 1950.

[Min98] N. Mineo, K. Yamada, K. Nakamura, S. Sakai, T. Ushikubo, "60-GHz band electroabsorption modulator module", presented at OFC 98, pp. paper $\mathrm{ThH}$, 1998.

[Mit92] O. Mitomi, S. Nojima, I. Kotaka, K. Wakita, K. Kawano, M. Naganuma, "Chirping characteristic and frequency response of MQW optical intensity modulator", J. Lightwave Technol., vol. 10, no. 1, pp. 71-77, 1992.

[Mit94] O. Mitomi, K. Wakita, I. Kotaka, "Chirping characteristic of electroabsorption-type optical-intensity modulator", IEEE Photon. Technol. Lett., vol. 6, no. 2, pp. 205-207, 1994.

[Miy91] Y. Miyajima, T. Sugawa, Y. Fukasaku, "38.2 dB amplification at 1.31 um and the possibility of 0.98 um pumping in PR+3-doped fluoride fiber", presented at topical Meet. Opt. Ampl. Their Applic, pp. paper PdP1, 1991. 
[Moo95] D. G. Moodie, D. Wake, N. G. Walker, D. Nesset, "Efficient harmonic generation using an electroabsorption modulator", IEEE Photon. Technol. Lett., vol. 7, pp. 312-314, 1995.

[Mor96] K. Mori, T. Morioka, M. Saruwatari, "Wavelength-shift-free spectral inversion with an optical parametric loop mirror", Opt. Lett., vol. 21, pp. 110-112, 1996.

[Mor97] J. Mork, A. Mecozzi, "Theory of nondegenerate four-wave mixing between pulses in a semiconductor waveguide", IEEE J. Quantum Electron., vol. 33, pp. 545-555, 1997.

[Mor98] T. J . Morgan, J. P. R. Lacey, R. S. Tucker, "Widely tunable four-wave mixing in semiconductor optical amplifiers with constant conversion efficiency", IEEE Photon. Technol. Lett., vol. 10, no. 10, pp. 1401-1403, 1998.

[Mor98a] T. J. Morgan, R. S. Tucker, G. Yoffe, "Passive optical equalization of wavelength converters based on four-wave mixing in semiconductor optical amplifiers", IEEE Photon. Technol. Lett., vol. 10, no. 4, pp. 522524, 1998.

[Muk90] T. Mukai, T. Saitoh, "Detuning characteristics and conversion efficiency of nearly degenerate four-wave mixing in a $1.5 \mathrm{~mm}$ travelling-wave semiconductor amplifier", IEEE J . Quantum Electron., vol. 26, pp. 865875, 1990.

[Noe97] L. Noel, D. Wake, D. G. Moodie, D. D. Marcenac, L. D. Westbrook, D. Nesset, "Novel techniques for high-capacity $60-\mathrm{GHz}$ fiber-radio transmission systems", IEEE Trans. Microwave Theory Tech., vol. 45, no. 8, pp. 1416-1423, 1997.

[Oga91] H. Ogawa, Y. Kamiya, "Fibre-optic microwave transmission using harmonic laser mixing, optoelectronic mixing, and optically pumped mixing", IEEE Trans. Microwave Theory Tech., vol. 39, pp. 2045-2051, 1991.

[Ols87] R. Olshansky, "RF multiplexing techniques applied to video distribution in local networks", presented at ECOC 87, pp. 122, 1987.

[Ols89] R. Olshansky, V. A. Lanzisera, P. M. Hill, "Subcarrier multiplexed lightwave systems for broadband distribution", J . Lightwave Technol., vol. 7, no. 9, pp. 1329-1341, 1989.

[Ols94] R. Olshansky, "Single sideband optical modulator for lightwave systems", U.S. Patent 5301 058, 1994.

[Oue87] F. Ouellette, "Dispersion cancellation using linearly chirped Bragg grating filters in optical waveguides", Opt. Lett., vol. 12, no. 10, pp. 847-849, 1987. 
[Pap97] P. B. Papazian, G. A. Hufford, R. J . Achatz, R. Hoffman, "Study of the local multipoint distribution service radio channel", IEEE Trans. Broadcasting, vol. 43, no. 2, pp. 175-184, 1997.

[Par96] J. Park, A. F. Elrefaie, K. Y. Lau, "Fiber chromatic dispersion effects on multichannel digital millimeter-wave transmission", IEEE Photon. Technol. Lett., vol. 8, no. 12, pp. 1716-1718, 1996.

[Par97] J. Park, W. V. Sorin, K. Y. Lau, "Elimination of the fibre chromatic dispersion penalty on $1550 \mathrm{~nm}$ millimetre-wave optical transmission", Electron. Lett., vol. 33, no. 6, pp. 512-513, 1997.

[Par97a] J. Park, A. F. Elrefaie, K. Y. Lau, "1550-nm transmission of digitally modulated $28 \mathrm{GHz}$ subcarriers over $77 \mathrm{~km}$ of nondispersion shifted fiber", IEEE Photon. Technol. Lett., vol. 9, no. 2, pp. 256-258, 1997.

[Pas97] D. Pastor, J. Capmany, J. Marti, "Reduction of dispersion induced composite triple beat and second-order intermodulation in subcarrier multiplexed systems using fiber grating equalizers", IEEE Photon. Technol. Lett., vol. 9, no. 9, pp. 1280-1282, 1997.

[Pat94] D. M. Patrick, R. J. Manning, "20 Gbit/s wavelength conversion using semiconductor nonlinearity", Electron. Lett., vol. 30, no. 3, pp. 252-253, 1994.

[Pep80] D. M. Pepper, A. Yariv, "Compensation for phase distortions in nonlinear media by phase conjugation", Opt. Lett., vol. 5, pp. 59-60, 1980.

[Pet90] K. Petermann, "FM-AM noise conversion in dispersive single-mode fibre transmission lines", Electron. Lett., vol. 26, no. 25, pp. 2097-2098, 1990.

[Pet91] K. Petermann, "Laser diode modulation and noise", Kluwer Academic Publishers, 1991.

[Phi91] M. R. Phillips, T. E. Darcie, D. Marcuse, G. E. Bodeep, N. J. Frigo, "Nonlinear distortion generated by dispersive transmission of chirped intensity-modulated signals", IEEE Photon. Technol. Lett., vol. 3, no. 5, pp. 481-483, 1991.

[Phi99] M. R. Phillips, D. M. Ott, "Crosstalk due to optical fiber nonlinearities in WDM CATV lightwave systems", J . Lightwave Technol., vol. 17, no. 10, pp. 1782-1792, 1999.

[Pol98] V. Polo, J. Marti, F. Ramos, J. M. Fuster, "Millimetre-wave optical harmonic mixer employing a single Mach-Zehnder electro-optic modulator", presented at EuMC'98, paper MD-4, 1998.

[Pol99] V. Polo, J. Marti, F. Ramos, D. Moodie, "Mitigation of chromatic 
dispersion effects employing electroabsorption modulator-based transmitters", IEEE Photon. Technol. Lett., vol. 11, no. 7, pp. 883-885, 1999.

[Pol99a] V. Polo, F. Ramos, J . Marti, J . M. Fuster, "Demonstration of dispersiontolerant $34 \mathrm{M}$ bit/s data transmission in electro-optically upconverted 28 GHz LMDS fiber-optic link", presented at IEEE MTT-S, paper WE 4B-2, 1999.

[Ral94] J. Ralston, S. Weisser, K. Eisele, R. Sah, E. Larkins, J . Rosenzweig, J . Fleissner, K. Bender, "Low-bias-current direct modulation up to $33 \mathrm{GHz}$ in InGaAs/GaAs/AIGaAs pseudomorphic MQW ridge-waveguide devices", IEEE Photon. Technol. Lett., vol. 6, pp. 1076-1079, 1994.

[Ram00] F. Ramos, J. Marti, "Frequency transfer function of dispersive and nonlinear single-mode optical fibers in microwave optical systems", IEEE Photon. Technol. Lett., vol. 12, no. 5, 2000.

[Ram98] F. Ramos, J. Marti, V. Polo, J . M. Fuster, "On the use of fiber-induced self-phase modulation to reduce chromatic dispersion effects in microwave/millimeter-wave optical systems", IEEE Photon. Technol. Lett., vol. 10, no. 10, pp. 1473-1475, 1998.

[Ram98a] F. Ramos, J . Marti, "Compensation for fiber-induced composite secondorder distortion in externally modulated lightwave AM-SCM systems using optical-phase conjugation", J . Lightwave Technol., vol. 16, no. 8, pp. 1387-1392, 1998.

[Ram99] F. Ramos, J. Marti, V. Polo, "Compensation of chromatic dispersion effects in microwave/millimeter-wave optical systems using four-wavemixing induced in dispersion-shifted fibers", IEEE Photon. Technol. Lett., vol. 11, no. 9, pp. 1171-1173, 1999.

[Ram99a] F. Ramos, J. Marti, "Comparison of optical single-sideband modulation and chirped fiber gratings as dispersion mitigating techniques in optical millimeter-wave multichannel systems", IEEE Photon. Technol. Lett., vol. 11, no. 11, pp. 1479-1481, 1999.

[Rei92] J.J. O'Reilly, P. M. Lane, R. Heidemann, R. Hofstetter, "Optical generation of very narrow linewidth millimetre-wave signals", Electron. Lett., vol. 28, pp. 2309-2311, 1992.

[Ric95] D. J . Richardson, R. P. Chamberlin, L. Dong, D. N. Pane, "High quality soliton loss-compensation in $38 \mathrm{~km}$ dispersion-decreasing fibre", Electron. Lett., vol. 31, no. 9, pp. 1681-1682, 1995.

[Roy94] A. Royset, L. Bjerkan, D. Myhre, L. Hafskjaer, "Use of dispersive optical fibre for characterisation of chirp in semiconductor lasers", Electron. Lett., vol. 30, no. 9, pp. 710-712, 1994. 
[Sab95] R. Sabella, E. Iannone, E. Pagano, "Effect of phase noise in compensation of distortion in long-haul optical transmission by optical phase conjugation", presented at EFOC\&N 95, pp. 217-220, 1995.

[Sco97] S. Scotti, L. Graziani, A. D'Ottavi, F. Martelli, A. Mecozzi, P. Spano, R. Dall'Ara, F. Girardin, G. Guekos, "Frequency conversion realized by FWM in SOAs: performance dependence on device-length and pumpwavelength", COST 240 Workshop, Prague, 1997.

[Sch94] M. Schiess, H. Carldén, "Evaluation of the chirp parameter of a MachZehnder intensity modulator", Electron. Lett., vol. 30, no. 18, pp. 15241525, 1994.

[Sch94a] H. Schmuck, R. Hofstetter, R. Heidemann, "Advanced fibre-optic distribution of $140 \mathrm{Mbit} / \mathrm{s} \mathrm{mm}$-wave signals at $36 \mathrm{GHz}$, presented at ECOC 94, 1994.

[Sch95] H. Schmuck, "Comparison of optical millimetre-wave system concepts with regard to chromatic dispersion", Electron. Lett., vol. 31, no. 21, pp. 1848-1849, 1995.

[Sch96] H. Schmuck, R. Heidemann, "Hybrid fibre-radio field experiment at 60 GHz", presented at ECOC 96, 1996.

[Sch97] H. Schmuck, "Carrier-to-noise limitations in optical mm-wave links due to phase-induced-intensity-noise", Electron. Lett., vol. 33, no. 14, pp. 1236-1237, 1997.

[Set98] S. Y. Set, S. Yamashita, M. I bsen, R. I. Laming, D. Nesset, A. E. Kelly, C. Gilbertas, "Ultra-high bit rate optical phase conjugation/wavelength conversion in DSF and SOA with novel configuration incorporating inline fibre DFB lasers", Electron. Lett., vol. 34, no. 17, pp. 1681-1683, 1998.

[Sha91] N. K. Shankaranarayanan, S. D. Elby, K. Y. Lau, "WDMA/SubcarrierFDMA lightwave networks: limitations due to optical beat interference", J . Lightwave Technol., vol. 9, no. 7, pp. 931-943, 1991.

[Shi87] N. Shibata, R. P. Braun, R. Waarts, "Phase-mismatch dependence of efficiency of wave generation through four-wave mixing in a singlemode optical fiber", IEEE J . Quantum Electron., vol. 23, no. 7, pp. 12051210, 1987.

[Shi90] N. Shibata, K. Nosu, K. I washita, Y. Azuma, "Transmission limitation due to four-wave mixing in single-mode optical fibers", J . Select. Areas Commun., vol. 8, pp. 1068-1077, 1990.

[Shi96] W. Shieh, E. Park, A. E. Willner, "All-optical wavelength shifting of microwave subcarriers by using four-wave mixing in a semiconductor optical amplifier", IEEE Photon. Technol. Lett., vol. 8, no. 4, pp. 524- 
526, 1996.

[Smi97] G. H. Smith, D. Novak, Z. Ahmed, "Overcoming chromatic-dispersion effects in fiber-wireless systems incorporating external modulators", IEEE Trans. Microwave Theory Tech., vol. 45, no. 8, pp. 1410-1415, 1997.

[Smi97a] G. H. Smith, D. Novak, Z. Ahmed, "Technique for optical SSB generation to overcome dispersion penlties in fibre-radio systems", Electron. Lett., vol. 33, no. 1, pp. 74-75, 1997.

[Smi97b] G. H. Smith, D. Novak, C. Lim, K. Wu, "Full-duplex broadband millimetre-wave optical transport system for fibre-wireless access", Electron. Lett., vol. 33, no. 13, pp. 1159-1160, 1997.

[Smi98] G. H. Smith, D. Novak, C. Lim, "A millimeter-wave full-duplex fiberradio star-tree architecture incorporting WDM and SCM", IEEE Photon. Technol. Lett., vol. 10, no. 11, pp. 1650-1652, 1998.

[Sot99] H. Sotobayashi, K. Kitayama, "Effects of asymmetric power change on BER performance using midway optical phase conjugation for fading cancellation in $60 \mathrm{GHz}$ millimetre-wave optical DSB signal transmission over $100 \mathrm{~km}$ non-dispersion-shifted fibre", Electron. Lett., vol. 35, no. 12, pp. 992-993, 1999.

[Sot99a] H. Sotobayashi, K. Kitayama, "Cancellation of the signal fading for 60 $\mathrm{GHz}$ subcarrier multiplexed optical DSB signal transmission in nondispersion shifted fiber using midway optical phase conjugation", J . Lightwave Technol., vol. 17, no. 12, pp. 2488-2497, 1999.

[Sri95] R. C. Srinivasan, J . C. Cartledge, "On using fiber transfer functions to characterize laser chirp and fiber dispersion", IEEE Photon. Technol. Lett., vol. 7, no. 11, pp. 1327-1329, 1995.

[Ste90] M. Stern, J. P. Heritage, R. N. Thurston, S. Tu, "Self-phase modulation and dispersion in high data rate fiber-optic transmission systems", J . Lightwave Technol., vol. 8, no. 7, pp. 1009-1016, 1990.

[Sum96] M. A. Summerfield, R. S. Tucker, "Optimization of pump and signal powers for wavelength converters based on FWM in semiconductor optical amplifiers", IEEE Photon. Technol. Lett., vol. 8, no. 10, pp. 13161318, 1996.

[Sun95] C. Sun, S. Pappert, R. Welstand, T. Zhu, P. Yu, Y. Liu, J . Chen, "High spurious-free dynamic range fiber link using a semiconductor el ectroabsorption modulator", Electron. Lett., vol. 31, pp. 902-903, 1995.

[Suz92] M. Suzuki, H. Tanaka, Y. Matsushita, "InGaAsP electroabsorption modulator for high-bit-rate EDFA system", IEEE Photon. Technol. Lett., vol. 4, no. 6, pp. 586-588, 1992. 
[Tan98] J. M. Tang, K. A. Shore, "Influence of probe depletion and cross-gain modulation on four-wave mixing of picosecond optical pulses in semiconductor optical amplifiers", IEEE Photon. Technol. Lett., vol. 10, no. 11, pp. 1563-1565, 1998.

[Tho95] J. L. Thomas, "Cable television proof-of-performance", New J ersey: Prentice Hall, 1995.

[Tsu98] K. Tsujikawa, K. Nakajima, Y. Miyajima, M. Ohashi, "New SBS suppression fiber with uniform chromatic dispersion to enhance fourwave mixing", IEEE Photon. Technol. Lett., vol. 10, no. 8, pp. 11391141, 1998.

[Ven93] A. M. Vengsarkar, W. A. Reed, "Dispersion-compensating single-mode fibers: Efficient designs for first- and second-order compensation", Opt. Lett., vol. 18, pp. 924-926, 1993.

[Wab95] S. Wabnitz, "Nonlinear enhancement and optimization of phaseconjugation efficiency in optical fibers", IEEE Photon. Technol. Lett., vol. 7, no. 6, pp. 652-654, 1995.

[Wak92] D. Wake, I. C. Smith, N. G. Walker, I. D. Henning, R. D. Carver, "Video transmission over a $40 \mathrm{GHz}$ radio-fibre link", Electron. Lett., vol. 28, no. 21, pp. 2024-2025, 1992.

[Wak93] D. Wake, L. D. Westbrook, N. G. Walker, I. C. Smith, "Microwave and millimetre-wave radio fibre", BT Technol. J ., vol. 11, no. 2, pp. 76-88, 1993.

[Wal92] N. G. Walker, D. Wake, I. C. Smith, "Efficient millimetre-wave signal generation through FM-IM conversion in dispersive optical fibre links", Electron. Lett., vol. 28, pp. 2027-2028, 1992.

[Wan92] J. Wang, K. Petermann, "Small signal analysis for dispersive optical fiber communication systems", J . Lightwave Technol., vol. 10, no. 1, pp. 96-100, 1992.

[Wan95] Z. Wang, A. Li, C. J. Mahon, G. J acobsen, E. Bodtker, "Performance limitations imposed by stimulated Raman scattering in optical WDMSCM video distribution systems", IEEE Photon. Technol. Lett., vol. 7, no. 12, pp. 1492-1494, 1995.

[Wat93] S. Watanabe, T. Chikama, G. Ishikawa, T. Terahara, H. Kuwahara, "Compensation of pulse shape distortion due to chromatic dispersion and Kerr effect by optical phase conjugation", IEEE Photon. Technol. Lett., vol. 5, no. 10, pp. 1241-1243, 1993.

[Wat93a] S. Watanabe, T. Naito, T, Chikama, "Compensation of chromatic dispersion in a single-mode fiber by optical phase conjugation", IEEE Photon. Technol. Lett., vol. 5, no. 1, pp. 92-95, 1993. 
[Wat94] S. Watanabe, G. Ishikawa, T. Naito, T. Chikama, "Generation of optical phase-conjugate waves and compensation for pulse shape distortion in a single-mode fiber", J . Lightwave Technol., vol. 12, no. 12, pp. 21392146, 1994.

[Wat94a] S. Watanabe, T. Chikama, "Highly efficient conversion and parametric gain of nondegenerate forward four-wave mixing in a single-mode fiber", Electron. Lett., vol. 30, no. 2, pp. 163-164, 1994.

[Wat96] S. Watanabe, M. Shirasaki, "Exact compensation for both chromatic dispersion and Kerr effect in a transmission fiber using optical phase conjugation", J . Lightwave Technol., vol. 14, no. 3, pp. 243-248, 1996.

[Way99] W. I. Way, "Broadband hybrid fiber/coax access system technologies", Academic Press, San Diego, 1999.

[Wed94] B. Wedding, "Analysis of fibre transfer function and determination of receiver frequency response for dispersion supported transmission", Electron. Lett., vol. 30, no. 1, pp. 58-59, 1994.

[Wei86] J. A. C. Weideman, B. M. Herbst, "Split-step method for the solution of the nonlinear Schrodinger equation", SI AM J. Numer. Anal., vol. 23, pp. 458-507, 1986.

[Wel95] R. Welstan, C. Sun, S. Pappert, Y. Liu, J. Chen, J. Zhu, A. Kellner, P. $\mathrm{Yu}$, "Enhanced dynamic range property of Franz-Keldysh effect waveguide modulator", IEEE Photon. Technol. Lett., vol. 7, pp. 751-753, 1995.

[Wil94] F. W. Willems, W. Muys, J. S. Leong, "Simultaneous suppression of stimulated Brillouin scattering and interferometric noise in externally modulated lightwave CATV systems", IEEE Photon. Technol. Lett., vol. 6, no. 12, pp. 1476-1478, 1994.

[Wil95] B. Wilson, Z. Ghassemlooy, I. Darwazeh, "Analogue optical fibre communications", IEE, London (UK), 1995.

[Wil96] F. W. Willems, W. Muys, J . C. van der Plaats, R. Nuyts, "Experimental verification of self-phase-modulation induced nonlinear distortion in externally modulated AM-VSB lightwave systems", Electron. Lett., vol. 32, no. 14, pp. 1310-1311, 1996.

[Wu94] W. Wu, P. Yeh, S. Chi, "Phase conjugation by four-wave mixing in single-mode fibers", IEEE Photon. Technol. Lett., vol. 6, no. 12, pp. 1448-1450, 1994.

[Yam90] S. Yamamoto, N. Edagawa, H. Taga, Y. Yoshida, H. Wakabayashi, "Analysis of laser phase noise to intensity noise conversion by chromatic dispersion in intensity modulation and direct detection optical-fiber transmission", J. Lightwave Technol., vol. 8, no. 11, pp. 
1716-1722, 1990.

[Yam97] T. Yamamoto, M. Nakazawa, "Highly efficient four-wave mixing in an optical fiber with intensity dependent phase matching", IEEE Photon. Technol. Lett., vol. 9, no. 3, pp. 327-329, 1997.

[Yam98] S. Yamashita, S. Y. Set, R. I. Laming, "Polarization independent, allfiber phase conjugation incorporating inline fiber DFB lasers", IEEE Photon. Technol. Lett., vol. 10, no. 10, pp. 1407-1409, 1998.

[Yao98] X. S. Yao, "Brillouin selective sideband amplification of microwave photonic signals", IEEE Photon. Technol. Lett., vol. 10, no. 1, pp. 138140, 1998.

[Yao98a] X. S. Yao, "Phase-to-amplitude modulation conversion using Brillouin selective sideband amplification", IEEE Photon. Technol. Lett., vol. 10, no. 2, pp. 264-266, 1998.

[Yar79] A. Yariv, D. Fekete, D. M. Pepper, "Compensation for channel dispersion by nonlinear optical phase conjugation", Opt. Lett., vol. 4, no. 2, pp. 52-54, 1979.

[Yon91] H. Yonetani, T. Watanabe, K. Kamite, T. Takada, K. Shima, "Analysis of dispersion induced distortion in multichannel analog transmission", presented at ECOC 91, pp. 577-580, 1991.

[Yos93] H. Yoshinaga, "Influence of stimulated Brillouin scattering on nonlinear distortion in SCM video transmission", Electron. Lett., vol. 29, pp. 1707-1708, 1993.

[Zak72] V. E. Zakharov, A. B. Shabat, "Exact theory of two-dimensional selffocusing and one-dimensional self-modulation of waves in nonlinear media", Sov. Phys. J ETP, vol. 34, no. 1, pp. 62-69, 1972.

[Zha95] X. Zhang, F. Ebskamp, B. F. J orgensen, "Long-distance transmission over standard fiber by use of mid-way phase conjugation", IEEE Photon. Technol. Lett., vol. 7, no. 7, pp. 819-821, 1995.

[Zha96] X. Zhang, B. F. J orgensen, "Analysis of optical phase conjugation using four-wave mixing in a dispersion-shifted fibre", IEE Proc.Optoelectron., vol. 143, no. 3, pp. 195-199, 1996.

[Zha97] S. L. Zhang, J. J. O'Reilly, "Effect of dynamic stimulated Brillouin scattering on millimeter-wave fiber radio communication systems", IEEE Photon. Technol. Lett., vol. 9, no. 3, pp. 395-397, 1997.

[Zho94] J. Zhou, N. Park, J. W. Dawson, K. J. Vahala, M. A. Newkirk, B. I. Miller, "Efficiency of broadband four-wave mixing wavelength conversion using semiconductor traveling-wave amplifiers", IEEE Photon. Technol. Lett., vol. 6, no. 1, pp. 50-52, 1994. 
[Zys96] G. Zysman, R. Thorkildsen, D. Lee, "Two-way broadband access", BT Technol. J., vol. 1, no. 1, pp. 115-129, 1996. 